\title{
dUTlungs of
}

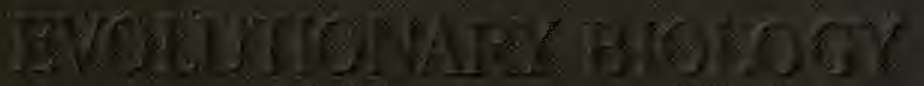

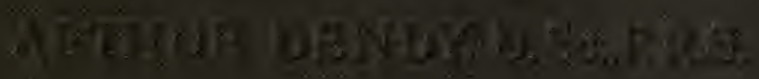


$12 / 6 \mathrm{~m}$

1446

$57 x$

a 1101

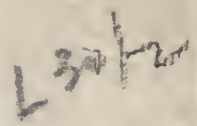




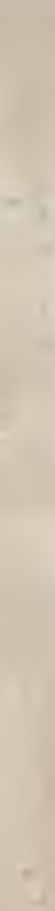







\section{OUTLINES OF}

\section{EVOLUTIONARY BIOLOGY}





\section{OUTLINES OF}

\section{EVOLUTIONARY BIOLOGY}

BY

AR'THUR DENDY, D.Sc., F.R.S.,

Professor of Zoology in the University of London (King's College); Zoological Secretary of the Linnean Society of London;

Honorary Member of the New Zealand Institute; formerly

Professor of Biology in the Canterbury College

(University of New Zealand), and Profesșor of

Zoology in the South.African College,

Cape Town.

\section{LONDON}

CONSTABI.E \& COMPANY I'TD

10 ()RANGE STREET LEICESTER SQUARE W.C. 



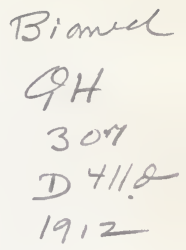

\section{PREFACE}

Biology, the fundamental science of living things in all their manifold relations, is a study which, at the present time, is but little encouraged by educational authorities in this country. It has no place in the ordinary school curriculum and even in our Universities it has been thrust into the background, partly because University authorities devote so much of their attention nowadays to subjects which are considered more likely to bring in a direct pecuniary reward to the student, and partly because of the immense elaboration of the various branches of biological science, such as Zoology, Botany, Physiology, Comparative Anatomy and Embryology, that has taken place in recent years, and the claims of these to more or less separate recognition.

The student, if he studies Biology at all for its own sake, which is seldom the case, usually confines himself almost entirely to one or other of these branches, which he finds treated more or less as an independent science, with an extensive literature of its own, and he runs a grave risk of losing sight of the general principles which underlie all and from which all derive their chief educational value.

The medical student, it is true, usually takes a year's course in what is called Biology, but his curriculum is, perhaps unavoidably, dominated by the type-system and by what is thought likely to be of direct service to him in his future anatomical and physiological studies, so that in the brief time which the medical authorities allow him to devote to the scientific foundation of his professional work he has but little opportunity for a philosophical treatment of the subject.

When we at length come to realize the meaning of Man's position as, for the time being, the highest term of a great evolutionary series which stretches far back into the dawn of 
the earth's history, and to appreciate the importance of the fact that he derives his existence from the same ultimate sources and is subject to the same natural laws as all the other living things with which he shares the earth, we shall perhaps see the necessity for making Biology, in the widest sense of the term, one of the foundation stones of our educational system.

In the meantime those who wish to familiarize themselves with the rapidly accumulating results of biological investigation and the bearing of these results upon human problems ought not to be debarred from so doing by want of the necessary knowledge of fundamental facts and principles, and it is largely with a view to the requirements of such students that the present work is offered to the public.

That even the elementary study of biological theory should, wherever possible, be preceded by a systematic course in Zoology and Botany, based upon the type-system and including laboratory work, is, no doubt, indisputable. Unfortunately, under existing conditions, regular laboratory work is, for most people, impossible. We are apt to forget, however, that in reality we all of us spend our lives in a biological laboratory, where we are surrounded by living organisms which we can hardly avoid studying. In this way we learn much of the nature of living things and are to some extent prepared for the study of biological principles.

The problems of life, however, cannot be satisfactorily solved if we confine our attention to the higher and more familiar forms of plants and animals. Man, in particular, is far too complex a type to begin with in a philosophical treatment of the subject. The logical method of study is, no doubt, to follow as closely as possible the course which we believe to have been taken in the actual evolution of living things, beginning with the simple and ending with the complex. This method, of course, is attended with certain practical difficulties, mainly due to the microscopic size of the more primitive organisms, but these difficulties are not insurmountable and need not be considered in relation to the present work.

As I wish this book to be of use to those who have had no special biological training, as well as to students who have taken the ordinary first year's course, I have, in the earlier chapters, dealt in a very elementary manner with the structure and functions of both plants and animals. I have described Amceba 
and Hrmatococcus in considerable detail and used these familiar organisms as pegs on which to hang some elementary ideas with regard to the nature of living things and the differences between animals and plants. Otherwise I have as far as possible avoided the type-system as being altogether unsuitable for a work of this kind, though of course I have been obliged to refer to numerous different organisms in illustration of special points.

It was only by rigidly excluding from the earlier part of the book everything that was not considered essential to the understanding of general principles that it has been possible to find space for even a brief presentation of the evidence upon which the theory of organic evolution rests, and for a discussion of the principal factors which appear to have co-operated in determining the course of that evolution.

Although the entire work is intended to be of an elementary character, it has been impossible, in connection with the theory of heredity, to avoid, on the one hand, a considerable amount of cytological detail, and, on the other, some discussion of theoretical speculations of a highly controversial nature. In dealing with these vexed questions, which underlie the whole problem of organic evolution, I have endeavoured to present the views of opposing schools of thought as fairly as possible, but I must confess that I have ventured to lay considerable stress upon ideas which, though widely accepted elsewhere, have not as yet met with much appreciation in this country, though that they will do so in the future can hardly be doubted.

By way of introduction to the discussion of the factors of organic evolution a chapter has been devoted to the views of Buffon, Erasmus Darwin and Lamarck, and another to those of Charles Darwin, Robert Chambers and Alfred Russel Wallace, and I have endeavoured to present the opinions of these classical authors as far as possible by means of quotations from their own writings.

In a work such as the present the employment of numerous technical terms is, of course, unavoidable, but it is hoped that the meaning of these is sufficiently explained in the text, and the use of the index should obviate any difficulties in this respect, especially if the book is read systematically from the first chapter on wards.

It is a pleasure to record my thanlis to many colleagues who have ungrudgingly helped me in various ways. Amongst these 
I should like especially to mention Professor Poulton, Professor Herbert Jackson, Dr. Stapf, Dr. Daydon Jackson, Dr. Sibly and Professor G. E. Nicholls; while to Mr. R. W. H. Row, of the Zoological Department at King's College, I am much indebted for the trouble which he has taken in reading through the proof-sheets and for many valuable suggestions.

I owe a sincere acknowledgment to my publishers, Messrs. Constable \& Co., for their generosity in the matter of illustrations. Most of these are either entirely new or, in some cases, specially re-drawn from original memoirs. I have made extensive use of photomicrography for microscopic subjects, and in the preparation of the photographs at King's College have received much assistance from Dr. Rosenheim, Mr. Row, and my assistant Mr. Charles Biddolph.

For the loan of the blocks from which the remainder of the illustrations have been printed, or for permission to copy, I am indebted to the generosity of the following :-

Messrs. George Allen and Son, "The American Journal of Science,"

Mr. Edward Arnold,

Messrs. George Bell and Sons,

Messrs. A. and C. Black,

The Cambridge University Press,

The Clarendon Press,

Messrs. Constable \& Co.,

Messrs. Duckworth \& Co.,

Herr W. Engelmann,

Herr Gustav Fischer,

“'The Journal of Experimental Zoology,'

Messrs. Kegan Paul, Trench, Trubner \& Co.,

The Council of the Linnean Society of London,

Messrs. Longmans, Green \& Co.,

Mr. John Murray,

"The Quarterly Journal of Microscopical Science,"

The Council of the Ray Society,

Messrs. Smith, Elder \& Co.,

The Trustees of the British Museum,

Messrs. T. Fisher Unwin,

Messrs. F. Warne \& Co.,

Verlag des Bibliographischen Instituts, Leipzig und Wien. 
I must also express my gratitude to the numerous authors whose work I have made use of, and whose names are mentioned in the appropriate places.

I am indebted to the Council of the Royal Society of Arts for permission to make use of the Aldred Lecture which I delivered before the Society in 1909, and which is to a large extent reprinted from their Journal in Chapter XIV.

\section{ARTHUR DENDY.}

Kixg's College, LoNdon, December, 1911. 


\section{CORRIGENDA.}

1. 35", line --for "her" real "it-."

". "9-jor "the" read "life."

" "10-for "her-elf" reanl - it-elf." 


\section{CONTENTS}

\section{PART I.-THE STRCCTUPE AND FUYCTIONS OF ORGANISMS-THE CELL THEORI}

\section{CH.IPTER I}

Introductory: The nature of life-The living organism viewed as a machine-The essential functions of the living body -The source of energy in living things

\section{CHAPTER II}

Amoeba as a typical organism-The properties of protoplasm .

\section{CHAPTER III}

Hrematococeus-The differences between animals aod plants . .

\section{CH.IPTER IV}

The cell theory-Cunicellular organisms-Differentiation and division of labour-Co-operation-The transition from the unicellular to the multicellular condition-The early derelopment of multicellular animals and plants .

\section{CH.APTER $\Gamma$}

The cell theory as illustrated by the histological structure of the higher animals and plants-Iimitations of the cell theory-The cell as the phrsiological unit . . . . . . . .

\section{CH.IPTER VI}

The multiplication of cells-Mitotic and amitotic nuclear division

\section{PART II.-THE ETOLUTION OF SEX}

\section{CHAPTER VII}

Limitation of the powers of cell-dirision-Rejureneacence by conjugation of gametes-The origin of sex in the Protista 


\section{CHAPTER VIII}

Sexual phenomena in multicellular plants-The distinction between somatic cells and germ cells-Alternation of sexual and asexual generations-Suppression of the gametophyte in flowering plants .

\section{CHAPTER IX}

Sexual phenomena in multicellular animals - Structure and life history of Hydra and Obelia-Alternation of generations-The coelomate type of structure-Secondary sexual characters-The evolution of sex

\section{CHAPTER $\mathrm{X}$}

Origin of the germ cells in multicellular animals-Maturation of the germ cells -Reduction of the chromosomes-Sex determination in insects-Different forms of gametes-Mutual attraction of the gametes-Fertilization and parthenogenesis .

\section{PART III.-VARIATION AND HEREDITY}

\section{CHAPTER XI}

Variation-Meristic and substantive variations-Fluctuations and mutations-Somatogenic and blastogenic variations-Origin of blastogenic variations

\section{CHAPTER XII}

Heredity-General observations-Darwin's theory of pangenesis and Weismann's theory of the continuity of the germ plasm-The nucleus as the bearer of inheritable characters

\section{CHAPTER XIII}

The inheritance of acquired characters and the mnemic theory of heredity .

\section{CHAPTER XIV}

The Mendelian experiments in hybridization-The doctrine of unit characters and the purity of the gametes-Galton's law of inheritance

\section{PART IV.-THE THEORY AND EVIDENCE OF ORGANIC EVOLUTION: ADAPTATION}

\section{CIIAPTER XV}

Organic evolution versus special creation-Spontaneous generation and biogenesis - The origin of living things . 


\section{CHAP'TER XVI}

The continuity of life-The conception of species-The principles of taxonomy-The taxonomic evidence of organic evolution

\section{CHAPTER XVII}

Connecting links-Homology and analogy - Convergent evolutionChange of function-Testigial structures-Reversion . . .

\section{CHAPTER XVIII}

Ontogeny - The recapitulation hypothesis-Interpretation of the ontogenetic record-Palingenetic and cænogenetic characters . .

\section{CHAPTER XIX}

The stratified rocks - Geological periods - The age of the habitable earth - The geological record-The succession of the great vertebrate groups .

\section{CHAPTER XX}

Fossil pedigrees-Ancestry of birds, horses, elephants and whales .

\section{CIIAPTER XXI}

Geographical distribution-Areas of distribution-Barriers to migration-Means of dispersal-Changes in the physical conditions of the earth's surface - The evidence afforded by the study of geographical distribution with regard to the theory of organic evolution .

\section{CIHAPTER XXII}

Adaptation to environment in animals - Deep sea animals-The colouration of animals - Protective and aggressive resemblances-Warning colours-Mimicry-Epigamic ornamentation . . . . .

\section{CHAPTER XXIII}

Adaptation to the environment in plants-Alpine plants, desert plants and lianes-The modification of flowers in relation to insectfertilization

\section{PART V.-FACTORS OF ORGANIC EVOLUTION}

\section{CHAPTER XXIV}

Tiews of Buffon, Erasmus Darwin and Lamarck .

\section{CHAPTER XXY}

Robert Chambers and the "Vestiges of Creation"-Natural selection - The views of Charles Darwin and Alfred Russel Wallace. 


\section{CHAPTER XXVI}

Selection not confined to the organic world-Illustrations of the action of natural selection in the struggle for existence--Degeneration -Flightless birds-Extermination of the Morioris - Sedentary animals-Parasites-Co-operation of natural selection and the so-called Lamarckian factors of evolution - The influence of internal secretions upon growth-Increase in size beyond the limits of utility

\section{CHAPTER XXVII}

Artificial selection-Continuous and single selection-The mutation theory of the origin of species - Mutual adaptation - Unit characters - Isolation - Physiological selection - Non-adaptive characters-The evolution of man .

INDEX 


\section{OUTLINES OF EVOLUTIONARY BIOLOGY}

\section{PART I.-THE STRUCTURE AND FUNCTIONS OF ORGANISMS-THE CELL THEORY}

\section{CHAPTER I}

Introductory : The nature of life-The living organism viewed as a machine - The essential functions of the living body-The source of energy in living things.

Thene are many ways in which the extremely heterogeneous materials of which the earth is composed may be classified. Physicists often speak of them as solid, liquid or gaseous respectively, but we know that one and the same substance may exist in any of these conditions, changing from one to the other in accordance with changing conditions of temperature and pressure. Chemists, on the other hand, tell us that all substances are made up of certain definite chemical elements, occurring free or in a state of combination with one another, and that the almost infinite variety of gases, liquids and solids with which we are familiar has arisen mainly from this power of combination in very diverse but perfectly definite ways. The tendency of modern research, however, is to show that even the so-called chemical elements are not really so elementary in their nature as has been supposed, and it is quite conceivable that they may all have a common origin.

Our present purpose is to investigate a condition of matter which altogether transcends the classifications of the chemist and the physicist, a condition in which it exhibits those peculiar phenomena which we regard as manifestations of Life, and the study of which constitutes the science of Biology. It is not 
that living matter differs fundamentally from not-living matter in chemical or physical characters. On the contrary we recognize in it exactly the same chemical elements-or rather some of them-as we find in other constituents of the earth, and these elements appear to obey precisely the same chemical and physical "laws" in both cases, but in the living body they are associated and combined with one another in such a manner as to give rise to substances much more complex than any found outside the animal and vegetable kingdoms, and possessed of peculiar properties which raise them to an altogether higher plane of existence. The complex substances in question constitute protoplasm, which is the one essential constituent of every living thing, upon the peculiar properties of which the life of the organism depends.

We may begin our investigations into the nature of this life by examining the well-worn but none the less valuable analogy of the flame of a candle. Chemists and physicists have taught us that flame consists of incandescent matter, raised to a high temperature by the process of combustion, or chemical union with the oxygen of the atmosphere, and that the flame can exist only so long as the combustion goes on and a sufficiently high temperature is thereby maintained to render the burning matter luminous, or in other words to produce those vibrations of the invisible and intangible ether which we recognize as light. The flame, then, is the outward and visible sign of certain chemical and physical processes, of the action and reaction between the material which is being burnt and the atmosphere which surrounds it.

Similarly biologists have learnt to recognize life as the expression of the constant interaction which goes on between the living organism and its environment, or, in the words of Herbert Spencer, "the continuous adjustment of internal relations to external relations." So far as we have yet been able to analyze it, this interaction also consists of chemical and physical processes, amongst which combustion plays a large part; but the whole business is vastly more complex than the processes involved in the production of a flame, and so far many of its details have defied analysis.

We may vary our analogy by looking upon the body of a living organism, whether plant or animal, as an extremely elaborate engine or machine, whose existence depends upon a perfect 
adaptation to its environment, and whose action consists in continual self-adjustment to changes in that environment. What we call the life of the organism consists of the sum total of all the activities which it thus exhibits. The question at once arises: How, then, does a living organism differ from a mere man-made machine? and this question is one which it is by no means easy to answer. An organism, however, is not merely a piece of apparatus which has the power of maintaining itself for a longer or shorter period in a state of equilibrium with its environment and thereby preserving itself from destruction, for it also has the power of reproducing its kind by a process of selfmultiplication. In the ease of an artificial machine, where there is little or no automatic adjustment, the forces of the environment very soon get the upper hand; the metal work becomes corroded by oxidation, or worn away by friction, and presently the whole affair comes to a standstill. Oxidation and friction, and innumerable other chemical and physical agencies also tend to destroy the machinery of the living body, but for a longer or shorter period they are held in check by automatic processes of repair and renewal, and when the inevitable end does come it is usually not until the organism has produced at least enough offspring to take its place in the struggle for existence.

One of the most brilliant writers of the nineteenth century, Samuel Butler, has indulged in the somewhat fantastic suggestion that some day the construction of machines might be so perfected that they also would be able to reproduce their kind, and the little steam-engines would be seen playing about the door of the engine shed. It certainly does not seem possible that machines will ever multiply in this way, but should they do so, and should they at the same time be able to feed and grow, it is difficult to see why they should not be as much entitled to be called living organisms as any of the plants and animals which inhabit the earth to-day. They would, however, still be totally different from plants and animals both in structure and composition.

One of the most remarkable and characteristic features of the living things which inhabit this earth is that they are all com. posed of very similar materials, which are very different in their nature from any which we should be likely to choose in the construction of a machine. In making an engine we select those substances which seem best calculated to resist the destructive 
action of the environment; hard and rigid metals which will bear heavy strains, and as far as possible such as will be proof against the chemical action of the atmosphere; and we do our best by means of oil and paint to protect even these from injurious influences.

A living body may also have its hard protective structures, as the shell of the oyster and the scales of the fish, or its rigid levers, as the bones in our own limbs, but the really essential part of the organism is built up of just those materials which are most liable to destruction by chemical and physical agencies-of that almost liquid and extremely unstable substance which we have already referred to as protoplasm, and of its various derivatives. The experience of every day teaches us how rapidly the bodies of animals and plants decay when they are left exposed to the atmosphere after life has become extinct; and this decay is simply the destruction caused by the disintegrating forces of the environment. A disabled steamboat may lie rusting on the shore for many years without undergoing much change, but the dead body of a stranded jelly-fish thrown up beside it will become disorganized and disappear in the course of a few hours, and yet the jelly-fish when alive was undoubtedly the more complex and perfect piece of apparatus.

The body of an organism, moreover, undergoes destruction not only after death; it is always undergoing destruction, and its very life depends upon its destruction, just as the flame of a candle depends upon the destruction of the candle. But as it is destroyed it is constantly built up again; new protoplasm is formed and new tissues take the place of those which are worn out. The life of the organism is, in fact, the outcome of the constant struggle between destructive and constructive forces, and the keener the struggle the more vigorous will be the lifejust as the flame will be brighter or hotter in proportion to the activity of the combustion to which it owes its existence.

Life, like the flame, is a manifestation of energy, and the living body is, like the steam-engine, a machine for transforming one kind of energy into another. Moreover, the ultimate source of the energy is the same in both cases. That of the steamengine is derived from the combustion or oxidation of coal, which contains stores of energy derived millions of years ago from the light and heat of the sun by the green plants which flourished in the vast forests of the Carboniferous epoch. The green plants 
of to-day still derive supplies of energy from the same source and lock up in their leaves and stems what they do not themselves expend, while the animals in turn derive their energy from the green plants upon which they directly or indirectly feed. Hence both plants and animals are ultimately dependent upon the sun for their existence.

Even the most superficial examination is sufficient to demonstrate the fact that the body of any of the more familiar animals or plants is, as we have already indicated, an extremely complicated thing. Whatever may be the degree of complexity, however, and however much one organism may differ from another in details of structure, whether it be a microscopic alga or an oak tree, an Amœba or a man, there are always certain things which have to be done, certain actions or functions which have to be performed, in order that its life may be maintained.

In the first place, the organism must safeguard itself as far as possible from the destructive influences of its environment. It must not only be able to protect itself from such physical agents as heat and cold, mechanical impact and friction, but it must be able to select a situation where life is possible, and to escape from other organisms by which it is liable to be attacked. All this involves the expenditure of energy in some form or another; it may be in the manufacture or secretion of protective envelopes or shells, such as we find even in some of the simplest Protozoa, or it may be in actively moving away from the source of danger. Thus it appears that the very first thing necessary for the maintenance of life is the expenditure of energy. This energy, though ultimately derived from the sun, is, as we have already seen, derived immediately from the combustion of fuel, very much as in the case of a steam-engine, but with the important difference that in the living organism the fuel is, at any rate to a large extent, the actual substance of which the body is composed. In this respect the comparison with a candle is especially apt, for it is the combustion of the actual substance of which the candle is composed that liberates the energy manifested in the light and heat of the flame.

Now combustion is, of course, simply another name for what chemists term oxidation, or combination with the element oxygen, a process which is often accompanied by the liberation of a considerable amount of energy in the form of heat and light, though this is by no means always the case. In the 
higher animals sufficient heat is evolved to maintain the temperature of the body at a level considerably above that of the surrounding atmosphere, and such animals are accordingly termed "warm-blooded"; in plants, on the other hand, and in the "cold-blooded" lower animals, the amount of heat evolved is not as a rule sufficient to raise the body temperature to any great extent, if at all. Heat, however, is only one form in which energy may be manifested, and in living organisms it is, as a matter of fact, much more conspicuously manifested in the form of motion, especially in animals, while in not a few cases even a low temperature combustion may liberate energy in the form of light, as in the glow-worm and numerous other luminous animals and plants.

When a piece of charcoal is burnt in the air it enters into combination with the elementary oxygen gas of the atmosphere, and another invisible gas which we term carbon dioxide, or carbonic acid, is produced, in accordance with the equation-
C
(Carbon)

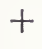
$\underset{(\text { Oxygen })}{\mathrm{O}_{2}}=$
$\mathrm{CO}_{2}$
(Carbon Dioxide).

In this process energy is set free in the form both of heat and light. We must now inquire a little more carefully whence this energy really comes, for although this is a question primarily for the chemist and physicist it is also clearly one which the biologist cannot afford to leave unanswered.

In accordance with the principles of the conservation of energy and the indestructibility of matter we believe that the quantities of energy and matter which exist in the universe are fixed and constant. Neither energy nor matter can be created and neither can be destroyed, though each may express itself in a great variety of ways and change more or less readily from one mode of expression to another. Thus, as we have already seen, the energy of the sun's rays may be utilized in building up the bodies of green plants, and locked up, as it were, in the substances of which these are composed.

We also know that different chemical elements have a very strong "affinity" for one another, their atoms tending to unite and form compound molecules when they are brought within the sphere of each other's attraction. Once united the $y$ can only be separated again by the expenditure of energy, and when they unite a corresponding amount of energy is 
liberated. We may say, for example, that in the elements carbon and oxygen, so long as they remain separate, a certain amount of energy remains latent. We call this potential energy. When the carbon and oxygen atoms are allowed to come together and unite, this potential energy of chemical affinity is liberated as kinetic energy, and manifested in the form of light and heat.

It is from the potential energy of chemical affinity that the energy of a living organism is immediately derived. Protoplasm, the fundamental constituent of both plants and animals, contains chemical compounds of extremely complex structure, composed of many elements and containing a large amount of potential energy locked up in them. Moreover, these proteids, as they are termed, are extremely unstable bodies, readily breaking up on oxidation into simpler and more stable combinations and thus liberating energy. It is the presence of these unstable proteids which confers upon protoplasm its peculiar fitness to form what has been so aptly termed by Huxley "The physical basis of life." They play the part of the gunpowder in a cartridge, ready to produce a manifestation of energy as soon as the proper stimulus is applied.

It is, then, the breaking up of proteids, or of some other complex substances, usually by recombination of their constituents with oxygen, which furnishes the constant supply of energy which an organism requires. This process, however, can only go on so long as the supply of combustible matter on the one hand and of oxygen on the other is adequately maintained, and this brings us to the consideration of two of the most important functions which every living organism must perform, nutrition and respiration.

Under the head of nutrition we must include all those processes which are concerned in building up the body, in making good the waste of substance necessitated by the expenditure of energy and thus providing new stores of fuel for the use of the organism. The first step in nutrition is the taking into the body of suitable food material. In the case of the typical animal this material must contain in some form or other all the necessary supply of potential energy, locked up in more or less complex and unstable chemical compounds such as it can obtain only from the bodies of other organisms. The green plant, on the other hand, by virtue of the chlorophyll 
which it contains, has the power of absorbing energy directly from the sun's rays and using this to build up the complex proteids from very simple constituents. The feeding of the organism, whether plant or animal, is comparable to the stoking of the engine, but with this difference, that the food material, unlike the fuel in the engine furnace, has usually to undergo complex chemical processes, which may actually result in the formation of new protoplasm, before it is available as a source of energy.

Supplies of energy are, of course, useless unless they can be liberated when required. The fuel must be burnt, and for this purpose, as we have already said, a supply of oxygen gas is necessary, and the function which is concerned in providing this supply we call respiration. The term respiration, however, is one in the employment of which we shall have to exercise a certain amount of care. It is naturally associated in our minds with the mechanical act of breathing which we ourselves perform. We can see a man breathing, but we cannot see an oak tree breathing; nevertheless the oak tree performs the function of respiration just as efficiently as the man. We have to learn to dissociate the essential part of this function, which is common to all living things, from the subsidiary complications which have been introduced in the case of the higher animals during the course of their evolution from lower forms.

Respiration, in the scientific acceptance of the term, is simply the exchange by the organism of the carbon dioxide gas which has been formed in the body in the process of combustion for the oxygen gas which is required for that combustion. It is therefore a double function-oxygen being taken in and carbon dioxide got rid of by one and the same process. This process is, in its essential features, an extremely simple one, depending upon the physical principle of osmosis or diffusion, in accordance with which two gases of different densities tend to change places, until equilibrium is established, whenever they are placed in the necessary relations with one another, as when they are separated only by some membrane through which both can pass.

Carbon dioxide, however, is not the only waste product resulting from the breaking up of the complex proteids, for these also contain hydrogen, nitrogen, sulphur and phosphorus, and other products of decomposition are accordingly formed 
which contain amongst them all of these elements. These must also be eliminated from the body, and this process of elimination of waste products constitutes the function of excretion. This function may be performed in a variety of ways and by a variety of organs. In so far as the carbon dioxide is concerned it is, as we have already seen, an essential part of the function of respiration. Urea, on the other hand, a nitrogenous substance which is perhaps the most characteristic waste product in the higher animals, is eliminated by special excretory organs, such as the kidneys. In the case of the higher plants the waste products are for the most part stored up in the leaves and got rid of when these are shed.

We have thus seen that the supply of energy to a living

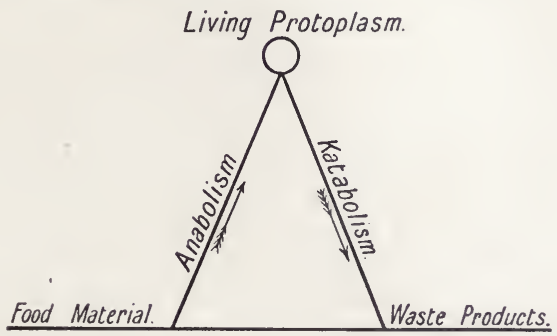

FIG. 1.-Diagram of Metabolism.

organism, and therefore also its life, depends upon a series of complex chemical processes which take place within the body. All these processes collectively are spoken of as metabolism, and we may distinguish between two sets of metabolic changes: those which are constructive and lead to the building up of new living protoplasm out of food material, and those which are destructive and lead to the decomposition of the body substance, the liberation of energy and the formation of waste products. The former are termed anabolic and the latter katabolic.

We may roughly illustrate these elementary conceptions of the chemical processes which take place in the living body by the accompanying diagram, in which a mass of living protoplasm is represented as balanced in a very unstable position on the apex of a triangle. It is constantly undergoing destruction, 
accompanied by the liberation of energy and resulting in the formation of waste products, substances which, in falling down one side of the triangle to a lower level, to a greater or less extent lose their potential energy. On the other side of the triangle, however, complex food material, containing fresh supplies of potential energy, is supposed to be taken in, or, in the case of green plants, actually built up from simple constituents by the energy of the sun's rays, and used in repairing the waste of the protoplasmic body.

If the constructive processes proceed more vigorously and rapidly than the destructive, if the food supply is abundant and the expenditure of energy comparatively low, the body may grow, though, as we shall see presently, only within certain limits. If the reverse is the case and the expenditure exceeds the income, the body may dwindle away and finally die. If it is to remain in a condition of healthy equilibrium a just balance must be maintained between the two sides of the account.

Perhaps the most characteristic property of living things is, as we have already suggested, the power of reproduction. This is the last resort of the organism in the struggle for existence. The individual, which owes its very life to the perishable nature of its body, always succumbs to the destructive influences of the environment sooner or later, but before yielding to the inevitable it will, under normal conditions, have produced offspring which will carry on the struggle for another generation.

The phenomenon of reproduction is intimately associated with that of growth, and may be traced back to the division of a simple ancestral mass of protoplasm into two parts whenever its size increased to such an extent that the ratio of surface to volume became too small for the necessary intercourse between the organism and its environment. With this division of the protoplasmic body the proper proportion is restored, and hence reproduction by multiplication of protoplasmic units may be looked upon as primarily the direct consequence of superabundant nutrition.

We have now learnt to look upon an animal or a plant as a complex and extremely delicate piece of mechanism, constantly employed in collecting energy directly or indirectly from the sun's rays and in using that energy to maintain an incessant struggle against the destructive forces of its environment. This incessant getting and spending, winning and losing, constitutes 
what we call the life of the organism. In considering what is the meaning of all this we must remember that, primarily at any rate, every living thing exists for its own benefit, and that living, like virtue, is its own reward. The organism also, however, exists for the benefit of future generations, to which it may hand on the lamp of life before its own flame is finally extinguished. There is a race life as well as an individual life, and we cannot realize too clearly that in the economy of nature the former is of infinitely greater importance than the latter.

The ideas which we have just been considering are by no means of modern origin. More than three centuries ago the philosopher Descartes endeavoured to explain the human body as a machine, but as a machine under the control of the "soul," which he curiously located in that part of the brain known as the pineal gland. His ideas of physiology, however, were, naturally, of the crudest description, and immense strides have been made in this direction since his time. Chemists and physicists have helped us much towards a correct understanding of the living mechanism, but when they have done their best it may well be that the question "What is Life?" will still remain unanswered, and that we may still have to take refuge in the idea of an unknown "soul" to explain the difference between living and not-living things. The "soul" of Descartes" philosophy corresponds more or less closely with the "vital force " of some more recent writers and the "entelechy" 1 of others, but whatever term we employ it must be rather as a cloak for our ignorance than as an expression of any definite opinion as to what it is that really animates the living body.

1 Vide Driesch, "The Science and Philosophy of the Organism," (London, A. \& C. Black, 1908), Vol. 2, pp. 137, 138. 


\section{CHAPTER II}

Amœba as a typical organism-The properties of protoplasm.

Is illustration of the general principles dealt with in the foregoing chapter we may now consider a definite concrete example of a living organism. Probably none is better. suited for this purpose than the familiar Amœeba, which may be regarded as a kind of pocket edition of a typical animal.

Amœbæ may be found creeping about on the mud at the bottom of ponds and ditches. Although of microscopic size and, usually at any rate, invisible to the naked eye, they are by no means the smallest or simplest of living things, but exhibit within the narrow limits of their gelatinous bodies a considerable amount of structural differentiation.

In general appearance (Fig. 2) an Amœba resembles nothing so much as an irregular speck of translucent jelly, but if we watch it for a few minutes under the microscope we soon find that it is something more than this. If in an active and healthy condition it never maintains the same shape for long together, but manifests an ever-changing irregularity as it slowly creeps about from place to place, throwing out irregular projections of its body first in one direction and then in another and withdrawing old projections as new ones are put forth.

The viscid substance of which the entire body is composed is protoplasm, but this protoplasm is not homogeneous throughout; on the contrary, it exhibits a characteristic differentiation into parts or organs, which can be more or less readily distinguished from one another and each of which has its own duties or functions to perform.

Inasmuch as it consists of but one protoplasmic unit, however, we may speak of the body of an Amœba as a single cell. ${ }^{1}$ As in all other typical cells, the most fundamental aifferentiation

1 The origin and meaning of this term will be discussed more fully in a later chapter. 
which it shows is into cell-body and nucleus. The cell-body forms by far the greater part of the organism, and the

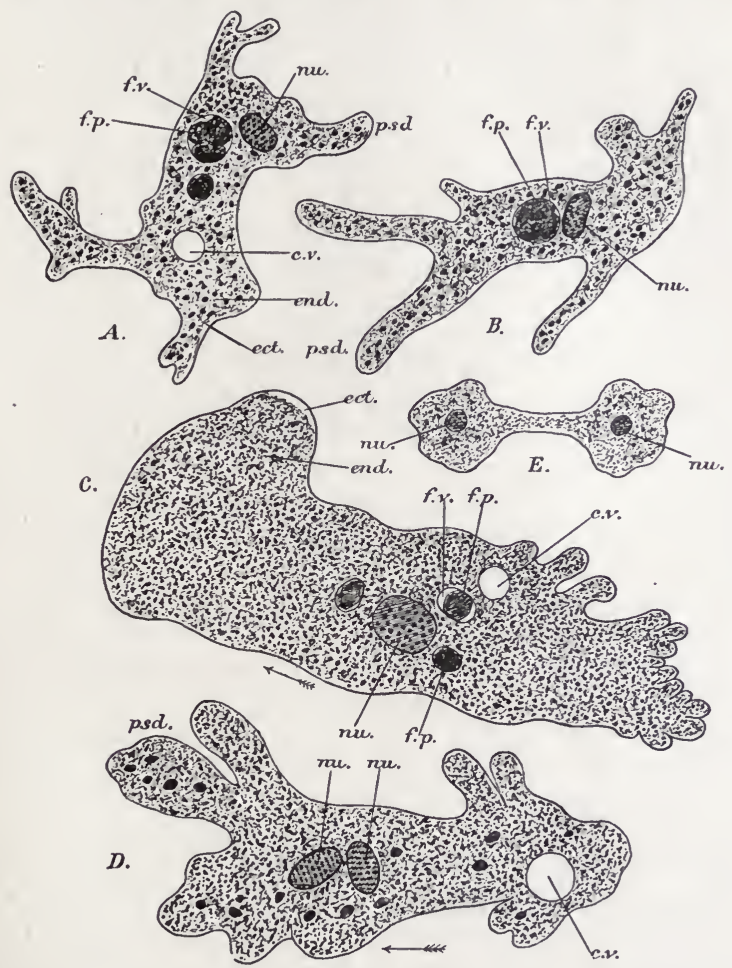

Fig. 2.-Amoba.

$A, B$. The same individual in two phases of active movement, showing change of form.

$C$. Another specimen, of a different species. Note the numerous short projections at the hinder end due to contraction, while at ect. the commencement of a new pseudopodium is indicated by a thickening of the ectoplasm.

$D$. A specimen with two nuclei.

$E$. Diagram of reproduction by simple fission.

c.v. Contractile vacuole; ect. ectoplasm or ectosarc; end. endoplasm or endosarc; f.p. food particles; f.v. food vacuole; $n u$. nueleus; $p s d$. pseudopodium. (The arrows show the general direction in which the animal is moving.)

protoplasm of which it is composed is often distinguished as cytoplasm. 
The nucleus (Fig. 2, nu.), composed of a somewhat different variety of protoplasm sometimes known as karyoplasm or nucleoplasm, is a very definite body of more or less rounded form, sometimes shaped like a bun, and easily distinguishable from the cytoplasm even in the living animal by its more highly refractive character. Its position is by no means constant, for it floats about from place to place in the interior of the almost liquid cell-body.

The cytoplasm is very imperfectly differentiated into inner and outer portions. The former, in which the nucleus is lodged, is often called the endosarc or endoplasm (Fig. 2, end.), the latter the ectosarc or ectoplasm (Fig. 2, ect.). The ectoplasm must be regarded as a feebly developed protective layer ; it is the part which comes into direct relation with the surrounding water and through which all intercourse between the Amœba and its environment must take place. Though soft and gelatinous, it is a good deal firmer and denser than the endoplasm, and it is also more transparent, for the endoplasm contains imbedded in it numerous more or less opaque particles of various kinds which give it a coarsely granular character. Most of these particles are minute, but others are generally present of comparatively large size and enclosed in drops of clear liquid. These latter are food particles (Fig. 2, f.p.) undergoing digestion, and they can frequently be identified as the bodies of other organisms which the Amoba has taken in, microscopic plants or animals smaller than itself. The drops of liquid in which they occur are termed food vacuoles (Fig. 2, f.v.).

Another spherical drop of liquid (Fig. 2, c.v.) may be observed somewhere near the surface of the cell-body. This is perfectly clear and contains no solid particles; moreover it undergoes a rhythmical dilatation and contraction, gradually increasing to a maximum size and then suddenly disappearing owing to the discharge of its contents into the surrounding water. If the spot where this "contractile vacuole" disappears be carefully watched another drop of liquid is seen gradually to accumulate there, and the process is repeated.

We are told that in the early days of chemistry, before the highly specialized apparatus which is now used was thought of, the originator of the atomic theory performed his experiments with the ordinary domestic crockery. So also an Amoba is able to perform, with the extremely simple apparatus at its disposal, 
all those actions or functions which are really essential for the maintenance of life.

In the higher animals the primary differentiation of the body is into an outer protective and an inner digestive layer, each of very complex structure. The Amoba accomplishes the same end in its own primitive manner by the differentiation into ectoplasm and endoplasm. In most of the higher animals, again, we find very well developed organs of locomotion in the form of limbs. The Amœba has no permanent organs of locomotion at all but merely temporary projections of the body, the so-called pseudopodia (Fig. 2, psd.), which are put forth when required. In both cases, however, movement is effected in essentially the same way, by contraction and expansion of the living protoplasm. In the higher animals this power of contraction is localized in the muscles, which are highly specialized for the purpose and have no other duties to perform, while in the Amœeba any part of the body, or at any rate of the ectoplasm, may contract or expand as occasion requires.

In the process of formation of a new pseudopodium we see first a thickening and protrusion of the clear ectoplasm (Fig. 2, C., ect.), accompanied by a streaming in of the endoplasm, and the latter seems to bulge out the ectoplasm as it flows forwards. The pseudopodium is withdrawn again by a reversal of the process, the endoplasm streaming out from it into the central mass of cytoplasm and the ectoplasm contracting after the retreating endoplasm. Thus at the posterior end of an actively creeping Amøeba one frequently sees numerous blunt projections which are the last remnants of retracted pseudopodia (Fig. 2, C.). The shape of the pseudopodia when fully extended differs very much in different kinds of Amœbæ. In some species they are comparatively short, thick and blunt, as in our illustration, while in others they are very long and slender, radiating outwards from the body of the animal in all directions. They are used by their possessor not only as organs of locomotion but also as tactile organs and, as we shall see directly, for the capture of prey.

'The protrusion and retraction of pseudopodia imply, of course, the expenditure of energy, and this energy must be derived from the combustion of the body of the Amoba. In this way the protoplasm is gradually used up, and, unless the animal is to die of starvation, it must be replaced, which brings us to the consideration of the manner in which an Amœba performs the 
important function of nutrition. As it crawls slowly about the pseudopodia come into contact with all sorts of solid particles in the surrounding water. Some of these will be inorganic, grains of sand and so forth, others will be the dead or living bodies of other organisms, sometimes much smaller than the Amœba itself. 'The Amoba has the remarkable power of distinguishing amongst these different kinds of particles those which are good for food from those which are not. How it does so we do not know; we can only say that the presence of a particle which is good for food stimulates the living protoplasm in a way quite different from that in which it is stimulated by the presence of a mere grain of sand. In the latter case the Amœba will simply pass to one side and avoid the object; in the former it will put forth pseudopodia which will close around and envelop it. The food particle is thus passed through the ectoplasm into the interior of the body. There is no definite mouth, but food is taken in wherever it happens to come into contact with the surface of the body, and the aperture closes up after it. Thus a temporary mouth is formed as occasion demands. Similarly there is no permanent digestive cavity or stomach, but merely a temporary food vacuole into which a digestive fluid is doubtless secreted by the surrounding protoplasm.

Digestion, as in higher animals, is essentially a process of solution, whereby those parts of the food which are digestible are dissolved and rendered capable of - diffusing from the digestive cavity into the surrounding body. The higher animals make use chiefly of three classes of food material, proteids, carbohydrates (e.g., starches and sugars) and fats. It is said that Amceba can only digest proteids, which of course it must obtain from the protoplasmic bodies of other organisms. When digestion is complete a certain amount of insoluble residues from the food will remain over; these constitute the fæces and have to be got rid of. 'There is, however, no permanent vent or anus, and the fæces are cast out through the ectoplasm at the hinder end of the body as the animal crawls along.

Owing to the minute size of the whole organism there is no need for a complex circulatory system, such as is found in the higher animals, for the distribution of the digested food; it merely soaks into the surrounding protoplasmic body from the food vacuoles, and, by anabolic changes which are not fully understood, is converted into new, living protoplasm. 
It is not easy, if it be possible at all, actually to observe the process of respiration in so small an animal as an Amoeba, but we know perfectly well from the analogy of higher organisms what must take place. Oxygen is required for the combustion of the protoplasm from which the energy of the organism is derived, and this oxygen occurs in a state of solution in all ordinary water which is exposed to the air. At the same time carbon dioxide, or carbonic acid gas, must be produced as one of the products of the combustion, by oxidation of the carbon in the protoplasm. This waste product $\left(\mathrm{CO}_{2}\right)$ will first of all be dissolved in the water which forms the greater part of the bulk of the living organism, while at the same time the water by which the animal is surrounded may be regarded as a very dilute solution of oxygen. The outermost layer of the ectoplasm may be looked upon as a thin membrane separating the two solutions.

We know from experiment that whenever two gases, or solutions of gases, of different density, are separated from each other by a thin organic membrane, they will pass through that membrane in opposite directions until a state of equilibrium is established between the two. This process of osmosis or diffusion is, as we have already seen, the essential feature of respiration in all plants and animals, although probably the purely physical process is controlled to some extent by the living protoplasm.

In the case of the Amoba then, the carbon dioxide diffuses out through the ectoplasm into the surrounding water while the oxygen from the surrounding water diffuses in, and the necessary exchange of gases is brought about. No specialized organs of respiration, such as we meet with in the higher animals, are required. The whole surface is a respiratory surface, and all parts of the interior are within reach by the simple process of diffusion, aided doubtless by the circulation of the semiliquid protoplasm which is constantly going on inside the body.

Other waste products must be formed by the combustion of the protoplasm in addition to carbon dioxide. What these are we do not exactly know in the ease of the Amœba, but it is evident that they must contain nitrogen, which is one of the essential constituents of all proteids. These waste products must be got rid of by some process of excretion, and it is usually supposed that they are passed in a state of solution to the contractile vacuole and thence periodically expelled to the exterior, so that 
the contractile vacuole is probably to be regarded as the special excretory organ of the animal.

In all but the lowest animals there is a more or less specialized nervous system, whose function it is to place the different parts of the body in communication with one another and, through the mediation of the sense organs, with the external world or environment. The action of this system depends primarily upon one of the fundamental properties of living protoplasm, the power of responding to stimuli by some definite change in its own condition. The stimulus is, in the first instance, supplied by some factor of the environment, such as light, heat, electricity or mechanical impact. It may appear to originate in the central nervous system itself, but this is probably secondary. It has the effect of liberating stored energy in those parts of the organism which are sensitive to that particular stimulus, in somewhat the same way that the stimulus of heat may have the effect of liberating the stored energy in a charge of gunpowder. The living molecule has, indeed, actually been described as explosive. In both cases potential energy is converted into linetic energy, and the effect which is produced may be out of all proportion to the amount of energy represented by the liberating stimulus itself.

In the higher animals, then, the stimulus received from the external environment, whatever its nature may be, acts primarily upon some special sense organ or receptor, whence it is transmitted along highly specialized tracts of tissue, the nerves, to some part of the central nervous system, where it usually gives rise to what we call a sensation. The central nervous system, again, not only has the power of receiving stimuli through afferent or sensory nerves, but also of sending stimuli through efferent nerves to the various organs of the body, whereby their functions are controlled and regulated. The contraction of muscles and the secretion of glands are all controlled in this manner, and the entire working of the body is co-ordinated by the action of the nervous system.

In the Amøba, however, we see no trace of a special nervous system, nor of sense organs, but nevertheless the organism is certainly capable of receiving and responding to stimuli; in other words it is irritable. Thus the protoplasmic body responds by contraction to the stimuli of mechanical impact, heat, light, electricity and chemical reagents, and, as we have already seen, 
the presence of particles which are good for food causes the protrusion of pseudopodia in a definite and purposive manner. Probably the whole of at least the ectoplasm is to some extent sensitive to stimuli of certain kinds, and it is also probable that stimuli may be conducted from one part of the body to another without the existence of special nervous tracts.

One of the most difficult problems in connection with the physiology of Amceba-and indeed of any living organism-is that of automatism. Does an Amœba do anything really automatically or spontaneously, or are all its actions the result, direct or indirect, of the application of external stimuli to the explosive molecules of living matter? Is the organism merely a machine run by the environment, or is it something more? Here, of course, at the very beginning of our investigations, we are face to face with the old question, already referred to, of the existence of an animating principle or "soul," which exercises some sort of control over the physical and chemical processes upon which the life of the organism depends. This is a question which perhaps falls within the province of the philosopher rather than that of the biologist. The theory of vitalism, by postulating the existence of some such special vital force in all living things, undoubtedly enables us to avoid many difficulties, but it is doubtful if it really explains anything. As a matter of fact the more we study living organisms by actual observation and experiment, the more fully are we able to interpret their behaviour in terms of chemistry and physics, but this is a very different thing from saying that chemistry and physics will ultimately yield a complete explanation of vital phenomena.

It is quite possible, for example, that the movements of the Amœba may all ultimately be interpreted in such terms, for Bütschli has shown that they can be closely imitated by minute artificially prepared drops of oil-foam surrounded by water. The substance of which these droplets are composed is of course totally different chemically from protoplasm and is in no sense alive, but it seems highly probable, if not certain, that since purely physical processes (amongst which surface tension seems to play an important part) are capable of producing strikingly amœboid movements in the oil-foam, they may also be largely, if not solely, responsible for the similar phenomena of movement in the living protoplasm of the Amœba itself, which seems closely to resemble an oil-foam in its physical properties. 
Like other organisms, the Amøeba sometimes undergoes a period of rest, during which its various activities are more or less completely suspended. Under these circumstances the pseudopodia are withdrawn, the body is rounded off and a protective envelope or: eyst is secreted by the protoplasm. This, however, is only a temporary state, perhaps necessitated by unfavourable conditions of the environment, and sooner or later the organism emerges from its retirement and resumes its activity.

If in the course of its wanderings the Amcba meets with an abundant supply of food and takes in more than is actually required to make good the waste of protoplasm; if, in other words, anabolism preponderates over katabolism, the organism may increase in size by growth, by the addition of new particles of protoplasm in excess of those which are used up. These new particles are deposited, not on the surface, but throughout the whole mass of protoplasm, between those which are already formed. Thus growth takes place, not by accretion, as in a crystal or a snowball, but by intussusception, and we have here a characteristic though by no means absolute distinction between the growth of not-living and that of living matter.

As in all organisms, however, there is a limit to the size which the body may attain, and this limit varies with different species of Amœba. It depends primarily, no doubt, upon the necessary relation between surface and volume. As we have seen, all interchange between the organism and its environment has to be maintained through the surface, and a given area of surface cannot supply the wants of more than a certain volume of protoplasm. As the animal grows the volume must necessarily increase in a much higher ratio than the surface, and the proportion between the two is rapidly altered. This is probably not the whole explanation of the limitation of growth in an Amœba, the problem being doubtless complicated by other factors, but we may take it as quite certain that increase beyond a certain size, if possible, would inevitably result in death. Such a calamity is avoided by the simple expedient of dividing into two parts whenever the limit of safety is reached. The nucleus divides first and the two halves move away from one another, then the protoplasm constricts into a bridge between the two nuclei, the bridge narrows and finally ruptures, and instead of one Amoba there are now two, each exactly resembling the parent (Fig. 2, E.). 
This simple fission of a single protoplasmic unit, or cell, forms, as we shall see later on, the essential feature of ordinary reproduction throughout the animal and vegetable kingdoms. It will be observed that in this process generation succeeds generation without the intervention of anything which we can speak of as death. There is indeed no room for death in the history of these simple organisms, unless it be death by accident, for every time fission takes place the entire body is used up, and nothing is left over to die. Nor is there any distinction to be drawn between parent and offspring, for the two new individuals are in all respects similar to one another, and neither can be said to precede the other in point of time.

We have now become sufficiently well acquainted with the nature of protoplasm to profit by a more detailed examination of its physical and chemical properties. We have seen that, as it occurs in the body of an Amœeba, it is a viscid, more or less liquid, colourless substance, almost transparent but exhibiting, under moderately high powers of the microscope, a granular appearance due to the presence of numerous minute and more or less opaque particles. These particles may be regaried as impurities, and indeed protoplasm can never be obtained in a perfectly pure state, for it is constantly undergoing chemical change, both constructive and destructive, and the impurities owe their origin partly to the food materials which are taken in and partly to katabolic processes which give rise ultimately to waste products.

Even apart from these impurities, however, the protoplasm itself never exhibits a perfectly uniform structure. It is by no means homogeneous but shows a more or less distinct differentiation into different portions, as, for example, into nucleoplasm and cytoplasm, ectoplasm and endoplasm, and so forth. In other words it is an organized substance. Moreover, it has a characteristic minute structure or texture which can to some extent be recognized under high powers of the microscope and concerning the interpretation of which different observers are as yet by no means all agreed. According to Professor Bütschli it is a kind of microscopic foam, consisting of exceedingly minute drops of a more liquid substance separated by very thin layers of denser material, and thus resembling physically such a foam as can be prepared from a mixture of oil, salts of various kinds, and 
water. If this really be a correct account of the minute structure of living protoplasm it helps us, as we have already seen, to explain its characteristic movements in terms of well known physical phenomena.

Other competent observers, however, maintain that the appearance of foam-structure is a delusion and that what Bütschli interprets as thin sheets separating the droplets from one another are in reality very delicate fibres arranged in a network. These fibrillæ are supposed to be contractile and thus to be responsible for the movements of the protoplasm as a whole. But whence comes the contraction of the fibrillæ?

Various considerations, again, and especially the phenomena of heredity, oblige us to postulate for protoplasm an even more minute fundamental structure than the microscope is capable of revealing to us. It is, in all probability, made up of ultramicroscopic material units, each composed of a group of molecules, which units, or "biophors," must themselves be regarded as living bodies capable of nourishing themselves, growing and multiplying by division.

It is difficult to form a satisfactory idea of the chemical composition of protoplasm because it is impossible to analyze it in the living condition; indeed, in the living condition it is constantly undergoing chemical change, and the moment it dies it ceases to be protoplasm. It is certain, however, that it is not a definite chemical compound, but a mixture of several distinct substances: proteids, mineral salts and water. Moreover, different samples of protoplasm, taken from different organisms or from different parts of the same organism, may differ widely from one another in chemical composition. Thus the difference between nucleoplasm and cytoplasm is largely a chemical one, depending to some extent upon the relatively large amount of phosphorus present in the former.

By far the most characteristic and important of the chemical constituents of protoplasm are, of course, the proteids. These form a remarkable class of substances which do not occur in nature except in the bodies of plants and animals. They are definite chemical compounds containing the elements carbon, hydrogen, oxygen, nitrogen, sulphur and frequently phosphorus, and they have an extremely complex and unstable constitution, readily splitting up on oxidation into simpler and more stable compounds and therely liberating kinetic energy. Many 
different kinds of proteids are known to us and have received special names; such are the albumen which occurs in the white of an egg, the casein which is met with in cheese, the legumin which is characteristic of peas and beans, the gliadin and glutinin of flour, and so forth.

These proteids are, for the most part at any rate, colloid substances, that is to say they are more or less gelatinous and incapable of diffusing through organic membranes. This may be accounted for if we assume that the highly complex molecules of which they are composed are too large to pass through the very minute pores which occur in such membranes and which readily allow of the passage of the molecules of simpler, crystalloid substances. The colloid nature of the proteid constituents of protoplasm plays a very important part in determining its properties and behaviour. Crystalloid mineral salts and other diffusible substances in a state of solution can pass through a cell-wall or membrane by osmosis, and thus the living protoplasm receives fresh supplies of nutriment, but the colloid proteids are as a rule formed inside the cell and cannot usually pass out again until they have undergone some chemical change whereby they are rendered diffusible.

The mineral salts which we find in the protoplasm, usually. in a state of solution, are of very various kinds, compounds of sodium, potassium, calcium and other elements with various inorganic and organic acids, such as sulphuric, hydrochloric, malic and citric acids.

Finally, water must always be present in living protoplasm and usually forms a very large percentage of the whole mass.

Whatever view we may take with regard to the question of vitalism, there can be no doubt that the most distinctive property of living protoplasm is its power of controlling chemical and physical processes so as to make them yield results different from those which would be obtained if we were dealing with not-living matter. The various processes upon which depend the functions of movement, nutrition, respiration and excretion all appear to be controlled in this manner, but the general principle is perhaps most beautifully illustrated in the case of many of the lower animals and plants, in which the protoplasm secretes a protective or supporting skeleton of some mineral substance, such as silica, or carbonate of lime. Silica, for example, in the inorganic world, occurs abundantly in a state of solution in water, from which it 
may be deposited in different forms, in shapeless masses as in the case of flints, or in symmetrical crystals as in some specimens

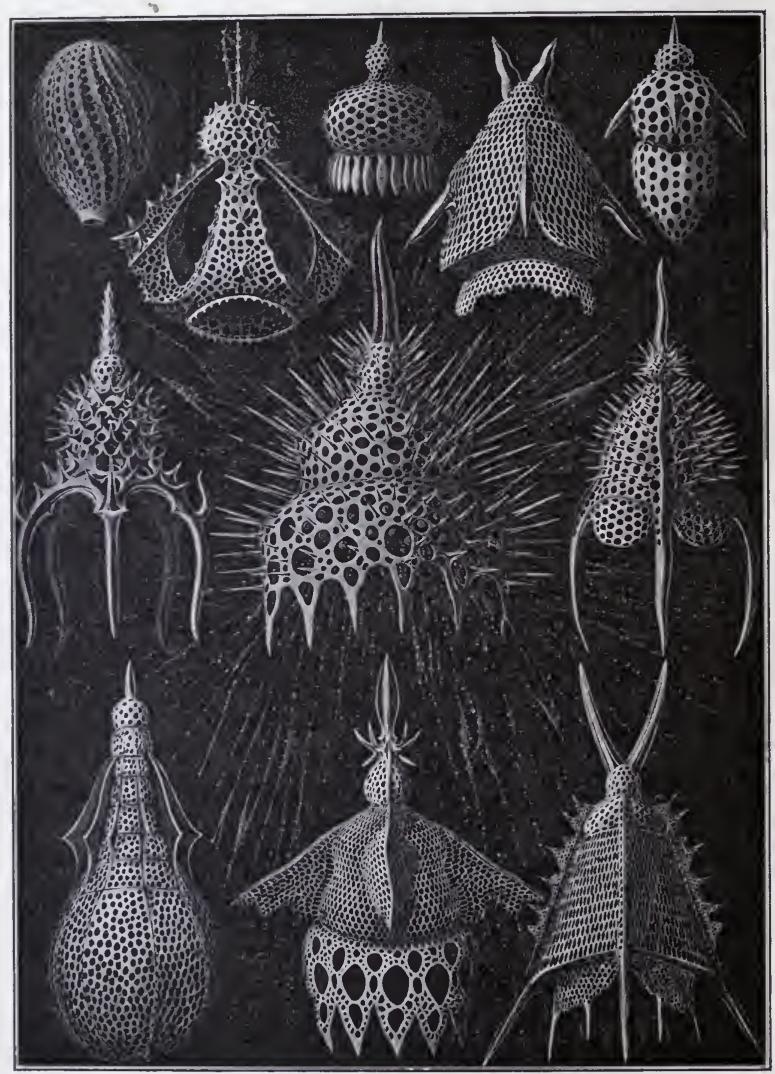

FIG. 3.-Different forms of Radiolarian Skeletons. In the central figure the protoplasmic pseudopodia are seen coming out from the openings in the shell. (From Haeckel's " Kunstformen der Natur.")

of quartz, whilst the beautiful opal, chemically speaking, is merely a hydrate of silica, or silicic acid. 
Many simple unicellular organisms, such as the Radiolaria (Fig. 3) amongst animals, and the diatoms amongst plants, have

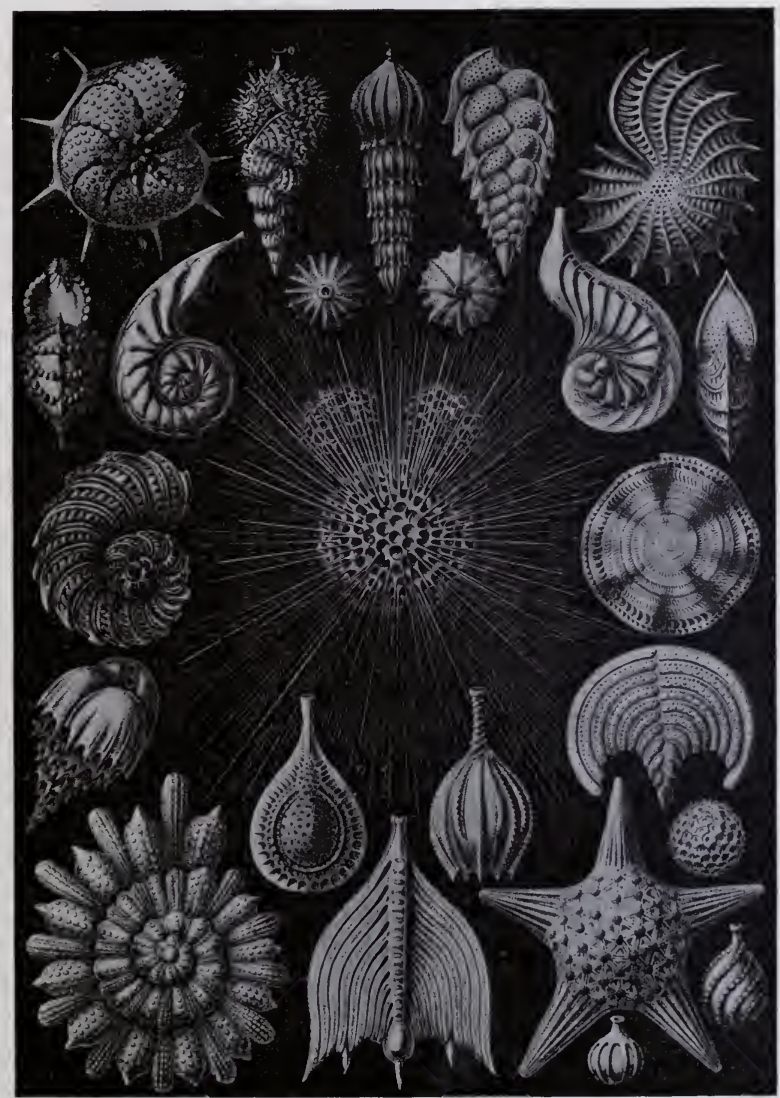

FIc. 4.-Different forms of Foraminiferan Skeletons. (From Iraeckel's "Kunstformen der Natur.")

the power of taking up dissolved silica from the water in which they live and using it for building skeletons. These skeletons, 
however, which are really composed of opal, do not consist either of shapeless masses or of geometrical crystals, but assume beautifully symmetrical forms which vary with each particular kind of organism and which are wholly different from any forms occurring in the inorganic world. The same is true of those somewhat more highly organized members of the animal kingdom, the siliceous sponges, whose skeletons consist of spicules of opal (Fig. 88), often of most beautiful and characteristic shape, and each one as a rule formed by the activity of the living protoplasm within the compass of a single cell.

Whilst many unicellular organisms and many sponges thus manufacture for themselves skeletons of a siliceous character, others, living perhaps in the same water, secrete skeletons which are composed of carbonate of lime. Such, for example, are the Foraminifera, the dead calcareous shells of which (Fig. 4) accumulate to-day at the bottom of the deep sea in many places in the form of ooze, while in the Cretaceous period of the earth's history they played the principal part in building up the chalk cliffs on the south coast of England. Each of these microscopic shells, which are often of extreme beauty and frequently ornamented with elaborate patterns, is formed as a protective envelope by a simple protoplasmic organism closely resembling an Amoba.

It is evident, then, that we must attribute to living protoplasm a very remarkable power of selection or choice, for it is able, as it were, to pick out certain materials from its environment for its own purposes and to reject others. We have already seen this in the case of the selection of food materials by the Amœba; we see it again in the selection of the materials for skeleton formation in other simple organisms. The fact that one organism will select silica while another selects carbonate of lime from the same sample of sea water and for the same purpose, must correspond to some deep-seated difference in the pro:oplasm of which they are composed, and illustrates very well the diverse potentialities of this remarkable substance. 


\section{CHAPTER III}

IIæmatococcus - The differences between animals and plants.

IN striking contrast to Amœba, which, though primitive, is nevertheless a very typical example of an animal organism, stands Hæmatococcus or Sphærella, which, by botanists at any rate, is regarded as a very simply organized member of the vegetable kingdom. Like Amœba, this organism is of microscopic size, consisting of only a single cell or protoplasmic unit.

Hamatococcus pluvialis (also known as Spherella lacustris) occurs in temporary pools of stagnant rain-water or, in the resting condition, in dried-up mud or dust. Though individually invisíble, or barely visible, to the naked eye, it may occur in such dense associations as to give the water a bright red (or sometimes green) colour and form a red crust on the sides of the vessel in which it is cultivated. A closely related, if not identical, species (Hcematococcus nivalis) is the cause of the red patches which are sometimes observed on the snow-fields in Arctic regions. Cultivation is easy, and the same stock may be kept going for many years and multiplied indefinitely. Twenty years ago or more I had a. sample given to me in Australia, descendants of which are now flourishing in full vigour in England. It can be dried up when not required and when wanted again in the active condition needs only to be supplied with fresh rain-water and placed in the sun.

In the resting condition each individual consists of a spherical protoplasmic body (Fig. 5, A) of a bright red or green colour, or sometimes partly green and partly red, with a more or less centrally placed nucleus (nu.). It differs from an Amøba in the presence of a very distinct, firm cell-wall (c.w.) on the outside, as well as in its definite shape and characteristic colour. The cellwall is composed of cellulose, one of a group of chemical compounds known as carbohydrates. These compounds are all characterized by the fact that they contain only three elements, carbon, hydrogen and oxygen, the hydrogen and oxygen occurring 
in the same proportions as in water $\left(\mathrm{H}_{2} \mathrm{O}\right)$. Thus the chemical formula for cellulose is $\left(\mathrm{C}_{6} \mathrm{H}_{10} \mathrm{O}_{5}\right)_{n}$, while that for glucose or grape sugar, another carbohydrate, is $\mathrm{C}_{6} \mathrm{H}_{12} \mathrm{O}_{6}$. The presence of very definite cell-walls, composed of cellulose and formed as a secretion by the living protoplasm, is very characteristic of vegetable as contrasted with animal organisms.

The protoplasm which lies inside the cell-wall is, as we have already said, either red, green or parti-coloured. The green
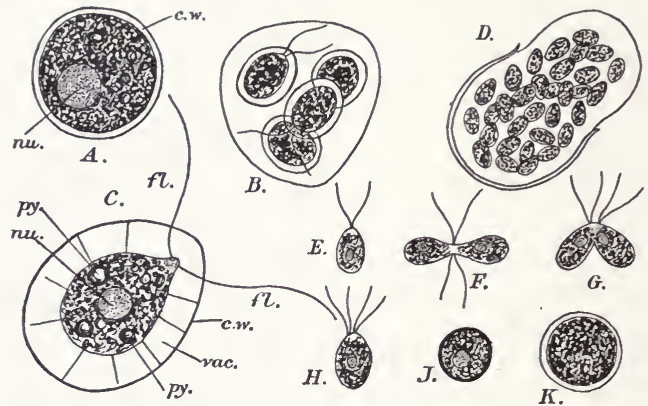

F1G. 5.-Structure and Life-history of Hamatococcus pluvialis.

$A$. Resting stage with thick cell-wall.

$B$. Division into four motile zoospores within the old cell-wall.

C. Free-swimming zoospore.

$D$. Division of the resting cell into 32 microzooids or gametes.

$\boldsymbol{E}$. Free-swimming gamete.

$F-G$. Conjugation of two gametes.

$H$. Zygote with four flagella, formed by conjugation.

$J$. Zygote with flagella withdrawn.

$K$. Resting cell formed from the zygote by secretion of a thick cell-wall.

$c . w$. cell-wall; $f$. flagellum; $n u$. nucleus; $p y$. pyrenoid ; vac. vacuole.

(Figs. $D-K$ adapted from Peebles.)

colour is due to the presence of that extremely characteristic vegetable pigment known as chlorophyll, a remarkable product of the activity of the living protoplasm with which we are all familiar in the ease of ordinary green plants. The red pigment, known as hæmatochrome, is but a slight chemical modification of the green chlorophyll, and the one may readily be converted into the other. If some nitrogenous substance, such as a small quantity of manure water, be placed in a vessel containing red Hæmatococcus, the latter will in a short time assume a bright green colour, whence we may conclude that the red colouration is probably an effect of nitrogen starvation. 
When a dried-up sample of Hæmatococcus is supplied with fresh rain-water and placed in the sunlight it undergoes a remarkable change. The protoplasmic body within the cell-wall undergoes division, first into two and then into four parts (Fig. 5, B). This is effected by a process of simple fission exactly comparable to that which we have already described in the case of Amœba. The four parts or daughter cells (sometimes called zoospores) are for a short time kept together within the old cellwall, but presently this ruptures and they escape into the surrounding water.

It will now be seen that these so-called zoospores differ greatly in structure from the resting Hæmatococcus. Instead of being spherical they are more or less oval or pear-shaped in outline (Fig. 5, C). Each has secreted a new cellulose wall of its own $(c . w$.$) , but this is separated from the main protoplasmic body by$ a considerable space, or vacuole, filled with water (vac.), across which stretch delicate threads of colourless protoplasm, which keep the protoplasmic body in position. The main mass of protoplasm is coloured red or green, as before, and contains the nucleus $(n u$.$) . At one end it is drawn out into a kind of beak,$ from which two very long and slender threads of colourless protoplasm $(f l$.) pass outwards, through minute apertures in the cellulose wall, into the surrounding water. Owing to their whip-like form and characteristic lashing movements, these are termed flagella.

It is by the very rapid movements of the flagella that the locomotion of the active Hæmatococcus is effected. They are carried in front, and the body of the organism is pulled through the water by their action much as a boat is pulled by a pair of sculls, at a rate which, though very slow when judged by our own standards, appears very rapid when considered in relation to the minute size of the organism. The movements of the flagella are somewhat complex and of an undulatory kind. They appear to be entirely automatic, but it seems probable that they must be performed in response to stimuli which we are unable to recognize. The flagella are much more definite and highly specialized structures than the pseudopodia of an Amoba. Like the latter, however, they owe their value as organs of locomotion to that inherent power of contraction which is one of the most characteristic features of living protoplasm. It is probable that each really consists of several very slender filaments, lying 
side by side, and that the complex undulatory movements are due to alternating contractions and relaxations of these.

The presence of a firm cell-wall makes the protrusion of pseudopodia in the case of Hæmatococcus quite impossible, and at the same time prevents the organism from taking in any solid food, for there is no aperture through which such food could pass. It must therefore depend entirely for its food supply upon substances which are able to pass through the cell-wall in a state of solution. These substances are all very simple chemical compounds, consisting of certain mineral salts and carbon dioxide gas, which, amongst them, contain all the elements necessary for the formation of protoplasm. They are, however, very stable bodies, with little or no affinity for oxygen gas and little potential energy. They cannot, therefore, by themselves supply the energy which the organism requires for its vital activities. Energy has to be supplied from the environment and the simple food materials thereby partially deoxidised and combined together in more complex and less stable molecules containing stores of potential energy which can be liberated by oxidation as required.

The energy which Hæmatococcus uses for the building up of its complex molecules is, as we have already observed for green plants in general, the radiant energy of the sun, in the form of light. The process is known as photosynthesis, and can only take place in organisms which possess chlorophyll or some functionally equivalent pigment, such as hæmatochrome. In some way or other the pigment absorbs the energy of the light rays and renders it available for the process of constructive metabolism (which in plants is also spoken of as assimilation).

The first step in this complex process involves a chemical decomposition, carbon dioxide, obtained in solution from the surrounding water, being decomposed with evolution of free oxygen gas.

We have already seen that in the combustion of charcoal the reverse of this takes place, carbon and oxygen combining to form carbon dioxide and the act of combination being accompanied by the liberation of energy. It is obvious that if energy is liberated in the one process a corresponding amount must be absorbed in the other.

When a glass jar of water containing Hæmatococcus, or any green aquatic plant, is placed in bright sunlight the decomposition 
of carbon dioxide in the plant takes place so rapidly that minute bubbles of oxygen may often be seen rising to the surface of the water. If, for example, we cut off a leafy branch of the common Canadian water weed, known as Elodea canadensis, and fix it under water in such a jar, it is possible to arrange the experiment so that a regular stream of small oxygen bubbles will be given off from the cut end, and it is further possible to adjust the experiment so delicately that the interposition of a dark screen between the jar and the sunlight will cause the immediate cessation of the. stream of bubbles, which will start again the instant the screen is removed. This simple and beautiful experiment clearly demonstrates the dependence of the process of decomposition of carbon dioxide upon the presence of sunlight.

The oxygen liberated in this way is not (with the exception of a relatively small quantity used in respiration) required by the organism, and is accordingly at once discharged into the surrounding medium. The carbon, on the other hand, is needed for the manufacture of new protoplasm. It is never actually set free as carbon, but its molecules are probably recombined-under the influence of the sunlight-with the molecules of water to form the carliohydrate known as glucose or grape sugar. This process may be represented by the equation-
$6 \mathrm{CO}_{2}$
$+6 \mathrm{H}_{2} \mathrm{O}=$
$6 \mathrm{O}_{2}$
$\mathrm{C}_{6} \mathrm{H}_{12} \mathrm{O}_{6}$
(Carbon Dioxide)
(Water)
(Oxygen)
(Glucose).

It is probable that a simpler compound, possibly formaldehyde $\left(\mathrm{CH}_{2} \mathrm{O}\right)$, is formed as an intermediate product, while, on the other hand, the glucose appears to be rapidly converted into starch, which is the first visible product of the process of photosynthesis in the plant cell.

Starch, like glucose and cellulose, is a carbohydrate, and, though differing in many of its chemical and physical properties, has the same general formula as the latter $\left(\mathrm{C}_{6} \mathrm{H}_{10} \mathrm{O}_{5}\right)_{n}$. This means simply that the elements carbon, hydrogen and oxygen are present in the same proportions as in cellulose, but they must be linked together differently in the molecule.

The first step in the actual construction of the proteid molecule is then the combination of carbon with the elements hydrogen and oxygen to form a carbohydrate. In the higher plants starch first appears in the chlorophyll-containing cells of the leaves in 
the form of starch grains, which may afterwards be converted into sugar again and then, in solution, transferred to other parts of the plant, where it is redeposited and stored up, once more in the form of starch grains, for future use, as in the potato and in starchcontaining seeds such as peas and beans.

Both starch and chlorophyll are, at any rate usually, formed in the cell in connection with specialized portions of the protoplasm known as plastids. These are regarded as living bodies which are specially concerned in the formation of chlorophyll, starch and other substances. When they contain chlorophyll they are termed chloroplastids, and in the higher plants they take the form of numerous minute "chlorophyll corpuscles" of definite shape, which occur in abundance in the cells of all green parts, and in connection with which the starch grains are formed (vide Fig. 26). In Hæmatococcus practically the whole central mass of cytoplasm is coloured by the chlorophyll (or hæmatochrome) and may perhaps be regarded as a single large chloroplastid. The starch, however, is collected around small, specialized, proteid bodies imbedded in the general mass of cytoplasm. These are known as pyrenoids (Fig. 5, C, py.).

We have thus seen how the green plant obtains the carbon, hydrogen and oxygen which it requires for the manufacture of protoplasm. Other elements, however, have to be combined with the molecules of carbohydrate before proteids can be formed. These are nitrogen, sulphur and, sometimes at any rate, phosphorus, all of which are obtained by green plants by the decomposition of mineral salts-nitrates, phosphates and sulphates-which exist in solution in the water or damp soil in which the plant grows. In the higher plants these substances are taken up by the root-hairs and transmitted to the leaves by a system of vessels and tracheids analogous to the circulatory system of animals. In such a plant as Hæmatococcus they simply diffuse into the protoplasmic body from the surrounding water by the process of osmosis. Exactly what happens when they meet with the carbohydrates we do not know, but further chemical combinations must take place under the influence of sunlight, which finally result in the formation of new proteid molecules which are added to the already existing protoplasm.

Respiration in Hæmatococcus probably takes place exactly as in Amœba, but it is more easily studied in the case of the higher plants. In daylight the process is greatly obscured by 
the absorption of carbon dioxide and the evolution of oxygen gas which accompany photosynthesis. In darkness, however, the gaseous interchange which forms the essential feature of respiration can readily be detected, carbon dioxide, produced by oxidation of the protoplasm, being given off and oxygen taken in.

No special organ of excretion has been observed in Hæmatococcus, though a contractile vacuole occurs in closely allied forms, and waste products must simply diffuse into the surrounding water through the permeable cell-wall.

We have already seen that Hæmatococcus multiplies itself by simple fission within the old cell-wall. This process usually results immediately in the production of four new individuals. Under favourable circumstances it may be repeated very rapidly, without the organism going through any true resting stage, so that in a short space of time the number of active zoospores may be very largely increased. The individuals thus produced are usually all of the same form, and ultimately of the same size, as the parent. Occasionally, however, a somewhat different process of multiplication takes place. Instead of dividing into four relatively large zoospores a resting individual may divide into thirty-two or sixty-four much smaller "microzooids" (Fig. 5, D), which differ from the ordinary active form in the absence of the characteristic cell-wall with its underlying vacuole.

The microzooids (Fig. 5, E) swim actively about by means of their flagella. Sooner or later, however, they come together in pairs (Fig. 5, F, G), and the members of each pair fuse completely with one another to form a single individual (Fig. 5, H) with four flagella, which presently loses its flagella, secretes around itself a thick cell-wall, and enters upon the resting state (Fig. 5, J, K). From this resting individual new generations will be produced by the ordinary method of division into zoospores.

We have here an excellent illustration of what is usually termed sexual reproduction, the essential feature of which is the union or conjugation of two sexual cells or gametes (in this case the microzooids) to form a single cell, the zygote, which is the starting point of a fresh series of cell generations, This important process will be discussed more fully in a subsequent chapter.

We have spoken of Amoba as an animal, and, as we have seen, many people regard Himatococcus as a plant. We must next endeavour to find out what it is that really differentiates a plant from an animal. Of course amongst the more highly organized 
members of the animal and vegetable kingdoms we can point to many obvious distinctions. The higher plants are fixed and stationary, while the animals move about from place to place by means of special organs of locomotion. The animals have complex digestive, respiratory, excretory, nervous and sensory organs, which are wanting in the plants. Lastly, the animals have no chlorophyll and cannot therefore, like the green plants, obtain their supplies of energy directly from the sun's rays by photosynthesis, but must depend upon the potential energy contained in the complex molecules of their food, which they obtain ready made from the bodies of other organisms.

Amongst the lower organisms, however, we find that most of these distinctions disappear. Thus many of the lower plants move about actively while many of the lower animals, such as the sponges, hydroids and corals, are fixed and stationary in the adult condition, though still showing their animal nature in other respects, such as their method of nutrition. This mixture of what were once regarded as distinctively animal and vegetable characters in such forms as the corals and hydroids gave rise to the name "zoophytes," or animal-plants, by which these organisms were known to the older naturalists.

When we descend to forms still lower in the scale of organization, consisting each of a single cell, we find that every distinction may disappear except that of the presence or absence of chlorophyll and the mode of nutrition immediately dependent thereon:--plant-like or holophytic as in Hæmatococcus, animallike or holozoic as in Amoba. ${ }^{1}$

It cannot be maintained, however, that even these characters form an absolute distinction between plants and animals, for, in the first place, many undoubted plants, such as the Fungi, have lost their chlorophyll by degeneration, and, in the second place, while botanists claim Hæmatococcus and the forms closely related to it as plants, zoologists claim them as animals, chiefly because they are so closely related in structure to other unicellular flagellates which contain no chlorophyll that we cannot refuse to include them in the same group.

1 The nutrition of any typical green plant is holophytic, that of any typical animal holozoic. The latter term implies the taking in of solid food derived from the bodies of other organisms, and is thus distinguished from the saprophytic type of nutrition met with in many of the lower animals and plants (e.g. Fungi), which consists in the absorption of liquid food derived from the decaying bodies of other
organisms. 
The explanation of the difficulty really lies in the fact that both plants and animals originally sprang from common unicellular ancestors which were neither the one thing nor the other. The first appearance of chlorophyll initiated the great cleavage between the animal and vegetable kingdoms. Thenceforward the two great groups developed each along lines of its own. By virtue of their chlorophyll the green plants became the great proteid manufacturers of the world, and the animals became dependent upon them for their food supply. Animals are largely dependent upon green plants in another respect also, for the latter, as we have seen, split up the carbon dioxide, formed as a waste product in the respiration of both groups, and thus set free fresh supplies of the necessary oxygen.

Closely correlated with the differences in their mode of nutrition are the great differences in the mode of life of the higher plants and animals. Plants have no need to move from place to place in search of food, which they obtain from the air and the soil, and the supplies of which are constantly renewed by wind and rain. Highly organized animals, on the other hand, would soon exhaust their supplies if they remained always in the same place, and it is doubtless the necessity for actively seeking out and even contending one with another for fresh supplies that has brought about the wonderful elaboration and perfection of their organization, while the fact that in so many eases they devour one another, instead of remaining directly dependent upon vegetable organisms, must have greatly intensified the struggle for existence and correspondingly increased the rate of progress in their evolution. 


\section{CHAPTER IV}

The cell theory-Unicellular organisms-Differentiation and division of labour-Co-operation-The transition from the unicellular to the multicellular condition-The early development of multicellular animals and plants.

WE have seen that, although Hæmatococeus and Amcba differ widely from one another in various respects, they nevertheless exhibit a fundamental agreement in structure, for each consists essentially of a single nucleated mass of protoplasm, each is a single cell.

The history of the term cell is a curious one, and affords a good illustration of the manner in which our scientific conceptions

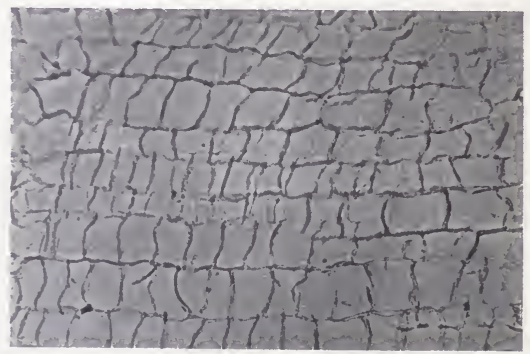

Fig. 6. - Thin Section of a Bottle Cork, showing cellular Structure, $\times 170$. (From a photograph.)

gradually become modified and improved as our knowledge increases. Nothing was known of cells before the invention of the microscope, but in the latter half of the seventeenth century this invention opened up an entirely new field of research, and enabled the earlier microscopists to lay the foundations of the modern science of biology. To Robert Hooke has been assigned the credit of first observing the cellular structure of vegetable tissues, and his observations, published in 1665 , were soon afterwards 
confirmed by Nehemiah Grew, who published his great work on the Anatomy of Plants in $\mathbf{1 6 7 2 .}$

It is now a matter of common knowledge that many vegetable tissues, such as cork and pith, when seen in section under the microscope, exhibit a honeycomb-like appearance, being composed

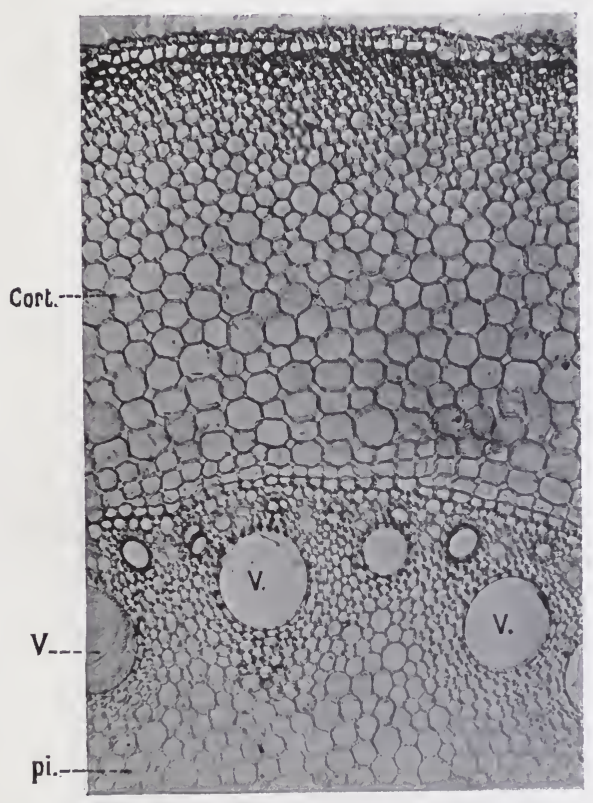

Fra. 7.-Part of a cross Section of a Maize Root, showing cellular Structure, $\times 84$. (F'rom a photograph.)

Cort., cortex; pi., pith; V., vessels.

of rectangular or polygonal, or it may be spherical chambers separated from one other by firm walls. This structure is very well shown in Fig. 6, which represents part of a thin section of an ordinary bottle cork, and in Fig. 7, which represents part of a transverse section of a root of maize. It was the resemblance to a honeycomb that led to the application of the term cell to these chambers. The earlier observers naturally attached most 
importance to the cell-walls, which indeed are alone visible in dead tissues such as dry cork and pith.

It was not until 1846 that von Mohl first gave the name protoplasm to the slimy contents of the cells in living tissues. It then gradually became evident that this protoplasm was the really vital constituent of the cell, and that it was identical in nature with the substance of which minute naked organisms such as the Amœba are composed, and which was already known by the name sarcode, given to it by Dujardin in 1835.

The cell theory, first propounded in a very imperfect form by Schleiden and Schwann, about the year 1838, rapidly developed, during the course of the nineteenth century, into one of the most fertile generalizations of natural science. At the present day the term cell is extended to protoplasmic units which may have no cell-walls at all, and to which therefore it is etymologically quite inapplicable, and for a long time a cell has been defined simply as a single nucleated mass of protoplasm.

In accordance with the cell theory such nucleated masses of protoplasm are the organic units of which the bodies of all living things are built up. The simpler organisms, such as Amœba and Hæmatococcus, consist each of a single unit only, and are therefore said to be unicellular, while the more complex forms, both of animals and plants, consist each of many such units united together in a multicellular body. Moreover, we now know that cells never originate de novo but multiply by division, so that each one is the immediate descendant of a pre-existing cell, a very important fact which was emphasized by Virchow in his often quoted phrase "Omnis cellula e cellula."

The zoologist includes under the name Protozoa all those unicellular organisms which he claims as members of the animal kingdom, whilst the unicellular plants are relegated to the domain of the botanist under the name Protophyta, but, as we have already seen, it is impossible to draw a rational line of demarcation between these two groups and they are often included together under Haeckel's term Protista.

The outstanding feature in all these simple forms of life is that the single cell is a complete and self-supporting organism. It has to perform all the necessary vital functions for itself, by means of such simple organs, temporary or permanent, as can be produced by differentiation within the microscopic limits of its 
protoplasmic body. ${ }^{1}$ It is surprising what a high degree of organization, as indicated by complexity of structure, may be attained in such a case.

Differentiation and division of labour aretheresults of progressive evolution, and at the same time the means by which further progress is effected. Even in Amœba and Hæmatococcus we see clearly enough the operation of these two great principles. Many unicellular organisms, however, exhibit a far higher degree of organization. The Protozoon, Paramocium (Fig. 8), so common in infusions of decaying vegetable matter, swims actively about by means of innumerable short vibratile cilia which project all over the surface of the body. It has a definite mouth, through which

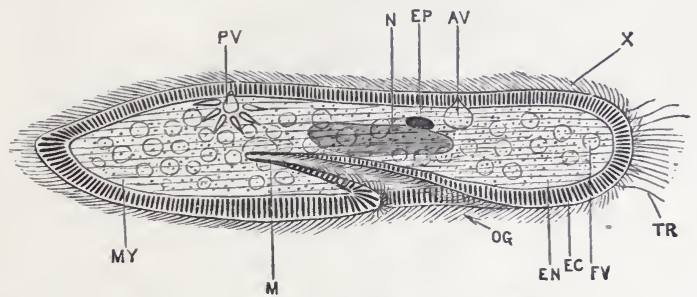

Fig. 8.-- Paramoecium aureliu, $\times$ 300. (From Marshall and Hurst's "Practical Zoology.")

$\mathrm{AV}$, anterior contractile vacuole (dilated) ; EC, ectoplasm with trichocysts; EN, endoplasm; EP, micronucleus; FV, food vacuole; M, mouth; MY, contractile fibrillæ; $\mathrm{N}$, meganucleus; $\mathrm{OG}$, groove leading to mouth: $\mathrm{PV}$, posterior contractile vacuole (contracted); TR, discharged trichocyst threads; $\mathrm{X}$, cilia.

solid food particles are taken in, and a less definite anal spot at which fæcal matter is ejected. It has special weapons of offence or defence (trichocysts) which can shoot out from the surface of the body long threads when the animal is irritated. It has two contractile vacuoles, each with a system of radiating canals discharging into it, and it has two nuclei, large and small (meganucleus and micronucleus), which appear to fulfil different functions and each of which doubtless has a complex structure of its own. How complex the structure of the nucleus may be we shall be better able to judge when we come to speak of the phenomena of nuclear division in a subsequent chapter.

1 The organs into which a single cell may be differentiated are sometimes spoken of as organellæ, but if we define an organ as any part of an organism which is specialized for the fulfilment of some particular function, it is quite unnecessary to distinguish the organs of a single cell by a special term. 
Paramœeium and many other Protozoa, again, have specialized contractile threads of protoplasm lying just beneath the surface of the body, comparable to the muscle fibres of higher animals, which enable them to perform movements of a different kind from those effected by flagella or cilia. By means of such contractile fibres, localized mainly in a long stalk, the bellanimalcule, Vorticella (Fig. 9, 10), can instantaneously draw in its ciliated disc and pull itself out of harm's way on the approach of danger. Other examples of these highly organized ciliate Protozoa (Infusoria) are shown in Fig. 9.' These forms should be compared with the Radiolaria and Foraminifera represented in Figs. 3 and 4.

Another very important factor in the progress of organic evolution is supplied by the principle of co-operation between different organic units. This is illustrated to some extent in the process known as colony-formation met with in certain Protozoa and Protophyta. Carchesium, Epistylis and Zoothamnium (Fig. 9, 11-15), for example, are colony-forming Infusoria closely related to Vorticella. Vorticella itself multiplies rapidly by simple longitudinal fission. The bell-shaped protoplasmic body at the end of the stalk divides into two parts, one of which swims away, attaches itself to some foreign object, and develops a new stalk. If, instead of separating, the two daughter cells remained together and went on dividing, and if this division also extended each time to the upper part of the stalk, the organism would presently arrive at a branching, tree-like condition. This is what has happened in Carchesium, Epistylis and Zoothamnium, and also in various other Protozoa.

This arborescent type of colony-formation, moreover, is by no means the only one met with amongst the Protista. It is characteristic of stalked forms. Other forms, which are not stalked, may give rise to free-swimming or floating, solid or hollow aggregates of plate-like or it may be spherical shape. The development of such colonies is perhaps nowhere better seen than in the small group of unicellular organisms to which Hæmatococcus belongs, the members of which are known, on account of their plant-like character and their possession of flagella, as Phytoflagellata.

Hæmatococcus, or the closely related Chlamydomonas, may be taken as the starting point of the series. These two are always solitary, the individuals separating completely from one another 


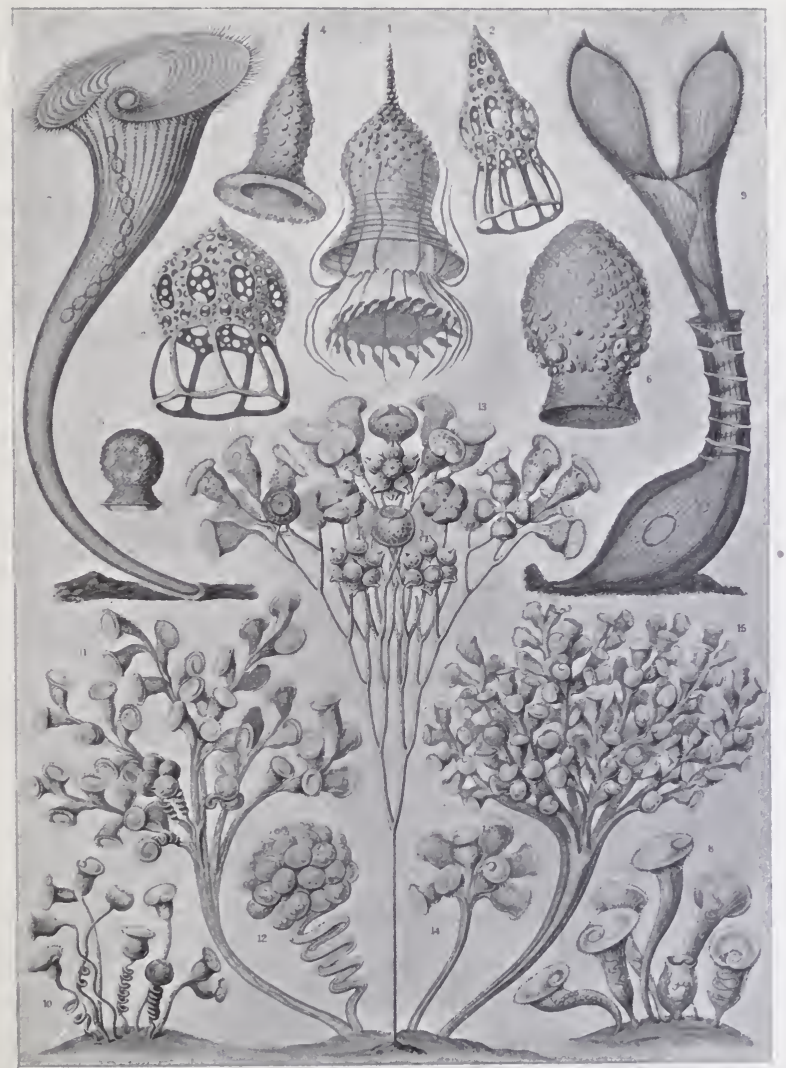

Fıg. 9.-Various forms of Ciliate Protozoa (Infusoria), highly magnified. (From Haeckel's "Kunstformen der Natur.")

1, Codonella ; 2, 3, Dictyocysta (shell only); 4, Tintinnopsis (shell only); 5, Cyttarocyclis (shell only); 6, Petalotricha (shell only); 7, 8, Stentor; 9, Freia; 10, Vorticella ; 11, 12, Carchesium; 13, Epistylis; 14, 15, Zoothamnium. 
after each cell-division. In Pandorina (Fig. 10), however, which is another common fresh-water organism, the individuals produced by fission remain together and form a solid ball, composed of from sixteen to sixty-four cells, enclosed in a gelatinous envelope. These little colonies swim about actively by means of their flagella, which project from the surface in pairs, one pair belonging to each individual. Multiplication is usually effected by the division of each individual cell into sixteen, so that as many daughter colonies are formed as there were cells in the parent colony, and these daughter colonies finally separate

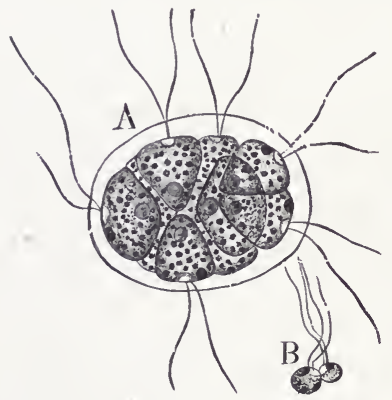

FIG. 10.-Pandorina morum, $\times 400$. (From Vines' "Botany.")

A, free-swimming colony; $\mathrm{B}$, conjugation of two gametes.

from one another. Eudorina (Fig. 40) forms slightly larger colonies of a similar kind, but with the component individuals somewhat widely separated from one another by the gelatinous matrix. Finally, in Volvox (Fig. 11), one of the most familiar and beautiful of the microscopic fresh-water organisms, we find the individual cells, each one still closely resembling a Hæmatococcus, arranged side by side in the gelatinous wall of a hollow sphere, with their flagella projecting from the surface, while daughter colonies are frequently seen swimming about freely in the interior of the sphere.

In all these colonies the gelatinous matrix or ground substance is formed as a secretion by the cells which it serves to hold together. It is noteworthy that the connection between the individual cells is much more intimate in Volvox than it is in the lower types, for they are all united together by extensions of their protoplasmic bodies which give a reticulate appearance to the wall of the sphere. Volvox, moreover, attains a relatively large size, and there may be as many as 22,000 cells in a single colony, though about half that number appears to be more usual.

We meet with another example of the formation of hollow spherical colonies in the case of the beautiful Radiolarian, 
Sphærozoum (Fig. 12), which floats about in the surface waters of the open ocean and has in each of its component cells a supporting skeleton of branched siliceous spicules.

The co-operation of a larger or smaller number of cells to form a colony at once opens up new possibilities with regard to

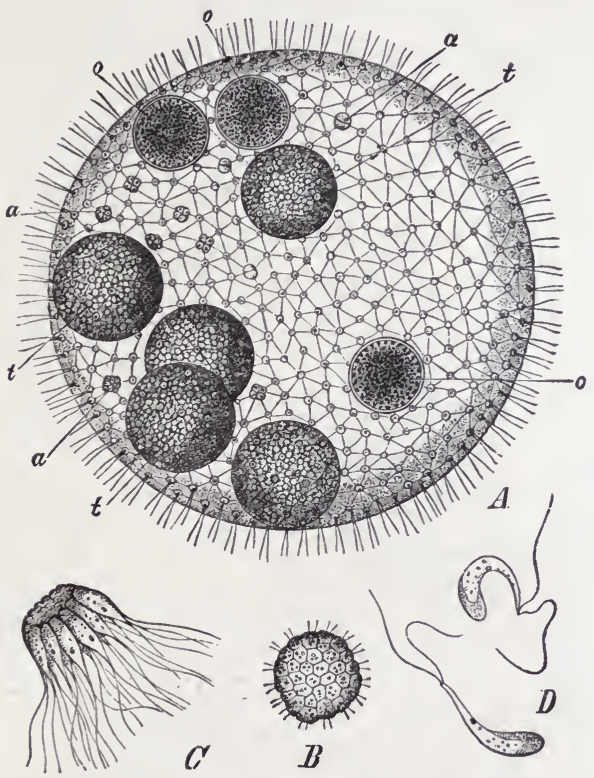

Fig. 11.-Volvox aureus. (From Weismann's "Evolution Theory," after Klein and Schenck.)

$A$, mature colony containing daughter colonies $(t)$ and ova $(o) ; B$, group of 32 developing spermatozoa seen end on; $C$, the same seen sideways; $D$, mature spermatozoa, $\times 824$.

differentiation and division of labour. If a sufficiently good understanding, so to speak, can be established between the different members (or zooids) of the colony it will no longer be necessary for each one to do everything for itself. At the expense of becoming mutually dependent upon one another they will be able to specialize in different directions, some identifying themselves with one necessary duty or function and some with 
another. As a result of this specialization the various functions may be much more efficiently and economically performed, but a no less important result will be that the different individuals will no longer be able to lead independent lives-if separated from one another they will perish because unable to perform for themselves individually all the functions which are necessary for their existence.

In colonies of Protista we meet with little if any of this differentiation and division of labour; from the physiological point of view the cell units remain almost if not quite independent of one another, and that is why they are still classed

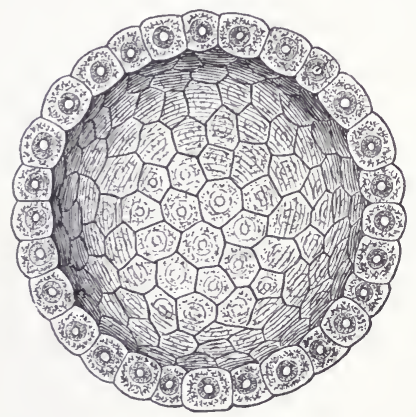

Fig. 12.-A colony of Sphærozoum, cut in half. (After Haeckel, in "Challenger" Report.) amongst the unicellular organisms. There can be no doubt, however, that it was this habit of colony-formation that led to the origin of true multicellular animals and plants-Metazoa and Metaphyta-from unicellular ancestors. The component cells of a colony gradually became integrated to form an individual of a higher order, and this process was accompanied by that differentiation and division of labour which in course of time led to the astonishing complexity of structure which characterizes the higher members of both the animal and vegetable kingdoms. In contrast to this complexity of the organism as a whole we shall find that the individual cells of one of the higher animals or plants are usually much simpler in structure than the more highly organized Protista. After what we have said about differentiation and division of labour the reason for this should be sufficiently obvious.

Perhaps no more convincing demonstration of the applicability of the cell theory to the higher plants and animals could be given than that which is afforded by the study of development, for, however highly organized a plant or an animal may be in the adult condition, it always commences its individual existence as a single cell-the fertilized ovum or zygote-and attains the 
adult state by means of a longer or shorter series of cell-divisions. The earlier cell-divisions constitute what is termed the segmentation of the ovum, and result in the formation of a number of daughter cells, which exhibit little or no differentiation amongst themselves and are known as blastomeres. Sooner or later, however, differentiation sets in and leads to the formation of more or less highly specialized tissues or cell aggregates.

In the primitive, fish-like Amphioxus, ${ }^{1}$ for example, the ovum (Fig. 13, I) is a spherical, nucleated cell about $\frac{1}{250}$ th inch in diameter. After fertilization by a male gamete or spermatozoon it divides first into two equal and similar embryonic cells or blastomeres (Fig. 13, II) by a vertical cleavage. Another vertical cleavage, at right angles to the first, divides each blastomere into two smaller ones (Fig. 13, III). This is followed by a horizontal cleavage, which results in the formation of eight cells, in two tiers of four each, the four upper ones being slightly smaller than the four lower (Fig. 13, IV). The blastomeres go on dividing and presently arrange themselves in the form of a hollow sphere, whose wall is composed of a single layer of cells (Fig. 13, VII). The embryo has now reached the blastula (or blastosphere) stage of its development, a stage which is passed through by all multicellular animals whose life-history follows a typical course unmodified by secondary features.

If we allow for the fact that the cells all remain together instead of separating from one another after each division, it is obvious that the segmentation of the fertilized ovum into blastomeres is identical with the process of multiplication by fission in such a protozoon as Amœba. The fact that each blastomere is equivalent to a single protozoon and multiplies in a similar manner has been experimentally demonstrated in an extremely interesting way by Herbst, whose observations were made upon the development of the common sea urchin (Echinus). $\mathrm{He}$ found that, if the eggs are allowed to develop in sea water from which every trace of calcium has been removed, the blastomeres actually do separate after each division and give rise to 808 individual cells, which swim about separately like so many flagellate Protozoa instead of remaining united together and co-operating with one another to form the normal blastula. The blastula stage itself, of course, corresponds very closely in general features with such a protozoon colony as we meet with in the case

1 The external appearance of the adult Amphioxus is represented in Fig. 118. 


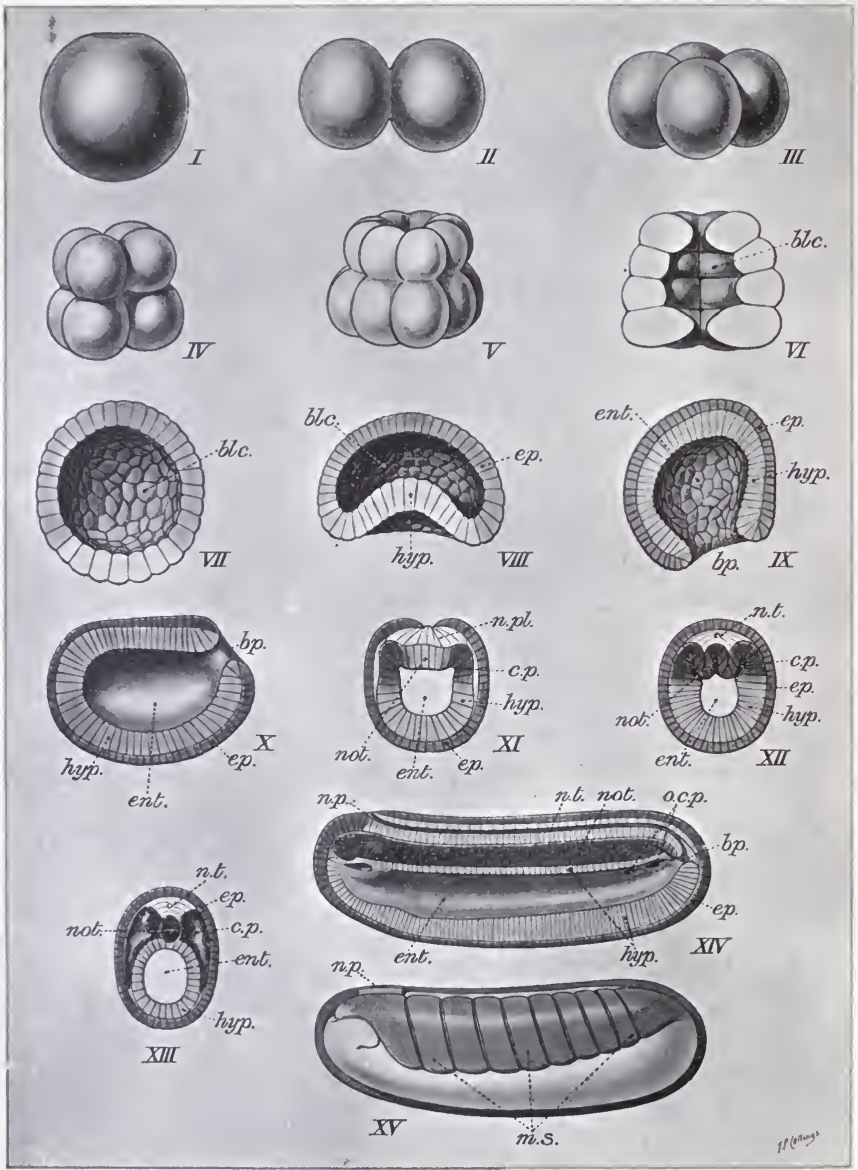

Fig. 13.-Early Developmentı of Amphioxus. (Adapted from Ziegler's Models, after Hatschek.)

$I$, the fertilized egg; II, two blastomeres formed by the first cleavage; III, stage with four blastomeres; $I V$, stage with eight blastomeres; $V$, stage with sixteen blastomeres; $V I$, stage with thirty-two blastomeres, cut in half vertically; $V I I$, blastula or blastosphere stage, cut in half; VIII, early stage of gastrulation, cut in half; 
of Volvox or Sphærozoum (compare Figs. 11 and 12), a point to which we shall have occasion to return in a later chapter.

In the blastula of Amphioxus the cells are still all very much alike, except that those at one pole of the sphere are somewhat larger than the others. Differentiation, however, now sets in in a very marked manner, and the cells thereby become divided into two distinct groups. That portion of the wall of the hollow blastula which is formed by the larger cells becomes pushed inwards or invaginated (Fig. 13, VIII), much as a tennis ball may be pushed in by the pressure of the thumb, until it comes into contact with the inner surface of the remainder of the wall. In this way the original cavity (blastocol) is obliterated and the embryo takes on the form of a double cup (Fig. 13, IX, X). The cavity of this cup is an entirely new formation. It is the primitive digestive cavity of the animal and is known as the enteron or gastral cavity. Its mouth gradually contracts to a narrow aperture, the blastopore. The outer layer of cells forming the wall of the cup is termed the epiblast and the inner the hypoblast, and the two are continuous with one another all round the blastopore. The stage now reached is spoken of as the gastrula stage.

In nearly all Metazoa the blastula stage of development is followed by one exhibiting the essential features of the gastrula, or at any rate some indication thereof. The primary differentiation of the component cells of the body into an outer epiblast (which becomes the ectoderm of the adult) and an inner hypoblast (which becomes the endoderm of the adult), the one serving for protection and for the maintenance of all the necessary relations with the external environment, and the other surrounding a gastral cavity and concerned with the digestion of the solid food which the animal captures, is closely correlated with the characteristic animal or holozoic method of nutrition.

In later stages of development, in all animals higher than the

$I X$, young gastrula in longitudinal section; $X$, older gastrula in longitudinal section; $X I, X I I$, XIII, transverse sections of older embryos, showing the formation of the coelomic pouches, notochord and neural tube; $X I V$, longitudinal section of embryo of about the same age as $X I I ; X V$, side view of embryo of same age as $X I V$ with the epiblast stripped off from one side to show the mesoblastic somites formed from the coelomic pouches.

$b p$. blastopore ; blc. blastocol or segmentation cavity ; c.p. coelomic pouch; ent. enteron ; $e p$. epiblast; hyp. hypoblast; m.s. mesoblastic somites; not. notochord ; n.p. neuropore or anterior opening of neural tube; n.pl. neural plate; n.t. neural tube (central nervous system, formed by folding of neural plate); o.c.p. openings of cœlomic pouches into enteron. 
Coelenterata (a group which includes such forms as jelly-fish, sea anemones and corals), a third layer of cells makes its appearance between the first two and forms the mesoblast (giving rise to the mesoderm of the adult). In Amphioxus this mesoblast arises as a series of hollow, pouch-like outgrowths of the hypoblast (Fig. 13, $\mathrm{XI}, \mathrm{XII}, c . p$. ), and the cavities which these pouches contain give rise to the body cavity or coelom, while their walls give rise to mesodermal tissues.

In accordance with what is commonly known as the germlayer theory, these three layers of cells, epiblast, mesoblast, and hypoblast, can be recognized in the embryos of all the higher animals, and from them all the various parts of the adult body are derived. The epiblast gives rise to the outer skin or epidermis, the nervous system and the essential parts of the sense-organs; the hypoblast gives rise to the lining epithelium of the alimentary canal and its various outgrowths, including the digestive glands, while from the mesoblast arise the various connective and skeletal tissues, the blood-vessels and the essential organs of reproduction. The cells of which each tissue is composed acquire a characteristic structure of their own, and in this way the histological differentiation of the body is gradually completed.

With the early stages in the development of Amphioxus, described above, we may compare the corresponding processes in the life-history of a flowering plant. Here the ovum, instead of being at once liberated from the parent, undergoes all the earlier stages of its development within the so-called ovule, which, with the contained embryo, will ultimately be set free as the ripe seed (compare Chapter VIII.).

As a definite example we may take the common weed known as shepherd's purse, Capsella bursa-pastoris (Fig. 14). The ovum lies in a cavity in the ovule termed the embryo-sac. It is at first a single nucleated mass of protoplasm without any cell-wall. After fertilization it divides into two cells separated by a wall, and the process is repeated by a series of divisions parallel to the first one until we have a row of cells. The cell at one end of this row, known as the basal cell (Fig. 14, A, B, b.c.), is larger than the others and is attached to the wall of the embryo-sac. At the opposite end is a rounded cell, called the embryonic cell, from which the body of the young plant will be chiefly formed. The remainder of the row, including the basal cell, is known as 
the suspensor, and serves for the attachment and nutrition of the embryo, which at first develops entirely at the expense of the parent, having no means of feeding itself. All the cell-divisions

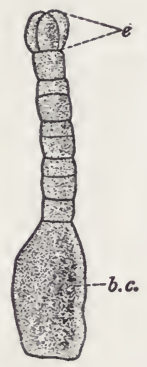

A

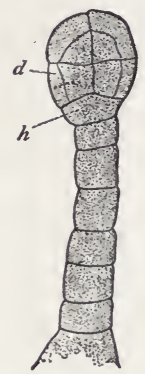

C

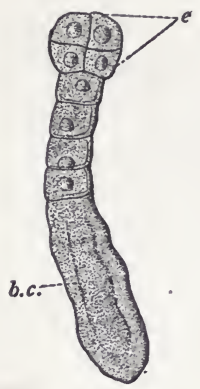

B

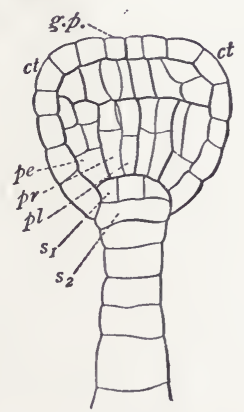

D

Fir. 14.-Four stages in the early Development of a Flowering Plant, C'apsella bursa-pastoris, $\times 200$. (From S'cott's "Structural Botany," after Hanstein.)

b.c., basal cell of suspensor; ct., cotyledons growing out; $d$, dermatogen; $e$, embryonic group of cells; g.p., growing point of stem; $h$, uppermost cell of suspensor; $p e$, periblem ; $p r, p l$, cells of plerome; $s_{1}, s_{2}$, cells derived from $h$.

so far have taken place in planes parallel to one another, thus giving rise to a single row of cells, but the embryonic cell now divides into four parts by the formation of two cell-walls at right angles to each other and to the preceding divisions (Fig. 14, $A, e)$, and each of these again divides into two by the formation 
of a wall parallel to the earlier divisions (Fig. 14, B,e). The embryo now consists of eight cells or " octants." The next divisions are parallel to its surface and cut off a superficial covering of cells, known as the dermatogen (Fig. 14, C, d), from which the whole of the epidermis of the adult shoot will be derived. The mass of rapidly dividing cells within this soon becomes differentiated into two parts, the plerome (Fig. 14, D, $p r, p l$ ), lying in the axis of the embryo, and the periblem (Fig. 14, D, pe), lying between the plerome and the dermatogen. The plerome will give rise to the vascular system of the plant and the tissues associated therewith, while the periblem will give rise to the cortical tissues of the stem and root and the mesophyll or middle layer of the leaves. The periblem of the root, and the root-cap, are really formed from the uppermost cell of the suspensor (Fig. 14, C, $h$ ). By further cell-multiplication and differentiation in the three primary layers-dermatogen, periblem and plerome-all the various tissues of the adult plant are produced.

Thus we see that in the higher plants and animals alike the development of the individual from the fertilized egg consists in the first place of a process of cell-division, and in the second place of differentiation between the cells thus produced, accompanied by grouping of the differentiated cells to form the more or less sharply defined tissues of the adult. 


\section{CHAPTER V}

The cell theory as illustrated by the histological structure of the higher animals and plants-Limitations of the cell theory-The cell as the physiological unit.

IN all the higher animals and plants the constituent cells of the adult body are grouped in more or less well defined tissues, which originate from the fertilized ovum in the manner indicated in the last chapter, and the cells of each tissue co-operate with one another in the fulfilment of some common function. The study of the microscopic structure of tissues is termed histology.

As examples of animal tissues we may take blood, epithelium, fat, cartilage, muscular tissue and nervous tissue, as met with in typical vertebrates.

Blood is exceptional in that it is a liquid tissue, a condition which is of course necessary in order that it may circulate through the blood-vessels and perform its functions as the distributor throughout the body of food material and oxygen, and the carrier of carbon dioxide and other waste products from all the various parts of the body to the special organs of respiration and excretion. Floating in the liquid portion, or plasma, are found two kinds of cells, the white and red blood-corpuscles.

The white corpuscles, or leucocytes (Fig. 15, a.), closely resemble Amœbæ. They are colourless, nucleated cells, exhibiting amoboid movements, and they have the remarkable power of creeping through the thin walls of the blood-capillaries into the surrounding tissues. Like the Amoba they feed, in part at any rate, by taking in and digesting the bodies of other minute organisms, and they grow and multiply by simple fission. They exhibit a much greater degree of independence than most of the cells of the body and can even live outside the body for a time if kept in suitable culture media and at the proper temperature. Their most important function appears to be to defend the body from the attacks of bacteria and other harmful micro-organisms. 
If these gain entrance into the tissues at any weak spot they are set upon by the leucocytes and literally devoured. This process is known as phagocytosis, and in this capacity the leucocytes are often spoken of as phagocytes. It is obvious that the health of the body must depend largely upon the activity of the phagocytes and their efficiency in dealing with disease-producing " germs."

The white blood-corpuscles, then, differ in no essential particular as regards their structure and mode of life from so many Protozoa. It is true they cannot live permanently outside the

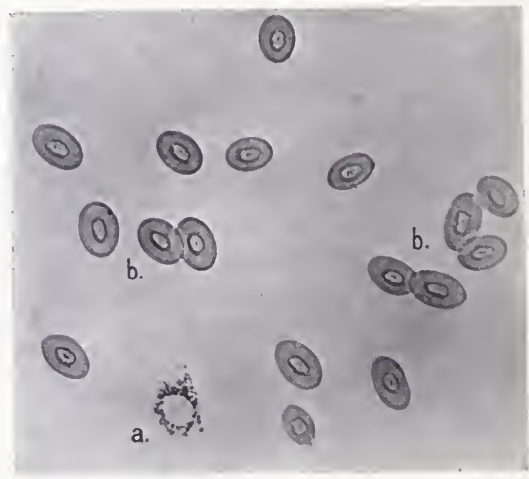

FIG. 15.-Blood Corpuscles of the Frog, $\times$ 326. (From a photograph.)

a., white corpuscle or leucocyte; b., red corpuscles or hæmatids. The nuclei appear light-coloured in the photograph owing to their having been stained blue in the preparation.

body, but that is also the case with many parasitic Protozoa which live in the blood of other animals. That they are not independent organisms, but form an integral part of the body in which they occur, is, however, obvious from the fact that they have a common origin with all the other tissues from the developing ovum.

The red corpuscles, or hæmatids, are very different bodies. They float passively in the blood-stream and serve as the carriers of oxygen gas from the respiratory organs to the various tissues. Unlike the leucocytes they have definite and constant outlines, though, owing to the flexible nature of the thin cell-membrane by which they are enclosed, they may undergo temporary distortion. 
Individually of a pale yellow colour, and so small as to be quite invisible to the naked eye, they occur in such vast numbers as to give the blood its characteristic scarlet or purple colour. It is estimated that in a cubic millimetre of human blood there are about five millions of these red corpuseles.

In the frog the hæmatids are flattened oval cells about $0.02 \mathrm{~mm}$. in longer diameter, with a centrally placed nucleus (Fig. 15, b.). In man they are a good deal smaller, and circular in outline, like biscuits, and, as in all the Mammalia, the nucleus

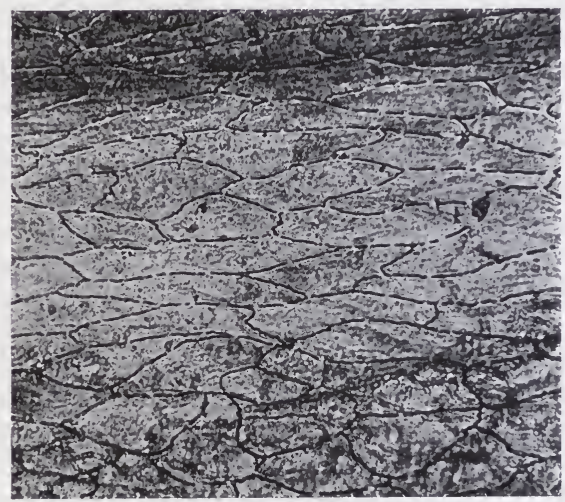

Fig. 16.-Epithelium from the Mesentery of a Frog, $\times 280$. (From a photograph.)

The underlying tissues are seen indistinctly through the transparent epithelial cells, whose outlines only are visible.

has entirely disappeared. They owe their red colour, and their power to act as carriers of oxygen, to the presence in them of a peculiar pigment known as hæmoglobin, with which the oxygen appears to enter into a state of loose chemical combination from which it is easily liberated again when required by the tissues. They may indeed be regarded as mere bags of hæmoglobin, formed from highly specialized cells which have lost all power of independent existence. They cannot even multiply by division, but, as the old ones are worn out, they are replaced by the formation of new ones from less specialized cells in various parts of the body. 
The term epithelium is applied to any layer of cells covering a free surface. An epithelium is therefore primarily a protective layer, but it frequently becomes modified for other purposes. It may, for example, become glandular, certain of its cells taking on the function of secretion, or it may become sensory, with cells specially adapted for the reception of stimuli. It may consist of a single layer of cells or of several layers one above the other.

A good example of the single-layered type is found in the peritoneal epithelium which covers the surface of the mesentery or membrane supporting the intestines in the cœlom or body cavity. Fig. 16 represents a portion of such an epithelium in which the cell-outlines have

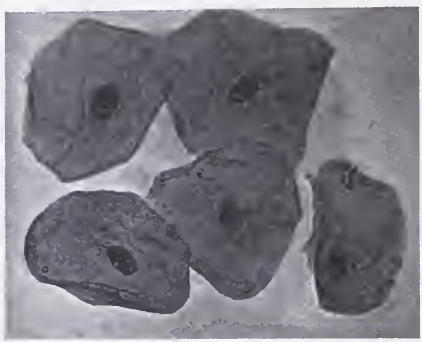

Fig. 17.-Five isolated Epithelial Cells from theinner Surface of the human Cheek, $\times 420$. (From a photograph.)

The nuclei are stained darkly. been rendered very distinct by staining with silver nitrate; the nuclei, however, are not shown by this method. Each cell has the form of a thin, flat, polygonal plate, and they all fit accurately together at their edges. With this Figure should be compared Fig. 28, A, which represents a single-layered epithelium prepared in such a way as to show both nuclei and celloutlines.

If we gently scrape the inside of the cheek with some clean, blunt instrument, and examine the milky-looking product under the microscope, we shall find that it contains a number of flattened, scale-like bodies (Fig. 17), either entirely separated from one another or still more or less connected together by their edges, and probably to some extent overlapping. These also are epithelial cells, which have formed part of the special epithelium known as the epidermis, which is derived from the epiblast or external cell-layer of the embryo and covers the outer surface of the body. If we examine our preparation more carefully we shall find that the cells have an irregularly rounded contour and that they measure about $0.08 \mathrm{~mm}$. in diameter. There is a more or less centrally placed nucleus (which appears dark in the figure owing to the manner in which it has been stained) and the 
cytoplasm is granular. They are, however, dead, or at any rate moribund cells. Owing to the constant friction to which the

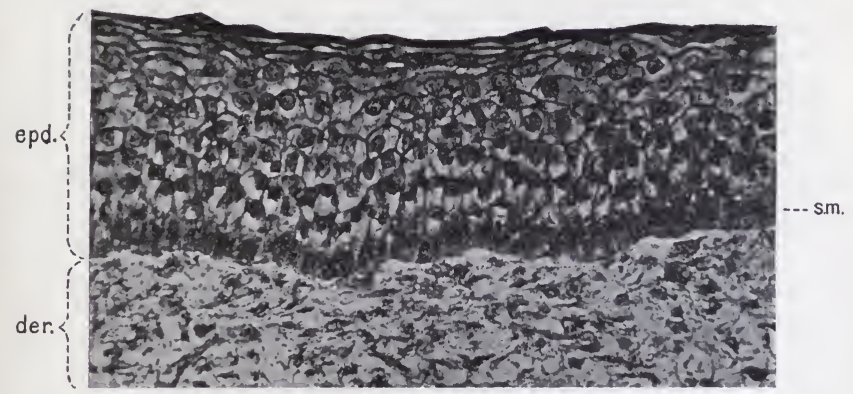

FIG. 18. - Vertical Section of Stratified Epithelium from the Mouth of a fotal Cat, $\times$ 280. (From a photograph.)

der., dermis; epd., epidermis; s.m., Stratum Malpighii, or layer of actively dividing cells.

surface of the body is exposed such cells are always being rubbed off, and it is these which, by accumulating in places where the friction is less severe, form the so-called scurf of the hair.

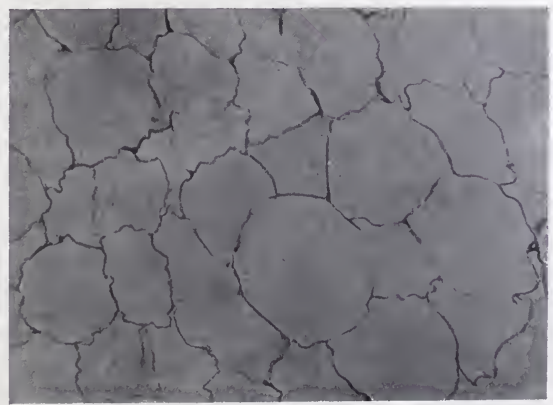

Fia. 19.-Section of Adipose Tissue from which the Fat has been dissolved out, leaving the thin-walled Cells empty and shrivelled, $\times 175$. (From a photograph.)

As they are worn away their places are taken by other cells which arise from a deeply situated layer, at the lower limit of the 
epidermis, in which cell-division goes on actively throughout life. In this way a many-layered or stratified epithelium is formed, as shown in Fig. 18, which represents a small portion of a thin vertical section through the epidermis (epd.) in the mouth of a foetal cat. At the lower limit of the epithelium, resting immediately upon the connective tissue of the dermis (der.), is seen the layer of actively dividing cells (s.m.). The cells cut off from this layer are gradually pushed outwards, becoming flattened and scale-like as they approach the surface.

Fat, or adipose tissue, consists of an aggregation of more or

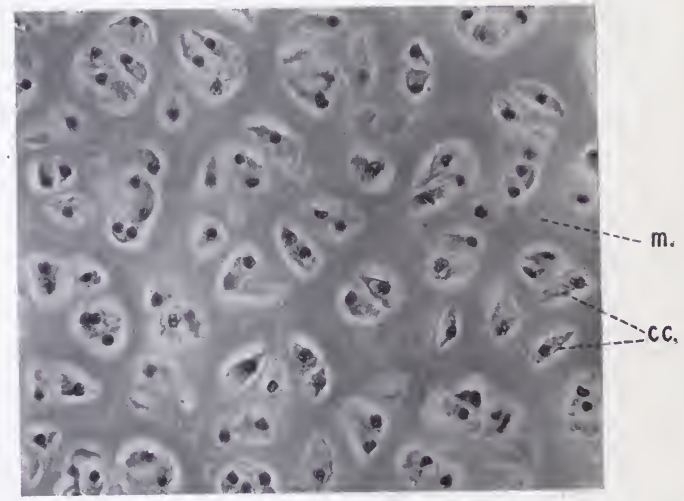

FIG. 20.-Section of Cartilage, showing the Cartilage Cells (c.c.) imbedded in the transparent intercellular Matrix or Ground Substance $(\mathrm{m}),. \times 390$. (From a photograph.)

less globular cells, swollen out by the accumulation within them of drops of oil. If the oil is dissolved out by suitable reagents the empty cells are left with their cell-walls or membranes in a somewhat shrivelled condition, as shown in Fig. 19, and the tissue now bears a curious resemblance, when seen in section, to vegetable parenchyma, such as is seen in sections of pith (compare Fig. 7).

Cartilage, or gristle, is one of the skeletal tissues, serving for the support of the body and the protection of special organs. In some of the lower vertebrates, such as the dog-fish, it forms practically the whole of the internal skeleton, but in higher forms 
it is to a greater or less extent supplemented or even replaced by bone. It consists mainly of a tough, translucent matrix, or intercellular substance, which is formed as a secretion by the cartilage cells and in which the latter are imbedded at wide intervals (Fig. 20). In this respect it differs greatly from the epidermis, in which the cells lie close together and little or no intercellular substance is developed. The cartilage grows by repeated division of the cells which it contains and the secretion of additional intercellular matrix between them. The frequent arrangement of the nucleated cells in pairs, as shown in the illustration, is an indication of recent celldivision. Bone is a more complex tissue than cartilage and is further strengthened and hardened by the deposition of calcareous salts, chiefly phosphate of lime, in the matrix.

Muscular tissue is specialized in a totally different direction from any of the foregoing. Its function is to contract, and by so doing to bring about the various movements of which the higher animals are capable. There are two very distinct kinds of muscular tissue, the one comparatively simple and the other much more complex in structure. The former, which is known as unstriped muscle (Fig. 21), consists of greatly elongated cells, the musclefibres, associated in sheets or bundles. Each has a centrally placed nucleus and its cellular nature is at once obvious. The wall of the alimentary canal, outside its lining epithelium, is composed chiefly of muscle-fibres of this

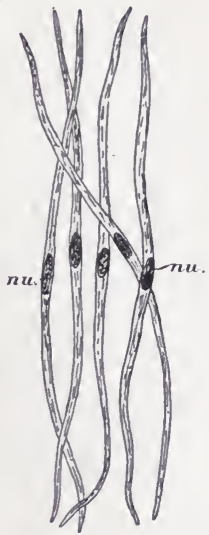

Fig. 21.-Unstriped Muscle Fibres from the Wall of the Rabbit's Intestine, $\times 300$.

nu., nuclei. kind. Their rhythmical and co-ordinated contraction causes the characteristic peristaltic movement whereby the onward passage of the food is secured. These and similar movements effected in other organs by the action of unstriped muscular tissue take place quite independently of the will-whence the term "involuntary" is often applied to this type of muscle.

Striped or striated muscular tissue is usually under the control of the will, and is hence often spoken of as "voluntary," but it is found in the higher animals wherever very sharp, precise movements are required, as for example in the walls of the heart, the 
contraction of which serves to pump the blood through the bloodvessels. It is more especially associated, however, with the movements of the limbs, the bones of which form a system of levers operated by the muscles which are attached to them. It differs greatly in minute structure from unstriped muscle, though consisting essentially of greatly elongated, nucleated fibres endowed with remarkable powers of contraction. These fibres, and the fibrillæ into which they are subdivided, are characterized by a transverse striation of alternate light and dark bands. 'I'heir structure is very complex and in the fully developed muscle it is difficult if not impossible to recognize the limits

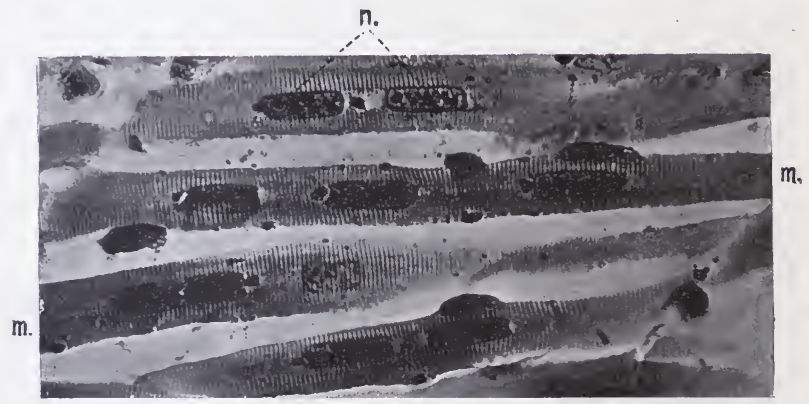

Fig. 22. - Striped Muscle-Fibres (m.) from the Tail of a larval Axolotl, showing their nuclei (n.), $\times 560$. (From a photograph.)

between the constituent cells. Fig. 22 represents a number of striated muscle-fibres from the tail of a larval axolotl, in which each fibre is seen to be provided with several distinct nuclei.

'The nervous system, as we have already pointed out, serves to place the different parts of the body in communication with one another and exercises a controlling and co-ordinating influence over the whole, while through the mediation of the special organs of sense it keeps the organism in close touch with its environment. The tissue of which it is composed (Fig. 23) consists of nerve-cells and nerve-fibres, but the fibres are merely outgrowths of the cells. A cell and fibre together form a neuron -a single unit of the nervous system. The nerve-cells, or rather their bodies, occur chiefly in the brain and spinal cord, which constitute the central nervous system, but also in small 
local aggregations, or ganglia, in various parts of the body. The nerve-fibres extend outwards from the central nervous system in long, slender bundles, the nerves, which are distributed to the various organs.

The body of a nerve-cell contains the nucleus and is usually much branched into slender processes or dendrons (Fig. 24), which are quite distinct from the nerve-fibre and are supposed to afford the means of transmitting impulses between one nervecell and another, with the dendrons of which they interlace.

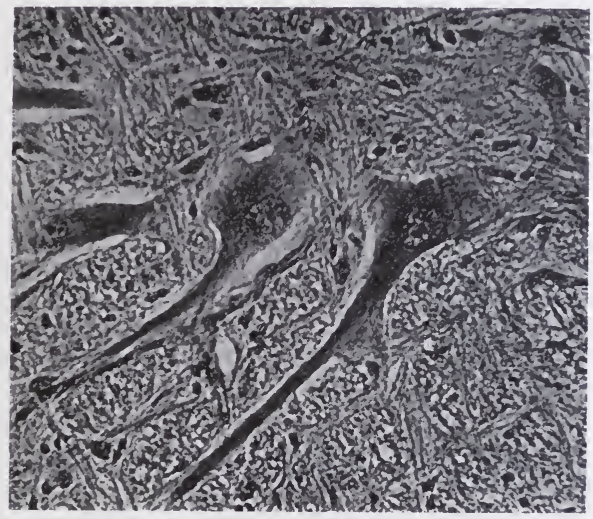

FIG. 23.--Nervous Tissue, as seen in a thin Section of the Brain (Medulla oblongata) of the Monk Fish." Two large nucleated Nerve Cells are shown imbedded in a Mass of smaller Cells and Fibres, $\times 168$. (From a photograph.)

Like other higher specialized tissue-cells of the animal body the neurons have lost the power of multiplication by division. Moreover there appears to be no provision, in some adult vertebrates, for their renewal when worn out or injured. A certain number are formed in the course of the development of the embryo and these have to serve the animal for the whole of its life.

All the different kinds of cells met with in the body, a few of which have been thus briefly deseribed, are derived from the apparently simple unicellular ovum by repeated subdivision and gradual differentiation. The functions which in an Amœba are all performed by a single protoplasmic unit are in one of the 
higher animals distributed amongst thousands of millions of such units, arranged, so to speak, in regiments and armies, each group with its own duties to perform and all co-operating for the common good under the supervision and control of the central nervous system. The marvellous perfection of the whole machinery is the result of that differentiation and division of labour which was first rendered possible by the union and co-

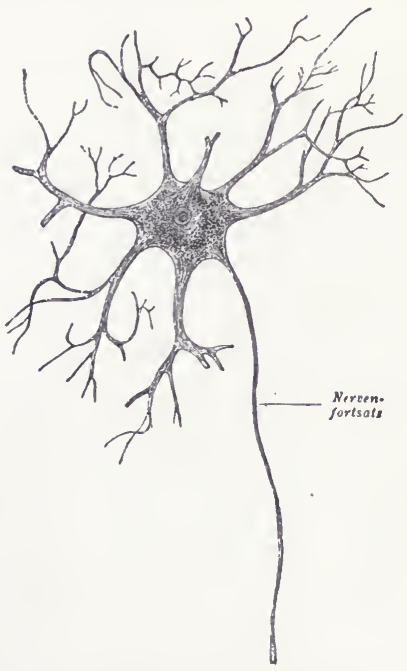

Fig. 24.-Diagram of a Neuron, (From Hertwig.)

Nervenfortsatz, a nerve-fibre coming off from the body of the cell. operation of the individuals of a protozoon family to form a multicellular body.

Turning now to the higher plants, we shall find that, in accordance with their much lower degree of functional activity, their organization is far less elaborate than in the higher animals. In correlation with their stationary habit all those organs and tissues which are specially concerned with locomotion are absent, and in further correlation with this character there are no nervous system and no special organs of sense. Of the functions concerned with the life of the individual - that is, other than reproductive functions - that of nutrition is alone highly developed. The entire plant is little more than a piece of apparatus for extracting carbon, water and mineral salts from the air and soil, and converting these, with the aid of the sun's rays, into organic substances. Although the higher plants often attain a much larger size than any animals, this does not indicate a higher degree of organization, for it is brought about simply by the repetition of similar parts-such as roots, branches and leaves-and the accumulation of dead cell-walls in the form of wood and bark. As we have already seen, a green plant, instead of spending the energy which it derives from the sun on its own 
activities, stores most of it up in the complex chemical compounds which it manufactures.

Nevertheless, though the degree of histological differentiation is not nearly so high as it is in the higher animals, we find in the higher plants also a considerable variety of cells and tissues, derived, as we have already pointed out, from the dermatogen, periblem and plerome of the embryo.

We may illustrate this point by a study of some of the cells and tissues which occur in the well-known spiderwort of our gardens, Tradescantia virginica. If we examine the flowers of this plant we shall find that the stamens are covered with long
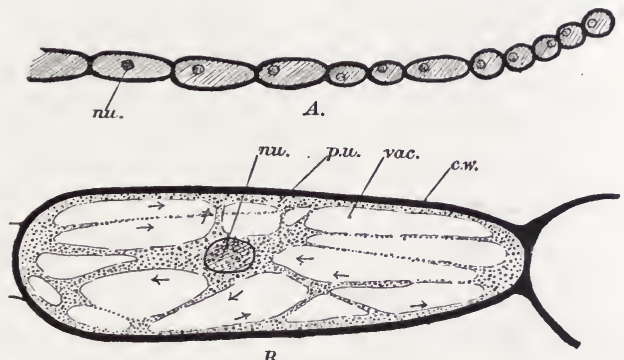

$B$.

Fig. 25.-Structure of a Hair from a Stamen of Tradescantia virginica.

A. End of a hair as seen under a low power of the microscope. The hair is made up of a single row of cells.

$B$. A single cell more highly magnified.

$c . w$. cell-wall; $n u$. nucleus; $p . u$. primordial utricle; vac. vacuole filled with coloured or colourless cell-sap.

slender hairs. It will be convenient to make these hairs the starting point of our inquiry. If we study them first under a low magnifying power we shall see that each hair (Fig. 25, A) is made up of a single row of cells, arranged like the beads in a necklace; most of the cells are elongated, but towards the apex of the hair they become short and spherical.

If we now concentrate our attention on one of the larger cells and study it carefully under a moderately high power of the microscope, we shall find that it exhibits the appearance shown in Fig. 25, B. It measures about $0.27 \mathrm{~mm}$. in length by $0.08 \mathrm{~mm}$. in breadth and consists of a thin-walled bag filled with living protoplasm. The wall (c.w.) is transparent and colourless and is composed, as in Hæmatococcus, of cellulose. 
The granular, colourless protoplasm does not fill the interior of the cell in a uniform manner, but is arranged partly as a thin lining to the cell-wall, known as the primordial utricle $(p . u$.$) , and partly in irregular strings which branch and anas-$ tomose and stretch across the cavity of the cell in various directions. These strings of protoplasm tend to converge towards an irregular mass in which the nucleus is situated. The nucleus itself (nu.) is a nearly spherical body of denser protoplasm, about $0.024 \mathrm{~mm}$. in diameter. The extensive space which lies inside the primordial utricle and between the strands of protoplasm is filled with a more fluid liquid known as the cell-sap. It is to this cell-sap that the flowers of Tradescantia owe their colour; if the flowers are blue the cell-sap will be found to be blue and if they are white it is because the cell-sap is colourless.

The most striking feature of the cell which we are examining still remains to be noticed. The protoplasm is in constant movement. This is at once evident from the characteristic streaming of the small granules which it contains. Both in the primordial utricle and in the network of threads a constant circulation is kept up, though not in a very definite manner, as the threads themselves are constantly undergoing slow changes in their arrangement. The arrows in Fig. 25, B indicate approximately the course taken by the streaming protoplasm at the time when the drawing was made.

T'he streaming of the protoplasm appears at first sight to be an essentially vital phenomenon, but it is probably merely the mechanical result of chemical and physical processes going on in the cell, such as the diffusion of various substances in solution from one cell to another, which must take place in the process of nutrition. If the cell is killed by the addition of alcohol the physical and chemical conditions are at once altered and the movement ceases; the protoplasm is coagulated and, if we are dealing with a cell containing coloured cell-sap, the nucleus absorbs the colouring matter with great avidity and becomes deeply stained, while the cytoplasm stains only very slightly or not at all. We are thus able to make the cell stain itself differentially, without the aid of any extraneous colouring matter, the cell-sap acting as what is termed a nuclear stain.

It will be necessary to restrict our further observations on Tradescantia to the structure of the leaf (Fig. 26). 'The leaves 
of this plant are long and narrow, somewhat resembling those of grasses. There is a pronounced midrib and the so-called veins
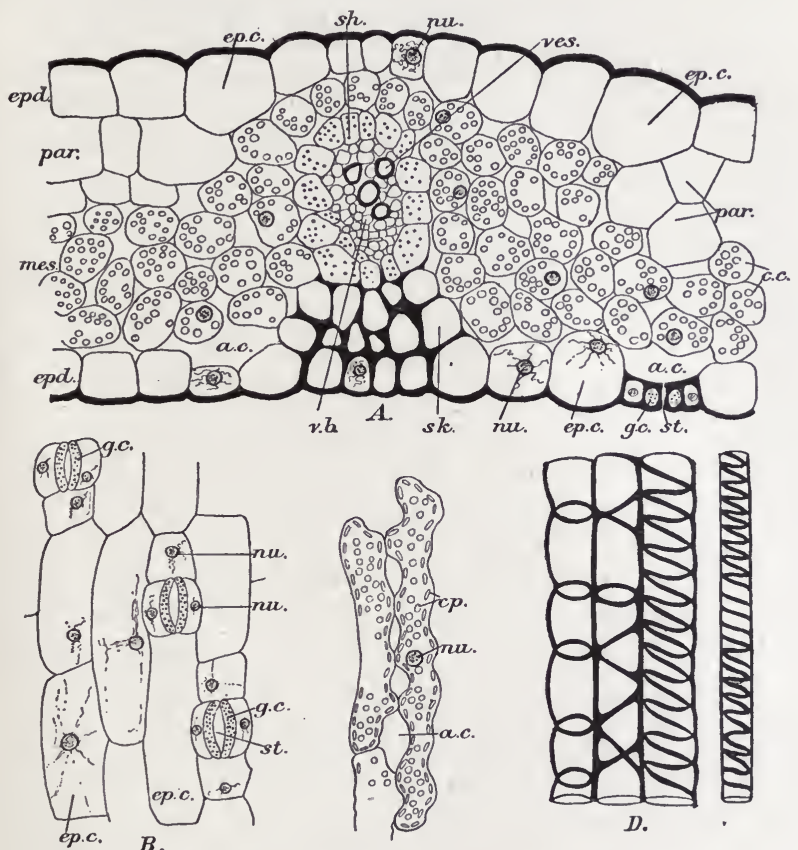

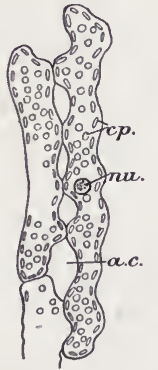

c.

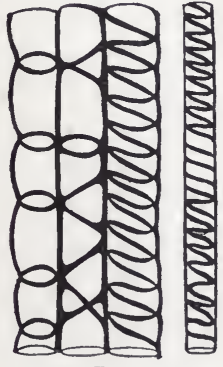

D.

Fig. 26.-Histology of the Leaf of Tradescantia virginica.

A. Part of a transverse section of the leaf.

$B$. Piece of the epidermis stripped from the lower surface of the leaf.

$C$. Cllorophyll cells from the mesophyll, as seen in a longitudinal section of the leaf.

$D$. Portions of four vessels from a vascular bundle, with spiral and annular markings, as seen in a longitudinal section of the bundle. (All more or less highly magnified.)

a.c. air-cavity ; c.c. chlorophyll cell; $c p$. chloroplastids ; ep.c. epidermic cell ; epd. epidermis; g.c. guard-cell; mes. mesophyll; nu. nueleus; par. colourless parenchyma ; $s h$. sheath of vascular bundle, composed of thin-walled cells containing starch grains; $s k$. thick-walled skeletal tissue; st. stoma; $v . b$. vascular bundle; ves. vessels.

run parallel with one another from base to apex, as in all typical Monocotyledons.

If we cut a thin transverse section of a living leaf and examine it under the microscope in a drop of water (Fig. 26, A) we 
shall see at once that it is made up of three principal tissuesystems, the epidermis, the mesophyll and the vascular bundles. The epidermis (epd.) covers the upper and lower surfaces in the form of two single layers of cells, the mesophyll (mes.) occupies the space between these two layers, and the vascular bundles $(v . b$. corresponding to the veins of the leaf-are imbedded in the mesophyll at fairly regular intervals (only one is shewn in the figure). We must examine each of these tissue-systems separately, and in order to gain a correct idea of the form and arrangement of the cells of which they are composed it will be necessary to study them from various points of view.

The epidermis may be stripped off bodily from the surface of the leaf and then exhibits under the microscope the appearance shown in Fig. 26, B. It is composed of elongated cells, placed side by side in a single layer. The amount of protoplasm which these cells contain is very small, but the nucleus (nu.) is frequently conspicuous. Their external walls are specially thickened in relation to their protective function, a feature which can only be seen in sections (Fig. 26, A). At frequent intervals little slit-like openings oceur in the epidermis. These are the stomata (st.) which lead into air-spaces in the mesophyll. Each stoma is bounded by a pair of specially modified epidermic cells known as the guard-cells (g.c.) - the only epidermic cells containing chlorophyll-which have the power of opening and closing the stoma like a pair of lips and thus regulating the amount of aqueous vapour which passes through the stomata in the process of transpiration. On the outer side of each guardcell lies another epidermic cell of much smaller size than the ordinary kind, and these, together with the guard-cells, form a kind of roof (or floor) to the air-cavity (Fig. 26, A, a.c.).

The mesophyll contains the chlorophyll-bearing cells, by which the assimilation of carbon dioxide is effected and which are at once recognized by their green colour. They appear more or less round or oval in transverse sections (Fig. 26, A, c.c.), but are really considerably elongated, parallel to the length of the leaf, as shown in Fig. 26, C. They come in contact with one another by numerous short protuberances, between which lie the spaces in which the air, containing carbon dioxide and aqueous vapour, circulates. Each contains a nucleus (nu.) and numerous small, biscuit-shaped green bodies, the chloroplastids or chlorophyll corpuscles (cp.), imbedded in the cytoplasm. 
In certain places the green mesophyll cells are interrupted by groups of cells containing no chlorophyll. At intervals beneath the upper epidermis we see masses of large, thin-walled, colourless cells forming a parenchyma or ground-tissue ( $p a r$.), while beneath the vascular bundles we find bands of very thick-walled cells $(s k$.) which play an important part in the mechanical support and strengthening of the leaf.

The vascular bundles are surrounded each by a sheath of thinwalled cells $(s h$.$) containing numerous small starch grains.$ Within this lie the bast or phloem and the wood or xylem, composed of the elongated tubular elements through which the sap circulates. The raw sap consists of water with mineral salts in solution, and ascends through the xylem, while elaborated sap, containing the proteids which have been manufactured in the leaf under the influence of sunlight, descends through the phloem. For our present purposes we may confine our attention to certain of the xylem elements, known as the spiral and annular vessels. These consist really of dead cell-walls, forming long narrow tubes each composed originally of a row of cylindrical cells placed end to end. In the course of their development the transverse dividing walls between these cells are absorbed, the protoplasm disappears, and nothing remains but a long hollow tube whose walls are strengthened by spiral or annular thickenings. Portions of these vessels are represented separately in Fig. 26, D ; in A they are seen only in transverse section (ves.).

Such are the principal kinds of tissue met with in a typical flowering plant, and such is the way in which it carries out the principles of co-operation, differentiation and division of labour amongst its constituent cells.

To most people it will probably appear that the fundamental truth and general applicability of the cell theory are sufficiently firmly established by considerations such as those with which we have been dealing. It has, however, certain undoubted limitations, and upon these limitations some biologists are inclined to lay a good deal of stress.

Thus, from the point of view of the cell theory, we regard the cell as the organic unit; there are, however, units of a lower order, of which the cell itself is composed. The chloroplastids of one of the higher plants, for example, exhibit a good deal of individuality, being capable of independent growth and multiplication, while, as we shall see later on, it is necessary for 
theoretical reasons to postulate the existence of some such bodies as Professor Weismann's biophors as the primary units of which living protoplasm is built up.

Then, again, although it may be questioned whether any absolutely unnucleated organisms, such as Haeckel's Monera, really exist, there can be no doubt that the most simply organized living things known to us, the Bacteria, which probably stand a long way below the point where the animal and vegetable kingdoms part company, and which are the most abundant of all living organisms, do not show that sharp differentiation into cell body and nucleus which is so characteristic of typical cells,

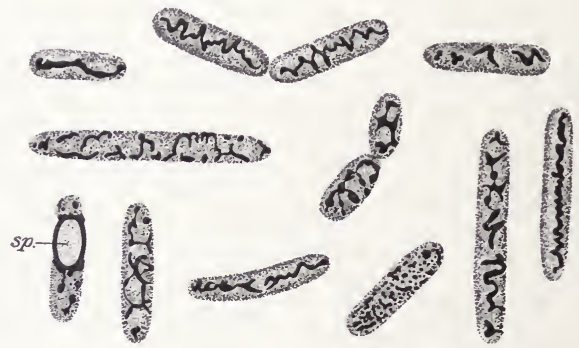

FIG. 27.-Bacillus saccobranchi. Bacteria from the Blood of a Finh (Saccobranchus) stained so as to show the distribution of the chromatin (nuclear) material, which is represented in black, and which.may be arranged in small scattered granules thronghout the cell, or in an irregular network, or in an irregular, more or less twisted rod, $\times 2000$. (After Dobell in the "Quarterly Journal of Microscopical Science.")

the nuclear constituents being more or less scattered throughout the eytoplasm (Fig. 27).

A difficulty of another kind is met with in the fact that in a good many cases the division of the nucleus is not followed-at any rate not immediately-by corresponding division of the cytoplasm. Some Amœbæ constantly have two nuclei, and we sometimes get relatively large masses of protoplasm containing many nuclei formed by repeated division. These are termed syncytia. We meet, in fact, with all degrees of separation of the cytoplasm into distinct cells, and a great many of the cells even of highly developed plants and animals may remain connected together throughout life by thin strands of protoplasm. We have already noticed an example of this continuity of the protoplasm in the 
case of Volvox; another is shown in Fig. 28, B, which represents a syncytial epithelium.

On the other hand, in some of the lower organisms-the Myxomycetes, slime-fungi or Mycetozoa, as they are variously called-numerous originally separate Amœba-like individuals may fuse together to form plasmodia, which may continue to feed and grow and undergo nuclear division until they form great sheets of living protoplasm containing perhaps hundreds or thousands of nuclei (Fig. 29). 'l'hese and similar facts, however, interesting and instructive as they undoubtedly are, cannot be regarded as constituting a serious invalidation of the cell theory.

There is no more fundamental or more stimulating conception in the domain of biological science than that of the continuity of life as formulated by this theory. We have to imagine the whole organic world as consisting of a continuous stream of living protoplasm, which commenced to flow many
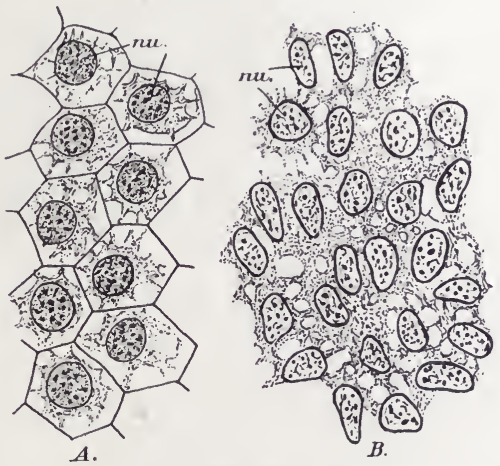

Fig. 28.

A. A single-layered epithelium with very distinct celloutlines, from the brain of a reptile (Sphenodon punctatus), $\times 750$.

$B$. A syncytial epithelium, without cell-outlines, from another part of the brain of the same animal, $\times 750$.

$n u$. nuclei.

millions of years ago and has continued without interruption ever since. At every cell-division the stream branches and physical continuity is more or less completely interrupted, but this in no way invalidates the conclusion that if all living things did not actually have a common origin in a single primordial protoplasmic unit, they probably at least originated from several such units which themselves arose under unknown conditions from inorganic matter.

The modern science of cytology, which is contrasted with histology as the study of individual cells rather than that of tissues or cell combinations, and which is yielding such important 
results both for pure biology and for medical science, owes its origin and development entirely to the elaboration of the cell

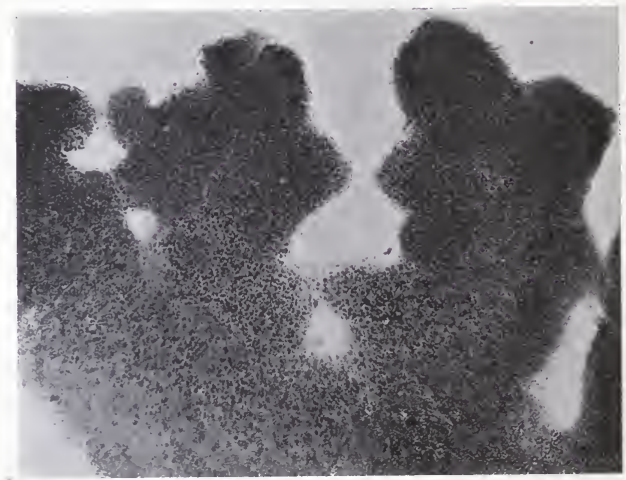

Fig. 29. - Part of the Plasmodium of a Mycetozoon (Badhamia utricularis), $\times$ 50. (From a photograph.)

The small dark spots are the very numerous nuclei.

theory under the influence of improved methods of microscopical investigation.

There is another point of view with regard to the living cell which we may briefly refer to in this place. It is not only the morphological or structural unit of the body but also the physiological or functional unit. All the essential vital processes talie place within the bodies of cells, and all the different materials required for and produced by these processes pass in and out of them. Each cell may be looked upon as a microscopic laboratory in which the complex chemical reactions comprised under the term metabolism take place, and although, in the higher organisms, the cells have become mutually dependent upon one another, yet each retains a certain degree of physiological as well as of morphological individuality. 


\section{CHAPTER VI}

The multiplication of eells-Mitotic and amitotic nuclear division.

We have already seen that the possibilities of structural differentiation within the limits of the individual cell are by no means exhausted by the distinction between cytoplasm and nucleus, but it is only when we come to study in detail the process of cell-division that we begin to gain any adequate conception of the fundamental complexity of the organic unit. We have hitherto spoken of this process, as it occurs for example in Amœba, as though it were a simple matter, initiated by constriction of the nucleus into two parts and concluded by a corresponding division of the cytoplasm. The researches of the last forty years, however, rendered possible by the improvements in microscopical apparatus and micro-chemical technique, have taught us that in the vast majority of cases the process of celldivision is one of extreme complexity, accompanied by remarkable phenomena which reveal a previously unsuspected degree of structural differentiation within the nucleus itself. To these phenomena Schleicher in 1878 gave the name karyokinesis, for which Flemming, in 1882, proposed to substitute mitosis. Both these terms are still in common use.

In a typical cell (Fig. 30) the cytoplasm (cyt.) is a semi-liquid substance usually enclosed in a thin cell-membrane (animal cells) or a thicker cell-wall (plant cells). It exhibits a microscopic structure which is variously interpreted as reticular (fibrillar), alveolar (foam-like), or simply granular, the probability being that the real truth is expressed by a combination of these different views. It may or may not contain plastids of various kinds (e.g. chloroplastids in green plants).

The nucleus (nu.), consisting of the so-called nucleoplasm or karyoplasm, is usually a spherical, more or less centrally situated body enclosed in a definite nuclear membrane (n.m.). Within this membrane the karyoplasm is differentiated into various constituents. In the first place there is a network or reticulum of 
delicate protoplasmic threads, known as the linin network, the meshes of which are filled with a clear, probably liquid groundsubstance known as nuclear sap. 'These two together seem to differ but little from the cytoplasm which lies outside the nucleus.

The most characteristic constituent of the nucleus is another substance, to which, on account of the readiness with which it becomes coloured by certain dyes, the name chromatin ${ }^{1}$ has been given. This substance usually occurs in the form of small granules (ch.g.) scattered over the linin network, so that when very close together they appear to form a chromatin network,

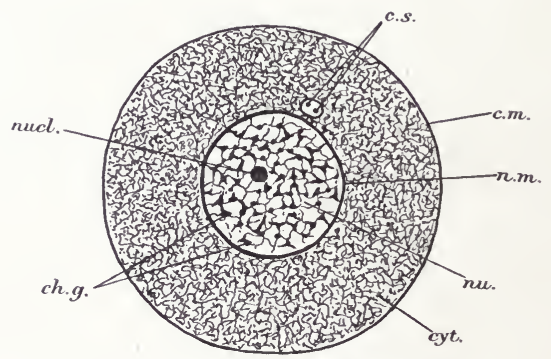

FIG. 30.-Diagram of a typical Cell.

ch.g., chromatin granules; $c . m$, cell-membrane; $c . s$. , centrosomes lying in centrosphere cyt., cytoplasm; n.m., nuclear membrane; nu., nucleus ; nucl., nucleolus.

while not infrequently a specially large aggregation of chromatin substance forms a nucleolus or karyosome (nucl.).

Owing to the presence of this chromatin the nucleus as a whole, under low powers of the microscope, appear's to be deeply coloured by such stains as various preparations of logwood and carmine and the basic aniline dyes. We have already had occasion to observe this staining property in the case of the hair-cells of Tradescantia, where, it will be remembered, the nucleus becomes deeply stained by the coloured cell-sap as soon as the cells are killed by the action of alcohol. Other stains, again, affect the cytoplasm rather than the nucleus, and these various chemical reactions enable us to differentiate fairly sharply between the different constituents of which the cell is composed, though it is a matter of some doubt exactly how far the structure of the

1 Greek $\chi \rho \hat{\omega} \mu \alpha$, a colour. 
living protoplasm really corresponds to the appearances exhibited in our preparations.

The differences in their staining reactions of course indicate corresponding differences in chemical composition between the chromatin and the cytoplasm, and analysis has shown that the chromatin is characterized by the presence of relatively large quantities of phosphorus. This is contained in the complex nucleinic acid, with which various albuminous bodies may be combined to form the chromatin substance.

When a typical animal cell is about to divide another structure makes it appearance, usually just outside but occasionally inside the nucleus. This is the centrosome (Fig. 30, c.s.), a very minute body which has peculiar staining properties and which is surrounded by a differentiated area of protoplasm known as the centrosphere or attraction sphere (Fig. 31, A, csph). It has been questioned whether a centrosome and attraction sphere are always present or whether they make their appearance only when the nucleus is about to undergo mitosis. This process certainly seems to be initiated by the centrosome, which may divide into two parts long before the nucleus itself commences to do so, so that two centrosomes often appear alongside the so-called resting nucleus (Figs. $30 ; 31, \mathrm{~A}$ ). Presently the two centrosomes move away from one another, both still keeping close to the nucleus, and each is now seen to be surrounded by its own attraction sphere (Fig. 31, B). Around each attraction sphere delicate threads or fibrillæ of the cytoplasm become radially arranged to form a star or aster, and the rays of the asters which lie between the two centrospheres combine to form a spindle (Fig. 31, C, ksp).

In the meantime remarkable changes have commenced in the nucleus itself. The chromatin granules, together with the linin by which they are apparently held together, have arranged themselves in the form of a long coiled thread, the spireme (Fig. 31, B), and presently the nuclear membrane begins to disappear (Fig.31, C, km), being apparently dissolved in the general protoplasm. In this way the distinction between cytoplasm and nucleoplasm is obliterated.

The spireme thread breaks up into a number of short lengths known as chromosomes (Fig. 31, C, chrs), the actual number being, with certain exceptions, a constant character for each species of plant or animal. The centrosomes at about this 
time take up their positions at points corresponding to two opposite poles of the original nucleus, with the spindle of fine

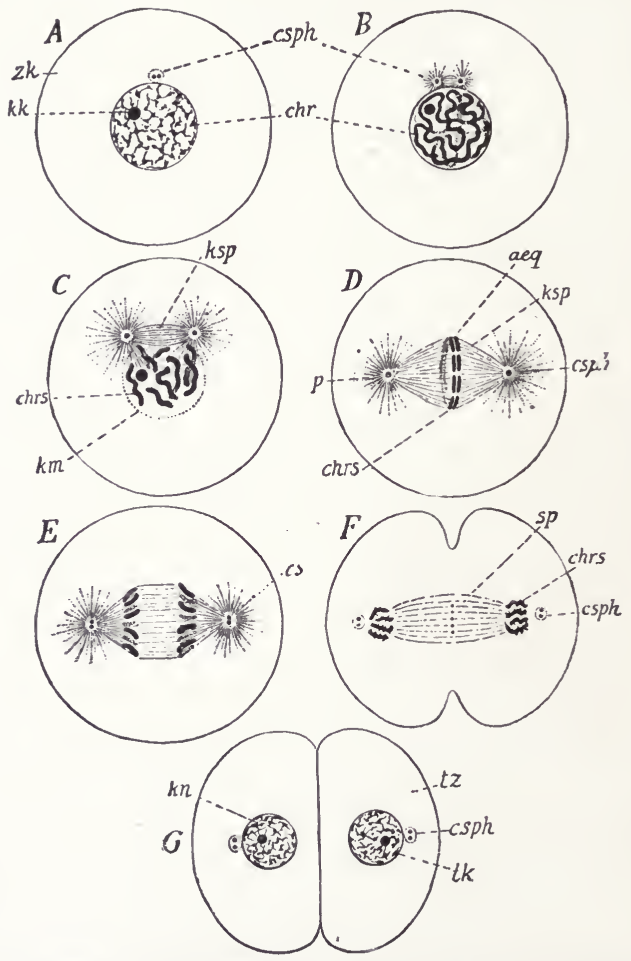

FIG. 31.-Diagram of the principal Stages in the mitotic Division of the Nucleus in a typical Animal Cell. (From Weismann's "Evolution Theory," adapted from E. B. Wilson.)

aeq, equatorial plate; $c h r$, chromatin ; chrs, chromosomes; $c s$, centrosome ; $c 8 p h$, centrosphere, containing one or two centrosomes; $k k$, nucleolus; $k m$, nuclear membrane; $k n$, nucleus; $k s p$, sp, nuclear spindle; $p$, aster; $t k$, daughter nucleus; $t z$, daughter cell; $z k$, cytoplasm forming the cell body.

protoplasmic fibres stretched between them (Fig. 31, D), and the chromosomes "go on the spindle," arranging themselves in a so-called equatorial plate across its widest part (Fig. 31, D, aeq). 
The shape of the chromosomes varies much in different cases; they may be more or less spherical, but they are frequently short, rod-like bodies, often shaped like a $\mathbf{V}$ (Fig. 32, A). Each one is composed, like the spireme thread from which they are derived, of an aggregation of chromatin granules, held together by a linin basis. The chromatin granules are sometimes arranged like the beads on a necklace (Figs. 32, B ; 77), and are known as chromomeres. The number of the chromosomes also varies greatly, from as low as two in a variety of the horse-worm (Ascaris) to as many as one hundred and sixty-eight in the shrimp Artemia. In cases where the chromosomes are very small each one may perhaps be equivalent to only a single chromomere.

Either before or after taking up its position in the equatorial
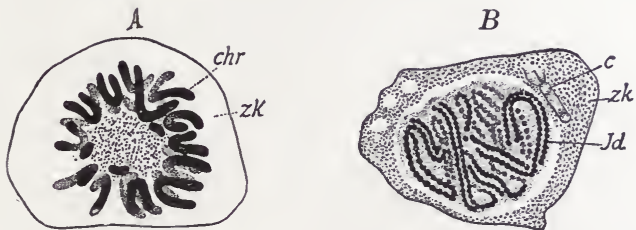

FIG. 32.-Sperm-mother-cells of a Salamander, during Mitosis. In $A$ the chromosomes are shown; in $B$ the spireme thread is split lengthwise, and also shows very clearly the chromomeres of which it is made up. (From Weismann's "Evolution Theory," after Hermann and Drüner.)

$c$, dividing centrosome ; $c h r$, chromosomes; $J d$, chromomeres; $z k$, cytoplasm.

plate, each chromosome splits longitudinally into two parts (Fig. 31, D), in fact the splitting can sometimes be observed in the spireme thread even before it breaks up transversely into chromosomes (Fig. 32, B). The result of this splitting is that the number of chromosomes is doubled ; but the danghter chromosomes very soon separate into two equal groups, one of which moves towards each centrosome (Fig. 31, E). Each group contains one of the two halves of each parent chromosome.

Having migrated to opposite poles of the spindle the two groups of daughter chromosomes there form the foundations of two new nuclei (Fig. 31, F). The chromosomes break up into granules again; a new nuclear membrane is formed, whereby a portion of the general cytoplasm is separated off to form the linin network and ground-substance of the nucleus; the asters and nuclear 
spindle more or less completely disappear--though the centrosome may certainly persist in some cases if not in all - and the newly constituted nucleus (Fig. 31, G, $t k$ ) enters upon a longer or shorter period of inactivity accompanied by growth.

In the meantime the cytoplasm which constitutes the cell-body has also divided into two parts in a plane which passes through the middle of the nuclear spindle and at right angles to its length. In animal cells this division is usually effected by a constriction which starts from the outside (Fig. 31, F, G) and in plant cells by the deposition of a cell-plate (Fig. 34, E, c.p.) in the equator of the nuclear spindle. This cell-plate forms the foundation of the double cell-wall which will separate the two daughter cells; it must not, of course, be confounded with the equatorial plate formed temporarily by the chromosomes.

Various attempts have been made to explain the dynamics of this remarkable process of mitosis or karyokinesis, the essential features of which are always much the same though the details vary considerably in different cases. The centrosomes, with their centrospheres, asters and spindle, sometimes spoken of collectively as the achromatic figure, are usually regarded as a special mechanism for bringing about the equitable partition of the chromatin substance between the two daughter nuclei. This substance is evidently so important that no rough and ready division will suffice. It is probable, as we shall see later on, that the chromomeres of which each chromosome is composed have different properties, and that it is necessary, in ordinary celldivision, not only that the chromosome as a whole shall be divided into two parts but that each daughter nucleus shall have its share of each individual chromomere (compare Fig. 77). In other words a qualitative as well as a quantitative division of the chromatin material has to be effected, and this is secured by the longitudinal splitting of the chromosomes. A transverse division would only result in the separation of the chromomeres into two groups, but the longitudinal division involves each one.

According to some observers the fibres of the nuclear spindle are actively contractile and actually pull the two halves of each split chromosome asunder. Others maintain that the centrosomes attract the chromosomes in somewhat the same way as the poles of a horse-shoe magnet attract iron filings sprinkled between them.

Of late years the electro-magnetic explanation has been coming 
more prominently to the front. Thus Gallardo has suggested that the chromatin substance is charged with negative and the cytoplasmic colloids with positive electricity, while the centrosomes are capable of aequiring a positive potential higher than that of the general cytoplasm. Increase of this potential causes the centrosome to divide and the radiations which form the asters and spindle indicate lines of force in the cytoplasm. The two daughter centrosomes, inasmuch as they bear like charges of electricity, repel one another. In a similar way the chromosomes divide under the influence of their high negative charges and the two halves of each repel one another and are at the same time attracted by the positive centrosomes. The two new groups of negatively charged chromosomes then attract the positive eytoplasm in opposite directions and thus the division of the cell body follows upon that of the nucleus.

Whatever may be the physical explanation of these complex phenomena, we must think of them as lying at the root of all normal processes of growth and multiplication in the higher plants and animals. With comparatively rare exceptions, some of which will be mentioned later on, every one of the innumerable series of cell-divisions initiated by the fertilized ovum, and continued throughout life in the growth and repair of tissues, is accompanied by complicated processes similar to those above described. The process of cell-multiplication, however, is frequently confined in adult organisms to certain regions. Thus, as we have already seen, in the higher animals the growth of the epidermis depends upon cell-divisions which go on only in its deepest layer, the stratum Malpighii (Fig. 18, s.m.). Most of the cells in the body sooner or later lose the power of division, but they are then usually short-lived, as in the case of those cells which form the outer layers of the epidermis and which rapidly become converted into more or less horny scales to be cast off on reaching the surface.

The majority of the tissues are thus renewed throughout life by the mitotic activity of some unspecialized cell-group, a high degree of specialization in the tissue cells of the higher organisms being always, as we have already seen in the case of red blood corpuscles and nerve cells, accompanied by the loss of the power of multiplication.

The limitation of cell-multiplication to definite circumscribed regions of the body is perhaps best seen in the case of the higher 
plants, where the various meristematic or actively dividing tissues remain in an undifferentiated embryonic condition and give rise to those additions to the permanent tissues whereby growth is effected. Such actively dividing meristem is found at the growing points of stems and roots, where it serves to bring about growth in length, and in the cambium, which serves, by the addition of new elements to the wood and the bast, to bring about growth in thickness.

The microscopic appearance of such a meristematic tissue,

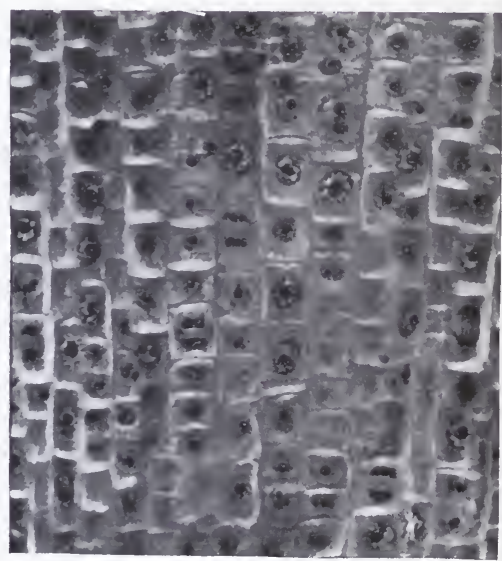

A.

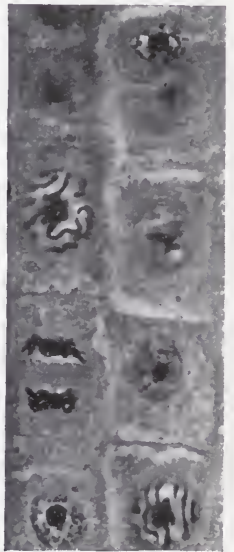

B.

FIG. 33.- Part of a longitudinal Section of the actively growing Root of a Hyacinth (Galtonia candicans) showing the Nuclei of the Cells in various stages of mitotic Division, $A \times 280 ; \mathrm{B} \times 640$. (From photographs.)

when suitably stained and prepared for examination, is shown in Fig. 33, taken from photographs of part of a longitudinal section of the growing point of the root of a hyacinth (Galtonia candicans). The cell-walls are as yet thin and inconspicuous and filled with dense protoplasm, while the conspicuous nuclei exhibit all stages of mitosis, the whole forming a striking contrast to the dead tissues, such as cork and wood, of which the bulk of many plants is made up, and which consists merely of cell-walls without any protoplasmic contents ( $c f$. Figs. 6 and 7 ).

Mitosis in the cells of the higher plants is usually, though by 
no means always, characterized by the absence of recognizable centrosomes. The actual appearance of some of the principal stages in the process is shown more highly magnified in Fig. 34,

A.
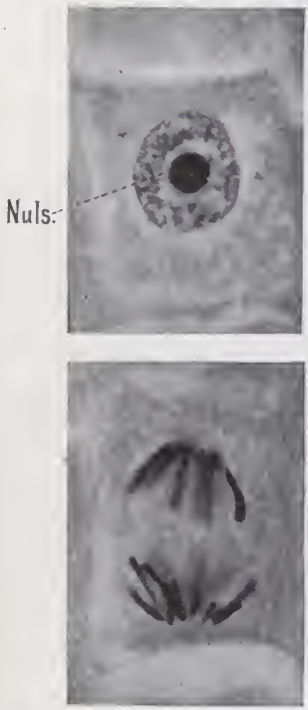

D.
B.
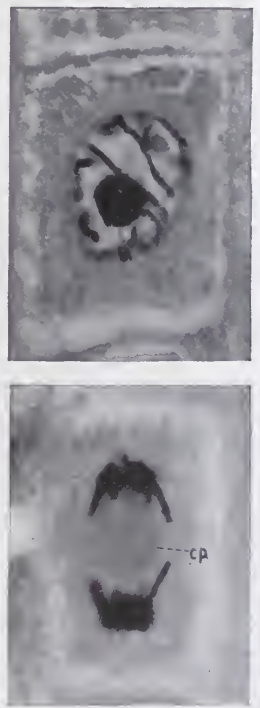

E.
C.
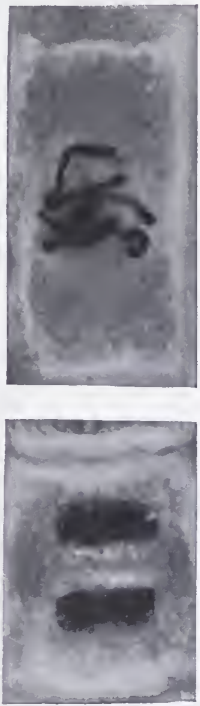

$F_{\text {. }}$

FIG. 34. - Six selected Stages in the mitotic Division of the Nucleus in the growing Root of Galtonia candicans, $\times 1120$. (From photographs.)

A. Resting nucleus with large nucleolus (Nuls.).

B. Spireme stage, with coiled chromatin thread.

C. The spireme thread has broken up into ehromosomes which are forming the equatorial plate.

D. The chromosomes have split longitudinally and the two groups of daughter chromosomes thus formed are passing to opposite poles of the spindle.

E. Formation of the cell-plate (c.p.) across the equator of the nuclear spindle.

F. Completion of the cell-division, and disappearance of the individual chromosomes in the daughter nuclei.

which represents a series of six selected stages arranged in proper sequence, reproduced from photo-micrographs.

Fig. 34, A represents the so-called resting stage of the nucleus, in which it will be noticed that there is, in addition to the minute, scattered chromatin granules, a large spherical chromatin nucleolus or karyosome (Nuls.). B shows the spireme 
stage, with the chromatin granules collected together in a long spirally coiled thread and the nucleolus still very conspicuous. C shows the group of chromosomes formed by transverse breaking
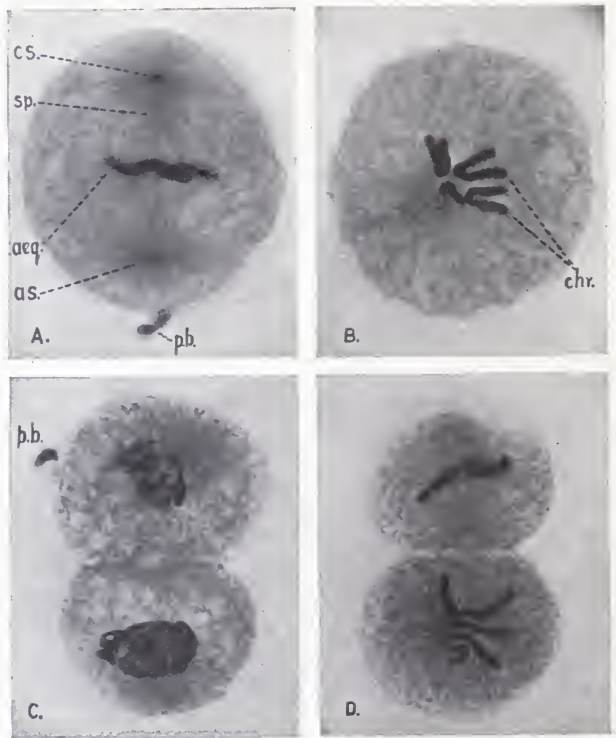

FIG. 35.-Mitosis in the segmenting Egg of the Horse-Worm (Ascaris megalocephala), $\times$ 770. (From photographs.)

A. Lateral view of the egg during the first cleavage; showing the nuclear spindle $(s p$.), the equatorial plate (aeq.), one of the two centrosomes (c.s.), the other being out of focus, and the asters (as.) formed by fine threads of protoplasm radiating from around the centrosomes. Polar bodies $(p . b$. $)$ are also shown.

B. The same stage viewed from one pole, showing the four $\mathrm{V}$-shaped chromosomes $(\mathrm{chr}$.) in the equatorial plate.

C. The first division is completed and the nuclei have again passed into the spireme stage. A polar body $(p . b$.$) is still visible.$

D. Each of the first two blastomeres has again reached the stage represented in A and $B$.

up of the spireme thread. The karyosome has now disappeared, having apparently been used up in the formation of the chromosomes. D shows the two groups of daughter chromosomes formed by longitudinal splitting of the parent chromosomes and retreating towards the two ends of the spindle, which is only 
faintly visible. $\mathrm{E}$ shows the commencement of the cell-plate (c.p.) across the middle of the spindle, and $\mathrm{F}$ the two young daughter cells each with a new nucleus. in which the chromosomes have again broken up into granules.

It is only by the examination of large numbers of examples that all the minute details of the process can be elucidated, but the main features as represented in the above figures can very easily be made out.

For comparison with the process of mitosis as seen in typical plants such as Galtonia, we may take the first division of the fertilized egg in the horse-worm, Ascaris, a classical subject from the study of which much of our knowledge of nuclear division in animal cells has been derived. In this case there are only four chromosomes, but they are large and conspicuous, and characteristically $\mathrm{V}$-shaped when forming the equatorial plate on the spindle.

Fig. 35 is again taken from actual photographs. In this figure, A represents a side view of the entire egg-cell during the division of the nucleus, with spindle ( $s p$.$) , asters (as.),$ centrosomes (one only of which, cs., appears in the photograph, the other being out of focus), and equatorial plate (aeq.). B shows a similar stage viewed from one pole, so that the spindle itself does not appear, but the four chromosomes forming the equatorial plate are distinctly visible. C shows the two daughter cells or blastomeres resulting from the first division of the egg, each with the nucleus preparing for further division, and D represents a later stage in which the nucleus of each daughter cell is again actually in process of division and shows the separate chromosomes very distinctly.

It must not be supposed that the phenomena of mitosis are by any means confined to the higher animals and plants ; they are observable throughout the animal and vegetable kingdoms, in unicellular as well as multicellular forms. The process has long been known to take place, for example, in at any rate some Amøebs, and it probably occurs wherever there is a clearly differentiated nucleus. 'The Bacteria and their allies, in which the chromatin granules are scattered throughout the cell body and there is no proper differentiation into cytoplasm and nucleus, apparently form exceptions to the general rule.

There are, however, even amongst the higher animals, some cases of cell-division which do not exhibit mitotic phenomena, but in 
which the nucleus appears simply to constrict into two or more parts (Fig. 36). This is known as direct or amitotic nuclear division.

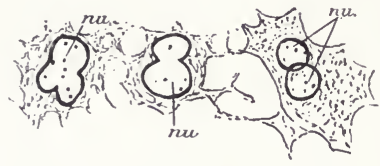

FIG. 36.-Amitotic nuclear Division as seen in Cells from the Cavity of the Paraphysis in Sphenodon, $\times 1000$.

nu. nuclei.

It is frequently met with in degenerating cells and pathological tissues, but it is doubtful if it ever occurs (in the higher organisms at any rate) in cells which are destined to undergo long-continued multiplication. We may therefore regard it as a more or less abnormal process with which we have no need to concern ourselves any further. 'The phenomena of mitosis, on the other hand, are thoroughly normal and practically universal, and, as we shall see later on, they are of the deepest significance from the point of view of the theories of heredity and variation. 


\section{PAR'T II.-THE EVOLUTION OF SEX}

\section{CHAPTER VII}

Limitation of the powers of cell-division-Rejuvenescence by conjugation of gametes-The origin of sex in the Protista.

By the process of cell-division an unbroken continuity has been established in the chain of living things from the earliest appearance of unicellular organisms to the present day. Every cell is the descendant of pre-existing cells and, in accordance with the theory of evolution, all cells which exist to-day, distributed amongst the bodies of countless millions of different organisms, could, if our knowledge were sufficiently complete, be traced back to a single ancestral cell.

It by no means follows from these considerations, however, that there is, under natural conditions, no limit to the ordinary process of cell-division. On the contrary it is well known that in any cell family, whether belonging to a unicellular or a multicellular organism, the power of multiplication tends to become exhausted, and, if that particular cell family is to continue its existence, has to be in some way renewed.

''ake, for example, an ordinary ciliate or flagellate Protozoon, which multiplies by simple fission. If a single individual be isolated and placed in water containing suitable food material, and kept under suitable conditions of temperature, light and so forth, it will multiply very rapidly, until possibly hundreds of generations of separate cells have been produced and the total number increased perhaps to millions. But under ordinary circumstances a time presently arrives when the individuals begin to show signs of exhaustion, accompanied by physical degeneration, and to slack off in their rate of multiplication. They may be stimulated to renewed activity for a time by special feeding or by constantly varying the culture medium, ${ }^{1}$ but in a

1 Mr. L. L. Woodruff has kept a culture of Paramcecium under observation for nearly three and a half years, taking precautions to prevent the possibility of conjugation, but constantly varying the culture medium. During this time more than two thousand generations of Paramceium were produced by repeated fission-an

B. 
state of nature the chief if not the only means by which the family can be kept from speedy extinction is conjugation, the

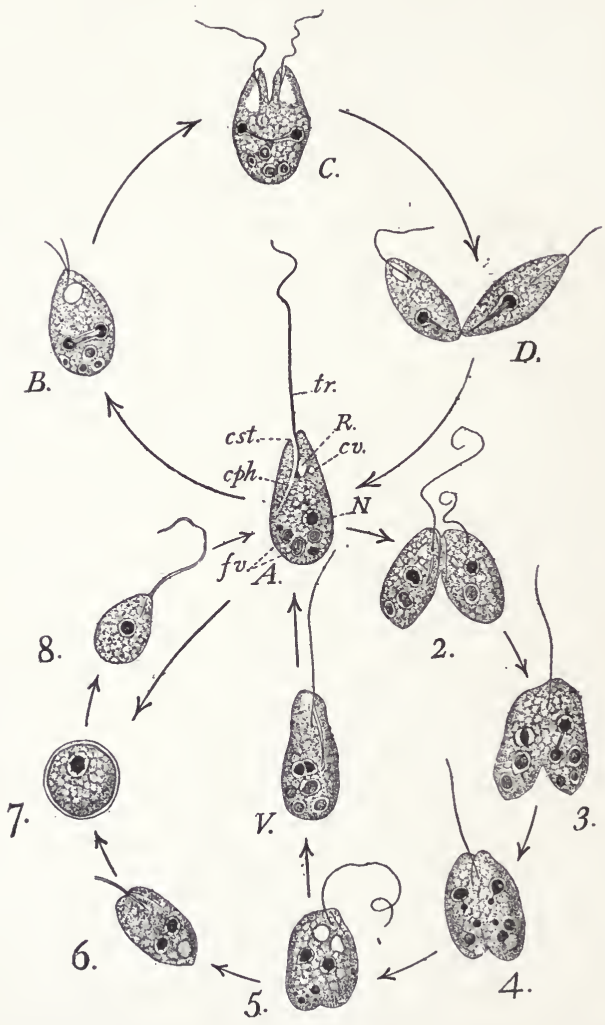

F1G. 37.-Life History of Copromonas. (From Bourne's "Comparative Anatomy," after Dobell.)

$c v$., contractile vacuole; $c p h$., cell pharynx; cst., cell mouth; $f v$., food vacuole; $N$., nucleus; $R$., reservoir; $t r$., flagellum. (For further explanation see text.)

exhausted individuals approaching one another and finally uniting in pairs. In this way they appear to become rejuvenated and arerage of about one division every fifteen hours--and at the end of the period the organisms were still in a perfeetly normal condition. 
their failing powers of multiplication by cell-division are completely restored.

For the purpose of studying this process of conjugation in its primitive simplicity we can hardly do better than take the minute flagellate form Copromonas, which is found in water frequented by frogs, from the excrement of which it derives its nutriment. The adult organism (Fig. 37, A) cousists of a very minute ovoid mass of protoplasm with a single flagellum ( $t r$.) springing from the narrow end. Alongside the base of the flagellum is a definite cell mouth (cytostome, cst.) through which solid particles of food are taken into the interior of the body. Close to this there is a contractile vacuole $(c r$.$) , accompanied by a "reservoir" (R)$ into which it discharges. The nucleus $(N)$ is situated nearer to the broadly rounded hinder end of the body, which may also contain a number of food-vacuoles ( $f v$.).

If the food supply be abundant the individual Copromonas will grow and presently divide into two by simple longitudinal fission, which commences at the narrow anterior end (Fig. 37, B-D). The division of the nucleus is said to be amitotic. The two daughter cells separate, feed, grow and repeat the process, and in this way a whole swarm of monads is produced. In the course of a few days, however, they appear to become exhausted and conjugation sets in, the individuals uniting in pairs (Fig. 37, 2-5). The result of each such union is a single larger individual, which may either undergo a period of rest within the protection of a special envelope or cyst (Fig. 37, 7), or at once assume the ordinary form and begin to multiply with renewed activity (Fig. 37, $\mathrm{V}$; For the continued existence of the species it is probably necessary that the encysted monads should at some time or another be swallowed by frogs and passed out again in the fæces, in order that they may be brought in touch with the necessary food supply.

We have here, as in the case of Hæmatococcus described in Chapter III., a perfectly typical example of conjugation ${ }^{1}$ occurring at longer or shorter intervals in the life cycle of the organism. The whole process consists in the union of two separate cells, known in this connection as gametes, to form a single cell known as the zygote, and it is of the utmost importance to observe that not only is there a union between the cytoplasm of the two gametes but the nuclei also unite to form a single zygote nucleus. Indeed,

1 Also known as syngamy or zygosis. 
as we shall see later on, it is the union of the nuclei which is the really important part of the business, for in some cases (e.g., Paramceium) the union of the two cell bodies is a merely temporary affair, a necessary preliminary to an exchange and subsequent union of nuclei.

In such simple cases as that of Copromonas we see all the essential features of the sexual process which occurs so constantly throughout the animal and vegetable kingdoms. It is evident that in itself conjugation is not a process of reproduction, for its immediate result is to halve the total number of cells instead of doubling it. It has in fact exactly the opposite effect to that of cell-division. It is a process which appears to be necessary for the rejuvenescence, at longer or shorter intervals, of exhausted cells, whereby they are endowed with renewed powers of multiplication by ordinary cell-division. At the same time it forms the starting point of all those remarkable structural modifications of the organism, whether unicellular or multicellular, which accompany the evolution of sex.

In Copromonas and in Hæmatococcus, although there is a true sexual process, there is apparently no sexual differentiation at all ; there is no distinction between male and female gametes; the two conjugating cells are exactly alike, and the conjugation is therefore said to be isogamous. In Copromonas, moreover, the gametes or sexual cells are indistinguishable from the ordinary individuals, every individual being at least a potential gamete. Starting from such a case as this we find, even amongst the unicellular plants and animals, every stage in the evolution of highly specialized male and female gametes, differing widely from the ordinary individuals and from each other. Conjugation will then take place between two dissimilar gametes, and is said to be anisogamous.

The first hint, so to speak, of sexual differentiation is to be observed in the behaviour of the conjugating gametes; it is a physiological rather than a structural or morphological phenomenon, and consists in the fact that one gamete is active while the other remains comparatively passive. We shall find that this distinction lies at the root of all sexual differentiation throughout the animal and vegetable kingdoms. The more active gamete is spoken of as male and the more passive as female. The passivity of the female is intimately associated with and probably to a large extent dependent upon the fact that it contains more 
cytoplasm and is therefore more heavily weighted than the male gamete. This cytoplasm, moreover, is in many cases densely charged with food material, which constitutes the capital with which the zygote, formed by the union of the two gametes, has to begin its new life cycle.

It is quite clear that the primary distinction between the sexes is a simple case of division of labour accompanied by a corresponding structural differentiation. Two ends have to be secured by the gametes. They must come together in order that they may conjugate, and therefore one or both must be capable of active locomotion. They must also contain between them sufficient material, either in the form of actual protoplasm or of some substance that can easily be worked up into protoplasm, to give the new individual which results from their union a fair start in life.

A cell body heavily weighted with food material is, however, clearly incompatible with great activity, so one of the two gametes remains unencumbered and becomes specialized as the active partner, charged with the duty of seeking out its mate and bringing about their union, while the other, more or less burdened with the necessary supplies, passively awaits the event. The conjugation of such differentiated gametes leads to a more satisfactory result than can be attained in cases of isogamy like that of Copromonas, for the new individual will have a better chance in life owing to the greater amount of capital with which it commences. Such sexual differentiation of the gametes finds its most complete expression in the formation of female ova and male spermatozoa, which are especially characteristic of the higher animals (compare Fig. 69), though they also occur in many plants and even in some unicellular forms. The process of conjugation in such a case is often spoken of as the fertilization of the ovum by the spermatozoon.

These considerations enable us to understand at once the great difference in size which usually distinguishes the male from the female gamete, whence the general terms microgametes and megagametes so often applied to them. The microgamete is as small as possible in order that its activity may not be impaired; the megagamete is swollen out with nutrient material.

We may illustrate these general principles by a brief description of a few more cases of conjugation amongst unicellular organisms.

Bodo, or Heteromita (Fig. 38), is a very minute flagellate monad 


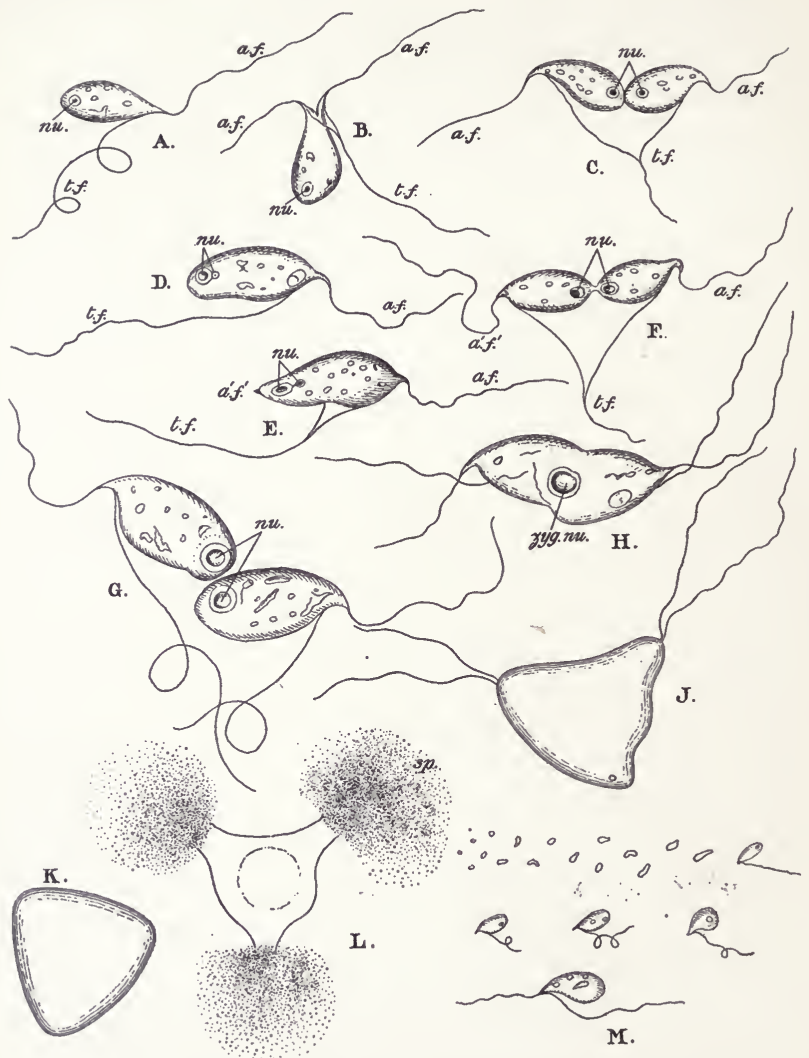

FIa. 38.-Life History of Bodo, or the Springing Monad, very highly magnified. (From Dallinger and Drysdale.)

A. Ordinary individual.

B., C. Multiplication by longitudinal fission (the nucleus divides and the cell body and both flagella split lengthwise).

D-F. Multiplication by transverse fission (the nucleus and cell body divide, the trailing flagellum splits lengthwise and a new anterior flagellum is budded out at one end).

G. Two gametes about to conjugate.

H. Conjugation.

J. Zygote formed by conjugation, with flagella still attached.

K. Fully forined zygote.

L. Escape of spores by rupture of the zygote wall.

M. Development of the spores.

$a . f$., anterior flagellum; $a^{\prime} . f^{\prime}$., new anterior flagellum sprouting out; $n u$., nucleus; sp., spores; t.f., trailing flagellum; zyg.nu., zygote nucleus. 
which occurs in long-standing infusions of cod's head. It differs from Copromonas chiefly in the possession of two flagella and in the absence of a cell mouth, all its food being taken in in a state of solution by diffusion through the thin cell membrane. The two flagella both spring from the beak-like anterior extremity. One (A, a.f.) extends forwards and by its movements enables the organism to swim actively about, the other (A, t.f.) hangs down and is trailed behind during active locomotion. The monad anchors itself by the trailing flagellum and then, by coiling and uncoiling the latter, executes characteristic springing movements. Asexual reproduction (i.e., reproduction without any sexual process) is effected by simple fission, which may be either longitudinal $(\mathrm{B}, \mathrm{C})$ or transverse $(\mathrm{D}-\mathrm{F})$.

There are no structurally differentiated gametes or sexual cells, but conjugation $(\mathrm{G}-J)$ is effected between two apparently similar individuals which are indistinguishable from the ordinary form. It is noteworthy, however, that one of the two gametes at the time of union is anchored, while the other swims actively up to it, and thus we get a slight indication of physiological differentiation into active and passive, or male and female. The male gamete also arises by a somewhat peculiar method of fission. Conjugation of the two gametes produces a zygote which has somewhat the shape of a triangular sac (K). The flagella disappear and in the interior of the sac cell-division goes on with great rapidity, giving rise to an immense number of very minute spores, which ultimately escape from the corners of the sac in the form of very fine dust $(\mathrm{L}, s p$.$) . Each spore no doubt is a minute nucleated cell, but it is$ so small that the nucleus cannot at first be made out. It grows by absorbing liquid food from the infusion in which it lives, and as it grows the nucleus becomes apparent, flagella are put forth, and the adult form is gradually attained (M). We have here a striking illustration of the fact that the most obvious result of conjugation is an increase of the power of cell-division.

As a case of complete morphological as well as physiological differentiation between male and female gametes in a unicellular organism we may take that of Coccidium schubergi, which occurs as a parasite in the intestine of a centipede (Lithobius forficatus). The life history of this remarkable protozoon is very complicated and it is not necessary for our purposes to describe it in detail. The adult organisms occur in the form of spherical nucleated cells, each actually inside one of the epithelial cells 
which line the intestine of the host and upon which the parasites feed (Fig. 39, A.). The latter increase in number very rapidly by a kind of multiple fission, and successive generations of parasites attack fresh epithelial cells of the host until the epithelium is more or less completely destroyed. After many generations have been produced asexually in this manner a sexual process sets in. Megagametes and microgametes are produced; the former by growth of an ordinary individual into a large spherical ovum, or egg-cell (Fig. 39, B., \& gam.), the latter by growth and division of an ordinary individual into a number
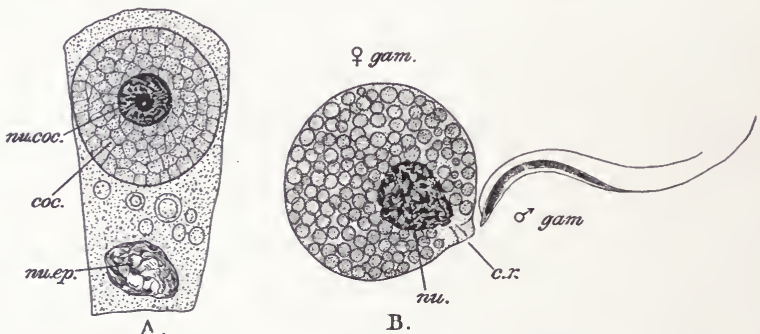

Fig. 39.-Coccidium schubergi, highly magnified. (After Schaudinn.)

A. A full grown Coceidium lying within an epithelial cell of the host.

B. Male and female gametes about to conjugate.

coc., the Coccidium; c.r., the cone of reception put out by the female gamete; $n u$, nucleus of female gamete; nu.roc., nucleus of adult Coccidium ; nu.ep., nucleus of epithelial cell of host; ð gam., male gamete or spermatozoon; $q$ gam., female gamete or ovum.

of very much smaller spermatozoa or sperm cells (Fig. 39, B., ơ gam.).

The ovum is densely filled with food granules and has no power of locomotion. The spermatozoon closely resembles a flagellate monad, being provided with a pair of flagella by means of which it swims actively about; the body, however, is long and slender and consists almost entirely of nuclear(chromatin) material. It seeks out the ovum, which exercises a peculiar attraction upon it, and the two conjugate, the spermatozoon boring its way into the ovum and their nuclei fusing to form the zygote nucleus. The only trace of activity, apart from nuclear phenomena, which the ovum exhibits is the protrusion of a small "cone of reception" (Fig. 39, B. c.r.) from the surface of the cell towards the approaching spermatozoon, which seems to indicate that the 
attraction is mutual. The process of conjugation is followed as usual by cell-division on the part of the zygote, which in this case results in the formation of a small number of comparatively large spores, enclosed in tough protective envelopes. From these spores new individuals are produced which, under favourable circumstances, commence the life cycle afresh. It is extremely interesting to observe that we have here, in a unicellular Protozoon, as complete a sexual differentiation of the gametes as we meet with in any of even the most highly organized plants and animals.

We must now briefly notice the sexual phenomena exhibited by those interesting Protista which we have already had occasion to refer to in Chapter IV. under the name Phytoflagellata. It will be remembered that in Hæmatococcus (Fig. 5) the process of simple fission sometimes results in the production of a relatively large number (32-64) of small individuals instead of the usual four comparatively large ones. These small individuals are specialized gametes, differing from the large ones not only as regards size but also in the absence of the characteristic cell-wall of the latter. There is, however, no differentiation into male and female. Conjugation is of the isogamous type and produces a zygote which grows into an ordinary resting cell which will presently begin to multiply actively by ordinary fission.

We have also seen that this organism forms the starting point of a series of forms, represented by the genera Hæmatococcus, Pandorina, Eudorina and Volvox, which illustrate progressive stages in the process of colony formation. The same series also shows us very clearly the differentiation between male and female gametes - the origin of sex in the vegetable kingdom. It will be remembered that Pandorina forms colonies of sixteen or thirtytwo cells enclosed in a common envelope (Fig. 10, A). A sexual multiplication is effected by each cell of the colony dividing into $2,4,8$, and finally 16 or 32 , which form a daughter colony within the parent, to be liberated presently by softening of the parental envelope. Occasionally, however, the individual cells of a colony divide each into eight gametes. These are small cells, each with a pair of flagella, which escape and swim about separately. They exhibit no clear distinction into male and female, but some are comparatively large, some small, and some intermediate in size. They conjugate in pairs (Fig. 10, B), and the two members of a conjugating pair are often, though apparently not always, of 
different sizes. It is probable that we have here a kind of foreshadowing of that sharp differentiation into large (female)
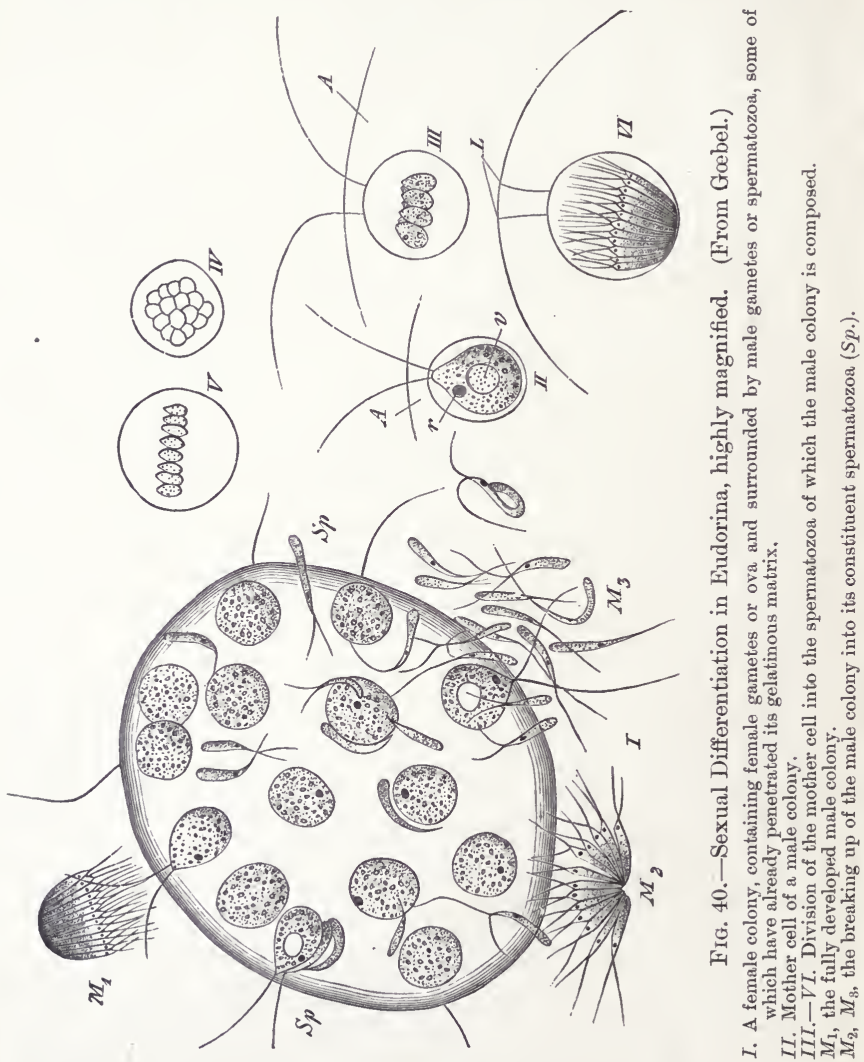

megagametes and small (male) microgametes of which we have already spoken.

In Eudorina, which forms colonies somewhat similar to those of Pandorina, this differentiation is already fully expressed (Fig. 40). The megagametes or ova ${ }^{1}$ differ scarcely at all from ordinary 
individuals. They are shown imbedded in the gelatinous matrix of the large colony on the left of the figure. The microgametes or spermatozoa, ${ }^{1}$ on the other hand, are much smaller, club-shaped bodies, having a characteristic yellowish colour and with a pair of flagella at the narrow, pointed end (Fig. 40, $\mathrm{M}_{3}$ ). They are produced in bundles of sixty-four by repeated division of a mother cell (Fig. 40, II-VI). Thus the male and female gametes, spermatozoa and ova, do not occur together in the same colony, but the colonies, when they consist of gametes and not of ordinary individuals, contain only one or the other kind. Hence the sexual differentiation is in this ease extended from the gametes themselves to the colonies which bear them, and we may recognize colonies of three kinds: (1) Asexual, which produce no gametes and reproduce by ordinary fission of all or any of the component cells, (2) male sexual, which produce microgametes or spermatozoa, and (3) female sexual, which produce megagametes or ova. The bundles or colonies of microgametes (Fig. 40, $\mathrm{M}_{1}, \mathrm{M}_{2}$ ) swim about actively by means of their flagella, apparently in search of the larger and much less active female colonies (Fig. 40, I). Having found such a colony the now separated microgametes (Fig. 40, $\mathrm{M}_{3}$ ) bore their way in, ultimately conjugating with the megagametes to form zygotes.

In any one colony of Pandorina or Eudorina the constituent cells are all of one kind, all ordinary asexual cells or all male gametes or all female gametes. In Volvox (Fig. 11) we meet with a further advance. Even in asexual colonies which do not produce gametes at all we find the cells differentiated in so far that some only are capable of giving rise (asexually) to daughter colonies, while in the female colonies only some of the cells form female gametes or ova (Fig. 11, A, o). The male gametes (Fig. 11, B-D) are very similar to those of Eudorina. 'They unite with the female gametes to form zygotes, which, after a period of rest, develop into new Volvox colonies.

In order to emphasize the fact that the process of conjugation is essentially a nuclear phenomenon we may now turn to the case of Paramœcium. The general appearance and structure of this protozoon have been described in Chapter IV. (Fig. 8). It multiplies by simple transverse fission, and under favourable conditions continues to do so until exhaustion sets in, when its

\footnotetext{
1 Often termed by botanists spermatozoids.
} 
failing powers are restored by conjugation. The conjugation in this case, however, is not quite like that which takes place in the other unicellular organisms which we have been studying, and the term conjugant may be applied, in preference to the term gamete, to the individuals concerned.

It may easily be observed that the union of the two conjugants (Fig. 41) is a merely temporary affair. They remain attached together by their mouth-bearing surfaces for a short
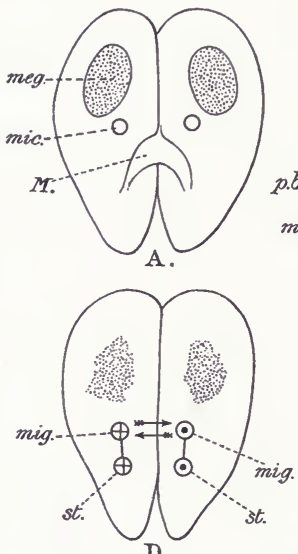

D.

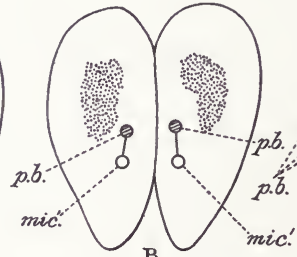

B.

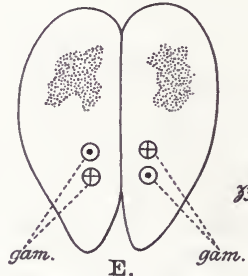

E. gàm.

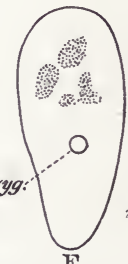

F.

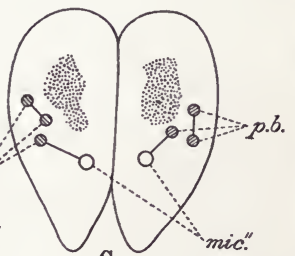

C.

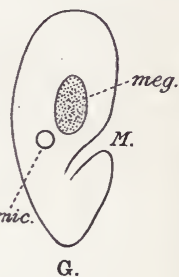

Fig. 41.-Diagram of the Process of Conjugation in Taramoecium.

gam., gametic nuclei; M., mouth; meg., meganucleus; mic., micronucleus; mig., migratory nucleus; $p . b .$, products of the division of the micronucleus which disappear (polar bodies); st., stationary nucleus; zyg., zygote nucleus. (For further explanation see text.)

time and then separate again and continue their independent lives. Before separating, however, they evidently undergo some kind of rejuvenescence whereby their vigour and power of multiplication are completely restored. 'This is accounted for by the fact that during the time of their union cert:in complex nuclear processes take place, the net result of which is an exchange of chromatin material between the two conjugants.

It will be remembered that Paramœeium differs from most Protozoa in the possession of two nuclei, large and small, or meganucleus and micronucleus (Fig. 41, A, meg. and mic.). The 
latter is alone concerned in the process of eonjugation, the meganucleus in the meantime breaking up and being absorbed into the cytoplasm, to be replaced in the manner described later on. We may confine our attention, therefore, to the behaviour of the micronucleus. In each conjugant this divides mitotically into two daughter nuclei (Fig. 41, B, mic.' and p.b.) each of which again divides, so that there are now four micronuclei (Fig. 41, C). Of these four three $(p . b$.$) go to the bad, being apparently absorbed$ into the cytoplasm, while the remaining one (mic.") divides once more, so that each conjugant has now again two micronuclei (Fig. 41, D). These two, though similar in appearance, differ strikingly in their behaviour, one of them remaining quiescent while the other passes over into the body of the other conjugant. They are therefore known respectively as the stationary (st.) and the migratory (mig.) micronuclei. In this way the migratory micronuclei of the two conjugants change places with one another, as indicated by the arrows, and the sole object of the temporary union of the two conjugants appears to be to enable this interchange to take place. When it has been effected a true conjugation occurs between the two micronuclei in each cell (Fig. 41, E, gam.), derived one from each conjugant. This is the real sexual process. The migratory and stationary nuclei are gametic nuclei and the result of their union is a zygote nucleus (Fig. 41, F, zyg.). Moreover, we have here again an evident distinction into male and female gametic nuclei, characterized in the usual way by the activity of the one and the passivity of the other. The two conjugants themselves, however, cannot be distinguished as male and female, for each produces both male and female gametic nuclei and may therefore be regarded as hermaphrodite.

After the interchange of gametic nuclei has taken place the two conjugants separate as ex-conjugants (Fig. 41, F). The zygote nucleus in each divides repeatedly by mitosis and from the daughter nuclei thus produced both micronuclei and meganuclei are formed. Presently the ex-conjugants themselves begin to divide once more by fission and the new micronuclei and meganuclei are distributed amongst the new individuals (Fig. 41, G).

The essential feature of this very complicated process is clearly the same as in the simpler cases which we have examined, and consists in the union of two nuclei belonging to different cells to 
form a single zygote nucleus which has renewed powers of multiplication by division. As already observed, the micronucleus alone takes part in the process, the meganucleus being merely concerned in the life of the individual and its asexual multiplication by simple fission. 


\section{CHAPTER VIII}

Sexual phenomena in multicellular plants-The distinction between somatic cells and germ cells-Alternation of sexual and asexual generationsSuppression of the gametophyte in flowering plants.

When we consider the habit of colony formation which is so common amongst the Protophyta, and which we have discussed in the cases of Pandorina, Eudorina and Volvox, we see at once that it is impossible to draw any strictly logical distinction between such primitive forms and the true multicellular plants or Metaphyta. The common fresh water alga, Spirogyra, for example, might be regarded either as a colony of single cells or as a very simple multicellular plant in which the constituent cells exhibit little or no differentiation amongst themselves. In any case it forms a very convenient starting point for the consideration of the sexual phenomena met with in Metaphyta, being in this respect actually in a much-more primitive condition than either Eudorina or Volvox.

The fully developed Spirogyra plants consist of long green filaments of hair-like dimensions, which float in loose slimy masses in clear fresh water. Each filament consists of a single row of cylindrical cells placed end to end, each cell being enclosed in a thin, transparent wall of cellulose (Fig. 42, c.w.), whereby its protoplasmic contents are completely separated from those of adjacent cells. The cytoplasm forms a thin primordial utricle (p.u.), lining the cell-wall and enclosing

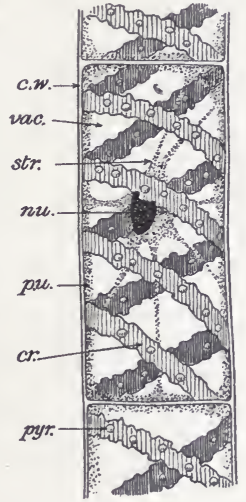

FIG. 42.-Part of a filament of Spirogyra, showing one complete cell and parts of two others; highly magnified.

$c . w .$, cell-wall; $c r$. , chromatophore; nu., nucleus; $p . u$., primordial utricle; pyr., pyrenoid ; str., strands of protoplasm; vac., vacuole.

a large vacuole (vac.) filled with colourless, watery cell-sap, in which a more or less central mass of cytoplasm, containing the 
nucleus $(n u$.$) , is suspended by slender radiating protoplasmic$ threads (str.). So far, both in structure and arrangement of its component cells, the plant closely resembles a single hair of Tradescantia (compare Fig. 25). It differs, however, in the

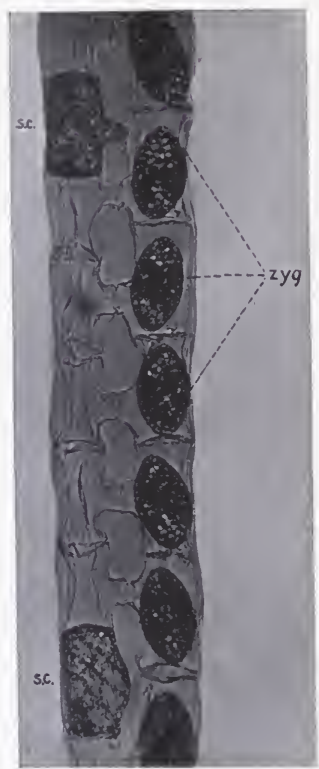

Fra. 43. - Conjugation in Spirogyra, showing in one Filament solitary Cells (s.c.) which have failed to mate, and in the other Zygotes (zyg.), × 82 . (From a photograph.)

presence in each cell of one or more conspicuous chloroplastids or chromatophores $(c r$.$) , coloured bright green$ by chlorophyll and wound spirally round and round inside the cell-wall, like pieces of ribbon. It is from these characteristic structures that the name Spirogyra is derived.

The filaments increase in length by transverse fission of the component cells. Every cell, however, or to speak more accurately its protoplasmic contents, must also be looked upon as a potential gamete. Conjugation in some species takes place between the cells of two filaments which are lying side by side, parallel with one another (Figs. 43, 44). In others it takes place between adjacent cells of the same filament, but we may confine our attention to the former case. The first indication of the process is seen in the formation of a small, hollow protuberance on the wall of one of a pair of cells which happen to be more or less opposite to each other (Fig. 44,a.). This is shortly followed by the formation of a similar protuberance on the wall of the other cell (b.). The two protuberances meet $(c$.$) and fuse$ together, and the cell-walls at the point of union are dissolved away (d.). Thus a hollow eanal is formed placing the cavities of the two conjugating cells in free communication with one another. In the meantime changes are going on in the protoplasmic contents of the conjugating cells, essentially similar in the two members of each pair but with one cell still taking the lead and the other lagging somewhat 
behind. The chloroplastid breaks up; the primordial utricle retreats from the cell-wall towards the middle, and the entire protoplasmic contents round themselves off ints a compact nucleated mass - the gamete (gam.). The time has now arrived for the all-important event; the gamete from one cell-chamber creeps through the canal into the other chamber and conjugates with the gamete which there awaits it (Fig. $44, e$.$) . The result is a$ zygote or zygospore (Figs. 43, 44, zyg.) which surrounds itself with a thick protective envelope within the cell-wall of the parent and after a period of rest germinates, giving rise by repeated transverse division to a new filament.

In this case it will be observed that the two conjugating gametes are morphologically alike, and we may therefore describe the process of conjugation as isogamous. As in the case of Bodo, however, there is a physiological distinction between the two as regards their activity; the gamete which takes the lead throughout the whole process and finally crawls through the canal of communication is obviously to be regarded as male, and its partner as female. Moreover, one entire filament may produce nothing but male gametes and may therefore be regarded as a male plant (Fig. $44, \delta$ ), while the other may produce

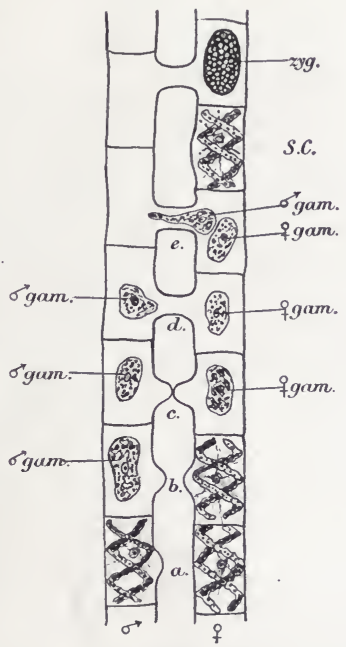

Fig. 44.-Diagram of two conjugating filaments of Spiro. gyra.

a.-e., pairs of cells in successive stages of conjugation; $\bar{\sigma}$ gam., male gametes; 9 gam., female gametes; S.C., solitary cell; zyg., zygote. only female gametes and therefore be regarded as a female plant (Fig. 44, $q$ ). In the closely related form, Zygogonium, there is no distinction of any kind between the two sexes, both gametes exhibit the same degree of activity and they meet each other halfway, in the connecting canal between their respective chambers, to form the zygote.

In Spirogyra, as we have just seen, every cell of the adult plant is a potential gamete or germ cell ; in the case of Volvox, it will 
be remembered, this is not so, certain cells only being concerned in the sexual process. We have here a kind of fore-shadowing of that distinction into somatic or body cells and sexual or germ cells which is so characteristic of the higher organisms, both plants and animals. We see a somewhat similar fore-shadowing

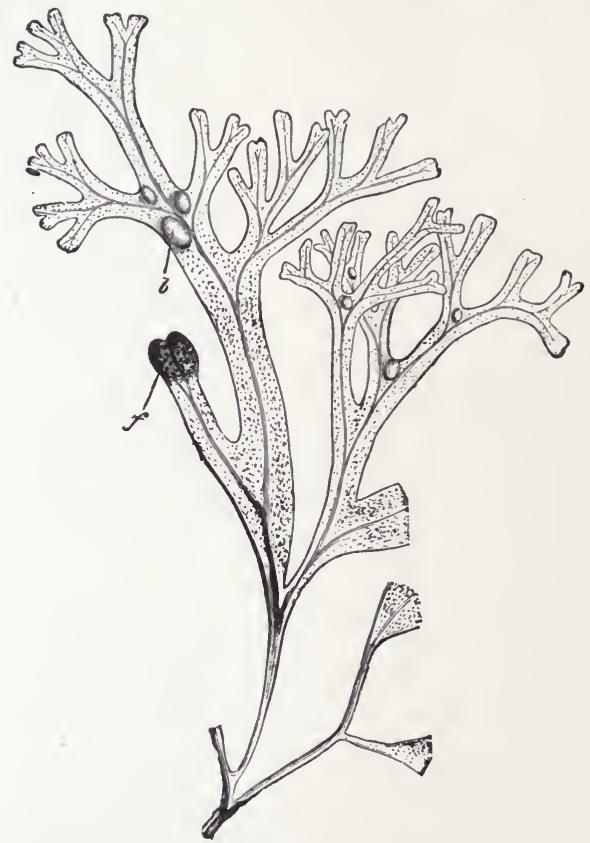

FIG. 45.-Fucus vesiculosus, about half nat. size. (From Vines' "Botany.") $b$, air bladders; $f$, fertile branch.

even in the unicellular Paramœcium, except that the distinction is here confined to the nuclei, the meganucleus being concerned with the life of the individual and the asexual process of ordinary cell-division, while the micronucleus is alone concerned in the sexual phenomena. We can, in this case, distinguish between somatic nucleus and germ nucleus, though not between somatic and germ cells. 
'The loss of the power to act as germ cells or gametes which the vast majority of the constituent cells in a typical multicellular organism usually suffer is undoubtedly one of the penalties which they have to pay for their high degree of specialization. The germ eells themselves, on the other hand, always remain in a more primitive, less specialized condition, and may, in fact, be regarded as so many unicellular Protista enclosed within the multicellular body. They resemble the free-living Protozoa and Protophyta also in that they exhibit a certain degree of independence and are in the majority of cases actually set free from the parent body as unicellular individuals;

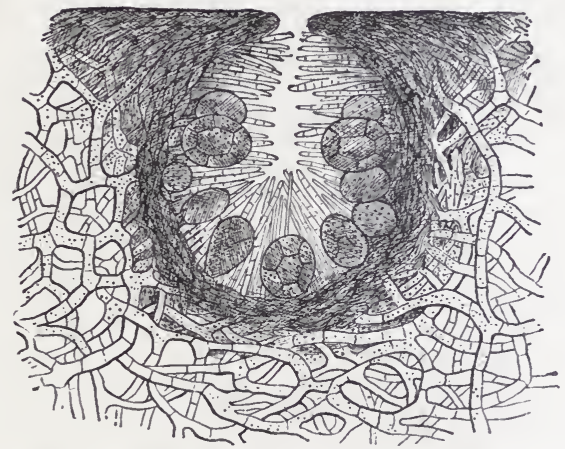

Fig. 46.-Fucus vesiculosus; Section through a female Conceptacle, $\times 50$. (From Vines' "Botany," after Thuret.)

short-lived, it is true, unless they happen to meet one another and conjugate, but nevertheless enjoying that liberty which is only possible to independent organisms.

All this is very beautifully illustrated in the case of the common brown seaweed or bladder-wrack, Fucus resiculosus (Fig. 45). The entire plant consists of flattened branches, copiously subdivided and attached by a root, uscially to some rock between tide-marks. Here and there the branches are swollen out to form air-bladders (b), which serve as organs of flotation. The histological structure of the plant is comparatively simple and need not detain us; we are concerned only with its reproductive processes.

Fucus resiculosus is diœeious or unisexual, there being distinct 
male and female plants. In both cases certain branches $(f)$, usually described as fertile, are characterized by the presence of numerous minute spherical pits, opening on to the surface by narrow mouths. These pits or conceptacles, one of which is represented in vertical section in Fig. 46, contain the sexual organs, male antheridia or female oogonia, as the ease may be, intermingled with hair-like structures known as paraphyses. 'The antheridia (Fig. $47, a$ ) are attached to the branching paraphyses in the male
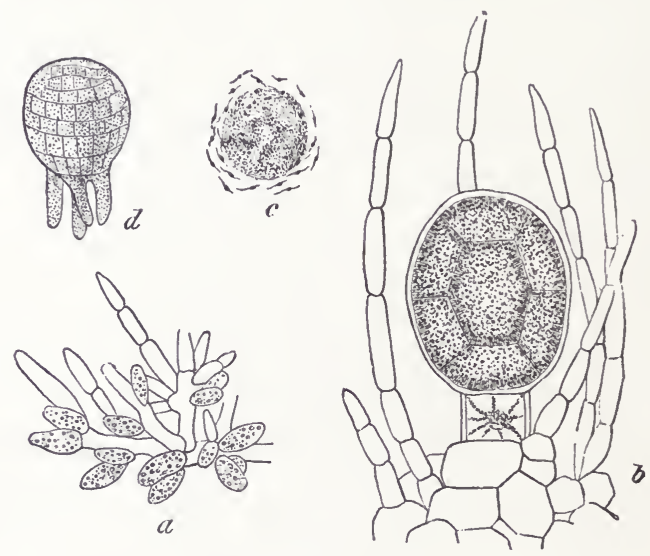

FIG. 47.-Fucus vesiculosus.

$a$, branching paraphysis from male conceptacle, bearing antheridia; $b$, an oogonium surrounded by unbranched paraphyses and with its contents divided into eight ova; $c$, a discharged ovum surrounded by spermatozoa, one of which will fertilize it; $d$, a developing embryo ; all $\times 160$. (From Vines' "Botany," after Thuret.)

conceptacles in the form of small sacs in which the male gametes (spermatozoa) are produced. These are minute, nucleated, pearshaped cells, each with two flagella; thiey are produced in large numbers in each antheridium and set free by rupture of the wall of the latter to make their way out of the opening of the conceptacle by their own activity.

The oogonia are oval sacs, a good deal larger than the antheridia, and occur amongst the hair-like paraphyses in the female conceptacles (Fig. 46). In each oogonium (Fig. 47,b) eight female gametes (egg cells, ova or oospheres) are formed by cell-division. These are spherical, nucleated cells, very much larger than the 
spermatozoa, owing to the great amount of cytoplasm which they contain. They are liberated by rupture of the oogonium and discharged through the opening of the conceptacle on to the surface of the plant. There they are found by the spermatozoa, which swarm around them in large numbers, endeavouring to conjugate (Fig. 47, c). Finally a single spermatozoon succeeds in boring its way into each large egg cell, and fertilization is effected by the union of the male and female nuclei. The zygote, well supplied with food material by the egg cell, begins to undergo cell-division immediately, forming a multicellular embryo (Fig. $47, d$ ) which attaches itself by roots and grows into a plant resembling the parents.

Here we have a perfectly typical case of differentiation of the gametes or germ cells into large passive female ova and small active male spermatozoa, and conjugation is anisogamous, the ovum being "fertilized" by the spermatozoon.

In the ferns, mosses and other more highly organized plants a new complication is introduced by the fact that two distinct forms of the plant alternate with one another in the life cycle. In one only of these forms, known accordingly as the gametophyte, does a sexual process occur; the other, known as the sporophyte, reproduces by means of unicellular spores, which are produced asexually and develop into new individuals without any process of conjugation. The gametes or germ cells, borne on the gametophyte, on the other hand, conjugate, and the zygote develops, not into another gametophyte but into a sporophyte, while, conversely, the spores produced by the sporophyte develop into gametophytes.

This alternation of sexual and asexual generations is a phenomenon of very wide-spread occurrence in the vegetable kingdom, and, as we shall see in our next chapter, something of the same kind occurs also in certain multicellular animals.

Take, for example, any ordinary fern. The conspicuous plant (Fig. 48) is the sporophyte. It is very highly organized and shows the typical differentiation into root, stem and leaf met with in all the higher groups of the vegetable kingdom. Some or all of the leaves sooner or later produce on their lower surfaces sporangia (Fig. 48, A, C), little sac-shaped structures in which the spores arise-by division of mother cells into fours. These spores are liberated, by rupture of the sporangia, in the form of fine brown dust, which may be carried to considerable distances by the wind. 


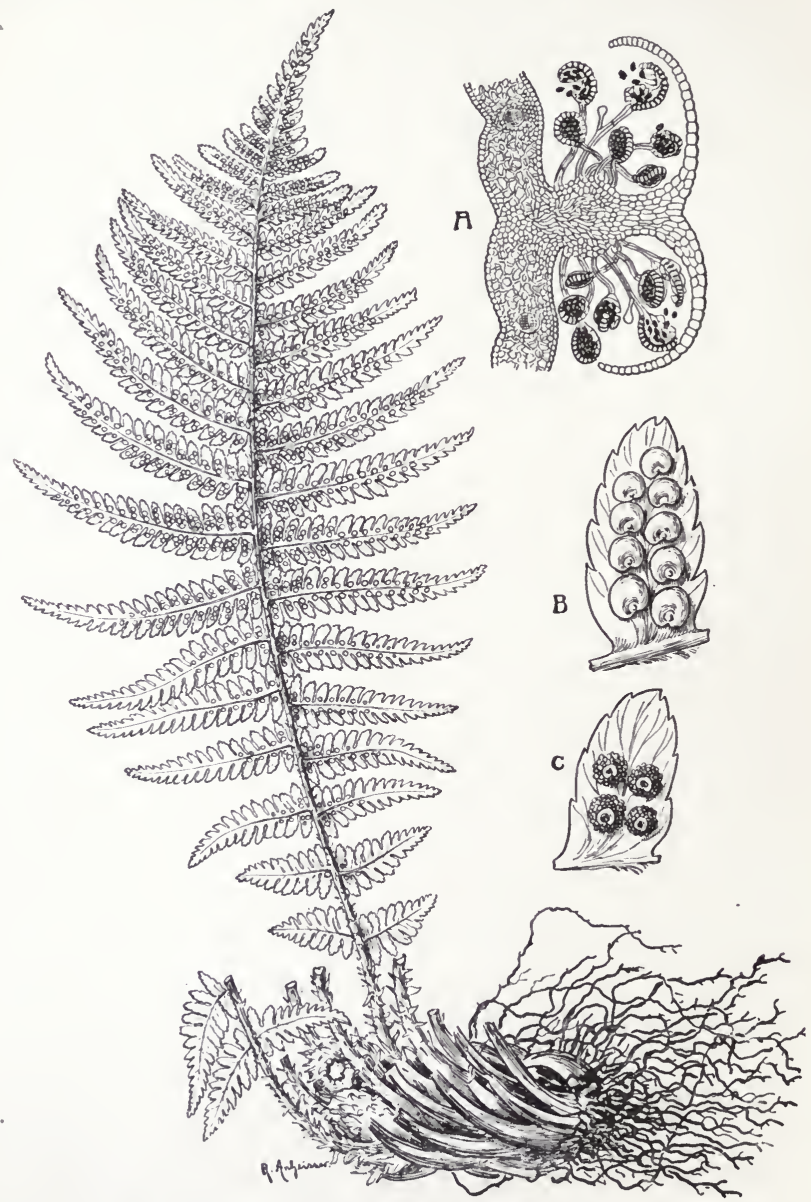

FIG. 48.--The Sporophyte Generation of a Fern, Aspidium filix mas. (From Strasburger.)

A, section through a sorus or group of sporangia, covered by the indusium ( $\times 20$, after $\mathrm{Kny}$ ); $\mathrm{B}$, lower surface of a pinna, showing the indusia covering the sori; $\mathrm{C}$, lower surface of a pinna with the sori exposed. 
If one of them alights on a suitable spot, in a moist and shady situation, it may germinate (Fig. 49). Its thick outer wall ruptures and a delicate tube is put forth, containing the protoplasm and nucleus. Celldivision takes place and results presently in the formation of the gametophyte.

The gametophyte of the fern (Fig. 50) is known as a prothallus. It is an independent, self-supporting plant, but much less highly orga-

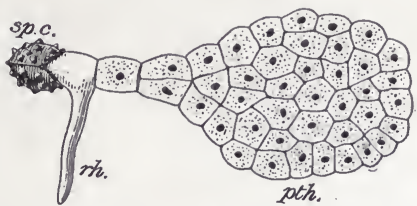

FIG.49.-Diagram of a young Prothallus ( $p t h$.$) formed by Germination of a$ Fern Spore.

$r h$., rhizoid or root hair; sp.c., spore coat. nized than the sporophyte, consisting usually of a green, heartshaped plate of cells, not more than perhaps a quarter of an inch in diameter, and attached to the substratum by delicate hair-like rhizoids. It develops no vascular system but never-

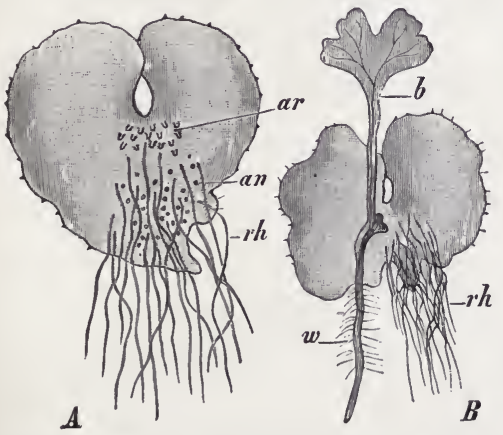

FiG. 50.--The Gametophyte Generation or Prothallus of a Fern, Aspidium filix mas, $\times 8$. (From Strasburger.)

$A$, lower surface of a sexually mature prothallus, showing antheridia $(a n)$, archegonia $(a r)$, and rhizoids $(r h)$.

$B$, an older prothallus with the young sporophyte generation or fern plant $(b, w)$ attached to it.

theless obtains its food in the same way as the sporophyte, absorbing water containing dissolved mineral salts from the soil by means of its rhizoids, and splitting up carbon dioxide, obtained from the air, by aid of its chlorophyll. Such prothalli are frequently to be found attached to the surfaces of flowerpots and walls in damp greenhouses and other places where ferns are grown.

The sexual organs, male antheridia (Fig. 50, an) and female archegonia (Fig. 50, ar), are, like the rhizoids, found on the lower surface of the prothallus, both usually occurring on one and the same plant, which is therefore monœeious or hermaphrodite. The antheridia (Fig. 51,a) are essentially similar 
to those of Fucus, being hollow sacs in which the male gametes are developed. The latter (Fig. 51, s.) are active spermatozoa,

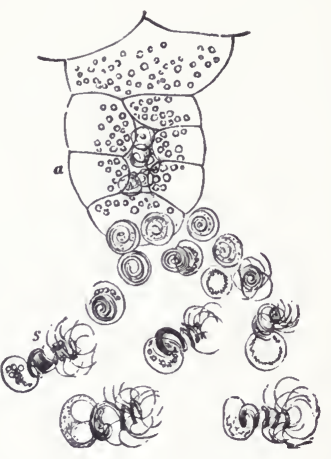

FIG. ó1.--Antheridium of a Fern, discharging Spermatozoa (Antherozoids) from its opening. highly magnified.

(From Vines' "Botany.")

$a$, antheridium; $s$, spermatozoon.

each having a spirally coiled body, consisting chiefly of chromatin material, and bearing a bunch of cilia at one end, by the vibration of which the gamete swims actively about in any dew or other moisture which may be deposited on the prothallus.

The archegonia (Fig. 52) differ considerably from the oogonia of Fucus, having a characteristic structure which is more or less accurately repeated in the corresponding organs of all the higher plants. Each consists of a hollow swollen venter, sunk in the tissue of the prothallus, and a long neck which projects from the surface, and the wall of which is composed of four rows of cells. The venter contains a single relatively large ovum or oosphere (Figs. 52, A, o, and $52, \mathrm{~B})$, above which an axial row of canal cells $\left(K^{\prime}, K^{\prime \prime}\right)$

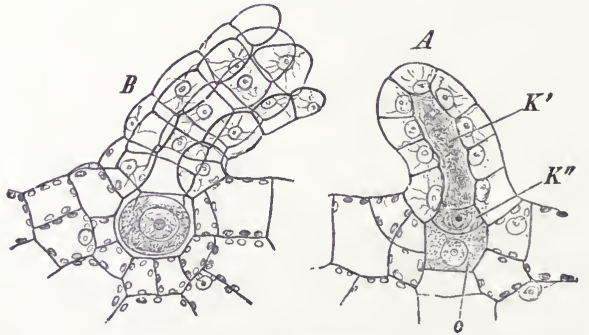

FIG. 52.-Archegonia of a Fern, Polypodium vulgare, $\times 240$. (From Strasburger.)

$A$, young, showing ovum (o) and canal cells $\left(K^{\prime}, K^{\prime \prime}\right)$, and with the end of the neck closed. $B$, mature, with the end of the neck open.

extends into the neck. When the ovum is ready for fertilization the canal cells degenerate into mucilage and the cells at the end 
of the neck separate so as to form an opening (Fig. 52, B). The spermatozoa appear to be attracted to the opening by an acid secretion discharged therefrom. One of them makes its way down the neck to the ovum and fertilizes it by the usual process of conjugation.

The zygote begins to develop, by cell-division, within the venter of the archegonium, and forms a young sporophyte, which for some time remains attached to the prothallus as shown in Fig. 50, B, drawing nutriment therefrom by means of a special temporary organ known as the foot. Presently, root, stem and leaf are developed and the sporophyte becomes self-supporting.

One very remarkable fact in connection with the sexual process in the fern remains to be noticed. The gametes, as we have seen, are normally produced in special sexual organs and are themselves perhaps as highly differentiated in relation to the function of conjugation as gametes ever are. It has been found, however, that if the normal sexual union between ova and spermatozoa be prevented a conjugation may take place between nuclei from adjacent vegetative cells of the prothallus, resulting in the formation of an embryo sporophyte by so-called apogamy. In these cases it is obvious that the sexual process is not really suppressed, but simply transferred to ordinary prothallial cells, which, though they do not conjugate under normal circumstances, have retained the power of so doing when occasion arises. It seems probable, however, that in some cases true apogamy, or suppression of the sexual process, occurs, the embryo sporophyte arising from the prothallus without any conjugation of gametes.

The gametophyte or prothallus of an ordinary fern is, as we have already seen, produced by the development of a unicellular spore (Fig. 49), and in most cases is monocious or hermaphrodite, bearing both male and female sexual organs and male and female gametes. In the less commonly known and not very fern-like "heterosporous" forms, however (Isoëtes, Salvinia and Marsilea), the gametophyte is diøeious or unisexual, there being distinct male and female prothalli, and this sexual differentiation affects not only the prothalli themselves but the spores from which these are developed. Hence in these forms we find small microspores which produce male prothalli, and large megaspores which produce female prothalli. The spores themselves are set free from the parent sporophyte, but the prothalli are very much 
reduced in size and never become free from the spores; they nevertheless develop antheridia and archegonia respectively, in which spermatozoa and ova are produced; and from the conjugation of these arise zygotes or fertilized ova which develop into new sporophytes.

We have briefly noticed these heterosporous ferns because, as regards the sexual phenomena which they exhibit, they constitute a very interesting connecting link between the ordinary (homosporous) ferns, which produce only one kind of spore, and the highest members of the vegetable series, the flowering plants.

In the flowering plants an alternation of sexual and asexual generations can still be traced, but here the gametophyte is so much reduced in size and has become so degenerate in structure that it is quite inconspicuous, and can only be detected by microscopical examination and recognized as constituting a distinct generation in the light of our knowledge of lower forms.

The flowering plant itself is the sporophyte, and it is heterosporous, producing microspores and megaspores. The pollen grains are the microspores, while the megaspores are represented by the embryo sacs enclosed within the ovules or unripe seeds. The microspores, like the spores of ferns, are set free from the parent sporophyte, the megaspores, however, are never set free as such, and in neither case does the gametophyte become free from the spore.

The terms pollen grain and embryo sac were applied to the structures in question long before their true nature as microspores and megaspores was recognized, and they have become so firmly established that it is hardly possible to avoid using them.

If we examine any typical, fully developed flower, such as is represented diagrammatically in Fig. 53, we shall find that it consists of four whorls or circlets of specially modified leaves. Beginning at the outside we find first the calyx $(K e)$, composed of a number of sepals, which usually, but by no means always, retain the green colour characteristic of leaves and serve mainly for the protection of the inner parts of the flower while in the bud; then the corolla $(K)$, composed of petals, which may be brightly coloured and serve to attract insects; then the androcium, composed of stamens $(a, f)$; and lastly, in the centre of the flower, the gynœeium or pistil $(n, g, F)$, composed of carpels. 'The stamens and carpels are often spoken of as the essential 
parts of the flower. They are really to be regarded as sporebearing leaves or sporophylls.

Each stamen consists usually of a long stalk or filament $(f)$, bearing an anther $(a)$ at its extremity. The anther is a bilobed structure, and each lobe contains two chambers, or pollen sacs, in which the pollen grains $(p)$ are formed by the division of mother cells into fours, just as the spores of an ordinary fern are developed within the sporangia. The pollen sacs are, in fact, nothing but sporangiaand microsporangia, because they contain microspores.

The pistil is formed of a varying number of carpels, which, either singly or united, give rise to a closed chamber below, the so-called ovary ${ }^{1}(F)$, surmounted by a longer or shorter style $(g)$, ending above in a rounded viscid surface, the stigma $(n)$. In the interior of the ovary, attached to the carpels, are developed the ovules $(S)$, which are nothing but sporangia (megasporangia) enclosed each in a double envelope (i). In each ovule a single embryo sac or megaspore $(\mathrm{em})$ is produced. Only one ovule

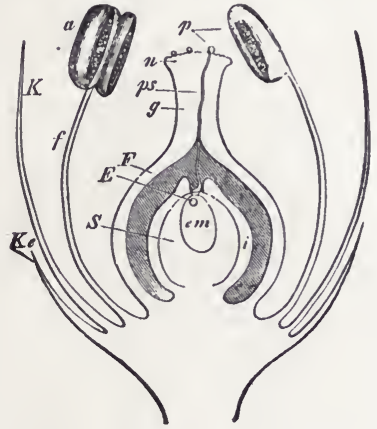

Fig. 53.-Diagram of a typical Flower in vertical Section. (From Vines' “ Botany.")

$a$, anther; $e m$, embryo sac; $E$, ovum ; $f$, filament of stamen; $F$, wall of ovary; $g$, style; $i$, integument of ovule; $K$, corolla; $K e$, calyx; $n$, stigma; $p$, pollen grains; $p s$, pollen tube; $S$, ovule.

is represented in the diagram, but there are usually a large number in each ovary.

Having thus briefly described the parts of the sporophyte with which we are immediately concerned, we must turn our attention for a few moments to the gametophyte. The male gametophyte, which never consists of more than a very small number of cells, is developed from the pollen grain or microspore. This latter is at first a perfectly typical unicellular spore, with a single nucleus surrounded by cytoplasm, and the whole enclosed in a thick protective cell-wall often ornamented with microscopic sculpture of various patterns. The commencement

1 This is a very unfortunate name because the structure in question is an entirely different kind of organ from the ovary of animals. 
of germination of this spore in typical cases (Fig. 54) is marked by the division of the nucleus into two. Around one of these two cytoplasm collects to form a naked "antheridial cell" (iil) ; the other, with the remainder of the cytoplasm, constitutes the "vegetative cell" $(k)$, which may or may not divide again. The antheridial cell divides into two "generative cells." The vegetative cell, or cells, represents the last vestige of the body of the male prothallus; the generative cells are male gametes. The germination of the pollen grain and development of the

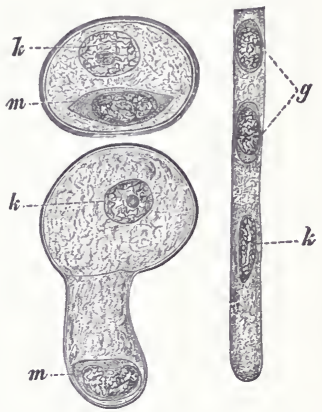

FIG. 54.-Germination of the Pollen Grain of Lilium martagon, $\times 375$. (From Strasburger, after Guignard.)

$k$, nucleus of the vegetative cell of the pollen tube; $m$, antheridial cell ; $g$, male gametes formed by division of the antheridial cell. male prothallus are completed by the putting forth of the pollen tube (Fig. 53, ps, Fig. 54), which takes place if the pollen grain is fortunate enough to alight upon the stigma of a flower of the right kind. The pollen tube forces its way through the loose tissue of the style to the ovary and comes into intimate relations with one of the ovules contained therein.

The female gametophyte is represented by a few cells formed by division of the megaspore (embryo sac), or rather of its nucleus, and to some extent of its cytoplasm, within the ovule. The process is a somewhat complicated one, but, without going into details, we may note that at the time when the ovule is ready for "fertilization" the embryo sac in a typical flowering plant (Angiosperm) contains seven cells, one of which is a female gamete (ovum or oosphere), while the others may be taken to represent the female prothallus, including a vestige of an archegonium. The arrangement of these cells is shown in Fig. $55(a t, e, k, s)$.

The embryo sac or megaspore $(E)$ is surrounded by a cellular layer known as the nucellus (Fig. 55, $K$ ), which represents the wall of the sporangium, and this in turn by two other coats (ai and ii), the outer and inner integuments of the ovule, which grow up around the nucellus. The entire ovule is attached to the wall of the ovary by a stalk or funiculus $(f)$, upon which it is 
frequently bent sharply round as shown in Fig. 55. Opposite to the spot where the funiculus is attached to the ovule an aperture is left in the integuments known as the micropyle $(\mathrm{m})$. It is through this micropyle that the tip of the pollen tube usually forces its way in search of the female gamete $(e)$, which lies at the end of the embryo sac close to it. The wall of the pollen tube and that of the embryo sac are absorbed where they come in contact with one another, and thus a passage is opened for the male gamete, which passes down the pollen tube into the embryo sac and there conjugates with the ovum. The zygote thus formed develops within the embryo sac into an embryo sporophyte ${ }^{1}$; the integuments of the ovule become hardened to form the seed coat; reserve food material, such as starch or oil, is stored up either in the embryo itself or in the endosperm ${ }^{2}$ around it, and the ovule and its contents separate from the parent sporophyte as the ripe seed (Fig. $56, \mathrm{~A})$.

When the seed ripens the development of the contained embryo is suspended for an indefinite period, to be resumed again if and when the seed finds

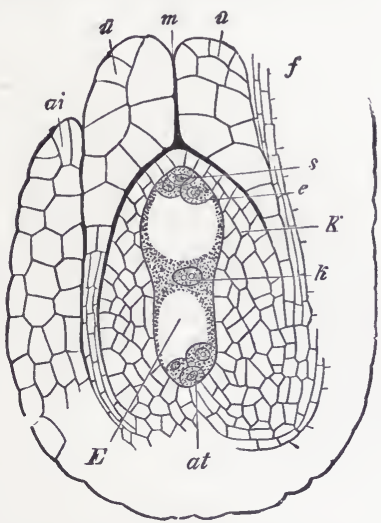

FIG. 55.-Diagram of the unfertilized Ovule of a Flowering Plant (Angiosperm) in longitudinal Section. (From Vines' "Botany.")

$a i$, outer integument; at, antipodal cells; $e$, ovum; $E$, embryo sac; $f$, funiculus; $i i$, inner integument; $k$, central or definitive nucleus of embryo sac; $K$, nucellus ; $m$, micropyle ; $s$, synergidœ. a suitable situation in which to germinate. The embryo may then continue its development into the adult sporophyte (Fig. 56, B).

It is interesting to notice that no less than three distinct generations take part in the formation of the seed. The seed coat belongs to the parent sporophyte, while the contents of the

1 Vide pp. $48-50$ (Fig. 14).

2 The second generative nueleus from the pollen tube conjugates with the nueleus in the middle of the embryo sac (Fig. 55 $k$ ), and the "endosperm " arises by repeated division of the nueleus resulting from this eonjugation. The whole process is somewhat complex and it is not essential to our present purpose to deseribe it in detail. 
embryo sac at first represent the female gametophyte, which disappears as the zygote develops into the embryo of another sporophyte. In this way a very intimate relation is established between each sporophyte generation and the one which precedes it, and the gametophyte is crushed out of existence, as an independent generation, between the two.

Accompanying this almost total suppression of the gametophyte we find a delegation of certain responsibilities connected with the sexual function to the asexual sporophyte, and the development by the latter of what may be termed vicarious sexual characters.
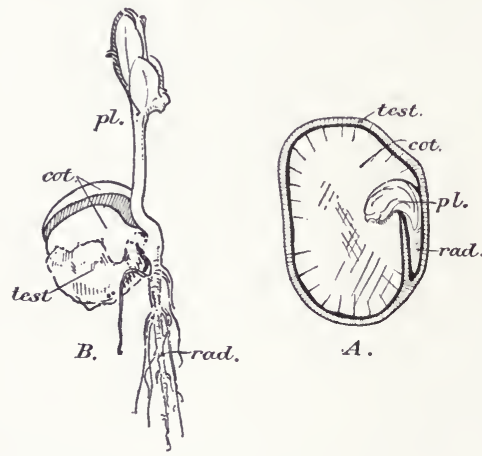

FıG. 50.-Garden Pea (Pisum sativum).

$A$, ripe seed split in half; $B$, seed germinating.

cot., cotyledons or seed leaves; $p l$, plumule or shoot of embryo; rad., radicle or root of embryo; test., testa or seed coat.

These characters find their expression in that most remarkable feature of all the flowering plants, the flower itself. Thus the use of the terms male and female may be extended in the first instance to the stamens and pistil, though these are really merely the spore-bearing leaves of the asexual generation. Similarly the transference of the pollen grains from stamens to stigma is often spoken of as the fertilization of the flower, though it is obviously not the true process of fertilization but only a necessary preliminary to the conjugation of the gametes, and is therefore more accurately spoken of as pollination.

It will have been observed from the foregoing description that not only is the male gametophyte of the flowering plant reduced almost to the point of disappearance, but the male gamete itself has apparently suffered great degeneration. It is no longer an active spermatozoon, swimming about by means of flagella or cilia, as in Eudorina or in the ferns, but a shapeless nucleated mass of protoplasm which has at the most a sort of amoboid power of locomotion. By far the greater part of the travelling which it has to accomplish in order to reach the ovum is 
effected while it is still enclosed within the pollen grain and at the expense of some external agency, for it is not until the pollen grain has alighted upon the stigma and the pollen tube is put forth that the "generative cell " begins to exercise its own feeble powers of locomotion.

The transference of the pollen to the stigma is effected usually in one of two ways, either by the action of the wind or by the agency of insects. Flowers which are pollinated in the first of these two ways are said to be anemophilous, and they are usually small and inconspicuous, as in the grasses and plantains, and many forest trees. It is a very extravagant method of pollination, involving the production of enormous quantities of pollen, most of which is wasted, for only a very minute percentage of the pollen grains will ever chance to alight upon stigmas. We realize this when we see the enormous quantities of yellow pollen dust which are blown off the pine trees in spring time. The entomophilous or insect-pollinated flowers, on the other hand, have hit upon a much more economical way of doing the business. The development of nectar or honey as a bait for their insect visitors, and of gaily coloured petals or sepals and sweet scents as a means of attracting them, and in many cases of elaborate mechanical contrivances to secure that the insect shall not obtain its reward without doing the work of pollination, all co-operate in bringing about the desired end, and the study of these various adaptations forms one of the most interesting chapters in biological science. We shall refer to it again when we come to deal with adaptation and natural selection.

In many cases a complete sexual differentiation is manifested by the entire flowers themselves, some having stamens without carpels and others carpels without stamens, and being spoken of as "male" or "female" flowers accordingly. We see this, for example, in the case of the vegetable marrow, where both kinds of flower are borne on the same plant, while in other cases, such as the weeping willow and the Japanese Aucuba, the whole plant may be either "male" or "female," producing flowers of the one kind only. Thus the terminology which strictly speaking is applicable only to the sexual gametophyte, has, as a matter of convenience, been extended to the asexual sporophyte in order to describe the secondary sexual characters which have been transferred to it in consequence of the suppression of the former.

The telescoping of successive generations one within the other 
- the embryo sporophyte into the ovule of the preceding sporophyte generation, with the gametophyte crushed in betweenis the most characteristic feature of the life cycle of the higher plants.

From the homosporous ferns upwards throughout the vegetable series it is obvious that the gametophyte is not nearly so well adapted in its organization to the conditions under which the higher plants have to live as is the sporophyte. There has apparently been a kind of rivalry between the two alternating generations, in which the gametophyte has had much the worst of it. The sexual function itself, however, is far too important to be altogether abandoned, and so the successful sporophyte has finally taken over many of the responsibilities connected therewith, while the poverty-stricken gametophyte has ultimately become entirely parasitic upon its rival. 


\section{CHAPTER IX}

Sexual phenomena in multicellular animals-Structure and life history of Hydra and Obelia-Alternation of generations-The colomate type of structure-Secondary sexual characters-The evolution of sex.

In multicellular animals or Metazoa, as in multicellular plants, a sharp distinction can usually be drawn between the somatic cells which build up the various tissues and are concerned with the life of the individual, and the germ cells or gametes which are concerned with the propagation of the race and which alone (in most cases) have the power of separating from the parent soma or body and giving rise to new individuals.

In nearly all the Metazoa the gametes are sexually differentiated into relatively large, passive ova and much more minute, active spermatozoa which swim about by means of flagella. The actual gametes arise by subdivision of undifferentiated primordial germ cells. In the sponges, whose organization has not advanced very much beyond that of complex colonies of Protozoa, the primordial germ cells are merely wandering amœboid cells, resembling the white blood corpuscles of vertebrates. Some of these round themselves off and give rise to more or less spherical ova, others divide into spermatozoa, and probably the entire sponge.itself is in most cases either male or female, producing one kind of gamete only. In the sponge the germ cells are not localized in definite organs but scattered singly or in groups throughout the gelatinous ground-substance of which the body is largely composed.

In the great majority of Metazoa, on the other hand, the germ cells are segregated in well-defined organs termed gonads. As a rule each gonad produces only ova, when it is known as an ovary; or spermatozoa, when it is known as a spermary or testis; only occasionally does it produce both, as in the çase of the ovotestis of the snail. T'he gonads may accordingly be spoken of as female, male, or hermaphrodite as the case may be, and the same terms are also applied to the animals themselves, a male or a 
female animal possessing either male or female gonads, while a hermaphrodite animal may either possess a combined ovo-testis or both ovaries and testes separately.

In illustration of these points we may briefly describe the structure and life history of the common fresh water polype,

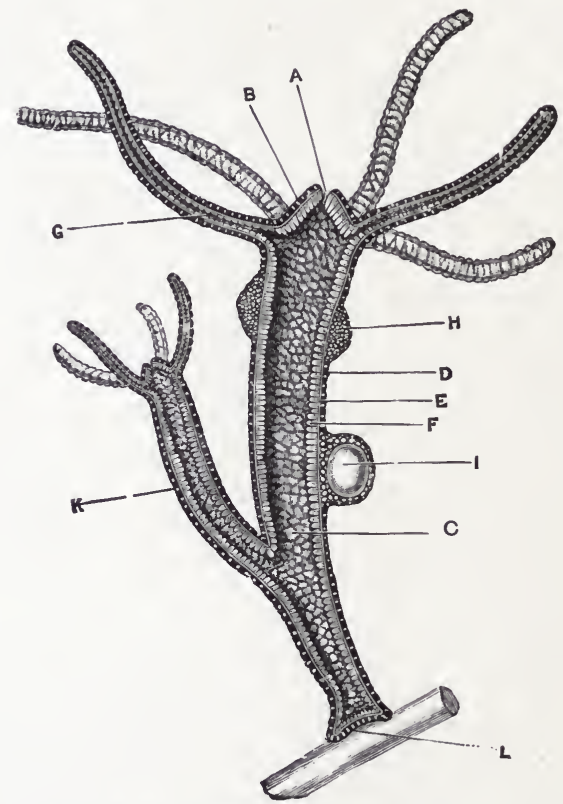

Fig. 57.-The fresh water Polype (Hydra) eut in half longitudinally and greatly enlarged. (From Marshall and Hurst's "Practical Zoology.")

A, mouth ; B, hypostome ; C, digestive cavity ; D, ectoderm ; E, mesogloea ; F, endoderm ; $\mathrm{G}$, tentacle; $\mathrm{H}$, testis; I, ovum in ovary; $\mathrm{K}$, bud; $\mathrm{L}$, foot.

Hydra (Fig. 57), so frequently found attached to aquatic plants in ponds and ditches. Hydra is a member of the great group Colenterata, which includes the sea-firs, jelly-fish, sea-anemones and corals, and which are distinguished by the fact that they retain throughout life the fundamental features of the gastrula (compare Fig. 13, IX, X). The body of a typical Cœelenterate animal consists essentially of a simple sac (Fig. 57) whose wall 
is composed of two layers of cells. The cavity of the sac (C) is the digestive or gastral eavity (enteron), and it has only a single opening to the exterior, the mouth (A), usually surrounded by a ring of tentacles $(G)$. The wall of the sac is solid and there is no body cavity or cœelom surrounding the digestive tube as in higher animals (Colomata). The outer cell layer (D) of the body wall is the ectoderm (epiblast of the embryo), the inner (F) is the

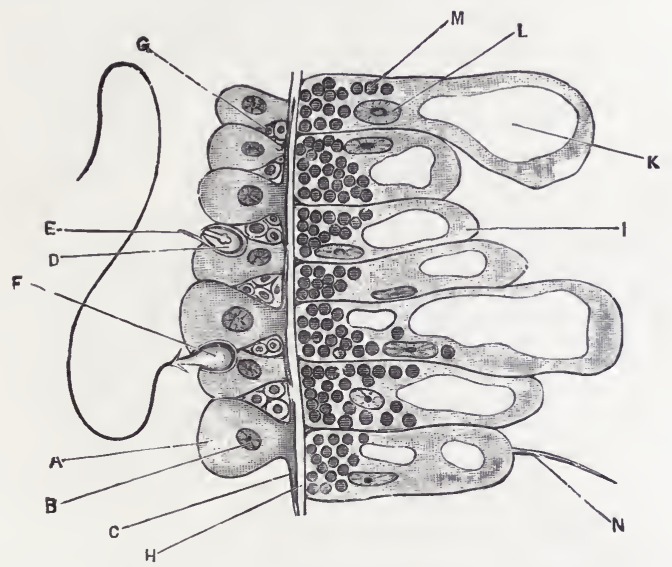

FIG. 58. - A small portion of a thin longitudinal Section through the Body Wall of Hydra viridis, $\times 800$. (From Marshall and Hurst's " Practical Zoology.")

A, a large ectoderm cell ; B, its nucleus; C, its muscle process; D, an undischarged thread cell ; E, its trigger process; $\mathrm{F}$, a thread cell with discharged thread ; $\mathrm{G}$, interstitial cells; $H$, mesogloea ; $I$, endoderm cell; $K$, vacuole; $L$, nucleus of endoderm cell; $\mathrm{M}$, green algal cells living in the endoderm cells; $\mathrm{N}$, flagellum of endoderm cell.

endoderm (hypoblast of the embryo) and between the two is a gelatinous, non-cellular supporting lamella, the mesoglœe (E).

Hydra itself is a very small form, but easily recognizable by the naked eye. The body is long and slender or short and thick, according to its state of contraction, and the same is true of the tentacles, which may be visible as mere knobs around the mouth at the unattached end of the animal, or as long slender threads extended through the water like fishing lines and serving for the capture of the minute organisms upon which the Hydra feeds. The mouth is situated on the top of a conical projection, 
the hypostome (Fig. 57, B), which lies within the circle of tentacles.

The endoderm, which immediately lines the gastral cavity, is made up of a single layer of relatively large cells (Fig. 58, I) whose function is digestive. The ectoderm is made up of several kinds of cells, some larger than others. The larger ones (A) are much broader at their outer than at their inner ends and the interstices thus left between the latter are filled up by small interstitial cells $(G)$. The endoderm cells and the larger ectoderm cells both send out prolongations of their bodies into the gelatinous mesoglœa $(\mathrm{H})$ which lies between them, and these processes, having the form of elongated fibres (C), are the seat of that power of contraction which Hydra possesses in such a high degree-they are in fact muscular.

Hydra has two very distinct methods of reproduction, asexual and sexual respectively. The former consists in a process of budding, little hollow outgrowths of the body being formed, which elongate, acquire mouth and tentacles, and for a time remain attached to the parent (Fig. 57, K). In this way temporary colonies of polypes may be produced, but sooner or later the buds separate and begin to lead independent lives.

Sexual reproduction is effected by means of ova and spermatozoa, which are essentially similar to those of higher animals. They are produced in gonads-ovaries and testes-and as both kinds of gonad usually occur in the same individual the animal is hermaphrodite. The testes (Fig. 57, H) take the form of little swellings situated at a short distance beneath the ring of tentacles and formed each by an accumulation of interstitial ectoderm cells. These are the primordial germ cells, by the division of which the spermatozoa are formed. The spermatozoon is perfectly typical, resembling. a flagellate protozoon, with an ovoid head consisting almost entirely of chromatin and a long cytoplasmic tail or flagellum by means of which it swims actively about when shed into the surrounding water by rupture of the testis.

There is usually only a single ovary, appearing as a larger projection from the body wall nearer to the attached end of the animal; it consists at first, like the testes, of a heap of primordial germ cells formed by the multiplication of interstitial cells. In each ovary, however, only a single cell develops into a mature ovum (Fig. 57, I), its sister cells, which may all be 
regarded as potential ova, being sacrificed for the benefit of the one; in fact they are simply devoured by the voracious egg
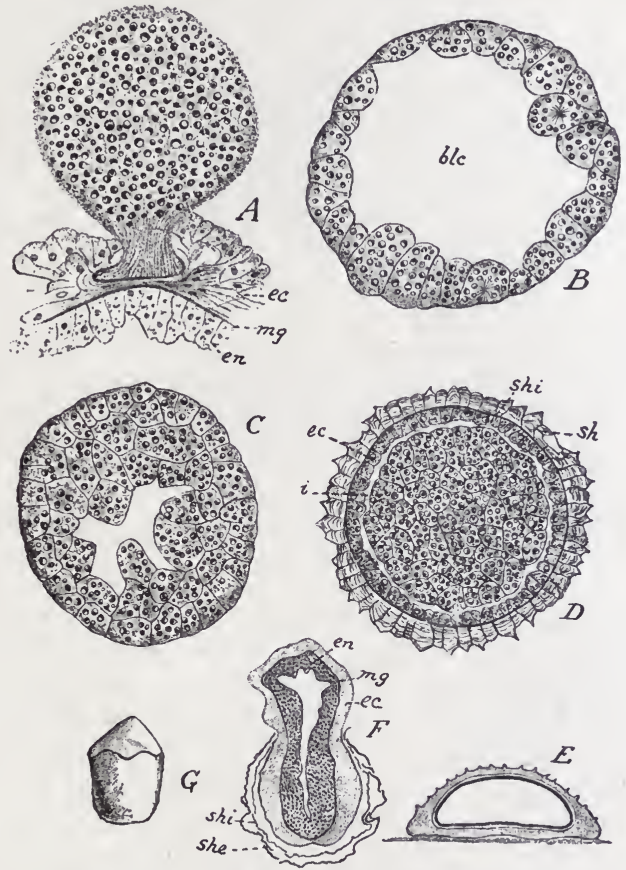

Fig. 59.-Development of Hydra. (From Bourne's "Comparative Anatomy," partly after Brauer.)

A, the mature ovum, full of yolk granules and still attached to the body wall of the parent; B, section of blastula or blastosphere produced by segmentation of the ovum; $\mathrm{C}$, the embryo becoming solid by migration into the blastocol of cells cut of from the wall of the blastula to form the hypoblast; D, solid embryo composed of epiblast and hypoblast and enclosed in a protective shell; $\mathrm{E}$, embryo flattening itself out within the shell; F, embryo emerging from the shell and with the gastral cavity appearing in the endoderm (hypoblast); G, empty shell after the escape of the embryo.

$b l c$, blastocoel ; $e c$, ectoderm (epiblast); en, endoderm (hypoblast); $i$, solid mass of hypoblast cells; $m g$, mesogloea ; sh, shell ; she, outer layer of shell; shi, inner layer of shell.

cell, which puts forth pseudopodia and feeds upon them like a hungry Amoba. In this way the ovum attains a relatively large size and its cytoplasm becomes loaded with yolk corpuscles 
which will serve later on for the nutrition of the developing embryo.

Some of the surrounding cells of the ectoderm at first

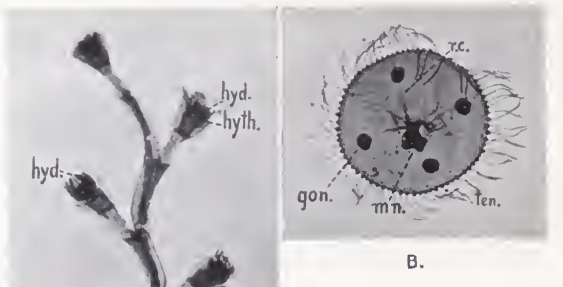

form a covering or envelope for the growing ovum, but this is presently ruptured and the mature egg is exposed on the surface of the body of the parent Hydra. There it is found by a spermatozoon, which is attracted towards it and by its own activity bores its way into the ovum. This act of fertilization is concluded in the usual manner by the fusion of the nucleus of the spermatozoon (male pronucleus) with that of the ovum (female pronucleus) to form the zygote nucleus.

The fertilized ovum or zygote (Fig. 59 , A) undergoes segmentation while still remaining attached to the parent Hydra. In this way a single-layered, hollow blastula (B) is formed, which becomes converted into a two-layered embryo by migration of cells into the in-

Fig. 60.-Obelia geniculata.

A, part of hydroid colony; B,

free-swimming medusa; both $\times 13$. (From photographs.)

bst., blastostyle; gon., gonad; gth., gonotheca ; hyc., hydrocaulus; hyd., hydranth; hyth., hydrotheca ; $m n$., manubrium; p.s., perisarc; r.c., radial canal; ten., tentacles.

terior to form the at first solid hypoblast or endoderm (C, D, i). The epiblast or ectoderm cells $(\mathrm{D}, e c)$ now secrete a thick horny protective envelope ( $s h$, shi) around the embryo, which falls off from the parent and undergoes a period of rest at the bottom of the pond. After a time the interrupted development is resumed, the horny envelope is ruptured, and the embryo escapes (F). The gastral cavity appears in the midst of the endoderm cells, the mouth is formed by perforation at one end, and the tentacles bud out. With the formation of the mouth the gastrula stage is reached, but it will be noted that 
this condition is arrived at by a somewhat different route from that which leads to the corresponding stage in Amphioxus (compared Fig. 13, I-X).

Closely related to Hydra are a large number of marine Cœlenterates, which, from their obviously animal nature combined with their plant-like mode of growth, were known to the older naturalists as zoophytes. One of the most familiar examples of these is Obelia (Fig. 60), which is frequently found attached to rocks or seaweeds near low water mark.

Obelia differs from Hydra in several interesting particulars. In the first place the asexual process of multiplication by means of budding takes place in a very regular manner, and the buds, instead of separating from the parent, remain connected together to form permanent colonies (Fig. 60, A) in which the constituent individuals or persons (sometimes called zooids) are arranged in a perfectly definite way. The colony is comparable to an arborescent colony of Protozoa such as Zoothamnium or Epistylis (compare Fig. 9, 11-15), but the individuals of which it is composed are units of a higher order than single cells.

In the second place the colony develops a common skeleton, secreted by the ectoderm, which takes the form of a slender tube of horny perisare ( $p s$. ) enclosing all the branches and expanding at the end of each into a little cup or hydrotheca (hyth.), occupied by a single zooid. Lastly the colony is polymorphic, the zooids exhibiting a certain amount of differentiation and division of labour amongst themselves.

A network of root-like branches at the base of the colony creeps over the substratum and serves for attachment. From this network, which is not shown in the illustration, arises a little forest of vertical stems, each of which has a characteristic zig-zag outline (Fig. 60, A). From each angle of the stem a short branch is given off which terminates in a single hydra-like zooid known as a hydranth (hyll.), enclosed in one of the horny cups or hydrothecæ, from the mouth of which its tentacles are extended into the water.

The structure of the hydranth is similar in all essential respects to that of Hydra. In the middle of the ring of tentacles is the mouth, situated on a projecting hypostome and leading into the digestive cavity, and the different hydranths of the colony are all placed in communication with one another by the tubular hydrocaulus, or common stalk ( $h y c$.), enclosed in the horny 
perisarc. These hydranths are the nutritive individuals of the colony, whose function it is to capture and digest the prey.

Very often we find, in the angle between a hydranth-bearing branch and the main stem, a short branch bearing an individual or zooid of a different kind, enclosed in a horny cup of totally different shape. These individuals are somewhat club-shaped; they have no mouth and no tentacles, and they are termed blastostyles (Fig. 60, A, bst.). The horny cup in which each is enclosed is urn-shaped, with constricted mouth, and is distinguished by the name gonotheca $(g t h$.$) . The function of the$ blastostyle is exclusively reproductive and it is entirely dependent for its nutrition upon digested food received from the hydranths through the hydrocaulus. It reproduces by budding, and the buds are often found attached to it in large numbers, within the sheltering gonotheca, as shown in the figure.

The colony itself increases in size by the formation of new buds in regular succession, alternately on the right and left sides, beneath what is, for the time being, the topmost hydranth of each main stem, each new bud giving rise to a hydranth-bearing branch which overtops its immediate predecessor. The buds formed on the blastostyle, on the other hand, do not develop into hydranths at all, but into another kind of individual known as a medusa, or medusoid person, which presently detaches itself from the parent and escapes through the mouth of the gonotheca as a free-swimming individual.

The medusa of Obelia (Fig. 60, B), though larger than the hydranths, is still very small, not more than about $\frac{1}{10}$ th of an inch in diameter. At first sight it looks very different from a hydroid individual (hydranth), but it can easily be shown to have the same fundamental plan of structure. It consists of a circular disk surrounded by a fringe of tentacles (ten:). From the middle of one surface projects a handle-shaped structure, the manubrium ( $m n$.), corresponding to the hypostome of the hydranth and bearing the mouth at its extremity. 'I'his mouth leads into a central digestive cavity from which four radial canals (r.c.) run outwards through the gelatinous ground-substance to a circular marginal canal. The medusa swims actively about by muscular contractions of the disk, and in doing so it assumes a bell-like shape with the manubrium projecting from the convex surface, like the handle of the bell.

Most medusæ-the larger of which are familiar to us as 
"jelly-fish"-are more definitely bell-shaped, but the manubrium lies in the hollow of the bell, like its clapper; in comparison with these the Obelia medusa may be said to turn itself inside out in the act of swimming. In accordance with their active life the medusæ have a fairly well developed nervous system and a number of sense organs around the margin of the bell. The point with which we are immediately concerned, however, is their mode of reproduction.

Unlike the hydranths and blastostyles of which the Obelia colony is composed the medusæ are sexual individuals, bearing sexual organs or gonads from which the gametes are set free. The gonads (Fig. 60, B, gon.) are masses of germ cells lying one beneath each radial canal, on the same side of the disk as the manubrium. The sexes are distinct, the medusæ being either male or female, and the gonads accordingly producing either spermatozoa or ova, which, when mature, are discharged into the water by rupture of the gonad.

The ova are fertilized by the spermatozoa and undergo segmentation much as in the case of Hydra, developing ultimately into a hydroid individual (hydranth), which by budding gives rise to a new Obelia colony.

It is obvious that we have here a case of alternation of sexual and asexual generations (metagenesis), comparable to that which occurs in the fern, except that the sexual generation arises from a multicellular bud and not from a unicellular spore. The sexual medusoid individual is produced by budding from the asexual hydroid, and itself gives rise, through the conjugation of gametes and the development of the zygote thus produced, to the asexual hydroid again. The process is somewhat complicated by the fact that the hydroid also produces other asexual individuals by budding, but this really amounts to little more than does the formation of numerous branches by the budding of one of the higher plants, for in the latter case also each bud may be regarded as, potentially at any rate, a perfect individual.

We may draw an even closer comparison between the alternation of hydroid and medusoid generations in the Coelenterata and that of sporophyte and gametophyte in the higher plants, for in the former, as in the latter, we see in many instances a more or less strongly developed tendency towards the suppression of the sexual generation (sometimes called the gamobium in animals).

In Tubularia, for example, the medusoid individual never 
separates from the parent colony, but remains attached to it as a bud, though still showing clear evidence, in its bell-like shape and in the presence of the manubrium and of radial and circular canals, of its medusoid nature. It is in fact merely a degenerate

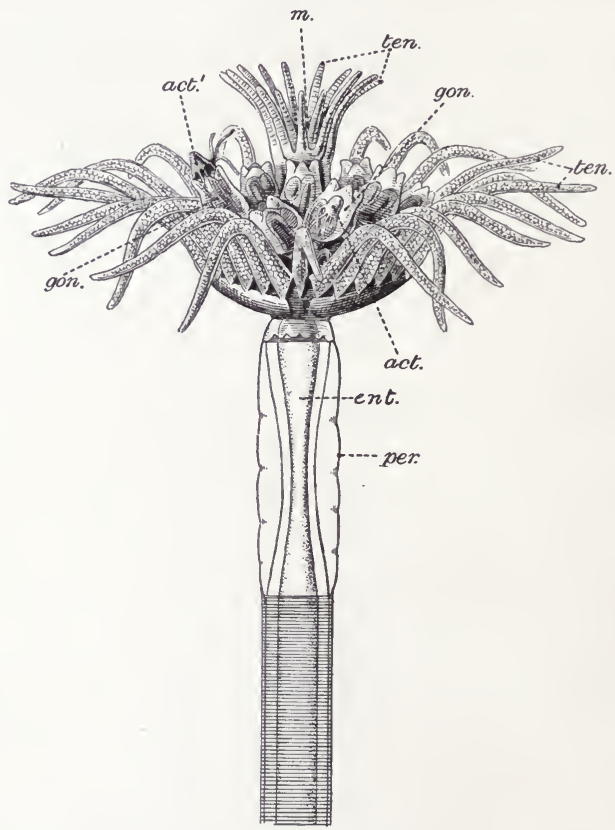

FIG. 61.-A single Hydranth of Tubularia, with medusoid Individuals (Gonophores) budded out between the two Circles of Tentacles; highly magnified. (After Allman.)

act., actinula larva enclosed in gonophore ; act.', actinula larva escaping; ent., enteron or digestive cavity ; gon., gonophore; $m$., mouth ; per., perisare; ten., tentacles.

medusa or gonophore (Fig. 61, gon.). It still retains its sexual function, producing either ova or spermatozoa, and in this case the fertilized ova actually develop into young hydroids or "actinula larvæ". (Fig. 61, act., act.') before escaping from the parent gonophore. We have here a telescoping of the successive generations quite comparable to what we find in a flowering 
plant, and again it is the sexual generation which has undergone reduction.

In other cases the medusoid bud or gonophore more or less completely loses its medusa-like structure and becomes a mere sac containing the gonad, and it has been suggested that in Hydra we may have the last stage in this progressive reduction of the sexual generation, the medusoid having altogether disappeared, after having transferred its sexual functions to the hydroid.

It seems almost incredible that the germ cells themselves which are originally produced by one generation should in this manner be transferred to what is really the preceding generation, but as a matter of fact we see all stages in this process in different genera of Hydrozoa. Even in Obelia and certain other medusæ the germ cells do not originate in the gonads beneath the radial canals but in the ectoderm of the manubrium, reaching their final position by a process of migration. In Eudendrium, where the medusoid is reduced to a mere vestige, the germ cells no longer originate in the sexual individual at all, but in the main stem of the hydroid colony, whence they migrate into the medusoid bud. If we imagine them ceasing to migrate from their place of origin in the hydroid, while the medusoid is no longer formed, we reach the condition of Hydra, with a complete transference of the sexual function to what should be the asexual generation.

In the cœlenterates, however, it is not always the asexual (hydroid) generation which predominates, for in some cases this is suppressed more or less completely and the medusa or jellyfish reproduces its own kind directly without the intervention of a hydroid phase.

In cœlenterate animals such as Hydra and Obelia, as we have already seen, the body consists of a single hollow tube whose wall is made up of only two cell layers, the ectoderm on the surface and the endoderm lining the digestive cavily, with a supporting layer of gelatinous consistency-the mesoglœa -between the two. In the case of the larger jelly-fish this inesoglœe attains a great thickness; it is, however, never a true cell layer like the ectoderm and endoderm, but rather of the nature of intercellular substance secreted by the cells on either side of it.

In Hydra and Obelia the germ cells arise from the ectoderm, 
and the gonads (Fig. 62, A, gon.) are accordingly situated close to the surface of the body and no special ducts are required for the conveyance of ova or spermatozoa to the exterior.

In the great group Colomata, which includes practically all animals higher in the scale of organization than the coelenterates, we find a different state of affairs. The mesoglcea has been replaced by a true cellular mesoderm (Fig. 62, B, mes.), formed of cells derived from ectoderm or endoderm, or from both, and in the thickness of this layer a cavity is developed, the cœlom or

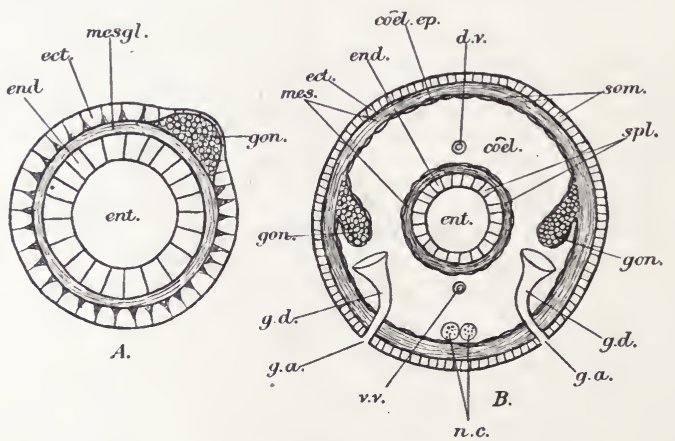

Fig. 62.-Comparison of Coelenterate and Invertebrate Cœlomate Types of Structure.

A. Diagram of a transverse section of a colenterate animal.

B. Diagram of a transverse section of an invertebrate colomate animal.

coel. coelom or body cavity; cœl. ep. coelomic epithelium lining body cavity; d.v. dorsal blood-vessel; ect. ectoderm; end. endoderm; ent. enteron or digestive cavity; g.a. genital aperture; g.d. genital duct (gonoduct); gon. gonad; mes. mesoderm; mesgl. mesogloea; n.c. double nerve cord; som. somatopleure or body wall; spl. splanchnopleure or gut wall; v.v. ventral blood-vessel.

body cavity (col.), which more or less completely surrounds the digestive tube or alimentary canal (compare the development of Amphioxus, Fig. 13, XI-XIII). The outer layer of the mesoderm unites with the ectoderm to form the body wall or somatopleure (som.), while the inner layer unites with the endoderm to form the gut wall or splanchnopleure (spl.), and the body thus acquires the form of a double tube. The body cavity is lined by a layer of epithelial cells (cœl. ep.), and it is from this cœlomic epithelium that the germ cells arise. The gonads (Fig. 62, B, gon.) therefore project into the body cavity and into this cavity the mature germ cells are primarily discharged. In the great majority of cases 
special genital ducts or gonoducts $(g . d$.$) are developed which$ pierce the body wall and serve for the passage of the germ cells to the exterior. The sexes are usually distinct in the higher forms (Vertebrata), but many of the invertebrate cœlomates (e.g., the earthworm) are hermaphrodite, the same individual bearing both male and female gonads (testes and ovaries) with the corresponding gonoducts (vasa deferentia and oviducts).

Fertilization of the ova by the spermatozoa, or in other words conjugation of the gametes, may take place either within the body of the parent, as in most terrestrial forms, or externally, as in a very large proportion of aquatic animals. In the former case special organs are developed for the transference of the spermatozoa from one individual to another, and such transference usually occurs even in hermaphrodite forms, which, as a rule, are incapable of self-fertilization. Further modifications may arise in connection with the nutrition of the embryo, which may remain within the body of the parent-in an enlarged portion of the oviduct known as the uterus-until it has reached an advanced stage of development. This takes place more particularly in the females of the higher vertebrates.

In connection with the sexual differentiation, more especially in the higher animals, numerous secondary sexual characters may arise which are not directly connected with the organs of reproduction. Such are the various ornamental outgrowths of hair, feathers and so forth, which distinguish the males of many vertebrates and are supposed to appeal to the æsthetic sense and thus to contribute towards the mutual attraction between male and female, and the special weapons, such as antlers and spurs, which male animals frequently develop and which are used in combat for the possession of the females.

Although not directly connected with the gonads these secondary sexual characters seem to depend for their development in some curious way upon the presence of these organs. Thus it is well known that if the testes be removed by castration the secondary sexual characters will not, in most cases at any rate, develop properly. We see an excellent illustration of this in the case of the antlers of the stag, which are confined to the male and do not develop at all if the animal be castrated in early youth, while if the operation be performed after the antlers are fully developed these are prematurely cast off and replaced by imperfect ones. 
How this intimate correlation between gonads and secondary sexual characters is brought about is still uncertain, but there is strong reason to suppose that it is due to the secretion by the gonad of some specific substance (hormone), perhaps of the nature of a ferment, which circulates throughout the bodychiefly no doubt in the blood-and controls the development of the characters in question. That internal secretions may act in this way upon organs remote from their own place of origin is well known and is strikingly exemplified in the case of the corpora lutea of the mammalian ovary. These structures appear on the surface of the ovary in the places whence ova have been discharged, and are apparently of a glandular nature. As the fertilized ova pass down the oviduct they begin to develop, and on reaching the uterus fix themselves to the wall of the latter, in which they become imbedded. The spot where fixation takes place is far distant from the ovary, but it has been demonstrated that if the corpora lutea on the surface of the latter be destroyed the embryos will not become fixed at all. There is a close correlation, then, between the presence of the corpus luteum and the fixation of the embryo, and this is explained by supposing that the corpus luteum secretes some substance which, circulating in the blood, reaches the uterus and stimulates its epithelial lining to respond to contact with the embryo. Moreover, the reaction, whatever its cause, appears to be mutual, for if the discharged ovum does not get fertilized the corpus luteum does not attain its full development and soon disappears.

We have now very briefly traced the evolution of sexual characters from their starting point in the male and female gametes of the Protista to their culmination in the higher plants and animals. We have seen how sexual differentiation, which primarily concerns the gametes themselves, is gradually extended to the colonies or to the multicellular individuals from which the gametes arise, or even to a preceding, originally non-sexual generation. The various structural modifications thus brought about are all directed towards one end, the conjugation of the gametes. The mutual attraction which undoubtedly exists between the gametes themselves is not sufficient, at any rate in the case of the more highly developed and complex organisms, where the distances between their places of origin are relatively very great, to secure their union.

In the flowering plants their own efforts are supplemented by 
all the elaborate devices for securing pollination, and by far the most active part in the process is played by external agencies, especially by those insects which have become the vicarious fertilizers of the flowers.

In the higher animals, on the other hand, the necessity for bringing the gametes into close proximity with one another has led tothe development of all those secondary sexual characters, both bodily and mental, which play so conspicuous a part in the drama of life. 'Throughout the whole course of this remarkable process of evolution, except in the case of certain obviously degenerate forms, we observe that same fundamental distinction between the sexes which we first noticed in the gametes of unicellular organisms, and which in the higher animals is extended with the sexual differentiation itself from the gametes to the complex multicellular body which bears them. The female is the more passive partner and is especially concerned with the nutrition and rearing of the offspring, and her bodily organization is especially adapted to her maternal functions. These functions constitute an inevitable handicap in the struggle for existence, and the females and young of the higher animals are in most cases largely dependent upon the less burdened and consequently more active and vigorous males for their protection.

The explanation of this progressive sexual differentiation is undoubtedly to be found in the advantages to be derived from division of labour and the accompanying possibilities of specialization. The origin of conjugation itself, upon which all sexual phenomena are based, is another, and more fundamental, question. At first, as we have seen, the conjugating gametes were apparently exactly alike one another and exhibited no visible sexual differentiation at all. The habit of conjugation probably arose from the necessity of making good some disturbance of equilibrium in the protoplasm of the cell. It has been supposed that, as the result of repeated fission, some condition of inequality was gradually set up amongst the daughter cells, whereby some of them came to have too much of one constituent and too little of another, while others were in the opposite condition. In this way the successive unicellular generations gradually became more and more enfeebled-as we saw in the case of Paramœcium-and, owing perhaps to some sort of polarization, those which had become modified in opposite directions came to exercise an attraction upon one another which resulted in conjugation and restoration 
of the proper equilibrium. It may be that from the very first the inequalities of fission resulted in the accumulation of more active protoplasm in some cells and a greater amount of reserve material in others, and that this was the starting point of the differentiation into male and female.

Although fusion of the nuclei (karyogamy) of the two gametes appears now to be the most important feature of conjugation, we must suppose that it was preceded by plastogamy ${ }^{1}$ or fusion of the cytoplasm, which obviously constitutes the natural preliminary to karyogamy. In some of the lower Protozoa such plastogamy has been sometimes observed unaccompanied by karyogamy, and it is possible that in some cases plastogamy alone is sufficient to bring about rejuvenescence and renewed activity in cell-division.

. 1 Sometimes called plasmogamy. 


\section{CHAPTER X}

Origin of the germ cells in multicellular animals-Maturation of the germ cells-Reduction of the chromosomes--Sex determination in insectsDifferent forms of gametes - Mutual attraction of the gametesFertilization and parthenogenesis.

In many multicellular animals the distinction between somatic cells and germ cells becomes manifest at a very early stage in the development of the individual. An extreme instance of this is seen in the parasitic round-worm of the horse, Ascaris megalocephala. Here the distinction in question precedes all other histological differentiation. The two cells or blastomeres into which the fertilized ovum first divides (Fig. 35, C, D) are originally similar to one another, but as they prepare for the next mitotic division of the nucleus a remarkable difference is, according to the observations of Professor Boveri, established between them. Both at first (in the case of the variety known as univalens) exhibit two elongated chromosomes (the variety bivalens, which is represented in Fig. 35, having four), but in one the thickened ends of the two chromosomes are thrown off into the surrounding cytoplasm, where they degenerate, while the more slender middle portions break up into a number of short pieces. Thus two differentiated cells are produced, one with two large chromosomes and the other with numerous small ones. The latter gives rise by its subsequent divisions to somatic cells only. 'The former is a primordial germ cell; for some five or six times it will divide like its parent cell into a somatic cell and a primordial germ cell, but after these early divisions the primordial germ cells will give rise to their own kind only, until the time comes for the production of the actual gametes. The somatic cells, on the other hand, will gradually become differentiated into all the various tissue cells of the adult.

Perhaps the most significant part of this remarkable process as observed in Ascaris is the elimination of chromatin material from the nuclei of the somatic cells when these are first 
differentiated from the germ cells. The result of this is that the germ cells alone retain the full complement of chromatin derived from the parents, and their nuclei are accordingly actually much larger than those of the somatic cells.

The differentiation into somatic cells and germ cells camnot usually be traced so far back in the development of the individual as in Ascaris, but in a great many animals the distinction can be recognized at a very early stage. In certain insects, for example, the primordial germ cells can be traced back to a large "pole-cell" which lies at one end of the segmenting ovum, and

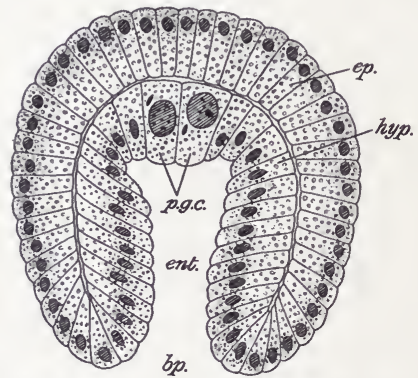

Fig. 63.--Section of the Gastrula of an Arrow Worm (Sagitta) showing the primordial Germ Cells. ( $\Lambda$ fter $O$. Hertwig.)

$b p$., blastopore ; ent., enteron; ep., epiblast; hyp., hypoblast; p.g.c., primordial germ cells. ment (Fig. 62, B). In plants also, in cases where there is a well developed gametophyte this appears to attain its full development before the germ cells are recognizable, and the entire life of the sporophyte is passed without any distinction between somatic and germ cells manifesting itself. Moreover, the fact that the ordinary cells of a fern prothallus can, on occasion, act as germ cells, ${ }^{1}$ prevents us from admitting any absolute distinction between the two categories.

The primordial germ cells may undergo extensive multiplication by ordinary mitotic division before giving rise to the actual gametes. In multicellular animals the process of gametogenesis (formation of gametes), which is either oogenesis or spermato- 
genesis according to whether ova or spermatozoa are produced thereby, is accompanied by nuclear phenomena of very great interest, whereby the maturation of the germ cells is effected. Before describing this process we must lay stress upon certain preliminary considerations.

As we have already seen, each kind of animal or plant is characterized by the appearance of a definite number of chromosomes in the nuclei of its cells at the time when these are undergoing division by mitosis. Although not absolutely constant in all cases the number is usually the same for all the different somatic cells of which the body is composed. It is usually an even number, and (with certain exceptions) it remains the same in successive generatious of individuals.

It will also be remembered that the zygote or fertilized egg from which the individual develops is formed by the conjugation of two gametes, ovum and spermatozoon, and that in this process the nuclei of the gametes, sometimes called the male and female pronuclei, unite, or at any rate co-operate as a single nucleus. Fig. 64 is

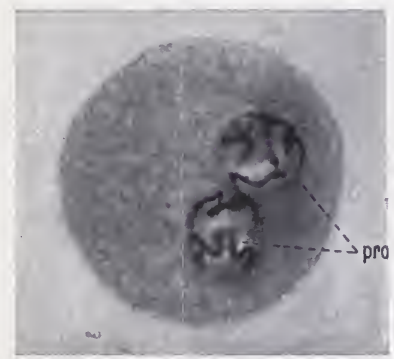

Fig. 64.-Ovum of the Horse Worm (Ascaris megalocephala) during the Process of Fertilization, showing the male and female Pronuclei (pro), $\times 770$. (From a photograph.)

taken from an actual photograph of an egg of Ascaris in process of fertilization; the spermatozoon has already entered the ovum and the male and female pronuclei (pro.) are seen lying side by side in the cytoplasm. Each pronucleus brings with it its own set of chromosomes, and hence the zygote nucleus has double the number of chromosomes possessed by either of the gametes. Thus it appears at first sight that every conjugation or sexual union of gametes (zygosis) must be accompanied by a doubling of the number of chromosomes, and we might therefore expect to find each successive generation with twice as many chromosomes in its nuclei as the preceding one. 'That this is not actually so depends (in the case of animals) upon the fact that the nuclei of the gametes contain only half the number of chromosomes characteristic of the somatic cells; if the 
somatic cells have eight the mature ovum or spermatozoon will have only four, and so on. This reduction of the number of chromosomes (meiosis) is the essential part of the process of maturation which the animal germ cells undergo, and it is
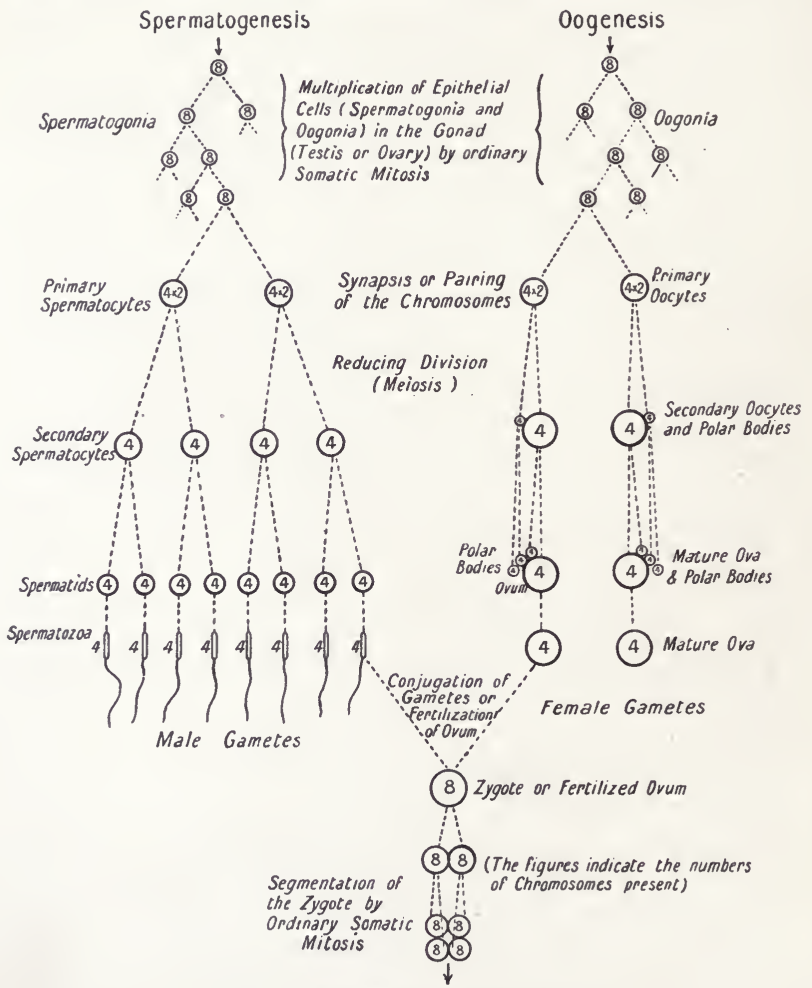

FIG. 65.-Diagram of Gametogenesis.

effected by a modification of the mitotic nuclear division, in which the chromosomes are separated into two groups, half the total number of entire chromosomes going into one daughter cell and half into the other.

In a typical case of spermatogenesis the first stage is the 
multiplication of cells (the so-called spermatogonia) derived from the germinal epithelium, in the testis, by ordinary cell-division. Let us suppose the number of chromosomes found in the nuclei of the somatic cells to be eight; it will, of course, remain the same so long as the character of the mitosis undergoes no change, each chromosome splitting into two at every nuclear division. Presently, however, we find the chromosomes arranged in pairs instead of all appearing separately in the mitotic figure, and the cells, which have increased considerably in size by the absorption of nutriment, may now be termed primary spermatocytes. This pairing of the chromosomes (synapsis) marks the onset of the "reducing division"; a nuclear spindle is formed, the paired chromosomes arrange themselves upon it, and the two members of each pair separate and travel towards opposite poles. Thus two new nuclei are formed each with only four chromosomes. The reduction is now complete and the new generation of cells, with reduced nuclei, may be termed secondary spermatocytes. One more mitotic division takes place, this time involving the splitting of each chromosome, so that there is no further reduction in their number, and giving rise to the minute spermatids, each of which develops a long, vibratile, eytoplasmic tail and forms a spermatozoon. Hence we see that each primary spermatocyte gives rise to four spermatozoa, with reduced nuclei containing half the number of chromosomes found in the somatic cells. The essential features of the whole process are re̊presented diagrammatically in Fig. 65 .

The process of oogenesis takes place in essentially the same manner; the so-called oogonia, ${ }^{1}$ derived from the germinal epithelium of the ovary, multiply and give rise to oocytes. Synapsis and reduction in the number of the chromosomes take place as they do in spermatogenesis, but, owing doubtless to the fact that it takes a comparatively large amount of cytoplasm to form the body of an egg, we find that only one perfect ovum arises from each primary oocyte, the other three forming the "polar bodies." Owing to their minute size as compared with the ovum itself, two of the polar bodies (Fig. 35, A, p.b.) appear to be cast out of the latter as it undergoes maturation, while the third is formed by division of the first; it will be elear, however, from a careful study of Figs. 65 and 68 that the

1 This term is very unfortunately chosen and must not, of course, be confounded with the same term as applied to the female organs of such plants as Fucus. 

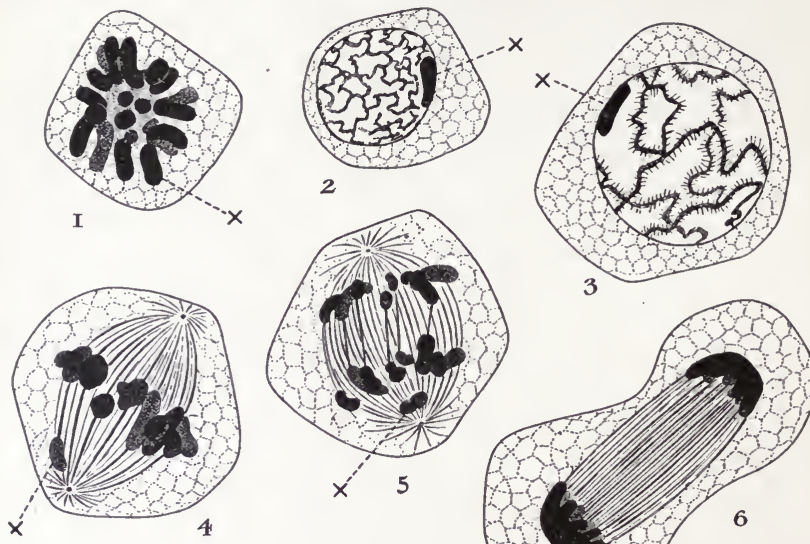

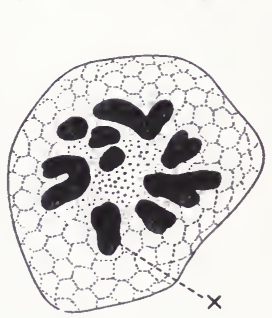

7
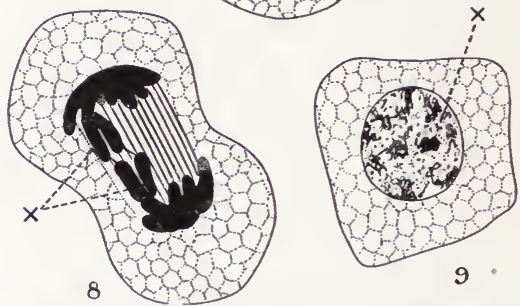

FIG. 66.-Some Stages in the Spermatogenesis of a Grasshopper (Stenobothrus viridulus). (Áfter Meek.)

1. A secondary spermatogonium in mitosis, with seventeen chromosomes (eight pairs and one accessory). Polar view.

2. Resting or growth stage following the mitosis of the secondary spermatogonium. The ordinary chromosomes have become resolved into a network but the accessory chromosome retains its individuality.

3. Primary spermatocyte formed by growth of 2 , preparing for mitosis.

4. Mitosis of the primary spermatocyte, showing the pairing (syuapsis) of the sixteen ordinary chromosomes on the nuclear spindle and the accessory chromosome unpaired. Side view.

5. Later stage in the mitosis of the primary spermatocyte, showing separation of the paired chromosomes. Side view.

6. Still later stage in the division of the primary spermatocyte into two secondary spermatocytes; the chromosomes massing at the two poles of the spindle (one mass only will contain the accessory chromosome). Side view.

7. A secondary spermatocyte in mitosis, slowing the reduced number of ordinary chromosomes (8) and the accessory chromosome. (The sister-cell would of course contain no accessory elıromosome). Polar view.

8. Later stage in the mitosis of the secondary spermatocyte; each chromosome (including the accessory one, which lags behind the others) has split into two parts and the two groups are separating. Side view.

9. A spermatid, formed by division of a secondary spermatocyte with an accessory chromosome. It will form a single spermatozoon.

$$
\times \text { The accessory chromosome. }
$$


process is merely one of repeated cell-division as in the ease of spermatogenesis. The three polar bodies consist almost entirely of chromatin and each of course contains the same reduced number of chromosomes as the ovum itself; they undergo no further development, however, and finally disappear. The truth of the view, now generally held, that the polar bodies are merely ova which have not sufficient cytoplasm to allow of their development, is demonstrated by the fact that in one of the turbellarian flat-worms, according to Francotte, the exceptionally large first polar body may occasionally be fertilized and actually develop as far as the gastrula stage.

The details of the process of gametogenesis vary very much in different cases, but the above outline may be regarded as generally applicable.

In a large number of insects it has been found that the male animal possesses an odd number of chromosomes in the somatic nuclei, due to the presence of a single "accessory" chromosome or " monosome," while the female possesses an even number (one more than the male owing to the presence of two "accessory" chromosomes). This leads to a curious complication in the process of spermatogenesis. The accessory chromosome (Fig. 66, X) can often be distinguished by its appearance from the others, and at the time of synapsis (Fig. 66, 4) it has no mate. Hence in the reducing division the chromosomes are separated into two unequal groups, one of which contains the accessory chromosome while the other does not (Fig. 66,5). Two kinds of spermatozoa are accordingly produced in equal numbers, one kind with an odd number of chromosomes and the other with an even number.

The matured ova, on the other hand, all have the same number of chromosomes, because the accessory chromosome has a synaptic mate. Fertilization of an ovum by a spermatozoon containing an accessory chromosome results in the production of a female animal with an even number of chromosomes in its somatic cells; fertilization by a spermatozoon which has no accessory chromosome results in the production of a male animal with an odd number of chromosomes in its somatic cells, as shown in Fig. 67.

Thus it appears that the chromosomes, at any rate in some cases, have a very important influence on the determination of sex, and that the latter is not, as has often been supposed, merely the result of nutritional and other environmental influences upon 
the developing organism, but a character of far more deeply seated origin.

It must not be forgotten that the chromosomes, as such, are only recognizable during the process of mitosis; in the resting condition of the nucleus they appear to be broken up into larger or smaller granules of chromatin scattered through the linin reticulum. The observations on accessory chromosomes above mentioned, and others to be referred to presently, point to the

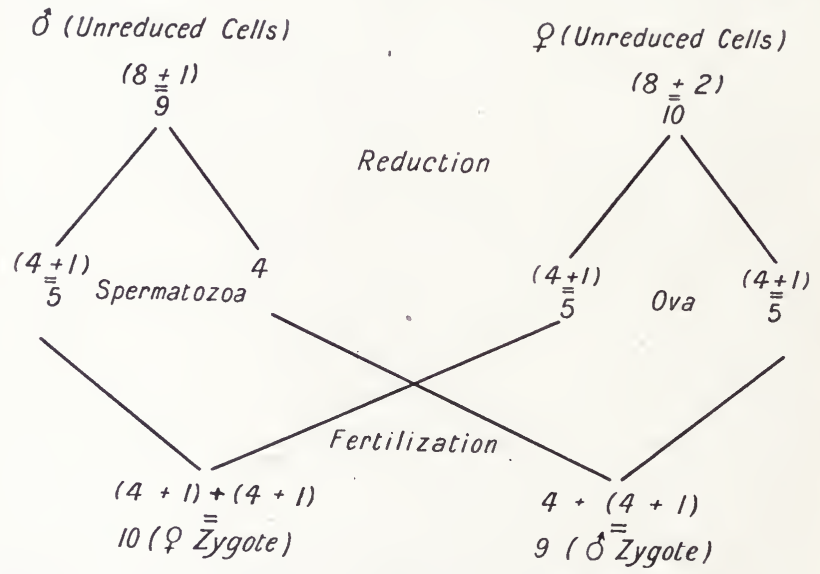

FIG. 67.-Diagram illustrating the Correlation between the Number of Chromosomes and the Sex in certain Insects. (The numbers of chromosomes given in this diagram are arbitrarily chosen and are obviousiy different from those which occur in Stenobothus, as shown in Fig. 66.)

conclusion, however, that, in spite of this, the different chromosomes preserve some sort of individuality from one cell-generation to another. In other words, we have reason to believe that the chromosomes which make their appearance at the onset of each mitosis are, taken each as a whole, the same as those which become disintegrated at the close of the preceding mitosis, though it is very possible that the constituent parts of the old chromosomes (chromatin granules, chromomeres or ids), after absorbing nutriment and increasing in size during the resting period, may come together in new combinations to form the new chromosomes each time division of the nucleus takes place. ${ }^{1}$

1 Tide Farmer, Croonian I.ecture, Proe. Royal Soc., Ser. B, Vol. 79, 1907. 
It is also highly probable, though by no means certain, that the chromosomes which are derived from the male parent remain throughout life distinct from those which are contributed by the female parent. According to this view every ordinary somatic cell has two sets of chromosomes, paternal and maternal respectively, and this again is strongly supported by the observations on the germ cells of insects above referred to, where all the chromosomes appear to be duplicated, with the exception of the accessory chromosome in the male animal.

There is reason for believing, therefore, that in ordinary cases every paternal chromosome in an unreduced nucleus has an equivalent or " homologous" mate derived from the female parent, and that the phenomenon of synapsis ${ }^{1}$ represents a pairing of these homologous paternal and maternal mates. The reducing division which follows on synapsis consists in the separation of the mates once more, one of each pair going to each daughter cell, so that the matured germ cells are left with a single instead of a double set of chromosomes.

If we assume that, as seems highly probable, the chromosomes of each paternal or maternal set are not all identical but differentiated amongst themselves-a differentiation which in some cases is actually visible, as shown in Fig. 66-and that one of each kind is necessary to make up the full complement of the nucleus of the gamete, the importance of the pairing of homologous chromosomes which takes place in synapsis becomes at once evident, for one of each pair goes to each daughter nucleus, which will therefore be certain to receive a chromosome of each kind instead of a chance assemblage. The chromosome of each kind which it receives, however, may be either the paternal or the maternal representative of that kind, and as these, though essentially homologous, may differ from one another to some extent in accordance with individual peculiarities of the parents from which they were derived, it will be seen that the matured gametes may differ widely amongst themselves in their nuclear constitution. 'This, as we shall see presently, is a very important matter from the point of view of the theories of heredity and variation.

This somewhat complex subject will be rendered more readily

1 The pairing of the chromosomes, for which we have used the term "synapsis," is spoken of by some writers as "syndesis," and by others as "conjugation." The use of the latter term seems likely to lead to eonfusion. 
intelligible by a careful study of Fig. 68 , which represents in a very diagrammatic manner the formation of the polar bodies and the distribution of the maternal and paternal chromosomes in the maturation of a typical animal ovum.

Periodic reduction of the number of chromosomes is clearly a necessary consequence of the sexual process, for a doubling of

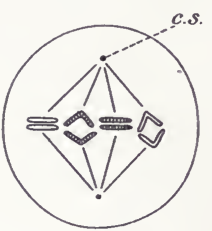

A

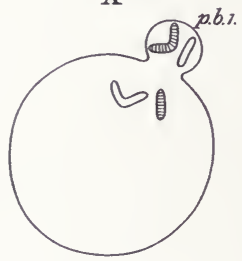

D

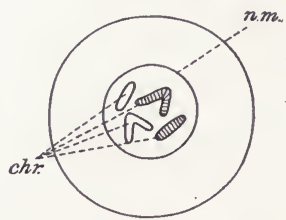

B

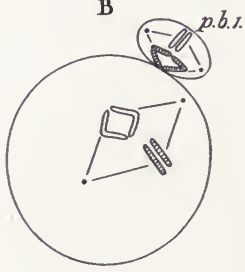

$\mathbf{E}$
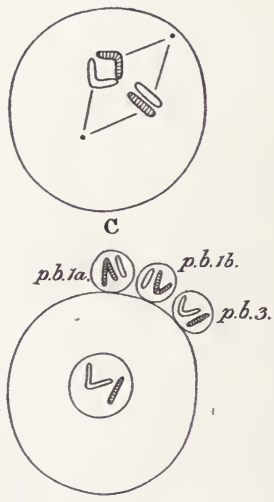

$\mathbf{F}$

FIG. 68.--Diagram of the Maturation of a typical Animal Ovum, showing the Behaviour of the Maternal and Paternal Chromosomes and the Formation of Polar Bodies. (The somatic number of chromosomes is supposed to be four; the maternal chromosomes are shaded and the patemal not, and the differences between the two of each set are indicated by their shapes.)

A, ordinary somatic mitosis in an oogonium, each chromosome split; B, daughter oogonium; C, synapsis in primary oocyte; D, reducing division, formation of first polar body ; E, commencement of formation of second (or third) polar body by ordinary mitosis and of division of the first; F, mature ovum with three polar bodies.

chr., chromosomes; c.s., centrosome; n.m., nuclear membrane; p.b. $1-3$, polar bodies.

the number at every zygosis or conjugation of gametes could not go on indefinitely without some such compensation. In animals, as we have seen, the reduction takes place during the maturation of the germ cells, but it is by no means necessarily associated with this process. In the ferns, where alternating sexual and asexual generations are represented by independent and well developed organisms, the reduction takes place in the process of sporeformation by the sporophyte. Hence the gametophyte (prothallus) to which the spore gives rise has in all its somatic cells 
the reduced number of chromosomes, while the sporophyte (fern plant proper), owing to the fact that it develops from a fertilized ovum and receives a set of chromosomes from each parent, has the full number, or, perhaps we should say more correctly, the double number.

Even amongst the Protozoa the phenomenon of nuclear reduction has been observed, and it probably occurs wherever the sexual

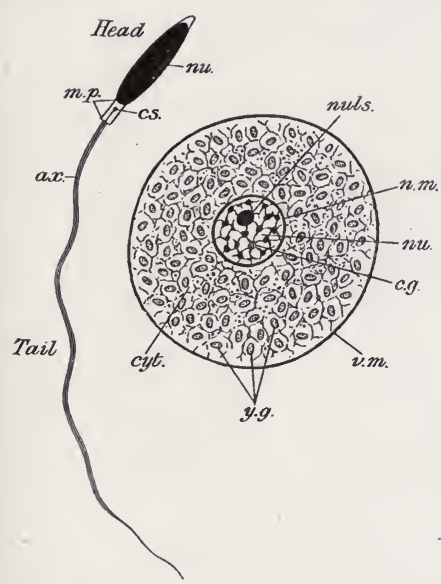

FIg. 69.-Diagram of typical Spermatozoon and Ovum, the former much more highly magnified than the latter.

ax., axial filament; c.g., ehromatin granules; cs., centrosome; cyt., cytoplasm; m.p., middle piece; n.m., nuclear membrane; $n u$. , nucleus; muls., nucleolus; v.m., vitelline membrane; y.g., yolk granules.

process takes place. Thus, in the case of Copromonas, the life history of which we have already dealt with, the nucleus of each of the similar gametes, previous to uniting with its mate to form the zygote nucleus, gives off two "polar bodies" which undergo degeneration in the eytoplasm (Fig. $37,3,4)$, and something very similar may be observed in the maturation of the ovum of Coccidium. In the conjugating Paramocium, again, the products of division of the micronucleus (Fig. 41, B, C) which undergo no further development may be regarded as polar bodies.

As we have already seen, the maturegametesthroughout both the animal and vegetable kingdoms usually exhibit a very strongly marked sexual di.norphism, which attains its fullest expression in the differentiation into active spermatozoon and passive ovum (Fig. 69).

A typical spermatozoon, as we have also pointed out, closely resembles a flagellate protozoon. It consists of a "head" and a "tail," connected together by a "middle piece" (m.p.). The head contains the nucleus (nu.), which almost entirely fills it, being covered with only a very thin envelope of cytoplasm. The middle piece contains a centrosome $(c s$.$) . The tail, or flagellum, is$ 
the active locomotor organ, by the movements of which the spermatozoon swims about; it contains an axial filament (ax.)

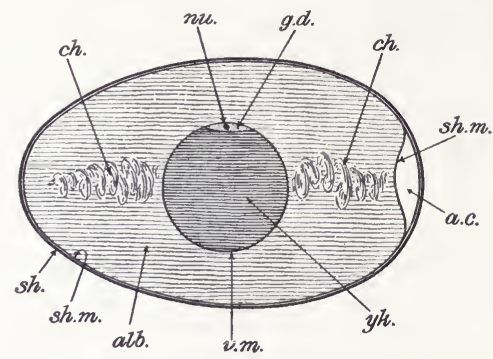

FIG. 70.-Diagram of the Structure of a Bird's Egg.

a.c., air chamber; alb., albumen; ch., chalazæ, twisted cords of dense albumen which serve to keep the "yolk" in position; g.d., germinal disk ; $n u$., nucleus; sh., shell; sh.m., shell membrane; v.m., vitelline membrane; $y$. .,"yolk."

and may or may not be provided with a lateral undulating membrane.

A typical ovum is a relatively large, spherical cell with a conspicuous nucleus ( $m$.) surrounded by a large quantity of cytoplasm (cyt.), and the whole enclosed in a delicate vitelline membrane (v.m.). The actual size of the ovum depends almost entirely on the amount of food material (deutoplasm or yolk), which is stored up in the cytoplasm in the form of granules (y.g.). In Amphioxus (Fig. 13, I) the amount of food material is very small, and the egg is only about $\frac{1}{2} \frac{1}{5} \mathrm{o}$ th inch in diameter.

An extreme contrast to the egg of Amphioxus is seen in that of a bird (Fig. 70), where the amount of yolk is enormously large and the active protoplasm is confined to a minute "germinal disk" (g.d.), containing the nucleus (nu.), which lies within the vitelline membrane $(v . m$.$) on the top$ of the "yolk" $(y k$.), while the ovum proper is entirely enclosed in accessory structures-the "white" or albumen ( $(l l b$.$) and the shell (sh.), with its lining$ membrane (sh.m.).

The mammalian ovum, on the other hand, is, like that of Amphioxus, very

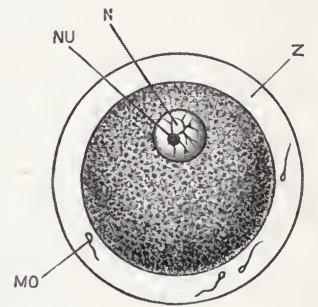

FiG. 71.-Ovum of a Rabbit, $\times 200$. (From Marshall's "Vertebrate Embryology," after Bischotf.)

MO, spermatozoa which bave penetrated the zona radiata; $\mathrm{N}$, nucleus; NU, nucleolus; $\mathrm{Z}$, zona radiata.

minute, in the rabbit (Fig. 71) again only about $\frac{1}{25}$ th inch in diameter. It is enclosed in an envelope known as the zona radiata $(Z)$, which lies outside the vitelline membrane, and it 
contains very little deutoplasm. This is correlated with the fact that it develops within the body of the parent at the expense of food material derived from the blood of the latter. There is reason to believe, however, as we shall see later on, that the small size of the mammalian egg is a secondary feature.

The plant egg-cell may also be loaded up with food material, so as to attain a large size, as in the green alga, Chara, where the contrast between the minute flagellate spermatozoon and the relatively gigantic ovum, filled in this case with starch grains, is very striking. In the higher plants, however, where, as in the case of the Mammalia, the developing embryo is not dependent for its nutrition upon food supplies stored in the eggcell, the latter remains quite small, as, for example, in the fern (Fig. 52) and the flowering plant (Fig. 55, e).

According to some authorities one of the most important differences between ovum and spermatozoon in animals lies in the fact that the centrosome of the former disappears finally during the process of maturation, the centrosome of the zygote being contributed by the spermatozoon alone. In view of the fact, however, that a definite centrosome is not usually recognizable at all in the higher plants we cannot attribute very great importance to its supposed absence in the animal ovum, and we shall also see presently that centrosomes appear in developing eggs which have not been fertilized by spermatozoa.

We have already had occasion to refer to the existence of some attractive force whereby the male and female gametes are brought together in conjugation. Many observers maintain that this is simply a case of positive chemotaxis, or the chemical stimulation of the protoplasm of one gamete by a specific secretion of the other in such a way as to cause them to respond by approaching one another (or by the male gamete approaching the female).

It is a well known fact that certain spermatozoa are attracted by specific chemical substances. Thus the free-swimming spermatozoa of ferns and mosses are attracted by weak solutions of malic acid and cane sugar respectively, and those of Coccidium are attracted by nuclear matter discharged from the ovum in the process of maturation.

There can be no doubt that, whether the attracting substance be secreted by the germ cells themselves or by some other part of the organism, chemotaxis sometimes plays a very important 
part in bringing the gametes together. So also, of course, do many other factors in various plants and animals, but we must distinguish between factors which act directly upon the gametes, such as chemotaxis, and those which act indirectly through the soma or body of the organism, as for example through the visual and olfactory senses of the higher animals.

It is probable, however, that chemotaxis itself is but a secondary factor which serves to bring the gametes within the range of one another's direct influence. Thus in Cocidium (Fig. 39) the chemotactic action seems to be exhausted after a certain number of spermatozoa have been attracted to the neighbourhood of the ovum and a fresh attraction appears to be exerted by the ovum itself or by its nucleus.

The term cytotropism, or cytotaxis, has been applied to the attraction which, according to some observers, is sometimes set up between two adjacent cells, and something of this kind probably comes into play in the mutual attraction of gametes. It can probably act only at very short distances, and hence the necessity for some preliminary means of attraction such as chemotaxis. That chemotaxis alone is not a sufficient explanation of the phenomenon in question is suggested by the case of Spirogyra. The conjugation of the gametes in this plant has already been described in Chapter VIII. It will be remembered that the process may take place between the cells of two filaments lying close together, parallel with one another (Figs. 43 and $44)$, and is then inaugurated by those of the male filament. Each of these cells which happens to lie opposite to a cell of the female filament puts forth a hollow protuberance of its wall, which is presently met by a similar protuberance from the wall of the female cell, the two projections uniting to form a canal through which the protoplasmic body of the male gamete creeps inside the cell-wall of the female gamete to conjugate with the latter. It sometimes happens, however, that, owing to inequalities in the sizes of the cells, there may be a cell in one filament which lies between two cells of the opposite filament and for which there is no mate, all the adjacent cells being coupled. In such cases the solitary cell (Figs. 43 and 44, S.C.), if it exhibits any of those remarkable activities which are shown by the conjugating cells on either side of it, merely makes preliminary advances which are prematurely checked, as though there were a competition for partners in which it was unsuccessful. Here it is obvious that 
in the case of two cells lying opposite to one another, though not in contact, and though each is enclosed in a firm cell-wall, some stimulus is transmitted from one to the other which calls forth a definite response manifested in the formation of the connecting canals and the conversion of the protoplasmic contents of the cells into gametes. The insufficiency of the principle of chemotaxis to account for these phenomena appears to be indicated by the fact that cells which have no mates do not form either complete connecting canals or gametes, though exposed equally with their more fortunate neighbours to the influence of any chemical substances dissolved in the surrounding water. The only explanation appears to be that the solitary cell cannot attract, or at any rate retain, the attention of a mate to stimulate it to complete the process of conjugation. ${ }^{1}$

The cytotropic attraction of the gametes, as we have already observed, probably depends upon some difference of polarity between the two. That it is mutual is demonstrated by such cases as that of Zygogonium (see p. 97) and by the fact that even when the ovum is too heavily laden with food material to take any active part in the process of conjugation it yet in many cases puts out a definite "cone of attraction" towards the advancing spermatozoon, as seen in Coccidium (Fig. 39). What the real nature of this primary attraction between the gametes is we do not know ; it may ultimately be explicable in terms of some force already known to us, or it may be one of those cases where it will be convenient to cloak our ignorance by the assumption of some special vital force of which we know nothing.

Although as a general rule an egg does not develop unless fertilized by a spermatozoon, this is by no means always the case, and many instances are known of parthenogenesis or the development of unfertilized eggs. This may either be a normal occurrence in the life cycle or it may be artificially induced.

Natural parthenogenesis occurs chiefly in insects, especially amongst the aphides or plant lice. In these animals males and perfect females appear only in the autumn. Fertilized eggs are then laid which hibernate through the winter and hatch in the spring, producing imperfect viviparous females. In these imperfect females eggs are formed which develop parthenogenetically within the body of the parent and give rise to fresh generations of viviparous forms. 'This reproduction by means of unfertilized eggs

\footnotetext{
1 See, however, the footnote on p. 189.
} 
is repeated again and again throughout the summer months and thus the aphides multiply with great rapidity. In the autumn, however, males and perfect females are again produced. The viviparous imperfect females, as well as the males, are generally winged, the perfect females are wingless. We have here another kind of alternation of generations, in which forms which reproduce parthenogenetically alternate with others which exhibit the normal sexual process; to this type of alternation the term leterogeny is sometimes applied. A very large number of parthenogenetic generations may intervene between two sexual ones.

Another well-known case of parthenogenesis is that of the hivebee, where the eggs laid by the queen may either be fertilized or not, in the former case giving rise to females (workers or queens) and in the latter to males (drones). Other instances occur amongst those parasitic flat-worms known as flukes (Trematoda) and in some Crustacea (Cladocera, the so-called water fleas).

The most remarkable cases of natural parthenogenesis, however, are those to which the special term pædogenesis has been applied, in which the imperfect females do not even wait to attain maturity before producing their offspring, but actually do so in the larval condition, as in Chironomus and some other two-winged flies (Diptera).

In general we may say that parthenogenesis occurs in cases where it is desirable to take advantage of a brief season of favourable conditions to multiply the race as rapidly as possible. It is necessary to make hay while the sun shines. When adverse conditions set in, such as the advent of winter in the case of the aphides, or discharge from the body of the host in the case of parasitic flukes, the vast majority of the race will perish, but a sufficient number will be able to protect themselves in some way (like the encysted cercariæ of the fluke), or a sufficient number of fertilized and protected eggs will be produced (as in the aphides) to tide over the evil time and form the starting points for fresh generations at the first favourable opportunity.

It seems possible, also, that in some cases parthenogenesis may be continued indefinitely without fertilization ever occurring, for in certain species of minute rotifers and crustaceans no males have as yet been observed.

Recent researches have shown that parthenogenesis can be artificially induced in cases where it does not occur naturally at 
all, and Professor Loeb ${ }^{1}$ goes so far as to maintain that the problem of fertilization is really one of physical chemistry. $\mathrm{He}$ holds that the development of the egg is to be regarded as a chemical process which depends mainly on oxidation, and finds that the unfertilized eggs of various animals (sea-urchins and worms) will undergo development (at any rate up to a certain point) after exposure to the action of certain chemical reagents. The unfertilized eggs of a sea-urchin, for example, developed into larvæ after being placed for two hours in sea water, the osmotic pressure of which had been raised about $60 \%$ by the addition of some kind of salt or sugar, but this "hypertonic" solution must contain a sufficient quantity of free oxygen. In another case the unfertilized eggs of the worm Chætopterus were stimulated to develop into larvæ by the mere addition of potash and acids, without the osmotic pressure of the sea water being raised.

Exactly what takes place under these circumstances we do not know, and any speculation on this point is perhaps somewhat premature, but it is quite clear from the experiments of Loeb and other workers in the same field that we can no longer regard fertilization as an indispensable condition of development even in the case of eggs which do not naturally exhibit the phenomenon of parthenogenesis. These experiments may also throw some light upon the process of normal fertilization, especially as regards the nature of the actual stimulus which causes the fertilized egg to begin segmenting.

The casting out of polar bodies during the maturation of the ovum led many of the earlier observers of this phenomenon to believe that the matured ovum is incapable of development because it has an imperfect nucleus, the importance of the nucleus as taking the lead in cell-division having been established at a comparatively early date. The imperfect nucleus of the matured ovum was termed the female pronucleus, and it was supposed to be converted into a perfect segmentation nucleus by union with the male pronucleus brought into the egg by the spermatozoon (Fig. 64), and the power of cell-division was supposed to result from this completion of the nucleus. The observations upon which this belief was based were perfectly correct, but the conclusions drawn from them have not been sustained by recent investigations, for in cases of artificial parthenogenesis development

1 "Die chemische Entwicklungserregung des tierischen Eies" (künstliche Par. thenogenese). Berlin, 1909.

B. 
takes place in spite of the fact that reduction of the nucleus of the ovum has occurred in maturation and has not been made good by union with the nucleus of a spermatozoon.

In cases of parthenogenesis it is clear that the developing organism is provided only with maternal chromosomes, but we are now also acquainted with cases which form the exact converse to this, only paternal ehromosomes being present. Such cases may arise when an ovum is artificially enucleated, so that all the maternal chromosomes are removed, and then fertilized by a spermatozoon. It has actually been found possible in this way to induce the development of enucleated eggs, and the phenomenon, our knowledge of which we owe mainly to Delage, is known as merogony. Although it is obvious that, as a result either of artificial parthenogenesis or of merogony, the developing organism may start life with only half the normal number of chromosomes, it is possible that, as maintained by Delage, this number may be subsequently doubled in some way.

It cannot therefore be the union of male and female pronuclei that furnishes the stimulus to development; this union, or amphimixis as Weismann terms it, has another significance, and, as we shall see later on, is most probably connected with the transmission of inherited characters from parent to offspring.

Boveri, followed by other observers, has put forward the view that the unfertilized egg cannot develop because the centrosome, which is to be regarded as the dynamical centre of the cell, has been eliminated during the process of maturation. It is the spermatozoon that, in the act of fertilization, brings with it the centrosome upon the activity of which the cell-divisions of the fertilized egg depend. This is probably perfectly true in cases of normal fertilization and development in animals, but the view that the centrosome of the spermatozoon supplies the essential stimulus to development seems to be hopelessly negatived by the phenomena of parthenogenesis, in which new centrosomes undoubtedly arise in unfertilized eggs.

It seems therefore as if we were for the present thrown back upon Loeb's hypothesis of a chemical stimulus, which he maintains for normal fertilization as well as for artificial parthenogenesis. In the former case the necessary chemical substances are supposed to be brought in by the spermatozoon. Loeb believes that there are two of these substances. One, which he terms a lysin, is supposed to bring about the formation on the 
surface of the egg of the "fertilization membrane," while the other is supposed to prevent the cytolysis or disintegration of the ovum, induced by the formation of the fertilization membrane, from going too far. It remains to be seen whether this "lysin theory" will stand the test of time.

What it is that stimulates the unfertilized ovum to develop in normally occurring parthenogenesis we do not yet know. In most cases of this kind the process of maturation seems to differ more or less from that which takes place in eggs which are destined to be fertilized by a spermatozoon, and it may be that these differences have something to do with the power of the egg to develop parthenogenetically, but the discussion of this very difficult problem is altogether beyond the scope of the present work. 


\section{PART III.-VARIATION AND HEREDI'TY}

\section{CHAPTER XI}

Variation-Meristic and substantive variations-Fluctuations and mutations-Somatogenic and blastogenic variations-Origin of blastogenic variations.

The term variation is used in more than one sense; it may be defined in the first instance as the process whereby closely related organisms come to differ amongst themselves. It is a matter of everyday experience that neither animals nor plants exhibit absolutely fixed and constant characters, which are handed on without alteration from parent to offspring. This is very well seen in the case of human families, in which there is rarely any difficulty in distinguishing the different members by more or less pronounced and characteristic individual traits. One may be fair and another dark, one short and another tall, one with brown eyes and another with blue, one clever and another stupid, and so on. In this way they vary amongst themselves and deviate from the common parents of the family often to a very considerable extent.

We cannot, however, avoid extending the use of the term variation from the process itself to the results of that process, and speaking of organisms as exhibiting variations, but this usage is not likely to cause any confusion.

Variations are of many kinds and may be classified in different ways according to the point of view from which we regard them.

Meristic variations, which are variations in the number of the repeated parts of an organism, are sometimes contrasted ${ }^{1}$ with substantive variations, which depend upon the structure (including shape, size and colour) of the organism or its parts.

Small fluctuating or continuous variations, which fluctuate

1 Bateson, "Materials for the Study of Variation." (Macmillan \& Co., London, 1894.) 
about a mean or average condition, are contrasted with larger discontinuous variations, or mutations.

Somatogenic variations, which affect the body of the organism and are acquired in the life-time of the individual, are contrasted with blastogenic or germinal variations, which arise as a consequence of some modification in the germ cells.

These three methods of classification obviously overlap one another. Thus a meristic variation may be continuous or discontinuous, somatogenic or blastogenic, and so on, but it will be convenient to deal with each group separately.

Meristic or Numerical Variations. In a very great number of organisms certain parts are repeated with a greater or less degree of regularity and constancy, and it is upon this repetition that the symmetry of the organism very largely depends. An ordinary star-fish, for example, is radially symmetrical, with five similar arms or rays radiating from a common centre; meristic variation, however, not infrequently gives rise to six-rayed individuals. The common jelly-fish, A ureliı aurita, again, usually has four principal radii, but specimens are occasionally found with two, three, or six.

All vertebrate animals, on the other hand, and many invertebrates, are bilaterally symmetrical, and at the same time metamerically segmented, i.e. with repetition of similar parts in linear series one behind the other. The vertebral column, for example, is made up of a larger or smaller number of morphologically equivalent units, the vertebræ, to which the ribs are attached in linear series on each side, and the limbs are arranged in two pairs, in each of which the same fundamental structure can be traced. Many instances occur in different animals of variation in the number of vertebræ, and in man the occasional occurrence of thirteen ribs instead of the normal twelve on each side is well known. Cases of polydactylism, or the development of extra digits on hand or foot, also come under the head of meristic variation.

In the vegetable kingdom, where the repetition of similar parts, such as the leaflets of leaves, is even more conspicuous than amongst animals, meristic variation is again a common phenomenon. A clover leaf, for example, normally consists of three leaflets, but the occasional discovery of a specimen with four has been a source of satisfaction to superstitious folk from time immemorial. We meet with similar variations in the number 
of petals in flowers and the number of flowers in inflorescences. An example of this will be given later on in the case of the grape hyacinth.

Substantive Variations. Examples of this type of variation are seen in the shape, size and colour of the human body and of its various parts. The total height of the individual, the shape of such features as the nose, ears and fingers, the colour of eyes and hair, are all subject to very great variation. The same is true of the shape and size of leaves and the colour of many flowers. Numerous physiological variations, which doubtless depend upon unrecognized and perhaps unrecognizable differences in structure, may also be classed under this head, as for example the variations in the egg-laying powers of fowls and the milkgiving powers of eows, and in the percentage of sugar present in the roots of the sugar beet.

Fluctuating Variations. The variations which come under this heading are also spoken of as normal, individual or continuous. They may be either meristic or substantive. They are characterized by the fact that they pass into one another by very minute or even insensible gradations, which fluctuate on either side of a mean or average condition, so that the extent to which a given organism (or part of an organism) varies can be graphically represented in the form of a curve. Fluctuating meristic variations lend themselves most readily to this graphic method of treatment, for all we have to do is to count the numerically varying parts in a sufficiently large number of cases and plot our curve accordingly.

The manner in which this is done is represented in the accompanying diagram (Fig. 72), which expresses graphically the results obtained by counting the number of flowers in 202 inflorescences of the common grape hyacinth (Muscari sp.). The number of flowers in an inflorescence ranged from 20 to 42 , and the number of inflorescences showing each of the different numbers of flowers ranged from 1 to 29 .

The base line of the diagram is divided into a number of equal parts (abscissæ) corresponding to the number of flowers (from 20 to 42). At one side an ordinate is erected on the base line and divided into a number of equal parts corresponding to the number of the individual inflorescences (from 1 to 29) bearing the different numbers of flowers. Lines are then ruled parallel to the base line and to the ordinate respectively, so as to divide the 
paper into a number of equal squares (or the proceeding may be simplified by working with already squared paper). The diagram is completed by shading in an appropriate square for each inflorescence, the position of the square being determined by the number of flowers which it contains, and by the number of

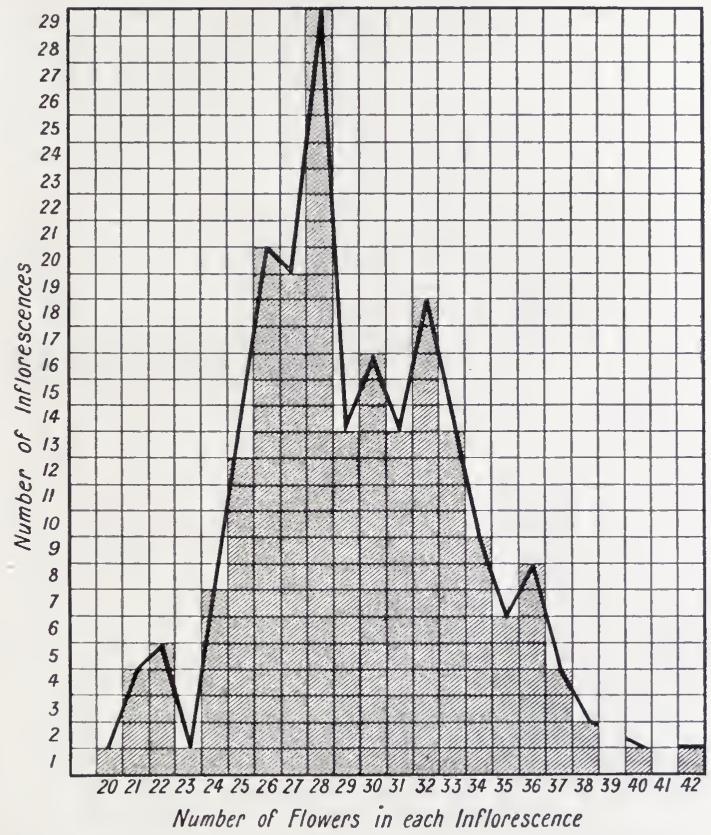

Fig. 72.-Fluctuating Variation in Inflorescences of the Grape IIyacinth.

similar inflorescences which have preceded it in the enumeration. Thus an inflorescence with twenty-five flowers must be represented by a square added to. the column above the number 25 on the base line, and so on. If now we join the middle points of the tops of all the columns by straight lines, we get a zig-zag line which represents the curve of variation as determined by our actual observations. It is obviously a very imperfect result, but its 
imperfection is undoubtedly due in the main to the fact that the number of observations upon which it is based has not been sufficiently large to give a fair average.

The larger the number of individual cases examined and recorded the more closely will our zig-zag line approximate to a regular curve. Even as it is, with all its imperfections, it shows certain very characteristic features. It rises steeply in the middle and falls away from the highest point, at first suddenly and then more gradually, on either side. This is a graphic representation of the facts, (1) that the most frequently occurring number of flowers in the inflorescence (28) lies not very far from midway between the two extremes, and (2) that the

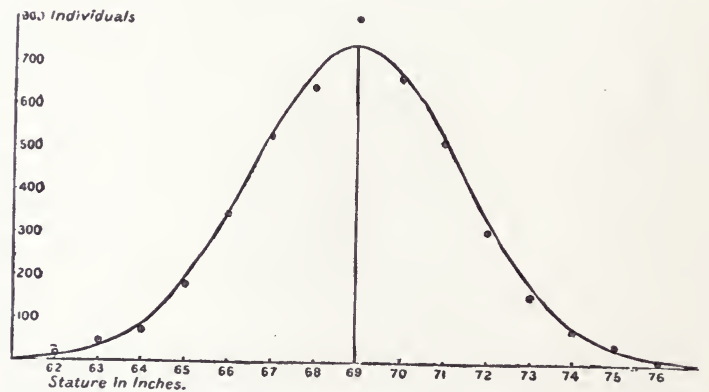

FIG. 73.-Fluctuating Variation in Human Stature. (From Lock.)

more the number of flowers deviates from this mean on either side the smaller is the corresponding number of inflorescences; or, to put it more generally, the number of individuals exhibiting any given degree of deviation from the mean condition of the species is inversely proportional to the amount of that deviation.

Fig. 73 is a diagram constructed on the same principles as the above, but based upon totally different material and a very much larger number of observations. It is taken from Mr. R. H. Lock's work on "Variation, Heredity and Evolution," and shows the variation in stature observed amongst 4,426 British members of the University of Cambridge. The dots in this diagram correspond to the middle points of the tops of the vertical columns in the preceding one. The statures are reckoned in the nearest 
whole numbers of inches. It is clear that a zig-zag line formed by joining the dots in the diagram would approximate fairly closely to the curve actually drawn.

Such a normal curve of variation agrees very closely with what mathematicians term the curve of frequency of error, which is the graphic representation of the mode of occurrence of chance deviations from a mean or average and may be derived from the theory of probability.

Such a curve may be experimentally produced by drawing a vertical line on a target and firing a large number of shots at it. There will be a more or less strongly marked tendency for the shots to hit the line, depending upon the skill or otherwise of the marksman. Most of them will probably strike to the right or left of the line and fairly near it, but a few will probably be very wide of the mark on either side. If the distances of the striking places from the vertical line be measured and tabulated the result may be expressed in the form of a curve which, if the number of shots be large enough, will probably closely resemble the curves described above.

The fact that the normal curve of fluctuating variation for any kind of organism is practically identical with the mathematical curve of frequency of error suggests very forcibly that the variation in question is due to chance or accident causing each individual in the course of its development to depart more or less from the mean or average condition of the species to which it belongs. 'These deviations must depend upon numerous factors. They are to some extent, no doubt, due to the direct influence of the environment, such as the effect of nutrition upon the size of the organism, but they may also depend largely upon the varying characters of the germ cells from which the organism develops, and especially upon the permutations and combinations of characters which happen to take place in the maturation of the germ cells and in their sexual union or amphimixis.

In short, the general tendency is doubtless for each individual to conform to the type of the species to which it belongs, but many accidental circumstances combine to prevent the realization of this tendency and deviations from the type (or mean) take place in accordance with the laws of chance.

Mutations. The term mutation, or discontinuous variation, is applied to the process whereby new and more or less conspicuous characters appear suddenly and spontaneously, without any 
obvious reason. In extreme cases the organisms exhibiting such mutation are often spoken of as sports or monstrosities; the latter term, however, is also frequently applied in the case of purely artificial modifications, which must not be included under this heading. Such artificial modifications are not, at any rate as a rule, inherited by future generations, while true mutations usually, if not invariably, are.

Mutations differ from fluctuating variations not only in that they usually deviate more widely from the type of the species, but also in the much greater rarity of their occurrence and in the fact that they do not fluctuate about the old mean or average condition; nevertheless it may be questioned whether we can logically draw a hard and fast distinction between the two.

Mutations may be either meristic or substantive. Human beings with six fingers or toes, in place of the normal five, are not infrequently met with, and this meristic mutation (hexadactylism) is well known to be transmitted by heredity.

The classical instance of the Ancon or otter sheep, on the other hand, affords one of the best known examples of substantive mutation. To quote the words of Huxley," "It appears that one Seth Wright, the proprietor of a farm on the banks of the Charles River, in Massachusetts, possessed a flock of fifteen ewes and a ram of the ordinary kind. In the year 1791, one of the ewes presented her owner with a male lamb, differing, for no assignable reason, from its parents by a proportionally long body and short bandy legs, whence it was unable to emulate its relatives in those sportive leaps over the neighbours' fences, in which they were in the habit of indulging, much to the good farmer's vexation." The inheritance of this peculiarity was so strong that this single individual actually became the starting point of a new breed.

Mutations, more or less pronounced in character, are also not infrequently met with in the vegetable kingdom. Foxgloves, for example, are sometimes found in which some or all of the petals are converted into stamens, and it has been proved by experiment that this peculiarity is handed down from parent to offspring.

Professor Hugo de Vries has, as is well known, made a special study of mutation amongst plants. In the year 1886 this disguished botanist found a large number of specimens of the evening 
primrose, Enothera lamarckiana, growing in a field near Amsterdam, whither they had made their way from a neighbouring garden. The plants were in a state of intense variability and their seeds gave rise to several quite distinct new forms, which, if they had occurred in a state of nature, would have been considered as separate species. ${ }^{1}$ Professor De Vries attributes great importance to mutations as the starting points of new species, which he believes to arise in this sudden manner rather than by fluctuating variation. ${ }^{2}$ We shall discuss this point in a subsequent chapter.

The difference between fluctuating variation and mutation is sometimes illustrated by means of the model known as Galton's polygon (Fig. 74). A thick slab of wood is cut into the form of a polygon, with unequal sides and capable of resting in a position of more or less stable equilibrium upon any of its edges, the

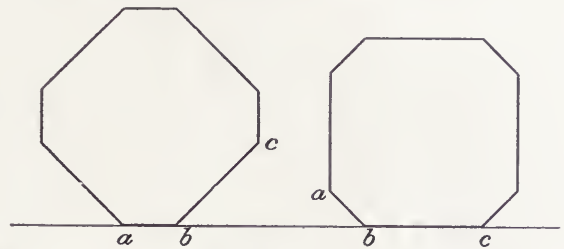

Fig. 74.-Model of a Polygon in two Positions, illustrating the Iifference between Fluctuating Variation and Mutation.

degree of stability depending upon the position of the centre of gravity above the edge upon which it rests.

The polygon may be supposed to represent an organism, or rather a number of successive generations of an organism, whose stability (or adherence to type) tends to be more or less disturbed by the unknown factors which cause variation. If the model be pushed it may be made to rock backwards and forwards on either side of a mean or average position, and if the oscillation does not exceed certain limits it will return to rest in that position. This oscillation may be compared to fluctuating variation. If the disturbing force be sufficiently great, however, the model will topple over into a new position of stability and come to rest on another

1 Considerable doubt has, however, been thrown upon the interpretation of these observations, the results being conceivably due to the splitting up of some unknown hybrid form, snch as is well known to take place in other cases (see Chapter XIV.).

2 "The Mutation Theory." Trans. by Farmer and Darbishire. London, 1910. 
edge. This may be compared to the process of mutation or discontinuous variation, whereby an organism acquires a new type of structure.

Concerning the forces which bring about mutation in organisms we know little or nothing. It is possible that in some cases the mutation may be gradually prepared within the germ cells long before it manifests itself-the final upsetting of the equilibrium only taking place on the addition of the last straw. If such be the case there is no need to suppose that mutations differ essentially in nature from small, fluctuating variations. Tower's observations on the artificial production of mutations in certain beetles, however, indicate very clearly that such modifications may apparently arise quite suddenly as the result of some change in the environment acting directly upon the germ cells of the parent. These observations will be referred to again at the close of the present chapter.

Somatogenic Variations. Characters are said to be somatogenic or "acquired" when they arise in the life-time of the individual exhibiting them and owe their origin to the direct influence of the environment upon the soma or body. They are, usually at any rate, not transmitted by heredity to succeeding generations, except perhaps to a very limited and inappreciable extent. ${ }^{1}$ Under this heading are included the effects of use and disuse of organs, and numerous cases in which modifications of the body are artificially produced, as well as those in which they are due to natural causes.

Amongst the effects of use and disuse we may mention, on the one hand, the enlargement or the atrophy of special organs consequent upon the extent to which they are employed, and, on the other, the effects of education. The muscles of an athlete may be greatly increased in size by constant use, and similarly if one of the two kidneys be removed the other, having more work thrown upon it, becomes enormously enlarged; but we should not expect these modifications to be handed on to the next generation. Children have been taught to speak ever since man first became differentiated from his speechless ancestors, but every child has to learn the art anew and if brought up amongst foreigners will come to speak a language different from that of its parents.

The small feet of Chinese ladies and the slender waists of many Europeans are artificially produced somatic modifications

\footnotetext{
1 This may be, however, a very important exception.
} 
which are well known not to be inherited. They are merely distortions, effected by easily recognizable mechanical agencies.

Much more remarkable and difficult to understand are those cases in which the addition of specific chemical reagents to the water in which aquatic larvæ are developing produces such definite and extensive modifications of structure as to give rise to veritable monstrosities. The "Lithium larvæ" of sea-urchins and frogs have long been known and more recently Stockard has described ${ }^{1}$ the "Magnesium larva" of the fish Fundulus leteroclitus (Fig. 75). He found that, when the developing embryos of this fish are subjected to the influence of magnesium salts dissolved in sea water, a large percentage of them acquire a "cyclopean" character, with a single median eye in place of the ordinary pair. Such embryos may hatch and swim about in a perfectly normal manner, but it is not known whether they can be reared to the adult condition. These observations seem to indicate that the cyclopean monsters which sometimes oceur in man and other mammals may also be somatogenic variations due to some unknown environmental influence.

Blastogenic or Germinal Variations. In this category are included those variations which are believed to owe their origin to some modification in the germ cells from which the organism exhibiting them has developed.

It is important to observe that the term congenital, sometimes used in this connection, is not synonymous with blastogenic, for it is obvious that animals which, like the mammalia, remain within the womb of the mother during the early stages of development, may come to develop purely somatogenic characters before birth, due to environmental influences acting in utero (e.g. poisoning of the foetus due to parental alcoholism).

It is very doubtful, as we have already said, whether we can really draw any absolute distinction between blastogenic and somatogenic characters, and it seems by no means impossible that somatogenic modifications may sooner or later make an impression upon the germ cells and thus ultimately become blastogenic. This point, however, will be discussed later on.

Blastogenic modifications are from their very nature as attributes of the germ cells handed on by heredity from generation to generation. All true mutations must be regarded as 1 " Journal of Experimental Zoology," February, 1909. 


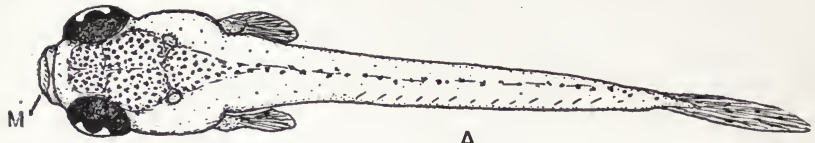

A.
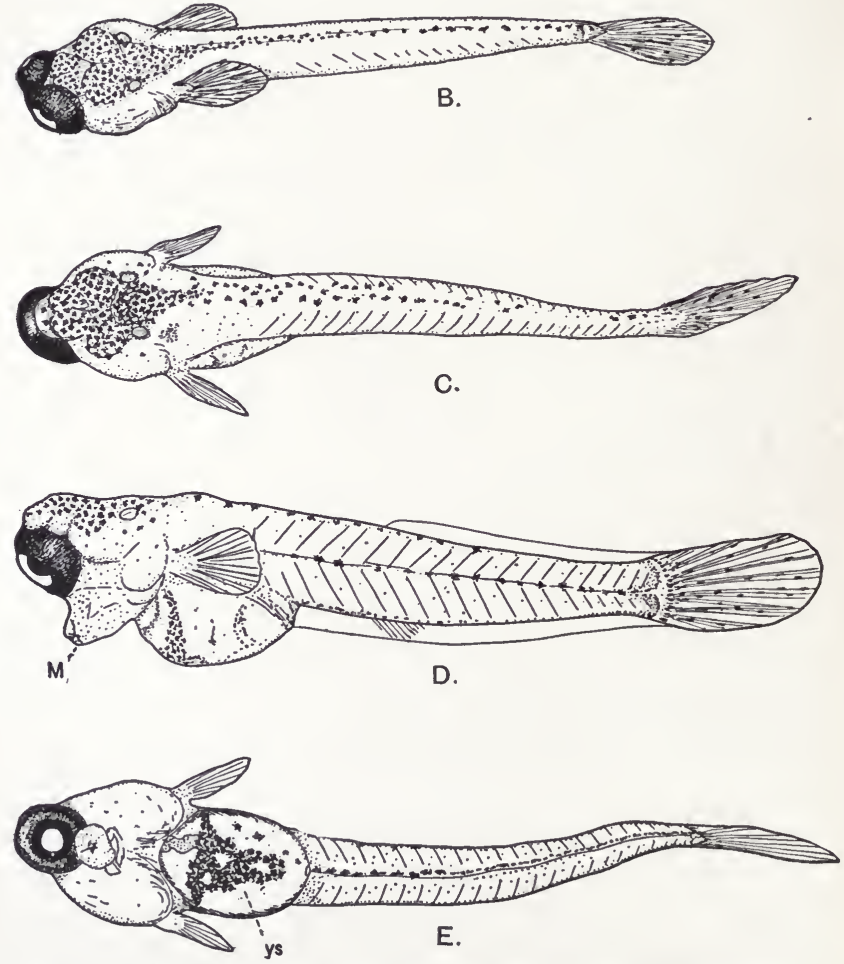

Fig. 75. - Free-swimming Larvæ of Fundulus heteroclitus. (From Stockard.)

A. Normal larva, with anteriorly placed mouth (M).

B. Incompletely cyclopean larva, with the two eyes joined and occupying the position usually taken by the mouth.

C. Completely cyclopean larva, with single antero-median eye. Dorsal aspect.

D. Lateral aspect of same, showing the ventral mouth (M).

E. Ventral aspect of same; $y s$, yolk sac. 
blastogenic and many so-called fluctuating variations may also perhaps belong to the same category.

The modifications of the germ cells by virtue of which the offspring come to differ to a greater or less extent from their parents are, as we have seen, often attributed in large measure to permutations and combinations of different characters which take place in the sexual process (amphimixis) and the preceding nuclear reduction. It has long been suspected, however, that the germ cells themselves, apparently independently of the body in which they are enclosed, may be influenced by the environment to which an animal or plant is exposed, and the observations of Tower ${ }^{1}$ upon beetles of the genus Leptinotarsa may be referred to in this connection.

This observer considers that all permanent variations in these beetles, so far as can be discovered, arise in the germ cells and are in no wise the results of inherited somatic modifications. He attributes their appearance to the direct action of the environment upon the germ plasm and supports his views by a series of very interesting experiments. He subjected the parents to environmental stimuli of various kinds during the growth and maturation of their germ cells, and then, after the ova had been fertilized, allowed the development of the young to take place under normal conditions. The parents, having already reached their final state, were not themselves visibly affected by the stimuli, but a large percentage of the offspring showed surprising modifications which were strictly inherited. These modifications appear to be in no way adaptive. They seem to bear no relation to the nature of the stimulus which calls them forth and to be of no value to the organism in the struggle for existence.

We may cite one example of Tower's experiments. Four males and four females of Leptinotarsa decemlineata (the potato beetle) were exposed during the earlier part of the laying period (the eggs being matured and laid in successive batches) to extremely hot, dry conditions accompanied by low atmospheric pressure. The eggs were removed as soon as laid and reared under natural conditions. From 506 larvæ thus reared 96 adult beetles were obtained, of which 82 were of a form known as pallida, 2 of a form known as immaculothorax, and the remainder unmodified. During

1 "An Investigation of Evolution in Chrysomelid Beetles of the Genus Leptinotarsa," by William Laurence Tower. (Publications of the Carnegie Institution, Washington, 1906.) 
the later part of the laying period the same parents were kept under normal conditions and yielded 319 eggs from which 61 normal beetles were obtained and none of the other forms, and these normal beetles continued to breed true for three generations, after which they were killed.

The two specimens of the immaculothorax form obtained in the earlier part of the experiment unfortunately died from disease, as also did all but two of the pallida. The remaining two, however, both being male, were crossed with normal females, yielding hybrid offspring with the decemlineata characters dominant, and these hybrids, breeding inter se, gave offspring which separated out in a characteristic Mendelian fashion ${ }^{1}$ into pallida, decemlineala and lybbids again. There can be no. question therefore that the pallida characters, first due to modification of the germ cells by the action of changed environment, were strictly heritable.

It should be observed that the forms pallida and immaculothorax also occur occasionally, but rarely, in a state of nature as sports or mutations, a fact which suggests that sports or mutations in general may owe their existence to the apparently direct action of the environment upon the germ cells. It is, of course, possible, or even probable, that the change in the environment merely acts as a kind of liberating stimulus, which enables characters already latent in the germ cells to express themselves in the developing organism, which, under normal conditions, they are unable to do.

We shall have to return to the question of the origin of blastogenic variations in future chapters.

1 See Chapter XIV. 


\section{CHAP'TER XII}

Heredity - General observations - Darwin's theory of pangenesis and Weismann's theory of the continuity of the germ plasm--The nucleus as the bearer of inheritable characters.

When we study the life histories of the unicellular Protista we find ourselves face to face with the problem of heredity in its simplest form. The Amøba divides into two parts by simple fission, the division of the cell body being preceded by that of the nucleus. The two daughter cells exactly resemble one another, and, except in point of size, also resemble the parent, while the latter ceases to exist as an individual in the very act of reproduction. Here we may suppose that we are dealing with a division which is qualitative as well as quantitative, that every organ possessed by the parent cell is divided into two similar parts and the total inheritance thus fairly apportioned between the offspring, which will therefore be exactly similar to one another and will need only to feed and grow in order to become exactly similar to the parent.

The young Amweb may be supposed to resemble the parent because they arise by duplication of the parent and of its organs ${ }^{1}$ moreover, there is a perfect continuity of the living substance, or protoplasm, of which the body is composed from one generation to the next, and the whole of the body of the parent is used up in providing the bodies of the offspring. Thus, although the individuality of the parent comes to an end, the Amœba never dies, for there is never anything left over to die, and, barring accident, it goes on multiplying for ever. We have here a typical illustration of the so-called immortality of the Protista.

The case is very different amongst the higher plants and animals. Here, as we have already seen, each individual starts

1 We cannot, however, say this of all Protista, for in many cases the division takes place asymmetrieally and entirely new organs have to be formed by one or both of the daughter cells, as, for example, in the transverse fission of Bodo (Fig. 38, D-F). In such cases it looks as if the nucleus might be the real seat of the inherited tendencies, and as if it wcre able, by its influenee, to mould the daughter eell into the form of the parent. 
its life as a single cell-the fertilized ovum or zygote-which is strictly comparable to a unicellular protistan. Like the Amœba it divides (under favourable cireumstances) repeatedly, but the products of division, instead of separating from one another and going each its own way as an independent unicellular organism, all remain together and co-operate with one another to form a multicellular body of greater or less complexity. The cells of which this body is composed become differentiated and specialized in various directions. In so doing the vast majority of them lose their faculty for independent existence, and when their powers of division have become exhausted the tissues into which they are combined become worn out and ultimately die. Thus the body or soma, as a whole, must suffer death sooner or later. The only cells which are, even potentially, exempt from this fate are the germ cells. These, instead of becoming highly specialized constituents of the soma, remain in the condition of more or less independent Protista, and have the power of separating sooner or later from the parent body. . Like most of the Protista they have also the labit of conjugating in pairs and thereby renewing their powers of cell-division, and thus arise the zygotes or fertilized eggs from which the new individuals take their origin.

The eggs from which the individuals of different kinds of plants and animals arise are for the most part extraordinarily similar to one another. The ovum of a rabbit, as we have already seen, is a minute nucleated mass of protoplasm about $\frac{1}{250}$ th inch in diameter, that of a human being is a similar but somewhat larger cell, and if the ova of a hundred different kinds of mammals were mixed together it would be an extremely difficult, if not impossible task to sort them all out, even after the most minute microscopical examination. Even where conspicuous differences exist, as between the eggs of a mammal and those of a bird, these are due almost entirely to the development of accessory features, such as protective envelopes and food-yolk, which have little to do with the nucleated mass of protoplasm which constitutes the really vital part of the egg. Yet each kind of fertilized egg, if it develop, will give rise to an organism resembling the parent from which it was itself derived. Moreover, the resemblance will not be merely a general one, it will be specific, and probably even more than specific, for it may include minute individual characters peculiar to one or other of the 
parents. Everyone is familiar with cases of this kind. It may be some abnormality of fingers or toes, or a lock of white hair in some special situation in a dark-haired man, or even some trifling nervous habit, that is thus indelibly impressed upon the organism and handed on from one generation to another.

Inasmuch as the only possible connection between parent and offspring is (in most cases) throngh the germ cells, it follows that there must be something in these germ cells which, so to speak, represents all the inheritable characters of the parents and is capable of giving rise to a repetition of these characters in the course of individual development.

T'wo sharply contrasted views as to what takes place in the development of the egg were maintained by the older embryologists, and, in a modified form, survive to the present day. The so-called "evolutionists," or "pre-formationists," maintained that the egg contains in itself a complete miniature of the organism into which it develops, and that the process of development consists simply in an unfolding ("evolution") and growth of this miniature. This idea, of course, carried to its logical conclusion, involves the further supposition that every egg contains in miniature the bodies of all future generations, like nests of boxes one within the other.

In opposition to this view the upholders of the theory of "epigenesis" maintained that there is no pre-formation of organs in the egg but that the different parts of the adult organism become gradually differentiated from the simple undifferentiated ovum during the course of development. This view, which is said to have originated with Aristotle and was strongly supported by the great pioneer embryologist C. F. Wolff about the middle of the eighteenth century, almost entirely superseded the crude ideas of the pre-formationists, but at the present day the latter are being revived to some extent, but in a more refined form, as a result of modern experiments in embryology. It cannot be disputed that in some cases certain parts of the adult organism can be traced back to corresponding portions of the egg, which cannot therefore be entirely undifferentiated, and it is probable that in the end the truth will be found, as in so many other cases, to lie in a compromise between the two extreme views.

The great problem which has to be solved by any theory of heredity is-How do the apparently simple germ cells of a multicellular organism come to be representative of all the other 
cells of the body, so that when they develop they will give rise to all those different kinds of cells arranged in the same way as in the parent? We must now briefly consider some of the numerous attempts which have been made to answer this question.

Darwin, in 1868, put forward his theory of Pangenesis as a provisional hypothesis to explain the facts of heredity, and this theory, though it seems never to have met with any large measure of acceptance, is of considerable historical interest. ${ }^{1}$ He supposed that all the constituent cells of which the body is composed not only multiply by ordinary cell-division, so as to build up the various tissues, but also, throughout life, give off extremely minute "gemmules" which wander through the body and are collected in vast numbers in the germ cells. The gemmules, although so small as to be invisible even with the highest powers of the microscope, are supposed to be capable of absorbing nutriment and multiplying by division, and each one is supposed, in some mysterious and unexplained manner, to represent the particular cell of the body from which it was derived, and to be capable, at the proper time and in the proper place, of impressing the character of its parent cell upon a corresponding cell of the new organism which develops from the germ cell.

In Darwin's own words :-

"The development of each being, including all the forms of metamorphosis and metagenesis, depends on the presence of gemmules thrown off at each period of life, and on their development, at a corresponding period, in union with preceding cells. Such cells may be said to be fertilized by the gemmules which come next in due order of development. Thus the act of ordinary impregnation and the development of each part in each being are closely analogous processes. The child, strictly speaking, does not grow into the man, but includes germs which slowly and successively become developed and form the man. In the child, as well as in the adult, each part generates the same part. Inheritance must be looked upon as merely a form of growth, like the self-division of a lowly organized unicellular organism. Reversion depend's on the transmission from the forefather to his descendants of dormant gemmules, which occasionally become developed under certain known or unknown conditions. Each animal and plant may be compared with a bed

\footnotetext{
1 For a complete exposition of the theory see Darwin's "Animals and Plants under Domestication" (Ed. 2, Vol. II., Chapter XXVII.).
} 
of soil full of seeds, some of which soon germinate, some lie dormant for a period, whilst others perish." 1

Darwin did not recognize tlie modern distinction between somatogenic characters, which are acquired by the body or soma during its individual life-time, and blastogenic or germinal characters, which are supposed to originate in the germ cells; or rather, in accordance with his theory of pangenesis, he believed that somatogenic modifications might be transferred to the germ cells and thus become blastogenic. In other words, he was a firm believer in the inheritance of acquired characters, a doctrine which, as we shall see presently, is now much discredited, and he endeavoured to explain by means of his theory how such characters may be transmitted from parent to offspring.

Suppose some part of the body in a particular multicellular individual were to become nodified by use or disuse, or by the direct action of the environment. Then the gemmules given off from the modified cells would also be affected in a corresponding manner and would carry information of the change to the germ cells. It would be as if some constituency with many representatives changed its political opinions and instead of sending conservative members to the House of Commons took to sending liberals. When the proper time came the new representatives would vote according to their instructions; but we must also suppose that the old ones could never be turned out and that there would be a struggle between the two. At first the old ones would be the more numerous and would outvote the new ones; presently, however, the new ones, being constantly reinforced, would come to outnumber the old ones and perhaps be able to give effect to the altered views of their constituency. As Darwin says, "It is generally necessary that an organism should be exposed during several generations to changed conditions or habits, in order that any modification thus acquired should appear in the offspring." It would probably be more in accord with the facts if, instead of "several generations" we said "a large number of generations." In this sense, we may well believe that acquired characters can be inlıerited, without expecting to be able to demonstrate such inheritance by eutting off the tails of a few generations of mice.

The theory of pangenesis certainly explains a great deal, but it involves so many unproval)le assumptions as to the nature 
and behaviour of the "gemmules" that it cannot be accepted as more than what Darwin himself termed it, a provisional hypothesis or speculation.

It is interesting to observe that Darwin finds the cause of variation in the direct influence of the environment, including under that term the effects of use and disuse upon the organism. In this respect he agrees with the views of Lamarck and differs widely from those of Weismann and many other modern biologists, who deny, either totally or in part, the possibility of the inheritance of acquired or somatogenic characters. It will have been noticed that the theory of pangenesis is of an essentially pre-formationist character, for it assumes the existence, within the fertilized egg, of an immense number of material particles (gemmules) which in some way or other represent the different inheritable characters of the body.

The celebrated theory of heredity which we owe to Professor Weismann ${ }^{1}$ is based upon what he terms the "Continuity of the Germ Ilasm." The general idea of continuity is, of course, by no means a new one ; indeed the protoplasmic continuity of parent and offspring, through the germ cells, must form the material basis for the transmission of characters on any theory of heredity. Weismann, however, gives much greater precision to the idea than any of his predecessors. He identifies the chromatin of the nucleus as the actual hereditary substance, the bearer of all inherited tendencies, and draws a very sharp distinction between the somatic cells which, with almost endless diversity of form and function, build up the body of one of the higher plants or animals, and the germ cells, which play little if any part in the life of the individual in which they are lodged but are destined, under favourable circumstances, to give rise to the next generation.

We have already seen that the germ cells are frequently separated from the somatic cells at a very early stage in development. It may be that the distinction between the two is actually inaugurated by the very first division of the fertilized ovum, as in the horse worm, Ascaris megalocephala (p. 129), or it may be recognizable at the gastrula stage, as in the arrow worm, Sagitta

1 For full accounts of this theory sce the English translations of Weismann's two chief works on the subject, "The Germ Plasm" (Contemporary Science Series, 1893) and "The Evolution Theory" (Edward Arnold, 1904). 
(p. 130), but such cases appear to be very exceptional and the segregation of the germ cells usually takes place much later and at different stages of the development in different species of plants and animals. The exact time at which the separation of the two groups of cells takes place, however, does not seriously affect the argument. In any case the ultimate distinction between germ cells and somatic cells is supposed to lie in the fact that the former retain each a complete sample of the ancestral germ plasm, in which at any rate all the essential characters of the organism are in some way or other represented, while the latter, by a series of differential divisions, gradually undergo a further segregation into the different tissue cells of the adult, each of which contains (in an active condition) only a sample of that part of the ancestral germ plasm which is appropriate to its own particular requirements.

Hence the germ cells, complete in themselves like so many Protista, alone retain the power of giving rise to new generations of complete individuals. The somatic cells have sacrificed this power to their need for specialization. Certain phenomena, however, such as the regeneration of lost parts in many animals and the propagation of plants by buds and cuttings, necessitate the supposition that some at any rate of the somatic cells must retain a more complete sample of the ancestral germ plasm than is necessary for their own development. For the hereditary substance of any particular cell Weismann adopts Nägeli's term "idioplasm," and in order to account for the phenomena just referred to he is obliged to postulate the existence, in the somatic cells in question, of "accessory idioplasm," which is only called into activity under exceptional conditions, as, for example, when it becomes necessary for a crab to regenerate a lost limb or for an earthworm to renew a portion of its body which has been bitten off by a bird or chopped off by a spade.

Weismann's theory involves the assumption of great complexity of structure in the germ plasm, which, as we have already seen, le identifies with the chromatin substance of the nucleus of the germ cells. He finds it necessary to assume the existence, not only of " determinants," which correspond more or less closely to Darwin's gemmules, and each of which is supposed to be responsible for the development of some special inherited feature of the organism, but also of structural units respectively of a lower and a higher order. Thus each determinant is supposed 
to be made up of "biophors," which are themselves the lowest vital units, but each of which is in turn made up of molecules in the chemical sense of the term; while, on the other hand, the determinants are supposed to be grouped in "ids," each of which is a complete ancestral germ plasm, theoretically sufficient in itself to determine all the different characters of an entire individual. The ids may in some instances correspond to the chromosomes, but these appear generally to be composite bodies ("idants") made up each of a large number of ids (chromomeres). The determinants, and of course the biophors also, are far below the limits of visibility even with the aid of the most powerful microscope; the ids, however, frequently appear during the process of mitosis and may give the spireme thread or the chromosomes into which it divides a characteristic beaded appearance (Fig. 32, B). Biophors, determinants, ids and idants must all be looked upon as living entities, growing by the absorption of nutriment and multiplying by division.

In accordance with Weismann's theory the germ cells themselves may be regarded as so many unicellular organisms, which multiply by fission and periodically, if they chance to meet with mates, conjugate with one another. Their cytoplasm as well as their chromatin is directly continuous from generation to generation just as it is in a dividing Amoba, and theoretically there is no reason why the constant succession of germ cells should ever be interrupted by death. The soma, or body, however, stands in a very different position. It may be regarded as a kind of appendage thrown off from the chain of germ cells after each conjugation, and resulting from the fact that most of the cells arising from the segmentation of the zygote not only remain together in intimate association with one another but become specialized in various directions and co-operate with one another to form a complex multicellular individual. Having exhausted its powers of growth and renewal this individual body sooner or later dies; but the germ cells periodically renew their powers of cell-division by conjugation and, under favourable conditions, go on for ever.

According to Weismann, inherited characters are transmitted not from soma to soma but from germ cell to germ cell, by virtue of the continuity of the germ plasm. The soma has little if any influence upon the germ cells which it contains beyond that which is involved in supplying them with protection and nourishment. 
There is no other means of communication between the soma and the germ cells, and hence somatogenic characters, which are acquired in the life-time of the individual body as the direct result of the action of the environment (including use and disuse of organs), cannot be transmitted to the germ cells and therefore cannot be inherited. The only characters which can be inherited are blastogenic characters, which arise by modification of the germ plasm in the germ cells themselves.

This denial of the transmission of so-called acquired characters constitutes the most important difference between the theories of heredity propounded by Weismann and Darwin. Both these theories postulate the existence of ultra-microscopical material particles, determinants or gemmules, but Weismann's theory allows of no transference of such particles from soma to germ cell, only from germ cell to germ cell and from germ cell to soma. There is supposed to be no mechanism for the transmission of somatogenic characters to the next generation. According to the older view the germ cells give rise to the soma and the soma to the germ cells alternately. According to the newer one the germ cells give rise to the soma and at the same time to the next generation of germ cells, while the soma gives rise to nothing but itself and ultimately perishes.

The contrast between the two views is clearly expressed in the accompanying diagram (Fig. 76), in which, for the sake of simplicity, the complication introduced by the process of conjugation of the germ cells has been ignored.

The inheritance of somatogenic characters being denied, Weismann is obliged to seek the origin of variations from some source other than the action of the environment and use and disuse. We shall return to this point presently. In the meantime we must point out that Weismann's theory harmonizes very well with the phenomena of mitosis, and especially with the remarkable modifications of those phenomena which accompany the maturation of the germ cells.

The entire process of mitosis serves to emphasize the importance of the chromatin substance of the nucleus. It is evidently of the utmost consequence that this substance should be aceurately apportioned between the daughter cells. We accordingly find the elaborate mechanism of centrosomes and nuclear spindle, and a splitting of each individual chromosome, which takes place longitudinally when the chromosomes themselves happen to be 
elongated in form. If, as Weismann maintains, and as can actually be demonstrated in many cases, each elongated chromosome is made up of a row of chromomeres or ids, which may be supposed to differ to some extent from one another as to the determinants which they contain, it is obvious that longitudinal splitting of the chromosome is the only way in which a qualitative as opposed to a mere quantitative division of the hereditary
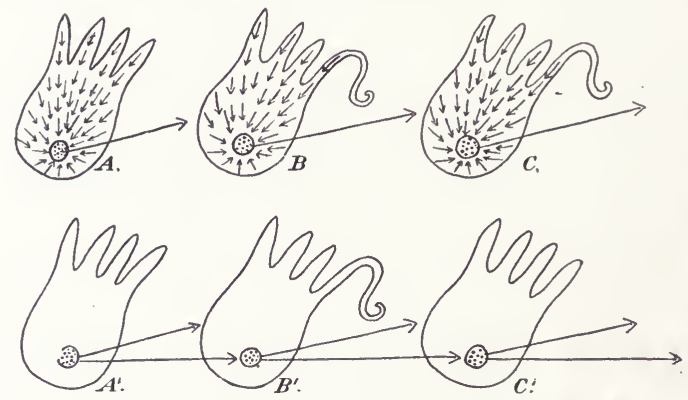

FIG. 76.-Diagram to illustrate the contrast between Darwin's Theory of Pangenesis and Weismann's Theory of the Continuity of the Germ Plasm.

The figures represent an imaginary organism with four processes given off from the soma or body, which is supposed to contain only a single germ cell (dotted). Three generations are represented, and for the sake of simplicity the complication introduced by the periodical eonjugation of male and female germ cells is omitted. Figs. A, $B$, C show how an aequired character-the elongation of one of the processes as a result of its use for some special purpose-may be supposed to affect the germ cells through the migration of gemmules (indicated by the small arrows), and thus be transmitted by leredity in accordance with the theory of Pangenesis. Figs. A', B', $\mathrm{C}^{\prime}$ show how such an acquired eharacter is, in accordance with Weismann's theory, unable to make any impression upon the germ cells and is therefore not transmitted by heredity. In the first case the germ cells of each generation are supposed to arise from the soma; in the second case they are supposed to arise directly from the preceding generation of germ cells, which also gives rise to the soma in which they are enclosed, as indicated by the large arrows.

substance can be brought about. This mode of division, then, otherwise difficult to explain, is fully intelligible in accordance with Weismann's theory.

It is not necessary to suppose, however, that such division always results in identical daughter chromosomes. We may assume either that all the individual ids divide into similar halves, containing similar determinants (as represented very diagrammatically in Fig. 77, A), in which case the two daughter chromosomes will be exactly alike, or that some or all of the ids 
divide each into two dissimilar halves containing different determinants (Fig. 77, B), when the daughter chromosomes will be unlike each other. In the former case the division is said to be integral and in the latter differential. It is by differential division that Weismann believes the histological differentiation of the soma to be brought about.

When we consider the phenomena of maturation and fertilization we find them in no less striking harmony with Weismann's views. We have already pointed out, in Chapter X., that each particular species of plant or animal is, as a general rule, characterized by the appearance of a definite and constant number of chromosomes in all the cells of the body during the process of mitosis. At some period in the life-cycle, however, in typical plants during the process of spore-formation and in animals during the maturation of the ova and spermatozoa, this number is reduced to half by separation of the entire chromosomes into two. groups, one of which passes to each of two daughter cells. Thus the mature germ cells have only half the number of chromosomes characteristic of the species (or, in the case of typical plants, of the sporophyte generation). The full number is made up again by the union of male and female gametes to form the zygote or fertilized egg.

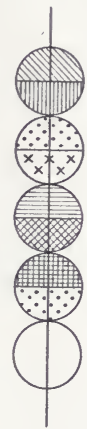

A

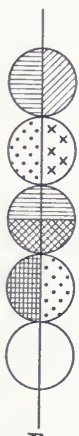

B

Fig. 77. - Diagram of (A) integral and (B) differential Iivision of a Chromosome consisting of five Ids or Chromomeres.

To the combination of the maternal and paternal chromosomes in the nucleus of the zygote Weismann has given the name amphimixis, and he sees in this mingling of ancestral germ plasms the cause of that mixture of paternal and maternal characters which we commonly find in animals and plants. If, for the sake of simplicity, we imagine that each chromosome consists (as appears to be sometimes the case) of only a single id or chromomere, and that eight of these are present in the nucleus of the mature germ cell, we may represent the effect of repeated amphimixis upon the constitution of the nucleus by means of the diagram (Fig. 78), which shows how the ids must become more and more diversified in each successive generation. In this 
diagram A represents an unreduced nucleus composed of sixteen ids, eight paternal and eight maternal, all the paternal and all the maternal ids respectively being supposed to be alike. After reduction and union with another mature germ cell containing also only one kind each of paternal and maternal ids, but both differing in some respect from those of its mate, the nucleus of the next generation will contain four kinds of ids, two paternal
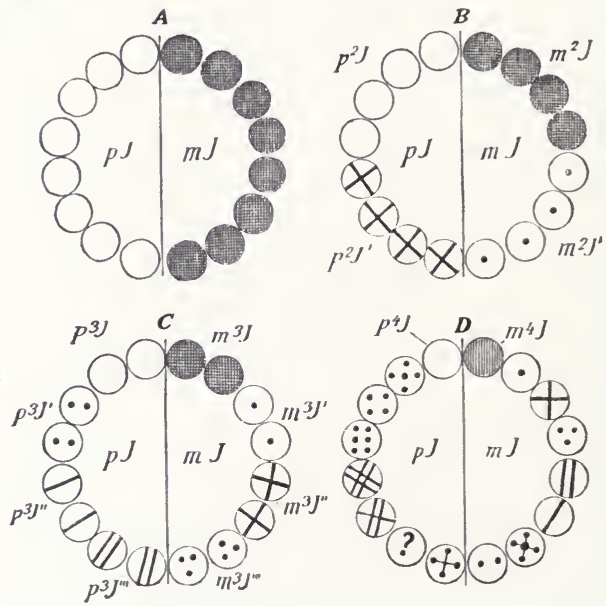

F19. 78.-Diagram illustrating the Composition of the Germ Plasm (Chromatin Substance of the Nucleus) out of Ancestral Ids, and the Effect thereon of repeated Amphimixis. (From Weismann's " Evolution 'Theory.")

$A-D$ the uureduced nucleus, containing sixteen chromosomes (each consisting of a single id), of four snccessive generations. In $A$ the germ plasm consists of only two kinds of ids; in $B$ of four; in $C$ of eight, and in $D$ of sixteen. $m J, p J$, maternal and paternal ids.

and two maternal, as shown in $\mathrm{B}$, while in the next generation there may be eight kinds, as shown in $\mathrm{C}$, and in the next sixteen, as in D.

We must, then, in accordance with the views of Weismann, look upon the germ plasm of any one of the higher organisms as being made up of a larger or smaller number of ids, each one representing the inheritance received from some more or less remote ancestor on the paternal or maternal side, though of course 
it is quite possible that there may be a number of ids of the same kind. When, during the process of maturation, half the total number of chromosomes (each made up of one or more ids) are eliminated from the germ cell, it appears to be largely a matter of chance which shall go and which shall remain, and the nature of the new combination of ids resulting from the process of amphimixis must also be a matter of chance, depending upon what luck the germ cells happen to have in their mating.

As has been well said, a new shuffling of the cards must take place in each generation. The characters of the organism developed from any zygote will depend upon the hand dealt out to it in the processes of reduction and amphimixis, and as it can rarely, if ever, happen that any two hands will be exactly alike, so it will rarely, if ever, happen that any two organisms, however closely related, will exactly resemble one another in all their characters. Indeed, the only cases in which even an approximation to exact resemblance is known, at any rate amongst the higher animals, are those of "identical" twins, and the explanation of these is that they have arisen by an integral division of a single fertilized ovum, followed by separation of the first two daughter cells or blastomeres thus produced.

We therefore find in the processes of reduction and amphimixis, in the permutation and combination of ancestral characters, an abundant source of variation. This, however, is not supposed to explain fully the origin of variations, and Weismann accordingly invokes the aid of another hypothesis, his theory of "Germinal Selection." In accordance with this theory the determinants of which the ids are composed are supposed to be differently situated with regard to their facilities for obtaining the nutriment necessary for their growth and multiplication. There is a kind of struggle for existence going on amongst them. Those which are more successful in obtaining supplies, having once got a start, will tend to supplant those which are less successful. Some will become weaker and some stronger, and thus, as the result of differences in nutrition, variation is set up amongst the determinants themselves. If these vary it follows that their determinates, or the parts which they control in the developing organism, will vary also. ${ }^{1}$

1 There would seem, however, to be a serious objection to the theory of germinal selection in the fact that the nuclous of any given germ cell contains many ids, and that similar determinants must as a rule recur in each id. We ean hardly suppose that the corresponding determinants in each id are always subject to precisely the 
In this way Weismann seeks to avoid the necessity of believing in the transmission from parent to offspring of modifications which result from the direct action of the environment upon the body itself, without, however, altogether denying that external influences, and especially nutrition, may act upon the germ plasm through the body and thus cause modifications in the offspring.

We have seen that Weismann's theory of the continuity of the germ plasin involves the acceptance of the chromatin of the nucleus as the actual material basis of hereditary transmission. 'There can be no doubt that the phenomena of ordinary mitosis in the case of tissue cells, and those of maturation and fertilization in the case of the germ cells, strongly support this view, while the simple fact that the spermatozoon, consisting almost entirely of chromatin substance and with a minimum of cytoplasm, contributes equally with the ovum to the characters of the offspring in normal cases, seems almost conclusive as to the predominating importance of the chromatin in this respect. Some observers, however, still maintain that the cytoplasm plays a very important part in heredity.

It is probable that much light will be thrown upon this question by the development of that extremely important branch of biological science known as experimental embryology, which is as yet in its infancy. We have already pointed out that in certain cases eggs containing no nucleus may be fertilized by spermatozoa, and may then develop up to a certain point, and that this process is termed merogony. Some years ago Boveri claimed to have fertilized enucleate fragments of the eggs of one genus of seaurchins (Sphrechinus) with the sperm of another genus (Echinus), and obtained larvæ with only paternal characters. He concluded from this experiment that the nuclear substance is alone responsible for the transmission of inherited characters. Unfortunately it seems that his results are open to a different interpretation, and they have been severely criticized. They certainly cannot be regarded as by any means conclusive.

More recently Godlewski has succeeded in fertilizing eggs of the common sea-urchin (Echinus) with sperm of the feather star (Antedon), belonging not only to a distinct genus but to a widely

same advantages or disadvantages of position. It seems nuch more likely that variations in this respect in different ids would tend to neutralize one another, the kind of determinant which is unfavourably sitnated in one id being favonrably situated in another, so that each kind wonld, on an average, have the same chance of nutrition. 
different order of echinoderms. The larvæ of these two types are easily distinguished even in early stages of development. The larvæ produced by fertilization of normal eggs of Echinus with sperm of Antedon are said to be of the maternal type. This result is in itself very remarkable, but Godlewski was also able to fertilize enucleate egg fragments of the sea-urchin with sperm of the feather star. Eight larve produced in this way reached the blastula stage, but only four developed as far as the gastrula. These four, however, were again of the maternal type, and could only be distinguished by their size from those of the pure Echinus culture. From these experiments Godlewski concludes that cytoplasm as well as chromatin must be concerned in the transmission of hereditary characters, for no maternal chromatin was present in the eggs from which the larvæ developed. Whether these results, which are in direct opposition to those of Boveri, will be confirmed or refuted by further observations remains to be seen. IValker, in his recent work on heredity, ${ }^{1}$ has accepted Godlewski's conclusions and made use of them in support of the theory that the chromosomes are the bearers of individual characters only, while racial characters may be transmitted by " the whole protoplasm of the cell."

1 "Hereditary Characters and their Modes of Transmission," by C. E. Walker (London, Edward Amold, 1910). 


\section{CHAPTER XIII}

The inheritance of acquired characters and the mnemic theory of heredity.

No biological question during the last fifty years has given rise to more acute and vigorous controversy than that of the inheritance or non-inheritance of " acquired" characters. The following paragraph may be quoted to show the manner in which Weismann stated the case and endeavoured to give precision to the terminology employed:-

"By acquired characters I mean those which are not preformed in the germ, but which arise only through special influences affecting the body or individual parts of it. They are due to the reaction of these parts to any external influences apart from the necessary conditions for development. I have called them 'somatogenic' characters, because they are produced by the reaction of the body or soma, and I contrast them with the 'blastoyenic' characters of an individual, or those which originate solely in the primary constituents of the germ ('Keimesanlagen '). It is an inevitable consequence of the theory of the germ-plasm, and of its present elaboration and extension so as to include the doctrine of determinants, that somatogenic variations are not transmissible, and that consequently every permanent variation proceeds from the germ, in which it must be represented by a modification of the primary constituents." 1

As already pointed out, the view here expressed is directly opposed to that of Lamarck and Darwin, who believed that characters acquired in the life-time of the individual, either as a result of the use or disuse of organs or of the direct action of the environment, might be handed on by heredity from parent to offspring, and Darwin's theory of pangenesis was essentially an endeavour to imagine some mechanism by which such transference of acquired characters might be effected.

We may at once agree with Weismann that blastogenic characters alone are transmitted from parent to offspring, but the real question is-Can a somatogenic character be converted into,

1 "The Germ-Plasm: A Theory of Heredity," by August Weismann (Contemporary Scienee Series, 1893), 1. 392. 
or give rise to, a blastogenic one? In other words-Can a modification of the body or soma, arising in the life-time of the individual and itself in no way due to inheritance, affect the germ cells in such a way that the offspring developed from them will exhibit a corresponding modification of its soma?

It is useless either to deny or to assert the possibility of the inheritance of such characters on any purely a priori grounds. The fact that no satisfactory mechanism for the transference of such characters from parent to offspring has yet been demonstrated does not justify us in denying the possibility of such transference. Our decision must depend upon an unbiassed examination of the evidence which can be brought forward on each side.

It is, of course, obvious that inasmuch as any organism differs to a greater or less extent from its ancestors, the differences being as a general rule greater in proportion to the remoteness of the particular ancestor with which it is compared, the differentiating characters must have been acquired, in the ordinary sense of the word, during the interval which separates the two generations in question. For example, there can be no reasonable doubt that birds are descended from ancestors which were reptilian in character and had no feathers. Feathers have unquestionably been acquired somehow or other during the progress of the bird's evolution. This, however, is not the sort of acquisition the inheritance of which is in dispute. Weismann and his followers would deny altogether that feathers originated as somatogenic characters; they would say that certain apparently fortuitous modifications in the constitution of the germ cells themselves were responsible for the first appearance of feathersprobably in an extremely rudimentary form-and that this new character proving to be of value in the struggle for existence was preserved and fostered by natural selection until after a long process of evolution the elaborate plumage of existing birds was perfected.

In striking contrast to such a case as the above we have innumerable cases of the more or less sudden appearance of somatic characters during the life-time of an individual as the obvious result of the action of some external or environmental influence, or of the use or disuse of some organ by its possessor, and it is to such cases that Weismann and his followers would, rightly or wrongly, confine the discussion. 
Artificially or accidentally produced mutilations afford a very good example of such obviously somatogenic characters, and with regard to the inheritance of these Weismann's position is clearly summed up in the following passage:- "As far back as the eighteenth century the great philosopher Kant, and in our own day the anatomist Wilhelm His, gave their verdict decidedly against such allegations, and absolutely denied any inheritance of mutilations; and now, after a decade or more of lively debate over the pros and cons, combined with detailed anatomical investigations, careful testing of individual cases, and experiment, we are in a position to give a decided negative and say there is no inheritance of mutilations." I It is, however, as already pointed out, all a question of evidence, and we may here quote a definite case in order to show the nature of the evidence with which we have to deal :-

"A female (and very prolific) cat, when about half-grown, met with an accident. "Her fine, long tail was trodden on and had a compound fracture, two vertebræ being so displaced that they ever after formed a short offset between the near and far end of the tail, leaving the two out of line. At first I noticed that out of every litter of kittens some had a tail with a querl in it.' With successive litters the deformity increased, until ' not a litten of the old cat had a straight tail, and it grew worse in her progeny, until now we have not a cat with a normal tail on the premises, (in a cat-population of six or eight, exclusive of young kittens). 'The tails are now in part mere stumps, some have a semicircular sweep sideways, and some have the original querl. Perhaps the deformity was somewhat aggravated by in-and-in breeding and by artificial selection practised by my Chinaman, who, with the perversity of his race, preferred the crooked tails, and thus preserved them in preference to the normal kittens. There are no other abnormally-tailed cats in the neighbourhood." "

Professor Brewer quotes this remarkable case ${ }^{2}$ from "that keen observer and eminent scientist, Professor Eugene W. Hilgard of the University of California," along with others of a like kind from various sources.

It is of course essential to the stability of Weismann's theory as a whole that evidence such as this should be rebutted. I am not aware that he has anywhere criticized this particular case, but his general remárks in somewhat similar cases may be quoted with advantage in this connection:-

\footnotetext{
1 "The Evolution Theory" (London, 1904), Vol. II., p. 65.

2 Vide Cope's "Primary Factors of Organic Evolution" (Chicago, 1896), pp. 432-3.
} 
"In the first. place, the assertion that congenital stump-tails in dogs and eats depended on inherited mutilation proved to be unfounded. In none of the cases of stump-tails brought forward could it even be proved that the tail of the relevant parent had been torn or cut off, much less that the occurrence, in parents or grandparents, of short tails from internal causes was excluded. At the same time anatomical investigation of such stump-tails as occur in cats in the Isle of Man, and in many Japanese cats, and are frequently found in the most diverse breeds of dogs, showed that these had, in their structure, nothing in common with the remains of a tail that had been cut off, but were spontaneois degenerations of the whole tail, and are thus deformed tails, not shortened ones (Bonnet).

"Experiments on mice also showed that the cutting off of the tail, even when performed on both parents, does not bring about the slightest diminution in the length of tail in the descendants. I have myself instituted experiments of this kind, and carried them out through twenty-two successive generations, without any positive result. Corroborative results of these experiments on mice have been communicated by Ritzema Bos and, independently, by Rosenthal, and a corresponding series of experiments on rats, which these two investigators carried out, yielded the same negative results.

"When we remember that all the cases which have been brought forward in support of an inheritance of mutilations refer to a single injury to one parent, while, in the experiments, the same mutilation was inflicted on both parents through numerous generations, we must regard these experiments as a proof that all earlier statements were based either on a fallacy or on fortuitous coincidence. This conclusion is confirmed by all that we know otherwise of the effects of oft-repeated mutilations, as for instance the well-known mutilations and distortions which many peoples have practised for long, sometimes inconceivably long, ages on their children, especially circumeision, the breaking of the incisors, the boring of holes in lip, ear, or nose, and so forth. No child of any of these races bas ever been brought into the world with one of these marks ; they have to be re-impressed on every generation." 1

It cannot be seriously questioned that in the majority of cases mutilations are not visibly inherited, but the fact that no one has as yet succeeded in producing experimentally an inheritable mutilation does not prove that such never occur accidentally.

Weismann's arguments can hardly be regarded as conclusive against such strong evidence as that afforded by Professor Hilgard's

1 Weismann, "The Evolution Theory" (London, 1904), Vol. II., pp. 65-6. 
cats. Moreover, there appears to be considerable difference of opinion as to whether or not the effects of circumcision are ever inherited. Darwin, in discussing this point,' admits that it is possible that all the recorded cases of apparent inheritance of such effects may be accidental coincidences, but the fact that there are such recorded cases prevents us from accepting the sweeping generalization which Weismann makes in the last sentence of the paragraph above quoted.

Darwin himself was convinced that the effects of operations are sometimes inherited by the well known and often quoted case of Dr. Brown-Séquard's guinea-pigs. In these animals not only did epilepsy appear in the offspring of parents which had been rendered epileptic by injury to the nervous system, but certain structural modifications (e.g. loss of toes) appeared in the offspring when corresponding structural modifications had been produced in the parent (indirectly) by similar injury. Weismamn, however, endeavours very ingeniously to explain away these observations, and the correctness of Brown-Séquard's results has been seriously impugned.

It cannot be denied, however, that the primî facie evidence for the occasional transmission of such characters as those produced by mutilation is very strong. We may allow much for coincidence, but well authenticated cases like that of the cats above referred to, and like the following, are too numerous to be all explained away in this manner :-

"A person, when a boy of ten years, cut the terminal phalange of the little finger of his left hand with a sickle. The joint was not injured, nor was the function of the finger seriously impaired. There was, however, an obvious deformity. The finger was ill-shaped and crooked, and the nail abnormal. He married and had two children, the first a son, with normal fingers, the second a daughter, who had the little finger of the corresponding (the left) hand deformed from birth in the same manner. The function of the finger was not seriously injured, but the deformity was precisely the same in shape, even to the malformation of the finger-nail. She died at thirty, without children, consequently no observation on a succeeding generation could be noted. None of his other kindred had malformed fingers, nor had any ancestor of the child for at least three generations, and there was no knowledge of any such in the more remote ancestry.

1 "Auimals and Plants under Domestication" (2nd Ed., 1882), Vol. I., p. 467. 
" (This case was related to me in full detail by the father with the deformed finger, and with whom I was personally acquainted. He was an eminent physician, the president of a large and reputable medical college, and his name is well known to the profession.)" 1

It seems only reasonable, then, to admit that suddenly acquired somatogenic characters are occasionally, though probably very rarely, transmitted by heredity in a sufficiently pronounced form to be recognizable, but even if this were not so we should not be justified in eoncluding that all the characters which an organism exhibits have their origin in the first instance in modifications of the germ plasm. It must be remembered that a great many somatogenic modifications are the result of what we may call the normal action of the environment, and that such action may extend over very long periods of time, in which many thousands of generations may be produced. In such cases the stimulus, whatever it may be, to which the organism responds by modification in bodily structure, is repeated a vast number of times, and therefore seems much more likely to produce an inheritable effect than a single accidental or experimental injury. A single drop of water falling on a stone makes no visible impression, but if the dropping goes on for a long time the stone will gradually be worn away, and it is by no means essential to a belief in the heritability of acquired characters that such eharacters should be immediately inherited in full perfection. It may be merely a question of time.

It is a well known fact that the "habit" and even the structure of a plant are largely determined by the conditions under which its growth takes place. ${ }^{2}$ A plant growing in a hothouse may acquire a very different habit from another of the same species growing in the open air. Many alpine or sub-alpine plants have become adapted to their peculiar environment by various highly characteristic modifications, amongst which a reduction in the size of the leaves is one of the most conspicuous, and such plants may be induced to change their mode of growth by simply removing them to sufficiently warm and sheltered situations, in which their habit comes to approach that of their lowland relatives. In such cases it is difficult to say how far

1 This is another of the striking cases collecterl by I'rofessor Brewer and quoted in Cope's "Primary Factors of Organic Evolution," pp. 433-4.

2 The reader should consult the quotations from Lamarck bearing upon this point given in Chapter XXIV., especially the case of Ranuncu'us uquatilis. 
the habit of the plant, whether alpine or lowland, is really inherited, or how far it may be produced afresh in each generation as a direct response to environmental stimuli.

It appears, however, from the observations of Bordage, ${ }^{1}$ that peach trees in the climate of Reunion gradually acquire an almost evergreen habit, and that this character is hereditarily transmitted, being exhibited by seedlings of the modified trees when grown in situations where the peach is usually deciduous. Dr. Bordage considers, and it appears to us that he has good reason for so doing, that his observations and experiments definitely prove, in the case of plants, the hereditary transmission of characters acquired under the influence of a change of climate.

To take another example, it has been shown that in rats and mice certain modifications in bodily structure are produced as the result of raising or lowering the temperature to which the young animal is exposed during its growth, and such modifications appear to be inherited. Sumner ${ }^{2}$ found, as the result of a large number of careful measurements, that mice reared in a warm room (about $21^{\circ}$ C.) differed considerably from those reared in a cold room (about $5^{\circ} \mathrm{C}$.) as regards the mean length of the tail, foot and ear, which were longer in the former than in the latter. The same differences occurred to a recognizable extent in the offspring of the warm room and cold room parents, although these offspring were all reared together in a common room under identical temperature conditions.

Observations such as these, which are rapidly accumulating, lead us to hope that the question of the inheritance or noninheritance of somatogenic characters which have undoubtedly arisen in response to the direct action of the environment may, before long, be answered conclusively. They doubtless require confirmation and extension, but they afford very strong evidence in favour of the inheritance of such characters. It has been suggested that in such cases the stimulus of changed conditions affects the body and the germ cells simultaneously and in a parallel manner, rather than that the body is modified first and then influences the germ cells, but such a suggestion seems like a last attempt to avoid at all costs the necessity for believing in the

1 Edmond Borlage, "A propos de l'hérédité des caractìres acquis" (Bulletin Scientitique de la France et de la Belgique. Tome XLIV, Paris, 1910).

${ }^{2}$ Francis B. Sumwer, "An Experinental Study of Somatic Morlitications and their Reappearance in the Offspring," (Archiv für Entwicklungsmechanik der Organismen, Bd. 30,1910 ). 
transmission of "acquired" characters, and is quite as difficult to accept as the latter, while, as Sumner points out, it does not affect the question of the importance of the environment in determining the course of evolution.

Professor Henslow ${ }^{1}$ believes that the direct action of the environment, in its widest sense, coupled with the responsive power of protoplasm, is the sole and efficient cause of adaptive variations in plants, without any aid from natural selection. This of course implies a firm belief in the inheritance of somatogenic modifications. Although these may be acquired slowly throughout the course of a long series of successive generations, they may become gradually more and more fixed and permanent, until finally they attain a degree of stability which entitles them to be considered as truly blastogenic.

Professor Eigenmann ${ }^{2}$ has arrived at very similar conclusions as a result of his careful study of animals which live in dark caves. A very characteristic feature of such animals is the bleaching which they undergo, due to the loss of pigment. Professor Eigenmann regards this character as due in the first place to the direct influence of the environment (i.e. the absence of light) upon the individual. He tells us "The bleached condition of animals living in the dark, an individual environmental adaptation, is transmissible, and finally becomes hereditarily fixed." In Amblyopsis, one of the blind eave fishes, the fixation of this character has become so complete that even when the young are reared in the light they develop without pigment-the originally somatogenic character has apparently become blastogenic. In the well known European Proteus - a tailed amphibian which lives in subterranean waters-on the other hand, it appears that the bleached condition has not yet become hereditarily established, for this animal becomes darker when exposed to the light. Professor Eigenmann points out that " natural selection cannot have affected the coloration of the cave forms, for it can be of no consequence whether a cave species is white or black."

We do not know how many generations it may take to effect the fixation of a character acquired slowly under the influence of a constantly repeated or continuous environmental stimulus, but the fact that human beings have not yet learned to speak

1 "Origin of Plant Structures" (International Scientific Series, Vol. I.XXVII., $1895)$.

2 "Cave Vertebrates of America. A Study in Degencrative Evolution" (Washington : Carnegic Institution, 1909). 
without being taught shows that the number may, in some cases at any rate, be very large. Speech, however, is a comparatively recent acquisition in the human race and many of the higher animals perform actions without being taught which their ancestors may very well have originally learnt to perform by observation or experience. It would be extremely difficult to explain the nest-building habits of lirds, and other so-called "instincts," except as having been originally acquired in this way in the life-time of the individual.

On the whole, then, the available evidence seems to indicate that suddenly and exceptionally acquired characters, such as mutilations, are occasionally but only rarely inherited to such an extent as to be recognizable, while, on the other hand, characters which are due to the continued action of some external stimulus, extending perhaps over many generations, in the long run become so firmly impressed upon the organism that they affect the germ cells as well as the somatic cells and thus become truly blastogenic.

We must look upon the germ cells as highly conservative bodies, possessed of great inertia, which can only be induced very slowly in normal circumstances, or by some sudden revolution, of the nature of which we know nothing, in abnormal circumstances, to alter their constitution. This conservatism is no doubt of great value as a check upon the inheritance of accidental and unfavourable modifications. The adaptation of the individual to its particular environment is to a large extent provided for by somatogenic modifications which arise in its own life-time. What suits one individual, however, may not suit its successors, which may have to live under more or less widely different conditions, and for progressive evolution what is needed is an average adaptation of the race to an average environment. Hence the germ plasm in its evolution lags behind the soma and is only very slowly impressed with those characters which experience has shown to be of permanent value to the race. It is a kind of second chamber which acts as a useful check upon too radical tendencies.

That the germ plasm is to some extent capable of modification by environmental stimuli, however, is clearly shown by the experiments of Tower referred to in Chapter XI., but it is extremely difticult to see how such stimuli as he mentions could affect it except by acting primarily upon the soma in which the germ cells are enclosed. Then the soma might be modified in response to the 
stimulus in the first instance, and this modification, whatever its nature (and it might of course be quite unrecognizable by us), might affect the germ cells - in which case the germ cells could not be entirely shut off from the influence of the soma.

The chief obstacle in the way of our belief in the inheritance of acquired characters lies, as we have already seen, in the difficulty of imagining any mechanism adequate to bring about the conversion of somatogenic into blastogenic modifications. If, as Weismann insists, the germ cells are really incapable of being influenced by the body, the difficulty does indeed seem insurmountable; but there seems to be no valid reason why we should follow Weismann in this respect. Nor does it seem necessary that we should adopt any theory which postulates the migration of material particles, such as the gemmules of Darwin's pangenesis, in order to get over the difficulty.

Herbert Spencer long ago indicated the direction in which the solution of this problem must be sought. "It is," he says, "an unquestionable deduction from the persistence of force, that in every individual organism each new incident force must work its equivalent of change; and that where it is a constant or recurrent force, the limit of the change it works must be an adaptation of structure such as opposes to the new outer force an equal inner force. The only thing open to question is, whether such re-adjustment is inheritable; and further consideration will, I think, show, that to say it is not inheritable is indirectly to say that force does not persist. If all parts of an organism have their functions co-ordinated into a moving equilibrium, such that every part perpetually influences all other parts, and cannot be changed without initiating changes in all other parts-if the limit of change is the establishment of a complete harmony among the movements, molecular and other, of all parts; then among other parts that are modified, molecularly or otherwise, must be those which cast off the germs of new organisms. The molecules of their produced germs must tend ever to conform the motions of their components, and therefore the arrangements of their components, to the molecular forces of the organism as a whole; and if this aggregate of molecular forces is modified in its distribution by a local change of structure, the molecules of the germs must be gradually changed in the motions and arrangements of their components, until they are re-adjusted to the aggregate of molecular forces. For to hold that the moving equilibrium of an 
organism may be altered without altering the movements going on in a particular part of it, is to hold that these movements will not be affected by the altered distribution of forces; and to hold this is to deny the persistence of force." 1

In other words, the whole complex system of forces which determines the constitution of the germ cells must be in a state of equilibrium with the system of forces which determines the constitution of the body, and any disturbance in the latter must be met by a corresponding disturbance in the former. The same idea may be expressed by saying that modifications of the soma act as stimuli, which make more or less permanent impressions or "engrams" upon the germ cells in much the same way that stimuli of various kinds received through the sense organs make impressions upon certain cells in the brain. In the case of the brain cells the original impressions may become dormant and be revived later on as memories, which are a kind of reproduction of the phenomena to which the impressions were originally due. In the case of the germ cells it is supposed that the engrams also become dormant, but are aroused to activity again in the course of the development of these cells into new organisms, exerting their influence in such a manner as to bring about a kind of reflection, in the body of the offspring, of the parental characters. This is the central idea of the "Mnemic" 2 theory of heredity, associated more especially with the names of Hering, Samuel Butler $^{3}$ and Semon. ${ }^{4}$

According to some authorities it is through a continuity of protoplasm from cell to cell, or especially through the nervous system in the case of the higher animals, that stimuli are transmitted from the soma to the germ cells, but the assumption of any definite material paths for such transmission seems an altogether superfluous encumbrance of the theory.

It has long been known that it is possible to send messages, or if we like to call them so, stimuli, from one place to another without any material means of communication. The very existence of life on the earth depends upon stimuli received in the form of

1 "Prineiples of Biology" (London, 188t), Vol. II., p. 387.

2 From the Greek $\mu \nu \eta \mu \eta$, memory.

"See Butler's "Life and Habit" (2nd Edition, London, 1878) and "Unconscious Memory" (New Edition, London, 1910), the latter of which contains a translation of Hering's lecture "On Memory as a Universal Function of Organized Matter."

4 Semon, "Die Mneme als erlaltendes Prinzip im Wechsel des organisehen Geschehens," 3rd Ed., Leipzig, 1911. 
light and heat from the sun across an intervening space of almost inconceivable extent which is supposed to contain nothing but the hypothetical and intangible ether. To account for such transmission we postulate the occurrence of vibrations or undulations in this ether, and the truth of this theory may now be regarded as conclusively demonstrated. The stimulation of the sensory cells of the retina by certain of these vibrations gives rise, when transmitted along the optic nerve to certain cells in the brain, to the sensation which we recognize as light, while other vibrations are perceived by us as heat.

The rapid development of wireless telegraphy in recent years has familiarized us with another type of vibration in the ether, by taking advantage of which we are able to send messages over immense distances from one instrument to another, without the aid of wires or any other material connections. The Röntgen rays, again, can make impressions upon sensitive photographic plates after passing through solid objects which are quite impervious to the ordinary light vibrations.

In view of these facts it seems absurd to deny that the living cells of the soma, in which doubtless complex vibrations, possibly comparable to those which are responsible for the phenomena of light and electricity, are constantly going on, may conceivably influence the germ cells without our being able to demonstrate the existence of material connections by which the necessary stimuli might be transmitted.

The different means of communication between one cell and another in the living body may be compared to the different methods by which messages are transmitted between distant members of a civilized human community. The circulation of fluids (such as the blood) distributes throughout the body definite substances secreted by certain cells, which may act as stimulants upon other and far distant cells. We may compare this to the transmission of messages by letter post. The nervous system of the higher animals, and probably also the delicate threads of protoplasm which so frequently connect one cell with another both in animals and plants, provide a means of communication which is closely comparable to ordinary telegraphy over conducting wires. Why should we deny the possibility that a third means of communication, analogous to wireless telegraphy, may also exist?

Darwin's hypothesis of pangenesis depends upon the 
existence of the first of these means of communication. So also does the modern theory of hormones, chemical substances which are believed to exercise a most important controlling influence over parts of the body that may be far remote from the place where they themselves are secreted, ${ }^{1}$ and the aid of which has also been invoked to account for the phenomena of heredity. Others, again, as we have seen, have called in the aid of the second means of communication to account for the transmission of stimuli from somatic to germ cells, but their view is hardly supported by what we know of the arrangement of the nervous system throughout the animal kingdom, and the difficulty of accepting it is still greater in the case of plants, in which no definite nervous system is developed. The third method of communication, however, seems open to no objection, and, whether it may be supplemented by the others or not, seems amply sufficient to account for the facts.

The faculty of receiving and responding to stimuli of various kinds is one of the most characteristic features of living protoplasm. In the higher animals this function is more or less concentrated in definite sense cells, each of which is adapted for the reception of stimuli of one particular kind. The visual sense cells are adapted for the reception of stimuli from the light-vibrations of the ether, the auditory sense cells are stimulated by vibrations of the air or water in which the animal lives, the olfactory sense cells are stimulated by the chemical action of material particles, and the tactile cells by the mechanical stimuli of contact and pressure. In such an organism as the unicellular Amceba, on the other hand, all parts of the superficial protoplasm are probably sensitive to stimuli and no special organs of sense are developed. We may well suppose, therefore, that the undifferentiated germ cells of the multicellular animals and plants are sensitive to stimuli received from the somatic cells, though it is impossible in the present state of our knowledge to determine the nature of these.

It is not difficult to demonstrate that one cell may actually be stimulated by another without the existence of any protoplasmic connection between the two, as, for example, in the mutual attraction of male and female gametes. Such stimulation, it is true, is usually attributed, mainly at any rate, to the secretion of some specific chemical substance which diffuses into the surrounding water, and classed accordingly under the head of 
chemotaxis. In the case of Spirogyra, however, discussed in Chapter $\mathrm{X},{ }^{1}$ it certainly seems as if chemotaxis were not sufficient to account for the phenomena.

Belief in the possibility of the transmission of acquired characters from somatic to germ cells by no means obliges us to throw over Weismann's theory of determinants. Indeed it seems necessary to postulate the existence in the germ plasm of some such material primordia as the responsible agents in the transmission of heritable characters in order to account for the Mendelian phenomena of hybridism, and especially the existence of interchangeable unit characters, which will be dealt with in the following chapter. Moreover, the theory of determinants, as we have already seen, harmonizes very well with what we know of the microscopic structure of the germ plasm and its behaviour in cell-division and conjugation.

It may be that it is the determinants themselves that are influenced by stimuli received from the somatic cells. It is even possible that each different kind of determinant is "tuned" to respond to vibrations of a particular wave-length, emanating from corresponding determinants in the nuclei of somatic cells of a particular kind. Modifications of these somatic cells may then, by altering the character of the vibrations in the determinants of their own nuclei, affect the corresponding determinants in the distant germ cells in a similar manner. By means of some such hypothesis as this, which would be strictly in accordance with modern physical ideas on the subject of the transmission of electrical energy from one group of electrons to another at a distance, any a priori difficulty in accepting the possibility of the inheritance of acquired characters might be removed.

The case of neuter insects has frequently been adduced as a serious stumbling block in the way of the theory of the origin of permanent, heritable characteris from somatogenic modifications, for these neuters possess features of their own which are not shared by the males and perfect females and which, as the neuters do not themselves produce offspring, they cannot transmit directly to future generations. According to Weismann's theory these characters are supposed to result from fortuitous favourable variations of the germ plasm of the parents, which are accumulated and intensified under the influence of natural

1 Pp. 142-3. I have perhaps lairl too much stress upon this case; chemotaxis may have more to do with it than appears at first sight. 
selection, the welfare of the insect community as a whole, rather than that of its constituent individuals, being in this case the determining condition through which natural selection operates. The case of such communities, however, is exactly analogous to that of individuals, except that the single cells are replaced by complete and separate multicellular units. The whole community may be looked upon as one individual of a higher order, and the problem of the transference of stimuli from the bodies of the neuters to the germ cells of the perfect insects differs in no essential respect from that of the transference of similar stimuli from somatic cells to germ cells in an ordinary individual. The close association in which members of such communities live may be supposed to facilitate the transfer of such stimuli (arising from modification of the somatic cells of the neuters in response to the environment) to the germ cells of the males and perfect females, without the aid of material conductors.

In the present state of our knowledge, of course, any suggestions which may be put forward on this subject must be regarded as mere hypotheses, incapable of demonstration, and as such they will doubtless appear to many people to be unwarrantable. If, however, they serve to show that there is no a priori reason for denying the possibility of the transmission of somatogenic characters to the germ cells, and thence to future generations, they will serve a useful purpose.

We must, however, again point out that, under normal circumstances, it probably takes many generations before any impression produced by modification of the soma upon the germ cells becomes so deeply ingrained as to find full expression in the offspring produced by their development. The impression, once made, appears to be equally difficult to remove, and hence has probably arisen that conservatism or inertia of the germ cells to which we have already alluded. Occasionally, however, it appears that a suddenly acquired somatogenic character at once makes a deep impression upon the germ cells. Why this should be so we do not know, but it is perhaps no more remarkable than the well known fact that the germ cells themselves may occasionally throw aside their conservatism and give rise to sports or mutations. ${ }^{1}$

It is well known that, apart altogether from the highly specialized brain cells, living protoplasm frequently has the power

1 It is possible, however, that the suddenness of a mutation is apparent rather than real, at any rate in some cases; compare p. 156. 
not only of receiving and responding inmediately to stimuli of various kinds, but also of storing up impressions or engrams for future use. This is demonstrated quite clearly by the phenomena known as "after-effects" in various plants and animals. One of the best known examples of such after-effects is seen in the daily periodicity of plant growth. The light of the sun acts as a restraining or inhibiting stimulus upon the rate of growth of ordinary plants. In consequence of this the plant grows most rapidly in the early hours of the morning after a prolonged exposure to darkness, and most slowly in the afternoon, after long exposure to daylight. It has been shown that this daily periodicity, or variation in rate of growth in correspondence with the periodic variation in environment, is continued when the plant is kept in perpetual darkness, and the direct stimulation of changing environment thereby rendered impossible. In other words, the plant, though entirely devoid of any nervous system in the ordinary sense of the term, establishes a habit, which must depend upon something analogous to memory on its part.

The mnemic theory of heredity is based, as we have seen, upon a comparison of the phenomena of inheritance with those of memory. The latter are reasonably explained by supposing that impressions received by certain cells of the brain may be stored up as "engrams", in these cells for use on future occasions, when, under appropriate stimulation, they give rise to mental conditions corresponding to those produced by the original stimuli. The stimulus which evokes a memory is commonly the repetition of some stimulus which was originally associated with the thing remembered. Thus the sight of a person whom we have not seen for a long time, or even of his portrait, will evoke a whole train of memories connected with that person.

The degree of accuracy with which we remember any occurrence depends partly upon the nature of the occurrence itself and partly upon the frequency with which it has been brought under our notice. The first time we take a walk we may have to pay attention to every turning and every signpost in order to find our way at all, but if we take the same walk many times we at length become so familiar with it that we may be thinking of other things the whole time and not consciously notice a single landmark from start to finish. As Samuel Butler has most forcibly pointed out, the more perfect memory becomes the less is it accompanied by consciousness of the things remembered, as when 
a skilled musician, playing a composition for perhaps the hundredth time, is quite unconscious of the individual actions which he performs by memory in striking the notes, while a beginner, who has only played the same piece once or twice before, may be acutely conscious of every note which he strikes. The development of an individual organism from the egg is, according to the mnemic theory, merely an unconscious repetition, by memory, of acts which have been performed many thousands of times by its ancestors, and, as in the performance of a piece of music, each successive act constitutes the stimulus which calls forth the next.

The fertilized ovum may be looked upon as being charged with the latent memories of past generations. The unconscious memory of what it did last time it was a fertilized ovum prompts it to divide into two cells, this re-arrangement of its constituents supplies another stimulus which prompts it to a further division, and so on through all the stages of ontogenetic development. In the words of Professor Francis Darwin, "the rhythm of ontogeny is actually and literally a habit." 1

Progressive evolution takes place owing to the fact that each successive generation may add a little bit to the record on its own account, this addition being the result of some new experience due to some difference in its environment as compared with the environment of its predecessors.

There are, then, two distinct sets of factors which determine the individual development of any organism, first the inherited tendencies, or " engrams," and second the stimuli provided by the environment during its own life-time. An egg placed under very unusual conditions, as, for example, too low or too high a temperature, may be unable to develop at all, but if the change of conditions be but slight the organism may adapt itself, educate itself and furnish itself with a new store of experiences, which in course of time may be impressed upon the germ plasm and thus added to the record and handed on to future generations.

We have already had occasion to notice, in the case of artificially produced cyclopean fish larvæ, how an alteration of the environment not sufficient altogether to prevent development may so far overcome the inherited tendencies of the organism as to give rise to the production of monstrosities (p. 157).

1 Presidential Address to the British Association, Dublin, 1908. 
Such cases clearly prove the immense influence of the environment in determining the character of the organism. If we admit that inherited tendencies also owe their origin in the first instance to the action of the environment on previous generations, it is difficult to avoid the conclusion that every organism is ultimately indebted for all its characters to the action of external stimuli upon responsive protoplasm. 


\section{CHAPTER XIV}

The Mendelian experiments in hybridization - The doctrine of unit characters and the purity of the gametes-Galton's law of inheritance.

Whatever view we may adopt as to the mechanism of heredity there can be no doubt as to the existence of a material foundation for the transmission of characters from parent to offspring. We know that the protoplasm which forms the material basis of all living things is continuous, by means of the germ cells or gametes, in an unbroken stream from one generation to another. At each conjugation of gametes, however, two such streams are mingled, and the offspring receives part of its initial stock of protoplasm from one parent and part from the other, each part bringing with it all the potentialities which may have been derived from a long line of ancestors. Bearing these facts in mind, we must now direct our attention to some results which have been obtained by the "Mendelian" method of attacking the problem of heredity.

In the early part of the nineteenth century much attention was paid by horticulturists to experiments on the hybridization of plants. It was found that, within certain limits, it was possible to fertilize the flower of one variety of plant with the pollen of another variety, and in this manner to alter the character of the offspring. Many crosses or hybrids between different varieties were thus obtained, differing in various ways from the parent plants. It was also found that by repeatedly fertilizing the flowers of one variety with the pollen of another the descendants of the first could be entirely changed into the second. This clearly proved that all the distinctive characters of the male parent were in some way represented in the pollen grains, and could be transmitted by these to future generations, but it also proved something more, it proved that it was possible to eliminate the special characters of the female parent, or at least to prevent them from manifesting themselves in the offspring.

As a rule it is only possible to bring such experiments to a 
successful issue when working with closely related species or varieties. The flower of one kind of orchid, for example, may perhaps be fertilized by the pollen of a different orchid, but the pollen of such a plant as a lily would probably not have the slightest effect upon it.

The first observer to throw a clear light upon the meaning of the remarkable results obtained by hybridization was Gregor Johann Mendel, a native of Austrian Silesia, born in 1822, whose work has recently attracted so much attention. As Abbot of Brünn, he was the happy possessor of a garden, and presumably also of that peace and quiet which are so essential to intellectual work. He was not content with merely casual experiments; he had time to think about what he was doing and he attacked the problem in the true scientific spirit. Unfortunately for science, however, his seclusion was a little too complete. He was content to publish his results in $\mathbf{1 8 6 5}$ and 1869 in the proceedings of a local natural history society, where they remained buried and almost unnoticed for more than thirty years. Thus, by the irony of fate, our own illustrious countryman, Charles Darwin, although a contemporary of Mendel, probably never heard of those remarkable discoveries which bid fair to solve some of the problems in which he himself was so deeply interested.

In order to gain an insight into the nature of Mendel's work we cannot do better than turn to his original memoir, entitled "Experiments in Plant Hybridization," of which an excellent translation has been published by Professor Bateson, ${ }^{1}$ one of the leading exponents of what is now termed Mendelism. Even fifty years ago, experiments in hybridization were, as we have already seen, no new thing. Mendel had many predecessors in this line of research, but it was reserved for him to introduce exact statistical methods into the work, and it is to these methods that he owed his success. It was already known that hybridization, or the crossing of distinct species and varieties, might result in the production of several distinct types of offspring from the same hybrids. Mendel was not content with this knowledge: he proceeded by numerous and often repeated experiments, extending over several years, to classify the offspring produced by hybridization, to follow out their descendants from generation to generation, and above all to find out the exact numerical proportions in which the different types appeared in

1 Vide Bateson, "Mendel's Principles of Heredity" (Cambridge, 1909). 
successive generations. It was the discovery of these proportions that furnished the clue to the mystery.

Mendel found the material which he required for his experiments chiefly in the numerous garden varieties of the common edible pea. How these varieties first originated we do not know. Possibly they arose as sudden and apparently spontaneous variations, or mutations. No less than twenty-two such varieties were chosen for experiment.

The next thing was to select certain differentiating characters upon which to concentrate attention, and this is a very important point. Of course, all the varieties agreed with one another in most of their characters; in other words, they were all edible peas exhibiting the specific characters of the plant known to botanists as Pisum sativum, but they differed in numerous minor features. Of these differentiating characters Mendel selected seven for the purposes of his experiments, amongst which I need mention only two: (1) the form of the ripe seed, which, roughly speaking, may be either round and smooth or angular and wrinkled; and (2) the difference in colour of the seed-contents or cotyledons, which may be either yellow or green and which usually determine the colour of the seed as a whole.

The two differentiating characters of each pair were artificially united by cross-fertilization, the flowers of a round-seeded pea being fertilized by the pollen of a wrinkled-seeded pea, those of a green-seeded pea by the pollen of a yellow-seeded pea, and so on.

In this way a number of hybrid plants, represented in the first instance by seeds, were obtained. ${ }^{1}$ These hybrids constituted what modern writers term the $\mathrm{F}^{\mathbf{l}}$ or " first filial" generation, and the curious fact was observed that every hybrid closely resembled one of the two parents, instead of being, as is frequently the case in other hybrids, intermediate in character between them. Further experiments with the offspring of the hybrids, however, showed that, although only one of the two contrasted characters manifested itself in the hybrid, the other was there also in a latent or dormant condition, and appeared again in

1 It must be remembered that the pea-seed consists chiefly of the young plant in a dormant condition, and that its characters are therefore, with the exception of those appertaining to the seed-coat, which are not considered in this chapter, those of the sporophyte generation which follows the conjugation of the gametes. The faet that the complete life-cycle of the plant really includes two generations, a well developed sporophyte and a vestigial gametophyte, does not affect the Meudelian conclusions. 
subsequent generations. The character which appears in the hybrid is said to be dominant, while that which is suppressed is said to be recessive. In the case of these peas, then, one character is always dominant over the other in the hybrid, and, moreover, it is always the same character, and it does not matter whether it is derived from the male or from the female parent. Thus the round form of seed is dominant over the wrinkled, the yellow colour of the seed-contents over the green, and so on.

Having obtained the hybrids, the next step was to follow the history of the offspring throughout suecessive generations. For this purpose the flowers of the plants raised from the hybrid seeds were allowed to fertilize themselves with their own pollen, no further crossing being permitted. The seeds thus obtained (constituting the $\mathrm{F}^{2}$ or " second filial" generation) were now again apparently of two kinds, resembling the two original parent forms from which the hybrid was produced. Moreover, these two kinds occurred in definite proportions, three of the dominant to one of the recessive-three round seeds to one wrinkled, three yellow to one green, and so on. Of course, these proportions are only averages, and, in order to eliminate errors of chance, large numbers of observations must be made-the greater the number the more reliable the result. To take an actual example from Mendel's work, 7,324 seeds were obtained in the second trial year from the hybrids between round and wrinkled. Of this number 5,474 were found to be round, and 1,850 wrinkled-showing the ratio of 2.96 to 1 . Again, out of 8,023 seeds produced by the hybrids between green and yellow, 6,022 were yellow and 2,001 green, the ratio being $3 \cdot 01$ to 1 . These experiments have often been repeated during recent years, especially by Mr. A. D. Darbishire, ${ }^{1}$ and it is found that the average ratio of three to one is always maintained.

The significance of this proportion is not at first sight obvious. It is necessary to continue the experiment for at least another generation in order to gain further insight into the matter. We have as the result of the self-fertilization of our hybrids apparently only two kinds of seeds or plants, which we may call $\mathrm{D}$, showing the dominant character, and $\mathrm{R}$, showing the recessive character, in the proportion of $3 \mathrm{D}$ to $1 \mathrm{R}$. If these

1 Some beautiful illustrations of Mendelian results in both plants and animals are given in Mr. Darbishire's recent work "Breeding and the Mendelian 1)iscovery" (Cassell \& Co., Id., 1911). 
plants are again eultivated and allowed to fertilize themselves we find that every one of the R's breeds true, and no matter how many generations we raise they will always remain of the recessive type. These are now called "extracted recessives." When, however, we cultivate the plants showing the dominant character in the same way we soon find that, though similar to one another externally, they are not in reality all alike, for one-third of them will yield nothing but D's, while the remaining two-thirds will yield D's and $R$ 's in the same proportion of 3 to 1 as the original hybrid. Moreover, the D's obtained from the one-third will continue to breed true from generation to generation, and do what we will we can never get an $\mathrm{R}$ out of them again. They are called "extracted dominants."

It is obvious then, from these experiments, that the apparent D's are not all true D's, but that two-thirds of them contain the recessive character in a latent or dormant condition, and are, therefore, still hybrid in composition. If we indicate those which we can thus prove by breeding to contain the recessive character by the letters $\mathrm{D}(\mathrm{R})$, we can sum the whole story up in the following simple diagram :-

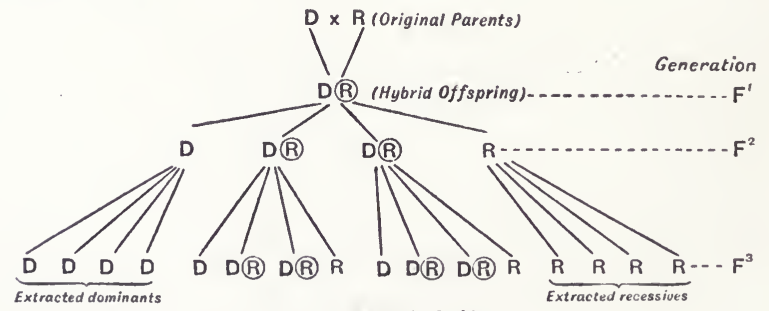

Fig. 79.--Monohybridısm.

Thus we see that from generation to generation of the offspring of the hybrid there goes on a constant sorting out into three categories, the two original parental types and the hybrid form being always produced in definite proportions. If we assume that each kind produces on an average an equal number of offspring, we shall see from the diagram that in the course of a few generations of undisturbed reproduction by far the greater number of the plants will have finally reverted, in equal proportions, to one or other of the original types, but a certain number 
will always remain in the hybrid condition and will continue to split up as before in the characteristic Mendelian proportions. We see, then, from Mendel's experiments, that in the case of peas, where one of the two contrasted characters is dominant over the other, the offspring of the first. hybrid appear in the proportion of three apparent dominants to one recessive, but that further analysis shows that the real proportion is

$$
1 \mathrm{D}: 2 \mathrm{D}(\mathrm{R}): 1 \mathrm{R}^{1}{ }^{1}
$$

This proportion has since been observed not only in peas but in a large number of other cases, including animals as well as plants. It is evidently a phenomenon of very common occurrence, though we cannot as yet say that it occurs universally whenever two forms with contrasted characters are crossed. Moreover, as we have already noticed, the phenomenon of dominance is not always shown, and the hybrid may exhibit a character intermediate between those of the two parents or different from either. The occurrence of Mendelian proportions, however, is sufficiently frequent to demand explanation, and this explanation we must now seek.

Suppose we take a large number of black and an equal number of white counters, all alike in shape, size and weight, and after shaking them up thoroughly in a bag, draw them out two at a time, and one on top of the other, without looking. Each pair that we draw out may consist of white over white, black over white, white over black, or black over black. If we draw out a sufficiently large number of pairs entirelyat random in this way and then count them, we shall find that they occur in the proportion:-1 W W : $1 \mathrm{~B} \mathrm{~W}: 1 \mathrm{~W} \mathrm{~B}: 1 \mathrm{~B} \mathrm{~B}$, or, since $\mathrm{B} \mathrm{W}$ and $\mathrm{W}$ B may be looked upon as the same, $1 \mathrm{~W} W: 2 \mathrm{~W} \mathrm{~B}: 1 \mathrm{~B} \mathrm{~B}$, or one all white, two white and black, and one all black; this being, of course, only what is to be expected in accordance with the mathematical law of probability. This is also the same as the simple Mendelian proportion, and at once suggests that the latter may, perhaps, be explained in a similar way as the result of random union of characters in accordance with the laws of chance.

We know that each individual plant or animal produced by sexual reproduction is formed by the union of two germ cells or gametes. Now these gametes are formed in very large numbers

1 This is sometimes written more fully $1 \mathrm{D} 1): 2 \mathrm{D}(\mathrm{R}): 1 \mathrm{R} \mathrm{R}$. The reason for so doing will be obvious from what follows. 
in the parent organisms, and if we suppose that each one contains only one of the two contrasted characters with which we are experimenting, and if we further suppose that the two kinds are produced in equal proportions and that they unite at random, then, in accordance with the law of chance, we should expect to get in the offspring the proportion of one with the one character, two with both characters and one with the other character, exactly as Mendel found in his experiments. ${ }^{1}$ There seems to be no other possible explanation of the Mendelian phenomena.

The conclusions to be drawn from these results are of fundamental importance. In the first place, we learn that small individual characters may be separately represented in the germ cells and separately transmitted from parent to offspring. This indicates that the entire organism may be built up of a number of "unit characters," and if we can once establish the general occurrence of such unit characters we shall have taken a long stride towards the understanding of the laws of heredity.

In the second place, we may conclude from these experiments that, as regards the unit characters with which we are dealing, we have a complete segregation amongst the germ cells or gametes, each of which carries only one of each pair of contrasted characters (technically termed allelomorphs), and in this respect it makes no difference whether the germ cell be male or female. Thus we arrive at what is sometimes termed the doctrine of the "purity of the gametes"-purity, that is to say, with regard to each character of any such contrasted pair, and without reference to the other innumerable characters which must be represented in the germ cells in order that the whole complex structure of the organism may be developed from them.

We may now examine an example of a class of practical problems which may be solved by the application of such simple Mendelian principles.

Fowl-fanciers are well acquainted with a particular breed of fowl known as the blue Andalusian. It has long been known that this kind of fowl cannot be made to breed true. Some of

1 A little consideration will show that if the contrasted characters are represented in equal proportions of both male and female gametes, the facts that a male must always unite with a female, and that the actual number of sperm cells produced is usually very much larger than the number of ova, will not affect the result. 
the offspring will be white and others white with black splashes, the remainder being like the parents. It has been shown, moreover, that the three kinds of ehicken occur, on an average, in definite proportions, a quarter being black, a half blue, and a quarter white splashed with black. Here we have the familiar Mendelian proportion $1: 2: 1$, suggesting that the blue Andalusian is really a hybrid, and that the so-called "wasters " are the parent forms. It is easy to prove that this is the ease, for if the two kinds of waster are mated we invariably get the blue Andalusian again. 'The old idea of the so-called practical breeder would have been to go on destroying the wasters and earefully selecting the blues in order to maintain the "purity of the breed." We now know, however, that there is no such thing as a pure blue Andalusian breed; the blue is really a hybrid, and you can get more blues by mating the wasters than by breeding from the blues themselves. This case is also interesting as affording an example of a hybrid which differs in character from either of the parent forms.

If only a single pair of alternative characters or allelomorphs is dealt with in the experiment the case is termed one of monohybridism, and such cases yield the proportion 1:2:1 (or apparently $1: 3$ in cases of dominance) amongst the offspring of the hylorid, i.e. in the $\mathrm{F}^{2}$ generation. It will frequently happen, however, that the two varieties which are united in the formation of the hybrid will differ from one another as regards more than one pair of contrasted characters. We may illustrate this by a case of dihybridism, in which two pairs of contrasted characters are involved. The case selected is one which shows us how, under certain cireumstances, we ean obtain a hybrid exhibiting an entirely new combination of characters, which in spite of its hybrid nature will continue to breed true.

Suppose a gardener had only two kinds of peas, one with round green seeds and the other with wrinkled yellow seeds, and that he wished to obtain peas with wrinkled green seeds. Mendel has shown us how he may get what he desires, both surely and speedily, from the material already in his possession.

The necessary procedure will be evident from the following scheme :-

Let $\mathrm{R}=$ round, $\mathrm{W}=$ wrinkled, $\mathrm{Y}=$ yellow, and $\mathrm{G}=$ green. We know from previous experiments that $R$ is dominant to W, and $\mathrm{Y}$ to $\mathrm{G}$. 
R G crossed with WY gives the hybrid, R (G) (W) Y, which, owing to the dominance of $\mathrm{R}$ and $\mathrm{Y}$, will appear in the form of round yellow seeds (the brackets around the $G$ and $W$ indicating the recessive nature of green and wrinkled). That is not what our gardener wants, and if he knew no better he would naturally be

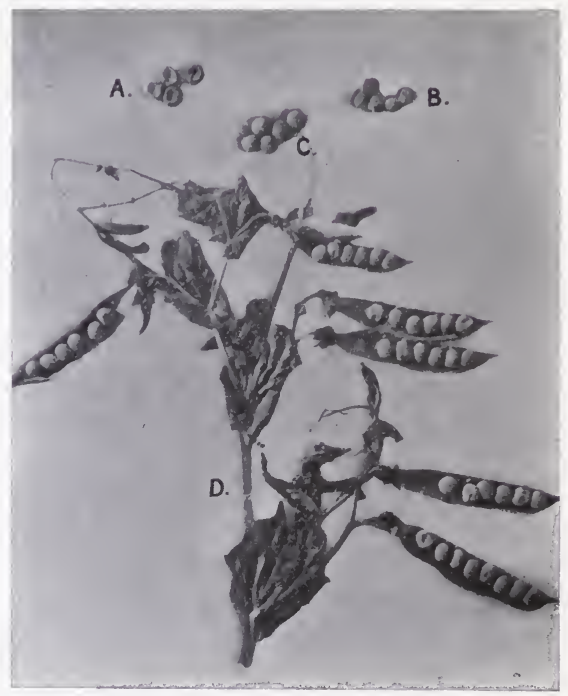

FIG. 80.-An Example of Dihybridism in Peas.

$A$, wrinkled yellow peas; B, round green peas; C, hybrid peas (F1 generation, round and yellow in appearance) produced by crossing $A$ and $B ; D$, plant of the $F^{1}$ generation, with seeds of the. $\mathbf{F}^{2}$ generation, produced by self-fertilization and of the four different kinds, round yellow, round green, wrinkled yellow, and wrinkled green, lying in the pods. (Plotographed from a preparation by Mr. Darbishire, who has reproduced the same subject in colours in his work on "Breeding and the Mendelian Discovery").

much disappointed with the result. If, however, the hybrids be allowed to fertilize themselves and reproduce, then their offspring will appear in the apparent proportions :-

$$
9 \mathrm{R} \mathrm{Y}: 3 \mathrm{R} \mathrm{G}: 3 \mathrm{WY}: 1 \mathrm{WG},
$$

no account being taken of the non-apparent recessive characters. The meaning of the proportion $9: 3: 3: 1$ exhibited in this 
case wil be readily understood from the accompanying table (Fig. 81) :-

\begin{tabular}{|c|c|c|c|c|}
\hline \multicolumn{2}{|c|}{$\begin{array}{l}\text { Germ } \\
\text { eells }\end{array}$} & $\mathrm{R} \mathrm{G}$ & W Y & W G \\
\hline \multirow{2}{*}{$\stackrel{\downarrow}{\downarrow} \mathrm{Y}$} & R Y & $R(G)$ & (W) Y & $(\mathrm{W})(\mathrm{G})$ \\
\hline & R Y & R Y & R Y & R Y \\
\hline \multirow{2}{*}{$\mathrm{R} \mathrm{G}$} & $\mathrm{R} \mathrm{Y}$ & R G & (W) $\mathrm{Y}$ & (W) $\mathrm{G}$ \\
\hline & R (G) & R G & R (G) & $\mathrm{R} \mathrm{G}$ \\
\hline \multirow{2}{*}{ W Y } & R Y & $\mathbf{R}(\mathrm{G})$ & W Y & W (G) \\
\hline & (W) $\mathrm{Y}$ & (W) $\mathrm{Y}$ & W Y & W Y \\
\hline \multirow{2}{*}{ W G } & R Y & $R$ G & W Y & W G \\
\hline & (W) (G) & (W) $\mathrm{G}$ & W (G) & W G \\
\hline
\end{tabular}

Fig. 81.-Dihybridism.

The constitution of the hybrid (in so far as the characters in question are concerned) is $R(G)(W) Y$. Each of its germ cells, however, contains one character, and one only, from each contrasted pair. Each may, therefore, contain $\mathrm{R} Y, \mathrm{R} G, \mathrm{~W}$ Y or W G. These different kinds of germ cells will occur on the average in equal numbers, and on self-fertilization of the hybrid flowers they will unite in pairs at random. The possible ways in which such union may take place are shown in the table, and if allowance be made for the phenomenon of dominance, in accordance with which $G$ disappears from view whenever it meets $\mathrm{Y}$, and $\mathrm{V}$ whenever it meets $\mathrm{R}$ (as indicated by the brackets in the table), we get the apparent proportion of 9 round yellow seeds, 3 round green seeds, 3 wrinkled yellow seeds, and 1 wrinkled green seed. The number of wrinkled green seeds will be small at first, but they will breed true, containing as they do only the extracted recessive characters, for if a dominant character were present it would necessarily show itself.

Thus we see that one way of producing new forms of plants and animals is by the artificial combination of characters which already exist in different varieties. These elementary or unit characters can be brought together by the process of hybridization, and new organisms produced in somewhat the same 
way as that in which the chemist is able to produce new compounds by analysis and synthesis from other substances.

Probably no man has made more successful use of the process of hybridization in the production of new and valuable forms of plant-life than Luther Burbank, at his celebrated Californian nurseries. Mr. Burbank himself is unfortunately not a writer, and for a scientific account of his work we are indebted to Professor Hugo de Vries, who in his book on "Plant Breeding"1 describes what he himself saw and heard during his visits to Mr. Burbank's farms. Some idea of the commercial value of Mr. Burbank's work may be formed from the fact that special companies have been formed for the propagation and sale of some of these wonderful hybrids. White blackberries, stoneless prunes, "plumcots" and thornless cacti are only some amongst the many novelties which he has produced by crossing different varieties by hybridization and thus combining two or more desirable qualities in one plant.

Take, for instance, the case of the stoneless prune. It had somehow or other come to the knowledge of Mr. Burbank that about 200 years ago there existed in France a plum known as the "Prune sans noyau." He succeeded in obtaining specimens of this fruit, but it proved to be of little or no commercial value owing to its poor quality. Its one valuable feature was its stonelessness, and Burbank set to work to transfer this character, by hybridization, to a variety of good quality. He succeeded, and there appears to be no reason why the stoneless character should not be similarly implanted upon all the different varieties of plums now in cultivation.

Similarly, the "plumcots" are hybrids between plums and apricots, which Professor de Vries speaks of as "most delicious and beautiful fruits."

It must be admitted that Burbank's work is practical rather than scientific. He apparently pays no attention to the law of Mendel in his operations, and just goes on hybridizing until he gets what he wants. In this process enormous numbers of plants which do not fulfil his requirements have to be destroyed for every one that is worth preserving.

$\mathrm{He}$ is not necessarily concerned with the production of pure breeds; of forms, that is to say, which will breed true from seed,

1 Hugo de Vries, " Plant Breeding " (London, Kegan Paul, Trench, Trïbner \& Co., Id., 1907). 
or, in other words, of forms which will hand on their desirable qualities to future generations by heredity. This does not matter in the case of fruit trees and other plants which can be propagated by buds independently of sexual reproduction. As a general rule, a fruit tree cannot be relied upon to come true from seed, the characters which it has received from different ancestors not being permanently combined, but separating out and undergoing fresh combinations in the sexual process, probably in accordance with Mendelian principles. The seedlings will therefore be "degenerate," as horticulturists say, and will no longer exhibit those valuable qualities which depend upon the confluence in one individual of particular lines of ancestry.

The majority of Burbank's productions could not survive in a state of nature at all; they are essentially artificial and have to be artificially propagated by means of buds or cuttings. In this respect they are quite different from the pure races which it is possible to produce by hybridization carried out in accordance with Mendelian principles, in which new and permanent combinations of unit characters, capable of being transmitted by heredity, are effected.

Professor Bateson, Professor Biffen, Mr. Hurst and others have lately done much to demonstrate the possibilities of progress in this direction, and the value which the application of the Mendelian principles of heredity must have from the economic point of view. We know now that such cereals as wheat and barley obey the Mendelian laws of hybridization as regards various important characters. So also do horses as regards the colour of their coats, and human beings as regards the colour of their eyes, and in some other respects, especially as regards the inheritance of certain diseases. In short, it appears certain that Mendelian principles have a very general application, and the economic value of the knowledge of such principles must be enormous. Professor Biffen has shown, for example, that susceptibility and insusceptibility to that destructive disease in wheat known as "rust," behave as Mendelian characters, and that it is possible by a very simple process of hybridization to confer immunity from this disease upon naturally susceptible varieties.

From the theoretical point of view the great value of the Mendelian experiments lies in the possibilities which they present, at any rate in certain cases, of analyzing the constitution of the hereditary substance (germ plasm), and thereby gaining some 
insight into the real nature of heredity. We may regard the existence of " unit characters" as being conclusively demonstrated by these experiments, and the fact that we are able to interchange these characters and to add and subtract particular characters to and from the organism seems to indicate very clearly that the germ plasm must contain material primordia or "factors," as they are often called, which are responsible for the development of these characters.

In so far as they demonstrate the existence of such factorswhich evidently correspond very closely with Weismann's hypothetical determinants - the Mendelian experiments may be taken as affording confirmation of Weismann's theory of the constitution of the germ plasm. Their results also harmonize very well with those of recent eytological investigations. We have seen that there is strong evidence for regarding the chromatin substance of the nucleus as the material basis of heredity. We have further seen that this chromatin is very complex in structure and that the chromosomes, or units of the highest order of which it is composed, can in many cases be optically resolved into chromomeres or units of the next lower order. According to Weismann's theory these chromomeres or ids are in their turn made up of the determinants. Moreover the associations and redistributions of chromosomes which take place in the process of "reduction" and in the conjugation of the germ cells seem to afford ample opportunity for those permutations and combinations of factors the occurrence of which is demonstrated by the Mendelian experiments. As Professor Farmer observes, "The facts of meiosis ${ }^{1}$ are seen to fall completely into line with conclusions drawn from experiments on breeding as far as the numerical distribution of characters is concerned." 2

The doctrine of the "purity of the gametes" teaches us that any given mature germ cell contains only one member of any given pair of alternative factors or primordia (allelomorphs). This may be accounted for by the pairing of the chromosomes and the subsequent halving of their number which take place at some period or other prior to the formation of the gametes. There is good reason to believe that each member of such a pair of chromosomes is homologous with or morphologically equivalent

1 I.e. the phenomena accompanying the reduction in the number of chromosomes which takes place periodically in all typical organisms (vide Chapter X.).

${ }^{2}$ Croonian Lecture. Proceedings of the Royal Society, B, Vol. 79, 1907. 
to the other, the difference between them lying in the fact that one is paternal and the other maternal in origin. ${ }^{1}$ Reduction (or halving of the total number of chromosomes) is accomplished by the distribution of the two members of each pair to different daughter cells. Hence if the paternal and maternal chromosomes contain different alternative factors (or determinants), derived from different parents, so also will the gametes when these are formed.

Suppose we represent the two members of any pair of factors by the letters $a$ and $b$, then any one gamete may contain either $a$ or $b$, but not both. When the gametes unite in conjugation the zygote will again have both maternal and paternal chromosomes and there will be three possibilities as to its constitution in regard to the characters in question. If a gamete containing $a$ happens to unite with another containing $a$ the zygote will contain the pair $a$, and will be termed in Mendelian phraseology a "homozygote." 2 If a gamete containing $b$ happens to unite with another containing $b$ the zygote will contain the pair $b b$ and will again be a homozygote. If, on the other hand, a gamete containing $a$ unites with another containing $b$ the zygote will contain the pair $a b$, and such a zygote is termed a "heterozygote" or hybrid.

In accordance with what is termed the "presence or absence hypothesis" one of the two " factors" in an allelomorphic pair may be merely negative in character ; i.e. it may have no real existence as a material primordium or determinant. In the words of Professor Bateson, "All observations point to a conclusion of great importance, namely that a dominant character is the condition due to the presence of a definite factor, while the corresponding recessive owes its condition to the absence of the same factor. This generalization, which so far as we yet see, is applicable throughout the whole range of Mendelian phenomena, renders invaluable assistance in the interpretation of the phenomena of Heredity. The green pea, for instance, owes its recessive greenness to the absence of the factor which, if present, would turn the colouring matter yellow, and so forth." 3

It is obvious that if, in our general formula, we take $a$ to represent the dominant factor and $b$ the recessive, we might

1 Compare Chapter $\mathrm{X}$.

2 This term has been extended to the fully developed organism which arises from the zygote.

${ }^{3}$ Bateson, "Mendel's Principles of Heredity" (Cambridge, 1909), pp. 53-54. 
substitute not $a$ for $b$ without altering the result, and the differencess between the paternal and maternal chromosomes of any given pair may depend merely upon the absence from one or the other of one or more factors which are present in its mate.

Mendelian phenomena are often greatly complicated and rendered very difficult of interpretation by the fact that the activity of a given factor, or its power to influence the development of the zygote, may be dependent upon the presence of another factor belonging to a different "allelomorphic pair." In other words, a given character may depend not merely upon the presence of a single factor in the zygote but upon the co-operation of two or more factors, and if these factors happen to be separated in the process of reduction and do not happen to come together again in the union of the gametes to form the zygote, then the character in question ${ }^{1}$ will not appear in the organism developed from the zygote. In this way it may happen that a character which was present in the ancestors of a particular organism may disappear for many generations and then suddenly re-appear as the result of some cross or hybridization in which the necessary factors happen to be brought together again in the zygote. In this way are explained those cases of "reversion" to ancestral types which Darwin found to occur so frequently as the result of cross-breeding.

One of the most thoroughly investigated cases of this $\mathrm{kind}^{2}$ is that of certain white sweet peas belonging to the variety known to horticulturists as "Emily Henderson." Plants of this variety are not really all alike but differ from one another in the shape of their pollen grains, and when the two kinds, distinguishable only in this way, are crossed, the offspring are frequently found to possess purple flowers resembling those of the wild Sicilian pea from which our cultivated varieties have been derived. It is not necessary to go into the somewhat complicated analysis of this ease in any detail, but it has been demonstrated by the researches of Professor Bateson and Professor Punnett that the re-appearance of the lost colour in the hybrid is due to the re-union in the zygote of certain factors which had become separated at some period or other in the ancestral history of the white-flowered parents.

The existence of interactions of this kind between the different factors or determinants in the germ plasm, giving rise often to

1 Professor Bateson terms such characters "compound charactcrs."

2 Bateson, op. cit., pp. 89 et seq. 
very complex results as regards the characters of the offspring, is a sufficient explanation of the fact that many cases of hybridism are at present incapable of interpretation in terms of the Mendelian theory, and such cases cannot be legitimately used as arguments against the validity of that theory. It is obviously impossible to explain the results of any particular cross until we have some knowledge, not only of the extremely complex constitution of the parental germ plasm on both sides, but also of the way in which the factors which meet in the zygote may be expected to influence one another.

Mendelian phenomena, of course, can occur only as a result of crossing or hybridization at some stage or other of the ancestral history, and it does not seem likely that such crossing has had any very great influence upon the evolution of plants and animals in a state of nature. In cases of monohybridism, as we have seen above, the hybrid form in the course of a few generations would probably be quite swamped by the numerical preponderance of the pure extracted forms which have reverted to one or other of the original parental types, the hybrid itself never being permanently fixed. New and permanent combinations may, of course, sometimes arise naturally through dihybridism, but they would at first be produced in very small numbers and could only be expected to survive in the struggle for existence if they liappened to possess some material advantage over the parent forms. Thus it appears that in a state of nature the results of hybridization tend to be automatically eliminated.

Attention has frequently been called to the alleged discrepancy between the results of the Mendelian experiments and those obtained by the late Sir Francis Galton and others by statistical methods based upon the quantitative estimation of characters in a number of successive generations. As a result of his investigations Galton was able to formulate the following "Law" of inheritance:- "The two parents contribute between them on the average one-half, or $(0.5)$ of the total heritage of the offspring; the four grand-parents, one-quarter, or $(0 \cdot 5)^{2}$; the eight greatgrandparents, one-eighth, or $(0 \cdot 5)^{3}$, and so on. Thus the sum of the ancestral contributions is expressed by the series $\{(0 \cdot 5)+$ $(0 \cdot 5)^{2}+(0 \cdot 5)^{3}$, \&c. $\}$, which, being equal to 1 , accounts for the whole heritage." 1

This series has been modified by Professor Karl Pearson for

1 Proceedings of the Royal Society of London, Vol. LXI.. 1897, p. 402.

B. 
certain reasons into which we cannot enter in this place, but it may be taken as a substantially correct expression of the results obtained by biometrical inquiry. If we remember that Galton's Law merely expresses the average results which may be anticipated from the interbreeding of a large population, in which hybridization probably plays a very small part, it does not appear in any way antagonistic to the Mendelian theory. It is of course not applicable to individual cases of hybridization, where we are concerned, not with small variations in the same characters, but with the permutations and combinations of alternative character units.

Intimately bound up with Galton's Law of Inheritance is another important generalization known as the Law of Filial Regression, which we owe to the same distinguished philosopher. The law of ancestral inheritance teaches us that on an average the individual derives half its characteristics from its immediate parents and the remaining half from its more remote ancestry. If the immediate parents, or one of them, happen to depart from the average condition of the race in respect of any character there will be a tendency on the part of the offspring to inherit the deviation in question; but not to the same extent, for the influence of the more remote ancestry will tend to counteract that of the parents and cause a partial return-or regressiontowards mediocrity. As a result of his statistical investigations Galton estimated that the offspring of parents exhibiting a marked deviation from the average would tend to inherit that deviation to the extent of only one-third of its magnitude in the parents (the mean of the two parental deviations being taken as the standard of comparison). In the words of Professor Karl Pearson, "It is the heavy weight of this mediocre ancestry which causes the son of an exceptional father to regress towards the general population mean ; it is the balance of this sturdy commonplaceness which enables the son of a degenerate father to escape the whole burden of the parental ill. Among mankind we trust largely for our exceptional men to extreme variations occurring among the commonplace, but ... if we could remove the drag of the mediocre element in ancestry, were it only for a few generations, we should sensibly eliminate regression or create a stock of exceptional men." 1

1 Grammar of Science, 1900 , pp. 456-457. 


\section{PART IV.-THE THEORY AND EVIDENCE OF ORGANIC EVOLUTION: ADAPTATION}

\section{CHAPTER XV}

Organic evolution versus special creation-Spontaneous generation and biogenesis - The origin of living things.

Aт the present day we see the surface of the earth teeming with hosts of living things, incalculable in number and of endless diversity in form and structure. Every situation where life is possible is occupied by plants or animals of some kind or other, all specially adapted in bodily organization to the conditions under which they have to maintain their existence. From the bleak and inhospitable summits of high mountain ranges to ocean depths which can be measured in miles; from the perpetually frozen circumpolar regions to the torrid zone on either side of the equator, living things abound. Seas, rivers, lakes, dry land and air have all alike been taken possession of by representatives of the animal and vegetable kingdoms. A single drop of water may contain thousands of organisms, and every chalk-cliff, coalseam or peat-bog testifies to the countless myriads which have lived in past times and whose remains have contributed in no small measure to the formation of the earth's crust.

In the animal kingdom alone it is probable that at least a million different kinds or species are living on the earth at the present day. Some half million or so have already been discovered, named and more or less imperfectly described, while every exploring expedition brings back many which have never before been seen.

Nevertheless there must have been a time when no living things whatever existed on the earth. According to the nebular hypothesis our planet is still gradually cooling from a molten state, preceded probably by an incandescent gaseous condition, which must have rendered the existence of any protoplasmic organism a physical impossibility. Protoplasm becomes 
coagulated and thereby destroyed as living substance at comparatively low temperatures, and cannot have been present on the earth until the surface of the latter had not only solidified but also cooled down to something approaching its present degree of heat.

Living things must therefore have first put in an appearance at some definite period well advanced in the earth's history. According to the biblical account given in the Book of Genesis their advent was due to a series of special acts of creation performed by the Creator at the appropriate time, and this account forms the basis of the doctrine of Special Creation which still survives amongst uneducated people. Upholders of this doctrine maintain that each kind of plant or animal was not only created as a separate kind and in a state of full perfection, but was specially designed to suit the conditions under which it was placed, and in this way the marvellous adaptation of plants and animals to their environment was supposed to be accounted for. The fish was created to swim in the sea, the horse to run on dry land, the monkey to climb in the tree and the bird to fly in the air, and each was constructed in accordance with its foreseen requirements. Moreover, each species was supposed to be immutable, propagating its own kind by reproduction but never changing into a different kind. ${ }^{1}$

Such a view of the origin of living things could only have arisen in a state of almost complete ignorance of the phenomena which have to be accounted for, and at the present day it has been entirely superseded, as a scientific theory, by the doctrine of Organic Evolution. This doctrine teaches us that species or kinds are not immutable but are subject to changes whereby one may give rise to another, and that all existing species are the more or less modified descendants of pre-existing ones. Modern biologists, moreover, maintain that the further back we trace the ancestral history of living things the less diversity of structure do they show, until finally, if we could trace them back to their origin, we should find all the different lines of descent converging towards a common starting point far back in geological time. We shall see presently that the adaptation to environment which forms such a characteristic feature of all living organisms can be explained just as well in accordance with the theory of organic

1 Compare the quotation from Linnæus on p. 222 (1st footnote) and that from Buffon on p. 3.69 . 
individuals arise by some process of reproduction from pre-existing parent individuals. This is represented in the upper parts of both diagrams, where a $1-8, \mathrm{~b} 1-7$, c $1-4, \mathrm{~d} 1-5$ and e $1-5$, on each side, are supposed to represent small groups of individuals of the species A B C D and E respectively, each group being descended from some common ancestor (or pair of ancestors) which itself belonged to the species.

The study of this diagram is alone sufficient to afford strong presumptive evidence in favour of the view of the evolutionist as against that of the upholder of special creation, for the evolutionist in his imagination extends backwards into the past the processes which he sees taking place constantly at the present day, and endeavours to account for the origin of species in accordance with what he knows to be true of the origin of individuals. In other words, the diagram expressing the idea of organic evolution is consistent throughout, whereas that which represents the idea of special creation is made up of two incongruous portions, and is therefore less likely to be correct. The great weakness of the doctrine of special creation, however, lies in its failure to explain countless facts of comparative anatomy, embryology, geographical distribution and palæontology, all of which are readily explicable in terms of evolution. We shall deal with some of these facts in subsequent chapters.

It is obvious, then, that in order to be logically consistent the evolutionist need not postulate more than a single starting point for the evolution of the whole organic world, though he is not obliged to limit himself in this way should evidence be forthcoming that there has been more than one starting point. This brings us to the consideration of the extremely difficult and at present quite insoluble problem of the origin of the first living organisms.

Here again we may first inquire whether our experience of what takes place at the present day throws any light upon the problem. Many people in the past have held, and some few still maintain, that living things may, even now, arise from not-living matter. This is known as the doctrine of "Spontaneous Generation" or abiogenesis. One of the earliest expressions of this idea is met with in classical mythology, in the story of the birth of Aphrodite from the sea-foam ; and Vergil, in the Georgies, accepts the principle involved therein apparently in perfectly good faith.

Vergil's account of the manner in which a swarm of bees may 
be produced from the decomposing body of a dead ox affords a very striking illustration of that want of scientific training in observation and reasoning which has led to so many erroneous beliefs. He tells us, with much elaboration of detail, that if the body of an ox is well beaten and enclosed in a suitable chamber a swarm of bees will arise from it within a certain number of days. We now know perfectly well what may really happen under these circumstances. In the first place the supposed bees are not bees at all, but drone-flies, which superficially resemble bees, though easily recognizable as belonging to a totally different order of insects by the fact that they possess only two wings instead of four, and consequently any husbandman who followed Vergil's instructions must have been grievously disappointed in his expectations of honey. In the second place the drone-flies are not spontaneously generated from the body of the ox, or from anything else, but are hatched out, first in the form of maggots, from eggs which were laid by pre-existing flies. These maggots, having fed abundantly on the decaying carcase, presently undergo their metamorphosis and emerge as flies.

Many similar instances of alleged spontaneous generation, all resting upon gross ignorance of the real facts of the case, might be collected from the writings of ancient and mediæval authors. The cruder stories, which could be easily disproved by simple observation, were soon cast aside as fables under the influence of modern scientific methods. The invention of the microscope, however, and the consequent revelation of a new world of hitherto invisible organisms, led to a revival of the doctrine of abiogenesis. It was noticed that organic infusions, even after boiling, presently became densely filled with various kinds of micro-organisms, especially Bacteria, and as the boiling was supposed to have killed any organisms that might have been in them at first it was argued that living things arose in them de noto, by spontaneous generation. This was, however, merely a revival of Vergil's story of the swarm of bees in a more refined form. Had Vergil's ox been protected from flies none of the alleged bees would have been produced, and the careful experiments of such observers as Tyndall and Pasteur have conclusively demonstrated that if the organic infusions are adequately protected from the countless microscopical germs which float in the air they will remain free from living organisms, and consequently from putrefaction, for an indefinite period, provided always that 
they are themselves first properly sterilized. This sterilization, however, cannot always be effected by a single boiling, for the germs of some organisms are extremely resistant to heat and special precautions have to be taken accordingly.

Every story of alleged spontaneous generation has, so far, failed to stand the test of scientific criticism and experiment, and accordingly biologists are now almost unanimous in maintaining the contrary doctrine of biogenesis, which teaches that, at the present day at any rate, living organisms arise only by reproduction from pre-existing living organisms. Nevertheless there must, as we have seen, have been a time when there were no living things on the earth, and therefore living things must have either reached the earth from some outside source or have arisen on the earth itself from matter which was previously not-living, or in other words, by spontaneous generation. It seems as if we had got to take our choice between these two alternatives, neither of which is at all easy to accept.

It has been maintained by some that organic life is eternal and is transferred from one world to another-possibly on meteoritesin the form of minute germs or "Cosmozoa," but no one has ever seen such Cosmozoa, and it is difficult to imagine any which would be capable of surviving such a journey. It is perhaps less difficult to believe that under certain conditions, of which we at present know nothing and which perhaps no longer exist on the earth's surface, living protoplasm may have arisen from inorganic matter as the result of chemical and physical processes. In this connection we must remember that protoplasm-the physical basis of life-contains no chemical elements that are not also found in inorganic matter and is, as a matter of fact, constantly being built up from inorganic constituents in the bodies of living organisms, though apparently only by the action of pre-existing protoplasm.

Whether physical and chemical forces, such as we are acquainted with, alone sufficed to bring about the transformation of not-living, inorganic material into living protoplasm, or whether, as some people suppose, some unknown "vital force" was (and still is) involved in the process, must remain, for the present at any rate, an open question.

The time has evidently not yet arrived for solving the great problem of the origin of life, but we may, at any rate, legitimately speculate upon the nature of the living organisms which first 
appeared on the earth. In the first place we may safely assume that they were extremely simple in structure, for it is generally agreed that the evolution of the higher forms of life has been accompanied by a gradually increasing complexity of organization. It is also certain that they cannot have been animals, for animals, as we have seen in a previous chapter, are dependent for their food supply upon other living things, being themselves unable to build up the proteid molecule from inorganic constituents. Green plants are the great proteid manufacturers at the present day, but only by virtue of the fact that they contain chlorophyll, which enables them to utilize the energy of the sun's

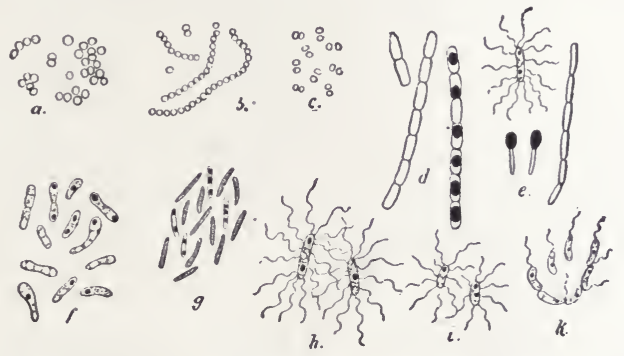

Fig. 83.-Different Forms of Disease-producing Bacteria, $X$ about 1500 . (From Strasburger, after Fischer.)

$a$, pus cocci ; $b$, erysipelas cocei ; $c$, gonorrhœa cocci ; $d$, bacilli of splenic fever; $e$, bacilli of tetanus; $f$, bacilli of diphtheria; $g$, tubercle bacilli; $h$, typhoid bacilli; $i$, colon bacilli ; $k$, cholera bacilli.

rays in the process of photosynthesis. Now chlorophyll itself is a very complex substance, and we cannot suppose that the first living things already possessed it. If, then, they had no other organisms to feed upon, and if they possessed no chlorophyll, how did they obtain the energy necessary to enable them to maintain life at all?

The simplest and at the same time the smallest organisms known to us at the present day are the Bacteria (Fig. 83), many of which are so minute as to be hardly visible even under the highest powers of the microscope. These Bacteria are neither plants nor animals, but occupy a position lower than either. T'hey have not even attained to the dignity of perfect cells, for they exhibit no proper differentiation into cell body and nucleus, the chromatin substance, which in a typical cell is aggregated in the nucleus, 
being scattered in minute granules throughout the cytoplasm (ride Fig. 27). They contain no chlorophyll and the colourless protoplasm is enclosed in a very thin and delicate cell wall. In form they vary greatly, being sometimes spherical, sometimes rod-shaped and sometimes corkscrew-shaped, and frequently they occur united together in chains. Some of them swim about by means of cilia or flagella, others are motionless, and they multiply by simple fission and also by the formation of spores, which have extraordinary powers of resistance and thus serve to secure the wide dispersal of these organisms by air and water.

A great number of different kinds of these Bacteria are already known to us, and doubtless a vast number still remain to be discovered. They occur practically everywhere; earth, air and water

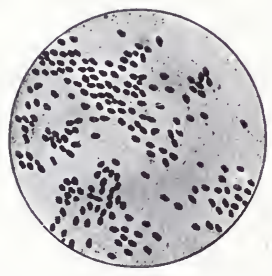

FIG. 84. - Nitrusumonas europar, $\times 600$. (From Percival's "Agricultural Bacteriology.") are full of them, and they exercise a most profound influence-sometimes injurious and sometimes beneficial-upon the lives of other living things. Some of them are the active agents in the putrefaction and fermentation which are rapidly set up in dead organisms, and others are responsible for many of the most grievous ills which living flesh is heir to. Thus most of them live either as saprophytes (upon dead organic matter) or as parasites (upon living organisms) and obtain their supply of energy from already formed proteids and other complex chemical compounds.

Some, however, are said to live free in the soil and to be able to derive their food supply, and with it their energy, from purely inorganic substances. 'T'hese are called nitrifying Bacteria. One form, Nitrosomonas (Fig. 84), converts salts of ammonia into nitrites, and another, Nitrobacter, converts nitrites into nitrates. In this way they perform an important function in preparing the food material in the soil for the use of green plants, but their chief interest for us lies in the fact that they are able, in some manner which we do not understand, to build up their own proteid molecules directly from inorganic constituents without the aid of chlorophyll. They show us that such a thing is at any rate possible, and that therefore the first living things may have been able to maintain their existence without either possessing chlorophyll or having other organisms to feed upon. 
It seems likely that of all the organisms known to us these Bacteria come nearest to the first living things, and yet they probably stand very far from them and represent a much later stage in the process of evolution. Minute and apparently simple as the Bacteria are, it seems more than probable that the first living things were very much smaller and simpler, so that even if we had them under the highest powers of our microscopes we should be unable to recognize them. Weismann has suggested that they may have been single biophors, i.e. vital units of the first order, such as he believes to constitute the ultimate living particles of protoplasm, and he has actually proposed the name "Biophoridæ" for these hypothetical freeliving primordial organisms.

These considerations throw a new light upon the question of spontaneous generation, for if living matter is first formed in such ultra-microscopic particles and can only be recognized as living matter after it has reached a comparatively high stage of evolution, it is obvious that we are not entitled to say that it is never formed from not-living matter at the present day. We cannot see it being formed, and we probably never shall see it being formed, but it is possible that it is still being "spontaneously generated" all the same. We are not logically obliged, as we said before, to content ourselves with a single starting point for organic evolution, and it would be quite impossible to prove that all the different kinds of Bacteria, the simplest organisms known to us, have descended from a single ancestor. They may equally well have been derived from a number of ancestral protoplasmic units which originated independently from inorganic, not-living matter. If such an event can have taken place once it may have taken place many times, and may still be taking place around us, though the imperfect means of observation at our disposal will not allow us to demonstrate the fact. We may safely affirm, however, that no living organism which we are at present capable of recognizing as such has arisen, or ever will arise, by spontaneous generation, but that all organisms known to us have been derived from pre-existing organisms by some process of reproduction; that, in the course of long ages, they have undergone slow changes, whereby they have become more and more diversified and usually more complex in structure, and that in this way the evolution of the animal and vegetable kingdoms has been brought about. 
In concluding this chapter it may be well to remind the reader that the idea of organic evolution is no novelty, but can be traced back to the philosophy of ancient Greece. It will be more convenient, however, to postpone what we have to say about the history of the evolution theory to a later chapter and in the meantime to familiarize ourselves with the theory itself and the evidence upon which it is based. 


\section{CHAPTER XVI}

The continuity of life-The conception of species-The principles of taxonomy-The taxonomic evidence of organic evolution.

IN accordance with the theory of evolution we may picture to ourselves the entire animal and vegetable population of the earth, both past and present, as forming one vast, tree-like organism, all parts of which, if we knew enough about their history, could be traced into actual, although of course not simultaneous, protoplasmic connection with all other parts. This tree commenced its growth far back in geological time, and its branches became ever more and more ramified in succeeding ages, and more and more diversified in character as they diverged from one another. Only the youngest twigs of the tree, however, are still actually alive, being represented by the animal and vegetable population of the earth for the time being. All the older parts have died away, most of them without leaving any traces of their former existence, and thus has arisen the actual discontinuity which is found to occur between the surviving groups of organisms at any given period.

The tendency to structural variation which all organisms exhibit, whatever may be its cause, is responsible for the progressive diversity which is gradually set up between the different branches of the organic tree. The combined action of the forces of heredity and variation bring about "descent with modification." How it is that such modification always leads, in the long run, to a more or less perfect and often very specialized adaptation of both plants and animals to the conditions under which they live will be discussed subsequently. At the moment we have to deal with things as we actually find them.

In any scheme of zoological or botanical classification the lowest unit must, of course, be the individual. The untrained observer, acquainted only with the more familiar plants and animals, sees no difficulty in arranging these in apparently 
sharply circumscribed groups. A child collecting flowers by the wayside will tell you without difficulty how many kinds he has found, and these kinds-speaking generally-correspond to the different species recognized by the botanist.

Even amongst the higher and better known plants and animals, however, the so-called species are not always sharply distinguishable from one another, for we not infrequently meet with intermediate forms, or connecting links, between what are usually recognized as distinct species. Linnæus himself, who pronounced most explicitly in favour of the doctrine of special creation, ${ }^{1}$ was forced by his profound knowledge of the animal and vegetable kingdoms to recognize this fact in the case of certain groups of plants ${ }^{2}$; and when we come to study some of the lower members of the animal kingdom, as, for example, the sponges, the difficulty of distinguishing species sharply from one another frequently becomes so great that the definition of any particular one is little more than a matter of individual opinion. The more we study the animal and vegetable kingdoms, in short, the more clearly is the fact impressed upon us that if we could have before us all past and present individuals we should find it impossible, except in an arbitrary manner, to arrange them in species at all, for each kind would be found to be connected with others by a series of small gradations.

According to Darwin, the differences which separate existing species from one another have, at any rate in most cases, arisen by the gradual accumulation of small successive variations. If this be the case the fact that we are able logically to distinguish such species from one another at all can only be due to that disappearance of the older portions of the organic tree whereby discontinuity has arisen between the surviving branches. In any case it is quite certain that such destruction of former connecting links has played a very large part in the separation of those groups of individuals to which the term species is usually applied. This process is clearly illustrated in the accompanying diagram (Fig. 85), in which each enclosed area is supposed

1 " Species tot sunt, quot diversas formas ab initio produxit Infinitum Ens; quæ deinde formæ seeundum generationis inditas leges produxere plures, at sibi semper similes, ut Species nune nobis non sint plures, quam quæ fuere ab initio."

Linnæus. Gen. Plant. (1737).

2 "Species Rosarum difficillime limitibus cireumseribuntur et forte natura vix eos posuit." Linnæus. Spee. Plant., Ed. 2, p. 705 (1762). I am indebted to my friend and colleague at the Limnean Society, Dr. B. Daydon Jaekson, for showing me these quotations. 
to represent the limits of an existing species. Within each one the individuals differ but slightly from one another, not more, perhaps, than might be expected amongst the offspring of the same parents. The three species represented have, however, become more or less widely separated from one another by the dying away of the branches from which they arose, and which are represented in the diagram by dotted lines.

Darwin has told us that he looked "at the term species as one arbitrarily given, for the sake of convenience, to a set of individuals closely resembling each other, and that it does not essentially differ from the term variety, which is given to less distinct and more fluctuating forms. 'The term variety, again, in comparison with mere individual differences, is also applied arbitrarily, for convenience' sake." 1

This, of course, is no definition of the term species and was not intended as such. A definition in accordance with the above views might, however, be given as follows:- "A species is a group of individuals that closely resemble one another

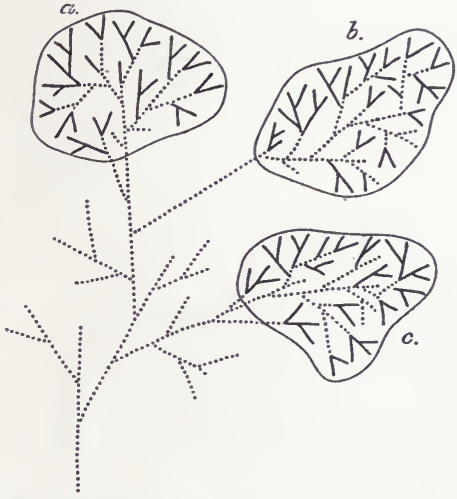

Fia. 85.-Diagram to illustrate the Separation of Species by the Dying out of connecting Links.

owing to their descent from common ancestors, which has become more or less sharply separated from all other eo-existing species by the disappearance of intermediate forms." Such a definition would be in accord with the practice of many systematic naturalists, who are in the habit of uniting species previously considered as distinct whenever intermediate forms are found, the former species being reduced to the rank of varieties of one and the same species.

It is obvious that the element of time must be taken into consideration in forming such a conception of species. At any

1 "Origin of Species." Ed. 6, p. 42. The reader should compare the very similar views of Lamarck on the species question, given in Chapter XXIV. 
given time the species living on the earth would form naturally limited groups, which we should be able to define more or less sharply if we had sufficient knowledge of the then existing fauna and flora to enable us to trace their boundaries. If, however, we were to include extinct forms in our survey, it is obvious that the number of existing species which we should recognize would be inversely proportional to the extent of our palæontological information, for the gaps between the survivors would gradually be filled up by the discovery of intermediate fossil forms.

Naturalists have long recognized the fact that there is another way in which more or less sharply defined groups of individuals may arise, and that is by the occasional and sudden appearance of " sports," or " mutations " as they are now generally called, ${ }^{1}$ which " breed true," handing on their special peculiarities from generation to generation. Darwin was of opinion that such sports differ only in degree from the ordinary variations to the accumulation of which he attributed the chief importance in the evolution of species. At the present day, as we have already had occasion to point out, some observers, and especially Professor Hugo de Vries, ${ }^{2}$ consider them to be totally different in kind from fluctuating or small successive variations, and regard them as affording the sole means by which new species originate.

According to the mutation theory any species may, from time to time, throw off such sports, either singly or in groups, and they are from the first distinguishable by well defined, though it may be minute, characters from the parent form. These mutations are regarded by de Vries as constituting " elementary species," which are already fully characterized and will not change again unless by giving rise to fresh mutations. De Vries maintains that many of the species of flowering plants as defined by Linnæus are really " aggregate species," each made up of a larger or smaller number of such elementary species. The common weed known as Draba verna, for example, regarded by Linnæus as constituting a single species, occurs under about 200 more or less distinct forms. All of these so-called elementary species are believed to come true from seed and to have arisen as sudden mutations.

1 Compare Chapter XI.

2 Vide "The Mutation Theory," by Hugo de Vries. English translation by Farmer and Darbishire (Kegan Paul, Trench, Trübner \& Co. Ld., London, 1910). 
According to this view a newly arisen species, at its first origin, is already sharply distinguished from the parent species and the extinction of intermediate forms is not required to separate the two.

The elementary species, however, though apparently constant in their characters, are often so similar to one another, and they are moreover so numerous, that for practical purposes of classification it would be undesirable to regard them as distinct species in the ordinary sense of the word. It is far more convenient to maintain the older view that they are merely varieties or subspecies, and their occurrence in no way invalidates our conception of the slow, tree-like evolution of the organic world. If de Vries be right as regards the importance he attributes to them as the sole means by which species have originated, which is extremely doubtful, it merely means that evolution has taken place in a rather more jerky fashion than we previously supposed. It will be necessary to return to this question when we come to discuss the factors of organic evolution. ${ }^{1}$

It is not sufficient for the purposes of classification to arrange the individual plants or animals with which we are acquainted in their respective species. These species in turn must be arranged in groups of higher order, which are termed genera. Each genus stands to the species included within it in much the same relation that the species themselves stand in with regard to individuals, and related genera, though often sharply definable, are only so by virtue of the disappearance of connecting links. The characters by which genera can be distinguished from one another are of a more deep-seated and fundamental nature than those which distinguish species. Individual opinion, however, differs greatly as to the exact limits which should be assigned to each, and it is a common thing for an old established genus to be subdivided into several as the result of the discovery of new species and the more exhaustive study of old ones. ${ }^{2}$ It is all a matter of convenience, and one may justifiably make a separate genus for any group of closely related species which can be distinguished by some common character from all other co-existing groups of species. Many naturalists, however, consider that the mutual relationships of species are more accurately expressed by making use of subgenera as intermediate groups between genera and species.

1 Vide Chapter XXVII.

2 A striking illustration of this is afforded by the recent history of the old genera Peripatus and Amphioxus, each of which is now subdivided into several.

B. 
Genera in turn are grouped in families, separated from one another by still more fundamental characters, and with subfamilies as intermediate groups where necessary. Families are grouped in suborders and orders, orders in subclasses and classes, classes in phyla (or subkingdoms), and phyla in kingdoms. Of kingdoms only two are recognized, animals and plants, and even these cannot be sharply separated from one another, because amongst the lowest forms of life (Protista) the distinction between animals and plants, as we have already seen, ceases to exist.

The arrangement of individual organisms in species, and the grouping of these to form genera, families, orders, classes, phyla and kingdoms, constitutes the work of the systematist and is usually spoken of as classification, while the study of the principles in accordance with which classification should be carried out forms a special branch of biological science sometimes known as taxonomy.

The first and greatest of the modern systematists was Karl von Linné, now usually known as Linnæus, a Swedish naturalist who was born in 1707 and died in 1778. In his great work, the "Systema Naturæ," which is now recognized as the starting point of modern systematic zoology and botany, he described all the species of plants and animals then known, and endeavoured to arrange them in a "natural system " in accordance with their mutual resemblances and differences. As an aid to the accomplishment of this formidable task he invented the binomial system of nomenclature, in accordance with which each kind of plant or animal is referred to by the name of the genus as well as by that of the species to which it belongs. The necessity for the constant repetition of longer or shorter descriptions as a means of identification every time there is occasion to refer to a particular species is thus avoided, while at the same time the use of the generic name makes it possible to employ the same specific name in a number of different genera without risk of confusion, a very important point in consideration of the enormous number of species which have to be distinguished from one another.

The binomial system of nomenclature has proved so well adapted to its purpose that it has survived practically unchanged to the present day, although the number of known species has increased enormously in the interval and the Linnæan system of classification has undergone profound modification at the hands 
of later investigators. Very many of the more familiar plants and animals are, however, still known by the names which Linnæus gave to them.

It still frequently happens that newly discovered species have to be named and new genera defined, and various attempts have been made to formulate a code of rules for the guidance of those engaged in this work. This is necessary in order to secure, so far as possible, uniformity of method, and to avoid confusion such as would arise by giving the same name to different genera or different species within a genus, or different names to the same genus or species. The chief points so far agreed upon are that generic and specific names (as well as those of larger groups) should be given in either Latin or Greek and that strict attention should be paid to the law of priority, the name first given taking precedence over any which may be proposed subsequently, as is often the case owing to ignorance of the work of previous writers.

The greatest latitude has, however, been allowed in the past with regard to the coining of generic and specific names. Even the resources of the Latin and Greek languages have proved hardly sufficient to meet all requirements, and many generic names have been given which, though euphonious enough, have no meaning whatever. It is said that one eminent naturalist (Dr. Gray) used to make up such names by drawing letters out of a hat, and it is certain that Dr. Leach derived the generic names of a whole series of parasitic crustacea (Cirolana, Conilera, Nerocila, \&c.) from anagrams on his wife's namê, Caroline. 'The most satisfactory names, on the other hand, are those which convey some definite information as to the characters of the genus or species concerned or as to the place where it is found.

If we have to classify a number of inorganic bodies we may set about our work in a variety of ways. We may arrange them according to colour, or size, or weight, or shape, or texture, or chemical composition, or according to the places they come from, the precise method adopted depending upon what it is that we wish to express by means of our classification.

It is exactly the same with plants and animals, the way in which we classify them will depend upon our point of view. A fisherman classifies fishes largely according to their food-value, and various other animals according to whether or not they are good for bait, and such classification serves its purpose quite satisfactorily. 
The aim which the zoologist or botanist sets before himself in classification is the expression of the "natural affinities" of the plants or animals investigated. The existence of such natural affinities was clearly recognized long before the explanation of them was known, and even by upholders of the doctrine of special

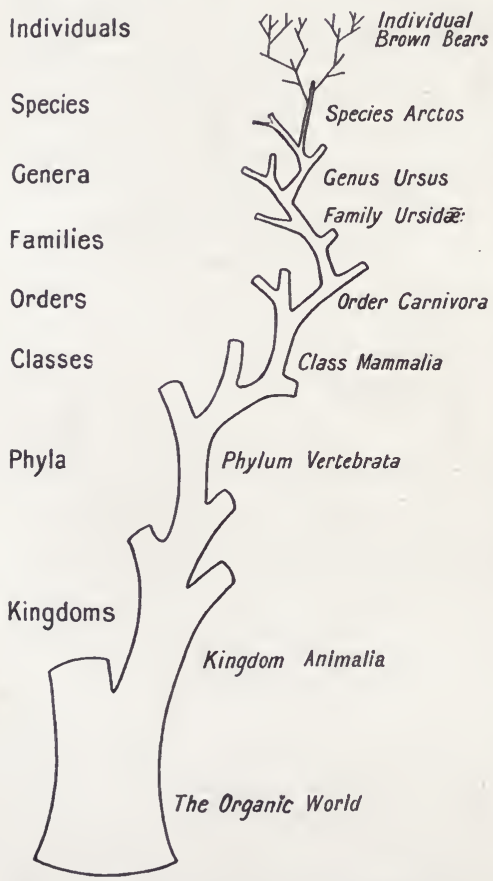

Fig. 86.-Diagram to show the Tree-like Form assumed by a natural Classification. creation. Thus it was well known to Linnæus that individuals fall naturally into species, that species may be grouped in genera, genera in families, and so on in ever widening circles. It also soon came to be recognized that natural affinities could be bestdetermined by taking into account as many characters as possible instead of relying merely on one or two. A system of classification based on a small number of characters only is always more or less artificial, and though it may serve its purpose for a time it will have to be amended by future workers who are able to bring a more complete knowledge to bear upon the problem. Thus it comes about that our views on the classification of the animal and vegetable kingdoms are always undergoing change, old systems are constantly being discarded as too artificial and more natural ones proposed in their stead.

As our knowledge progresses it becomes more and more evident that a natural classification assumes a tree-like form. The division of the whole organic world (with the exception of the 
lower Protista) into plants and animals is represented by the first branching of the tree, the division of each of these great kingdoms into phyla by the next branching, and the subdivision of phyla into classes, orders, families, genera, species and individuals by the further ramifications, as shown in the accompanying diagram (Fig. 86), which represents a mere fragment of the whole tree selected to show the systematic position of a single species of animal, the common brown bear, Ursus arctos.

This great truth affords one of the most striking pieces of evidence in favour of the theory of organic evolution, for the tree-like form assumed by a natural classification bears an unmistakable resemblance to the tree-like development of the whole organic world which evolutionists believe to have taken place. The two results represent, indeed, but slightly different aspects of the same truth; the resemblance between them is no mere coincidence, but the fact that we are able to classify organisms in a tree-like manner indicates very clearly that these organisms have been produced by tree-like evolution.

The systematist is now able to replace the vague groping after " natural affinities" by a much clearer conception of the aims of taxonomy. What he has to strive after is the elucidation of the actual pedigrees of existing organisms, the unravelling of what is termed their phylogeny or ancestral history. This means neither more nor less than the ultimate reconstruction of the whole vast tree of life. Piece by piece this is gradually being done, and it is the great German biologist, Ernst Haeckel, who has led the way in this particular department of biological investigation. Some of the main branches of the tree have already been reconstructed in a satisfactory manner, but it is a work of immense difficulty and practically unlimited extent, and at every step opinions differ as to the value of conflicting evidence and the interpretation of obscure facts. The greater part of the tree is dead and gone beyond recall, and such fragmentary information concerning extinct forms as may be supplied by the discovery of fossils can only be supplemented by indirect evidence derived from the study of existing organisms.

We must now inquire a little more fully into the relation between the tree-like form assumed by a natural system of classification, or the taxonomic tree, as we may term it, and the phylogenetic tree, which represents the actual pedigrees of organisms. In the former (Fig. 86) the main trunk of the tree, 
as we have seen, represents the entire organic world; in the latter it represents the remote Protistan ancestors from which both plants and animals have sprung. The first great branching represents in the one case the systematist's main division of organisms into vegetable and animal kingdoms, and in the other the gradual differentiation of the ancestral Protista (which at one

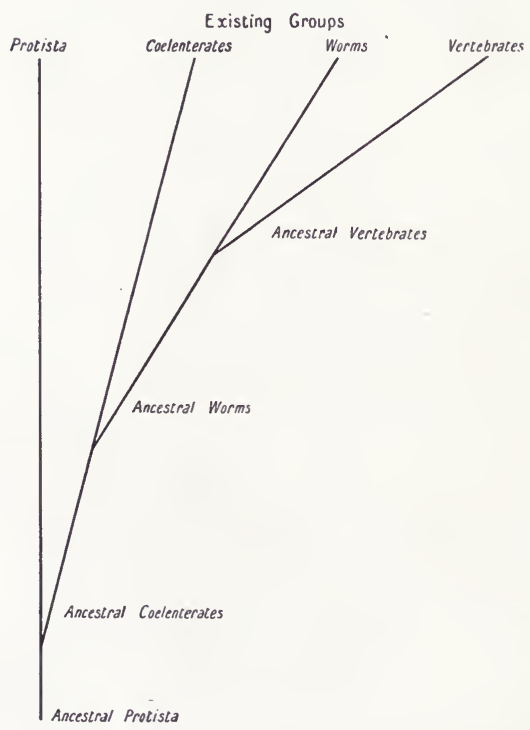

FIG. 87.-Diagram illustrating the Relation between Classification and Phylogeny. time constituted the entire organic world) into the primitive unicellular plants or Protophyta on the one hand and the primitive unicellular animals or Protozoa on the other; and so on with subsequent ramifications.

The parallelism between the two is sufficiently striking to justify us in believing that it would be complete if only our knowledge of classification and phylogeny were so; we should then doubtless see at once that the taxonomic tree and the phylogenetic tree are, after all, one and the same thing, for we should arrange all organisms strictly in accordance with the course of their evolution.

One point remains to be noticed in connection with the phylogenetic tree. The branching has been monopodial rather than dichotomous or polychotomous, each branch, in addition to giving off lateral branches, being itself continued on, so to speak, at each forking. The descendants of any given ancestral group did not all undergo modification to the same extent, but some adhered more or less closely to the ancestral condition and have continued to do so to the present day. Even the primitive 
ancestral Protista are still represented by descendants which probably differ but little from their remote progenitors and which have not yet reached the level of either plants or animals, and there are still living unicellular forms in abundance which, though they may perhaps be distinguished as plants or animals, have never been able to learn the secret of forming multicellular bodies. So it is with most of the great ancestral groups; they are represented still by descendants which have undergone comparatively little change in structure since remote geological periods. The algæ are still algæ, the colenterates are still cœlenterates and the fishes are still fishes, though each of these great groups has in past time given rise to descendants which have gradually become modified into higher types.

Many subordinate groups, such as the trilobites and ammonites and the winged reptiles of the secondary period, have of course become extinct during past geological ages, but the fact remains that even at the present day there still exist on the earth organic types which represent, in a more or less unmodified form, all the principal stages of organic evolution, and thus it is that, even if we had only living plants and animals to consider, our classification of the organic world would still assume a tree-like form, with the simplest organisms at the bottom and the most complex at the top of the tree. It would be difficult indeed to explain this fact in accordance with the theory of special creation and immutability of species.

The monopodial branching of the organic tree and the relation which the natural classification of existing animals bears to the phylogenetic or ancestral series is diagrammatically represented in Fig. 87. On the right hand side are shown some of the principal stages in the evolution of vertebrates from ancestral Protista, and at the top of the tree are shown the existing groups by which these stages are actually represented at the present day. Only a few stages are represented and the great majority of the lateral branches of the tree are supposed to have been lopped off. 


\section{CHAPTER XVII}

Connecting links - Homology and analogy - Convergent evolutionChange of function-Vestigial structures-Reversion.

Is presenting the evidence of organic evolution it is convenient to separate that derived from the study of comparative anatomy from that afforded by taxonomy, but the separation is not a logical one, for classification is necessarily based upon comparative anatomy, and much of the evidence might be equally well dealt with under either heading.

The mere fact that we are able to arrange existing organisms in progressive series, of which the extremes are connected by intermediate forms, in itself suggests that one form has been derived from another. This gradation is equally obvious whether we study entire organisms or confine our attention to their component parts or organs. It is illustrated very clearly by the accompanying diagram of the modifications exhibited by the siliceous spicules of certain sponges (Fig. 88). These microscopic organs of the sponge, each of which arises within a single cell, exhibit an astonishing diversity and beauty of form. The extreme types are as different from one another as we can well imagine, but in the particular group of sponges from which our illustration is taken (the Tetraxonida) all may be derived from the same four-rayed architype (number 1 in the centre of the figure) and numerous intermediate forms mark out the lines along which evolution has taken place. An analogous diagram, with a triaxonid architype, could easily be constructed for those remarkable deep-sea sponges known as the Triaxonida or Hexactinellida, and another for the calcareous sponges.

Amongst the higher animals also innumerable illustrations of the same general principle of gradation in structure, even in coexisting types, are met with, and are to be explained in the way indicated in the last chapter, as expressions of phylogenetic relationship. Take, for example, those now widely contrasted groups of vertebrate animals the Reptilia and the Mammalia. The 
reptiles as a group are distinguished from the mammals in many ways. Amongst other things they usually have a scaly epidermic covering, but they never have hair; they lay large,

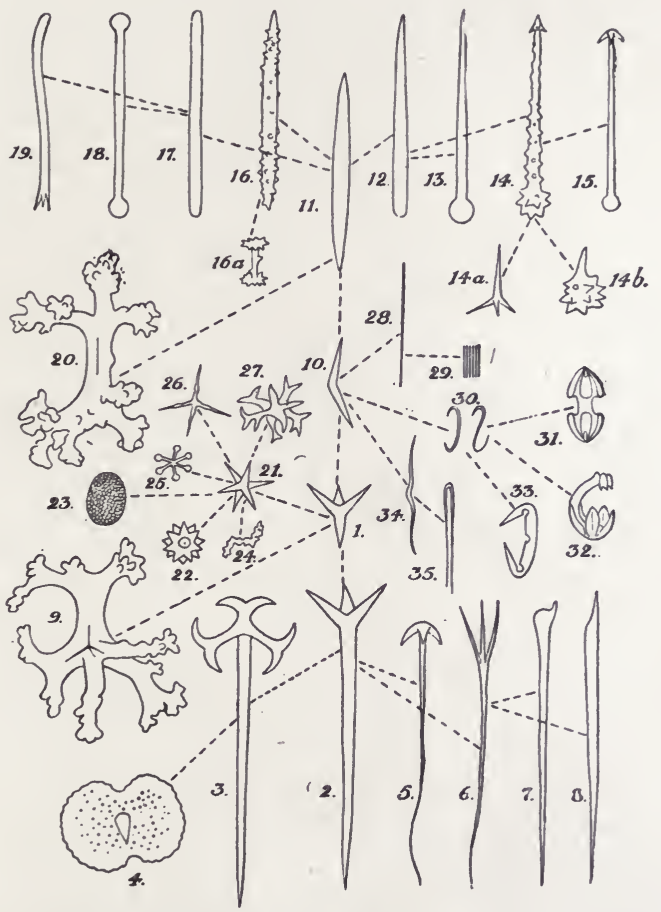

Fig. 88.- Series of tetraxon Sponge Spicules, showing how all may be derived from the same fundamental type, highly magnified. (From Dendy, Article "Sponges" in Encyclopiedia Britannica, Ed. XI.)

heavily-yolked eggs and do not suckle their young; they have a cloaca into which the urinary and genital ducts, as well as the alimentary canal, discharge; they have a very complex shoulder girdle (Fig. 89), consisting of a considerable number of more or less separate bones or cartilages, 
including suprascapula, scapula, coracoid, epicoracoid, clavicle and interclavicle.

The mammals, on the other hand, are characterized by the possession of an epidermic covering of hairs; they generally have very minute eggs almost destitute of yolk, and they always suckle their young; they usually have no cloaca but separate openings for the alimentary canal and urino-genital ducts, and the shoulder girdle (Fig. 90) is very greatly reduced, being often represented by only a single bone, the scapula, with a small

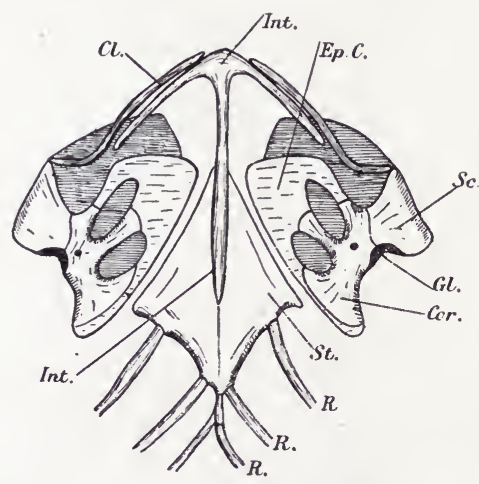

FIG. 89.-Shoulder Girdle and Sternum of a Lizard (Varanus) seen from below.

Cl. Clavicle; Cor. Coracoid; Ep. C. Epicoracoid; $G l$. Glenoid cavity; Int. Interelavicle; $R$. Ribs; $S c$. Scapula; $S t$. Sternum.

remnant or vestige of the coracoid completely fused with it, though a slender clavicle is sometimes present as well.

There exists in Australia and some of the adjacent islands, however, a small but very interesting group of animals known as the Monotremata, represented by the duck-billed Platypus (Ornithorlıynchus, Fig. 91) and the spiny anteater (Echidna, Fig. 92). The fact that these animals possess hair and suckle their young justifies us in classifying them amongst the Mammalia, but at the same time they exhibit certain characters in respect of which they differ from all typical mammals and agree with the reptiles. Thus they lay large, heavily-yolked eggs, the alimentary canal and urinogenital ducts discharge into a common cloaca, and the shoulder girdle (Fig. 93) is made up of a considerable number of separate bones almost exactly as in reptiles, most remarkable amongst which is the interclavicle, which is found in no other mammals.

Here then we have animals still existing which undoubtedly occupy an intermediate position between the reptiles and the typical mammals, and the three groups exhibit a progressive series of structural peculiarities. Such definite connecting links as the 
Monotremata, however, are more usually met with amongst the extinct animals of past geological periods. They indicate the paths along which the more highly organized groups have progressed during their evolution from more lowly organized ancestral groups, which latter may or may not still be represented by surviving forms at the present day.

It would be difficult to explain the occurrence of such graduated series of organs and organisms by the theory of special creation, and it would be no less difficult to explain in this way those remarkable facts of comparative anatomy which are grouped

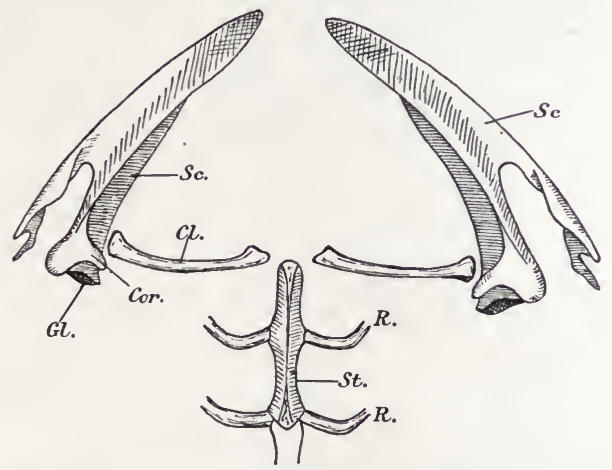

Fig. 90.--Shoulder Girdle and part of Sternum of a Rabbit, seen from below. Cl. Clavicle; Cor. Coracoid process of Scapula (=Vestige of Coracoid); Gl. Glenoid cavity; $R$. Ribs; Sc. Scapula; St. Sternum.

together under the terms homology and analogy (or homoplasy) to which we shall next refer.

We have already had occasion to point out that all living organisms are more or less perfectly adapted to the conditions under which they exist, and are accordingly provided with organs suitable for their various requirements. Thus all typical vertebrates have organs of locomotion formed from two pairs of limbs, but these differ very greatly in form and structure in accordance with their adaptation to very diverse conditions of life. In terrestrial vertebrates-amphibians, reptiles and mammals-both pairs of limbs usually take the form of ambulatory legs, adapted for moving the body on dry land. In birds the hind limbs are generally used for the same purpose and similarly constructed, but 
the fore limbs are modified to form wings for flying in the air ; and the same is true of the fore limbs of bats and of the extinct

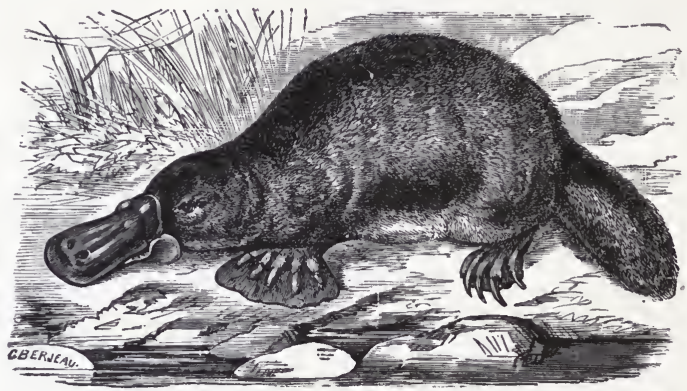

Fig. 91.-The Duck-billed Platypus (Ornithorhynchus anatinus). (From British Museum Guide.)

flying reptiles known as Pterosauria (pterodactyls). The Cetacea (whales and porpoises), the Sirenia (dugongs and manatees) and the seals, amongst mammals; and the turtles and extinct

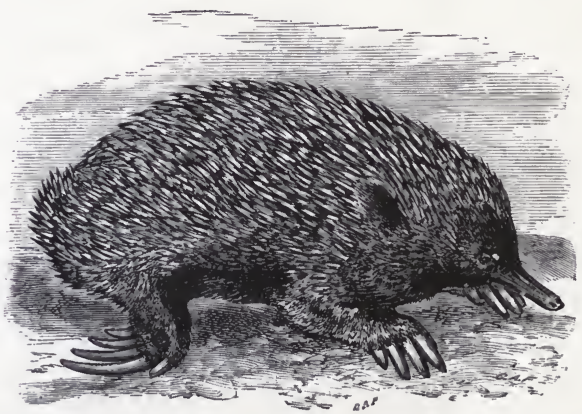

FIG. 92.-The Echidna or Spiny Anteater (Echiảna aculeata). (From British Museum Guide.)

Ichthyosauria and Plesiosauria amongst reptiles, on the other hand, have limbs which take the form of paddles and are specially adapted for swimming in water.

We find, then, three chief types of locomotor organs amongst 
the air-breathing vertebrates: ambulatory legs, wings and paddles, employed in totally different methods of locomotion and differing widely from one another in general form and appearance. Yet when we come to examine these organs closely we very soon discover the remarkable fact that all are constructed on essentially the same plan; all belong to what is known as the pentadactyl or five-fingered type of appendage.

We ourselves retain this type of limb-structure in a comparatively primitive condition (Fig. 94), although our fore limbs have taken on new functions as organs of prehension. The fore limb

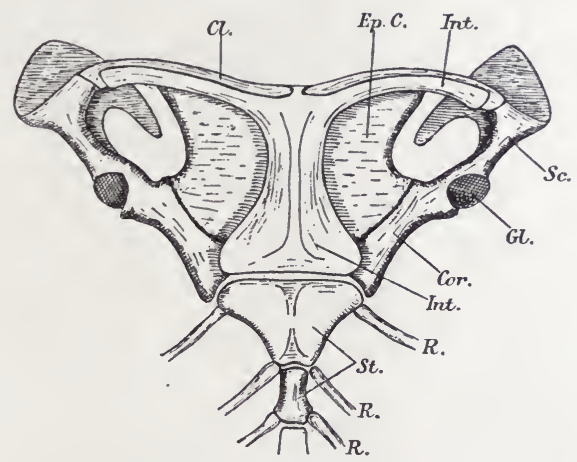

FIG. 93.-Shoulder Girdle and part of Sternum of Ornithorhynchus, seen from below.

Cl. Clavicle; Cor. Coracoid; Ep. C. Epicoracoid; Gl. Glenoid cavity ; Int. Interclavicle ; $R$. Ribs; Sc. Scapula; St. Sternum.

consists of arm, forearm, wrist and hand (manus) with five fingers ; the hind limb of thigh, leg, ankle and foot (pes) with five toes. Each segment of the limb is supported by an internal skeleton of bone which is clothed with muscle and skin. The bones are articulated with one another at the joints and moved like so many levers by the contraction of the muscles attached to them. The bone of the arm is the humerus and the corresponding bone of the thigh is the femur. In the forearm we find the radius and ulna and these are represented in the leg by the tibia and fibula. The wrist is made up of a number of small carpal bones and the ankle of a number of more or less similar tarsals. These small bones are arranged mainly in two rows, and the distal or far row 
affords support to the five metacarpal bones in the palm of the hand or the five corresponding metatarsal bones in the foot. Each metacarpal or metatarsal bone in turn forms the support of a series of phalanges which constitute the skeleton of the fingers or toes. The thumb and great toe have each two phalanges and each of the other digits has three.

When the human arm is stretched out at right angles to the long axis of the body, with the thumb towards the head, it is in the primitive position of the pentadactyl limb. The thumb and the radius then lie on what is called the preaxial or anterior border,

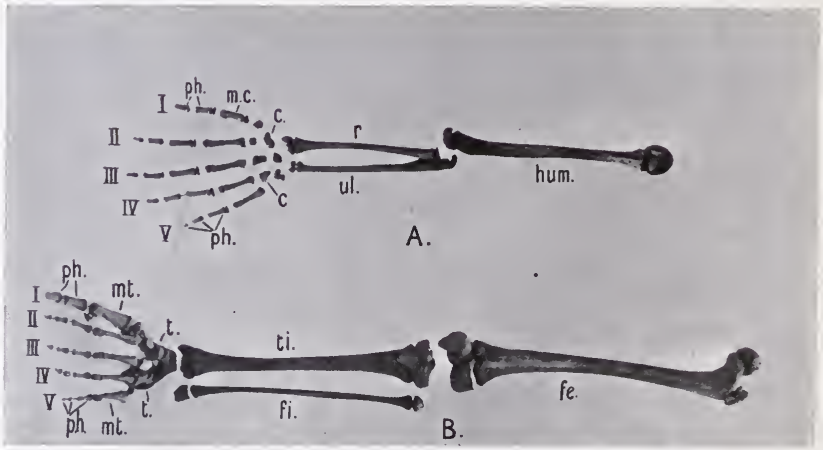

Fig. 94.- Skeleton of the fore $\operatorname{Limb}(\mathrm{A})$ and hind $\operatorname{Limb}(\mathrm{B})$ of Man, showing the pentadactyl Structure. (From photographs.)

c. carpals; fe. femur; fi. fibula : hum. humerus; m.c. metacarpals; mt. metatarsals ; ph. phalanges; r. radius; t. tarsals; ti. tibia; ul. ulna; I-V, digits.

the little finger and the ulna on the postaxial or posterior border of the limb. For convenience of comparison with other types the digits are numbered from in front backwards, the thumb being No. I and the little finger No. V. Similarly in the hind limb, which in man has become permanently twisted out of its primitive position, it is easy to show that the great toe is really the preaxial digit (No. I) and the little toe the postaxial (No. V). In Fig. 94 both limbs are represented in the primitive position.

Starting from the primitive pentadactyl type let us next inquire more closely how different groups of air-breathing vertebrates have solved the problems of locomotion on land, in air and in water.

In considering the modifications of the limbs adapted for 
locomotion on dry land we may conveniently confine our attention to the hoofed mammals or Ungulata. In the elephant the limbs remain, as in man, in a primitive condition, with five well developed digits in each. The entire limb is stout and massive and the foot ${ }^{1}$ remains very short and affords a broad support for

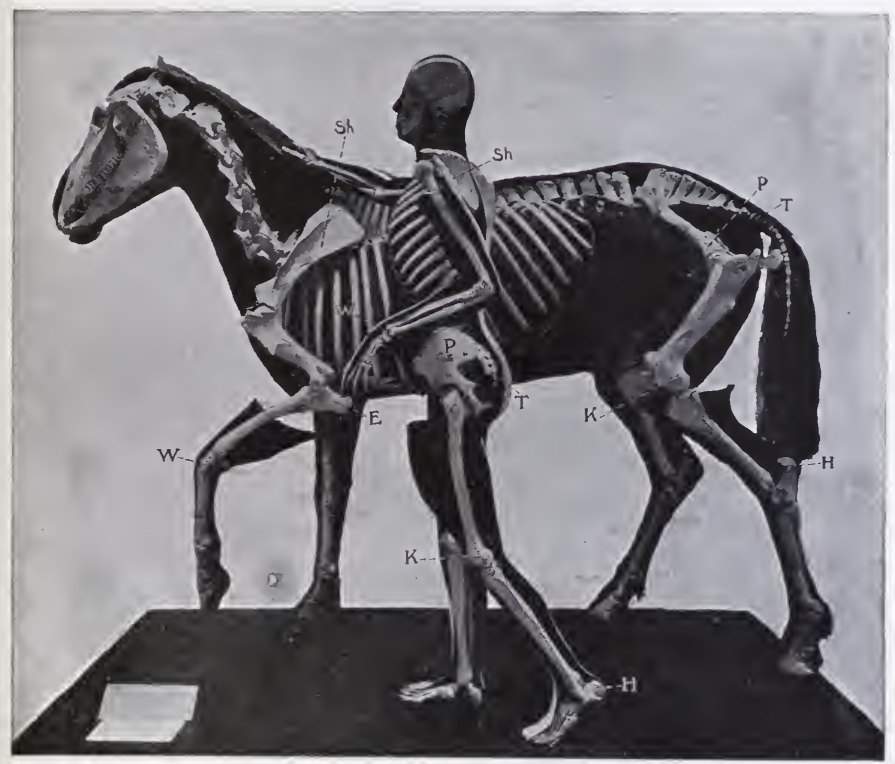

FIG. 95.- Skeletons of Man and Horse, photographed from a group in the Natural History Museum. (From Lankester's "Extinct Animals.")

E, Elbow bone (olecranon process of ulna); H, Heel bone in ankle (the hock of the horse); K, Knee joint (the stifle of the horse); P, Hip bone; Sh, Shoulder bone (scapula); T, Vertebræ of tail (coccyx in man); W, Wrist or carpus (the so-called knee in the horse's fore leg).

the heavy body. This type of limb is somewhat clumsy and not adapted for very rapid locomotion.

In the more typical ungulates we always find a reduction in the number of digits, accompanying an uplifting of the hinder part of the foot from the ground, until finally the animal comes to

1 The term foot in the case of quadrupeds is commonly used to include both manus and pes. 
walk and rest on the extreme tips of one or two toes only. The assumption of this " unguligrade" condition is accompanied by elongation of the limb and especially of the remaining metapodial bones (metacarpals and metatarsals), so that the wrist and ankle in the horse, for example, are uplifted high above the ground and form the so-called knee and hock respectively (Fig. 95).

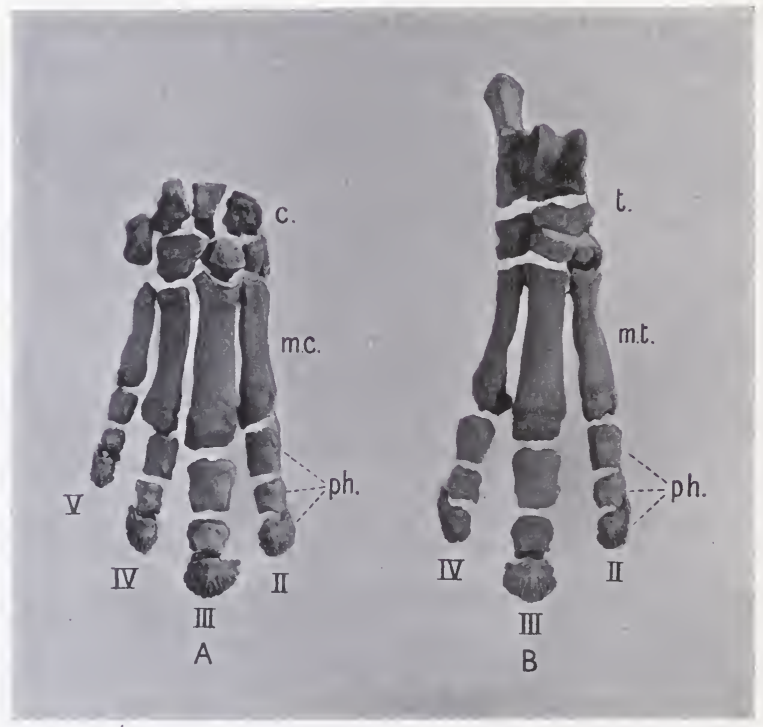

FiG. 96.-Skeleton of (A) the right fore Foot and (B) the right hind Foot of a Tapir, $\times \frac{1}{6}$. (From photographs.)

c. carpals; m.c. metacarpals; m.t. metatarsals; ph. phalanges; $t$, tarsals; II-V, digits.

It is also accompanied by a reduction of the ulna in the fore limb and of the fibula in the hind limb to mere vestiges. Thus the entire limb becomes long and slender and adapted for rapid locomotion by running on hard open ground.

The disappearance of digits from the manus and pes in the Mammalia follows a very simple law. The first to disappear is always the preaxial digit (No. I), which is the shortest of the series (having only two phalanges while the remainder have 
three each) and consequently the first to leave the ground as the hinder part of the foot is uplifted. The next to go is the postaxial digit (No. V) from the opposite side, the next No. II and the next No. IV.

In some ungulates the long axis of the foot passes through the middle digit (No. III) and in others between the third and fourth digits. The true ungulates are accordingly divided into two series, oddtoed or perissodactyl and even-toed or artiodactyl.

To the former belong the tapir (Fig. 96), with four digits in the fore foot and three in the hind; the rhinoceros, with four or three digits in the fore foot and three in the hind, and the horse (Fig. 97), with only the middle (third) digit remaining in both manus and pes, but with vestiges of Nos. II and IV in the functionless "splint-bones" which lie alongside the greatly elongated metapodial (= metatarsal or metacarpal) of No. III.

In the artiodactyl series (Fig. 98) we find the hippopotamus, with four welldeveloped toes on each foot; the pig, with four toes on each foot but the two outer ones greatly reduced; the deer, with the two outer digits still further reduced; the sheep and oxen, with the second and fifth digits reduced to small nodules of bone, and the camels and llamas, in which all traces of digits other
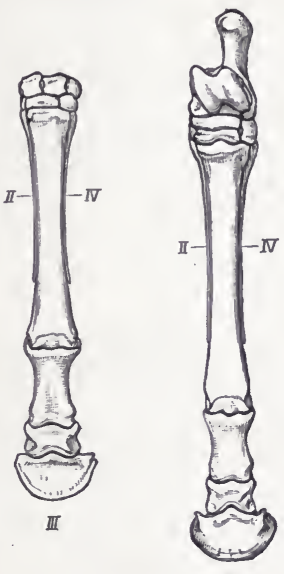

III

FIG. 97.-Fore and hind Feet of a Horse, showing reduction to a single functionul Digit (No. III) with Vestiges of two others (II and IV) in the form of splint bones, $\times \frac{1}{8}$. (From Lull, after Marsh.) than the third and fourth have completely disappeared. The gradual reduction of the number of digits in the artiodactyl series is accompanied by fusion of the two remaining metapodials to form a single "cannon bone" (well shown in Fig. 98, deer and camel), whereby the now greatly elongated and uplifted foot acquires much greater strength and rigidity.

It is very easy to see that all the different varieties of perissodactyl and artiodactyl limbs are modifications of one and the same primitive pentadactyl type. We must next 
consider the way in which this type has been adapted to form organs of flight in different vertebrate groups.

The wing of the extinct pterodactyls (Fig. 99) was formed
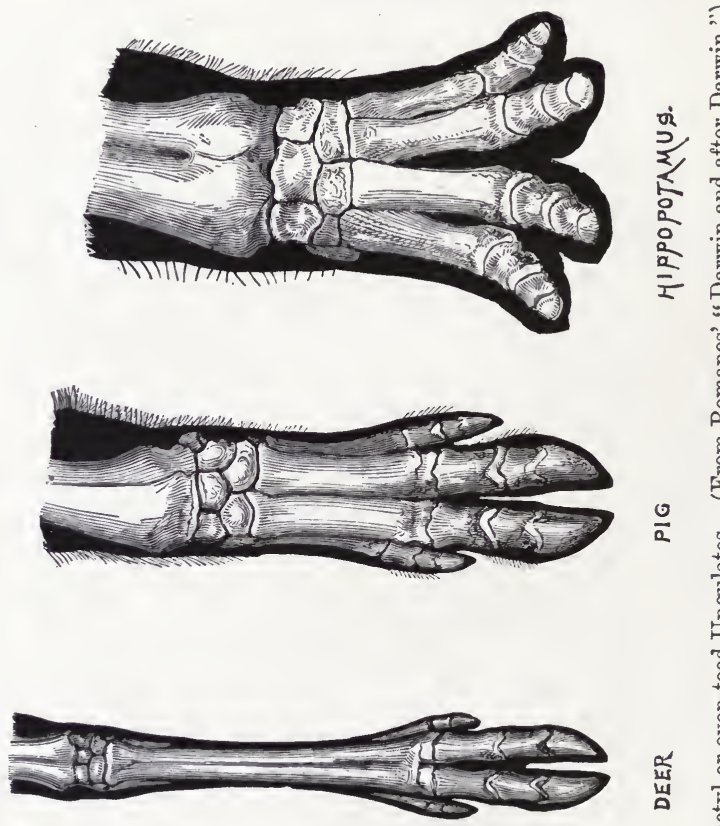

$\frac{1}{2}$

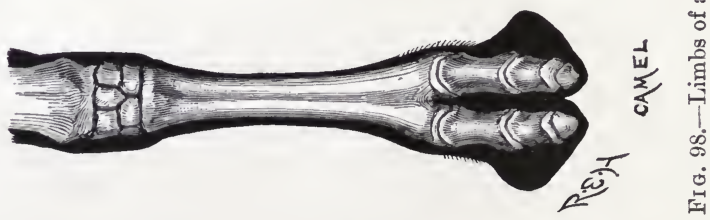

by an expansion of the skin of the body stretched between the fore and hind limbs, and the phalanges of the little finger were enormously elongated so as to aid in the support of its anterior 
margin. The surface of the flying membrane was probably covered with scales. In the bats (Fig. 99) four of the digits

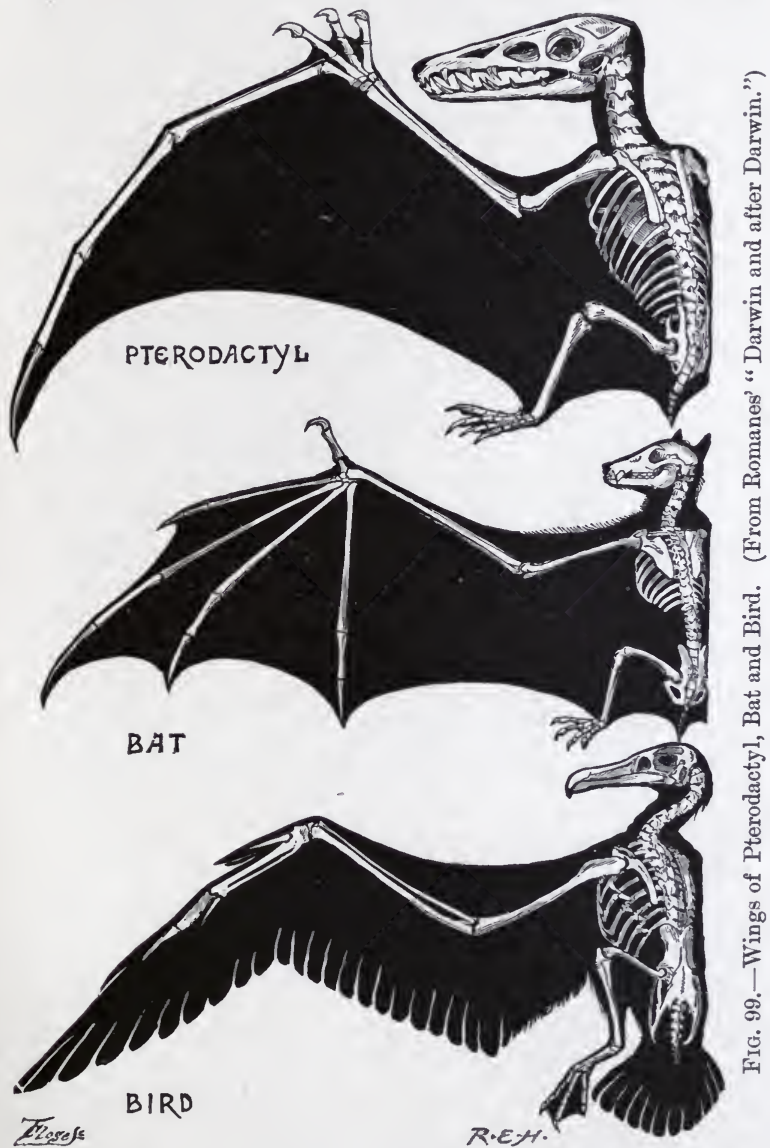

of the hand, Nos. II $-\mathrm{V}$, are elongated and take part in the support of the flying membrane, the surface of which is either naked or covered with fine hair. In the birds (Fig. 99) the 
flying membrane is formed chiefly of feathers, and the distal part of the wing is supported mainly by the second digit; the first and third digits are greatly reduced, and the other digits are absent. In all these different types of wing the skeleton is again clearly of the pentadactyl type, modified by enlargement, diminution or total suppression of certain of its constituent bones.

In the paddles of the modified aquatic mammals, such as the sirenians, seals (Fig. 100) and whales (Fig. 101), the whole limb

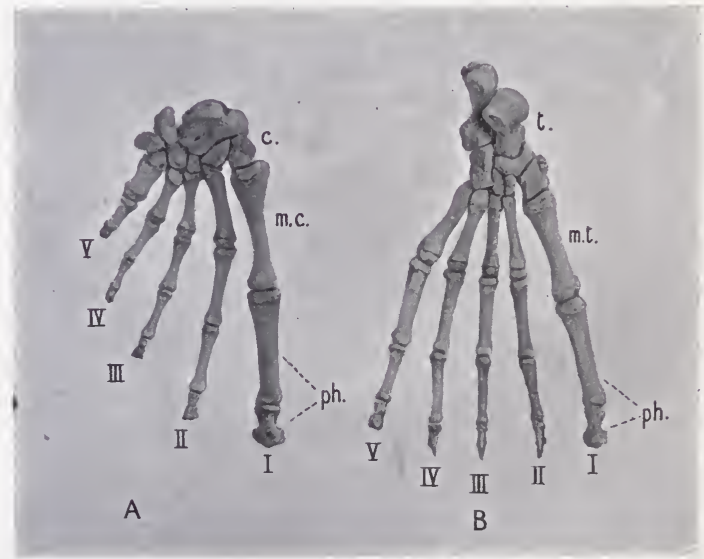

Fig. 100. - Skeleton of right fore Foot (A) and right hind Foot (B) of a Seal (Otaria hookeri), $\times \frac{1}{6}$. (From photographs.)

c. carpals ; m.c. metacarpals ; m.t. metatarsals; ph. phalanges; I-V, digits.

is very much shortened and flattened and the digits are enclosed in a common integument so as to offer a greater resistance to the water, but the skeleton still reveals the essential pentadactyl features, and the same is true of those aquatic reptiles, the turtles, plesiosaurians (Fig. 141) and ichthyosaurians (Fig. 142). In the whales, however, a curious increase in the number of phalanges (hyperphalangy) may often be observed, and in the ichthyosaurians supplementary small bones are developed in such positions that the entire skeleton of the paddle assumes the form of a mosaic pavement. 


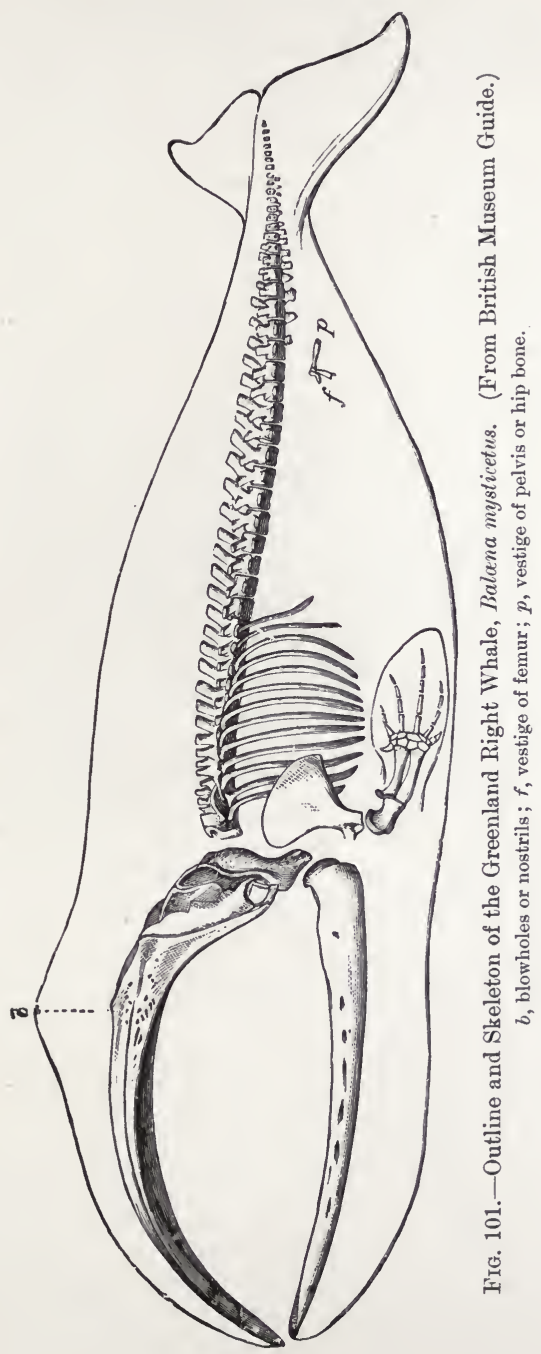


We can only explain the occurrence of the same type of skeleton-and that a very complex type-in all these different kinds of organs of locomotion on the assumption that it has been inherited from common ancestors. We cannot believe that one and the same type of skeleton was necessarily the most suitable for all these different cases, including ambulatory legs, wings and paddles, and was therefore specially and independently created for each. We must conclude rather that each organism receives a certain kind of material by inheritance from its ancestors and has to adapt it to its own requirements as best it may; has, in short, to cut its coat according to its cloth, and, whatever the

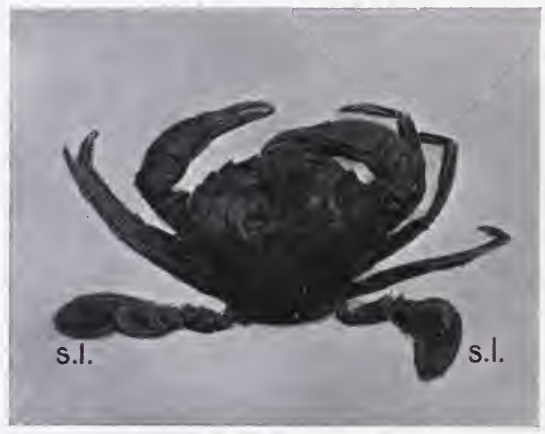

Fig. 102.-A swimming Crab (Portunus depurator), showing jointed ambulatory appendages and also swimming appendages (s.l.). (From a photograph.)

shape of the coat may ultimately develop into, the cloth will retain, more or less evidently, traces of the original pattern.

This conclusion is greatly strengthened when we turn to other groups of animals and see how they have solved the same problems with the aid of different materials. The vertebrates are not the only animals which have organs of locomotion in the form of jointed appendages. Many members of the great group Arthropoda-insects, crustaceans and spiders-have ambulatory limbs which externally bear considerable resemblance to those of vertebrates. If we examine these limbs, however, we find that they are constructed upon a wholly different plan. In the first place the skeleton is external instead of internal, and is composed 
of the hard cuticle secreted by the epidermis, there being no true bone. The muscles therefore lie inside the skeleton instead of outside. The number and arrangement of the limb-segments, again, is totally different, and there is, of course, no pentadactyl structure (compare Fig. 102).

Thus, with different material at their disposal, the arthropods have solved the problem of constructing an ambulatory appendage in a very efficient manner, but quite differently from the vertebrates. Many of them have also solved the problem of locomotion in water by modification of certain of the limbs to form paddles, as in the case of the swimming crab (Fig. 102). Many insects, on the other hand, have acquired the power of flight by means of wings, which, though bearing some external resemblance to those of vertebrates, are totally different in structure and origin not only from the latter but also from the other appendages of the arthropods themselves.

We are now in a position to define the meaning of the terms homology and analogy as used by biologists. Homologous organs are such as have the same essential structure, which they owe to inheritance from common ancestors, though they may be very differently modified in adaptation to different functions. The pentadactyl limbs of air-breathing vertebrates, however much they may differ amongst themselves, are all homologous organs in so far as their essential pentadactyl structure is concerned.

Analogous or homoplastic organs, on the other hand, bear only a superficial resemblance to one another, which they owe not to common ancestry but to adaptation of fundamentally different structures along similar lines for similar functions. The ambulatory appendages of arthropods and vertebrates are analogous but not homologous organs, so also are the wings of birds and insects.

The evolutionary process by which analogous but not homologous structures have come to resemble one another is sometimes spoken of as convergence, and the result may be looked upon as an illustration of the general principle that similar causes tend to produce similar effects. The necessity for the adaptation of different organs and organisms to the same environment and the same mode of life results in a superficial resemblance between the organs and organisms thus adapted.

One of the most familiar examples of convergent evolution 
is afforded by the whales, dolphins and porpoises (Figs. 101 and 161) with their extraordinary external ressmblance to fishes. 'The fore limbs, as we have already seen, are modified to form paddles, but they retain the pentadactyl structure and are thus totally different from the fins of fishes, which have never reached the pentadactyl stage. The hind limbs have completely disappeared, but vestiges of the limb bones and their supporting girdle, which in some cases are found buried deeply beneath the skin in the pelvic region (Fig. 101, $f, p$ ), still bear witness to their former presence. The powerful tail fin has obviously no real relationship to the tail fin of a fish, for it

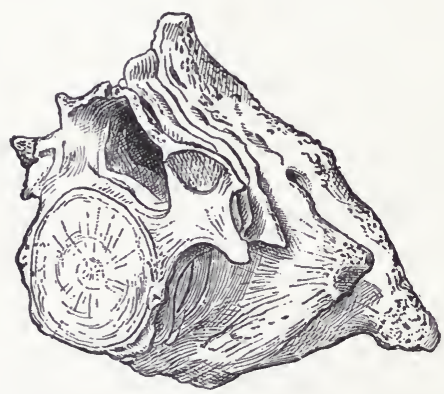

FIG. 103.-The seven cervical Vertebræ of a Whale, fused together in one Mass. (From Reynolds' "Vertebrate Skeleton.") throughout the mammalian series, but owing to the extreme shortness of the neck these vertebræ are all crushed together to form practically a single bone (Fig. 103). In the giraffe we also find seven cervical vertebræ, but they are all greatly elongated in accordance with the enormous length of the neck (Fig. 104). On the hypothesis of special creation we should certainly have expected the whale to have fewer vertebræ in its neck than the giraffe, and we can only suppose that the number seven has been inherited from some common mammalian ancestor. The resemblance of the whale to the fish, in short, is simply due to the fact that both have acquired the external form best adapted for an active aquatic life.

The wings of pterodactyls, bats and birds are equally good 
examples of convergent evolution within much narrower limits. They can only be regarded as homologous to the extent of all being

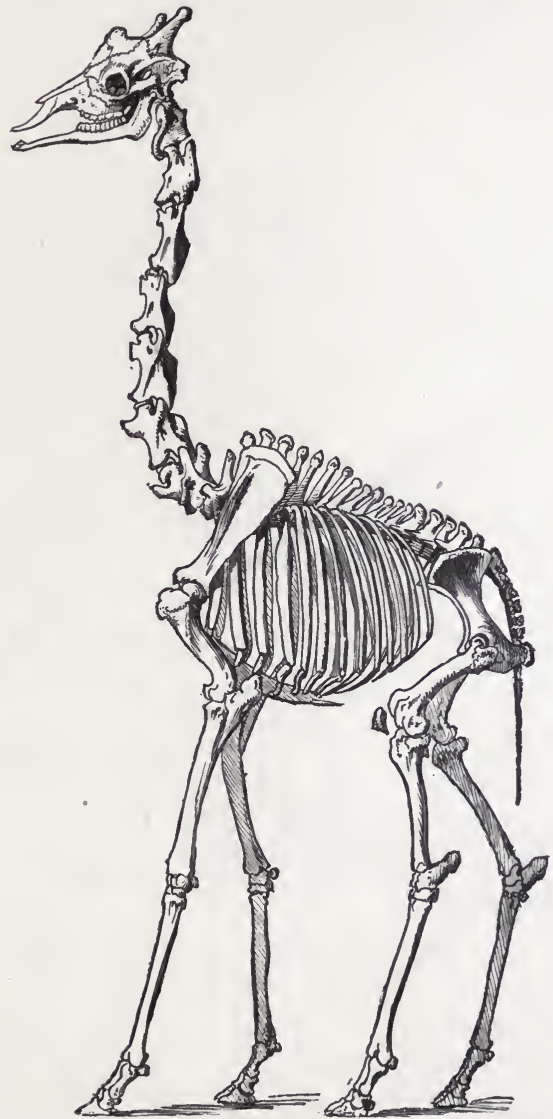

FIG. 104. Skeleton of the Giraffe, showing the seven separate and greatly elongated cervical Vertebra. (From Brehm's "Thierleben.")

pentadactyl. They do not owe their special characters as wings to descent from a common ancestral winged form, but have been 
evolved independently from more primitive pentadactyl types of limb.

We find analogous cases in other vertebrate groups. Several of the lizards, such as the so-called slow worm or blind worm (Fig. 105) and the "glass snakes" (Ophisaurus), have, by loss of their limbs, come so closely to resemble snakes as to be

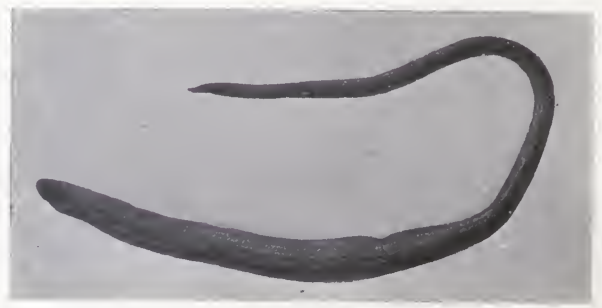

FIG. 105.-The Slow Worm, Anguis fragilis, $\times \frac{1}{3}$. (From a photograph.)

indistinguishable by most people. The Coeciliidæ (Fig. 106) amongst amphibians, the Amphisbænidæ amongst lizards and the Typhlopidæ amongst snakes have all adapted themselves to a burrowing underground life, like the earthworms. Their limbs have completely disappeared, the body has become cylindrical and worm-like and they have lost the use of their eyes, but though they

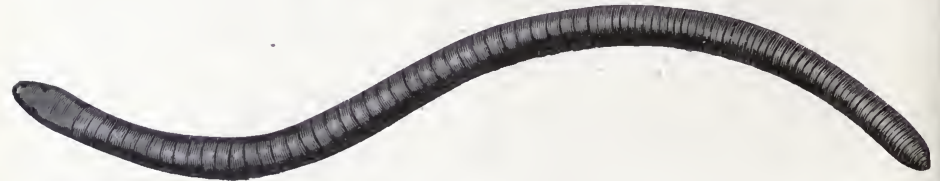

FIG. 106.-A worm-like, limbless Amphibian, Urcentyphtus africanus. (From British Nuseum Guide.)

have all come to resemble one another closely as the result of convergent evolution they are not in reality at all nearly related. The loss of limbs, the assumption of the worm-like form, \&c., have taken place quite independently in each group.

The mammalian fauna of Australia consists mainly of members of the single order Marsupialia or pouched mammals, but different representatives of this order have come, by convergence, to resemble closely members of widely different orders of 
mammals found in other parts of the world. The so-called marsupial wolf of Tasmania (Thylacinus) closely resembles a typical carnivore in its habits and general structure. Its teeth are adapted for a predaceous life, and the entire skull (Fig. 107, B), with its dentition, bears an extraordinary general resemblance to that of a dog (Fig. 107, A), being distinguishable only by details of structure which would hardly be noticed except by an

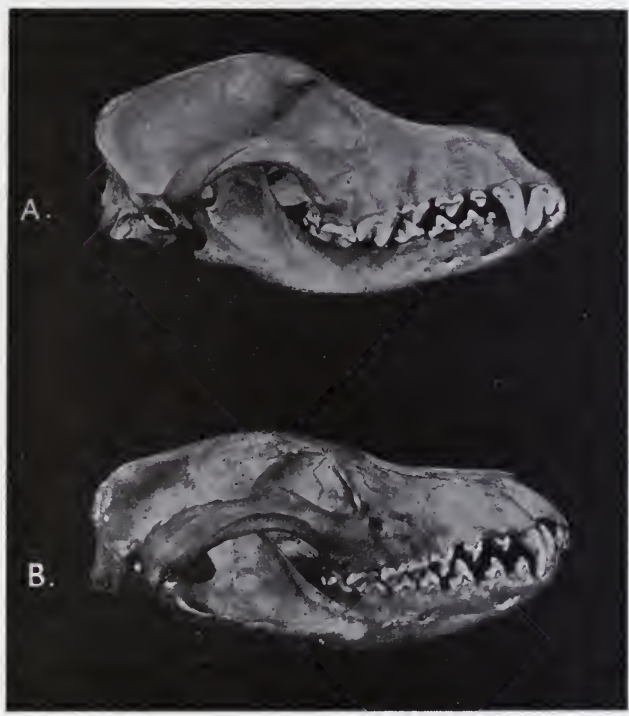

FiG. 107.-A. Skull of Dog, side view; B. Skull of Thylacine, side view. (From photographs.)

anatomist. These details, however, are quite sufficient to show that there is in reality no close relationship between the two. Thus the dog (Fig. 108, A, A $A_{1}$ ) has in each upper jaw three incisor teeth $\left(\mathrm{i}^{1}{ }^{1}-\mathrm{i}_{.^{3}}\right)$, one canine (c.), four premolars (p.m. ${ }^{1}-$ p.m. $\left.{ }^{4}\right)$ and two molars $\left(\mathrm{m}^{1}{ }^{1-}-\mathrm{m}^{2}\right)$; and the dentition of the lower jaw is the same except for the presence of a third molar (m.3), which is, however, in a vestigial condition. The thylacine, on the other hand (Fig. 108, B, B ), has four incisors $\left(\mathrm{i} .^{1}{ }^{1}-\mathrm{i}^{4}\right)$ in the upper and 

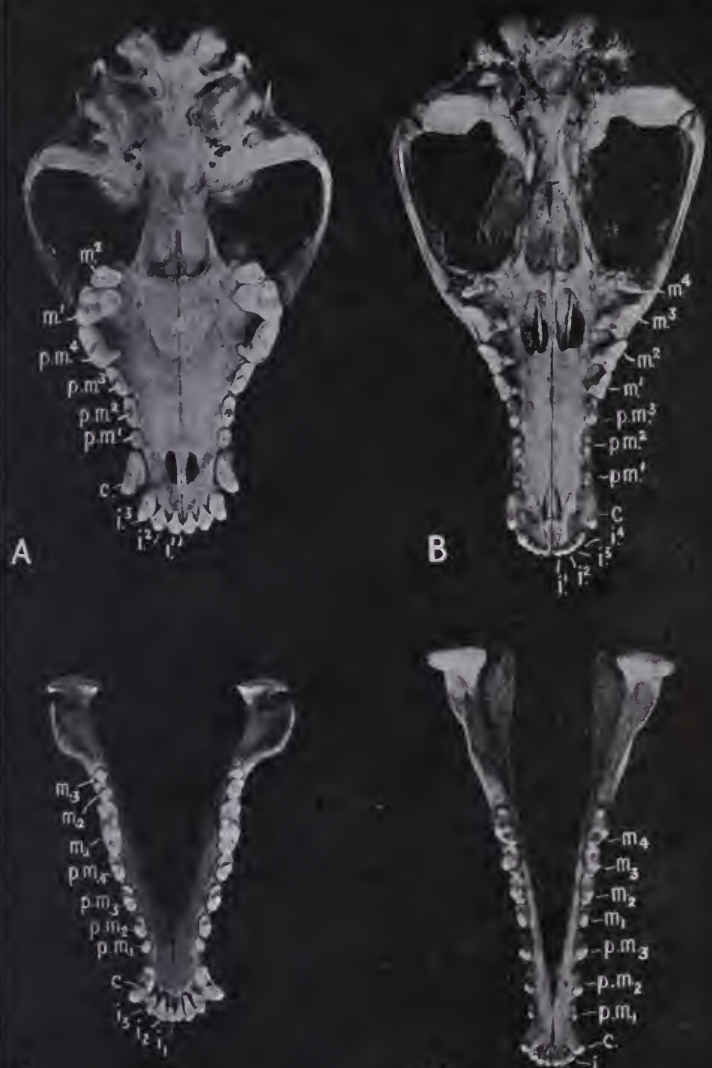

$A_{1}$

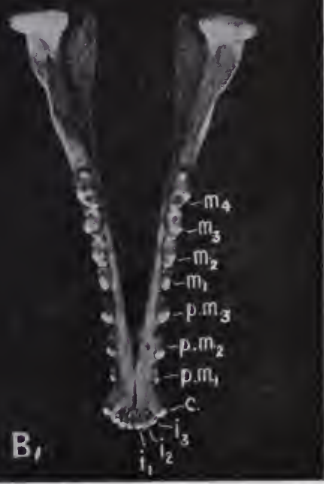

FIG. 108.-A, $A_{1}$, Skull and Mandible of Dog; B, $B_{1}$, Skull and Mandible of Thylacine, to show the arrangement of the teeth, \&c. (From photographs.)

$i_{.}{ }^{1}$. - i.4, incisors of upper jaw; $i_{.1}-i_{.3}$, incisors of lower jaw ; c., canine; p.m. ${ }^{1}-$ p.m.4,

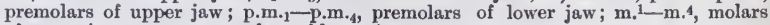
of upper jaw ; $\mathrm{m}_{\cdot 1}-\mathrm{m}_{\cdot 4}$, molars of lower jaw. 
three (i.1-i.s) in the lower jaw; and one canine (c.), three premolars (p.m.1-3) and four molars (m.1-4) in each jaw. The thylacine skull is further distinguished from that of the dog by the presence of large vacuities in the hinder part of the bony palate and by the strongly marked inflection of the angles of the mandibles. These two characters, both of which are shown in the illustrations, as well as the arrangement of the cheek teeth and other minor features which it is unnecessary to specify, are fundamental peculiarities of the great group Marsupialia and at once indicate the true affinities of Thylacinus.

'The case of the Australian marsupial mole, Notoryctes, is equally striking. The powerfully built digging limbs, the soft, close fur, the absence of external ears and the loss of sight, as well as the general shape of the body, all in adaptation to its burrowing habits, cause it to assume a wonderfully mole-like aspect, while in reality it comes nowhere near the true moles as regards genetic relationship.

Perhaps the most remarkable of all known cases of convergent evolution, however, is met with in the Ungulata. We have already seen that amongst the typical ungulate mammals of the present day we can distinguish two series, odd-toed or perissodactyl, and even-toed or artiodactyl. In the odd-toed series the reduction of the digits culminates in the horse, with a single perfect digit in each foot and vestiges of two others in the form of splint-bones. This extreme modification of the unguligrade type is so peculiar that it is difficult to believe that precisely the same line of evolution has been followed independently by two different groups of animals. Yet such appears to be actually the case. 'There is an extinct group of ungulates known as the Litopterna, whose remains have been described by Ameghino from Tertiary beds of Patagonia. The small size of the brain-cavity, the characters of the dentition, cervical vertebræ, carpus and tarsus, indicate that they were more primitive forms than the true Perissodactyla, and, as Dr. Smith Woodward observes, they reached their maximum of specialization at an earlier period than the latter. We find amongst them forms (Theosodon, Fig. 109, A, B) with three well-developed toes as in the rhinoceros, forms (Proterotherium, Fig. 109, C) with one welldeveloped toe and two small ones as in some of the extinct ancestors of the horse, and forms (Thoatherium, Fig. 109, D, E) with a single toe as in existing horses; and in all cases the axis 


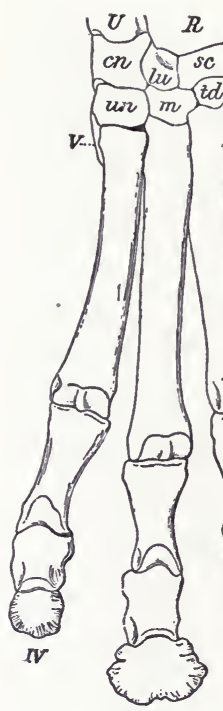

III

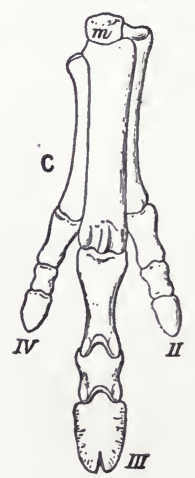

A
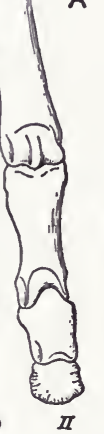

FIG. 109.-Feet of Ungulates belonging to the extinct group Litopterna, from Lower Tertiary deposits of Patagonia. (From Smith Woodward's "Vertebrate Palæontology," after Ameghino.)
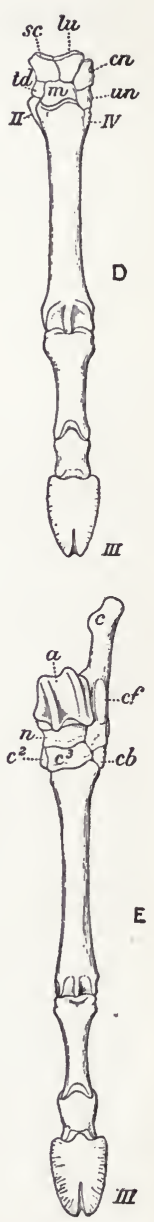

A, B, Theosodon lydekkeri; right fore and hind feet, $\times \frac{1}{3}$. C, Proterotherium intermixtum ; right fore foot, $\times \frac{1}{3}$.

D, E, Thoatherium crepidatum; left fore and hind feet, $\times \frac{1}{3}$. 
of the foot passes down the middle of the third digit. If we compare the foot of Thoatherium (Fig. 109, D, E) with that of a horse (Fig. 97) it is hard indeed to imagine that the animals possessing such closely similar and highly specialized limbs are not nearly related to one another. Expert palæontologists, however, tell us that they are not, and we must believe that the resemblance is due entirely to adaptation to a similar mode of life in the two cases. We shall have something to say as to how this adaptation was brought about in the case of the horse when we come to discuss the ancestral history of that animal in Chapter XIX.

It will be readily understood from the examples which we have been considering that the phenomena of convergence provide many pitfalls for the unwary biologist, and have led to many other mistakes in classification besides the popular error of placing the whales amongst the fishes. We have already noticed how the limbs of arthropods have come to bear a superficial resemblance to those of vertebrates, though so absolutely different in their essential structure that no anatomist would dream of regarding them as homologous organs. Many aquatic arthropods, belonging to the class Crustacea, like the lobsters, cray-fishes and crabs, breathe by means of gills which bear a superficial resemblance to those of fishes, but are again by no means homologous structures, and there are other resemblances between arthropods and vertebrates, due probably to convergence, which have led more than one observer to conclude that vertebrates are descended from arthropod ancestors, a conclusion which is by no means justified by the facts.

We may now further consider the process known as "change of function," in which an organ primarily adapted and used for one purpose takes on a new and altogether different duty and becomes modified accordingly. The lungs of air-breathing vertebrates, for example, are generally believèd to be homologous with the swim-bladder of fishes, for both arise in the same way as outgrowths of the front part of the alimentary canal, in the region of the throat. The swim-bladder of a fish is a hydrostatic organ; it is filled with gas, the amount of which can be regulated by suitable means, and assists the animal in maintaining its proper position in the water. In the dipnoan fishes, such as the Australian mud-fish (Neoceratodus, Fig. 110), the South American Lepidosiren and the African Protopterus, the walls of 
the swim-bladder have become highly vascular and it serves as an organ of respiration. Air is taken into it through the mouth, so that the blood circulating in its wall is oxygenated, and the arrangement of the heart and blood-vessels has become modified accordingly. Functional gills, however, are still present.

In the amphibians the swim-bladder has become completely converted into a pair of lungs, and in this way was rendered possible that great step in the evolution of the vertebrates, the migration from water to land. Thus in the air-breathing vertebrates the gills have been entirely supplanted and replaced functionally by the lungs, which are still to be regarded as homologous with, or morphologically equivalent to, the airbladder of fishes.

The hand of man affords another beautiful example of change

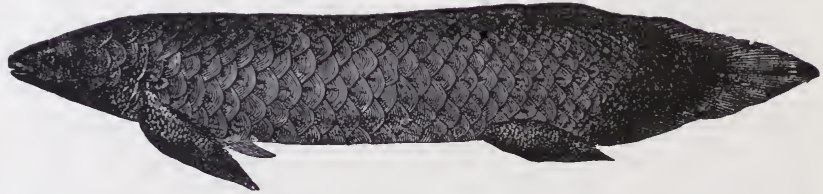

Fig. 110.--The Australian Mud-Fish, Neoceratodus forsteri, greatly reduced. (From British Museum Guide.)

of function-from locomotion to prehension-but it has undergone surprisingly little structural modification in the process. The proboscis of the elephant is also a very efficient organ of prehension, but it is formed by enormous elongation of the nose, which is primarily an organ for conveying air to and from the olfactory organs and lungs. Still more remarkable is the conversion of muscle fibres in the torpedo and the electric eel (Gymnotus) into powerful electric batteries, capable of giving severe shocks and therefore valuable as weapons of offence and defence. In this case the change of function is accompanied by very profound modifications in structure.

Wherever we turn we find that novel requirements are met, not by the sudden creation of new organs, but by the gradual modification of old ones. As we have already said, the organism has to do its best with the material which it has inherited from its ancestors; and yet the power of living protoplasm to meet every new requirement by a suitable modification of bodily structure 
appears to be almost unlimited. We must remember, however, that such modifications, in a state of nature at any rate, only take place very slowly and gradually.

Traces or vestiges of organs which have ceased to be of any use to their possessors persist with astonishing pertinacity in many organisms. Evidently their complete removal must be an extremely slow process. We have already noticed the persistence of vestiges of the pelvic girdle and leg bones in the whale (Fig. 101), of the reduced metapodials or splint bones in

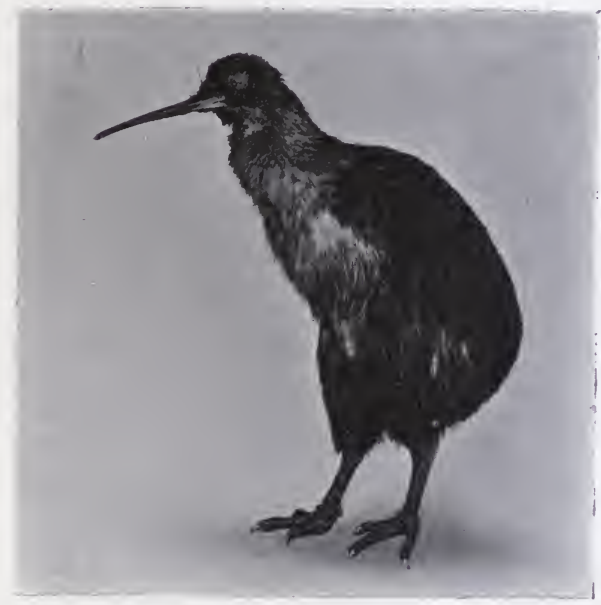

FrG. 111. $-A$ New Zealand Kiwi, $\times \frac{1}{6}$. (From a photograph of a stuffed specimen.)

the feet of the horse (Fig. 97), and of the coracoid in the typical mammalian shoulder girdle (Fig. 90). Unless we are to believe that such structures have been put where they are on purpose to mislead us we cannot possibly explain their occurrence on the theory of special creation. They are, at any rate in many cases, perfectly useless to their possessors, and the only rational way of accounting for their presence is by supposing them to be inherited from remote ancestors in which they were functional.

We may now briefly notice a few other instances of such $B$. 
vestigial structures. A characteristic feature of oceanic islands is the presence on them of birds which have more or less lost the power of flight. Such were the dodo of Mauritius, the solitaire of Rodriguez, and the gigantic moas of New Zealand. All these forms are now extinct, but we still find in New Zealand several flightless birds surviving. One of the most interesting is the kiwi or Apteryx (Fig. 111), a moderate-sized bird of nocturnal

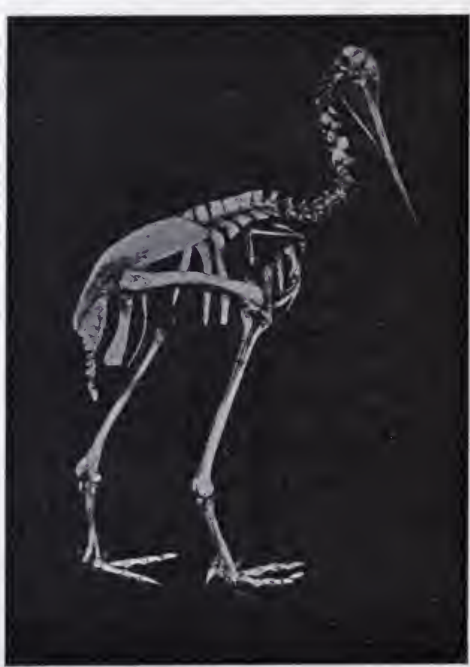

Fig. 112.- Skeleton of a Kiwi, showing the vestigial Wing Bones, behind which a piece of Black Paper has been placed, $\times \frac{1}{4}$. (From a photograph.) habits, now rapidly becoming exterminated. The wholebody is covered with coarse, hair - like plumage and externally shows no trace of wings. There is, however, a minute remnant of a wing present on each side, completely concealed in the plumage and entirely functionless as an organ of flight. Yet it still possesses the pentadactyl skeleton, though in a greatly reduced condition (Fig. 112). It is said that when the kiwi goes to sleep it still endeavours to tuck its long beak under its wing after the manner of other birds. In the extinct moas, to which the kiwis are perhaps related, even the last vestiges of the wings seem to have disappeared, for amongst the enormous quantities of the remains of these gigantic birds which occur in New Zealand no wing bones have ever been found. There is very strong reason to believe that the remote ancestors of existing vertebrates possessed an additional pair of eyes on the top of the head, behind the still existing lateral eyes. Traces of this second pair-the pineal or parietal eyes-are yet to be found in lampreys and lizards, and especially in that 
skin in the parietal foramen in the middle of the roof of the skull. It may to some extent still be functional, though probably of far less importance than in the extinct amphibia and reptiles of the Palæozoic and Mesozoic periods, in which the presence of a comparatively large parietal foramen indicates that the eye was better developed. In the case of the tuatara it has been demonstrated by anatomical and embryological investigation that the remaining pineal eye is the left-hand member of the original pair. In the lampreys it appears to be the right-hand member.

I'urning now to the frog, we sometimes find in this animal a minute light-coloured spot on top of the head (Fig. 115), between the paired eyes. 'Ihis marks the position of a little sac-shaped vesicle which lies

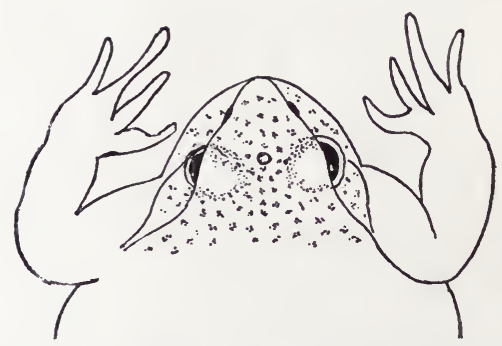

Fig. 115.-Head of Frog (Rana temporaria), showing Stieda's Organ between the lateral Eyes. (From Studnicka, after Stieda.) beneath the skin and is known as Stieda's organ, consisting merely of a number of undifferentiated cells surrounding a central cavity and attached to the end of a kind of stalk (Fig. 116). The vesicle represents the functionless vestige of a pineal eye and the stalk probably represents its disappearing nerve.

In the birds and mammals all trace of eye-like structure has disappeared from this region, but a vestige of one or both of the pineal eyes is probably to be recognized in the so-called pineal gland lying on the roof of the brain, which attained such celebrity in the seventeenth century owing to its identification by the philosopher Descartes as the seat of the soul.

In studying the mammalian dentition we again meet with plenty of illustrations of vestigial structures. The whale-bone whales (Balænidæ) have no teeth in the adult at all, but in the fœtus vestiges of teeth can be found imbedded in the gums. The young Australian duck-billed Platypus (Ornithorhynchus) has vestigial teeth which are entirely replaced by horny pads in the adult. The third molar in the lower jaw of the dog (Fig. 108, $\left.A_{1}, m_{.3}\right)$ is, as we have already seen, evidently in a vestigial 
condition and can be of very little if any use to its possessor, and there is reason to fear that in man himself the teeth are disappearing a good deal more rapidly than we could wish, though in this case the disappearance seems to be chiefly due to disease.

A better example of a vestigial structure in man is the coccyx at the hinder end of the vertebral column (Fig. 95), which represents the last remnant of the ancestral tail, and is occasionally accompanied by vestiges of the muscles by which an ordinary tail is moved. 'The hair on the chest, again, is a vestige of the

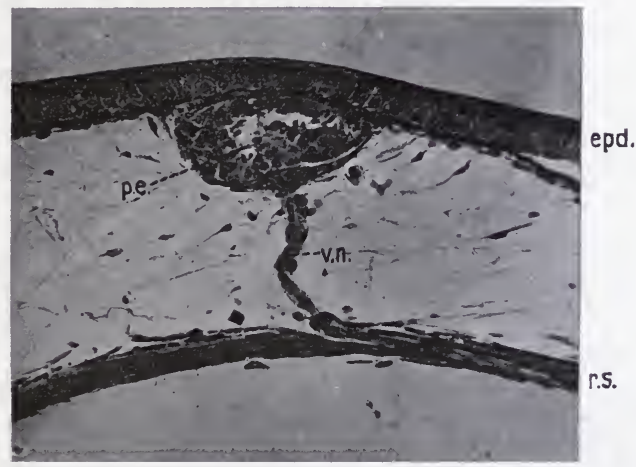

Fig. 116.-Section through the vestigial Pineal Lye of the Frog (Tadpole), $\times 168$. (From a photograph.)

epd., epidermis ; p.e., pineal eye; r.s., roof of skull; v.n., vestigial stalk and nerve.

hairy coat which once clothed the entire human body, as it still does that of the apes.

Examples of vestigial organs might be multiplied to an indefinite extent, but enough has perhaps been said to show that such structures are of very common occurrence and to indicate their value as evidences of organic evolution. 'They occur, of course, not only in the adult condition but also, as in the case of the embryonic gill-slits of air-breathing vertebrates, at earlier stages of development, but such cases will be more conveniently dealt with in the next chapter.

Closely akin to the occurrence of vestigial structures is another phenomenon known as atavism or reversion, by which we mean 
the sudden and sporadic reappearance of some ancestral structure which had either been completely lost or very greatly reduced. The elder Pliny has placed it on record that Cæsar the Dictator had a horse whose fore feet were like those of a man. This statement evidently refers to a more or less complete return to the ancestral pentadactyl condition. Marsh has given a figure of the fore foot of a horse in which the second digit is fairly well developed, with three perfect phalanges, though not so large as the third, while the first is represented by a splint bone, and a very similar case is exhibited in the Natural History Museum at South Kensington.

In man the occasional excessive development of the canine teeth is regarded as due to reversion to an ancestral condition similar to that of the anthropoid apes, in which the canine teeth are very large and used as weapons. The occasional occurrence in man of vestigial tail muscles, and even of a short tail, may also be regarded as due to reversion. Whether or not all such cases are capable of being explained on Mendelian principles, as suggested in Chapter XIV, it is impossible to say, but this does not affect their importance as evidence of the truth of the theory of organic evolution.

Although, in the present chapter, we have so far drawn our illustrations entirely from the animal kingdom, it must not be supposed that the same general principles cannot be equally well demonstrated in the case of plants. Indeed the whole chapter might be re-written entirely from the botanical point of view. Change of function is very well shown, for example, in the "pitchers" of Nepenthes and Sarracenia, formed from modified leaves and serving as traps for catching insects. Convergent evolution is illustrated by the strong superficial resemblance which exists amongst the various Alpine cushion plants belonging to widely different orders, all of which have assumed the form best suited for withstanding the peculiar hardships of their environment. The leaves of the parasitic dodder, reduced to tiny scales (Fig. 184), and the staminodes or functionless stamens of many flowers, again, might well serve as examples of vestigial structures. Considerations of space, however, forbid us to pursue this fertile line of inquiry any further. 


\section{CHAPTER XVIII}

Ontogeny-The recapitulation hypothesis--Interpretation of the ontogenetic record-Palingenetic and cænogenetic characters.

Is dealing with the cell theory, in Chapter IV, we have already laid stress upon the fact that every multicellular animal or plant commences its individual life as a unicellular egg or ovum, and gradually passes by slow stages of cell-division and differentiation into the adult condition. There are, of course, apparent exceptions to this rule in the case of animals and plants which reproduce by budding or by some analogous process, where the bud arises from a group of cells belonging to the parent, but even in these cases reproduction by means of germ cells is resorted to at more or less frequent intervals and the budding must be regarded merely as an additional method of multiplication interpolated in the life-cycle.

The individual organism, then, does not come into existence fully formed in all its perfection, but passes through a longer or shorter process of development to the adult condition. In fact it undergoes a process of individual evolution which constitutes its individual life-history or ontogeny, and the length and complexity of this process are proportional to the complexity of organization of the adult. Thus the complete life-cycle of many of the lower multicellular animals and plants is passed through in the course of a few months, while a man requires twenty years or more to attain his full development.

Although, for the sake of convenience, the ontogeny of any given organism may be divided up into a number of stages, yet these stages cannot in reality be sharply distinguished from one another. Even the division or segmentation of the ovum (Figs. 13 and 119), whereby the organism passes from the unicellular to the multicellular condition, does not take place suddenly but by means of the slow and complicated process of mitosis or karyokinesis (Fig. 31) ; and in cases where there is an apparently abrupt change, or metamorphosis, from one condition 
to another, as, for example, at a later stage, from the chrysalis to the butterfly, the development really progresses quite slowly and gradually internally, although the external appearance may for a long time remain unaltered and give the impression that it is completely at a standstill.

The fact that the young organism cannot commence its life at the stage reached by its parents, but has to make a fresh start from the beginning and go through a whole series of stages

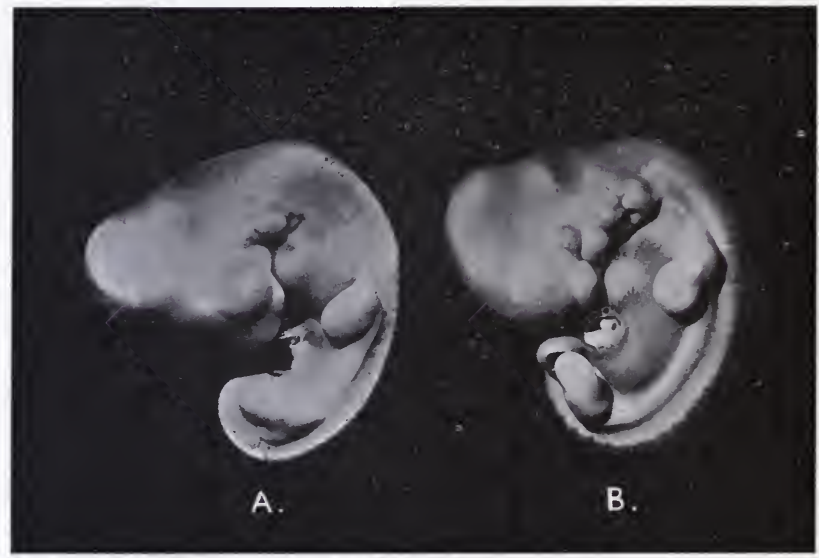

FIG. 117.-A. Chick Embryo of about $5 \frac{1}{2}$ days, $\times 5$. B. Rabbit Embryo of about 13 days, $\times 5$. In both cases the foetal membranes and yolk-sac have been removed. (From photographs.)

before reaching the adult condition, is very significant from the point of view of the evolutionist. Still more significant is the fact that different organisms all commence at the same stageas unicellular eggs-and come to diverge further and further from one another in structure as their development progresses.

If we examine a number of series of embryos belonging to different vertebrate types, no matter how widely the adult animals may differ from one another, we shall find that as we trace their life-histories backwards they gradually converge until, while still at a relatively advaneed stage of development, the different embryos come to resemble one another so closely that 
in many cases it is doubtful if even an experienced embryologist could distinguish between them. This great generalization appears to have been first reached by the embryologist von Baer, who in 1827 discovered the mammalian ovum. It may be illustrated by the chick and rabbit embryos represented in Fig. 117.

Von Baer's generalization contained the germ of what is now known as the Recapitulation Hypothesis, or, as Haeckel has termed it, the Biogenetic Law, which states that every organism in its individual life-history recapitulates the various stages through which its ancestors have passed in the course of their evolution. In other words, ontogeny, or the life-history of the individual, is a repetition of phylogeny, or the ancestral history of the race to which the individual belongs.

We have already referred, in Chapter IV (Fig. 13), to some of the earlier stages in the development of that primitive fishlike animal Amphioxus (Fig. 118). Let us next inquire how these stages may be interpreted in accordance with the recapitulation hypothesis. The unicellular ovum (Fig. 13, I) obviously represents the remote protozoon ancestors which were common to the whole animal kingdom, and which are also represented at the present day by independent unicellular organisms such as the Amoba. The segmentation of the ovum into primitive embryonic cells or blastomeres (Fig. 13, II-VI) represents the transition from the condition of the simple protozoon to that of the protozoon colony, in which the individual cells, instead of separating, as in the dividing Amœba, remain together, but still without undergoing any marked differentiation and division of labour. The arrangement of the blastomeres in the form of a hollow sphere, the blastula or blastosphere (Fig. 13, VII), with a single layer of cells surrounding a central cavity, represents the formation of such a protozoon colony as we see in the existing Volvox (Fig. 11) or Sphærozoum (Fig. 12). The process of gastrulation, whereby the single-layered blastula is converted into a two-layered gastrula (Fig. 13, VIII-X), with primitive digestive cavity (enteron) and primitive mouth (blastopore), represents the transition from the protozoon colony to the coelenterate stage of evolution, the latter being still represented at the present day by such forms as Hydra (Fig. 57), Obelia (Fig. 60), the jellyfish, the corals and all their numerous relations, which retain in their organization all the essential features of the gastrula, 
though generally complicated by the development of tentacles, skeleton, \&c. The development of cœlomic pouches (Fig. 13, $\mathrm{XI}-\mathrm{XIII}$, c.p.) as outgrowths of the primitive digestive cavity, and the conversion of these into mesoblastic somites (Fig. 13, $\mathrm{XV}$, m.s.), arranged serially or metamerically down each side of the body, mark the transition from the unsegmented and coelenterate condition to the metamerically segmented ${ }^{1}$ and cœlomate condition. The cavities of the cœlomic pouches form the cœlom or body cavity, which is at first transversely subdivided into compartments, as it still is in the adult earthworm, while their walls form the third germ-layer or mesoblast, lying between the epiblast which covers the surface of the body and

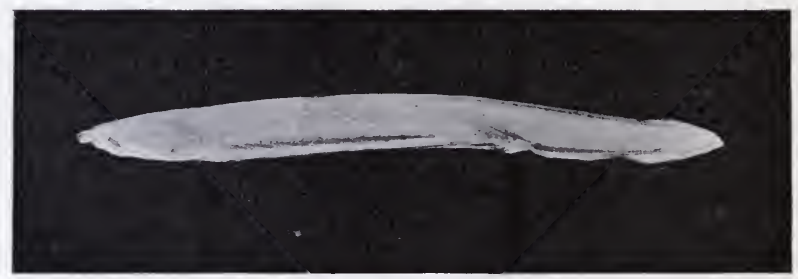

Fig. 118.-The Lancelet (Amphicxus larceolatus), $\times 2$. (From a photograph.)

the hypoblast which lines the digestive cavity. Along the middorsal line of the body a strip of epiblast sinks down (Fig. 13, $\mathrm{XI}, n . p l$.) and becomes folded into the form of a tube (Fig. 13, XII-XIV, n.t.), the rudiment of the central nervous system (brain and spinal cord), and beneath this tube a long strip of hypoblast becomes nipped off from the roof of the gut, forming the notochord (Fig. 13, XI-XIV, not.) or axial skeletal rodthe foundation around which in higher types the vertebral column is built up. A little later the front part of the gut becomes pierced by gill-slits for purposes of respiration and the primitive chordate condition is thus fully attained.

Amphioxus (Fig. 118) does not progress much beyond this stage in its development. It never acquires any limbs but swims about by lateral undulations of the body caused by the contraction

1 Metameric segmentation has, of conrse, nothing to do with the scgmentation of the ovum, but is the term applied to the transverse division of the body in many animals into a series of joints or segments arranged longitudinally, each of which typically repeats the essential features of all the others. 
of the lateral sheets of muscle derived from the mesoblast. No skull and no vertebral column are formed, the notochord remaining throughout life as almost the sole representative of the internal skeleton. 'I'he brain does not become distinctly differentiated from the spinal cord and no paired organs of sense are developed, nor does that division of the body into head and trunk, which is so characteristic of typical vertebrates, take place. Amphioxus is a chordate animal but it is not a true vertebrate. It probably, however, represents fairly closely a stage of evolution through which the ancestors of the vertebrates have passed, though it becomes somewhat modified by secondary features in the later stages of its development.

It is easy enough to interpret the earlier development of Amphioxus in terms of the recapitulation hypothesis and to recognize in the ontogeny certain stages of the phylogenetic history. The ontogenetic record, however, is by no means always so readily decipherable. It is generally more or less obscured by secondary features superposed upon it, much as an ancient picture may be concealed by another painted over it. These secondary features are characters which are developed in relation to the requirements of the embryo itself at its various stages, for it is not only the adult organism which becomes modified in structure in the course of evolution but all the stages of its life-history are likewise subject to adaptation.

In the first place nourishment has to be provided for the developing embryo, and this necessity is present from the very first division of the fertilized ovum. The blastomeres into which it divides cannot go about and seek their own food supplies like so many Amœbæ, they have sacrificed the power of leading independent lives for the sake of remaining together and building up a more or less complex multicellular body; but none the less they require feeding. Hence we find that all eggs contain a larger or smaller quantity of nutrient material stored up in the cytoplasm. 'This usually takes the form of definite granules or particles of food-yolk (deutoplasm), the amount of which varies immensely in different cases. If there is viery little the egg is said to be microlecithal as in Amphioxus; if there is much it is said to be megalecithal, as in the frog and still more so in the bird, and the size of the egg, as we have already had occasion to point out in Chapter X. depends almost entirely upon the amount of food-yolk which it contains. 
The possession of food-yolk is, of course, of immense importance to the embryo, for the stage which it is able to reach in its development before it has to begin to provide for itself will depend upon the amount of nutrient material which it has at its disposal. The presence of this food-yolk, however, must also act as a mechanical hindrance to the development, just as the supply of provisions which he carries with him must impede the progress of a traveller, though enabling him ultimately to accomplish a longer journey.

In Amphioxus the egg contains hardly any food-yolk and the process of segmentation and the formation of the blastula and gastrula take place in an almost perfectly typical manner. 'There is here practically no hindrance to development and no obliteration of the ontogenetic record. 'There is, however, just sufficient nutrient material to cause some of the cells-those which will invaginate to form the hypoblast of the gastrula-to be slightly larger than the remainder, which are destined to form the epiblast (Fig. 13, VII, VIII).

Let us compare with this the corresponding stages in the development of the frog (Fig. 119). Here the egg is, as we have already indicated, much larger, owing to the greater quantity of food-yolk which it contains. 'This is chiefly collected in the lower half of the ovum, while the upper half is deeply pigmented and thus readily distinguished. Segmentation begins as in Amphioxus by the formation of two vertical clefts, or cell-divisions, one after the other and at right angles to one another, whereby the four-celled stage is reached (Fig. 119, I-III). Each cleft begins at the pigmented pole and throughout the whole of the segmentation the yolk-laden half of the egg lags behind the pigmented half because of the mechanical hindrance which the food-yolk opposes to the process of cell-division. The first horizontal cleft (Fig. 119, IV) divides each of the four blastomeres into two daughter cells of very unequal size, an upper pigmented cell with little food-yolk and a much larger, lower, yolk-laden cell with little pigment. After this, segmentation continues to progress more rapidly in the upper than in the lower half of the egg (Fig. 119, V), until we reach the blastula stage (Fig. 119, VI), where we find the large yolk-laden cells forming the lower half of the wall of the hollow sphere and projecting into and partly obliterating its cavity, while the small pigmented cells form the upper half. 
The process of gastrulation is profoundly modified by the presence of the food-yolk, and instead of a simple invagination of the lower half of the blastula wall to form the hypoblast, we find the small (epiblast) cells, now arranged in several layers, spreading themselves over the yolk-containing cells until they completely enclose the latter except for a small circular area
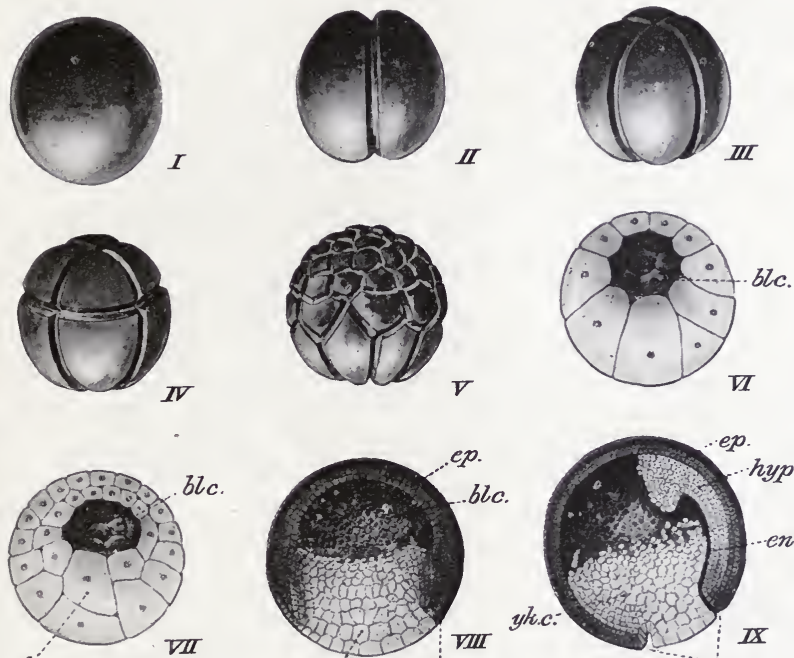

yłic.
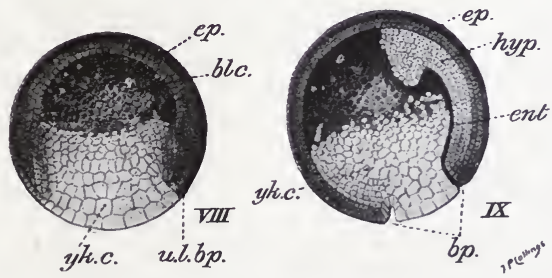

Fig. 119.- Early Stages in the Development of the Frog (partly from Ziegler's models and partly adapted from Marshall.)

$I$, the fertilized ovum; $I I-V$, segmentation of the ovum ; VI, blastula ; VII, modified blastula, with wall composed of more than one layer of cells; VIII, commencement of gastrulation; $I X$, the modified gastrula stage (VI-IX in vertical section).

$b l c$., blastocoel or segmentation cavity ; $b p$., blastopore; ent., enteron; ep., epiblast; hyp., hypoblast; u.l.bp., upper lip of blastopore; yk.c., yolk-containing cells.

which corresponds to the blastopore or aperture of invagination in Amphioxus blocked up by yolk cells (Fig. 119, VIII, IX). Indeed, practically the whole of the primitive digestive cavity or enteron in the frog's gastrula may be regarded as being filled with yolk cells, from which hypoblast will be derived, and it only gradually opens out later on in development as the yolk is used up. Hence the coelenterate stage of the ancestral history 
(Fig. 119, IX) is very much disguised in the ontogeny, simply owing to the presence of a large quantity of nutrient material in the egg.

In the development of the bird's egg (and also in that of reptiles, which is closely similar) it is still more difficult to recognize the process of gastrulation. The quantity of yolk (Fig. 70) is so enormous that it entirely prevents cell-division from taking place in the greater part of the egg, and segmentation is consequently confined to a small yolk-free area at one pole of the spherical mass, where it gives rise to a layer of cells known as the blastoderm. This blastoderm gradually spreads over the yolk and from it the embryo is formed by a kind of pinching-off process, the yolk remaining enclosed in a bag - the yolk-sac-attached to the lower surface of the embryo, until it is all absorbed by means of a special set of blood-vessels developed in the yolk-sac, and used up in the nourishment of the growing embryo. The embryo itself meanwhile becomes provided with special organs for its protection and respiration, the so-called foetal nembranes. Of these the amnion, formed by outgrowth of the embryonic body-wall, forms a sac filled with fluid, which surrounds the embryo at a distance and leaves it free room for growth, while the allantois, formed by outgrowth of the embryonic gut-wall, forms a highly vascular organ which comes into relation with the extremely porous shell and thus provides for the necessary gaseous interchange.

The yolk-sac, the amnion and the allantois are all organs which have been developed in accordance with the special requirements of the embryo, and which must therefore be left out of account in reconstructing the direct ontogenetic record.

In the dogfish also (Fig. 120) we find an excellent illustration of the formation of a special yolk-sac to contain the enormous supply of food material for the developing embryo, but in this case neither amnion nor allantois is developed.

The Mammalia, on the other hand, have adopted an improved method of nourishing their young. The developing embryos are retained within the body of the parent and obtain their food supply from the blood of the latter by means of a special organ known as the placenta, which is developed partly from the allantois of the embryo or "fœetus," and by which the latter is attached to the wall of the uterus. This method seems to have proved much more satisfactory than the provisioning of the egg 
with food-yolk, and we accordingly find that the mammalian ovum (Fig. 71) has become reduced in size again until it is no larger than that of Amphioxus, i.e. about $\frac{1}{2} \frac{1}{50}$ th inch in diameter. The early stages of segmentation have also gone back to the primitive type, but, strange to say, a yolk-sac is still formed although there is no yolk for it to contain-affording an admirable example of a vestigial embryonic organ. Here one record has been superposed upon another in the ontogeny and we have a recapitulation of an embryonic stage of the reptilian ancestors of the mammals. After birth, of course, the young

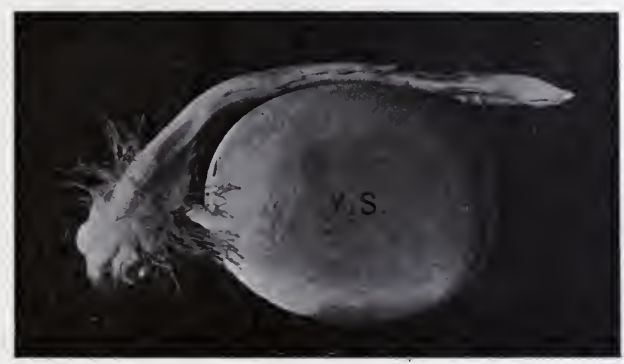

FiG. 120.-Embryo of a Dogfish, with Yolk-Sac (Y.S.) attached, $\times 2$. (From a photograph.)

mammal is nourished by the secretion of milk, and is thereby enabled to reach a very advanced stage of development before it has to begin to find its own food. The chick, owing to the very large amount of food-yolk with which the egg is supplied, is also able to reach a highly developed condition before it hatches and begins to look after itself.

With animals whose eggs contain insufficient food-yolk, and which are not provided with any other spacial means of nourishing the developing young, the case is very different. They are obliged to hatch and begin life on their own account while still a long way from the adult condition-when only a small part of their ontogenetic journey has been accomplished. Amphioxus, for example, hatches as a free-swimming ciliated embryo shortly after the gastrula stage has been passed through, and completes its development at the expense of food supplies which it obtains for itself. 
Embryos which, having hatched at a comparatively early stage of their development, lead independent, self-supporting lives, are commonly spoken of as larvæ, and amongst such larvæ we again find abundant instances of special larval organs developed in relation to their special requirements.

The tadpole of the frog (Fig. 121, 4-11) affords an excellent example of a free-living larva. It has certain special organs, such as its horny jaws and the suckers on the under surface of
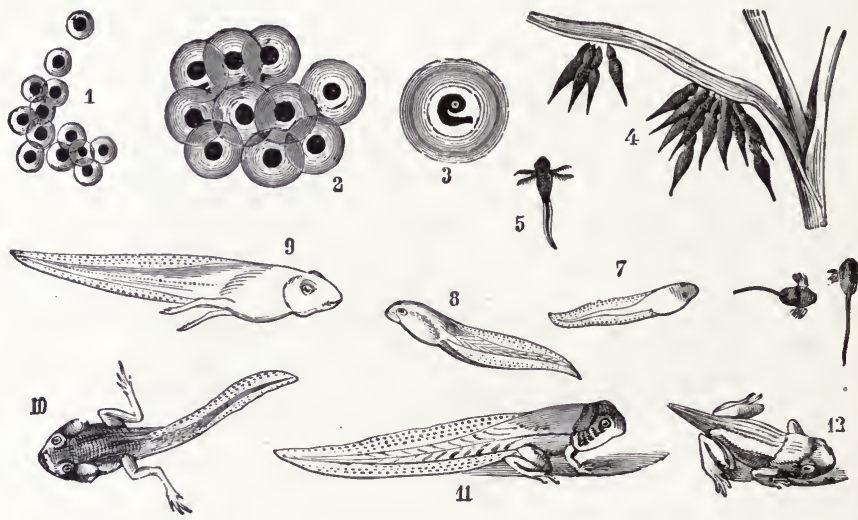

11

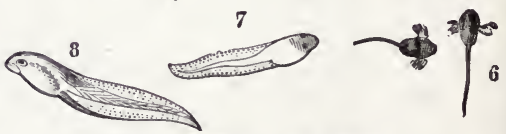

Fig. 121. Stages in the Life History of the Frog. (From Brehm's “Thierleben.")

1, freshly laid eggs; 2, eggs slightly magnified, adhering together by their gelatinous envelopes to form "spawn"; 3 , young tadpole still enclosed in the jelly; 4 , newly hatched tadpoles adhering by their suckers to a blade of grass; 5,6 , tadpoles with external gills; 7,8 , tadpoles with the gills concealed by the opereulum; 9,10 , tadpoles with conspicuous hind legs but with the front legs still concealed beneath the operculum; 11, tadpole during metamorphosis, with front legs protruded; 12, young frog with tail not completely absorbed.

(For microscopic details of the early stages see Fig. 119.)

the head, which are developed in relation to its larval life, but there can be no question about the phylogenetic significance of the tadpole stage as a.whole. In every essential feature of its organization the tadpole, before its legs develop, is a fish. It swims like a fish by means of its well developed tail, it breathes like a fish by means of its gills and gill-slits, and its internal structure is that of a fish. There can be no doubt that it represents a fish-like stage in the evolution of the frog.

In the tadpole, owing to its free-living, aquatic mode of life, 
the characteristic piscine organs are fully functional, but even in the embryos of higher air-breathing vertebrates-reptiles, birds and mammals-while enclosed within the egg-shell or within the womb of the parent, we can still recognize clear traces of a similar fish-like stage in their evolution, for gill-slits are present in all (Fig. 122, g.s.) and the arrangement of the principal veins and arteries is unmistakably fishlike.

Difficulties in the interpretation of the ontogenetic record arise not only from the addition of new features in the form of embryonic or larval organs but also from theabbreviation of the different stages through which the organism passes. The development of any of the higher organisms must be looked upon as a kind of compromise, due to the necessity of attaining as perfect a condition as possible in as short a time as possible, for the sooner the adult condition is reached the sooner will the organism be able to reproduce its kind, and hence any abbreviation of the earlier stages of development will be advantageous to the species and will be favoured by natural selection. For this reason the fish-like stage in the development of reptiles, birds and mammals is passed through very rapidly and the fish-like structure is

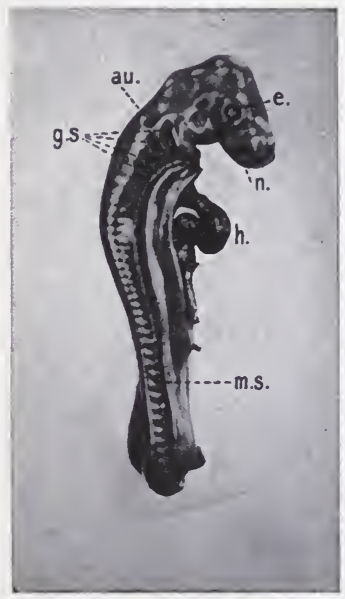

FiG. 122.-Chick Embryo of about 3 Days, with the Foetal Membranes and Yolk-Sac removed. (Photographed from one of Viegler's models.)

au., auditory organ; e., eye ; g.s., gill slits; h., heart; m.s., mesoblastic somites ; n., nasal organ. no longer fully developed. Although gill-slits are present the gills themselves are not, and other piscine characters are only represented by transient vestiges.

Turning now to some of the lower groups of the animal kingdom, we may briefly notice a beautiful illustration of the law of recapitulation afforded by the life-history of the common featherstar, Antedon (Fig. 123). This animal, found abundantly in comparatively shallow water off the British coast, belongs to the great phylum Echinodermata, which also includes the star-fish, 
sea-urchins and sea-cucumbers. The subdivision to which it belongs-the Crinoidea-is characteristically a deep-water group. It is also a group of great antiquity, the remains of crinoidssuch as the well-known "Encrinites" of the Carboniferous period - being abundant in certain Palæozoic formations.

Both the Palæozoic crinoids and the surviving deep-sea members of the group, such as Pentacrinus (Fig. 125), are stalked forms, the "calyx," with its radiating arms, being attached to

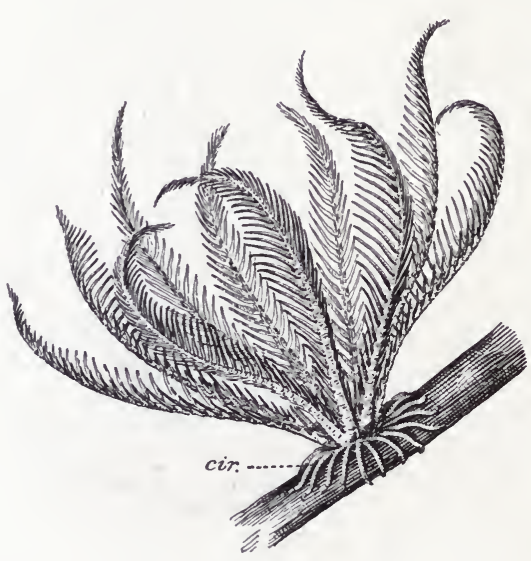

FIG. 123.

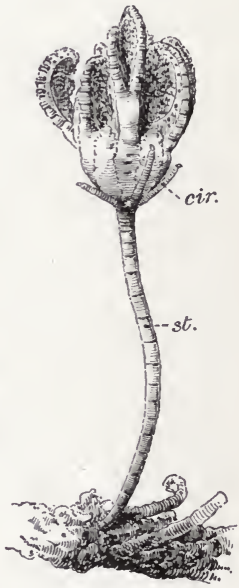

FIG. 124.

FIG. 123. - The Feather Star (Antedon bifida), nat. size.

FIG. 124.-Pentacrinoid Stage in the development of Antedon, $\times 14$. cir. cirri ; st. stalk.

the end of a long, jointed, calcareous stem, the lower extremity of which is permanently fixed to the sea-bottom. Antedon, and the other shallow-water crinoids of the present day, on the other hand (Fig. 123), have no stalks but in place thereof a number of slender "cirri" whereby they temporarily attach themselves to seaweed or other objects.

Now in the course of its development from the egg the featherstar passes first through a free-swimming larval stage and then through a fixed stalked stage (Fig. 124), known, from its resemblance to the deep-sea genus Pentacrinus, as the pentacrinoid 
stage. Later on the young animal develops cirri and falls off the end of the stalk, a ssuming once more an active modeoflife. Here we recognize very clearly in the pentacrinoid stage of the ontogeny a repetition of the $\mathrm{stalked}$

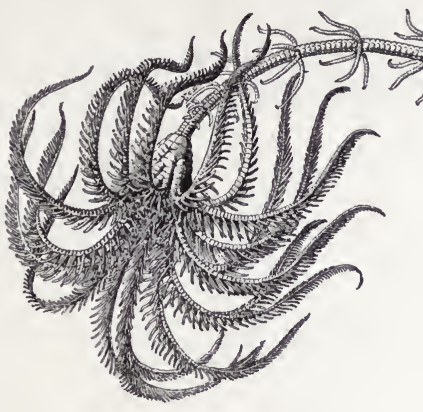

FIg. 125.-A Deep-Sea Crinoid, Pertacrimus (Isocrinus) decorus, about nat. size.

\section{condition}

through which the ancestors of the feather-star must have passed.

We also find amongst invertebrate animals abundant examples of special larval organs. The body of a caterpillar, for instance, is built up very largely of such structures, and it has to undergo an almost complete reconstruction within the protective envelope of the chrysalis before it reaches the adult condition and emerges as a perfect insect. It is therefore extremely difficult to interpret the caterpillar stage in terms of the ancestral history.

The ophiuroids or brittle stars (Fig. 126), so commonly met with between tide-marks, have remarkable, free-swimming "Pluteus" larvæ (Fig. 127), totally different in appearance from the adult. These larvæ occur in immense numbers in the surface waters of the ocean. They are provided with long slender arms supported on calcareous rods, which probably serve, in part at any rate, to protect the very

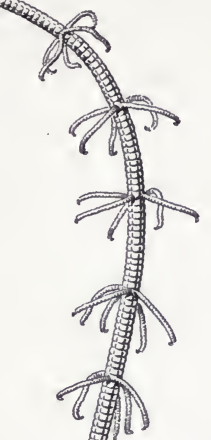
delicate body from the attacks of other small animals. Before attaining the adult condition the larva undergoes a complex 
metamorphosis, during which the long arms are completely lost and the form of the body entirely changed. The echinoids or sea-urchins pass through a very similar larval stage.

The free-swimming "Zoæa " larva of the crab (Fig. 128) is also provided with conspicuous defensive spines which are lost in the

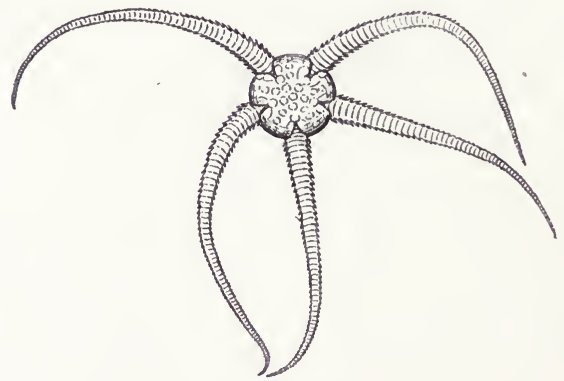

FIG. 126.

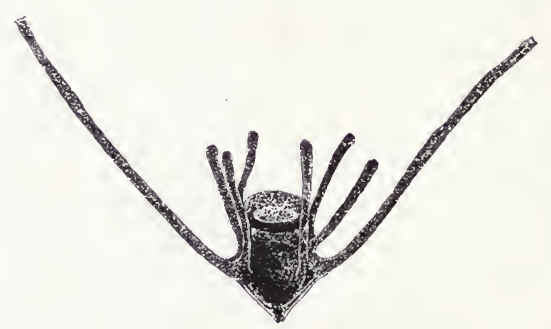

Fig. 127.

FIG. 126. - A trittle Star (Ophiura ciliaris), $\times \frac{3}{4}$.

Fig. 127. - The Pluteus Larva of a Brittle Star, $\times 62$.

adult condition (Fig. 102) and which have doubtless been developed in relation to the special conditions of the larval life.

In many cases the developinent of larval organs causes the young animal to differ so widely from the adult that the relationship of the two for a long time remained unsuspected, and hence the special names which many larvæ have received. In other cases, however, it is the adult animal which has become so profoundly modified in relation to special conditions of life as to 
obscure its genetic affinities, which may still be clearly indicated by embryonic or larval stages.

The common Ascidian, for example (Fig. 129), is a sac-shaped organism, permanently attached to rock or seaweed in the adult condition, which by the uninitiated would hardly be taken for an animal at all. Before their life-history was known the Ascidians were placed by zoologists amongst the "Molluscoida," a group of invertebrates supposed to resemble the mollusca or shell-fish. It was only the discovery that the typical Ascidians pass through an active tadpole stage in their development (Fig. 130), with a muscular tail, a notochord and a central nervous system all formed as in vertebrates, that demonstrated their true position as degenerate members of the great phylum Chordata (= Vertebrata in the widest sense of the term), the tadpole stage being, of course, a recapitulation of the ancestral, fish-like, chordate condition.

It will be readily understood from the foregoing illustrations that in endeavouring to reconstruct the ancestral history of an organism from the stages which it passes through in its indivi-

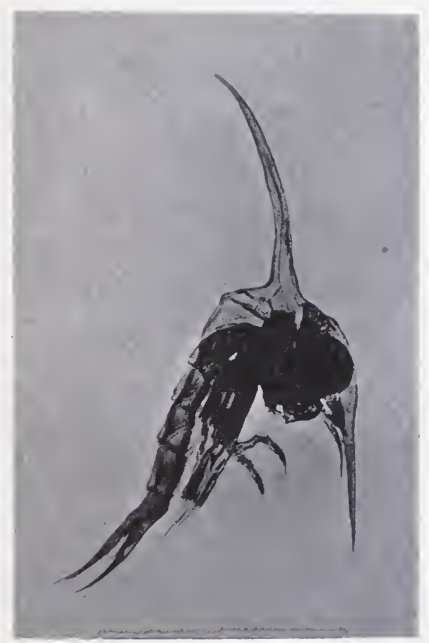

Fig. 128.-The Zorea Larva of a Crab (Portunus), $\times 30$. (From a photograph.) dual development, we have to distinguish very carefully between two sets of characters. In the first place there are characters which are due to inheritance from the adult condition of very remote ancestors and which really indicate stages in what we may call the direct line of evolution. These are termed palingenetic or ancient characters. Such, for example, are the essential features of the gastrula stage, which occurs so generally and which indicates a remote coelenterate ancestor (the Gastræa of Professor Haeckel) for at any rate the great majority of multicellular animals. 
Such also are the essential characters of the tadpole stage of the frog and of the ascidians, and the vestigial gill-slits of higher vertebrates, which indicate descent from some gill-breathing, fishlike form.

In the second place there are characters which have been acquired secondarily by the embryo or larva in relation to its

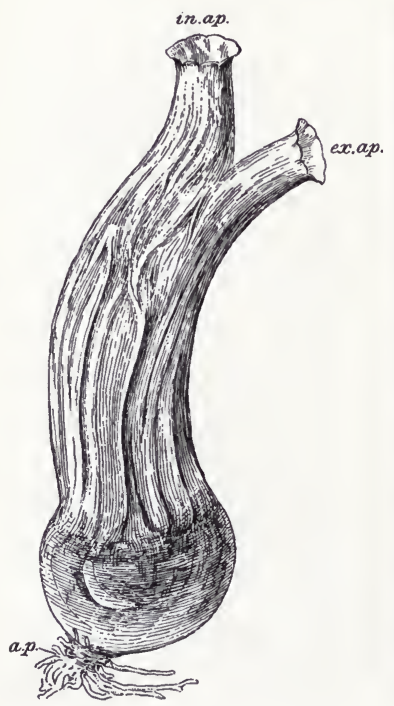

Fıg. 129.-A Simple Ascidian (Ciona intestinalis), $\times \frac{1}{2}$.

a.p., processes for attachment; in.ap., inhalant aperture; ex.ap., exhalant aperture.

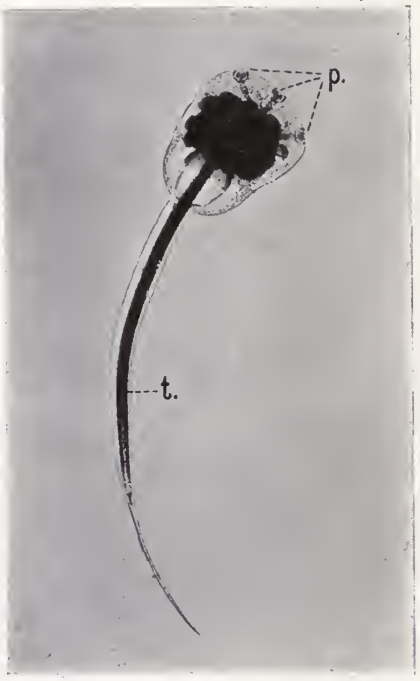

F1G. 130. - Tadpole Larva of a Compound Ascidian (Diplosoma crystallinum), $\times 35$. (From a photograph.)

p., papillæ for attachment; $t$., tail.

special embryonic or larval requirements. These are termed cænogenetic or modern characters, and it is these which tend more or less to obscure the ontogenetic record. Such are the presence of food-yolk, the foetal membranes of air-breathing vertebrates and the various special larval organs to which we have above referred. It is difficult, however, to draw a hard and fast line between palingenetic and cænogenetic characters, and we must always remember that embryonic stages, as well as the 
adult, undergo modification during evolution, and that some cænogenetic characters are very much more ancient than others.

The relation which exists between the phylogenetic and ontogenetic stages in the evolution of an organism are very diagrammatically expressed in Fig. 131, which is drawn with special reference to the familiar case of the frog. The vertical line is supposed to represent the ancestral history, some of the principal stages of which are indicated. The horizontal line at the top represents the individual lifehistory, with the stages indicated which represent those selected in the ancestral history. The ovum represents the protozoon ancestor; the blastula the hollow, spherical protozoon colony; the gastrula the cœlenterate; the metamerically segmented embryo the segmented worm, and the tadpole the fish-like stage. The oblique lines represent the lines of evolution of the different ontogenetic stages themselves, in the course of which cænogenetic characters, such as the acquisition of food-yolk by the ovum and of suckers and horny jaws by the tadpole, have arisen. The shorter horizontal lines repre-

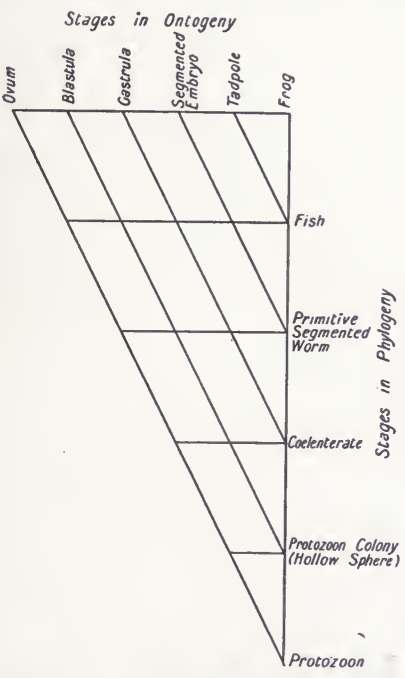

FIG. 131. - Diagram illustrating the Relation between Ontogeny and Phylogeny. sent the ontogeny at the different ancestral stages, and their increase in length from below upwards is obviously due to the successive addition of new stages to the ontogenetic record, while the shortness of the horizontal lines as compared with the corresponding portions of the vertical line may be taken to indicate, though very imperfectly, the abbreviation of the ontogeny.

In the vegetable kingdom we find illustrations of the law of recapitulation and of the complication of the ontogenetic record by the development of cænogenetic characters, quite as striking as any which we find amongst animals. Take, for example, the 
life-history of some of the Australian acacias, or wattles. These are leguminous plants and many of them have the pinnately subdivided leaves so characteristic of the order. Others, however, have the pinnate leaves in the adult entirely replaced by narrow "phyllodes," which closely resemble simple undivided leaves like those of some of the eucalypts, but are really only flattened leaf-stalks or petioles. The development of such phyllodes is no doubt to be regarded as an adaptation to the heat and drought of

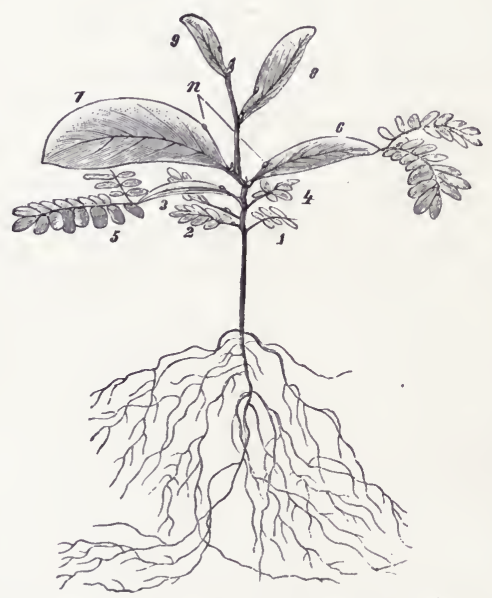

FIG. 132. - Seedling of a phyllode-bearing Acacia (Acacia pycnantha). (From Strasburger.) ('The cotyledons have already been shed.) the Australian climate, which delicately subdivided leaves of the ordinary leguminous type are ill-suited to withstand, and the resemblance of the phyllodes to true foliage leaves of the undivided type-and especially to those of the eucalypts - affords a very good illustration of the phenomenon of convergence. If, now, we examine the seedlings of a phyllode-bearing acacia (Fig. 132) we shall find that immediately after the cotyledons or seed-leaves they bear typical pinnate leaves, and then leaves of intermediate form, thus clearly recapitulating the ancestral condition from which the phyllode-bearing forms were derived. A very similar state of things occurs in the common European gorse or furze, another leguminous plant, in which the leaves of the adult have become modified to form spines, while those of the seedling still retain the ancestral ternate condition, somewhat resembling those of the clover.

The cotyledons or true seed-leaves of flowering plants, on the other hand, afford excellent examples of cænogenetic characters developed in relation to the special requirements of the embryo. 
They are very commonly modified to form receptacles for a store of food material for the young plant in the form of starch, as in the peas and beans (compare Fig. 56), and may never even leave the seed-coat or emerge above the ground. In such cases they develop no chlorophyll and differ absolutely from ordinary foliage leaves, without, of course, indicating any ancestral stage through which the latter have passed. Even when they emerge above the ground, as in the beech (Fig. 133), and become green, their form is usually widely different from that of the foliage leaves and appears to have been developed largely in adaptation to the necessity for close package within the narrow compass of the seed-coat, the shape of the cotyledons being governed, as Lord Avebury has pointed out, by that of the seed within which, in a dormant condition, they pass most of their lives.

In short, the embryological investigation of both animal and vegetable organisms leaves no doubt as to the general truth of the recapitulation hypothesis, and must convince any unbiassed

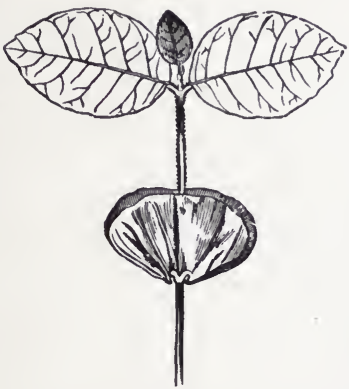

FIG. 133. - Seedling of Beech, showing the Cotyledons. (From Lubbock.)

observer that, however much modified it may be by abbreviation and by the superposition of secondary features, the life-history of the individual is essentially a condensed epitome of the ancestral history of the race. The law of recapitulation, indeed, may be regarded simply as a logical extension of the law of heredity, for every organism tends to inherit the characters not only of its immediate progenitors but of all its ancestors, and these characters appear in the individual life-history in the same order as that in which they first appeared in the ancestral history-in other words, ontogeny is a repetition of phylogeny and can only be explained in terms of organic evolution. 


\section{CHAPTER XIX}

The stratified rocks-Geological periods--The age of the habitable earth--

The geological record-The succession of the great vertebrate groups.

THE materials of which the solid crast of the earth is made up are, as it is perhaps hardly necessary to point out, extremely varied, but whatever may be their physical or chemical characters they are all spoken of by geologists as rocks. In accordance with their mode of formation these rocks may be divided into two chief categories, first, those which, like granite and basalt, have been formed directly by cooling of molten matter, of which the earth was perhaps at one time entirely composed, and, second, those which, like sandstone, shale, limestone and coal, have been formed by accumulation of detritus derived from pre-existing rocks or from the remains of dead organisms. The first are commonly termed igneous and the second sedimentary, the latter deriving their name from the fact that they have been deposited under water. Owing to the manner in which they have been laid down, usually in the form of gravel, sand, mud or ooze, which have subsequently become hardened and consolidated by the pressure of more recent deposits, the sedimentary rocks form a series of layers or strata of varying thickness superposed one upon another. The stratification must at first have been horizontal, but, owing to the irregular shrinkage of the earth's crust and to the action of volcanic upheavals, it has usually been more or less disturbed, so that the planes of bedding have been tilted at various angles and frequently exhibit a high degree of curvature or even contortion. Moreover, rocks which were originally laid down on the bed of the sea have in many cases come to form part of the dry land and even to be uplifted far above sea-level in the form of mountain ranges.

The formation of sedimentary rocks must have been going on ever since the earth became cool enough to admit of the condensation of aqueous vapour into water, and it is still going on in the same way at the present day. Every river is continually 
carrying down to the sea immense quantities of sand and mud, which will ultimately be deposited in the form of new strata; every cliff by the sea-shore is slowly or rapidly weathering away under the influence of atmospheric agencies-rain, frost, tides and wind; and far out, beyond the reach of the detritus derived from the land, the sea-bottom is being covered by the slow accumulation of ooze or mud derived almost entirely from the remains of marine organisms, while the activities of corals and other reef-building animals in their turn make no small contribution to the grand total.

The sedimentary rocks naturally contain the remains of vast numbers of organisms which flourished on the earth at more or less remote periods of its history, and the study of these fossils may justly be expected to yield results of the greatest importance from the point of view of the theory of organic evolution. We have here, in fact, the only really direct evidence of the course which the evolution of the animal and vegetable kingdoms has actually taken, and this evidence constitutes what is commonly known as the geological record.

The relative ages of the different sedimentary rocks and of the different geological formations which they build up are of course determined primarily by their position with regard to one another, each layer or bed being necessarily of more recent date than the one below it. ${ }^{1}$ The series of strata met with in any particular locality may, however, be very different from that which occurs in other localities, for while mud may be accumulating in one place, sandstone may be forming in another and limestone in a third, and over other areas, which lie above sea-level, rock formation will be replaced by destruction or denudation. Nevertheless, geologists have found it possible to compare the series of stratified rocks found in different parts of the world one with another, and, by the aid of the fossils which they contain, to identify a number of more or less well defined epochs in the geological history of the earth.

At the bottom of the stratified series lies an immense thickness of rocks which seem to have been originally sedimentary, but many of which have undergone profound changes due to pressure and heat since they were laid down. These rocks indicate a very long period of the earth's history, the

1 Except in a few cases where contortion has been so extensive as to reverse the relative positions. 
Geological Time-Scale, as indicated by the Stratified Rocks.

\begin{tabular}{|c|c|c|c|}
\hline \multirow[b]{2}{*}{ 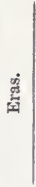 } & \multirow[b]{2}{*}{$\begin{array}{l}\text { Epochs, with relative duration } \\
\text { indicated by thickness of } \\
\text { stratified rocks. }\end{array}$} & \multirow[b]{2}{*}{$\begin{array}{l}\text { Some representative } \\
\text { British Formations. }\end{array}$} & $\begin{array}{l}\text { Range in Time of } \\
\text { Animal Groups. }\end{array}$ \\
\hline & & & 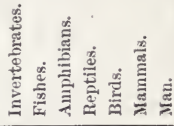 \\
\hline \multirow{5}{*}{ 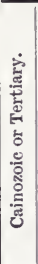 } & Kecent and Pleistocene 4000 feet. & Alluviun, Peat, boulder Ciay, dc. & $::$ \\
\hline & Pliocene 13000 feet. & $\begin{array}{l}\text { Norwich and Red Crag, Coralline } \\
\text { Crag, \&c. }\end{array}$ & $\vdots$ \\
\hline & Miocene 14000 feet. & & \\
\hline & Oligocene 12000 feet. & $\begin{array}{l}\text { Hamstead, Bembridge, Osborne } \\
\text { and Headon Beds. }\end{array}$ & \\
\hline & Eocene 20000 feet. & $\begin{array}{l}\text { Barton and Bagshot Beds, London } \\
\text { Clay, Thanet Sands, \&e. }\end{array}$ & \\
\hline \multirow{3}{*}{ 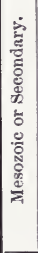 } & Cretaceous 44000 feet. & $\begin{array}{l}\text { Chalk, Greensand, Ganlt, Wealden, } \\
\text { \&c. }\end{array}$ & $\vdots$ \\
\hline & Jurassic 8000 feet. & Oulites, Lias. & $\vdots \vdots$ \\
\hline & Triassic 17000 feet. & $\begin{array}{l}\text { White Lias (Rhætic), Elgin Sand- } \\
\text { stone, \&c. }\end{array}$ & $\begin{array}{ccc}\vdots \\
\vdots \\
\vdots & \vdots \\
\vdots & \vdots \\
\vdots & \vdots & \vdots \\
\vdots & \vdots\end{array}$ \\
\hline \multirow{7}{*}{ 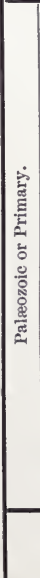 } & Permian 12000 feet. & $\begin{array}{l}\text { Maynexian Limestone, Red } \\
\text { Marls, \&c. }\end{array}$ & \\
\hline & Carboniferous 29000 feet. & $\begin{array}{l}\text { Coal Measures, Millstione Grit, } \\
\text { Mountain Limestone, \&c. }\end{array}$ & \\
\hline & Devonian 22000 feet. & Old Red Sandstone. & : \\
\hline & Silurian 15000 feet. & $\begin{array}{l}\text { Ludlow and Wenlock Shales and } \\
\text { Limestones, \&c. }\end{array}$ & $\begin{array}{l}\vdots \\
\vdots \\
\vdots\end{array}$ \\
\hline & Ordovician 17000 feet. & $\begin{array}{l}\text { Slates, Flags, Sandstones, \&c., of } \\
\text { Bala, Caradoc, Llandeilo, Skid. } \\
\text { daw, \&c. }\end{array}$ & \\
\hline & Cambrian 26000 feet. & Lingula Flags, Harlech Series, \&c. & \\
\hline & 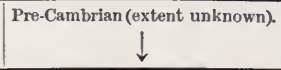 & $\begin{array}{l}\text { Archren Schists, Gneiss and } \\
\text { other Crystalline Rocks; largely } \\
\text { metamorplife. }\end{array}$ & ? \\
\hline
\end{tabular}


pre-Cambrian epoch, during which organic evolution must have been taking place, though only somewhat doubtful indications of organic remains have been met with. Probably the preCambrian rocks originally contained abundant fossils, which have been destroyed by the treatment to which they have been subjected. All the subsequent periods of the earth's history, however, are represented by rocks containing organic remains in greater or less abundance. These deposits indicate a division of the earth's history since pre-Cambrian times into three main eras-primary or palæozoic, secondary or mesozoic and tertiary or cainozoic. Each of these three is subdivided into a series of epochs, as shown in the accompanying table, in which are also mentioned some of the principal rock formations by which the different epochs are represented in the British Islands.

The thickness of the strata deposited during each period differs greatly in different parts of the world, and in North America the various formations appear to be much better developed than in North Western Europe. It is of the utmost importance that our estimates of this thickness should be as accurate as possible, for they constitute important data from which to calculate the age of the habitable earth and the duration of the different geological periods.

Sir Archibald Geikie, ${ }^{1}$ in 1892 , very cautiously estimated the total thickness of the stratified rocks, where most fully developed, from the bottom of the Cambrian onwards, as not less than 100,000 feet. He also estimated that it would take from 730 to 6,800 years to add a foot to this thickness by accumulation of material derived from the denudation of the land. Of course the actual time must vary very greatly according to the nature of the material deposited and the agencies at work. There is, however, no reason to suppose that denudation and sedimentation took place, on an average, more rapidly in past geological time than at the present day. If, then, we take the average between the most rapid and the slowest rate of denudation and sedimentation, and the thickness of the stratified rocks as calculated by Professor Geikie, we arrive at $376,500,000$ years as the age of the habitable earth exclusice of the pre-Cambrian period.

Sir Archibald Geikie appears, however, to have assumed for the purposes of his calculation that material derived from the wearing away of the land has been spread out over an equal area

1 Presidential Address, British Association, Edinburgh, 1892. 
of sea-bottom, so that every foot in depth worn off the land is taken to indicate a foot in thickness piled up in new deposits. This hardly seems to be a justifiable assumption, for in many localities sediment derived from a large area must be accumulated over a small one, and the increase in thickness of the newly forming rock must therefore take place at a much greater rate than the decrease in thickness of that which is being worn away.

Professor Sollas, in his presidential address to the Geological Society of London in 1909, gives the total maximum thickness of the sedimentary rocks from the bottom of the Cambrian to the top of the most recent formations as being no less than 253,000 feet. I have accepted this estimate in drawing up the geological timescale on page 284 , in which the supposed thickness of the deposits belonging to each of the main subdivisions of the three great eras is indicated both numerically and by the relative depth of the space allotted to each.

Professor Sollas, however, estimates a much more rapid rate of accumulation of these deposits — as high, indeed, as one foot per century - which, even with his own increased estimate of thickness, would give only $25,300,000$ years as the total lapse of time since the commencement of the Cambrian epoch. But he also points out that there is good reason for believing that the preCambrian epoch must have been as long as all the succeeding epochs put together, and the total lapse of time since the formation of the stratified rocks (including the pre-Cambrian) commenced might accordingly be estimated at 50,600,000 years.

We must also, of course, make abundant allowance for the fact that denudation has been going on at all times since the deposition of the stratified rocks commenced, and that the total thickness of these rocks has been enormously reduced thereby, the same material having been used over and over again in rock formation.

Where opinions differ so much, and where there is so much uncertainty as to the value of the data themselves, it is obvious that any estimate of geological time derived from the thickness of the sedimentary rocks can have only a doubtful value, and that it is highly desirable that such an estimate should be checked by making use of other sources of information.

Several other methods of estimating the age of the earth have been employed. Perhaps the most satisfactory is that which is especially associated with the name of Professor Joly, and which 
depends upon the amount of sodium present in solution in seawater. If we knew the total amount of sodium present in the ocean, and if we also knew the rate at which it has been accumulating since the ocean was first formed, it would be a simple matter to calculate the age of the ocean and therefore that of the stratified rocks, whose existence must have commenced with that of the ocean itself. The greater part at any rate of the salt contained in the sea must have been derived from the disintegration of the land and carried down by rivers. Estimates have been made, based upon careful analysis, of the amount of sodium contained in the sea and in the water of various rivers, as well as of the amount of water which flows into the sea annually, and Professor Sollas has come to the conclusion that " on a review of all the facts, the most probable estimate of the age of the ocean would appear to lie between 80 and 150 millions of years," which is considerably in excess of the estimate which he arrives at from the consideration of the thickness of the sedimentary rocks.

We cannot assume, however, that the earth has been habitable during the whole of this period, for when the ocean was first formed both it and the land must have been at far too high a temperature to admit of the existence of protoplasmic organisms. Possibly if we assume $100,000,000$ years as the total age of the habitable earth, and therefore as the time which has been available for the evolution of the organic world, we shall be as near as we can get to the truth in the present state of our knowledge.

We must now turn to the consideration of the geological record itself, and in the first place we may ask, what is to be legitimately expected from such a record? Have we any right to expect anything like a complete representation, by fossil remains, of the past history of the organic world? Obviously the answer to this question must be a decided negative. The imperfection of the geological record is due to many causes. It must have been imperfect when first laid down, for the great majority of organisms usually disintegrate and pass away without leaving any recognizable remains behind them at all. In most cases it is only hard skeletal structures that have any chance of being preserved. Bodies composed entirely of soft tissues, such as jelly-fish and many worms, are rarely represented by fossils. Even hard structures, such as animal skeletons, will only be fossilized if they happen by some 
fortunate chance to find their way to some place where rock formation of a suitable kind is going on. The remains of land animals may be carried by rivers to some sea or lake and buried in a suitable accumulation of mud or sand, but it is more likely that they will not. Marine animals, provided they have hard skeletons, have, of course, many more opportunities of attaining a lasting monument, and we often find great thicknesses of rock, such as chalk and limestone, composed almost entirely of their remains.

Even when the record has been successfully established, however, it is liable to destruction by various agencies. The

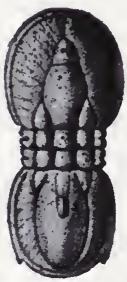

A.

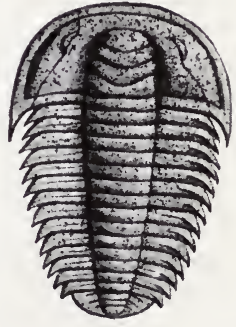

B.

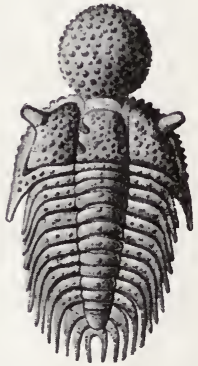

c.

FiG. 134.-Three Species of Trilobites. (From British Museum Guide.)

A., Agnostus princeps ; B., Olenus cataractes ; C., Staurocephalus murchisoni.

rocks containing it may be uplifted above sea-level and planed right away by sub-aërial denudation, or they may be sunk so far beneath later accumulations as to be profoundly altered by the action of heat and pressure, by which means any fossils which they contain may be completely destroyed. Then, again, we must remember that only a relatively small proportion of the earth's crust is accessible for investigation. Deep-lying rocks may be brought to the surface by upheaval, and denudation of overlying strata, or they may be reached by deep mining, but we know practically nothing of the strata which now lie beneath the bed of the sea, and even the dry land has only been tentatively scratched by inquisitive man in a few places.

Even were the geological record far less perfect than it actually is, therefore, its imperfection could not legitimately be 
used as an argument against the theory of organic evolution. From the nature of the case it must be imperfect and the surprising thing about it is that it should have yielded so much evidence as it has done. The earlier portions of the record, however, have apparently been completely destroyed. The fact that already in the Cambrian epoch highly organized invertebrate animals, such as trilobites (Fig. 134) and brachiopods, were in existence, shows us that evolution must then have been going on for an immensely long period. This period, as we have already indicated, is represented by the pre-Cambrian rocks, in which the organic remains have been so far destroyed as to be only recognizable in a few doubtful cases.

The investigation of what remains of the geological record has, however, yielded some very remarkable and conclusive results. In the first place we have learnt that life on the earth has been continuous without a break from the commencement of the Cambrian epoch to the present day, and in the second place we have learnt that the higher groups of animals and plants have appeared on the earth in exactly the order which we should expect on the assumption that each has arisen from some preceding and more lowly organized ancestral group.

In diseussing this point we may confine our attention to the Vertebrata, the evolution of which seems to have taken place entirely during post-Cambrian times. The most lowly organized group of true vertebrates existing at the present day is that of the Cyclosiomata, which includes the lampreys and hag-fishes. These are distinguished from true fishes by the absence of jaws, the mouth being suctorial, and by other primitive features. The skull and vertebral column remain cartilaginous throughout life and there are no paired limbs. Neither is any dermal armour of any kind developed. The only structures which they possess which seem at all likely to be preserved as fossils are the horny teeth. Certain bodies known as conodonts, which occur as fossils in strata ranging from the Lower Silurian to the Carboniferous, may perhaps represent such teeth. If so they are perhaps the earliest vertebrate remains as yet known. ${ }^{1}$

The remarkable group of fishes known as Ostracodermi, however, makes its appearance in the Upper Silurian and continues on to the Upper Devonian. These animals agree

1 Palæospondylus, from the Lower Old Red Sandstone of Scotland, is probably also a cyclcstome.

B. 
with the cyclostomes in the absence of true jaws and (in most known forms) of paired fins, and in the extremely primitive character of the vertebral column. They had also, like the cyclostomes, median fins, but they differed from the latter conspicuously in the presence of a strongly developed dermal armour,

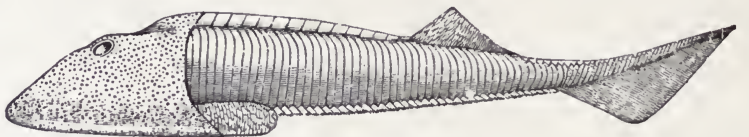

FIG. 135.--Restoration of Cephalaspis murchisoni, from the Iower Old Red Sandstone, $\times \frac{1}{2}$. (From British Museum Guide, after Smith Woodward.)

to the existence of which their fossil remains owe their preservation. As representative genera of this group we may mention Cyathaspis (Upper Silurian), Pteraspis (Lower Devonian),
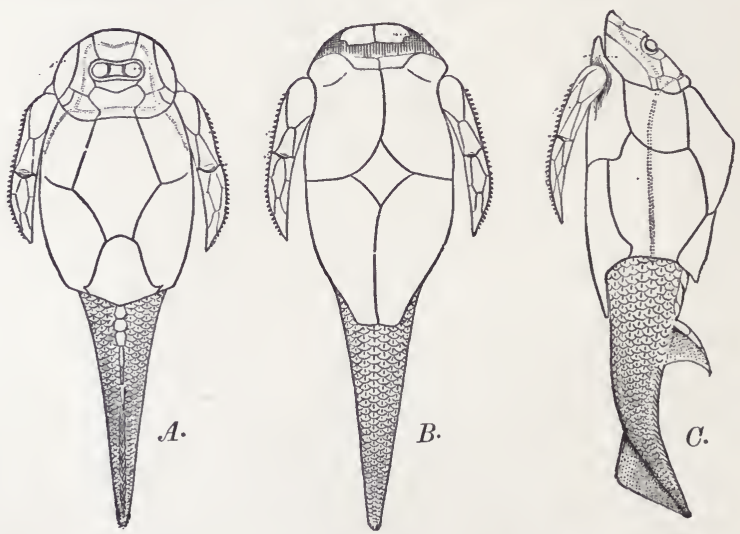

FIG. 136.-Pterichthys milleri, from the Lower Old Red Sandstone; restored by R. H. Traquair. A. Dorsal ; B. Ventral; C. Lateral views, $\times \frac{1}{2}$. (From Smith Woodward's " Vertebrate Palwontology.")

Cephalaspis (Upper Silurian and Lower Devonian, Fig. 135) and Pterichthys (Lower Devonian, Fig. 136).

The origin of the Ostracodermi is unknown, but their relationship to the cyclostomes has led to their inclusion with the latter in one class, the Agnatha (jawless vertebrates), which stands at the 
very bottom of the vertebrate series and was probably derived from some primitive chordate ancestor, not unlike Amphioxus, which had no hard structures to be handed down to us in a fossil condition. It is true that the ostracoderms bear a curious resemblance to certain extinct contemporaneous arthropods, the Eurypteridæ (Fig. 137), but this resemblance is probably merely superficial and due to convergence owing to similar conditions of life; it cannot be accepted as evidence of the origin of vertebrates from arthropodan ancestors, which, on other grounds, is extremely improbable.

The most primitive group of true jaw-bearing fishes is that of the Elasmobranchii, including the existing sharks, dog-fishes, skates and rays. These have well developed paired fins and a dermal armour of scales or denticles, but their primitive character is shown by the fact that the internal skeleton remains cartilaginous throughout life. Fragmentary indications of elasmobranchs occur already in the Upper

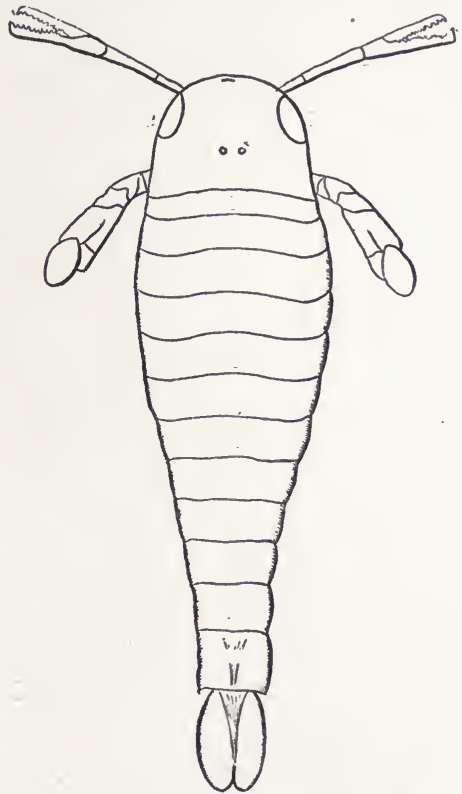

Fig. 137.-Iterygotus osiliensis, an Upper Silurian Eurypterid, dorsal view, reduced. (From Wood's "Palæontology," after Schmidt.)

Silurian, but the first satisfactory specimens have so far been met with in rocks of Lower Devonian age (Old Red Sandstone). A carboniferous form, Acanthodes, is represented in Fig. 138.

The remarkable Dipnoi, feebly represented by the mudfishes or lung-fishes of the present day-the Australian Neoceratodus (Fig. 110), the African Protopterus and the South American Lepidosiren-seem to have arisen during the 
Devonian period and to have attained their maximum of development and specialization during that epoch. This group is of especial interest, as we have already had occasion to notice, as indicating how the transition from an aquatic to a terrestrial mode of life was first rendered possible to vertebrate animals by the conversion of the swim-bladder into a pair of lungs. The dipnoids, however, have remained aquatic in habit, and retain their gills for aquatic respiration as well as being able to breathe air directly by means of their lungs.

The ordinary bony fishes (Teleostei), to which the vast majority of existing species belong, do not attain any prominence as a group before the secondary era, but they had fore-runners in some of the early ganoids which date back to lower Devonian times. The teleosts are the most specialized of all

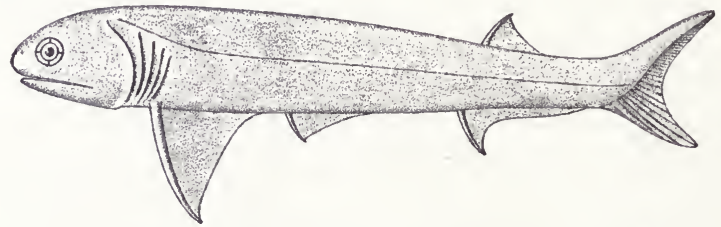

FIG. 138.--Restoration of Acanthodes wardi, from the Coal Measures of Staffordshire, $\times \frac{1}{3}$. (From British Museum Guide.)

fishes and it is not from such a group that we should expect the next great advance in organization to take its origin.

There are the strongest anatomical and embryological grounds for believing that the Amphibia, a group which at the present day includes the frogs, toads, newts and salamanders, are the descendants of fish-like ancestors which became adapted to an air-breathing mode of life by the development of lungs. The dipnoid fishes, which, as we have already seen, attained their maximum of development in the Devonian epoch, show us clearly enough how such a transition probably took place, and it was doubtless either from some dipnoid form, or from some other primitive type of fish which had also succeeded in converting its swim-bladder into lungs, that the Amphibia arose. The fact that amphibian remains first occur in Lower Carboniferous rocks is in complete harmony with this hypothesis as to the origin of the group. 
The earliest known amphibians are the Stegocephalia, socalled on account of the strongly developed bony armour which covered the head and formed the roof of the skull, as it did already in many of the earlier fishes. These Stegocephalia included both small, salamander-like forms such as Branchiosaurus (Fig. 139) and large crocodile-like creatures-the labyrinthodonts. Some of them survived into the Triassic
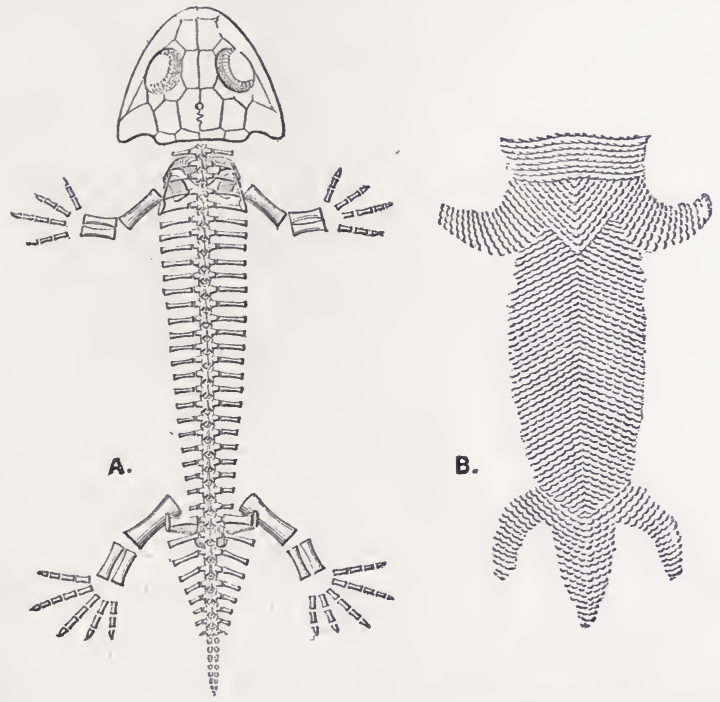

FIG. 139.-Branchiosaurus amblystomus; restoration of Skeleton (A) and ventral Armour (B) by H. Credner, nat. size. Lower Permian. (From Smith Woodward's "Vertebrate Palæontology.")

epoch, after which they appear to have become entirely extinct, and although a fair number of Amphibia still exist at the present day, the group must be regarded as having attained its maximum of development in late Palæozoic and early Mesozoic times. The existing frogs and toads are very highly specialized forms, completely adapted to a terrestrial life in the adult condition but for the most part resorting to water to deposit their eggs, which hatch out in the form of aquatic fish-like larvæ or tadpoles, 
breathing by means of gills (Fig. 121). The newts, on the other hand, though developing lungs, retain the fish-like form and the aquatic habit throughout life.

In one respect the imperfection of the geological record as regards the origin of the Amphibia from fishes is especially to be regretted. One of the chief distinctions between the two groups lies in the fact that whereas the Amphibia always have pentadactyl limbs (except when these have been lost by degeneration), the fishes have only fins, which are not pentadactyl. The conversion of the fin into a pentadactyl limb was no doubt an adaptation to the terrestrial mode of life, but neither comparative anatomy nor embryology nor yet the evidence of the geological record have so far enabled us to discover how the transformation of the one type of limb into the other actually took place. We can only hope that light may yet be thrown upon this difficult problem by the discovery of the fossil remains of intermediate forms.

The reptiles and the amphibians are closely related to one another, but existing reptiles are readily distinguished from existing amphibians in a variety of ways. For example, they produce much larger eggs, within which the young are nourished up to a very advanced stage of development by means of the yolk, while the embryo is provided with the special protective and respiratory fotal membranes known as amnion and allantois, which are not found in amphibians. A reptile, again, never develops gills at any stage of its existence, while an amphibian not only has gills in the larval but sometimes also in the adult state.

Fossil remains naturally afford no clue as to when the development of the fœtal membranes and the suppression of the larval gills took place, and we are thrown back entirely upon skeletal peculiarities as a means of distinguishing between extinct members of the two groups. From one point of view this is unsatisfactory, but the very difficulty which we experience in drawing a hard and fast line between the fossil reptiles and amphibians only serves to emphasize the fact that the one group has been derived from the other.

One of the chief osteological peculiarities which distinguish existing reptiles from amphibians is, as Dr. Smith Woodward observes, "the degeneration of the parasphenoid bone and its functional replacement in the basi-cranial axis by the pterygoids. 
. . If this feature in the palate has always been distinctive, Palcohatteria, from the Lower Permian of Saxony, is the earliest member of the class Reptilia hitherto discovered; and it is certain that during the Upper Permian age there were numerous reptiles both in Europe and America, probably also in South Africa." 1 So far as the evidence goes, therefore, the appearance of the Reptilia succeeds that of the Amphibia in exactly the way demanded by the theory of organic evolution.

The reptiles as a group attained their maximum of development in Mesozoic times, and of the nine great orders into which the class has been subdivided only four persist at the present day, and one of these four is represented by only a single

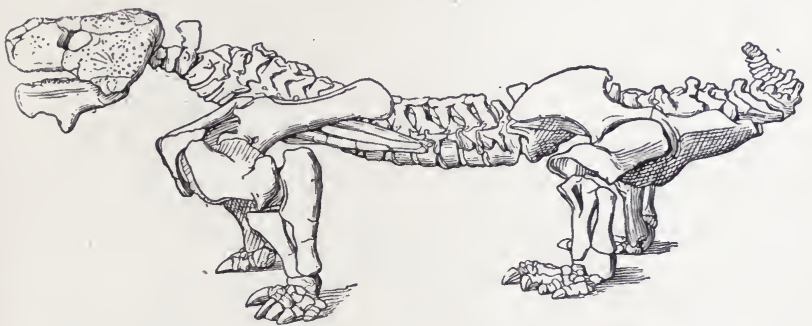

FIG. 140.- Skeleton of Pariasaurus baini, $\times \frac{1}{30}$. (From Smith:Woodward, after Seeley.)

species. This species, Sphenodon (=Hatteria) punctatus (Fig. 113), now confined to certain small islands off the coast of New Zealand, is the sole surviving representative of the order Rhynchocephalia, and not only constitutes the oldest existing type of reptile but also makes a very close approach to the oldest type known from fossil remains - the extinct Palæohatteria of the Lower Permian. The other existing orders of reptiles are the Chelonia (turtles and tortoises), the Squamata (lizards and snakes) and the Crocodilia (crocodiles and alligators).

Many of the extinct Secondary reptiles were far more highly specialized and remarkable than any existing forms. Different members of the class were adapted to the most diverse modes of life, the group having taken full possession of sea, land and air.

1 A. Smith Woodward, "Outlines of Vertebrate Palæontology for Students of Zoology" (Cambridge, 1898). 
The earliest and at the same time the least specialized of the extinct orders were the Anomodontia or Theromorpha, which Dr. Smith Woodward tells us were "directly intermediate in skeletal
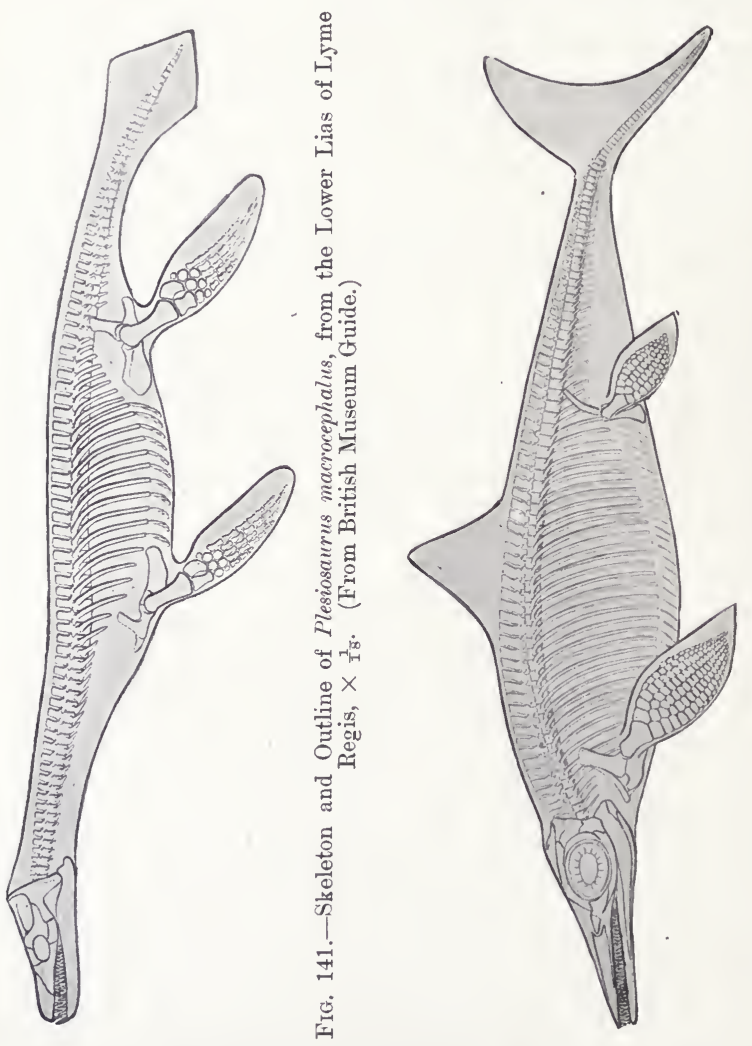

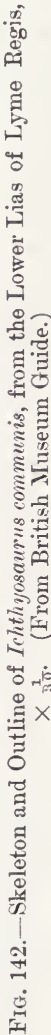

characters between the highest Labyrinthodonts (Mastodonsaurus and its allies) and the lowest Mammals (Monotremata)." These animals, whose remains have been found only in Permian and Triassic deposits, were terrestrial creatures with limbs modified for walking on dry land, so that they often bore a close general 
resemblance to mammals. One of the best known is the celebrated Pariasaurus (Fig. 140), described by the late Professor Seeley from the Karoo formation of Cape Colony. This animal attained a length of some ten or eleven feet.

The Sauropterygia or Plesiosauria (Fig. 141) were longnecked lizard-like forms, sometimes of large dimensions, which had become re-adapted to a marine life, and whose limbs, while retaining the typical pentadactyl structure, gradually became modified to form paddles. The group seems to have persisted

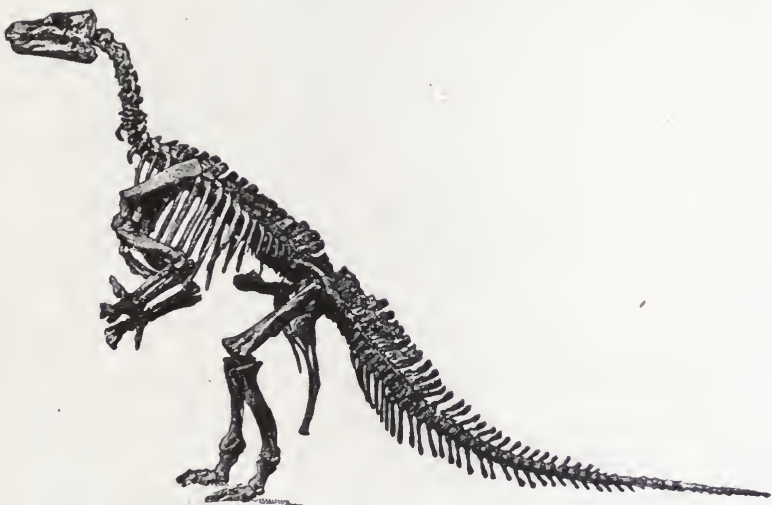

Fig. 143.--Skeleton of Iguanodon bernissartensis; from the Wealden of Belyium, $\times \frac{1}{80}$. (From British Museum Guide.)

throughout the whole of the Secondary period and shows a gradual evolution from the Trias onwards.

The Ichthyopterygia or Ichthyosauria (Fig. 142), which also range throughout the whole of the Secondary period, were more completely adapted to life in the ocean and acquired a remarkably fish-like form, like the whales, dolphins and porpoises amongst existing mammals.

The Dinosauria or Ornithoscelida formed an enormous group of land reptiles which seems to have arisen in the Triassic period and reached its climax in Jurassic and Cretaceous times. Some of them were carnivorous, others herbivorous; some walker on all fours and others on their hind legs, and they often attained gigantic dimensions. The well known Iguanodon(Fig. 143), from the 

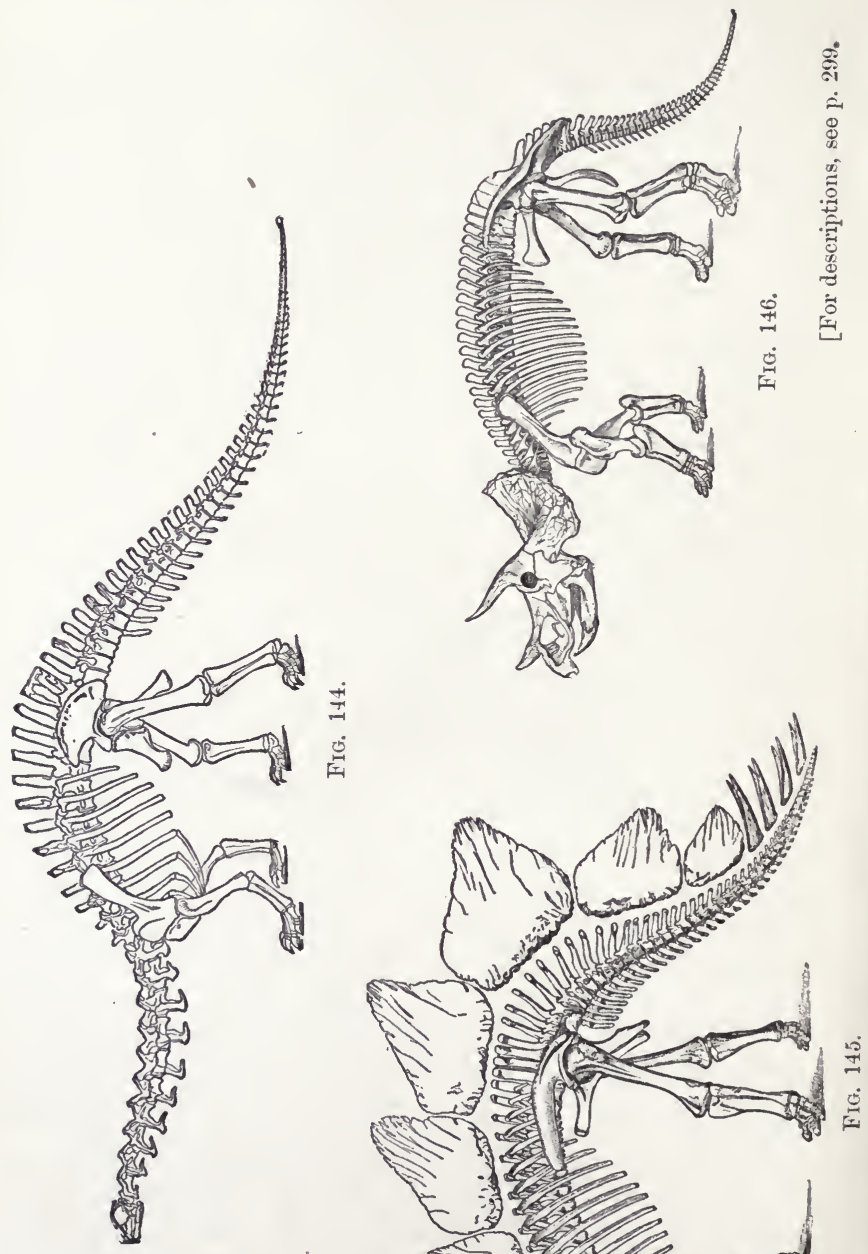

$\dot{0}$

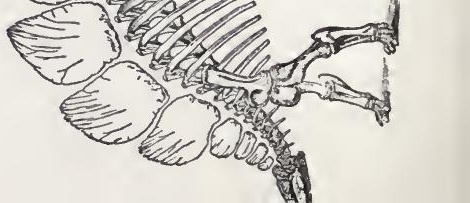


Wealden deposits of Europe, was a huge herbivore, which seems to have supported its massive body, chiefly at any rate, on its hind limbs, which were much more strongly developed than the frout pair. The American Brontosaurus (Fig. 144) and Diplodocus walked on all fours, and the latter attained a length of 80 feet, but a large proportion of this was taken up by the enormously long neck and tail. In both these forms the head was of astonishingly small dimensions in proportion to the rest of the body. Other Dinosaurs, such as Stegosaurus (Fig. 145) and Triceratops (Fig. 146), developed an extraordinary dermal armature of bony plates or spines.

Perhaps the most remarkable of all the extinct reptiles of the Secondary period, however, were the Ornithosauria (Pterosauria or pterodactyls), which at that time occupied the place now

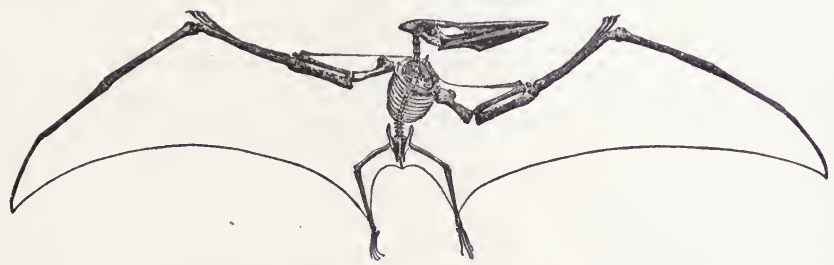

Fig. 147.- Skeleton and Outline of Pteranodon occidentalis, from the Upper Cretaceous of Kansas, U.S.A. $; \times \frac{1}{54}$. (From British Museum Guide.)

filled by the birds and bats. These animals were very perfectly adapted for flight, the wing being formed, as already described in Chapter XVII, by an extension of the skin supported by the arms, legs and tail, and especially by the enormously elongated fifth digit (Fig. 99). Some of these flying reptiles attained a very large size, the total expanse of the wings in Pteranodon (Fig. 147) being about eighteen feet.

The origin of birds from reptilian ancestors has been quite conclusively demonstrated on anatomical and embryological

FIG. 144.- Skeleton of Brontosaurus excelsus, from the Upper Jurassic of Wyoming, U.S.A.; $\times_{\frac{1}{5} \sigma^{\circ}}$ (From British Museum Guide, after O. C. Marsh.)

Fig. 145.- Skeleton of Stegosaurus ungulatus, from Jurassic of Wyoming; $\times \frac{1}{60}$. (From Smith Woodward's “Vertebrate Paløontology," after O. C. Marsh.)

FIG. 146. -Skeleton of 'Triceratops prorsus, from Cretaceous of Wyoming; $\times \frac{1}{80 .}$ (From British Museum Guide, after O. C. Marsh.) 
grounds, and Professor Huxley included both birds and reptiles in one large group, the Sauropsida. The structure of their wings shows, however, that birds cannot have arisen from pterodactyls but must have sprung from some less specialized form, mainly through the development of a dermal exoskeleton in the form of feathers, which constitutes the chief distinguishing feature of the group. It is, therefore, quite in accordance with expectation that the earliest known bird, Archæopteryx (Fig. 151), should have been found in the lithographic slates of Solenhofen in Bavaria, which are of Upper Jurassic age, and should exhibit characters intermediate between those of existing birds and reptiles. We shall have occasion to refer to this remarkable connecting link more in detail in our next chapter.

The Mammalia, so far as existing evidence indicates, appeared at an earlier date than the birds, but the earliest known mammals were far less highly specialized forms than birds, and although what appear to be mammalian remains have been found as low down as the Trias, the group did not attain much importance before the Tertiary epoch, when it replaced the reptiles as the dominant group of vertebrates. Opinions have been a good deal divided as to whether the mammals arose directly from amphibian ancestors or from some primitive group of reptiles. On the whole the evidence seems to be decidedly in favour of the latter view, which fits in very well with the first appearance of the mammals just when the reptiles were beginning to become dominant, and with the fact that, as we have already seen, some of the earlier reptiles were extraordinarily mammalian in aspect.

The earliest mammals were small forms of doubtful affinities. Dr. Smith Woodward remarks in this connection :- " It is as yet impossible to determine at what particular stage in the evolution of the vertebrate skeleton the lung-breathers first acquired the characteristic mammalian circulatory system, the milk-producing glands, and a dermal covering of hair," these being the principal features, apart from the skeleton, which distinguish mammals from reptiles at the present day. The difficulty is increased by the fact that some of the anomodont reptiles had acquired a dentition very similar to that of primitive mammals, while the earliest known mammals are represented by little more than a few fossil jaws and teeth.

The Mammalia, both living and extinct, may be divided into 
three main subclasses. (1) Prototheria, with the single surviving order Monotremata, represented by the Australian spiny anteater (Echidna, Fig. 92) and duck-billed Platypus (Ornithorhynchus, Fig: 91), which, as we have already seen, are intermediate both in anatomical structure and in their method of reproduction between the reptiles and the more typical mammals. (2) Metatheria, with the sole order Marsupialia, represented to-day by the Australian kangaroos, wombats, phalangers, thylacines and numerous other pouched mammals, and by the American opossums (Didelphyidæ) and Cænolestes. These are in some respects primitive forms, in which the young are born at a very early stage of their development (there being at the most only a very feebly developed placenta) and usually carried about, attached to the teat of the mother, in a marsupium or pouch. They are readily distinguished by osteological features, especially the structure of the jaws and teeth (Fig. 108), from the higher mammals. (3) Eutheria, the dominant mammals of the present day, with a large number of orders - edentates, whales, sirenians, ungulates, rodents, carnivores, insectivores, bats and primates; with well developed placenta and retaining the young in the womb until a very advanced stage of development. There can be no doubt that the Eutheria are on the whole more highly organized forms than the Metatheria and the latter than the Prototheria. This is especially indicated by the gradual acquisition and perfection of the characteristically mammalian method of nourishing the young by means of the placenta and mammary glands.

The order of appearance in time of the principal subdivisions of the class Mammalia, as indicated by the geological record, entirely supports the evolutionary hypothesis. The earliest known fossils which may possibly be referable to the class are of Triassic age. Tritylodon is represented by an imperfect skull from the Karoo formation of South Africa. This has generally been regarded as mammalian, but Dr. Smith Woodward, notwithstanding the fact that it has tuberculated grinding teeth with deeply cleft roots, considers that it probably belonged to an anomodont or theromorph reptile. Microlestes is represented by double-rooted, multituberculate teeth obtained from European Rhætic formations, and may also possibly be reptilian.

In deposits of Lower and Upper Jurassic age we find more convincing evidence of the existence of true mammals. The celebrated Stonesfield slate of Oxfordshire (Lower Jurassic) has 
yielded a fragment of a jaw, containing three small multituberculate grinding teeth, to which the name Stereognathus has been given, and one of the Middle Purbeck beds of Swanage (Upper Jurassic) has contributed the well known jaws of Plagiaulax (Fig. 148). Remains of closely related forms, supposed to have belonged to the same family (Plagiaulacidæ), have been discovered in Upper Jurassic and Upper Cretaceous formations of North America, and the family appears to have survived, both in Europe and North America, into early Tertiary times.

The Plagiaulacidæ and related forms have been grouped together in the extinct order Multituberculata, which Dr. Smith Woodward places provisionally amongst the Prototheria, side by side with the surviving order Monotremata, no remains of which are

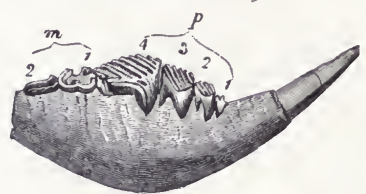

FIg. 148.-Mandible of Plagiaulax minor, $\times$ 4. (From Smith Woodward's " Vertebrate Palæontology," after Falconer.)

Microlestes). Mandibles of Phascolotherium (Fig. 149) and Amphitherium have been found in the Stonesfield slate, while Triconodon and Spalacotherium are similarly represented in the mammal bed at Durdlestone Bay near Swanage.

Throughout the whole of the Secondary period the mammals remained of insignificant size, and in a more or less primitive condition, such as is represented at the present day by the surviving monotremes and marsupials. The typical placental mammals (Eutheria) are not known to us from formations of earlier date than the Eocene. Then, all at once, they seem to have branched out in every direction and taken possession of land, sea and air just as the reptiles had done before them; whales replacing the Ichthyosauria and Plesiosauria, various groups of land mammals replacing the Theromorpha and Dinosauria, and bats sharing with the birds the kingdom of the air which had formerly belonged to the pterodactyls.

As in the case of their amphibian and reptilian predecessors, 
many of the mammalian groups in Tertiary times have run to great size; most of the larger forms, such as the primitive ungulate, Tinoceras (Fig. 150), of the American Eocene, and the giant ground sloth, Megatherium, of the American Pleistocene, are already extinct, but it must not be forgotten that the existing whales are amongst the largest animals that have ever lived, and in bulk at any rate will bear comparison with the largest of the great extinct reptiles.

The last term in the evolutionary series of the Mammalia is man, whose advent, so far as we at present know, dates back only

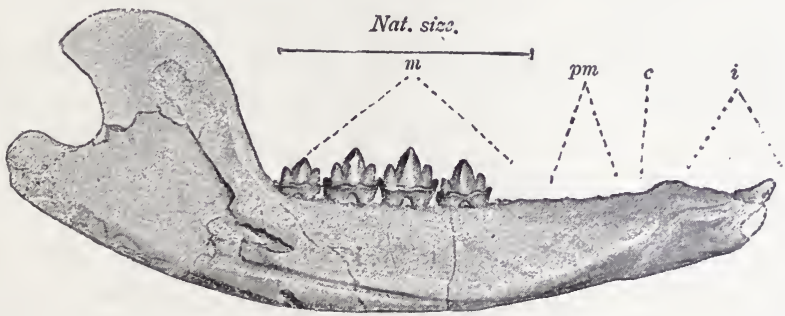

FiG. 149.-Mandible of Phascolotherium bucklandi; $\times 3$. (From Smith Woodward's "Vertebrate Palæontology," after Goodrich.)

to about the commencement of the Pleistocene or end of the Pliocene epoch. ${ }^{1}$

There is one more point that is well worth emphasizing about the evolution of the Vertebrata, as indicated not only by the geological record but also by the facts of comparative anatomy. Each successive great group appears to have arisen, not from the most highly specialized members of some preceding great group, but from comparatively undifferentiated forms. Thus the Amphibia arose, not from bony fishes, but from primitive dipnoids or ganoids; the reptiles arose, not from frogs or toads, but from primitive stegocephalian amphibia; the birds arose, not from pterodactyls, but from comparatively unspecialized reptiles; the mammals also arose from the more primitive reptilian forms, and man himself, whose advent undoubtedly marks the commencement of a fresh line of evolution, belongs to the order Primates, which in respect of bodily organization, as seen, for example, in the 
typical pentadactyl limbs, is far more primitive than many other mammalian groups.

Each great group seems to have begun in a small way, then developed rapidly, branching out in many directions and becoming the dominant group for the time being, only to dwindle away again and give place to some new and vigorous off-shoot. The dominance of any particular group has often been accompanied by the attainment of enormous size by its individual members, ${ }^{1}$

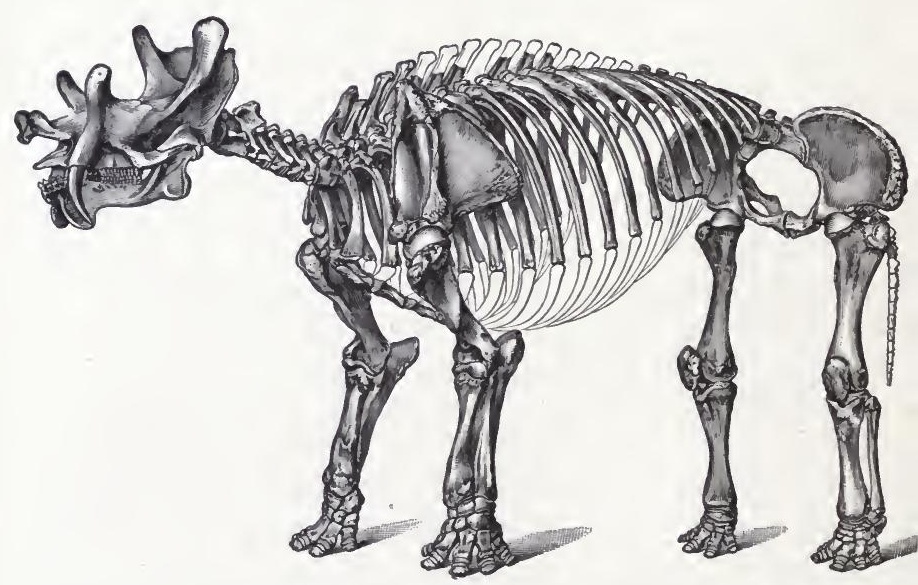

FIG. 150. Skeleton of Tinoceras ingens, from the Middle Eocene of Wyoming, $\times \frac{1}{30}$. (From British Museum Guide, after O. C. Marsh.)

and it is not impossible that this may have had something to do with its subsequent decline or complete extinction.

So far as the animal kingdom is concerned, we may perhaps say without exaggeration that the succession in time of the different groups, as indicated by the geological record, amounts to positive demonstration of the truth of the theory of organic evolution. In the case of plants the record is perhaps not quite so clear, but here also there can be no reasonable doubt that the great groups succeeded one another in a manner consistent with the theory, commencing with the algæ and ending with the flowering plants of the present day.

1 A possible explanation of this fact is suggested in Chapter XXVI. 


\section{CHAPTER XX}

Fossil pedigrees-Ancestry of birds, horses, elephants and whales.

In our last chapter we gave a brief outline of the general course of evolution amongst vertebrate animals as indicated by the geological record. We may now study in somewhat greater detail certain branches of the great phylogenetic tree which are especially well represented by fossil remains and therefore particularly instructive from the point of view of the evolution theory.

One of the most highly specialized groups of vertebrates that have ever existed is that of the birds. We have already pointed out that, on anatomical grounds, birds are classed together with reptiles as Sauropsida. 'They agree with reptiles in their method of reproduction by means of large, heavily yolked eggs, and in the presence, in the embryo, of the characteristic foetal membranes, amnion and allantois, as well as in certain morphological characters of the adult. They differ from reptiles, however, in many striking features. Thus they possess feathers, which almost (but not quite) completely replace the reptilian scales as a protective exo-skeleton. The anterior limbs are modified to form wings, constructed, as we have already seen in Chapter XVII, on an entirely different plan from those of flying reptiles. The digits of the hand are very greatly reduced; only one of them, and that in a vestigial condition, projects freely from the anterior border of the wing, forming the so-called "ala spuria" (Fig. 99). The true tail is greatly abbreviated and the caudal vertebræ reduced in number and to a large extent fused together to form the "ploughshare bone" which supports the tail feathers. Lastly, all existing birds have completely lost their teeth, which are functionally replaced by the horny beak.

The remains of the earliest known bird, Archæopteryx (Fig. 151), have been found in the celebrated lithographic stone of Solenhofen in Bavaria, of Upper Jurassic age, which, owing to its extremely fine grain, is peculiarly well suited for the preservation of even 
such delicate structures as feathers. This animal was about the size of a rook and the presence of well developed feathers and wings of the avian type is alone sufficient to show that we are dealing with a true bird. It still exhibits, however, a number of features which are usually met with in reptiles but have disappeared in modern birds. The digits of the anterior limb are not nearly so much reduced as in the latter, for three claw-bearing

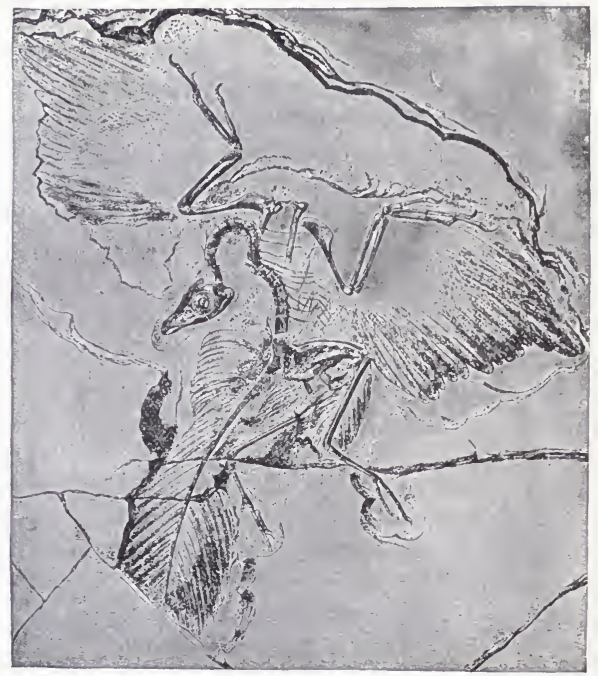

FIG. 151.-Fossil Remains of Archoeopteryx siemensi, showing the three fingers in each wing, the long tail, feathers, \&c. (From Lankester's "Extinct Animals.")

fingers project from the anterior margin of the wing; the tail is elongated like that of a lizard and supported by about twenty separate vertebræ each carrying a pair of feathers; and numerous teeth are present in the beak.

It is obvious that Archæopteryx represents a stage in the derivation of birds from reptilian ancestors, and this is exactly what we should expect of the earliest birds in accordance with the theory of evolution. Unfortunately, with the exception of a few other toothed birds of Cretaceous date, Archæopteryx is 
almost the only link in the pedigree of birds which has so far been discovered, and it teaches us nothing as to the origin of those characteristic avian structures, the feathers, which it possesses already in a fully developed condition.

It will be observed that Archæopteryx occupies a position between reptiles and typical birds exactly comparable with that of the Monotremata between reptiles and typical mammals (see Chapter XVII), the only difference being that the Monotremata still survive side by side with mammals of the most highly advanced type, while Archæopteryx has long since become extinct.

One of the most complete fossil pedigrees as yet known to us

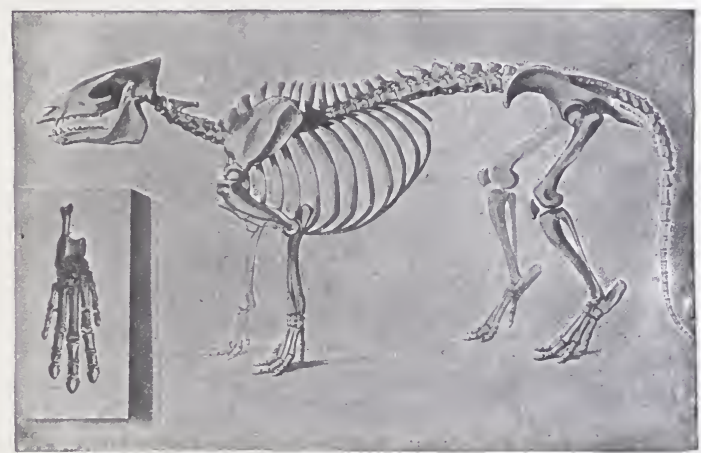

FIG. 152.-Skeleton of Phenacodus, a five-toed Eocene Ungulate. (From Lankester's "Extinct Animals.")

is that of the Equidæ or horse family. As we have already seen in Chapter XVII, the study of comparative anatomy indicates very clearly that the highly specialized single-toed limbs of the horse (Fig. 97) must have arisen from some primitive pentadactyl type by gradual suppression of all the digits except the middle one. Amongst the fossil remains of horse-like animals which abound in various tertiary formations of Europe and America we find a very complete series of stages in the evolution of the modern horse, which entirely confirms this conclusion. Our knowledge of this extremely interesting phylogenetic series is due largely to the late Professor O. C. Marsh and has been admirably summarized by Mr. R. S. Lull in the American Journal of Science. ${ }^{1}$

1 Series IV, Vol. XXIII, 1907. 


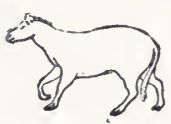

a

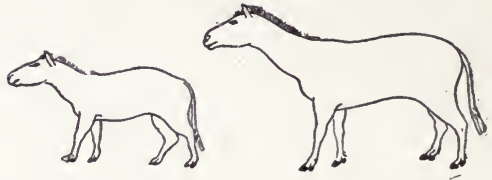

6

c

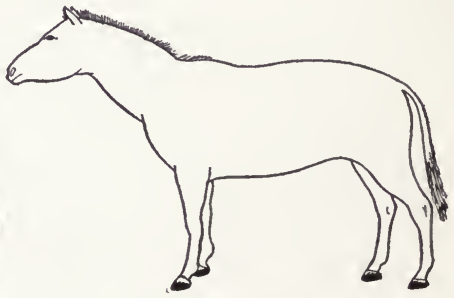

e

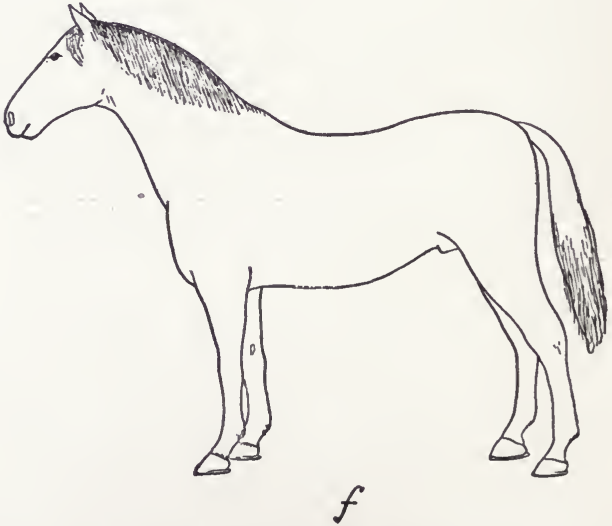

Fig. 153.-Outlines of Horses of different Geological Periods, showing their Relative Sizes. (From Lull.)

$a$, Protorohippus (Eocene); $b$, Orohippus (Eocene); $c$, Mesohippus (Oligocene); $d$, Merychippus (Miocene); $e$, Pliohippus (Pliocene); $f$, Equus (Recent). 
It is generally admitted that the Equidæ originated from the Condylarthra, a group of primitive, five-toed, ungulate mammals which made its appearance in early Eocene times, and the bestknown representative of which is Phenacodus (Fig. 152). The evolution of the horses appears to have taken place chiefly in America, though occasionally representatives of the group seem to have migrated to or from Europe, doubtless by a former land connection in the neighbourhood of Behring Strait. In comparatively recent times, however, the family became confined to the old world and was only re-introduced to America by human agency.

The course of their evolution has evidently been determined by the development of extensive, dry, grass-covered, open plains on the American continent. In adaptation to life on such areas structural modification has proceeded chiefly in two directions. The limbs have become greatly elongated and the foot uplifted from the ground, and thus adapted for rapid flight from pursuing enemies, while the middle digit has become more and more important and the others, together with the ulna and the fibula, have gradually disappeared or become reduced to mere vestiges. At the same time the grazing mechanism has been gradually perfected. The neck and head have become elongated so that the animal is able to reach the ground without bending its legs, and the cheek teeth have acquired complex grinding surfaces and have greatly increased in length to compensate for the increased rate of wear. As in so many other groups, the evolution of these special characters has been accompanied by gradual increase in size (Fig. 153). Thus Eohippus, of lower Eocene times, appears to have been not more than 11 inches high at the shoulder, while existing horses measure about 64 inches, and the numerous intermediate genera for the most part show a regular progress in this respect.

All these changes have taken place very gradually, and a beautiful series of intermediate forms indicating the different stages from Eohippus to the modern horse (Equus) have been discovered. The sequence of these stages in geological time exactly fits in with the theory that each one has been derived from the one next below it by more perfect adaptation to the conditions of life. Numerous genera have been described, but it is not necessary to mention more than a few.

The first indisputably horse-like animal appears to have been 
Hyracotherium, remains of which have been found in the London Clay (Lower Eocene). Another Lower Eocene genus was Eohippus, which seems to have arisen in Western Europe, possibly from a hyracotherian ancestry, and migrated, by way of Northern Asia, to America, where its remains occur in rocks of the same age. In this animal (Fig. 154) the fore foot had four well developed digits and the thumb was represented by a splint bone; in the hind foot the great toe had entirely disappeared and the fifth digit was represented only by a splint bone. In both fore and hind feet

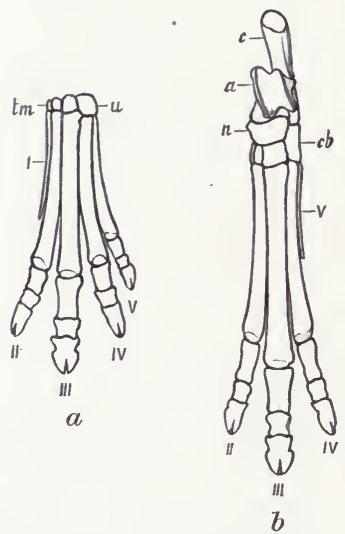

Fig. 154.- $a$, Fore Foot and $b$, Hind Foot of Eohippus pernix, $\times \frac{1}{2}$. (From Lull, after Marsh.) the third or middle digit was already conspicuously larger than any of the others.

Eohippus was succeeded by Protorohippus (Fig. 153,a), which was some 3 inches higher and had lost the vestigial thumb. Then came Orohippus (Fig. 153, b), again a little larger and with closely similar feet (Fig. 155), but with a considerable advance in the evolution of the grinding teeth. The last of the Eocene horses was Epihippus, still with four toes in front and three behind, but with the lateral toes further reduced in size and another distinct advance in tooth structure.

In Oligocene times there occurred in North America Mesohippus and Miohippus, and in Europe Anchitherium. Mesohippus (Fig. 153, c) was 18 inches or more in height, with three digits and a vestige of the fifth in the fore foot and three digits only in the hind foot (Fig. 156). Miohippus attained a height of 24 inches and closely resembled Mesohippus in the structure of its feet. Anchitherium is supposed to be a European derivative of Miohippus.

In the Miocene period the horses appear to have attained their maximum of development as a group, and a number of extinct American genera are distinguishable. Merychippus (Fig. 153, $d$ ), Protohippus and Neohipparion were still three-toed horses, though the lateral digits were now greatly reduced (Fig. 157). Pliohippus 
(Fig. 153,e), which continued on into Pliocene times and attained a height of 48 inches, had the second and fourth digits of each foot represented by mere splint bones as in modern horses, and had therefore already attained to the single-toed condition (Fig. 158).

In Pliocene times, however, we still find a three-toed horseHipparion-surviving in Europe, but the modern one-toed genus Equus (Fig. 153, $f$ ) also makes its appearance both in the old and new worlds, becoming extinct in the new world in Post-Pleistocene times until re-introduced from Europe by the agency of man.

The time occupied in the evolution of the genus Equus from its remote ancestor Eohippus is estimated by Professor Sollas at five or six millions of years. This period is sufficient to allow of a very slow and gradual change from one condition to the other. Allowing five years for each generation, Sollas arrives at the conclusion that somewhere about a million generations intervene between the two extremes. The total increase in height during this time has been 53 inches, and if this increase were spread fairly uniformly over the whole period

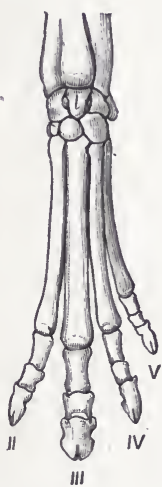

$a$

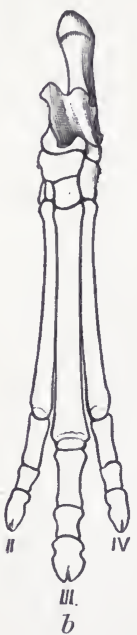

FIG. 155.- $a$, Fore Foot and $b$, Hind Foot of Orohippus agilis, $\times \frac{1}{2}$. (From Lull, after Marsh.) it would only mean about 0.00005 inch for each successive generation-an amount which would be quite imperceptible to human observers.

In reconstructing such a pedigree as that of the horse from palæontological evidence it is of course necessary to bear in mind that the great majority of extinct forms which come to light will almost certainly not be actually in the direct line of descent. Collateral branches will have been given off from the phylogenetic tree in various directions, and it is much more likely that any particular form discovered will belong to one of these branches than that it will belong to the main stem. This fact, 
however, by no means vitiates the general argument, for it is usually possible to pick out pretty accurately those which come into or near to the direct line, and even the collaterals afford valuable evidence as to the general course of evolution. We may safely say that the palæontological evidence amounts to a clear

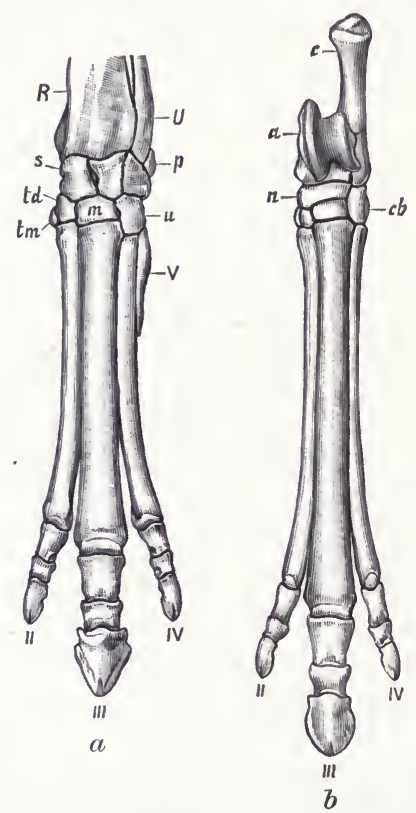

demonstration of the evolution of the horse from a five-toed ancestor along the lines indicated above.

The ancestry of the elephants is less well known than that of the horses, but recent discoveries in the Egyptian Tertiary formations, which we owe especially to the investigations of Dr. Andrews, have done much to elucidate the history of this remarkable group of mammals, and there can now be no doubt as to the main line of evolution which has led up to the existing Proboscidea. In some respects the elephants have remained in a somewhat primitive condition, as is indicated very clearly by the fact that all the digits remain well developed in both fore and hind feet. It is in the structure of the liead that they FIG. 156.- $a$, Fore Foot and $b$, Hind exhibit a high degree of specialFoot of Mesohippus celer, $\times \frac{1}{2}$. (From Lull, after Marsh.)

ization, marked particularly by the elongation of the snout to form a long prehensile trunk, by the enormous development of the occipital region of the skull, by the enlargement of the incisor teeth to form great tusks, by the shortening of the jaws and by the increase in size and complexity and the reduction in number of the cheek teeth. These changes have been accompanied by a huge increase in the size of the entire body, so that most of the elephants are amongst the largest of known land mammals, whether fossil or recent. 
Like the horses, the elephants probably originated from that primitive ungulate group, the Condylarthra. The earliest known form exhibiting proboscidean characters is Mœritherium, a tapir-like animal whose remains have been found in the Middle and Upper Eocene deposits of the Egyptian Fayûm. This was a comparatively small creature, about as large as a Newfoundland dog. It probably differed but little from other primitive ungulates, but the skull (Fig. 159, 1) already shows marked proboscidean tendencies. The position of the nasal bones, away back from the tip of the snout, indicates that there was in all likelihood a short proboscis. The occipital region of the skull is beginning to grow up and air cells are beginning to develop in the bones. The second pair of incisor teeth in each jaw are enlarged to form small tusks and the hinder cheek teeth are beginning to show an increase in complexity of structure. 'The total number of teeth however (36) is only eight short of the full typical mammalian dentition.

The next stage is represented by Palæomastodon (Fig. 159, 2) from the Upper Eocene of the same region, some species of which were little larger than Mœritherium
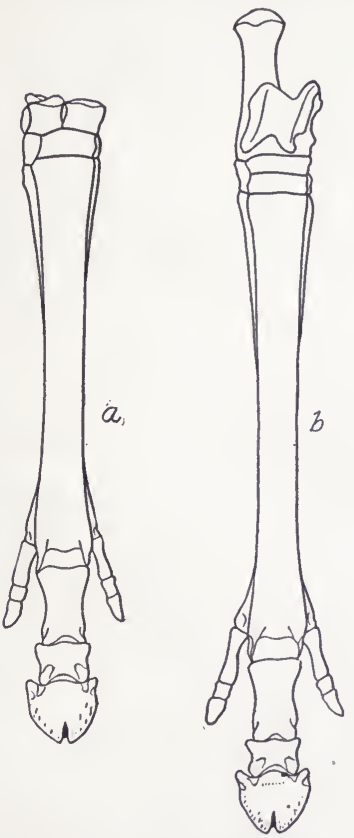

Fig. 157.- $a$, Fore Foot and $b$, Hind Foot of Neohipparion whitney $i, \times \frac{1}{4}$. (From Lull.) while others attained almost elephantine proportions. In this genus we notice a strong accentuation of the proboscidean characters. The occiput is higher, the nasal opening in the skull further back, the upper tusks better developed, the cheek teeth more complex; while the canines and all the incisors except the tusks in both jaws have disappeared. It will be observed that as yet there is no shortening of the jaws, but, on the contrary, the lower jaw has become 
considerably elongated, apparently serving as a support for the lengthening proboscis.

In T'etrabelodon angustidens, from European Miocene formations, this elongation of the mandible is much more marked, so that the lower jaw is much longer than the upper one and the short lower tusk comes to project almost as
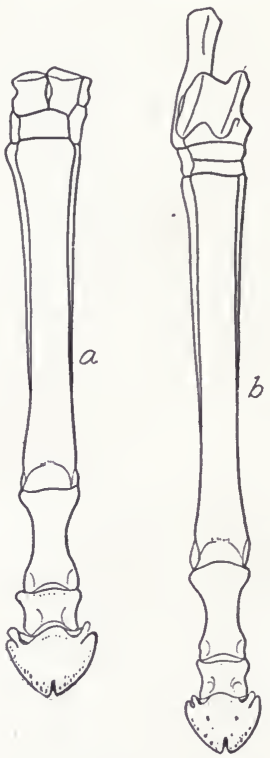

Fig. 158.- $a$, Fore Foot and $b$, Hind Foot of Pliohippus pernix, $\times \frac{1}{4}$. (From Lull.) far forward as the long upper one (Fig. $159,3)$. From this time onwards, however, the chin shortens, thereby allowing greater flexibility to the proboscis, so that in the lower Pliocene we find Tetrabelodon. longirostris (Fig. 159, 4) with the lower jaw only a little longer than the upper, leading the way to the mastodons and true elephants (Elephas), which also appeared in Pliocene times and in which the tusks have entirely disappeared from the greatly abbreviated mandibles while the cheek teeth have become enormously enlarged and complicated (Fig. 159, 5).

We have here a wonderfully perfect series of connecting links between the most primitive known ungulate mammals and the elephants. Only forms which appear to lie in or near the direct line of descent have been mentioned in the above brief account. Other modifications of the proboscidean type arose as lateral iffshoots from this main stem. One of the most remarkable of these is Dinotherium, with its great, downwardly directed lower tusks (Fig. 160), which appeared in Europe in the Pliocene period.

In the case of the Cetacea, a group which includes the whales, porpoises and dolphins, we have as yet only a much more fragmentary pedigree, but still quite sufficient to justify, on the palæontological side, the conclusion, already arrived at on anatomical grounds, that these extremely aberrant forms are the descendants of typical terrestrial mammals which have become re-adapted to an aquatic life and in accordance therewith have re-acquired a superficial resemblance to their much more remote 
Recent

$\begin{array}{ll}\text { Pleistocene } & \text { ELEPHAS } \\ \text { UnperPliocene } & \text { (short chin) }\end{array}$

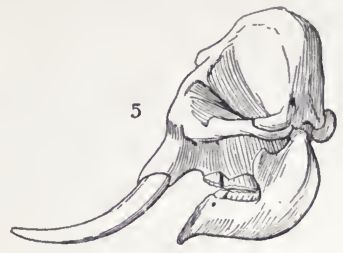

Lower Pliocene TETRABELODUN [LONGIROSTRIS STACE]

Unper Miocene (shorteningchin)

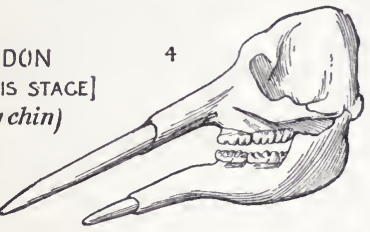

Middle Miocene

TETRABELODON

[ANGUSTIDENS STAGE]

Lower Miocene (longchin)

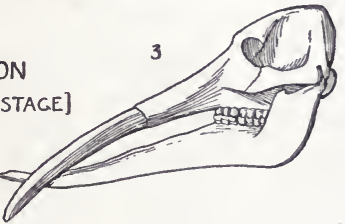
$\begin{array}{ll}\text { Utper Oligocene } & \text { Migration from Africa } \\ \text { into Europe-Asia }\end{array}$

?

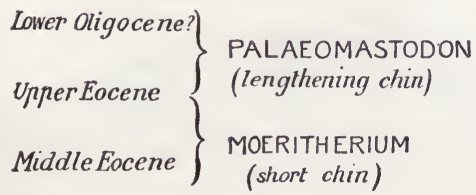

Lower Eocene

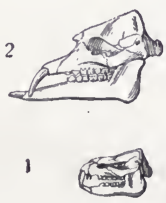

$?$

Fig. 159.- Some Stages in the Evolution of the Skull in the Proboscidea (From British Museum Guide.) 
fish-like ancestors. ${ }^{1}$ The fore limbs have become converted into paddles while the hind limbs have entirely disappeared externally

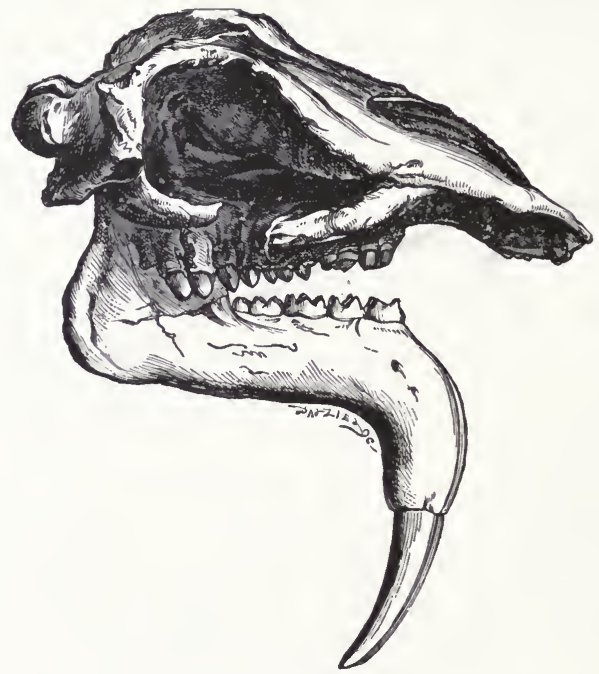

FiG. 160.-Skull of Dinotherium giganteum, Lower Pliocene, $\times \frac{\mathrm{T}}{15}$. (From Smith Woodward's “Vertebrate Palæontology," after Kaup.)

(Fig. 161). The tail has become flattened out into a horizontal fin and there is frequently a well developed dorsal fin. The skull

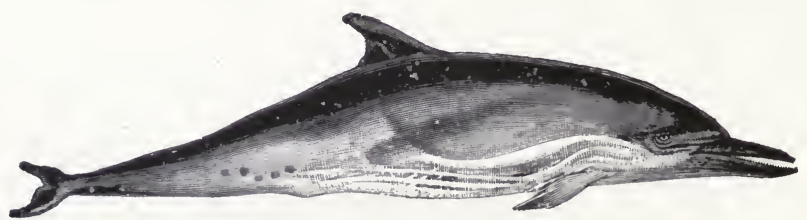

Fra. 161.-The Dolphin, Delphinus delphis, $\times \frac{1}{18}$. (From British Museum Guide.)

has undergone very curious changes. The brain case is rounded and strongly arched (Fig. 162) and the nasal apertures or blowholes lie far back, at or near the highest point of the head 
(Fig. 101, b), while the jaws have become greatly elongated. The teeth have in some cases completely disappeared, as in the whale-

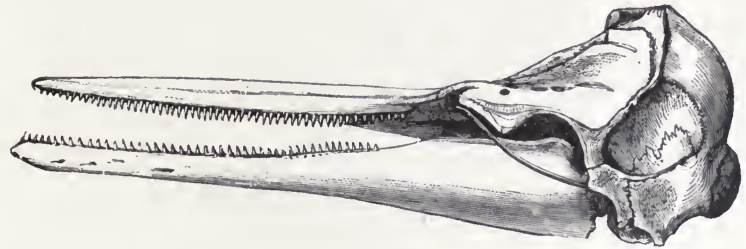

FIG. 162. - Skull of the Dolphin, $\times \frac{1}{5}$. (From British Museum Guide.)

bone whales (except for fotal vestiges), while in others they are present in large numbers but have lost the typical mammalian
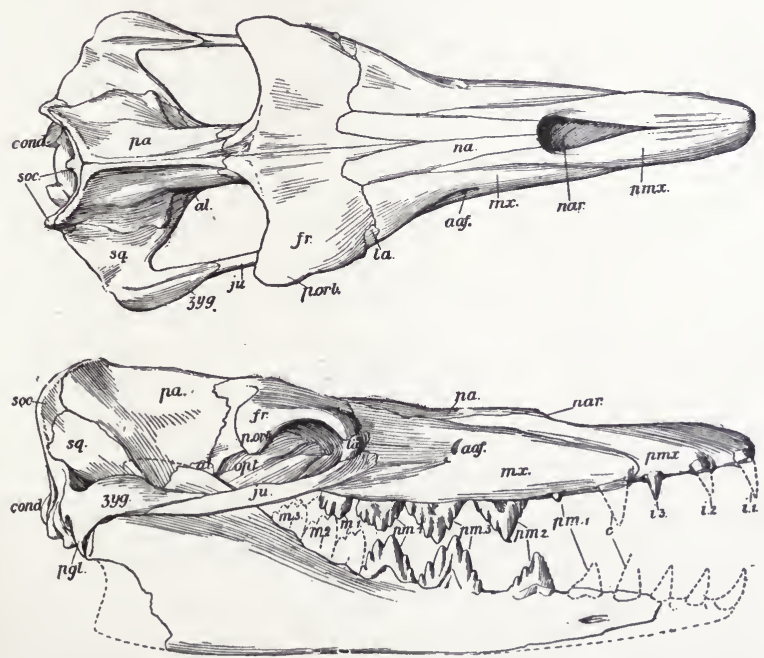

FIG. 163.-Dorsal and lateral views of the Skull of a primitive Whale, Prozeuglodon atrox, $\times \frac{1}{13}$. (From British Museum Guide.)

differentiation into incisors, canines, premolars and molars, being represented by a continuous and uniform series, all of which are conical in shape and single-rooted. Such teeth occur in both 
jaws of the porpoises and dolphins (Fig. 162) and in the lower jaw only of the sperm whale.

In the extinct shark-toothed Dolphins (Squalodontidæ), whose remains have been found in Miocene formations of Europe and America, the teeth are still differentiated into incisors, canines, premolars and molars, and the molars have double roots and compressed crowns with serrated edges.

Further back, in Eocene times, there existed, widely distributed over the northern hemisphere, a group of still more primitive whale-like animals known as Zeuglodontidæ. In these the seven vertebræ of the neck, which in existing whales are more or less fused together into a solid mass (Fig. 103), are all separate, and the typical dental formula is identical with that of primitive carnivorous land mammals, viz. i $\frac{3}{3}$, c. $\frac{1}{1}$, p.m. $\frac{4}{4}$, m. $\frac{3-2}{3}$.

The genus Prozeuglodon, from the Egyptian Eocene, approaches so closely in the characters of the skull and teeth (Fig. 163) to the primitive carnivores (Creodontia) of about the same period as to leave no reasonable doubt about the derivation of the Cetacea from that group, although it is quite possible that none of the extinct forms so far discovered are actually in the direct line of descent of any of the modern whales. 


\section{CHAPTER XXI}

Geographical distribution ${ }^{1}$-Areas of distribution-Barriers to migration - Means of dispersal - Changes in the physical conditions of the earth's surface-The evidence afforded by the study of geographical distribution with regard to the theory of organic evolution.

Iт is hardly necessary to remind the reader that each species of plant or animal, in a state of nature, is more or less sharply restricted to a certain portion of the earth's surface, the entire region over which it may be found, whether sea or land, being termed its area of distribution. Such areas of specific distribution are nearly always continuous, without any considerable gaps or intervals from which the species is entirely absent. This does not, of course, mean that the species necessarily occurs in all parts of its area of distribution at once, but that it is free to range over the whole of it and may accordingly be found in any suitable part of it at any time. It is necessary to introduce some such qualifying word as "suitable" in this connection, because each species is not only restricted in its range to a more or less well-defined geographical area, but can only live continuously in certain portions of that area, to the special conditions of which it is structurally and physiologically adapted and which constitute its habitat. Thus, for example, a fresh-water snail may perhaps range over an entire continent, but it would be useless to look for it except in fresh water. Individuals of a species may pass with more or less freedom, according to the nature of the case, from one habitat to another within the area of distribution, but it is only on rare and exceptional occasions that they are able to transgress the boundaries of the area itself.

True discontinuity in areas of specific distribution, as distinguished from mere discontinuity of habitats, is extremely rare. We have a good example of it, however, in the case of the marsh

1 The reader is referred to Dr. Wallace's classical volumes on "Island Life " and the "Geographical Distribution of Animals," and to Professor Heilprin's work on the "Distribution of Animals" in the International scientific Series (Vol. LVIII, 1887), for further information on this subject. 
tit (Parus palustris), which has two areas of distribution separated from one another by an interval of four thousand miles-in Europe and Asia Minor on the one hand and in Northern China on the other.

The size of the area over which a species may range varies immensely, in some cases comprising àn entire continent, or even more, and in others only a few square miles. Thus the leopard ranges over the whole of Africa and most of Southern Asia, while the Tuatara (Fig. 113) is confined to certain small islands off the coast of New Zealand, and certain species of humming birds are said to occur only on the volcanic peak of Chimborazo in the equatorial Andes.

An area of generic distribution is the sum of the areas of distribution of all the species which are comprised within the genus, and thus genera have usually a much wider geographical range than species. Families, again, have a wider range than genera and orders than families, and so on with groups of still higher value. In short, the more comprehensive the group the larger will be its area of distribution, until we find that the subkingdoms or phyla are cosmopolitan, ranging more or less over the entire world, wherever a suitable habitat is to be found.

The reason why species are rarely, if ever, cosmopolitan in their distribution is that they are confined within their own limited areas by the existence of physical conditions which constitute what are called barriers to migration. Such barriers either form absolutely insuperable obstacles to the passage of the species in question or they may be surmounted only at rare intervals and by some happy chance.

The nature of the barriers varies, of course, with the species concerned, and what is a barrier to one may be a high road to another. In the case of marine animals the principal barriers are continents and temperature conditions, while the deep sea itself acts as a barrier to the distribution of shore-dwelling or littoral forms. For terrestrial animals the chief barriers are seas, rivers, mountain ranges, deserts and climate generally; for fresh-water animals land and sea. In the case of plants the barriers to migration are very much the same.

It may be laid down as a general law that every organism, whether animal or vegetable, at some period or other of its existence is specially adapted so as to secure dispersal either by its own exertions or by the action of some external agency. By 
some means or another it is able, not only to spread itself over its own area of distribution, but also, when occasion offers, to extend that area by surmounting its barriers.

The lower terrestrial plants, such as fungi, mosses and ferns, are dispersed by means of spores, which, protected by special envelopes, may be widely distributed by the wind. In the higher plants the spores, as agents of dispersal, are replaced by seeds, which, usually still within the fruit, may be carried on the wind, floated on rivers or ocean currents, carried about entangled in the hair or feathers of animals, or actually eaten and passed out uninjured in the fæces. A great many seeds and fruits are specially modified in structure to secure their distribution in one or other of these ways, and the study of such adaptations constitutes one of the most interesting chapters in botanical science. We need only refer here to such fruits as the blackberry, whose succulence tempts the birds to eat them and carry the seeds, safely enclosed in their hard protective envelopes, to long distances; the various kinds of burs with their hooks for entanglement in fur and feathers; the winged fruits of the maple, elm and ash, and the thistledown of the thistles, adapted for floating on the wind.

The dispersal of plants is in all cases passive and dependent on external agencies, though sometimes aided by some purely mechanical device in the plant itself; in the case of animals it may take place either passively or actively, by the exertions of the animals themselves.

Beginningt with the marine fauna, we find that the larger forms-whales, porpoises, dolphins and fishes-owe their dispersal mainly to their own active powers of locomotion, while the smaller animals, especially the invertebrates, are largely dependent in this respect upon oceanic currents.

Even animals which, like the sponges and corals, are firmly fixed to the sea-bottom in the adult condition, have free-swimming larval forms (Fig. 164) whose own limited powers of locomotion may, under favourable circumstances, be enormously supplemented by the action of currents. Such larval forms, from the point of view of dispersal, play the part of the spores and seeds of plants, and they occur not only in cases where the adult is strictly sedentary in habit but also where its powers of locomotion are limited, as in many worms, snails, crabs (F'ig. 128), star-fishes, brittle stars (Fig. 127) and sea-urchins. The 
dispersal even of large and active fish, like the mackerel, is largely assisted by the action of currents upon the floating eggs, and this factor must be of still greater importance in the case of the comparatively sluggish bottom dwellers, such as the turbot and sole.

The more superficial waters of the open ocean are densely populated with pelagic animals and plants and with pelagic eggs and larvæ in various stages of development, all drifting more or less helplessly wherever the ocean currents may carry them, for their own powers of locomotion are usually quite insufficient to

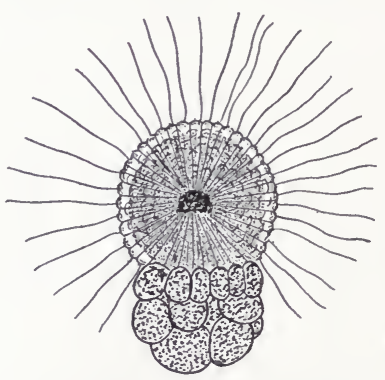

Fig. 164.-Free-swimming Larva of a Sponge, Grantia compressa; highly magnified.

(The larva swims by means of the rapid undulations of the numerous flagella with which it is provided.) enable them to pursue an independent course. This floating population is technically spoken of as "plankton" and its investigation, which is of great importance for the solution of practical fishery problems, has lately attracted a great deal of attention.

We must therefore regard all the great ocean currents, such as the Gulf Stream, as highways thronged with life of many kinds, including representatives of all the more important groups of marine animals, any one of which may be on its way to found a new colony and establish its own particular species in some region far distant from its original home. Some of the wanderers are only immature forms, belonging partly to shore-dwelling species, others are adult. Almost all exhibit some special adaptation to their pelagic mode of life. The fish-eggs are floated by oil-globules, and the larvæ of the crabs, brittle stars and sea-urchins are provided with defensive spines (Figs. 127, 128); but the most general and characteristic feature of all the pelagic host is transparency, whereby they are rendered inconspicuous and less likely to become the victims of the numerous enemies which feed upon the plankton. Adult jelly-fish, worms, molluses and crustaceans, and innumerable larval forms, all exhibit this same peculiarity.

The effect of ocean currents upon the distribution of marine 
animals is shown in a very interesting manner in the case of the Mediterranean and the Red Sea. In each of these there is a surface current constantly flowing in from the open acean and bringing in vast numbers of individuals, both larval and adult, which never find their way out again. Hence these almost enclosed seas form a kind of trap for marine animals and we accordingly find them to be inhabited by an exceptionally rich and varied fauna.

The range of marine species, though sometimes very wide, is usually more or less strictly limited, so that the shores of every continent have their own characteristic fauna and flora. This is no doubt partly accounted for by differences in climatic conditions, food supply and so forth, but it is mainly due to the fact that, in spite of the facilities for travel afforded by ocean currents, the dangers incidental to a long voyage from one continent to another are rarely surmounted, at any rate by shore-dwelling organisms. We know that many such forms flourish quite as well in some other part of the world as in their original home if they can once overcome the initial difficulty of migration. Thus the artificial introduction of the American oyster into British seas has accidentally brought with it the introduction of the remarkable limpet-like Crepidula, which attaches itself to the oyster shells and runs riot over the oyster beds on the Essex coast. That such occurrences may occasionally take place in a state of nature, and a species thereby be enabled to extend its area of distribution, there can be no reasonable doubt, for even American turtles have occasionally been carried by the Gulf Stream to the shores of Great Britain.

Amongst the higher forms of non-aquatic animals, and especially birds and mammals, their own powers of locomotion constitute the most important means of dispersal. Even these, however, are frequently transported for long distances by those external agencies which are chiefly responsible for the dispersal of less highly organized forms.

Leaving out of account, for the moment, the action of man, which has brought about immense changes in the geographical distribution of the existing fauna, the chief agents to be noticed in this connection are wind and water.

It is to the action of the wind that large numbers of winged animals-insects, birds and bats-owe the wide distribution which they enjoy. All actively flying land animals are liable to be 
carried out to sea in storms, and although the great majority of these will inevitably perish a few will occasionally manage to reach some distant haven where they may succeed in establishing a colony and thus extending the range of the species.

During westerly winds American birds not infrequently make their appearance on various parts of the coast of Europe, while north of the 58th parallel of latitude the polar winds trend in the opposite direction and with them we find a transference of European birds, by way of Iceland and Greenland, to the American continent. ${ }^{1}$ During storms, again, European birds are cast upon the Azores, about 1,000 miles from the nearest continental coast, and there is strong reason for believing that the little wax-eye (Zosterops lateralis) has been transported in this way from Australia to New Zealand, where it has succeeded in establishing itself.

Water currents may play an important part in the dispersal of two groups of terrestrial animals-those which occasionally swim and those which are liable to be carried away on icebergs or on floating masses of vegetation. Most quadrupeds swim well and even if not habitual swimmers may be forced to take to the water in times of flood. In this way they may cross large rivers and even get carried out to sea and perhaps to some neighbouring island, but they cannot cross large stretches of open ocean, and are accordingly never found, except when introduced by man, on islands far remote from any continent.

In polar regions the floating ice affords a means of dispersal to such animals as wolves and polar bears, while within the tropics floating islands or rafts formed of matted vegetation play the same part. Such islands have been observed floating out to sea from the mouths of large rivers like the Ganges, the Amazon, the Congo and the Orinoco. They serve as a means of transport to many different kinds of terrestrial reptiles, birds and mammals, and countless molluses, worms and insects, to say nothing of plants.

"If," says Sir Charles Lyell, "the surface of the deep be calm, and the rafts are carried along by a current, or wafted by some slight breath of air fanning the foliage of the green trees, it may arrive, after a passage of several weeks, at the bay of an island into which its plants and animals may be poured out as

\footnotetext{
1 Heilprin, op. cit., p. 47.
} 
from an ark, and thus a colony of several hundred new species may at once be naturalized." 1

As a definite example of this kind of dispersal may be mentioned the fact that in 1827 a large boa constrictor, twisted round the trunk of a tree, was carried by ocean currents from South America to the Island of St. Vincent, where it was destroyed after killing a few sheep.

A current flows from the North Island of New Zealand southwards to Chatham Island, four hundred miles distant from the nearest point on the New Zealand coast. This current carries considerable quantities of New Zealand timber to the island and its existence probably accounts for the fact that the planarian worm, Geoplana exulans, has been found both in the North Island of New Zealand and on Chatham Island, but, as yet, nowhere else. The land planarians habitually creep into the crevices of decayed timber, and their eggs are enclosed in tough, horny cocoons which may probably occasionally be transported even over wide stretches of sea.

Small terrestrial animals are, of course, often accidentally dispersed by human agency. Rats, mice and cockroaches have been carried nearly all over the world by ships, and snails, worms and other small creatures may be carried about with timber and earth, especially around the roots of plants. When I was in New Zealand I had some plants sent to me from England in a tightly closed tin box. When they arrived, after a voyage of some five or six weeks, I found an earthworm still alive in the tin. Many invertebrates have doubtless been unknowingly dispersed in this manner and great care has to be taken to make due allowance for such possibilities in studying problems of distribution. In exactly the same sort of way the seeds of many plants are accidentally dispersed over the world in ships' ballast, so that the same common European weeds occur in the neighbourhood of the ports along all the great routes of commerce.

The restrictions placed upon the dispersal of fresh water animals are more severe than in the case of either marine or terrestrial forms. One river system or one lake is separated from another by intervening land or sea which fresh water animals cannot as a rule cross by their own exertions. There are of course exception's, as in the case of the lampreys and eels,

\footnotetext{
1 Lyell's "Principles of Geology," Ed. 5, Vol. III., p. 44.
} 
and other fish which go down to the sea periodically, but for the most part the inhabitants of fresh water are largely dependent upon external agencies for their dispersal. Accordingly we find two groups of such animals, widely contrasted with one another as regards their distribution. Those which do not go down to the sea and which are not likely to be carried about by external agencies, such as most of the fishes, have usually restricted areas of specific distribution, and individual mountain lakes sometimes contain peculiar species of fish which are found nowhere else in

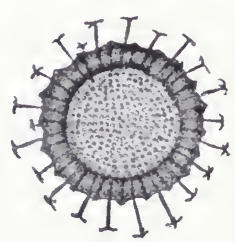

Fig. 16 .

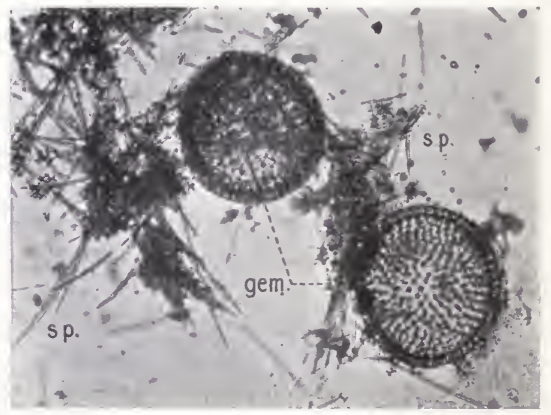

F1G. 166.

F1G. 165.-Gemmule or Statoblast of a Fresh Water Polyzoon, Cristatella mucedo, $\times 40$. (From Sollas.)

FIG. 166.-Two Gemmules of a Fresh Water Sponge, Ephydatia (Spongilla) fluviatilis, $\times 60$. (From a photograph.)

gem., gemmules; $s p$., spicules of the parent sponge.

the world. Galaxias nigothoruk, for example, is a small fish which occurs abundantly in lake Nigothoruk in Victoria (Australia). This lake is in a very isolated position in a mountainous region and the only outlet is by percolation underground. There appears to be no natural means by which the fish could be transferred to any other locality at the present time, and it is not known to occur elsewhere.

On the other hand many fresh water invertebrates, such as the Polyzoa, hydras and sponges, and above all the microscopic Protozoa, are remarkable for their wide distribution. Identical genera if not identical species of these groups occur almost all over the world, and the reason for this is not far to seek, for all 
of them have some special character which enables them to be easily dispersed by external agencies. The fresh water Polyzoa and sponges produce minute buds (statoblasts or gemmules) enclosed in hard protective envelopes (Figs. 165, 166), which are likely to be carried about in the mud on the feet of wading birds and mammals. The embryo of Hydra secretes its own protective envelope (Fig. 59, D-G) within which it passes through a period of rest embedded in the mud; while many of the Protista (e.g. Hæmatococcus) are capable of being dried up at some period or other of their life-history and carried about by the wind in the form of dust. Thus a sample of mud, taken from a pond and dried up, may, after an interval of many months, if again placed in water, give rise to an abundant fauna, amongst which even such highly organized forms as Crustacea (e.g. Apus and Branchipus, which lay specially protected eggs) will frequently appear.

We must remember that the present distribution of animals and plants is the outcome not only of the existing physical conditions of the earth's surface but also of conditions which obtained in past geological periods. From time to time these conditions undergo great changes, which may concern not only the climate of particular regions or of the entire world, but also the relative distribution of land and sea.

The earth has been subject, at various periods of its history, to climatic changes of two chief kinds, (1) cold or even glacial epochs in temperate regions, and (2) mild or warm epochs in aretic or antaretic regions. Probably alternations of these two extremes have been not infrequent, but the case of which we have most complete knowledge occurred in the Pleistocene period and is usually known as the "Glacial Epoch" par excellence.

There is clear evidence that during a pertion of the Pleistocene period a very large part of the northern hemisphere, which now enjoys a temperate climate, was covered with perpetual ice and snow and reduced to a condition resembling that of Greenland at the present time. Scandinavia and the whole of Northern Europe were buried beneath the ice-sheet, and the same is true of the northern part of North America.

The glacial epoch in the north must have driven the greater number of the northern plants and animals southwards, causing a keen struggle for existence in which many species were exterminated. Its influence was possibly intensified by the 
fact that the glaciation was not continuous but alternated with a succession of warm periods. The southern hemisphere also experienced a glacial epoch during which warm and cold periods alternated, and astronomers hold that the warm periods in one hemisphere coincided with cold ones in the other. It has been calculated that each warm or cold period lasted for about 21,000 years.

Other important changes in climate occurred long before the great glacial epoch. Thus the fossil remains of a luxuriant vegetation in Greenland and other northern localities indicate the occurrence of a mild arctic climate in Miocene times. Such a climate must have favoured migration between the old and new worlds by way of what is now Behring Strait, which may very well have been dry land at the time.

Owing to the gradual loss of the earth's heat by radiation and the consequent shrinkage and crumpling of the solid crust, variations in the level of the land are constantly taking place. Areas which are at present separated by sea may have been connected in former times and vice versâ, and there can be no doubt that the distribution of plants and animals has been profoundly influenced in this way. Many cases of discontinuity in distribution may be explained by the former existence of land connections which no longer remain. It is necessary, however, to be extremely careful how we invoke the aid of this principle, which, as an easy way out of difficulties, is apt to lead us into all sorts of unjustifiable speculations.

Those remarkable animals the lemurs, as a group, exhibit a very curious discontinuity in their distribution, occurring in Africa (and especially Madagascar) on the one hand and in Southern Asia on the other. To explain this distribution it has been suggested that in former times a continent-Lemuriaexisted in the Indian Ocean. Similarly, but with perhaps greater justification, it is believed by many people that the antarctic continent at one time extended much further north than at the present day, so as to afford, possibly with the aid of a chain of islands and with the co-operation of a mild antarctic climate, a route along which migration might take place between South America and Australasia. In this way may be explained certain remarkable points of agreement between the fauna and flora of Australia and New Zealand and those of South America. The genus Fuchsia, for example, is typically South American, 
but one or two species occur in New Zealand; and the same is true of Calceolaria. The evergreen beech forests of New Zealand must be extraordinarily like those which Darwin described in Patagonia. Even the same curious genus of fungus (Cyttaria) is found on the beech trees in South America, New Kealand and Tasmania. A fresh water lamprey, Geotria, also occurs both in New Zealand and South America and similar cases could be quoted from the invertebrate fauna.

It is very doubtful, however, whether such an extensive change in the configuration of the earth's surface as the submergence of an entire continent has ever taken place. According to Dr. Wallace, who is recognized as the greatest authority on the subject of geographical distribution, the existing continents and oceans as a whole are permanent features, although their outlines may be greatly affected by oscillations of the earth's crust.

Perhaps the strongest argument against the former existence of continents where we now have oceans lies in the fact that the average depth of the sea is many times greater than the average height of the land, no less than twelve thousand feet as compared with one thousand, for the great depths of the ocean extend over vast areas while the greatest heights of the land are narrow mountain ranges. Hence, although large areas of land might be submerged by a comparatively slight change of level, it would take an enormous movement to bring any extensive tract of the ocean bed to the surface.

In short, we are only justified in postulating the former existence of land in places where the ocean is comparatively shallow, but even this limitation leaves abundant opportunity for changes in the relative distribution of land and sea which would profoundly affect the distribution of plants and animals. The actual occurrence of such changes is abundantly proved by geological evidence and they are known to be going on at the present day in many parts of the world.

There is good reason to believe that the principal groups of terrestrial animals originated in the great northern land masses and that the southern peninsular areas of Africa, Australasia (now, of course, represented by detached islands) and South America have been peopled mainly by successive waves of migration from the north. We find in all these southern areas primitive, ancient forms of life. Marsupials at the present day 
are found only in Australasia and America, but the fossil remains of such animals are widely distributed over the northern hemisphere. The Onychophora, again, a small group of extremely primitive arthropods, which until recently were all included by zoologists in the single genus Peripatus (Fig. 167), are almost confined to Australasia, South Africa and South America, in all of which regions they are fairly abundant. It is more reasonable to imagine that the ancestors of the Onychophora migrated from the north, where the group las now become extinct, than to invent imaginary continents

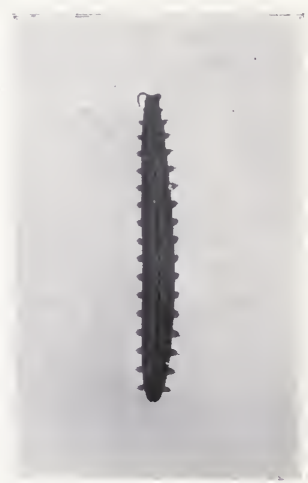

FIG.167.-Peripatuscapensis, from Cape Colony, $\times \frac{3}{5}$. (From a photograph.) across which they may have wandered, or even to suppose that they have been so widely distributed as we now find them by some external agency such as floating timber.

The geographical distribution of plants and animals would be quite inexplicable on the supposition that they had all been independently created and deposited where they now live. It is, however, easy enough to explain it on the theory that the earth has been peopled by the descendants of common ancestors which migrated from place to place as occasion permitted and at the same time underwent modification in many different directions. We may now briefly summarize the principal facts of distribution which justify us in holding this view.

(1) The extent of the area of distribution of any group of animals is directly proportional to its means of dispersal. 'T'hus flying animals are much more widely distributed than quadrupeds. Birds occur abundantly on oceanic islands, but the only mammals which occur there in a state of nature are bats and small forms like rats and mice which may be carried on floating timber. Nevertheless we know that when the larger mammals are transported by man to such localities they flourish exceedingly. Many Protozoa, again, which are readily blown about in the form of dust, are almost cosmopolitan even as regards their species.

(2) The degree of peculiarity of the fauna and flora of any 
area is proportional to the length of time for which and the extent to which that area has been isolated from other areas. Thus Australia, which has probably been separated from the Asiatic continent ever since the Cretaceous period, has a most peculiar fauna and flora. We have already referred to the numerous different kinds of marsupials-kangaroos, wombats, phalangers, native bears, native cats and so forth-which have not as yet been supplanted by the more recently developed groups of mammals found in other parts of the world. Australia is also still the home of those most primitive and reptile-like of all the mammals, the Monotremata (Figs. 91, 92). The Australasian forests, again, are composed principally of eucalypts of many different species, which are found nowhere else in the world. In New Zealand, which is even more isolated than Australia, we find no less peculiar inhabitants, including the wonderful tuatara (Fig. 113), the oldest surviving type of terrestrial vertebrate, together with the kiwi (Fig. 111) and other remarkable flightless birds.

The reasons why the degree of peculiarity of the fauna and flora of any region is proportional to the degree of geographical isolation are not difficult to find. On the one hand ancient types, such as the tuatara, the monotremes and the marsupials, may be preserved from competition with more modern forms long after they have been exterminated elsewhere. On the other hand,individuals accidentally introduced from distant areas at rare intervals will have few opportunities of breeding with others of the same species, and thus whatever variation occurs amongst them will be less liable to be swamped by intercrossing with the parent form. New races and ultimately new species will thus become established more readily in such areas than elsewhere. This principle of geographical isolation as a factor in the production of new species is of great importance and we shall have to refer to it again in a subsequent chapter.

The zoological or botanical affinities of the inhabitants of any given area, not only with one another but also with those of adjacent areas, are exactly what we should expect in accordance with the views which we are advocating. It is impossible to believe that the existing marsupials were (with the exception of the few American species) all specially created in Australasia when we know perfectly well that marsupials used to exist in Europe in past geological times and can still exist in Europe 
when transported there by human agency, and it is equally impossible to believe that such animals as sheep and rabbits, to which the Australian climate appears to be pre-eminently suited, were specially created in Europe and Asia but never in Australia. The existing condition of the Australian fauna is, however, easily explained on the supposition that it was originally derived from Asia at the time when marsupials and monotremes flourished in the north, and that the island continent became separated from the mainland before the more recent mammalian types, such as sheep and rabbits, had arisen on the latter. Divergent evolution within the limits of this isolated area is then quite sufficient to account for the immense variety of marsupials occurring there at the present day.

It is very instructive in this connection to contrast the condition of the fauna of a comparatively recently separated continental island, such as Great Britain, which is not far removed from its parent continent, with that of the fauna of a typical oceanic island which has never formed part of a continent at all and is very widely separated from any other land. The native or indigenous population of continental islands always exhibits a close relationship with that of the adjacent mainland, from which it was originally derived and with which it is still able to keep up a certain amount of intercourse. Such an island will contain indigenous quadrupeds, and the great majority of the species of plants and animals found in it will be identical with those of the mainland. True oceanic islands, on the other hand, such as St. Helena and the Sandwich Islands, are peopled entirely by waifs and strays which have gained access to them at rare intervals in one or other of the ways discussed in the earlier part of this chapter. They never contain large quadrupeds and, owing to their more or less complete isolation, the animals which do occur almost always belong to peculiar species found nowhere else in the world.

(3) Palæontological investigations have demonstrated that the present animal population of any tolerably isolated area is closely related to the population of the same area in comparatively recent geological periods. Thus in Australia we not only find that at the present day marsupials are by far the most characteristic features of the fauna, but also that the remains of extinct marsupials, many of which belong to genera and species different from any now living, are very abundant in the tertiary deposits 
of the same region. Similarly in South America at the present time the edentates (sloths, armadillos and ant-eaters) form the most characteristic mammalian group, and the tertiary deposits of that country have yielded the remains of a great number of extinct forms belonging to the same order. It would be very difficult to explain these facts on any theory of special creation, but we can easily understand how a group of animals, having once gained a footing in any area and finding itself secure and more or less cut off from communication with other parts of the world, would increase and vary, producing new species and ultimately becoming the dominant group in that particular region.

(4) Cases of discontinuous distribution are readily explicable on the theory of evolution and migration. Either individuals of the species in question have occasionally transgressed the barriers to their dispersal and established new and distant colonies, or possibly a large area of distribution has become broken up into a number of smaller ones by geographical or climatic changes rendering portions of it uninhabitable.

" Thus, for instance," says Romanes, " it is easy to understand that during the last cold epoch the mountain hare would have had a continuous range; but that as the arctic climate gradually receded to polar regions, the species would be able to survive in southern latitudes only on mountain ranges, and thus would become broken up into many discontinuous patches, corresponding with these ranges. In the same way we can explain the occurrence of arctic vegetation on the Alps and Pyreneesnamely, as left behind by the retreat of the aretic climate at the close of the glaçial period." 1

1 “ Darwin and after Darwin," Vol. I., p. 209. 


\section{CHAPTER XXII}

Adaptation to environment in animals-Deep sea animals-The colouration of animals - Protective and aggressive resemblances--Warning colours - Mimicry-Epigamic ornamentation.

In the last few chapters we have discussed a number of facts selected from that great and ever increasing mass of evidence which leads us to the inevitable conclusion that the present condition of the fauna and flora of the earth, with their almost endless diversity of plants and animals, is the outcome of a long process of organic evolution. It is desirable at this stage of our inquiry to emphasize the fact that this evolution, in the main, has been of a progressive character, and of such a character, moreover, as to maintain a more or less perfect harmony between the organism and its environment.

Adaptation in bodily organization and in corresponding function, whereby each kind of plant or animal is enabled to meet the constant demands made upon it and maintain its existence in the endless warfare of life, is the great outstanding feature of living things. So universal is this adaptation that we are apt to take it for granted, and any want of it is at once recognized as an exception and an anomaly. Anyone, for example, who watches the slow and clumsy movements of a tortoise cannot fail to be struck with the fact that the limbs of this animal are but ill-suited for purposes of locomotion, but even in this case there is compensation in that the tortoise carries its place of refuge about with it and has therefore little need to hurry itself.

We have seen in an earlier chapter how completely the pentadactyl limbs of air-breathing vertebrates may become modified from their primitive condition in correspondence with changes in the mode of life. The fore limbs, adapted in the first instance for locomotion on land, have become changed in the whales, seals and dugongs into paddles; in the pterodactyls, birds and bats into wings, and in man into organs of prehension. 
Indeed, given time enough, the power which an organism possesses of altering its bodily structure in accordance with new demands on the part of the environment seems, as we have already pointed out, to be almost without limits.

This plasticity is illustrated in the most striking manner in cases where the organism has been removed from what may be regarded as the normal environment of the group to which it belongs, and to which the great majority of the group are adapted, and come to live under new and very different conditions. Thus it is with the aquatic and aërial mammals, which, in encroaching upon the domains of the fishes and birds, have, by convergent evolution, come to resemble these in bodily form.

Wherever we turn we find freshillustrations of the same principle. At great depths of the ocean the conditions of life are very different from those which obtain in shallow water, and we find the animals which inhabit these abysses modified accordingly. Fig. 168 represents two deep sea sponges obtained by the "Challenger" expedition ; Cladorhiza longipinna from a
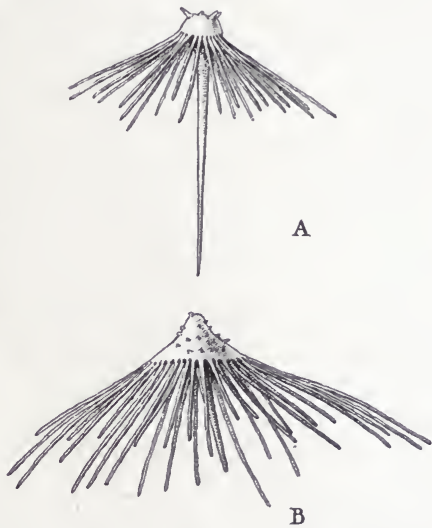

depth of 3000 fathoms in the North Pacific and Axoniderma mirabile from a depth of 2250 fathoms in the South Pacific. It will be seen at once that the form assumed by these sponges is very unusual and quite unlike that exhibited by their shallow water relatives. The great majority of the members of the group of sponges (the Tetraxonida) to which they belong are indeed by no means remarkable for symmetry of shape, but these two are beautifully symmetrical, their form at once suggesting that of a parachute, with a small conical body fringed by long radiating processes surrounding a central root-like projection. This "Crinorhiza form," as it is termed, is obviously an adaptation which serves 
to prevent the sponge from sinking into and being smothered by the soft mud or ooze which covers the bottom of the ocean at very great depths, and it is interesting to observe that species of several distinct though related genera have adopted the same device, thus affording a beautiful example of the phenomenon of convergence. Other sessile deep sea animals have found different means of overcoming the same difficulty, especially in many cases by the development of long stalks.

The absence of light at great ocean depths has led to the acquisition on the part of many of the deep sea fishes of brilliant phosphorescent organs, arranged like little lamps on various parts of the body. In some cases at any rate these serve to attract other animals upon which these fishes prey. Some of them, again, develop long and delicate feelers by aid of which they grope their way about in the dark.

In the brilliantly illuminated surface waters of the ocean conditions are very different, and here we find that the most favourite device for preserving life amidst a host of enemies is transparency, but we have already alluded to this in the preceding chapter and need not dwell upon it further. It is a phenomenon which falls under the head of protective colouration, of which we shall find better instances elsewhere.

The significance of the colouration of animals as a means of adaptation to environment is a subject which has in recent years developed into a special branch of biological science, and which already has a copious literature of its own. Professor Poulton, in his well known work on the Colours of Animals, ${ }^{1}$ has suggested an elaborate scheme of colour classification from this point of view. He distinguishes, in the first place, between apatetic (deceitful) colours, sematic (warning and signalling) colours, and epigamic colours (displayed in courtship), all of which afford marvellous instances of more or less highly specialized adaptation. We have not space to follow out the details of this classification but we shall presently refer to examples of all the more important types of colouration included therein.

Every observer of nature must have been struck with the general harmony of colouration which exists between animals and their surroundings. So complete is this harmony that our sense of hearing is frequently a better guide to the whereabouts of an insect, bird or mammal than our sense of sight. I

\footnotetext{
1 Interuational Scientific Series, Vol. LXVIII.
} 
remember standing with my gun in the midst of a dense patch of scrub in Australia and hearing the pademelons ${ }^{1}$ hopping about all around me. For a long time, however, I could see nothing but the trees. My native guide pointed out where I was to aim, but I only fired at a $\log$ from the side of which a pademelon hopped away. Again he pointed, and this time at a small white spot which I could just distinguish amongst the trees. I fired once more, aiming at the white spot, and sure enough a pademelon rolled over. It appeared that I had aimed at the white fur which occurs on the breast of the animal and which to the experienced eye of the native told all that he needed to know. It is often supposed that conspicuous patches of this kind serve as recognition marks between individuals of the same species, but it may be questioned how far the advantage of being recognized by a friend compensates for a disturbance of the colour harmony which reveals an animal to its enemies.

The type of colouration which aids in the concealment of an animal is termed, by Professor Poulton, cryptic. It belongs, of course, to the apatetic group. Concealment may be desirable either as a means of escape from enemies or for the purpose of ambuscading prey, or possibly for both. In the former case we may speak of it as protective resemblance (procryptic colouration), in the latter as aggressive resemblance (anticryptic colouration).

Protective resemblance is often of a very highly

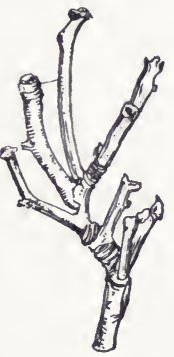

FIg. 169.--Jarva of the Brimstone Moth (Rumia cratae. gata) resting upon a Hawthorn twig; nat.size. (From Poulton.) specialized character, and may be due as much to adaptation in actual form as to adaptation in colour; frequently these two factors unite in producing the result, and a third may be added, viz., adaptation in habit or instinct. In the common stick caterpillars of the geometer moths we see all three factors co-operating. In colour and shape these caterpillars precisely resemble small twigs. They move about with a characteristic looping action amongst the leaves or branches of the bushes which they frequent, but when at rest they stiffen themselves up and stand out from the branch at the exact angle of a twig, and 
in this condition it is extremely difficult to detect them. Professor Poulton remarks :-

"These caterpillars are extremely common, and between two and three hundred species are found in this country; but the great majority are rarely seen because of their perfect resemblance to the twigs of the plants upon which they feed."

As will be seen from the illustration (Fig. 169), which represents the larva of the brimstone moth upon its food plant, the hawthorn, the caterpillar is enabled to main-

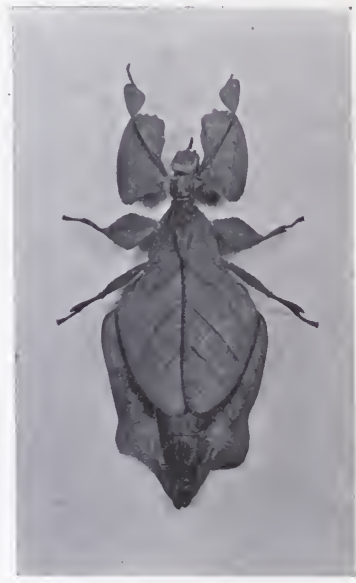

FIG. 170.-A Green Leaf Insect (Pulchriphyllium crurifolium, i), from Ceylon; $\times \frac{1}{2}$. (From a photograph.) tain its position for a long period by attaching its head to a twig by means of a silken thread.

Numerous moths so closely resemble in the colour and pattern of the upper surface of their wings the objects upon which they rest in the daytime, such as the bark of trees, that they are almost invisible, but perhaps the most perfect examples of protective resemblance are met with in the wonderful leaf insects. Fig. 170 represents an orthopterous insect, Pulchriphyllium crurifolium, from Ceylon. The whole insect is of a bright leaf-green colour, and not only are the wings shaped and veined so as to resemble leaves, but even the body and legs exhibit leaf-like outgrowths.

In the well known Indian leaf butterfly, Kallima (Fig. 171), the resemblance to a leaf is only seen when the insect comes to rest with its wings folded together above the body so as to expose their under surfaces. It is a dry, dead leaf which is imitated this time, and stalk and midrib, veins and colour markings, even down to such minutiæ as rust spots, are perfectly represented.

The Mantidæ or praying insects feed upon flies, \&c., which they capture with marvellous dexterity with their serrated claws. In some species the uniform green colouration doubtless serves, not only to protect them from their own enemies, but also to 
prevent them from being seen by their victims before they have come within range. Other species exhibit even more wonderful adaptations both in form and colour. Thus the South African IIarpax tricolor sits amongst the pink and white flowers of the heath, which are imitated by similarly coloured outgrowths of the insect, and there awaits the approach of its unsuspecting victims; while in Mozambique the terrible Idolum diabolicum

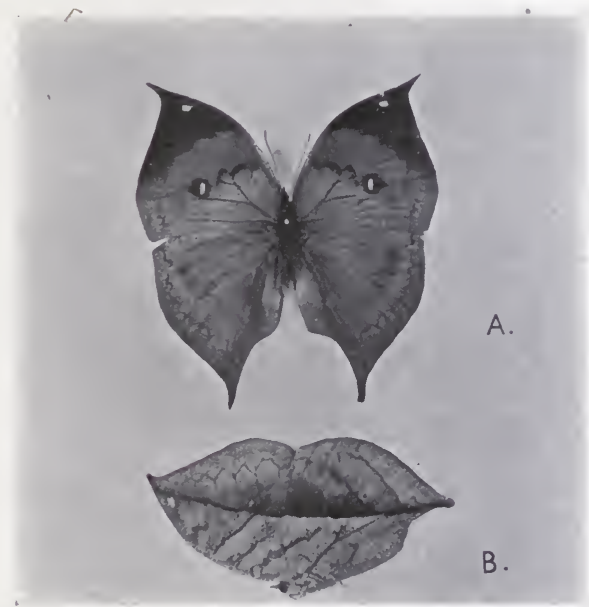

FIG. 171.-An Indian Leaf Butterfly (Kallima inachis); A., with wings expanded; B., with wings folded; $\times \frac{3}{7}$. (From a photograph.)

simulates, both in form and colour, a large flower, and thereby deceives and attracts other insects in search of honey.

It is no doubt amongst the almost innumerable species of the great group Insecta that cases of highly specialized adaptation for purposes of concealment or deception are most frequently met with. They also occur, however, and by no means uncommonly, in other groups of the animal kingdom. A familiar instance is afforded by the common British spider crab, now known as Macropodia rostrata, ${ }^{1}$ of which excellent illustrations (under the name Cancer Phalangium) were given by

1 I am indebted to my friend, the Rev. T. R. R. Stebbing. F.R.S., for information as to the correct nomenclature, \&c., of this species. 
Dr. Macculloch, in the Transactions of the Linnean Society, as far back as 1801. I am enabled by the courtesy of the Council of the Society to reproduce here, on a reduced scale, Dr. Macculloch's original plate (Fig. 172). The crab actually breaks off fronds of seaweed and attaches them to the long hairs of its body, thus disguising itself so effectually as to be quite unrecognizable except by careful examination. Dr. Macculloch was of opinion

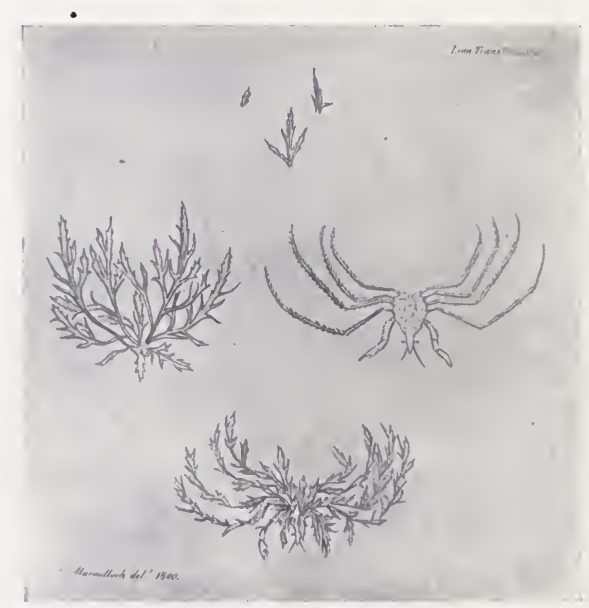

FiG. 172.-Reduced Facsimile of Dr. Macculloch's Plate of Macropodia rostrata, in the Transactions of the Linnean Society. On the left is shown a plant of the seaweed in which the crab dresses itself up; on the right the crab without the seaweed, and at the bottom the crab dressed up.

that this dressing up of the crab in seaweed was an artifice which assisted it in capturing its food (anticryptic), but it is much more likely that it is protective (procryptic). The late Professor Bell has told us how the slow and sluggish habits of the crab render it an easy prey to fishes, and the stomach of a thornback ray has been found entirely filled with them, so that there appears to be ample reason for them to seek concealment.

In the case of Macropodia the adaptation for concealment shows itself as an inherited habit or instinct more than in any modification of bodily structure, but such an instinct is probably 
itself the effect of some structural modification, however impossible to detect, in nervous tissue. In the Australian Phyllopteryx eques, a fish which is closely related to the curious sea-horse (Hippocampus) of our own coasts, we get precisely the same idea, so to speak, carried out in a different manner. Both Hippocampus and Phyllopteryx live amongst seaweed, to which they attach themselves by means of their curious prehensile tails. Hippocampus (Fig. 173) exhibits no special resemblance
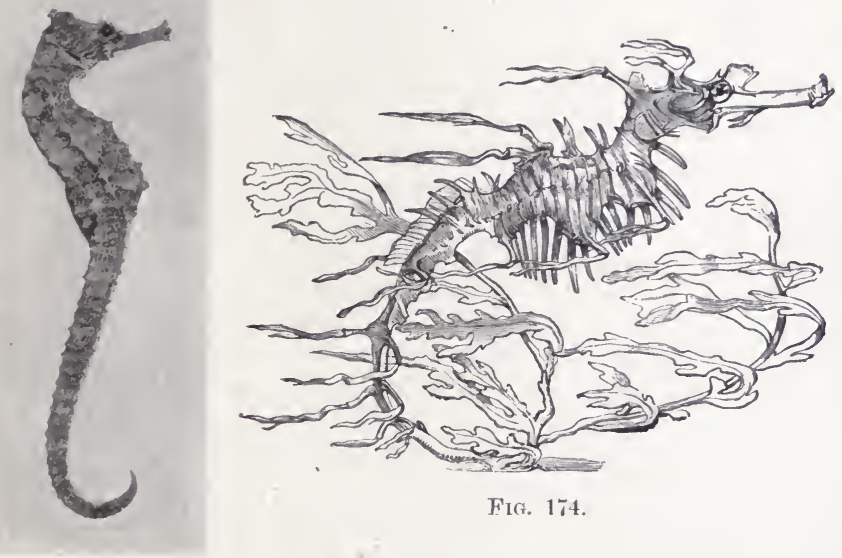

Fif. 174.

FIG. 173.

Fig. 173.-A Sea-horse (Hippocampus antiquorum), $\times \frac{2}{5}$. (From a photograph.)

FIG. 174.-Phyllopteryx eques, attached to seaweed. (From Günther's "Study of Fishes.")

to its surroundings, but in Phyllopteryx (Fig. 174) the body is covered with cutaneous outgrowths which float out in the water like fronds of seaweed and doubtless effect a most satisfactory disguise. This is certainly a less troublesome plan than that of dressing up in clothing borrowed from the outside world.

The well known colour changes of the chamrleon and of various flat fishes, not to mention numerous other instances which might be cited from different groups of the animal kingdom, are due to a complex apparatus, controlled by the nervous 
system, whose function it is to bring about a varying adaptation for concealment under varying conditions of the environment. How perfect the adaptation may be will be realized by all who have ever observed with what marvellous accuracy the colour markings of a turbot in an aquarium are made to match the sand or gravel upon which it is lying.

In striking contrast with the cryptic colouration by which an animal seeks, as it were, to avoid observation, are those numerous cases in which self-advertisement appears to be the main object in view. The British army, which only in recent years has learnt the advantages of khaki clothing when in the field, still exhibits some of the most startling instances of conspicuous colouration met with anywhere in the animal kingdom, though whether these examples should be classed under the head of warning colours, or regarded as belonging to the epigamic category, is perhaps an open question. We must, however, confine our attention in this place to a few examples of warning colours met with amongst the lower animals.

We have seen that both warning and signalling colours, or recognition marks, are spoken of as sematic. The former are further distinguished as aposematic and the latter as episematic. Aposematic colours are exhibited by many animals which possess some special means of defence and find it advantageous to advertize the fact. Wasps and hornets, with their conspicuous orange- and black-banded bodies, are excellent examples. Such animals do not seek to conceal themselves but rely upon their warning colours to remind their enemies that they had better leave them alone. It is not enough that they should possess the power of making themselves disagreeable; the fact must be clearly recognized, otherwise they would be constantly exposed to experimental attack, and suffer injuries for which any damage which they might inflict upon their pursuers would be but a poor consolation. Orange, red and black, owing to their great conspicuousness, especially when associated with one another, are the colours most frequently met with in this connection, and we find these colours, not only in noxious insects, but in various vertebrate animals, such as poisonous reptiles, toads and salamanders. The Gila monster (Heloderma suspectum), of Mexico and Arizona, is the only known poisonous lizard, and is conspicuously coloured in tints of blackish brown, yellow and orange, while other members of the same group are usually 
coloured so as to harmonize more or less perfectly with their surroundings.

If it is advantageous for a noxious species to advertize its true character, it is no less so for an innocuous one to advertize a false character, and gain credit for some power of making itself objectionable which it does not really possess. The practice of bluffing is by no means an exclusively human institution. 'Thus we find many insects, which in themselves are quite inoffensive, taking on the characteristic warning colouration of dangerous species. The drone-fly mimics the bee, and though they belong to widely different orders of insects, the one having only two wings and the other four, the resemblance is so close as to have given rise, as we saw in an earlier chapter, to the ancient belief in the spontaneous generation of bees from the carcases of oxen (on which, of course, drone-flies had deposited their eggs).

Most moths, as is well known, have opaque wings, covered with microscopic scales, but in the clear-winged moths (Fig. 175, A) the wings have partially lost their scales and become transparent, and this anomalous feature, combined with the colouration of the body, enables these perfectly harmless insects to mimic the dangerous hornets (Fig. 175, B). Even a harmless snake may mimic the warning colouration of a venomous

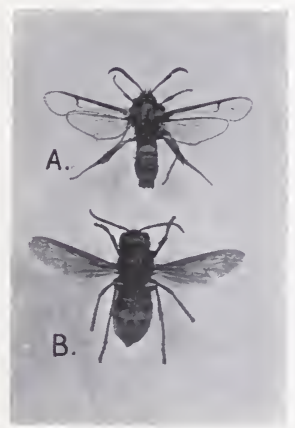

Fig. 175. - A., a clearwinged Moth (Sesia crabroniformis) mimicking B., a Hornet (Vespa crabro); both $\times \frac{3}{4}$. (From a photograph.) species, and thus secure for itself the respect which is properly due only to the latter.

It is not necessary that an animal should be capable of inflicting serious injury upon its enemies when attacked for it to secure immunity from pursuit as soon as recognized. Many butterflies and other insects, which are probably merely distasteful or nauseous (or perhaps actually unwholesome) to birds, exhibit aposematic or warning colouration. Amongst these we find curious associations - known as synaposematic groups, the members of which, belonging to distinct species and often by no means closely related to one another, seem to have combined 
together to share the expenses of a common advertisement and thereby reduce the cost to each. Young birds have to learn by experience which insects are good to eat and which are not. In making their experiments no doult they themselves suffer, but the subjects of the experiment are probably actually killed. Obviously, then, if one experiment can be made to serve for a number of different species of insects there will be a corresponding reduction in the death-rate, and hence it is that we

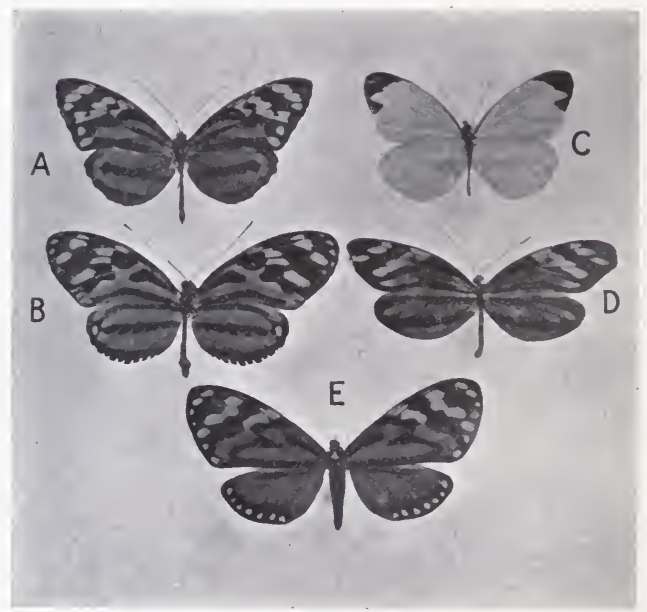

FIG. 176. - A Synaposematic Group of South American Lepidoptera, all $\times \frac{1}{2}$. (From a photograph.)

A, Tithorea harmonia; B, Heliconius ethilla; C, Perrhybris (Mylothris) malenka, ๙े ; D, Dismorphia praxinöe, + ; E, Pericopis angulosa.

find these groups of species all adopting the same type of warning colour, and thus coming to resemble one another very closely, although perhaps belonging to totally distinct families.

We may illustrate this somewhat complex phenomenon by reference to certain South American Lepidoptera which take part in the formation of such a synaposematic group. In Fig. $176 \mathrm{~A}, \mathrm{~B}$ and $\mathrm{D}$ represent butterflies belonging to three distinct families, while $\mathrm{E}$ is a motl, as may be seen at once by its thick body and the absence of terminal knobs on the antennx. 
All of these, in common with numerous other species which inhabit the same area, have adopted the same characteristic scheme of warning colouration, wherein the prevailing tints are black and orange.

In such a synaposematic group, or mimicry ring, it is usually possible to distinguish between certain species which seem to have led the way in the development of the warning colouration, and others which seem to have followed their example. In the particular case under notice the original " models" belong to the group Ithomiinæ, of which Tithorea harmonia (Fig. 176, A) is a representative. These are probably the most distasteful members of the combination to birds. They have been imitated by Heliconinæ, such as Heliconius ethilla (Fig. 176, B), Pierinæ ("whites"), such as Dismorphia praxinöe (Fig. 176, D), and Hypsidæ (a family of moths), as exemplified by Pericopis angulosa (Fig. 176, E), all of which may be regarded as mimics of the Ithomiinæ.

The case of the pierine mimics is particularly instructive, and shows very clearly that these forms really imitate other species, for the female is commonly a far more perfect mimic than the male, which often departs little, if at all, from the typical colouration of the group to which it belongs. Fig. 176, C represents a male pierine, Perrhybris (Mylothris) malenka, which is at once recognizable from its colouration as a "white," although even here, curiously enough, there is a faint trace of the warning colouraiion on the under surface of the hind wings. ${ }^{1}$ The female of the same species has the warning colouration well developed, as it is in both male and female of Dismorphia maxinöe.

So different are the males and females of some of these mimicking species that it would be difficult to believe, were it not for breeding experiments, that they are really specifically identical. 'I'he explanation of the difference is doubtless to be found in the fact that it is much more important, from the point of view of the species, that the females, heavily laden with the eggs upon which the existence of future generations depends, should be able to warn off the birds, than that the males should do so, for the latter, having once accomplished the fertilization of the eggs, is of no further value to the race.

1 Doubtless inherited incompletely from female ancestors, as. in the ease of the vestigial nipples of man. 
When an unquestionably harmless species mimics the warning colours of an undoubtedly noxious one, the case is sometimes spoken of as one of "Batesian" mimicry, after the distinguished naturalist, H. W. Bates, who added so much to our knowledge of the subject. In the case of a synaposematic group, or mimicry ring, however, it is often impossible to say whether any particular species is edible or not, and it may very well be that in some cases all are more or less inedible, though undoubtedly some, which are presumably the less objectionable forms, mimic others, which are presumably the more objectionable. This kind of mimicry, resulting in the development of a warning colour common to a number of inedible species, is sometimes distinguished as "Müllerian" mimicry, after the naturalist Fritz Müller, who first suggested the correct interpretation of the phenomenon.

Perhaps the most remarkable case of mimicry known amongst butterflies is that of certain species of Papilio found in Africa and Madagascar, which have formed the subject of exhaustive study by Trimen, Poulton and others. In Madagascar occurs Papilio meriones, a non-mimetic species in which the male and female (Fig. 177, A) closely resemble one another and both possess the "tail" on the hind wing which is such a characteristic feature of the genus. We may take it, then, that this is a primitive form. On the continent of Africa is found the widespread Papilio dardanus, with several subspecies. In these subspecies the male (Fig. 177, $\mathrm{B}_{1}$ ) retains the ancestral form, but in most of them the female is mimetic; it has lost the Papilio tail and closely mimies, both in shape and colour markings, some one or another of various species of butterflies belonging to different families which occur in the same region. Nor is this all, for the female is likewise polymorphic, and different individuals of the same subspecies resemble widely different models. Thus the subspecies merope is known to have three forms of female, a hippocoon form (Fig. 177, $\mathrm{B}_{2}$ ) which mimics the danaine butterfly Amauris niarius (Fig. 177, C), a trophonius form (Fig. 177, $\mathrm{B}_{3}$ ) which mimics another danaine, Limnas chrysippus (Fig. 177, D), and a planemoides form which mimics the acræine, Planema poggei. Our illustrations, which are reproduced from Mr. Trimen's original memoir, give a good idea of the form and pattern of some of these insects, but they lack the beautiful colouring of the original figures, which is 


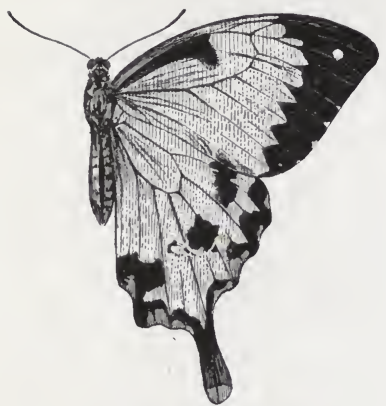

A. Papilio meriones 우

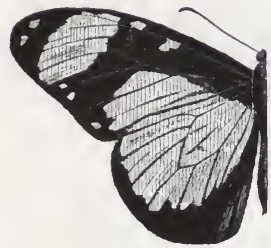

C. Amauris niavius

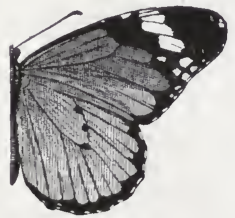

D. Limnas chrysippus.

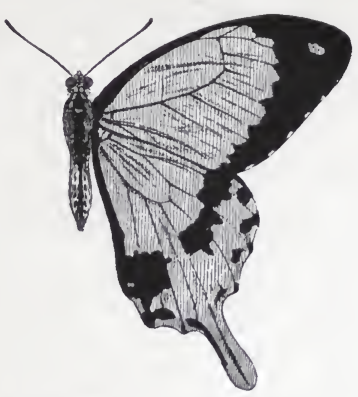

Bi. Papilio dardanus (merope) $\sigma^{*}$

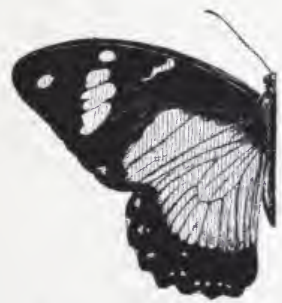

B2. Papilio dardanus (merope) 우 (hippocoon form)

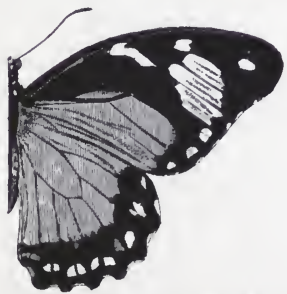

B3. Papilio dardanus (merope) ㅇ (trophonius form)

Fig. 177. - Mimicry in Butterflies. (After Trimen, from coloured plates in the Transactions of the Linnean Society, First Series, Vol. XXVI.) 
necessary in order to give a true idea of the close resemblance between mimics and models.

Breeding experiments carried out by Mr. G. F. Leigh with the subspecies cenea of Papilio dardanus have shown that the eggs laid by one and the same butterfly may give rise to at least

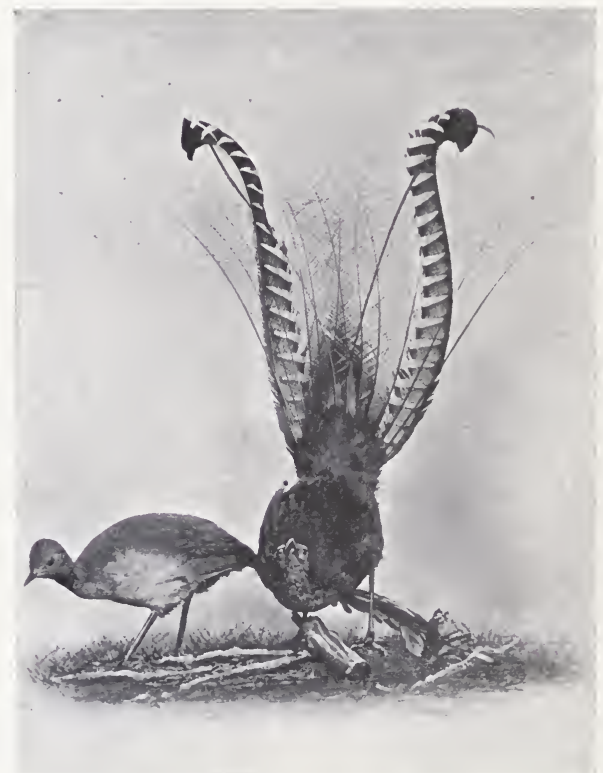

FIG. 178.-Male and Female of an Australian Lyre-Bird (Menura superba). (Photographed from specimens in the British Museum, Natural History.)

three different forms of mimetic female, as well as, of course, the male.

It is obvious that we have here the very opposite of a synaposematic group, for, instead of concentrating upon a single warning pattern, different individuals, even of the same species, adopt totally different patterns in imitation of totally different models. This case still requires a great deal of explanation, but concerning the facts there can be no doubt. 
Frequenters of any good museum of natural history will be familiar with plenty of examples of epigamic ornamentation. The highly elaborate and gorgeous plumage of many male birds, such as the peacock, the Argus pheasant, the Australian lyrebird (Fig. 178) and numerous species of humming birds, to say nothing of less conspicuous examples, affords the best illustration of this phenomenon. $r$ In all such cases the function of the ornamentation appears to be to gratify the æsthetic sense of the female during the period of courtship, and render her amenable to the attentions of her mate. There is no doubt that in many cases the cock bird deliberately displays himself to the best advantage before the admiring eyes of the hen, who is credited with a no less deliberate choice of the partner who most nearly approaches her ideal standard of beauty. In all these cases the plumage of the female is comparatively sombre and uninteresting. An elaborate and gaudy tail would be a disadvantage during the lengthy period of incubation, when it is desirable, both for her own sake and that of her offspring, that the female should be as inconspicuous as possible. Thus it is the male bird that has had to adapt his clothing to the requirements of the female, and she herself is unable to follow the fashions which she imposes upon her mate.

It is obvious that no theory of evolution can be regarded as satisfactory which does not offer some explanation of the origin of such highly specialized and precise adaptations as those which we have been considering in this chapter, and it was in order to emphasize the need for such explanation that we have laid so much stress upon them. We shall see in our next chapter that no less remarkable and precise adaptations for special purposes are also met with in the vegetable kingdom, and shall then pass on to seek the necessary explanation. 


\section{CHAPTER XXIII}

Adaptation to the environment in plants-Alpine plants, desert plants and lianes-The modification of flowers in relation to insect-fertilization.

Is plants no less than in animals we find adaptation to the conditions under which they have to live to be the most striking feature of their organization. We have already noticed, in dealing with the phenomenon of convergent evolution, the manner in which Alpine plants of many kinds tend to assume the compact cushion-like form which seems best suited to withstand the rigorous climate to which they are exposed. Wherever a plant may be found growing in a state of nature the character of the environment is sure to be reflected more or less obviously in its structure and habit. We see this equally clearly in the water-storing plants of desert regions-the cacti of America or the aloes of Africa-with their succulent stems or leaves and other structural modifications which enable them to withstand the effect of long-continued drought, and in the climbing lianes of tropical forests, whose one object in life appears to be to reach some elevation where they can expose their foliage to the light and air. Just as we find those air-breathing mammals which have taken to an aquatic life adopting the form and habits of fishes as being best suited to their changed conditions, so in the tropical forests of Queensland we find palms, members of a group which elsewhere are types of self-supporting independence, adopting the form and habit of climbing plants as the only means of coping with the exigencies of the situation.

If the adaptation amongst plants usually appears less remarkable than is often the casse in animals, it is because the relations of a plant to its environment are usually less complex than those of an animal. The greater activity of animals is associated with the development of highly specialized organs of locomotion, sense-organs and nervous system, all of which are alike subject to adaptation. Plants afford much more restricted opportunities for the effects of the environment to show themselves, and it is in their relations with animals, or to speak more 
accurately, in those organs which are immediately concerned with these relations, that adaptation becomes most complex and precise.

Even amongst animals, however, it would be difficult to find better illustrations of accurate adaptation to highly specialized conditions of the environment than those which we see in the wonderful structural modifications whereby the majority of flowers are adapted for pollination by insect agency. This process of pollination is commonly referred to as the "fertilization" of the flower, although, as we have seen in a previous chapter, the real' act of fertilization takes place inside the so-called ovule and consists in the conjugation of the male and female gametes, the sperm-cells and egg-cells.

The great majority of flowers produce both pollen and ovules, containing respectively the male and female gametes; in other words they are hermaphrodite. It is obvious that in such cases we have two possibilities with regard to fertilization, for the flower may either be fertilized by its own pollen or by that of some other flower. It may be either "self-fertilized" or " crossfertilized," and the cross-fertilization may be effected either by pollen from another flower of the same plant or by pollen from a different plant of the same kind, the latter being the more advantageous.

It seems at first sight a strange thing that when a flower is capable of self-fertilization such a roundabout and apparently unnecessary proceeding as cross-fertilization should ever take place at all. It has been shown, however, that cross-fertilization, if not absolutely necessary, is at any rate of very great advantage to the plant, or rather to the species, in which it occurs.

Charles Darwin experimented for eleven years on this subject, and proved conclusively that cross-fertilization yields better results than self-fertilization, both as regards the number of seeds produced and also as regards the quality of the offspring. It would be impossible to give an adequate account of his work in this place, but we may briefly notice one series of experiments and refer for the remainder to his classical volume on the "Cross and Self Fertilization of Plants." He started with the "Convolvulus major" (Ipomoea purpurea), the flowers of which are hermaphrodite and may be either cross- or self-fertilized in a state of nature. It is an easy matter, by artificially conveying 
the pollen on the tip of a feather, to bring about the fertilization in any way which may be desired. Ten flowers were thus selffertilized with their own pollen, while ten others were crossfertilized with pollen from a distinct plant. The crossed and self-fertilized seeds thus obtained were carefully cultivated under exactly the same conditions, and it was found that the plants raised from cross-fertilized seeds were taller than those raised from self-fertilized seeds in the proportion of 100 to 76 .

The same experiment was repeated with ten successive generations of the same plants, and always with the same result in favour of the cross-fertilized individuals. Moreover, it was proved at the same time that the fertility of the plants produced by crosis-fertilization was greatly superior to that of the selffertilized plants, a much larger quantity of seed being produced.

'These results, taken in conjunction with many others of the same kind, clearly proved the advantages of cross-fertilization. To quote Darwin's own words :-

"It has been shown ..... that the offspring from the union of two distinct individuals, especially if their progenitors lave been subjected to very different conditions, have an immense advantage in height, weight, constitutional vigour, and fertility over the self-fertilized offspring from one of the same parents."

The theoretical explanation of this advantage is not an easy matter, lout is probably to be sought in the admixture of two distinct series of hereditary tendencies in the offspring of a cross.

In a state of nature it is a very rare thing for plants to be exclusively self-fertilized, for, although self-fertilization may take place in some cases to a very large extent, and although some flowers are so constructed that self-fertilization alone is possible, yet there is nearly always at least an occasional cross by the introduction of pollen from a different plant.

Considering the advantages which arise from cross-fertilization, we need not be surprised to find that a very large number of flowers are provided with special adaptations whereby these advantages may be secured to them. We may notice first certain contrivances by means of which self-fertilization is more or less effectually prevented and the injurious effects of perpetual close inbreeding thereby avoided. These contrivances may be classed under three principal heads. 
(1) Separation of the Sexes.- In many flowers we find that only stamens or pistil are developed, never both, so that we get distinct male and female flowers, which renders self-fertilization absolutely impossible, though it does not necessarily prevent fertilization by pollen from other flowers on the same plant.

(2) Physiological Self-sterility. - In some flowers, to quote the words of Darwin, "the grules absolutely refuse to be fertilized by pollen from the same plant, but can be fertilized by pollen from any other individual of the same species." Again, in a large number of plants, although self-fertilization may take place, yet, if the pollen from another individual be brought to the stigma, it takes precedence, so to speak, of the flower's own pollen and renders the latter ineffectual; it is said to be prepotent.

(3) Dichogamy. - In a large number of flowers, in which both male and female organs are present, the stamens and pistil become mature at different times, so that self-fertilization cannot possibly take place and, physiologically speaking, the flowers are unisexual. Usually in these cases the pollen ripens and is all shed before the stigma is ready to receive it. The flower is then termed protandrous, as for example in the common pink (Dianthus). More rarely the stigma ripens and withers before the pollen is ripe, and such species are termed protogynous, as in the fig-wort (Scrophularia nodosa).

Of course it would be worse than useless to prevent selffertilization unless some means were provided at the same time for securing cross-fertilization. We saw in Chapter VIII that the principal agents in conveying pollen from one flower to another are wind and insects, and that flowers are accordingly distinguished as anemophilous or wind-fertilized and entomophilous or insect-fertilized. The fact that the former are usually very small and inconspicuous, as for example in the grasses, while the latter are large and brightly coloured, affords strong presumptive evidence that entomophilous flowers have become modified in relation to the insects which visit them in search of food or shelter. A great many different kinds of insects have this habit, bees, flies, butterflies, moths and even beetles; while in South America some of the humming birds in like manner play the part of pollen carriers.

We are in the habit of regarding bees as the most important insects concerned in the cross-fertilization of flowers, and in 
countries where they are plentiful this is perhaps the case. They visit the flowers in order to collect both honey and pollen, to be used as food for themselves and their offspring. The honey is usually found at the bottom of a long narrow tube formed by

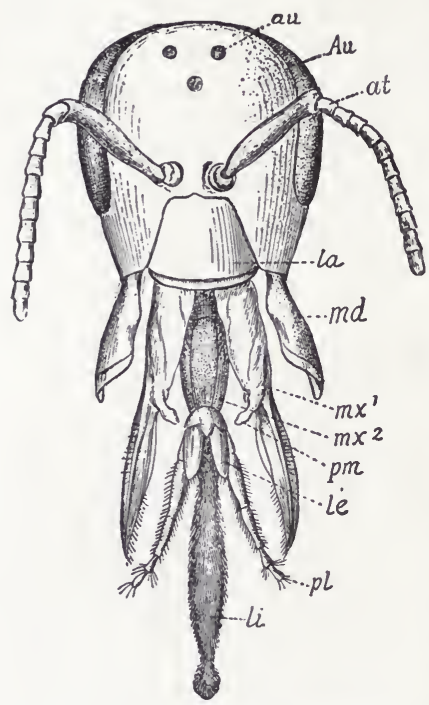

Fig. 179.--Head of a Bee, showing the complex Proboscis formed from modified Mouth Parts. (From Weismann's "Evolution Theory.")

$a t$, antennæ; $A u$, large compound eye; $a u$, ocellus; $l a$. labrum or upper lip; le, outer division of second maxilla (paraglossa); $l i$, ligule or tongue, formed by fusion of inner divisions of second maxillæ; $m d$, mandible; $m x^{1}, m x^{2}$, first and second maxillæ; $p l$, labial palp; p.m., maxillary palp.

the lower part of the corolla, so that in order to reach it the bee requires a correspondingly long and narrow instrument. This is provided in the shape of a very complicated proboscis (Fig. 179), formed by modification, especially elongation, of certain of the appendages surrounding the mouth, which in more primitive insects, like the cockroach, remain short and simple. When not in use the proboscis is neatly folded away beneath the head, but when a flower is visited it is unfolded and inserted into the tube containing the honey, which is then drawn up into the bee's stomach by means of a special sucking apparatus. In butterflies and moths also a somewhat similar proboscis is used for the same purpose, but it differs so much from that of the bee in details of structure as to indicate that it has been independently evolved from the primitive mouth parts of some remote insect ancestor. In both cases the mouth appendages have become specially adapted for the very special purpose of sucking honey, and the necessity for the fulfilment of the same function has led, as usual, to a superficial resemblance between the two types of proboscis. We have here, of course, another illustration of convergent evolution. 
For collecting pollen the bees make use of their legs, on which special collecting brushes and baskets are formed by the stiff hairs. The pollen is first moistened with honey to make it stick together, and then picked up on the collecting brushes, placed in the baskets on the hind legs and carried to the hive or nest.

Insects commonly visit arlarge number of flowers of the same kind in rapid succession, and, whether they intentionally collect pollen for their own purposes or not, it is evident that, after each visit to a flower containing ripe pollen grains, they will unintentionally carry away some of these, accidentally attached to various parts of the body. The pollen which is thus unconsciously carried away is equally unconsciously deposited on the stigma of the next flower visited, and thus cross-fertilization is effected. It may, of course, happen sometimes that the pollen is deposited on the wrong kind of flower, but that is of no consequence, for pollen is, with rare exceptions, quite incapable of fertilizing flowers of any but its own particular species.

Under these circumstances it will obviously be advantageous to a plant to make its flowers as attractive to insects as possible, and, just as it is desirable for a nauseous insect to inform the birds by means of warning colours of the fact of its unpalatability, so also is it desirable for flowers which have honey to offer in exchange for the service of pollination to make that fact known to their insect visitors by means of brilliant colours and strong scents. There can be no reasonable doubt that the colours serve to attract the insects and to enable them to recognize the flowers which they prefer, and that the same is true of the scents is very clearly indicated by the fact that certain flowers, such as various species of Arum and related genera, which are fertilized by carrion-loving flies, have managed to perfume themselves in accordance with the tastes of their visitors.

It is also desirable to ensure, by means of mechanical arrangements, that the insects shall not get their honey without paying for it, that is to say, without effecting fertilization, and this end is usually secured by concealing the honey at the bottom of a long tube, in such a manner that the insect must brush against the stamens and stigma before it can reach.it. Sometimes, however, the insects become a little too clever for the flower and steal the honey by biting a hole through the bottom of the tube without ever touching the stamens or stigma at all. In this way the red clover flowers are often robbed of their honey by the humble 
bee, Bombus terrestris, thus affording an example of imperfect adaptation.

We may now consider a few definite examples of the manner in which flowers may be specially adapted in structure so as to secure the advantages of cross-fertilization by insect agency.

If a number of plants of the common primrose, the oxlip, the cowslip or the polyanthus (species of the genus Primula) be examined carefully, it will be seen that in each case two quite different forms of flower occur. In other words the flower is dimorphic.

The two forms are known to

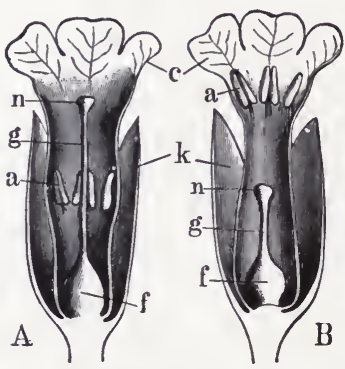

Fig. 180.-Heterostyled Flowers of the Oxlip (Primula elatior) in longitudinal section. (From Vines' “Botany.”)

A, long-styled; B, short-styled flower. a, anthers; c, corolla ; f, ovary ; $\mathrm{g}$, style; $\mathrm{k}$, calyx; $\mathrm{n}$, stigma. gardeners as "pin-eyed" and "thrum-eyed" respectively. In the pin-eyed flowers (Fig. 180, A) the style (g) is comparatively long and the stigma (n) appears as a round knob, like the head of a pin, in the centre of the flower, at the entrance to the long tube formed by the lower part of the corolla. The anthers (a) lie much lower down in the tube, so that they are invisible until the flower is cut open. In the thrum-eyed flowers(B) the positions of the stigma and anthers are reversed; the style being much shorter the stigma lies only half way up the tube, while the anthers appear in the centre of the flower, in the mouth of the tube. The two kinds of flower are always found on separate plants, and the long-styled and shortstyled plants are said to occur in about equal numbers under natural conditions. The pollen grains also differ in the two kinds of flower, those of the thrum-eyed being larger than those of the pin-eyed and of somewhat different shape. This particular kind of dimorphism, which is sometimes known as heterostylism, is extremely characteristic of the genus Primula, and it has recently been shown that the distinguishing features of the two forms are inherited in a Mendelian fashion.

The flowers of the primulas are fertilized by the agency of insects such as humble bees, and Darwin found that if insects were carefully excluded by covering the flowers with a net, little 
or no seed was produced. In order to reach the honey, which is secreted at the bottom of the long tube of the corolla, the bee thrusts its proboscis down the tube and in so doing brushes past the stigma and the anthers. If a pin-eyed flower be visited the pollen from the anthers is deposited comparatively low down on the proboscis. If a thrum-eyed flower be visited the pollen is deposited much higher up, in accordance with the more elevated position of the anthers. Thus the bees carry about two different kinds of pollen on two different parts of the proboscis, a fact which has been established by microscopic examination of the proboscis itself with the attached pollen.

If it be remembered that in the two different kinds of flower the relative positions of the anthers and stigma are reversed, it will be obvious that when a bee sucks honey from a long-styled flower the stigma will be touched and pollinated by that part of the proboscis which touches the anthers of a short-styled flower, and vice versâ. Hence it follows that pin-eyed flowers will be fertilized by pollen from thrum-eyed, and thrum-eyed flowers by pollen from pin-eyed.

Here, then, we have a very precise adaptation for a special kind of cross-fertilization, and Darwin further proved by his experiments that this is the only kind of fertilization which results in complete fertility. Cross-fertilization in these plants is, however, possible in no less than four distinct ways:-(1) A pin-eyed flower may be fertilized by pollen from another pin-eyed; (2) a thrum-eyed flower may be fertilized by pollen from another thrum-eyed; (3) a pin-eyed flower may be-fertilized by pollen from a thrumeyed; (4) a thrum-eyed flower may be fertilized by pollen from a pin-eyed. The first two methods have been termed by Darwin "illegitimate unions" and they result in incomplete fertility; the last two have been termed "legitimate" and result in complete fertility.

The benefit derived from the existence of the two kinds of flower lies in the intercrossing, not merely of two distinct flowers, but of two distinct plants, for it will be remembered that each plant bears only one kind of flower. Self-fertilization is not absolutely prevented in this case, for the anthers and stigma are mature at the same time in the same flower, but it is not likely to take place, and if it does it results in incomplete fertility and weakly offspring. But even if pollen should accidentally find its way to a stigma of its own plant it need not necessarily 
prevent cross-fertilization, for foreign pollen will be prepotent even if deposited on the stigma some time afterwards.

"To test this belief," Darwin observes, "I placed on several stigmas of a long-styled cowslip plenty of pollen from the same plant, and after twenty-four hours added some from a shortstyled dark-red polyanthus, which is a variety of the cowslip. From the flowers thus treated, thirty seedlings were raised, and all these, without exception, bore reddish flowers, so that the effect of pollen from the same form, though placed on the stigmas

twenty-four hours pre-

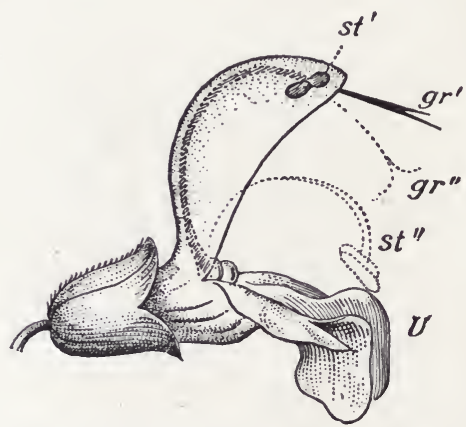

FiG. 181.--Flower of Salvia pratensis. (From

Weismann's " Evolution Theory," after H. Müller.)

$g r^{\prime}$ immature stigma; $g r^{\prime \prime}$, mature stigma; $s t^{\prime}$, anther-lobe concealed in the "helmet"; st", anther-lobes lowered; $U$, lower lip of corolla. viously, was quite destroyed by that of pollen from a plant belonging to the other form."

The flowers of the common sage, and of other species of the genus Salvia (Fig. 181), afford a no less striking example of profound structural modification in adaptation to the visits of insects. These flowers are protandrous, the stamens maturing and shedding their pollen before the stigma is ready to receive it, so that self-fertilization is absolutely prevented. Moreover, the stigma in young flowers (Fig. 181, $g r^{\prime}$ ) lies in such a position that it will not be touched by visiting insects, which crawl right under it in order to reach the honey at the bottom of the corolla-tube. In older flowers the style elongates and curves downwards, so that the stigma (Fig. 181, gr'") comes to lie in the mouth of the corolla-tube and must be brushed against by insects in search of honey.

The most remarkable feature of the flower, however, is a special mechanical contrivance for placing the pollen on the back of any insect which attempts to suck honey from it in its young or male condition. There are only two fully developed stamens, and these are most curiously modified in structure. Each anther 
consists as usual of two anther-lobes united together by a connective; but the connective is very greatly elongated, so that the anther-lobes are widely separated from one another instead of lying close together at the top of the filament. The anther-lobe at one end of each connective is imperfect and produces little or no pollen; the other is fully developed. The connective is attached to the top of the very short filament by a movable joint, so that it can be swung freely up and down in a vertical plane. It has a long limb which curves upwards inside the "helmet" of the corolla and terminates in the perfect anther-lobe, and a short one which bears the imperfect lobe, as shown in the figure. The two stamens lie side by side in the flower and the imperfect anther-lobes are held downwards in the mouth of the corollatube, exactly in the path of a visiting insect. The perfect antherlobes, on the other hand, are, at first, held up well above the level of the insect's back (Fig. 181, st').

When a large insect, such as a bee, visits the flower, it alights upon the large lower lip $(U)$, which, as in so many other flowers, affords a convenient platform and has doubtless been specially adapted to that end. The head of the insect is then thrust into the corolla-tube and pushes against the two imperfect anther-lobes. The connectives turn on their pivots like a see-saw and the two ripe anther-lobes $\left(s t^{\prime \prime}\right)$ are clapped down on the back of the insect and dust it with pollen. This happens when the insect visits a young flower. When an older flower, in the female condition, is visited, the pollen from the back of the insect is deposited on the mature stigma $\left(g r^{\prime \prime}\right)$, which now hangs directly in front of the entrance to the corolla-tube.

It is, however, in the highly specialized order Orchidaceæ that we find perhaps the most remarkable contrivances for ensuring cross-fertilization that are to be met with in the vegetable kingdom. Many of these have been described and illustrated by Darwin in his well-known work on the " Fertilization of Orchids."

In the common early orchis of Europe (Orchis mascula, Fig. 182) there are three sepals and three petals, and both sepals and petals are brightly coloured. 'The lower petal is very large and in front forms a tongue-like projection termed the labellum $(U)$, which serves as a landing platform, while behind it is produced backwards into a hollow spur or nectary $(s p, n)$ in which honey is secreted. The reproductive organs lie just above and partly in front of the entrance to the nectary, so that an insect, in 
poking its proboscis down to get at the honey, cannot fail to touch them. Both male and female organs are very much modified in structure, and united together to form the column $(C)$. There are three stigmas. Of these, however, only two $(B, n a)$ are functional, and they lie at the back of the entrance to the nectary. The third is specially modified to form a remarkable organ known as the rostellum $(B, C, v)$, which projects above the

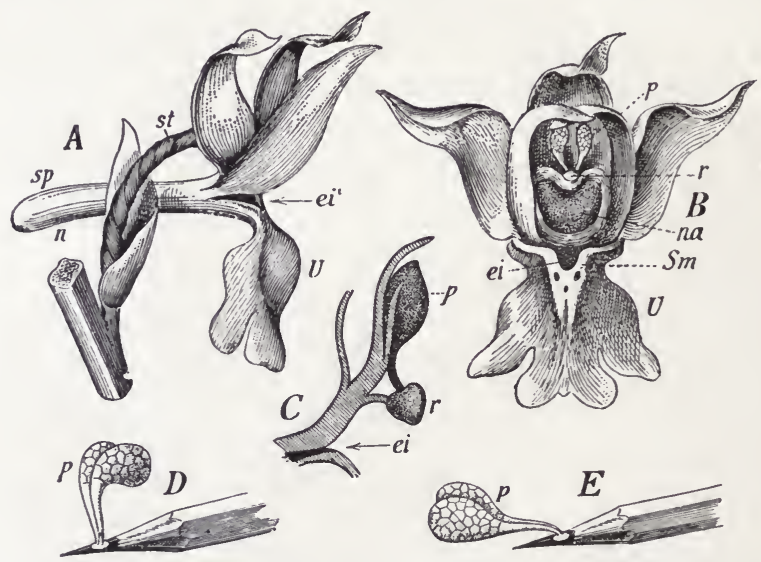

FIG. 182.-Adaptation in the Flower of Orchis mascula in relation to InsectFertilization. (From Weismann's "Evolution Theory.")

$A$, side view of flower; $B$, front view; $C$, vertical section through the column; $D$, pol-

linia removed on the point of a pencil and still standing erect; $E$, the same later on, bent forwards.

$e i$, entrance to nectary; $n$, nectary; $n a$, stigmatic surface; $p$, pollinia; $r$, rostellum; $S m$, honey guide; $s p$, spur; st, ovary (twisted); $U$, lower lip of flower (labellum).

other two in front of the mouth of the nectary. This rostellum, or beak, consists of an exceedingly sticky body covered over by a thin, membranous cap. The cap is so delicately adjusted that the slightest touch is sufficient to push it down and expose the sticky mass beneath, and it is so elastic that when the pressure is removed it springs back into its original position and again covers up the sticky substance.

There is only one perfect anther, consisting of two sacs which stand up above and behind the rostellum. Each sac contains a single coherent mass of pollen grains instead of the usual loose, 
powdery pollen. Each mass of pollen grains, or pollinium $(B, C, p)$, is a pear-shaped body provided with a short stalk or caudicle. The stalk is continued downwards into the rostellum as shown in $C$, where it ends in a membranous disc attached to the sticky substance. When the pollen is ripe the pollinia are exposed by rupture of the sacs in which they lie, so that in the mature flower they are only attached by means of their stalks or caudicles, loosely fixed by the sticky substance of the rostellum.

If we take any slender object, such as a pencil point, and poke it gently into the mouth of the nectary, we shall find on withdrawing it again that it will bring with it either one or both of the pollinia, attached to it by sticky cement. The pencil has, of course, touched the rostellum and pushed down the little cap which covers it. The sticky substance of the rostellum, thus exposed, has cemented on to the pencil the disc at the lower end of the caudicle, and thus the pollinium itself, or both of them, is pulled bodily out with the pencil. The cement rapidly hardens on exposure to the air.

When first-attached to the pencil the pollinium is in a nearly upright position $(D)$, so that if the pencil were inserted into another flower it would simply go back into its old place, without touching the stigma and of course, therefore, without effecting fertilization. After being exposed to the air for a short time, however, the disc by which the pollinium is attached to the pencil begins to contract, and in such a manner that the pollinium is pulled down until it comes to project straight forwards $(E)$. If now the pencil be inserted into a flower it will be found that the pollinium exactly strikes against one of the stigmas and dusts it with pollen.

In the economy of nature the proboscis of some insect takes the place of our experimental pencil. Bees and moths have frequently been observed with the pollinia of various species of Orchis attached to them; indeed Darwin figures one instance in which no less than fourteen pollinia are attached to the proboscis of a moth. Every time such an insect visits a flower in search of honey it will effect cross-fertilization by pushing the pollinia which it brings with it against the stigmas, and may perhaps carry off another pollinium into the bargain.

In this case one can hardly fail to be astonished at the number and complexity of the adaptations which have arisen in the flower for the purpose of ensuring cross-fertilization. There is 
the conspicuous colour of the sepals and petals, which serves as an advertisement; the formation of the long spur or nectary, with its secreted honey; the great development of the labellum to serve as a landing place, marked with the "honey-guide" ( $\mathrm{Sm}$ ) to point out the way to the visitor; the secretion of sticky cement by the rostellum; the formation of an elastic cap to keep the cement from being dried up before it is wanted; the agglutination of the pollen into solid masses, which serve to fertilize a large number of flowers in succession, losing a few grains at each contact; the remarkable form of these pollinia, with their adhesive discs and their peculiar relation to the sticky substance on the rostellum ; and, lastly, the wonderful mechanism by means of which the pollinia become bent downwards after their removal, so as exactly to adjust them for contact with the stigma of another flower!

In some species of plants certain parts of the flower are highly sensitive and respond to the slightest touch by a sudden and vigorous movement, going off, so to speak, like a spring trap or a hair trigger. This is well exemplified in a New Zealand orchid, Pterostylis trullifolia, as described by Mr. Cheeseman. In this flower the lower petal, or labellum, is highly sensitive, and when an insect alights upon it springs up and imprisons the visitor in a cage. The parts of the flower are so arranged that the insect can only escape by crawling through a narrow passage, and in such a manner that it must carry away the pollinia and also leave on the stigma some of any pollen which it may chance to bring with it.

A still more remarkable orchid, Catasetum, actually throws the pollinia at its insect visitors as soon as they touch the flower, and the tiny projectiles attach themselves to the intruder's head. Darwin has described how, on one occasion, when he experimentally irritated this flower, the pollinium was thrown for a distance of nearly three feet, when it stuck on to a window pane.

The presence of irritable structures which aid in crossfertilization by insect agency is, however, by no means confined to orchids. Candollea (Stylidium) graminifolia is a common Australian wild flower belonging to a totally different order, the Candolleaceæ. It is found growing on open heaths and sends up tall stems from the midst of a tuft of long, grass-like leaves, each stem bearing a large number of rather small pink flowers 
along its length. There are five petals, but only four are fully developed, and these spread themselves out in the form of a cross around the mouth of the tube in which the honey is secreted. The anthers and stigma are borne on the end of a long slender column which springs from the middle of the flower. Close to its base the column is bent downwards at a sharp angle so that it hangs out at one side of the flower, between two of the fully developed petals and resting upon the aborted petal as on a cushion. Near its apex the column is again bent at a sharp angle, this time upwards. The flowers are protandrous, the anthers becoming mature and shedding their pollen before the stigma is fully developed.

An insect, visiting the flower and poking its proboscis down the tube of the corolla in search of honey, must touch the column at the first bend. This is the irritable spot, and no sooner is it touched than the column springs over to the other side of the flower and brings the anthers or stigma, as the ease may be, down on the back of its visitor. In this way pollen is deposited upon or removed from the insect's back and cross-fertilization is effected. One of the most curious things about this case is that the column loses its irritability at nightfall and refuses to jump, however much you may tickle it.

The few examples of entomophilous flowers which we have now studied must suffice to give some idea of how wonderfully minute and perfect may be the adaptation of plants to highly specialized environmental conditions. The dominating factor of the environment here is, of course, the presence of insects, which can be pressed into service as pollen carriers, and it is obviously in relation to the requirements and tastes of these insects that the various adaptations which we have been describing have arisen. There can be little doubt that the insects select for their visits those flowers which please them best and which are most readily recognizable as the bearers of the coveted honey.

Although, as we have previously pointed out, some carrionfeeding flies prefer scents which are repulsive to ourselves, yet the vast majority of insect-fertilized flowers have scents and colours which we appreciate perhaps no less than the insects for whose gratification they primarily exist. As we shall see later on, these scents and colours, though now in many cases improved by human selection, doubtless arose in the first instance in response to insect selection. If we pursue this line of thought a 


\section{OUTLINES OF EVOLUTIONARY BIOLOGY}

little further, there seems good reason to suspect that man, who appeared upon the scene at a very much later date and whose ideas of what is beautiful are doubtless largely derived from the contemplation of flowers, may owe much of his æsthetic development to the fact that he has been educated by flower-loving insects. 


\section{PART V.-FACTORS OF ORGANIC EVOLUTION}

\section{CHAPTER XXIV}

Views of Buffon, Erasmus Darwin and Lamarck.

WE shall have occasion to point out in a subsequent chapter that many organisms exhibit characters which it is extremely difficult, if not impossible, to bring into any direct relationship with the environment, but this fact does not invalidate the generalization that the most striking feature of all living things is adaptation to the conditions under which they have to carry on their existence. In seeking for an explanation of the means whereby organic evolution has been effected, this fact must constantly be borne in mind, and, as we have already said, no theory can be considered adequate which does not take fully into account the phenomena of adaptation, and offer some reasonable explanation of the wonderful harmony which exists between living things and their surroundings.

We have said before that the theory of organic evolution is no new thing, but can be traced back even to the ancient Greek philosophers. In the middle ages such ideas were thrust into the background, along with other fruits of Greek and Roman intellectual activity, and to a large extent supplanted by the teachings of dogmatic theology. With the revival of scientific inquiry, however, the theory of organic evolution, as opposed to the doctrine of special creation and the immutability of species, again began to occupy a prominent place in the minds of thinking men, and a brief consideration of the views of some of the chief philosophical biologists of the last two centuries will perhaps form the most fitting introduction to this part of our subject.

The celebrated French naturalist, Buffon (1707-1788), who held the post of Superintendent of the Jardin des Plantes and, in conjunction with his colleagues, published a large number of volumes of Natural History, was one of those who had at any 
rate strong leanings towards the theory of organic evolution, although, unfortunately, he seems to have found it very difficult to give a frank expression to his views. The following translated extracts will serve to illustrate his position.

Speaking of the arbitrary character of our systems of classification he observes:-

"But Nature proceeds by unrecognized gradations and consequently she cannot lend herself completely to these divisions, since she passes from one species to another species, and often from one genus to another genus, by imperceptible shades: so that we find a great number of intermediate species and objects which we do not know where to place, and which necessarily upset the plan of the general system." 1

Buffon certainly appears in this passage as no believer in the immutability of species, and amongst the causes which bring about their modification he attributes great importance to the action of climate:-

"If we again consider each species in different climates, we shall find obvious varieties both as regards size and form; all are influenced more or less strongly by the climate. These changes only take place slowly and imperceptibly; the great workman of Nature is Time: he walks always with even strides, uniform and regular, he does nothing by leaps; but by degrees, by gradations, by succession, he does everything; and these changes, at first imperceptible, little by little become evident, and express themselves at length in results about which we cannot be mistaken." 2

In dealing with the animals of the old and the new worlds, and after speaking of the extinction of the mammoth, he says:-

"This species was certainly the foremost, the largest and the strongest of all the quadrupeds : inasmuch as it has disappeared, how many other smaller ones, weaker aud less remarkable, have had to succumb also, without having left us either witness or evidence of their past existence? How many other species, having become modified in their nature, that is to say, perfected or degraded by the great vicissitudes of land and sea, by the neglect or the culture of Nature, by the long influence of a climate become contrary or favourable, are no longer the same that they formerly were? And, moreover, the quadrupeds are, next to man, the beings whose nature is the most fixed and whose form is the most constant: that of the birds and of the fishes varies

1 Buffon, "Histoire Naturelle," Tom. I, p. 13.

2 Op. cit., Tom. VI, pp. 59, 60. 
more; that of the insects still more, and if we descend to the plants, which we must not exclude from animated nature, we shall be surprised at the promptitude with which the species vary, and at the facility with which they change their nature while taking on new forms.

"It would not be impossible then, that, even without reversing the order of Nature, all these animals of the new world may have been originally the same as those of the old, from which they may have been formerly derived; one might say that having been separated subsequently by immense seas or impassable lands, they would, in the course of time, have received all the impressions, suffered all the effects, of a climate itself altered in character by the same causes which brought about the separation; that in consequence they would in time have become dwarfed, changed their nature, \&c. But that should not prevent us from regarding them to-day as animals of different species: whatever may be the cause of this difference, whether it has been produced by time, climate and conntry, or whether it be of the same date as the creation, it is none the less real. Nature, I admit, is in a continual state of flux; but it is enough for man to seize her as she is in his own time, and to glance backwards and forwards in endeavouring to gain some glimpse of what she may have been in former times and of what she may become in the future." 1

This passage also seems to be a sufficiently clear declaration in favour of the theory of organic evolution and the action of the environment in modifying species. Buffon, however, by no means confined himself to the consideration of climate as a factor in the production of such modifications. The following quotation shows that he also realized the importance of the principles of use and disuse and the inheritance of acquired characters, which were destined to take such a prominent place in the subsequent writings of Lamarck:-

"The llama, which, like the camel, passes its life in bearing burdens and only rests by lying down upon its breast, has similar callosities which are perpetuated in the same way by generation. 'The baboons and the apes, whose most usual attitude, whether awake or asleep, is sitting, have also callosities beneath the region of the buttocks, and this eallous skin has even become adherent to the bones against which it is continually pressed by the weight of the body; but these callosities of the baboons and apes are dry and healthy, because they do not arise from the constraint of trammels or of the weight of a foreign burden, and because they 
are merely the effects of the natural habits of the animal, which remains seated more willingly and for a longer time than in any other posture : it is the same with these callosities of the apes as with the double sole of skin which we carry beneath our feet; this sole is a natural callosity which our constant habit of walking or resting upright renders more or less thick, or more or less hard, according to the amount of friction to which the soles of our feet are exposed." 1

It has been maintained that Buffon not only anticipated Lamarck's views as to the influence of the environment and the principle of use and disuse, but also those of Malthus and Charles Darwin with regard to the importance of the struggle for existence and the process of natural selection. That some such ideas were present in his mind seems sufficiently clear from the following passages. After speaking of the invasions of the Huns and Goths and other peoples he continues :-

"These great events, these conspicuous epochs in the history of the human race, are, however, only trifling vicissitudes in the ordinary course of living nature ; it is in general always constant, always the same; its movement, always regular, turns on two fixed pivots, the one the unlimited fecundity given to all species, the other the innumerable obstacles which reduce the product of this fecundity to a fixed quantity and at all times leave only approximately the same number of individuals in each species." ${ }_{2}$

"The causes of destruction, of annihilation and sterility follow immediately upon those of excessive multiplication; and, independently of contagion, the necessary consequence of too great an accumulation of any living matter in one place, there are in each species special causes of death and destruction, which we shall indicate in the sequel and which alone suffice to compensate for the excess of former generations." 3

"The least perfect species, the most delicate, the most heavily burdened, the least active, the least well armed, \&c., have already disappeared or will disappear." 4

Even if, on the strength of this last passage, however, we can claim that Buffon had conceived the idea of the survival of the

1 Op. cit., Tom. XIV, pp. 325, 326.

2 Op. cit., Tom. VI, p. 248.

3 Ibid., p. $25 \mathrm{I}$.

4 I translate this passage, which I have not found in the original, from a quotation given by Osborn in his interesting work "From the Greeks to Darwin " (Columbia University Biological Series). The student should refer to this work for the history of the theory of evolution. 
fittest, the mere question of priority is a matter of small moment. As a matter of fact it is now well known that this idea is very much older than Buffon, and can be traced back, as Osborn remarks, "to Empedocles, six centuries before Christ." ${ }^{1}$

Osborn also points out that:-

"Buffon's ideas regarding the physical basis of heredity are very similar to those of Damocritus, and certainly contain the basis of the conception of the Pangenesis theory of Darwin, for he supposes that the elements of the germ-cells were gathered from all parts of the body." 2

So far we have presented the views of Buffon as those of a thoroughgoing evolutionist. He had, however, apparently another side to his mind, which is extremely difficult to understand in the author of the foregoing passages. In the fourth volume of his Natural History, after discussing the possible modification of species, he continues :-

"But no, it is certain, by revelation, that all animals have participated equally in the grace of creation, that the two first of each species and of all the species came forth complete from the hands of the Creator; and we must believe that they were then much the same as they are now represented to us by their descendants." 3

The doctrine of special creation could hardly be more clearly expressed.

Of course it is possible, as Samuel Butler suggests in his interesting discussion of Buffon in "Evolution Old and New," that such passages as this may be ironical, but it seems more likely that Buffon vacillated between what was then regarded as religious orthodoxy and the more rational views which he knew so well how to express. Indeed, Butler himself remarks, ùpropos of another passage :-

"This is Buffon's way. Whenever he has shown us clearly what we ought to think, he stops short suddenly on religious grounds." 4

1 Op. cit., p. 117.

2 I bid., p. 135.

3 " Histoire Naturelle," Tom. 1V, p. 383.

4 "Evolution Old and New" (New Issue, Lordon, A. \& C. Fifield), p. 115. This work contains numerous passages translated from Buffon and Lamarck, and I have found it of great use as a guide to the more salient passages in the voluminous writings of the former. I have, however, in all cases made fresh translations from the French.

B. B B 
Buffon's inability to reconcile the logical consequences of his own inductions with his religious convictions is perhaps nowhere better illustrated than in the attitude which he adopted with regard to the position of man in the animal kingdom. Such an experienced observer as he was could not fail to realize the close agreement in structure between man and the higher apes :-

"We shall see, in the history of the orang utan that, were we to pay attention only to the form, we might equally well look upon this animal either as the highest of the apes or as the lowest of mankind; because, with the exception of the soul, he lacks nothing of all that we possess, and because he differs less from man in bodily structure than he differs from other animals to which the name of ape has been given." 1

"I admit that, if one should judge only by form, the ape species might be taken for a variety of the human species : the Creator did not think fit to make for the human body a model absolutely different from that of the animal ; He has included his form, like that of all the animals, in one general plan; but at the same time that $\mathrm{He}$ bestowed upon him this ape-like material form, $\mathrm{He}$ penetrated this animal body with His divine breath." 2

The ape, on the other hand, is a mere animal, and

" in spite of his resemblance to man, far from being the second in our species, he is not the first in the order of animals, since he is not the most intelligent." 3

There can be no doubt that the views of Erasmus Darwin (1731-1802) were largely influenced by the writings of Buffon, to which he repeatedly refers. Darwin's “Zoonomia" was published in $1794,{ }^{4}$ but, perhaps because it was mainly a medical work, it received but little attention from professional naturalists. The section dealing with "Generation" contains his views on evolution. A few quotations will suffice to illustrate these:-

"Owing to the imperfection of language the offspring is termed a new animal, but is in truth a branch or elongation of the parent; since a part of the embryon-animal is, or was, a part of the parent; and therefore in strict language it cannot be said to

1 "Histoire Naturelle," Tom. XIV, p. 30. The name "orang utan" was applied by Buffon to the ehimpanzee.

2 Ibid., p. 32.

8 Ibid., p. 37.

4 My quotations are taken from an edition published by B. Dugdale at Dublin iu 1800 . 
be entirely new at the time of its production; and therefore it may retain some of the habits of the parent-system."

Here we have clearly expressed the idea of continuity which plays such an important part in modern theories of heredity. Erasmus Darwin was, however, a "Spermatist," that is to say he believed

" that the embryon is procuced solely by the male, and that the female supplies it with a proper nidus, with sustenance, and with oxygenation; and that the idea of the semen of the male constituting only a stimulus to the egg of the female, exciting it into life, (as held by some philosophers) has no support from experiment or analogy."

It must be remembered that he wrote in the days before the cell theory had shed its illuminating rays over the science of embryology :-

"I conceive," says he, "the primordium, or rudiment of the embryon, as secreted from the blood of the parent, to consist of a simple filament as a muscular fibre,"

but this filament was not necessarily thread-like in form, for he adds :-

"I suppose this living filament, of whatever form it may be, whether sphere, cube, or cylinder, to be endued with the capability of being excited into action by certain kinds of stimulus.'

It thus absorbs nutriment and becomes organized by "accretion of parts," and this leads to the development of new kinds of "irritability." According to this view the appearance of a new organ precedes its use :-

" the lungs must be previously formed before their exertions to obtain fresh air can exist."

"From hence I conclude, that with the acquisition of new parts, new sensations, and new desires, as well as new powers, are produced; and this by accretion to the old ones, and not by distention of them."

But the exercise of these new powers in turn gives rise to the development of more new parts, which

" are formed by the irritations and sensations, and consequent exertions of the parts previously existing, and to which the new parts are to be attached."

"From this account of reproduction it appears, that all animals have a similar origin, viz. from a single living filament; and 
that the difference of their forms and qualities has arisen only from the different irritabilities and sensibilities, or voluntarities, or associabilities, of this original living filament; and perhaps in some degree from the different forms of the particles of the fluids, by which it has been at first stimulated into activity."

"From their first rudiment, or primordium, to the termination of their lives, all animals undergo perpetual transformations; which are in part produced by their own exertions in consequence of their desires and aversions, of their pleasures and their pains, or of irritations, or of associations; and many of these acquired forms or propensities are transmitted to their posterity."

Dr. Darwin thus passes from the discussion of what is now termed the ontogeny or development of the individual to that of the phylogeny or development of the race. From consideration of the former he endeavoured to gain some insight into the latter, and it may be fairly claimed that he thus anticipated what is known in modern biology as the Recapitulation Hypothesis:-

"From thus meditating on the great similarity of the structure of the warm-blooded animals, and at the same time of the great changes they undergo both before and after their nativity; and by considering in how minute a portion of time many of the changes of animals above described have been produced; would it be too bold to imagine, that in the great length of time, since the earth began to exist, perhaps millions of ages before the commencement of the history of mankind, would it be too bold to imagine, that all warm-blooded animals have arisen from one living filament, which the great First Cause endued with animality, with the power of acquiring new parts, attended with new propensities, directed by irritations, sensations, volitions and associations; and thus possessing the faculty of continuing to improve by its own inherent activity, and of delivering down those improvements by generation to its posterity, world without end!"

Erasmus Darwin was perfectly familiar with the idea of adaptation, as manifested, for example, in the colours of animals :-

"The colours of many animals seem adapted to their purposes of concealing themselves either to avoid danger, or to spring upon their prey. Thus the snake and wild cat, and leopard, are so coloured as to resemble dark leaves and their lighter interstices; birds resemble the colour of the brown ground, or the green 
hedges, which they frequent; and moths and butterflies are coloured like the flowers which they rob of their honey."

"A proboscis of admirable structure has been acquired by the bee, the moth, and the humming bird, for the purpose of plundering the nectaries of flowers. All which seem to have been formed by the original Tiving filament, excited into action by the necessities of the creatures, which possess them, and on which their existence depends."

In the following sentences we have at any rate a very close approach to the idea of natural selection which forms the keynote of the evolutionary theory as advocated by the illustrious grandson of the writer:-

"A great want of one part of the animal world has consisted in the desire of the exclusive possession of the females; and these have acquired weapons to combat each other for this purpose. . . . So the horns of the stag are sharp to offend his adversary, but are branched for the purpose of parrying or receiving the thrusts of horns similar to his own, and have therefore been formed for the purpose of combating other stags for the exclusive possession of the females; who are observed, like the ladies in the times of chivalry, to attend the car of the victor."

"The final cause of this contest amongst the males seems to be, that the strongest and most active animal should propagate the species, which should thence become improved."

Here the principle of selection seems to be clearly enough recognized, at any rate that form thereof which Charles Darwin afterwards distinguished as sexual selection.

The great French philosophical biologist, ${ }^{1}$ Jean Baptiste Pierre Antoine de Monet, Chevalier de Lamarck, was born in 1744 and died in 1829. His most celebrated work, the "Philosophie Zoologique," which contains the fullest expression of his mature views on the theory of organic evolution, was published in 1809, but these views appear to have been first announced to the world in the opening lecture of the Course of Zoology given at the Natural History Museum in Paris in 1800,

1 Lamarck was the originator of the term Biology for the Science of Living Things. 
and published in the following year in the form of a preface to the "Système des Animaux sans Vertèbres." 1

It seems impossible to doubt that Lamarck, like Erasmus Darwin, was largely influenced in his views by the writings of his great compatriot Buffon, with whom he was on terms of personal friendship, and to whom he refers in his published work. Whether or not he was acquainted with the works of Erasmus Darwin will probably never be known, but it is evident that both drew part at any rate of their inspiration from the same source. Owing largely to his official position as Professor of Invertebrate Zoology in the National Museum, Lamarck was able to bring forward a very imposing array of facts in support of his opinions, and though these opinions were but the natural development of those enunciated by his predecessors, he was able to place the theory of organic evolution on a much firmer and broader basis than it had previously enjoyed. He also doubtless derived great advantage from the fact that he was an experienced botanist as well as a zoologist, having published many important botanical works before he turned his attention more particularly to the zoological aspect of biology.

During the earlier part of his life Lamarck appears to have accepted the still prevalent doctrine as to the immutability of species, and it is perhaps significant that his conversion to evolutionary views seems to have followed very rapidly upon the extension of his investigations from the vegetable to the animal kingdom.

In the "Philosophie Zoologique" he maintains that the first living things arose by a process of spontaneous generation, which may still take place, and that from the starting points thus provided the entire animal and vegetable kingdoms as they now exist have arisen as the result of orderly and progressive evolution.

$\mathrm{He}$ devotes a large amount of space to the question of classification and the conception of species, and arrives at the following conclusions, which are, on the whole, in singularly close agreement with those of modern biologists:-

"But these classifications, of which several have been so happily imagined by naturalists, and the divisions and sub-divisions

1 Vide Packard's "Lamarek, the Founder of Evolution, his Life and Work" (Longmans, Green \& Co., 1901). This most interesting work contains translations of portions of Lamarck's writings, and has for the first time made the work of the great French philosopher available to the gencral reader in England and America. I have, however, thought it desirable to give fresh translations from the original French. 
which they present, are entirely artificial contrivances. Nothing of all that, I repeat, occurs in nature, in spite of the foundation which appears to be given to them by certain portions of the natural series which are known to us, and which have the appearance of being isolated. We may be certain that amongst her productions, nature has really formed neither classes, nor orders, nor families, nor genera, nor constant species, but only individuals which succeed one another and which resemble those which produced them. Now these individuals belong to infinitely diversified races, which shade off under all forms and in all degrees of organization, and each of which maintains itself without change so long as no cause of change acts upon it." 1

"The name species has been given to every collection of similar individuals which have been produced by other individuals like themselves.

"This definition is exact; for every individual that enjoys life always resembles very closely that or those from which it sprang. But to this definition has been added the supposition that the individuals which make up a species never vary in their specific character, and that consequently the species has an absolute constancy in nature.

"It is exactly this supposition that I propose to combat, because the clear evidence obtained by observation shews that it is unfounded.

"The supposition, almost universally admitted, that living bodies form species constantly distinguished by invariable characters, and that the existence of these species is as old as that of nature herself, was established at a time when observations were insufficient, and when the natural sciences were still almost non-existent. It is always contradicted in the eyes of those who have seen much, who have for a long time followed nature, and who have profitably consulted the great and rich collections of our Museum." 2

After speaking of the doctrine of special creation Lamarck continues :-

"Without doubt, nothing exists except by the will of the sublime Author of all things. But can we assign to Him laws in the execution of $\mathrm{His}$ will, and fix the method which $\mathrm{He}$ has followed in this respect? Has not His infinite power been able to create an order of things which should give existence 
successively to everything that we see, as to everything which exists and which is unknown to us?

"Assuredly, whatever may have been His will, the immensity of His power is always the same; and in whatever manner this supreme will may have been executed, nothing can diminish its grandeur." I

Lamarck seems to have been the first to insist upon the branching character of evolutionary series; after speaking of such series he goes on :-

"I do not wish to say thereby that existing animals form a very simple series, everywhere equally graduated; but I say that they form a branching series, irregularly graduated, and which has no discontinuity in its parts, or which, at least, has not always had, if it be true that, in consequence of some species having been lost, such discontinuity occurs anywhere. It results from this that the species which terminate each branch of the general series are connected, at least on one side, with other neighbouring species which shade into them." 2

Like Buffon, he lays great stress upon the action of the environment in modifying organisms :-

"Many facts teach us that in proportion as the individuals of one of our species change their situation, their climate, their manner of living or their habits, they thereby receive influences which little by little change the consistency and the proportions of their parts, their form, their faculties, even their organization ; so that everything in them participates, in the course of time, in the transformations which they experience.

"In the same climate, very different situations and exposures at first cause the individuals exposed thereto simply to vary; but, in the course of time, the continual difference in situation of the individuals of which I am speaking, which live and reproduce themselves successively in the same circumstances, causes in them differences which become, in some way, essential to their existence; so that, after many generations have succeeded one another, these individuals, which belonged originally to another species, find themselves in the end transformed into a new species, distinct from the other.

"For example, if the seeds of a grass, or of any other plant natural to a damp meadow, be transported by any chance, first to the slope of a neighbouring hill, where the soil, although more elevated, is still sufficiently cool to permit of the plant maintaining itself, and if afterwards, after having lived there and 
reproduced itself many times, it reaches, by slow degrees, the dry and almost arid soil of a mountainous region; if the plant succeeds in living there, and perpetuates itself for a succession of generations, it will then become so changed that the botanists who come across it will make of it a distinct species." 1

As Lamarck's views have so frequently been misrepresented it is incumbent upon us to make ourselves thoroughly acquainted with what he really meant by the action of the environment, and on this point, fortunately, he is very precise :-

"Here it becomes necessary for" me to explain the meaning which I attach to these expressions: The environment (les eirconstanees) influences the form and organization of animals, or in other words, as it becomes very different it changes, in course of time, the form and organization themselves by proportional modifications.

"Certainly, if people took these expressions literally they would attribute to me an error; for, whatever the environment may be, it does not directly effect any modification whatever in the form and organization of animals.

"But great changes in the environment lead, in the case of animals, to great changes in their requirements (besoins), and such changes in their requirements lead necessarily to actions. Now, if the new requirements become constant or very lasting, the animals then adopt new labits, which are as lasting as the requirements which have called them forth. . . .

"Now, if a novel environment, having become permanent for" a race of animals, has given to these animals new halits, or in other words has led them to new actions which have become habitual, there will have resulted therefrom the use of some particular part [of the body] in preference to some other, and in certain cases the complete disuse of some part which has become useless.

"None of these statements should be considered as hypothetical or as the expression of individual opinion; they are, on the contrary, truths which need only attention and the observation of facts to render them evident.

"We shall see immediately, by the citation of known facts which attest it, on the one hand that new requirements having made some part necessary, have really, by a series of efforts, caused this part to arise, and that afterwards its continued employment has little by little strengthened it, developed it, and in the end considerably enlarged it; on the other hand, we shall see that, in certain cases, the novel environment and new requirements having rendered some part quite useless, the 
complete want of employment of this part has caused it to gradually cease from developing like the other parts of the animal; that it has become reduced and attenuated little by little, and that at length, when this want of employment has been complete during a long period, the part in question has in the end disappeared. All this is positive; I propose to give the most convincing proofs of it.

"In plants, where there are no actions, and consequently no habits properly so-called, great changes in the environment have none the less led to great differences in the development of their parts; in such a way that these differences have caused certain of them to appear and develop, while they have caused many others to dwindle away and disappear. But here everything is effected by changes which take place in the nutrition of the plant, in its absorptions and transpirations, in the amount of heat, light, air and moisture, which it then habitually receives; finally, in the superiority which certain of the various vital movements may acquire over others." 1

Like his predecessors, and like those who followed him, Lamarck adduces in support of these views the remarkable modifications which have taken place in animals and plants under the influence of domestication :-

"That which nature does with the aid of much time, we do every day by suddenly changing, in relation to some living plant, the conditions under which it and all the individuals of its species, have existed.

"All botanists know that the plants which they transport from their native place in order to cultivate them in gardens, undergo, little by little, changes which, in the end, make them unrecognizable.

"Is not the cultivated wheat (Triticum sativum) a plant brought by man to the condition in which we now actually see it? Who will tell me in what country such a plant occurs naturally, that is to say except as the result of its cultivation in some neighbouring place?

"Where do we find, in a state of nature, our cabbages, our lettuces, \&c., as we now possess them in our kitchen gardens? Is it not the same with respect to the many animals which domestication has changed or considerably modified ?"2

We may next consider two examples of the kind of evidence which Lamarck brings forward in proof of the effects of the 
environment and of the use and disuse of organs upon plants and animals living in a state of nature :-

"The following fact proves, with regard to plants, how much the alteration of any important factor in the environment modifies the parts of these living bodies.

"So long as Ranunculus aquatilis is sunk beneath the surface of the water, its leaves are all finely divided and have their divisions hair-like; but when the stems of this plant reach the surface of the water, the leaves which develop in the air become broadened, rounded and simply lobed. If some runners of the same plant succeed in pushing their way into a soil which is merely damp, without being inundated, their stems are then short, and none of their leaves are divided into hair-like segments; thus arises Ranunculus hederaceus, which botanists regard as a species when they come across it." ${ }_{1}$

"With regard to habits, it is curious to observe the result thereof in the remarkable form and in the stature of the giraffe (Camelo-pardalis): we know that this animal, the tallest of the mammals, inhabits the interior of Africa, and that it lives in places where the earth, almost always arid and without herbage, obliges it to browse on the foliage of trees and to make continual efforts to reach it. As a result of this habit, maintained for a long time in all the individuals of its race, the fore limbs have become much longer than the hind ones, and the neck has become so much elongated that the giraffe, without standing up on its hind legs, raises its head and reaches a height of six metres (nearly twenty feet)." 2

The following passage must suffice to give some idea of Lamarck's views on the inheritance of "acquired" characters, which his theory necessarily implies, though not in the exaggerated sense of some modern writers :-

"These familiar facts are surely well suited to prove what is the result of the habitual use by animals of some particular organ or part ; and if, when we observe, in an animal, an organ specially developed, strong and powerful, anyone pretends that its habitual exercise has caused it to gain nothing, that its continued disuse would cause it to lose nothing, and that, in short, this organ has always been as it is since the creation of the species to which the animal belongs, I would ask why our domestic ducks can no longer fly like wild ducks; in a word, I would cite a multitude of examples relative to ourselves, which bear witness to the differences which result in our own bodies

1 op. cit., Tom. I, p. 230.

2 I bid., pp. 256-257. 
from the exercise or the want of exercise of any of our organs, although these differences are not maintained in the individuals of the next generation, for then their results would be much more considerable.

"I shall show in the second part that when the will determines an animal to some action, the organs which have to execute this action are forthwith stimulated by the affluence of subtle fluids (the nervous fluid) which become the determining cause of the movements which the action in question requires. A multitude of observations establishes this fact, which should no longer be called in question.

"It results therefrom that numerous repetitions of these acts of organization strengthen, extend, develop and even create the organs which are needed. It is only necessary to observe attentively what is happening everywhere in this respect to convince oneself of the actuality of this cause of organic development and modification.

"Now, every change acquired in an organ by a babit of use sufficient to have produced it, is maintained afterwards by generation, if it is common to the individuals which have united in the act of fecundation for the reproduction of their species. In short, this modification is propagated, and thus passes to all the individuals which follow and which are subjected to the same environment, without their being obliged to acquire it by the means which really created it.

"The mingling in reproductive unions, however, between individuals which have different qualities or forms, is necessarily opposed to the constant propagation of those qualities and forms. This is the reason why in man, who is subjected to so many different modifying circumstances, the accidental qualities or defects which he has chanced to acquire are not preserved and propagated by generation. If, when any peculiarities of form or any defects have been acquired, two individuals in this condition should always unite, they would reproduce the same peculiarities, and successive generations confining themselves to similar unions, a special and distinct race would then be formed. But the perpetual mingling between individuals which have not the same peculiarities of form causes all peculiarities acquired as the result of peculiar circumstances of the environment to disappear. Whence one may be certain that if human beings were not separated by the distances of their habitations, the mixed breeding would cause the general characters which distinguish the different nations to disappear." 1

In the light of modern knowledge these views on the subject of heredity are, of course, crude and inaccurate enough, but there 1 op. cit., Tom. I, pp. $25 \mathrm{e}-262$. 
is nothing absurd in them, and at the time when Lamarck wrote it would scarcely have been possible to formulate anything better.

It is, however, this assumption of the inheritance of somatogenic characters that has probably done more than anything else to prevent many modern biologists from accepting the so-called Lamarckian factors of evolution. Those who hold with Weismann that there is no possible mechanism by which a somatogenic character can be converted into a blastogenic one are forced to reject Lamarck's teaching, but the Weismannian assumption that there is no such possibility of inheritance of somatogenic characters rests upon no better foundation than the Lamarckian assumption that there is. We have dealt with this question at some length in an earlier chapter and need only now remember that, if there are many modern biologists who reject the Lamarckian factors because they cannot reconcile them with Weismannism, there are probably quite as many others who accept them becaúse they appeal irresistibly to their common sense, and because they refuse to believe that Weismann's difficulties are really insurmountable.

It is, of course, easy to ridicule Lamarck's views, and to say, for example, that he maintained that an animal could develop an organ by simply wishing for it, and ridicule of this kind has undoubtedly done much to hinder the due appreciation of his work. Such statements, however, are only made by those who have never paid adequate attention to the writings of the great French biologist.

It is evident from the passage last quoted that Lamarck also did not fail to perceive the importance of isolation as a factor in organic evolution, necessary to prevent the swampin effects of intercrossing upon newly arisen species or varieties.

In another place he discusses the influence of the struggle for existence in counteracting the effects of excessive multiplication, and in so doing just misses the idea of Natural Selection:

"Animals eat one another, except those which live only upon plants; but the latter are liable to be devoured by carnivores.

"We know that it is the stronger and the better armed which eat the weaker, and that the large species devour the smaller ones. Nevertheless the individuals of one and the same race rarely eat each other ; they make war on other races." 1 
Lamarck summed up his views as to the factors which have co-operated in organic evolution, at any rate so far as the animal kingdom is concerned, in a later work, the "Histoire Naturelle des Animaux sans Vertèbres," published in 1815, in the form of four "Laws," which may be taken as replacing the two "Laws" of the "Philosophie Zoologique." They are as follows :-

"First Law: Life, by her own forces, tends continually to increase the volume of every body which possesses it, and to extend the dimensions of its parts, up to a limit which she herself imposes."1

"Second Law : The production of a new organ in an animal body results from a new requirement which continues to make itself felt, and from a new movement which this requirement begets and maintains." 2

"Third Law : The development and efficiency of organs are constantly in proportion to the use of these organs." 3

"Fourth Law: All that has been acquired, traced out or altered in the organization of individuals during the course of their life, is preserved by generation, and transmitted to the new individuals which originate from those which have experienced these modifications." 4

With regard to the position of man in the animal kingdom Lamarck, unfortunately, does not seem to have been able, any more than Buffon, to divest himself of the fetters of religious orthodoxy. After pointing out at length the numerous ties by which man, in his bodily organization, is united to the lower animals, and especially his close relationship to the apes, he saves himself, so to speak, in the following paragraph :-

"Such would be the reflections which one might make if man, considered here as the pre-eminent race in question, were only distinguished from the animals by the characters of his organization, and if his origin were not different from theirs." 5

1 "Histoire Naturelle des Animaux sans Vertèbres," Tom. I, 1815, p. 182.

2 Ibid., p. 185.

3 1bid., p. 189.

4 1bid., p. 199.

5 "Philosophie Zoologique," Tom. I, p. 357. 


\section{CHAPTER XXV}

Robert Chambers and the "Vestiges of Creation"-Natural SelectionThe Views of Charles Darwin and Alfred Russel Wallace.

For half a century after the appearance of the "Philosophie Zoologique" the theory of organic evolution made but little progress. The gap, however, was to some extent filled by the publication, at first anonymously, of Robert Chambers' celebrated book, "Vestiges of the Natural History of Creation." This work first appeared in the year 1844 and rapidly passed through a large number of editions. Though the views of its author can hardly be said to mark any advance, but on the whole perhaps rather a retrogression, the work, which gave rise to much controversy, undoubtedly played a very important part in preparing the way for the reception of Charles Darwin's "Origin of Species." Chambers presented the evidence of organic evolution in a very convincing manner, laying great stress upon that afforded by the geological record and the facts of comparative anatomy and embryology, and he included mankind in his general scheme of evolution.

His views as to the modus operandi of organic evolution are probably expressed as clearly as such views could be in the following paragraphs :-

"The proposition determined on after much consideration is, that the several series of animated beings, from the simplest and oldest up to the highest and most recent, are, under the providence of God, the results, first, of an impulse which has been imparted to the forms of life, advancing them, in definite times, by generation, through grades of organization terminating in the highest dicotyledons and vertebrata, these grades being few in number, and generally marked by intervals of organic character which we find to be a practical difficulty in ascertaining affinities; second, of another impulse connected with the vital forces, tending, in the course of generations, to modify organic structures in accordance with external circumstances, as food, the nature of the habitat and the meteoric agencies, these being the 
"adaptations" of the natural theologian. We may contemplate these phenomena as ordained to take place in every situation, and at every time, where and when the requisite materials and conditions are presented-in other orbs as well as in this-in any geographical area of this globe which may at any time arise-observing only the variations due to difference of materials and of conditions." 1

"For the history, then, of organic nature, I embrace, not as a proved fact, but as a rational interpretation of things as far as science has revealed them, the idea of Progressive Development. We contemplate the simplest and most primitive types of being, as giving, under a law to which that of like-production is subordinate, birth to a type superior to it in compositeness of organization and endowment of faculties; this again producing the next higher, and so on to the highest. We contemplate, in short, a universal gestation of nature, analogous to that of the individual being; and attended as little by circumstances of a startling or miraculous kind, as the silent advance of an ordinary mother from one week to another of her pregnancy. We see but the chronicle of one or two great areas, within which the development has reached the highest forms. In some others, as Australia and the islands of the Pacific, development appears to have not yet passed through the whole of its stages, because, owing to the comparatively late uprise of the land, the terrestrial portion of the development was there commenced more recently. It would commence and proceed in any new appropriate area, on this or any other sphere, exactly as it commenced upon our area in the time of the earliest fossiliferous rocks, whichever these are. Nay, it perhaps starts every hour with common infusions, and in similar humble theatres, and might there proceed through all the subsequent stages, granting suitable space and conditions. Thus simple-after ages of marvelling-appears Organic Creation, while yet the whole phenomena are, in another point of view, wonders of the highest kind, being the undoubted results of ordinances arguing the highest attributes of foresight, skill, and goodness on the pait of their Divine Author." 2

Progressive evolution is here clearly attributed to some inherent tendency implanted in the first living things, and apparently the writer imagines that the same or closely similar results may have been arrived at along many different lines of evolution, each commencing at a distinct starting point and at a different time and

1 "Vestiges of the Natural History of Creation," 12th ed., 1884, pp. 201, 202.

2 I bid., pp. 230, 231. 
place from all the others. The author of this hypothesis evidently considers it a distinet improvement upon the views of Lamarek, which he very briefly discusses. He thinks that Lamarck attributes too much importance to the principle of use and disuse, which he regards as "obviously insufficient to account for the great grades of organization," though he admits that external conditions may have been "a means of producing the exterior characters." There can be little doubt, however, that, in relying upon a system of more or less definite and continuously operating natural causes as factors of organic evolution, Lamarck took up a far more scientific position than Robert Chambers.

In insisting upon the great importance of Natural Selection as a factor in organic evolution, Charles Darwin and Alfred Russel Wallace made a great advance upon the position of any of their predecessors.

We have seen in the last chapter that this principle had been hinted at by more than one writer about the close of the eighteenth and the commencement of the nineteenth centuries, and that it can even be traced back to the philosophy of ancient Greece. In the historical sketch which prefaces the later editions of the "Origin of Species," Charles Darwin himself quotes a translation from.Aristotle which shows sufficiently clearly that the idea was familiar to the great Greek biologist. In the same sketch he also quotes a passage from Dr. W. C. Wells, from a paper read before the Royal Society in 1813, in which the same principle is recognized in the most explicit and unmistakable manner.

It was not, however, until the year 1858 that the part played by natural selection in organic evolution began to be generally understood. In that year Sir Charles Lyell and Dr. J. D. Hooker communicated to the Linnean Society certain papers, ${ }^{1}$ written by Darwin and Wallace, which at once called prominent attention to the importance of this factor and contained the essential parts of the theory of natural selection as subsequently developed by both these writers.

Darwin's paper consisted of an extract from his as yet unpublished work, together with an abstract of a letter to

1 These papers have been reprinted by the Linnean Society in the volume published in connection with the Darwin-Wallace Celebration held on July 1st, 1908, and it is from this volume that the quotations which follow are taken.

B. 
Professor Asa Gray. A few quotations will suffice to indicate his views :-

"De Candolle, in an eloquent passage, has declared that all nature is at war, one organism with another, or with external nature. ... It is the doctrine of Malthus applied in most cases with tenfold force.... Even slow-breeding mankind has doubled in twenty-five years; and if he could increase his food with greater ease, he would double in less time. But for animals without artificial means, the amount of food for each species must, on an average, be constant, whereas the increase of all organisms tends to be geometrical, and in a vast majority of cases at an enormous ratio. Suppose in a cerlain spot there are eight pairs of birds, and that only four pairs of them annually (including double hatches) rear only four young, and that these go on rearing their young at the same rate, then at the end of seven years (a short life, excluding violent deaths, for any bird) there will be 2048 birds, instead of the original sixteen. As this increase is quite impossible, we must conclude either that birds do not rear nearly half their young, or that the average life of a bird is, from accident, not nearly seven years. Both checks probably concur. The same kind of calculation applied to all plants and animals affords results more or less striking, but in very few instances more striking than in man."

"Lighten any check in the least degree, and the geometrical powers of increase in every organism will almost instantly increase the average number of the favoured species.... Finally, let it be borne in mind that this average number of individuals (the external conditions remaining the same) in each country is kept up by recurrent struggles against other species or against external nature (as on the borders of the Arctic regions, where the cold checks life), and that ordinarily each individual of every species holds its place, either by its own struggle and capacity of acquiring nourishment in some period of its life, from the egg upwards; or by the struggle of its parents (in short-lived organisms, when the main check occurs at longer intervals) with other individuals of the same or different species.

"But let the external conditions of a country alter. If in a small degree, the relative proportions of the inhabitants will in most cases simply be slightly changed; but let the number of inhabitants be small, as on an island, and free access to it from other countries be circumscribed, and let the change of conditions continue progressing (forming new stations), in such a case the original inhabitants must cease to be as perfectly adapted to the 
changed conditions as they were originally. It has been shown in a former part of this work, that such changes of external conditions would, from their acting on the reproductive system, probably cause the organization of those beings which were most affected to become, as under domestication, plastic. Now, can it be doubted, from the struggle each individual has to obtain subsistence, that any minute variation in structure, habits, or instincts, adapting that individual better to the new conditions, would tell upon its vigour and health? In the struggle it would have a better chance of surviving; and those of its offspring which inherited the variation, be it ever so slight, would also have a better chance. Yearly more are bred than can survive; the smallest grain in the balance, in the long run, must tell on which death shall fall, and which shall survive. Let this work of selection on the one hand, and death on the other, go on for a thousand generations, who will pretend to affirm that it would produce no effect, when we remember what, in a few years, Bakewell effected in cattle, and Western in sheep, by this identical principle of selection?"

"In nature we have some slight variation occasionally in all parts;; and I think it can be shewn that changed conditions of existence is the main cause of the child not exactly resembling its parents; and in nature geology shews us what changes have taken place, and are taking place. We have almost unlimited time;"

"Another principle, which may be called the principle of divergence, plays, I believe, an important part in the origin of species. The same spot will support more life if occupied by very diverse forms. We see this in the many generic forms in a square yard of turf, and in the plants or insects on any little uniform islet, belonging almost invariably to as many genera and families as species. ... Now, every organic being, by propagating so rapidly, may be said to be striving its utmost to increase in numbers. So it will be with the offspring of any species after it has become diversified into varieties, or subspecies, or true species. And it follows, I think, from the foregoing facts, that the varying offspring of each species will try (only few will succeed) to seize on as many and as diverse places in the economy of nature as possible. Each new variety or species, when formed, will generally take the place of, and thus exterminate its less well-fitted parent. This I believe to be the origin of the classification and affinities of organic beings at all times; for organic beings always seem to branch and sub-branch like the limbs of a tree from a common trunk, the flourishing and 
diverging twigs destroying the less vigorous - the dead and lost branches rudely representing extinct genera and families."

The same paper also contains a sketch of the supplementary theory of sexual selection, which will be seen to agree very closely with the paragraph from Erasmus Darwin's “ Zoonomia" quoted in the last chapter :-

"Besides this natural means of selection, by which those individuals are preserved, whether in their egg, or larval, or mature state, which are best adapted to the place they fill in nature, there is a second agency at work in most unisexual animals, tending to produce the same effect, namely, the struggle of the males for the females. These struggles are generally decided by the law of battle, but in the case of birds, apparently, by the charms of their song, by their beauty or their power of courtship, as in the dancing rock-thrush of Guiana. The most vigorous and healthy males, implying perfect adaptation, must generally gain the victory in their contests. This kind of selection, however, is less rigorous than the other; it does not require the death of the less successful, but gives to them fewer descendants."

The publication from which these quotations are taken itself consists of extracts from Charles Darwin's manuscripts, selected with a view to explaining, as clearly as possible, his theory of naturai selection, and is therefore especially suitable for citation. In the following year (1859) the author's classical work, "The Origin of Species by means of Natural Selection," made its appearance, the first of that notable series of volumes on philosophical biology which have made his name so famous. In these works both the general theory of Evolution and the subsidiary theory of Natural Selection are elaborated and supported by an immense body of evidence drawn from published records and personal observations, and so successfully was this done that in a comparatively few years these theories met with general acceptance on the part, not only of scientific men, but also of the educated public. It must not be forgotten, either, that Charles Darwin applied the doctrine of organic evolution in a fearless and uncompromising manner to the origin of the human race.

Dr. Wallace's contribution to the Linnean Society symposium of 1858 was entitled "On the Tendency of Varieties to depart 
indefinitely from the Original Type." The following quotations will serve to show that his views on Natural Selection were closely similar to those of Charles Darwin.

"The life of wild animals is a struggle for existence. The full exertion of all their faculties and all their energies is required to preserve their own existence and provide for that of their infant offspring. 'I'he possibility of procuring food during the least favourable seasons, and of escaping the attacks of their most dangerous enemies, are the primary conditions which determine the existence botl of individuals and of entire species."

"Even the least prolific of animals would increase rapidly if unchecked, whereas it is evident that the animal population of the globe must be stationary, or perhaps, through the influence of man, decreasing. Fluctuations there may be ; but permanent increase, except in restricted localities, is almost impossible. For example, our own observation must convince us that birds do not go on increasing every year in a geometrical ratio, as they would do, were there not some powerful check to their natural increase. Very few birds produce less than two young ones each year, while many have six, eight, or ten; four will certainly be below the average; and if we suppose that each pair produce young only four times in their life, that will also be below the average, supposing them not to die either by violence or want of food. Yet at this rate how tremendous would be the increase in a few years from a single pair! A simple calculation will show that in fifteen years each pair of birds would have increased to nearly ten millions! Whereas we have no reason to believe that the number of the birds of any country increases at all in fifteen or in one hundred and fifty years. With such powers of increase the population must have reached its limits, and have become stationary, in a very few years after the origin of each species. It is evident, therefore, that each year an immense number of birds must perish - as many in fact as are born. . . . It is, as we commenced by remarking, ' a struggle for existence,' in which the weakest and least perfectly organized must always succumb.

"Now it is clear that what takes place among the individuals of a species must also occur among the several allied species of a group,-viz., that those which are best adapted to obtain a regular supply of food, and to defend themselves against the attacks of their enemies and the vicissitudes of the seasons, must necessarily obtain and preserve a superiority in population; while those species which from some defect of power or organization are the least capable of counteracting the vicissitudes of food 
supply, \&c., must diminish in numbers, and, in extreme cases, become altogether extinct."

"Most or perhaps all the variations from the typical form of a species must have some definite effect, however slight, on the habits or capacities of the individuals. Even a change of colour might, by rendering them more or less distinguishable, affect their safety; a greater or less development of hair might modify their habits. ... An antelope with shorter or weaker legs must necessarily suffer more from the attacks of the feline carnivora. ... If, on the other hand, any species should produce a variety having slightly increased powers of preserving existence, that variety must inevitably in time acquire a superiority in numbers. ... All varieties will therefore fall into two classes-those which under the same conditions would never reach the population of the parent species, and those which would in time obtain and keep a numerical superiority: Now, let some alteration of physical conditions occur in the district-a long period of drought, a destruction of vegetation by locusts, the irruption of some new carnivorous animal seeking 'pastures new'-any change in fact tending to render existence more difficult to the species in question, and tasking its utmost powers to avoid complete extermination; it is evident that, of all the individuals composing the species, those forming the least numerous and most feebly organized variety would suffer first, and, were the pressure severe, must soon become extinct. 'The same causes continuing in action, the parent species would next suffer, would gradually diminish in numbers, and with a recurrence of similar unfavourable conditions might also become extinct. The superior variety would then alone remain, and on a return to favourable conditions would rapidly increase in numbers and occupy the place of the extinct species and variety.

"The variety would now have replaced the species, of which it would be a more perfectly developed and more highly organized form. It would be in all respects better adapted to secure its safety, and to prolong its individual existence and that of the race. Such a variety could not return to the original form; for that form is an inferior one, and could never compete with it for existence. . . . But this new, improved, and populous race might itself, in course of time, give rise to new varieties, exhibiting several diverging modifications of form, any of which, tending to increase the facilities for preserving existence, must, by the same general law, in their turn become predominant. Here, then, we have progression and continued divergence deduced from the general laws which regulate the existence of animals, in 
a state of nature, and from the undisputed fact that varieties do frequently occur.'

It will be evident from the above sketch of the theory of Natural Selection, which I have thought it desirable to give in the actual words of its chief exponents, that adaptation is explained as the logical consequence of certain facts which can at any time be verified by direct observation.

(1) All organisms tend to increase in a high geometrical ratio; (2) there is, partly as a direct result of such increase, a keen struggle for existence, to which all organisms are more or less exposed and in which vast numbers perish without leaving offspring; (3) all organisms tend to vary in many directions; (4) variations, whether favourable or otherwise, tend to be transmitted by heredity from generation to generation; though, as we have already seen, there is at the present time much dispute as to whether variations of a certain kind ought not to be excluded from this generalization.

It follows inevitably from these premisses that in every generation there will be a more or less strongly pronounced tendency towards the elimination of those individuals which are least well adapted to their environment and a corresponding preservation and encouragement of those which are best adapted, or, in Herbert Spencer's celebrated phrase, a "survival of the fittest." This process, continued from generation to generation for countless ages, has resulted in that marvellous perfection of adaptation which we have seen to be such a striking feature of both plants and animals.

Charles Darwin himself, however, was not satisfied with natural selection as the sole factor concerned in bringing about progressive evolution and adaptation. Although, in the historical sketch which he added to the later editions of the "Origin of Species," he remarks :-

"It is curious how largely my grandfather, Dr. Erasmus Darwin, anticipated the views and erroneous grounds of opinion of Lamarck in his 'Zoonomia,',

and although he himself at first appears to have attached very little importance to Lamarck's opinions, yet we find in the last chapter of the sixth edition of the "Origin of Species " abundant evidence that he was obliged to admit the efficacy of the chief "Lamarckian" factor, the principle of use and disuse, in 
modifying species, and also, to some extent, that of the direct action of the environment:-

"Disuse, aided sometimes by natural selection, will often have reduced organs when rendered useless under changed habits or conditions of life; and we can understand on this view the meaning of rudimentary organs. ${ }^{1}$ But disuse and selection will generally act on each creature, when it has come to maturity and has to play its full part in the struggle for existence, and will thus have little power on an organ during early life; hence the organ will not be reduced or rendered rudimentary at this early age. 'The calf, for instance, has inherited teeth, which never cut through the gums of the upper jaw, from an early progenitor having well-developed teeth; and we may believe, that the teeth in the mature animal were formerly reduced by disuse, owing to the tongue and palate, or lips, having become excellently fitted through natural selection to browse without their aid; whereas in the calf, the teeth have been left unaffected, and on the principle of inheritance at corresponding ages have been inherited from a remote period to the present day." ${ }_{2}$

"I have now recapitulated the facts and considerations which have thoroughly convinced me that species have been modified, during a long course of descent. This has been effected chiefly through the natural selection of numerous successive, slight, favourable variations; aided in an important manner by the inherited effects of the use and disuse of parts; and in an unimportant manner, that is in relation to adaptive structures, whether past or present, by the direct action of external conditions, and by variations which seem to us in our ignorance to arise spontaneously. It appears that I formerly underrated the frequency and value of these latter forms of variation, as leading to permanent modifications of structure independently of natural selection. But as my conclusions have lately been much misrepresented, and it has been stated that I attribute the modification of species exclusively to natural selection, I may be permitted to remark that in the first edition of this work, and subsequently, I placed in a most conspicuous position-namely, at the close of the Introduction-the following words: 'I am convineed that natural selection has been the main but not the exclusive means of modification.' This has been of no avail. Great is the power of steady misrepresentation; but the history of science shows that fortunately this power does not long endure." 3

\footnotetext{
1 Often now called "vestigial organs."

2 "Origin of Species," Ed. vi. p. 420.

3 Ibid., p. 421.
} 
It is of the greatest interest to recognize the fact that Darwin himself saw nothing incompatible between the so-called Lamarckian factors of use and disuse and the direct action of the environment, and the principle of natural selection, but, on the other hand, that the one set of factors might supplement the other.

On the occasion of the unveiling of the statue of Charles Darwin in the Natural History Museum at South Kensington, Professor Huxley found occasion to observe that "science commits suicide when it adopts a creed." 1 This warning, it is to be feared, has not been heeded by all of Darwin's followers. Many of these have departed very far from the moderate and rational position of their leader and, while attributing to natural selection almost every advance which has been made in the evolution of the organic world, are, as we have already seen, obliged to justify their neglect of the "Lamarckian" factors by denying altogether the possibility of the inheritance of acquired characters, which Darwin, of course, freely admitted. Natural selection, in the hands of these enthusiasts, and in spite of Charles Darwin's efforts to maintain a just balance between this and other factors, has indeed become a creed.

Dr. Wallace from the fisst adopted an uncompromising attitude towards the opinions of Lamarck. In the Linnean Society paper from which we have already quoted he says:-

"The hypothesis of Lamarck-that progressive changes in species have been produced by the attempts of animals to increase the development of their own organs, and thus modify their structure and habits - has been repeatedly and easily refuted by all writers on the subject of varieties and species, and it seems to have been considered that when this was done the whole question has been finally settled; but the view here developed renders such an hypothesis quite unnecessary, by shewing that similar results must be produced by the action of principles constantly at work in nature. The powerful retractile talons of the falcon-and the cat-tribes have not been produced or increased by the volition of those animals; ${ }^{2}$ but among the different varieties which occurred in the earlier and less highly organized forms of these groups, those always survived longest which had the greatest facilities for seizing their prey."

1 Vide Herbert Spencer's "Faetors of Organie Evolution," p. 75.

2 Who ever said they had, except in the sense that an animal voluutarily uses its elaws on appropriate oceasions and that eonstantly repeated use causes them to 
Wallace's views ${ }^{1}$ also differ from those of Darwin in that, at any rate in later years, they have become strongly anthropocentric, and he now regards the whole of the organic world as having been designed by the Creator for the ultimate reception and benefit of mankind. He does not, it is true, go back to the old idea that species have been separately and specially created as we now find them, but he holds that the entire scheme of evolution was planned out in the mind of the Creator, and even suggests that the working out of this scheme may have been delegated by the Supreme Being to a body of "organizing spirits" :-

"At successive stages of development of the life-world, more and perhaps higher intelligences might be required to direct the main lines of variation in definite directions in accordance with the general design to be worked out, and to guard against a break in the particular line which alone could lead ultimately to the production of the human form." 2

Such speculations as this would render natural selection and all other natural factors of organic evolution superfluous, but we cannot profitably discuss them in a work like the present. We may point out, however, that they are in essential agreement with the views of the author of the "Vestiges of Creation," to which we have referred in the early part of this chapter, excepting that Robert Chambers did not venture to call in the assistance of subordinate "organizing spirits" to carry out the plans of the Creator.

increase in size and efficiency ? Lamarck did not suppose that an animal simply willed organs to sprout out of its body!

1 For a full exposition of these views the reader should refer to Dr. Wallace's "Darwinism" (London: Macmillan \& Co., 1889).

2 "The World of Life, a Manifestation of Creative Power, Direetive Mind and Ultimate Purpose," by Alfred Russel Wallace (London: Chapman and Hall, Ltd., 1910), p. 395. 


\section{CHAPTER XXVI}

Selection not confined to the organic world-Illustrations of the action of natural selection in the struggle for existence-Degeneration--Flightless birds-Lxtermination of the Morioris - Sedentary animals--Parasites-Co-operation of natural selection and the so-called Lamarckian factors of evolution-The influence of internal secretions upon growth - Increase in size beyond the limits of utility.

THE principle of selection is, of course, by no means confined to living things. The various bodies which make up the inorganic world owe theír actual form and arrangement largely to processes of selection which are constantly going on amongst them. The outline of the sea coast is the result of the selective action of atmospheric and tidal agencies upon the different kinds of rock of which it is composed. The softer parts are destroyed first, leaving the more resistant portions to stand out in the form of bluffs or promontories, and to illustrate in the inanimate world the principle of the survival of the fittest. We might even say that the prominent headlands exhibit adaptation, for if they were not adapted by their peculiar hardness to resist the disintegrating influences of the environment they would not be there, but would have perished with those portions of the land which formerly occupied the bays and inlets.

All things, in short, must be subject to the selective action of their environment, and we need not hesitate to attribute to natural selection a very large share in the modelling of the features of the organic world as we now see it. We know what we ourselves, by our so-called artificial selection, are able to do in this way. The chief difference between artificial and natural selection is that man selects for his own purposes and modifies organisms to suit his own ends, while Nature selects to the benefit of the species operated upon, which becomes thereby modified to its own advantage and preservation in the struggle for existence. But we cannot really draw a distinction between the two kinds of selection, for even in a state of nature organisms are often selected and modified to the advantage of other organisms. 
As we saw in a previous ehapter, the forms, colours and scents of many flowers are probably the result of unconscious selection by insects, extending over countless generations. It may be said that the advantage gained in this case is mutual ; the insect gets the honey and the flower gets fertilized. This of course is true, but exactly the same is true of human selection. 'The sheep gets the pasture and man gets the wool.

It seems impossible to explain on any other hypothesis than that of the natural selection and gradual accumulation of chance, favourable variations, those marvellous adaptations of animals which lead to protective resemblance and mimicry, for although we may admit that an organ which is actively employed may be modified by the efforts of an animal to maintain itself by the use of that organ, we can hardly extend the same principle to such passive features as colour and ornamentation, or the outgrowth of leaf-like dermal appendages and so forth. It may be questioned if, even with the aid of natural selection, we can fully account for all the wonderful phenomena of mimicry, for why, if it be an advantage to some species to adopt a common warning colour and band themselves together in synaposematic groups, should it be desirable for others to do just the reverse and split up into a number of differently coloured forms, each of which mimics some particular model? We can only say that we do not know all the factors of the environment, and that until we do our inability to solve the problem cannot be justly considered as an argument against the efficacy of natural selection.

It is, of course, extremely difficult, if not impossible, to obtain direct evidence of the action of natural selection in modifying species in a state of nature. Human life is all too brief to admit of our making very satisfactory observations concerning processes which extend perhaps over millions of years. Man has, however, in a comparatively short space of time, so changed the conditions of life for many of the lower animals as to lead, albeit unintentionally, to the more or less complete extermination of many species, and by studying these cases we may hope to arrive at sound conclusions as to what takes place in a state of nature. After all, mankind is a part of nature and we have no just reason for excluding his influence in our consideration of the factors which have brought about the present condition of the organic world.

It is well known that many of the birds of various remote 
islands have lost the power of flight. Such are the kiwi, the kakapo, the weka, the notornis and the already extinct gigantic moas of New Zealand; the dodo of Mauritius, and the solitaire of Rodriguez. Although belonging to several very distinct families of birds, including ratites, parrots, rails and pigeons, all the forms enumerated have undergone the same curious modification, resulting in the most extreme cases (the moas) in the complete loss of the wings, and in others in the reduction of those organs to a more or less vestigial condition. ${ }^{1}$

This convergence is clearly due to the similarity of the conditions under which these birds have had to live. One of the most characteristic features of oceanic islands is the absence from them of predaceous mammals, the natural enemies of birds, which have never been able to eross the great stretches of open ocean which separate such islands from the continental areas on which the Mammalia have been evolved. Birds, however, and even land birds, by virtue of their powers of flight, have been able to reach these islands at more or less frequent intervals and to establish themselves there. Finding abundance of food, which they could obtain near the ground, and finding themselves no longer under the necessity of constantly using their wings in order to escape from their enemies, some of these birds, though by no means all, gradually gave up flying and their wings underwent a slow process of degeneration in accordance with Lamarck's principle of disuse. No doubt such disuse, if continued only through a single lifetime, could scarcely produce a visible effect upon the next generation, but continued under the same conditions throughout thousands of generations it has brought about a permanent deterioration which can no longer be retrieved.

It is to be noted that this degeneration is the result of the removal of the organism, to a certain extent, from the struggle for existence. Natural selection can only act through the struggle for existence and upon those organs which are of value in the struggle. When the struggle ceases, natural selection ceases and degeneration sets in, for there is no longer any reason why a high standard of perfection should be maintained. All degrees of imperfection now have equal opportunities of propagating themselves. The inferior individuals are no longer weeded out, and the average condition of the species consequently deteriorates.

But observe what happens when a degenerate organism is

1 Compare Chapter XVII, Figs. 111, 112. 
once more exposed, by some unfortunate change. in its environment, to the old struggle from which it had escaped. This has actually taken place in the case of the flightless birds of New Zealand and other remote islands. With the advent of Europeans, predaceous mammals of many species-dogs, eats, rats, weasels, stoats and ferrets-have been let loose upon their helpless victims. These are once more exposed to a keen struggle for existence, while at the same time they have lost those very organs which are necessary to enable them to maintain themselves in that struggle, and natural selection, having regained her power, is rapidly exterminating them. It is not too much to say that in a few years' time there will be no flightless birds left in New Zealand except in special reserves where they are being protected by man.

It is highly instructive in this connection to contrast the condition of such a bird as the flightless parrot, or kakapo, with that of its relative the kea. The kakapo is a large, heavy bird of nocturnal habits and with practically no means of defence; it haunts the dense forest and is rarely seen except when hunted out by dogs. The kea, on the contrary, is one of the strongest fliers of the parrot tribe. It frequents high and more or less inaccessible mountain regions and since the advent of Europeans has learnt to make use of the sheep which they have introduced as an additional food supply. It is doubtful whether the utmost efforts of the sheep farmers, who annually expend large sums of money for the purpose, will ever enable them to exterminate the kea, and it is equally doubtful whether the efforts of the New Zealand Government to preserve the unique flightless birds will suffice to prevent the complete extermination of the kakapo within the next few years.

The aboriginal human population of remote islands has of course suffered not less than the lower animals from the invasion of their retreats by Europeans, although not always exclusively at the hands of the Europeans themselves. There are, perhaps, few more striking examples of the extermination of a primitive native race than that afforded by the rapid disappearance of the Moriori inhabitants of the Chatham Islands, some four hundred miles to the east of New Zealand, during the nineteenth century. ${ }^{1} \quad$ At the time of my visit to these islands, in

1 Compare Dendy, "The Chatham Islands : A Study in Biology "(Memoirs and Proceedings of the Manchester Literary and Philosophical Society, Vol. XLVI., 1902). 
January, 1901, there were only about a dozen pure-blooded individuals left; some of these were of great age, while the youngest was a lad of about 16, and they had all, I think, more or less completely adopted European manners and customs. Under these circumstances we are fortunate in possessing any reliable record of this interesting people, and that we do so is largely due to the energy and enthusiasm of Mr. Alexander Shand, who for more than thirty years lived amongst the Morioris and made a special study both of that race and of their Maori conquerors.

It appears from their language, customs and traditions, as well as from their physical characteristics, that the Morioris are closely related to the New Zealand Maoris. Their ignorance of the art of tattooing, and their very inferior artistic faculties in general, however, point to a very remote separation of the two races.

Like the Maoris they trace their origin to an unknown fatherland called Hawaiki, from which they must have emigrated to Chatham Island in canoes. In their new home they appear to have found the conditions of life remarkably easy, indeed, as the sequel shows, fatally so. With an abundant natural food supply of fruit, shell-fish, \&c., and with no enemies to contend with, they multiplied until the islands were thickly populated, while at the same time they doubtless became lazy and effeminate.

The discovery of the islands by the brig "Chatham," in 1790, may be said to have sealed the fate of the unfortunate Moriori, though it is doubtful whether any serious injury ensued until the advent of the whaling and sealing vessels in 1828 . 'These vessels brought with them many undesirable visitors, and probably were the means of introducing a disease which soon played havoc with the native race. On board some of the ships, moreover, were Maoris from New Zealand, who, on their return, painted such a glowing picture of the land of plenty, that a large number of their fellow-countrymen determined to emigrate to the islands en masse.

In order to effect this purpose they took possession of the brig "Rodney" at Port Nicholson, in New Zealand, about the beginning of November, 1835. They are said to have seized the crew and compelled the captain to transport them, about 900 in number, to their destination. At the time of the invasion the Morioris are supposed to have numbered about 2000, and had they 
attacked the new-comers on their first arrival, they might have exterminated them with little trouble and prolonged for an indefinite period the life of their own race. Unfortunately for themselves, however, they had lost the art of self-defence. Owing to the absence of competition they had, in this respect at any rate, undergone degeneration. Killing was actually forbidden by their laws, and peace had reigned too long and too securely to give place at once to war when the emergency arose.

Just as the flightless birds of New Zealand have more or less completely disappeared since the advent of carnivorous mammals, so the Morioris, their happy isolation once broken, fell an easy prey to the more virile Maoris. The latter proceeded to parcel out the conquered country amongst themselves, claiming not only the land but also the inhabitants thereof, many of whom were massacred under circumstances of unutterable atrocity, while the remnant were speedily reduced to the condition of slaves. Under the changed conditions which had suddenly arisen in their environment the Morioris were no longer fit to survive in the struggle for existence, they had become degenerate in a vital respect, and natural selection, as soon as opportunity arose, stepped in and eliminated them.

It would be easy to multiply illustrations of the great generalization that when removed from the struggle for existence all organisms tend to become degenerate, the organs or faculties which they no longer require atrophying and gradually disappearing for want of employment. We see this very clearly in the case of sedentary animals such as the ascidians (Figs. 129, 130). The young ascidian is a highly organized creature which swims actively about by means of a muscular tail, in the same way as the tadpole of a frog. Like the latter it has nervous system, notochord and sense organs-though the sense organs are of a type peculiar to itself-and is an undoubted chordate. It never, however, progresses further in organization, so as to attain the true vertebrate condition. On the contrary, it gives up its active life and withdraws as far as possible from the struggle for existence by fixing itself to some rock or seaweed and enveloping its entire body in a thick protective envelope, within which it undergoes extensive degeneration. The tail and notochord completely disappear, so do the sense organs, none of these being any longer required under the new conditions of life. The 
nervous system dwindles away to a mere ganglion, from which a few nerves come off, and the entire animal is reduced to the condition of a bag, with two openings through which the remaining organs obtain their food supply and communicate with the outside world by means of a stream of water maintained by ciliary action.

Still more conspicuous is the degeneration undergone by the great majority of parasites, whether animals or plants. Sacculina, for example, in the earlier stages of its existence, is an active crustacean which swims vigorously about by means of well developed appendages. It belongs to a group, the barnacles or cirripedes, which are notorious for sedentary habits and consequent degeneration in the adult condition. Saceulina, however, not content with a sedentary life, goes further down hill and becomes parasitic. It attacks crabs, and in the adult state is reduced to the condition of a large, irregularly shaped bag (Fig. 183) fixed to the under surface of the crab's abdomen by root-like pro-

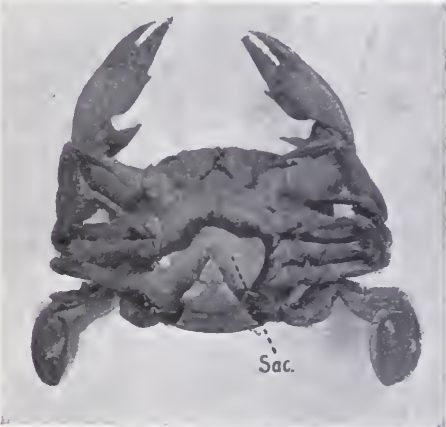

FIG. 183.-Lower surface of a Swimming Crab (Portunus depurator) with a Sacculina (Sac.) attached to it. (From a photograph.) cesses which penetrate the body of the host and extract nutriment therefrom. With the exception of these root-like processes, which are a special, cænogenetic development, adapted for nutrition under new conditions of life, the only organs which have not undergone degeneration are those of reproduction, for upon these depends the perpetuation of the race and upon these, therefore, natural selection is still able to retain her hold.

It is, indeed, a general rule amongst parasitic animals that the reproductive organs are largely developed and very complicated, for the conditions which have become necessary for the existence of these animals are so complex and highly specialized, while the chances of mating between different individuals for purposes 
of sexual reproduction are so remote, that the ova and spermatozoa have to be produced in vast numbers to compensate for the immense mortality which must take place amongst them and amongst the young animals to which they may give rise. Thus

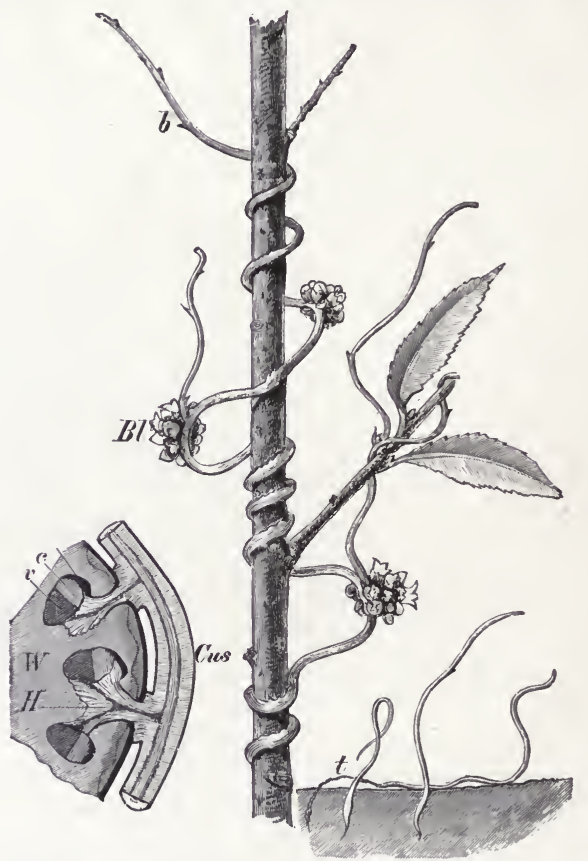

FIG. 184.-The Dodder, Cuscuta europaea. Part of a Plant parasitic on a Branch of Willow, with germinating Seedlings on the right and Section of Host and Parasite on the left. (From Strasburger.)

$b$, vestigial leaves; $B l$, flowers; $C u s$, stem of parasite in section; $H$, haustoria of parasite in section; $W$, stem of host in section, with vascular bundles $(v, c) ; t$, seedlings.

one of the most frequent results, or at any rate concomitants, of parasitism, and one which is well exemplified in the case of Sacculina, is hermaphroditism, which affords many more chances for the fertilization of the eggs than the unisexual condition.

We meet with precisely analogous phenomena in the case of many parasitic plants. In the dodder (Fig. 184) the leaves and 
roots have disappeared almost completely and no chlorophyll is produced, but special nutritive organs, the sucker-like haustoria, are developed on the slender, twining stems, and serve to extract the necessary food from the host plant. The flowers, however, upon which the perpetuation of the race depends, still remain in a well developed condition.

Both Sacculina and the dodder have lost the power of independent existence, and if, for any reason, they were to find themselves suddenly confined to an environment where there were no suitable hosts, -their races would inevitably become extinet. Nature would treat them just as she is treating the wingless birds, and make them pay the penalty for the degeneration which they have undergone.

It has sometimes been pointed out as an objection to the theory of natural selection that it cannot account for the first origin of favourable variations. The theory takes variations for granted and assumes that some will be favourable and some not, that the former will be fostered and accumulated from generation to generation and the latter ruthlessly eliminated. It is further alleged that variations are usually so slight at their first appearance that they can have no selective value, and that something is wanted to account for the increase of such variations along apparently definite lines of utility. The theory also takes the inheritance of variations for granted, and many people, as we have seen, consider nowadays that this is not altogether a justifiable proceeding, that while some variations undoubtedly are inherited, others, and amongst them many which would be likely to be of the greatest value to the organism, are not.

We have, then, to go much deeper than the idea of natural selection before we can reach a satisfactory working hypothesis as to the manner in which organic evolution has taken place. The problems of variation and heredity have already been dealt with in earlier chapters, and it will be unnecessary to discuss the matter now at great length, but there are certain points which we must recapitulate in this connection.

We have seen that somatogenic or bodily variations in the individual are undoubtedly brought about by the direct action of the environment and by the use and disuse of organs. We have also seen that blastogenic variations, which originate in the germ plasm, may likewise be brought about by the action of the 
environment (as in the case of the potato beetle as demonstrated by Tower), but that they probably also arise from the mingling of different streams of ancestral tendencies in the process of amphimixis or conjugation of gametes, and possibly in yet other ways with which we are not acquainted.

Of course, natural selection can only influence a species through variations which are capable of being inherited, and it is, as everyone knows, urged by many modern writers that somatogenic variations, due either to the direct action of the environment or to the use and disuse of parts, cannot be inherited and therefore have no significance in evolution, and that natural selection must content herself with such fortuitous and non-adaptive variations as may happen to arise in the germ plasm. This indeed seems an extreme view, and it is just here that the split between the extreme selectionists, who have gone far beyond Charles Darwin in this matter, and the followers of Lamarck arises. The curious thing about the controversy is that there is no inherent incompatibility between the views of the two schools. The theory of the inheritance, to a limited extent, of acquired characters, indeed, appears to be just what is necessary to supply the deficiencies of that of natural selection.

To say that acquired characters cannot be inherited because we cannot see them being inherited in our own brief lifetimes ${ }^{1}$ is like saying that a glacier does not move because we do not see it or feel it moving as we walk over it. I have endeavoured to show in an earlier chapter that it is not difficult to imagine a mechanism by which somatogenic characters may gradually be converted into blastogenic ones, and if this is in any way possible there is no reason why we should deny the possibility of their inheritance. No one, however, would be rash enough to suppose that all that an animal or plant acquires in its individual lifetime is transmitted to its heirs. Nature imposes a heavy death duty and takes away by far the greater part of the capital which has been accumulated by each individual. We may suppose, however, that a fraction remains, however unrecognizable by our limited powers, and that these fractions, accumulating under the same influences throughout thousands of generations, ultimately confer upon the organism as a birthright that adaptation which is essential to its existence.

1 As a matter of fact, it appears from recent experiments that in some cases we can see them being inherited (vide p. 182). 
Even the individual can do much in its own lifetime to adapt itself to its environment, and when the residua of all the individual adaptations are summed up by inheritance the result is such that we may well wonder how it can have been produced. Throughout the whole process, of course, natural selection must help by constantly weeding out inferiority, but it is probably the direct influence of the environment, including the use and disuse of organs in response to that influence, that is in most cases the determining factor in bringing about adaptation.

It may well be, however, that there are also cases in which natural selection alone, acting through the occurrence of purely fortuitous variations, has, in the struggle for existence, been sufficient to produce marvellous adaptations. This may have been the case with protective resemblance and mimicry in form and colour, and with the adaptation of flowers for fertilization by insects, in all of which it is difficult to see how the direct action of the environment or the use and disuse of organs could bring about adaptive modifications. But the fact that natural selection alone appears to have been sufficient in some cases must not prevent us from admitting the action of other factors in other cases. The fact that some carriages are pulled by motors affords no justification for asserting that other carriages may not be pulled by horses, or that the same carriage may not at one time be pulled by a motor and at another by a horse, or even by both together. Many factors must have co-operated to bring about such a marvellously complex result as the present condition of the organic world, and no sufficient reason has yet been shown for denying. ourselves the assistance of "Lamarckian" factors in our endeavours to discover the processes through which this result has come about. ${ }^{1}$

We may now turn our attention to a group of cases which certainly appear to support the view that the factors at work in determining any particular line of evolution are more complex than might at first sight be supposed. It is a fact well known to palæontologists that many widely separated groups of the animal kingdom have, during the course of their evolution, and especially towards the end of that course, shown a strongly marked tendency to enormous increase in size. We see this in the extinct eurypterids (Fig. 137), giants amongst the

1 "To insist on ascribing complex results to single causes is the well-known vice of narrow and untrained minds" (Morley's "Life of Gladstone," Vol. II., p. 68). 
arthropods; in the huge labyrinthodont amphibians; in many groups of reptiles of the Secondary period, some of which attained a length of 80 feet or more, and amongst mammals in the extinct Tinoceras (Fig. 150) and the still surviving elephants and whales. Comparative anatomists are familiar with similar phenomena exhibited by individual organs, such as the extraordinary development of horns and spines in many of the extinct reptiles referred to (Fig. 145), the immense tusks of

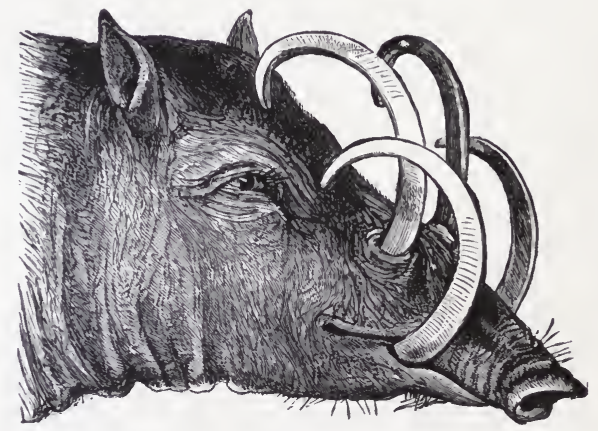

Fig. 185.-Head of Babirusa alfurus. (From Flower and Lyddeker's "Mammals Living and Extinct.")

the babirusa (Fig. 185), and the gigantic and grotesque beak and "helmet" of the hornbill (Fig. 186).

The exuberant development of some organs of this kind may possibly be attributed to the action of sexual selection, and indeed our daily experience of our own species seems to warrant us in believing that there is no limit to the grotesque results which may ensue from the unrestricted exercise of the rsthetic faculties of either sex, but it seems hardly reasonable to attempt to explain all such bizarre and monstrous productions in this manner.

In all the cases cited, and in many others which could be adduced, either the entire body or some particular organ appears to have acquired some sort of momentum, by virtue of which it continues to grow far beyond the original limits of utility, although perhaps in some cases a new use may be found which will assist the species in maintaining itself in the struggle for existence. 
An enormous increase of mere bodily size, however, seems in the long run to be always fatal to the race, whose place will be taken by smaller and presumably more active forms. The gigantic amphibians are all extinct, so are the really gigantic reptiles, and of the gigantic mammals only a couple of species of elephants

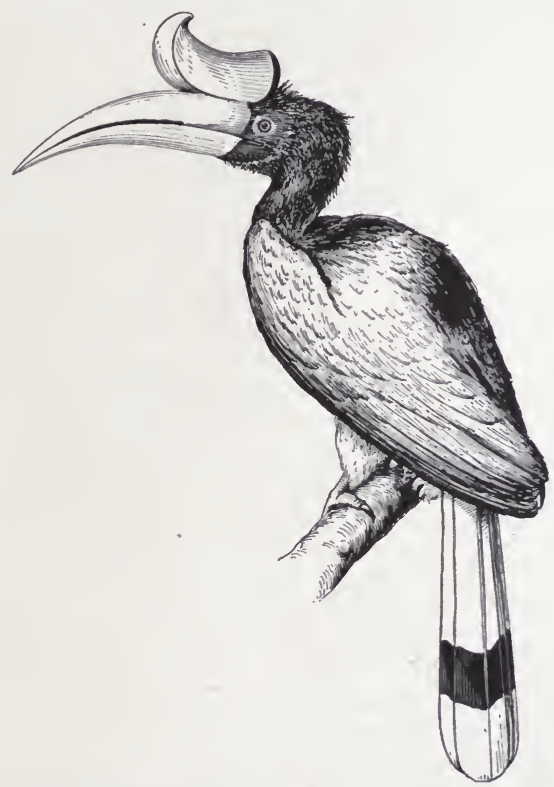

Frg. 186.-A Hornbill, Buceros rhinoceros, from North-West Borneo. (Drawn from a specimen in the British Museum, Natural History.)

and a few whales survive, all of which are being rapidly exterminated in competition with man.

There is perhaps some justification in recent developments of physiological science for the belief that a race of animals may acquire a momentum of the kind referred to; that some brake is normally applied to the growth of organisms and organs and that sometimes this brake is removed, leaving the organism to rush onwards to destruction like a car running away down hill.

Many modern physiologists hold the view that the growth of 
the different parts of the animal body is controlled by internal secretions, or hormones, the products of various glands. Thus we know that disease of the pituitary body in man may lead to acromegaly, one of the symptoms of which is great enlargement of certain parts. The most dreadful of all the diseases to which human beings are subject, cancer, is essentially due to an unrestrained multiplication of cells, and consequent abnormal growth of tissue, which may very possibly. be correlated with the extent to which some specific controlling secretion is produced in the body. In short, we are justified in supposing that in the individual growth may be normally inhibited or checked by specific secretions, and that in the absence of these it may continue far beyond the ordinary limits.

It is difficult to see any good reason why we should not apply this principle to the race as well as to the individual, and, paradoxical as it may appear, it even seems possible to explain both the growth of the organism as a whole and that of its various organs, beyond the limits of utility, as an indirect result of natural selection.

When a useful organ, such as the tusk of a wild boar, is first beginning to develop, or to take on some new function for the execution of which an increase in size will be advantageous, natural selection will favour those individuals in which it grows most rapidly and attains the largest size in the individual lifetime. If growth is normally checked and controlled by some specific secretion, or hormone, natural selection will favour those individuals in which the glands which produce this secretion are least developed, or at any rate least active. The process being repeated from generation to generation these glands (whatever may be their nature, and we use the term "gland " for any cell or group of cells which produces a specific secretion, whether recognizable as a distinct organ or not) may ultimately be eliminated, or at any rate cease altogether to produce the particular hormone in question. Moreover, this elimination may take place long before the organ whose growth is being favoured by natural selection has reached the optimum size. When it has reached this optimum it is certainly desirable that it should grow no larger, but is there now any means by which further growth can be checked? The inhibiting hormone is no longer produced; the brake has been removed, and further growth may be supposed to take place irrespective of utility, until, when 
the size of the organ gets too great to be any longer compatible with the well-leing of the race, natural selection again steps in and eliminates the race. The same argument of course applies to the size of the body as a whole as well as to that of its constituent parts.

It may be thought that many of the bizarre and almost monstrous characters under discussion, such, for example, as some of the excrescences of the dermal armature in extinct reptiles (Fig. 145), can never have had any value as adaptations, and that therefore natural selection could never have encouraged them to increase so much in size as to get beyond her control. Here, however, the principle of correlation comes in. Just as many totally different organs are affected by disease of the pituitary body, so the removal of the gland which controlled the development of some undoubtedly useful organ, such as a frontal horn, might at the same time permit the growth of all sorts of excrescences which have no adaptive significance.

Thus it appears not impossible that, the normal checks to growth being removed along certain lines by the action of natural selection, a definite direction might be given to the course of evolution, which the organism would continue to follow irrespective both of natural selection and of the principle of use and disuse. ${ }^{1}$

In the present state of our knowledge, however, the above suggestions can only be regarded as tentative. They are doubtless open to much criticism, and it is unfortunately impossible to subject them to the crucial test of experiment.

1 I have discussed this question at somewhat greater length in a paper read before the British Association for the Advancement of Science (vide Report of the Portsmouth Meeting, 1911). 


\section{CHAPTER XXVII}

Artificial selection-Continuous and single selection-The mutation theory of the origin of species - Mutual adaptation - Unit characters Isolation - Physiological selection - Non-adaptive characters - The evolution of man.

IT has long been recognized that much light may be thrown upon the problem of the origin of species by the careful study of the methods which mankind has adopted for the improvement of the various races of cultivated plants and domesticated animals. Many such races have been so greatly modified that, did they occur in what is commonly called a state of nature, we should be obliged to regard them as distinct species. The history of some of these is lost in antiquity and we have no positive knowledge of the methods by which the improvement of wild species was first effected. Wo may assume, however, with some degree of confidence, that the earliest breeders and cultivators would select for cultivation and propagation those individuals which offered them the most valuable qualities, and that they would reject such as exhibited marked signs of inferiority. This process, repeated from generation to generation through thousands of years, and aided in each generation by the direct effects of cultivation, could not fail to bring about conspicuous results. For an account of what has been effected in this manner the student should consult Charles Darwin's classical work on the "Variation of Animals and Plants under Domestication."

The almost unconscious efforts of our ancestors have given place in modern times to deliberate and systematic attempts to discover the principles upon which the improvement of cultivated races, both of plants and animals, should be based.

Perhaps no species of plants have been more improved by man than the various cereals upon which he relies so largely for his food supply. Professor de Vries, in his interesting book on "Plant-Breeding," 1 describes how such improvement has been

1 Kegan Paul, Trench, Trübner \& Co., London, 1907. 
effected in recent times. In the first place much care and thought have been devoted to carrying out experiments in accordance with the principle of continuous selection :-

"The general custom [in Germany] was to start such experiments from the best local or improved varieties by an initial choice of a certain number of typical heads. Such a group of selected plants was called the élite, and this élite had to be ameliorated according to the prevailing demands or even simply in accordance with some ideal model. Year after year, the best ears of the élite group were chosen for the continuance of the strain or family, and slowly, but gradually, its qualities were seen to improve in the desired direction. After some years, such a family might become decidedly better than the variety from which it had been derived. Then its yearly harvest would be divided into two parts, after having been sufficiently purified by the rejection of accidental ears of minor worth. 'I'he best ears were carefully sought out and laid aside for the continuance of the élite strain, but the remainder were sown on a distant field in order to be multiplied as fast as possible. By this means, after a multiplication during two or three generations, its product could be used as seed grain for the farm or sold to others for the same purpose. Each year the élite would, of course, give a new and better harvest which could be multiplied and sold in the same manner." 1

By this method improvement may undoubtedly be effected, but the selection has to be constantly repeated, otherwise the improved strain rapidly deteriorates again. Indeed it may be questioned whether it is possible in this way to effect any permanent improvement, at any rate in the case of cereals. One reason for this appears to be that we are dealing all the time, not with a single pure race, but with a mixture of distinct races. We must also remember that many of the characters which it is desired to perpetuate and increase may be the direct result of the cultural methods employed, and, as we have already seen, we cannot expect such causes to produce visibly heritable effects in the course of a few generations, whatever they might do in the long run.

We have had occasion to point out in an earlier chapter that, according to Professor de Vries, new species arise, in a state of nature, not by the accumulation in particular directions of small, fluctuating variations, but by the sudden appearance of those 
more conspicuous variations known as mutations. De Vries points out that many of the so-called Linnean species, such as Draba verna, are in reality made up of a large number of "elementary species" which have arisen in this manner, and certain results which have been obtained in experiments upon the improvement of cereals appear at first sight to afford considerable support to these views.

It has long been known that an ordinary field of wheat contains a larger or smaller number of "types," " mutations," or " elementary species," which can be recognized by the experienced eye, and it has been shown that if a single plant of one of these types be isolated it will produce offspring like itself and continue to breed true for an indefinite number of generations. Of course it is necessary that there should be no crossing with other types, but this is easily avoided, for, although accidental crosses may occur, the cereals, with the exception of rye, are usually self-fertilizing. Upon this knowledge is founded the method of single as opposed to continuous selection, a single selection of a suitable type being enough to establish the desired strain.

One of the first to make use of the method of single selections was Patrick Shirreff :-

" His first discovery was made in the year 1819. He observed a plant of wheat which surpassed its neighbors by its high degree of branching. It yielded 63 ears with about 2500 kernels. He saved the seeds, sowed them on a separate field and at considerable distances apart so as to induce in all the plants the same rich branching. He contrived to multiply it so rapidly that it took only two generations to get seed enough to bring it advantageously into the trade. He gave it the name of Mungoswell's wheat, and it soon became one of the most profitable varieties of Scotland. It has found its way into England and into France, where it is still considered one of the best sorts of wheat." 1

The same method has been subjected to severe tests and placed upon a thoroughly scientific footing at the Swedish Agricultural Station of Svalöf.

We have seen, in Chapter XIV, that hybridization may occasionally give rise to permanent races or strains exhibiting new combinations of characters, and that this takes place in 
accordance with Mendelian principles. It cannot be doubted that hybridization occurs occasionally in cultivated cereals, and Professor de Vries is of opinion that the occurrence of the different types or mutations is often the result of hybridization at various periods in the history of the race :-

"Experience, however, shows that in ordinary fields almost all possible combinations may be met with, and it is to be presumed that at least the greater number of them are due to crosses in previous and, perhaps, in long-forgotten years." 1

Some of these combinations, as might be expected, are not stable but split up into a number of varieties in the next generation, but also :-

"We may conclude that some, and perhaps many, of the types which may be selected and isolated in the fields and which prove to be constant races must be of hybrid origin." ${ }^{2}$

De Vries maintains that in the case of the cereals so many of these "types" now lie ready to our hand that all we have to do is to pick out those which we require and cultivate them in isolation from each other and from the remainder. ${ }^{3}$ Professor Biffen has shown, however, as we have already pointed out in Chapter XIV, that it is possible by intelligent artificial hybridization to produce yet other stable combinations or hybrids which may surpass in value any which have accidentally arisen in the past.

It appears, then, that many at any rate of the so-called mutations or types amongst cereals are due to hybridization. How far this applies to mutations in general it is quite impossible to decide. That it is not always so, however, appears to be proved by the occurrence of such mutations or sports as hexadactylism, which are known to be inherited and which cannot have arisen in this way.

De Vries tells us in another work that:-

"According to the theory of mutation species have not arisen gradually as the result of selection operating for hundreds, or thousands, of years but discontinuously by sudden, however small, changes. In contradistinction to fluctuating variations

1 De Vries, op. cit., p. 80.

2 Ibid., loc. cit.

81 bid., p. 50. It seems strange, considering that de Vries admits that many at any rate of the "types" have probably arisen by hybridization in the first instance, that he should attribute so little value to artificial hybridization as a means of improvement. 
which are merely of a plus or minus character the changes which we call mutations are given off in almost every manner of new direction. They only appear from time to time, their periodicity being probably due to perfectly definite but hitherto undiscovered causes.

"The theory of the inheritance of acquired characters comes under the heading of fluctuations. Acquired characters have nothing to do with the origin of species. Nor ean the theory of descent be applied to the solution of social problems." 1

There is here no suggestion of a hybrid origin for the mutations in question. If, however, as seems probable, a large proportion of so-called mutations are really the result of hybridization, and if, as we showed in Chapter XIV, hybrids tend to be automatically eliminated in a state of nature-though of course there is nothing to prevent a constant hybrid from being preserved if it happens to possess characters peculiarly favourable to its own existence-it does not seem likely that such mutations can have played any very great part in organic evolution. In any case there is no need to suppose that the theories of mutation and natural selection are mutually exclusive, for, however new characters may arise, they must be subject to the action of natural selection in the struggle for existence.

Professor de Vries' objection to small, fluctuating variations as the material upon which natural selection operates in the, modification of species appears to be based upon the view that such characters are acquired in the lifetime of the individual and cannot be inherited. If, however, we admit that a somatogenic character may, in the course of many generations and under the continued influence of the same conditions which originally called it forth, become converted into a blastogenic character, this difficulty entirely disappears.

It is extremely hard to believe that mutations, which, apart from the occurrence of hybridization, seem to occur very rarely and at long intervals, can have afforded sufficient opportunity for the production of all the marvellous adaptations which exist in nature. Take, for example, the mutual adaptations which we see between the length of the nectary in certain flowers and the length of the proboscis in the insects which fertilize them. We cannot suppose that either the elongated nectary or the elongated proboscis arose by sudden mutations, for unless these mutations

1 "The Mutation Theory." English Trans, Vol. I., p. 213. 
took place simultaneously in flower and insect, and in the same locality, and in a sufficient number of each, a supposition which, to say the least of it, is wildly improbable, the delicate correlation between the two would be thrown out of gear and the individuals exhibiting the mutations would be eliminated by natural selection because they were no longer sufficiently well adapted to the very special conditions of their environment.

We can only believe that the increase in length of nectary and proboscis took place so slowly that their reciprocal adaptation was never upset. A slight increase in the length of the nectary obliged the insect to poke further into the flower for the honey and thus increased the chances of fertilization. A slight increase in the length of the proboscis enabled the insect to get more honey and thus gave it a better chance of existence. After perhaps many thousands of generations, under the influence of natural selection, combined in the case of the insect with the effects of use and disuse, the present enormous lengths of proboseis and nectary have been attained, and no one doubts the fact that they have become blastogenic characters.

The same argument applies to all accurate adaptations to special conditions of the environment. How can we explain the facts of protective resemblance and mimicry except as due to the accumulation under the influence of natural selection of what Charles Darwin called slow successive variations? How, again, can the theory of mutation be applied to such cases as that of the flightless birds on oceanic islands? Who can doubt that the reduction of the wings and the loss of the power of flight has been brought about slowly and gradually as a result of disuse? and at the same time who would venture to argue that the flightless birds are not specifically distinct from their actively flying ancestors? If it be urged that mutations may be so small as to be almost imperceptible, then we must ask how do they differ from fluctuating variations ? and if we are told that they occur very rarely and do not fluctuate, that very answer is sufficient to show that they can hardly have given rise to adaptive modifications.

De Vries' theory of the origin of species by mutation is supposed to harmonize with the Mendelian principle of unit characters, but we have to ask ourselves, how do new unit characters arise in the first instance? It seems at least as probable that they arise by the gradual accumulation of slight 
fluctuating variations under the control of natural selection as that they originate in any other way that can be suggested in the present state of our knowledge; and even if these variations are at first purely somatogenic, we may suppose that in the course of many generations they gradually exert a cumulative influence upon the germ plasm until the latter, so to speak, topples over into some new position of equilibrium and a new unit character arises. We cannot, however, now add to what we have already said on this subject when dealing. with the theory of heredity.

One of the most important factors in bringing about divergent evolution is undoubtedly isolation. However a new character may have arisen it is liable to be swamped by the crossing of the individuals which possess it with others which do not possess it unless by some means or other the two groups are prevented from interbreeding. We cannot fail to see the importance of this principle when we study the fauna and flora of remote islands, and their relationships to those of the nearest continental areas or of other islands.

Take, for example, the case of the Chatham Islanids, which, as we have already seen, lie some 400 miles to the east of New Zealand. There can be very little doubt that these islands were formerly connected with the New Zealand mainland, and this connection probably continued into Pleistocene times, when a great depression took place which caused the two to be separated by a wide tract of ocean. All who have studied the question are agreed that the fauna and flora of the Chatham Islands are simply isolated detachments of those of New Zealand. Many species, especially of the plants, are identical with New Zealand species, but many others, though closely related to those of New Zealand, are considered by systematists to be specifically distinct, and they occur nowhere else in the world.

We have here an excellent illustration of the effects of geographical isolation, which we shall be able to appreciate better; perhaps, if we confine our attention to a few typical cases. The common New Zealand wood pigeon, Carpophaga (Hemiphaga) nove-zealandia, is represented on the Chathams by a species known as Carpophaga (Hemiphaga) chathamensis, differing but slightly from its new genus congener, and the New Zealand 
lizard, Lygosoma moco, is represented on Pitt Island (one of the Chathams) by a very similar form described by Mr. Boulenger under the name Lygosoma dendyi. The remarkable New Zealand lance-woods (Pseudopanax crassifolium and P. ferox) are represented on Chatham Island by the closely related Pseudopanax chathamicum, and the Chatham Island ribbon-wood also differs slightly from the common New Zealand species (Plagianthus betulinus).

The explanation of these differences is that the two portions into which each of the species mentioned became divided when the Chatham Islands were separated from New Zealand have diverged from one another and followed somewhat different lines of evolution. Owing, perhaps, to slightly different conditions of the environment, or to other causes which it is impossible to specify, one or both has become modified to a greater or less extent in its own particular direction and, owing to the geographical isolation, there has been no interbreeding between the two sections to keep them both in the same average condition.

The principle of isolation fully explains why the fauna and flora of oceanic islands in general are made up almost entirely of peculiar species found nowhere else in the world. The ancestors of these species were originally derived from some very distant, probably continental area, and their descendants have had few if any opportunities of interbreeding with the parent species, from which they have gradually diverged further and further under their new conditions of life.

Certain writers, such as Mr. Gulick and Dr. Romanes, have maintained that the mere separation of a species into two or more sections which are prevented from interbreeding would suffice to bring about divergent evolution, irrespective of whether or not the separate sections were exposed to different environmental conditions. It would probably be impossible to divide a species into two sections whose average qualities are identical, and :-

"No matter how infinitesimally small the difference may be between the average qualities of an isolated section of a species compared with the average qualities of the rest of that species, if the isolation continues sufficiently long. differentiation of specific type is necessarily bound to ensue." 1

1 Romanes, "Darwin and after Darwin," Vol. III. "Isolation and Physiological Selection," p. 13. 
If isolation is necessary for the establishment of new species by divergent evolution, why, it may be asked, do we find closely related species, which we must suppose to be descended from common ancestors at no very distant period, actually inhabiting the same areas at the present time? The answer is that there are other means by which groups of organisms can be prevented from interbreeding besides geographical isolation.

It used to be supposed that one of the best tests of the specific distinctness of any two forms was their incapacity for breeding together and producing fertile offspring. 1 The mule, it is true, is the offspring of parents belonging to two distinct species, the ass and the horse, but the mule is almost always sterile, and most well characterized species ${ }^{2}$ are incapable of breeding together at all. This fact has been regarded by many people as a most serious difficulty in the way of comparing the origin of species by natural selection with the results produced amongst domesticated plants and animals by artificial selection, for the products of artificial selection, however much they may differ from one another, if they have been derived from the same parent species will remain capable of breeding together with perfect fertility.

The solution of this difficulty is to be found in the theory of "Physiological Selection," which we owe to Mr. Gulick, Dr. Romanes and others. These writers point out that amongst the endless variations to which plants and animals are subject will be variations in the reproductive system, by which certain individuals will be rendered infertile when crossed with others of the same species, while remaining fertile with individuals which have varied in the same manner as themselves. In this way a species may be as effectively divided into two sections as by any geographical barrier, and under these circumstances divergent evolution may be expected to take place. According to this view the mutual sterility which, to a greater or less extent, undoubtedly does characterize distinct species in a state of nature, is the cause and not the result of their distinctness, and cannot be regarded as a reason for supposing that there is any essential difference between the processes of artificial and natural selection. In artificial selection it is merely another kind of

1 Although Lamarck pointed out a ccntury ago that this is really no criterion of specific distinction. The idea that it is so is clearly expressed by Buffon (" Histoire Naturelle," Tom. VI, 1756 , p. 16).

2 At any rate amongst the higher animals. 
isolation that has been employed to prevent the swamping effects of intercrossing; but it is an isolation that may be broken through at any moment, and if all the different varieties of some domestic plant or animal were turned loose to struggle for existence and interbreed with one another and with the parent species in a state of nature, they would probably in most cases very soon cease to have any separate existence.

The theory of natural selection, combined with that of the gradual inheritance of the effects of use and disuse and of other modifications brought about by the long-continued influence of the environment, affords a satisfactory explanation of the evolution of adaptive characters. Many if not all organisms, however, exhibit characters to which we can assign no adaptive value, which do not seem to be of any particular use to the organism in the struggle for existence, or which apparently might, so far as utility is concerned, be equally well replaced by any one of a number of alternative characters.

Amongst the microscopic Protozoa species are frequently distinguished from one another by minute differences in the form or ornamentation of the skeleton (compare Figs. 3 and 4). Different species of the genus Lagena (Fig. 4), ${ }^{1}$ amongst the Foraminifera, for example, exhibit different sculptured patterns upon their flask-shaped calcareous shells. Are we to suppose that it is of any consequence to the gelatinous, Amoeba-like inhabitant of the flask whether its shell be ornamented in one way rather than in another? Does one pattern help a unicellular foraminiferan or radiolarian more than another in the struggle for existence? The same argument applies to the extremely minute siliceous flesh-spicules or microscleres which occur scattered without order through the ground substance of many sponges, and the form of which is regarded by those who have studied the question as by far the most reliable guide, not only in the recognition of species, but also in the grouping of these species in genera and families. Take, for example, the wonderful chelæ (Fig. 187), characteristic of the family Desmacidonidæ. Different genera and species are distinguished by differences in the size, shape and number of

1 The two figures below the centre figure and two in the bottom right hand corner represent four species of Lagena. 
the teeth of these microscopic and apparently useless organsuseless at any rate so far as their generic and specific characters are concerned, for what can it matter to the sponge whether the number of the teeth be three or more or less, or whether the teeth at the two ends of the spicule be equal or unequal? Yet, in the course of evolution, such characters as these have become more

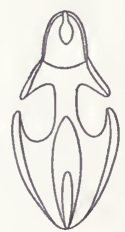

A.

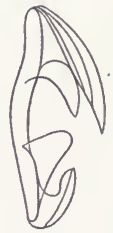

A

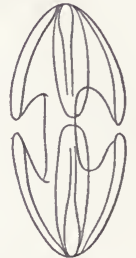

B.

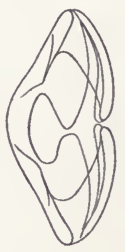

$\mathbf{B}^{\prime}$

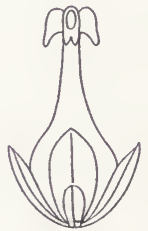

c.

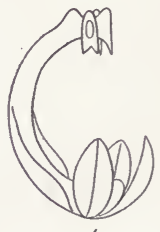

c.

Fig. 187.-Siliceous Spicules (Chelar) of Sponges. (After Ridley and Dendy, in "Challenger" Report.)

A. A', front and side views of chela of Esperella lapiduformis, $\times 360$.

B. B' $^{\prime}$, front and side views of chela of Esperiopsis pulchella, $\times 284$.

C. C', front and side views of chela of Cladorhiza (?) tridentata, $\times 360$.

or less fixed and constant and they are evidently handed down from generation to generation by the ordinary process of heredity. ${ }^{1}$

We pointed out in an earlier chapter that the external form of the entire sponge is, in some cases at any rate, explicable as an adaptation to peculiar conditions of the environment. We saw this is the case of the curious "Crinorhiza" form (Fig. 168) which prevents the sponge from sinking into the soft mud or ooze on which it rests. Let us glance for a moment, however, at another deep sea sponge, Esperiopsis challengeri, dredged up by the "Challenger" Expedition from a depth of

1 The reader should refer back to Fig. 88 fur other forms of sponge spicules. 
825 fathoms in the Malay Archipelago. In some respects this is the most remarkable sponge that has ever been discovered. Its form (Fig. 188) is absolutely unique and resembles rather that of some graceful plant than those of other sponges. It belongs to a group of sponges whose members occur mostly in much shallower water and are by no means distinguished by beauty or symmetry of shape. In spiculation, moreover, and other minute anatomical features, it exhibits no striking peculiarities; indeed, so closely does it agree with more ordinary species of Esperiopsis that it has not as yet been considered necessary to separate it generically. How then can we account for this wonderful form? Can we say that it is an adaptation to any special conditions of the environment? It hardly seems likely that this is the case, for we know that the relatives of this sponge get on well enough with all sorts of other forms, for the most part more or less irregular and ill-defined, and we know of nothing in the conditions under which it lives to make such a unique and beautiful form especially advantageous. The "Challenger" obtained no less than thirteen specimens of this sponge at the same place, and the form appears to be quite constant. No doubt the undisturbed condition of the sea at great depths favours symmetry of growth in sessile organisms like sponges, but why this particular and absolutely unique shape, so different from anything else that has ever been met with?

These are questions which we cannot

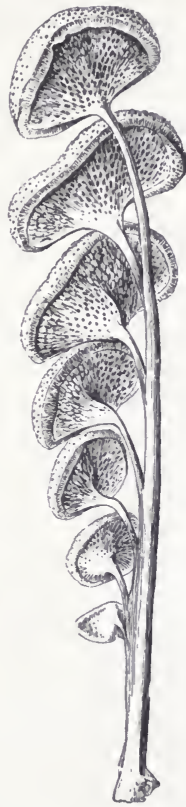

FIG. 188.-Esperiopsis challengeri, $\times \frac{1}{2}$. (After Ridley and Dendy, in "Challenger", Report.) answer, and it must suffice to point out that such cases can hardly be explained by the theory of natural selection. Many factors must combine in determining the course of evolution of any particular organism, and some of the characters which result from their interaction may perhaps have no more direct relation to the necessities of the organism in the struggle for existence than has the colour of a pebble to its continued existence on the sea-shore. 
It may even be questioned whether any large proportion of specific characters can have arisen through the action of natural selection. The characters by which we are accustomed to separate one species from another-such as minute differences in size, shape and colour-are usually so slight that we are hardly justified in attributing to them any adaptive value. They may, however, form the starting points from which, under the influence of natural selection and use and disuse, adaptive characters may subsequently arise.

The application of the principles of organic evolution to the problem of the origin and progress of the human race cannot be adequately dealt with in the present volume, while at the same time we cannot altogether ignore it, for not only is it in man himself that we find the most remarkable illustration of what has been accomplished by evolution, but the future progress of mankind must depend in large measure upon the correct understanding of the principles in question.

Buffon, more than a century ago, pointed out the close resemblance in anatomical structure between man and the higher apes, and it is clear that both he and Lamarck were only prevented by religious scruples from definitely maintaining the origin of the human species from ape-like ancestors, a view which at the present time is universally accepted amongst scientific men. This reluctance to admit the obviously close relationship of the human species with the apes was one of the evil results of the intellectual dishonesty and obscurantism of the middle ages. If we go back to the days of Carthage, we find that the explorer Hanno did not hesitate to speak of the "gorillas" 1 which he met with in Africa as hairy men and women of the woods, and although this was doubtless going too far in the opposite direction, it shows not only that he recognized the relationship but also that he approached the question with a mind entirely free from prejudice.

Man is one of the latest products of organic evolution, and his appearance upon the scene possibly does not date further back than Pliocene times. It is said that flint flakes of human workmanship have been discovered in early Pliocene deposits of Burmah,

1 Probably really chimpanzees. 
but perhaps the earliest actually human fossil so far known is a lower jaw of very massive form which was found in a deposit of late Pliocene or early Pleistocene age near Heidelberg, and described by Schoetensack in 1908 under the name Homo heidelbergensis. This name implies that in the opinion of its author the fossil man of Heidelberg was generically, but not specifically, identical with the human beings which now exist. The question of the distinction of species in the genus Homo, however, is, as in most other genera, a very difficult one, and opinions are divided as to whether only one or several species should be recognized amongst the existing races of mankind.

So close is the anatomical agreement between the genus Homo and the higher apes that there is little room for connecting links between them, the difficulty being rather to find any definite characters by which they can be separated than to discover reasons for bringing them together. Nevertheless, the gap, small as it is, has been filled by the discovery, by Dr. Dubois in 1894, of the remains of a semi-human, ape-like creature, to which the name Pithecanthropus erectus has been given. These remains were found in Pliocene strata in the island of Java and consist of a portion of a cranium, a thigh bone and two molar teeth. ${ }^{1}$

In deposits of Pleistocene age undoubtedly human remains become fairly abundant, and are found associated with the bones of other mammals, of which many, such as the mammoth, the cave bear and the woolly rhinoceros, are now extinet.

The chief factors which contributed towards the gradual transformation of ape-like into human creatures were doubtless the same as those which have operated in the evolution of other branches of the animal kingdom, namely, the efforts which the ancestral forms were obliged to make in order to maintain themselves in the struggle for existence and the natural selection of favourable variations. In no group of the animal kingdom do we see better illustrated the importance of Lamarck's principle of the effect of changed habits upon bodily organization. ${ }^{2}$

The anthropoid ancestors of man were undoubtedly, like

1 Some authorities regard these remains as truly human. The difference of opinion is itself very instructive.

2 Darwin, notwithstanding what he says in the sixth edition of the "Origin of Species" about the effects of use and disuse (quoted on p. 392), denies, in the "Deseent of Man" (Ed. 2, p. 619), that man has risell through his own exertions. I can see no reason for such a pessimistic view. 
existing Simiidæ, arboreal in habit. Their limbs, whose primitive pentadactyl structure indicates their origin from some little-specialized mammalian type, had ceased to be used exclusively as organs of locomotion on the ground and become adapted for climbing trees. Hence the opposable toe and thumb, which enabled their possessor to obtain a firm grasp of the branches. In the existing apes hand and foot are very similar, and both, as regards function, partake as much of the nature of hand as of foot, whence the name "Quadrumana" applied to the group by the older naturalists. In many of the lower apes or monkeys a long prehensile tail, which can be twisted round the branches, is of great assistance to the animals in their arboreal habits, but in the higher forms, such as the chimpanzee, the gorilla and the orang utan, the tail has already disappeared.

These tailless forms are still mainly arboreal, but when they have occasion to come to the ground they assume a semi-erect posture in locomotion. In walking they usually put their hands to the ground, but generally resting upon the backs of the fingers, instead of upon the palms as in the lower apes. ${ }^{1}$ Hence in these large old-world apes the hands and feet are more completely differentiated from one another.

The next step in the evolution of man was probably the gradual abandoning of arboreal habits and the assumption of a more completely erect attitude during locomotion. This appears to have been the real starting point of his human career. It was this change of habit which led to those structural modifications required to balance the body properly in its new position, and to the further differentiation between hand and foot, the latter being used to the complete exclusion of the former as an organ of locomotion and at the same time losing its prehensile character, so that one of the chief distinguishing features between man and the higher apes is that in man the great toe has ceased to be opposable.

In this way the hand was completely set at liberty for the purposes of a prehensile organ. It was, however, no longer used, as in the apes, chiefly for laying hold of branches in climbing and for conveying food to the mouth, but more for grasping loose objects and converting them into weapons or tools for different purposes. Hanno observed that the anthropoid apes which he met with in Africa defended themselves with stones,

1 Tide Beddard, "Mammalia " (Cambridge Natural History, Vol. X), p. 570. 
so that we can hardly say that the use of tools or weapons is an exclusively human attribute; but the hand of man is undoubtedly one of his most characteristic features, and by its aid man has been able to make for himself an unlimited number of what are really additional organs, derived not from his own body but from his environment.

To the experience gained by the exercise of the hands in so many different ways must also be attributed in large measure the extraordinary mental and moral development which, more than anything else, separates mankind from the apes. The constant exploitation of the environment stimulated and exercised the brain, which in turn suggested methods of employing the hands and the tools which they had constructed to ever greater advantage; and thus both hand and brain progressed until they attained their present wonderful state of efficiency.

The development of the brain has, however, long since taken the lead in human evolution and, considering the immense difference in intellectual capacity, it is surprising that there should be so little structural difference between the brain of man and that of the higher apes. As Huxley has pointed out :-

"It is only in minor characters, such as the greater excavation of the anterior lobes, the constant presence of fissures usually absent in man, and the different disposition and proportions of some convolutions, that the Chimpanzee's or the Orang's brain can be structurally distinguished from Man's." 1

Intellectual capacity appears, however, to depend mainly upon the size of the cerebral hemispheres, which is doubtless correlated with the number of nerve cells present, and in this respect the human brain is far in advance of that of any ape.

One of the most important characters which differentiate mankind from the apes is the faculty of articulate speech, but even this undoubtedly had its beginnings in the inarticulate sounds made by ape-like ancestors, either as spontaneous expressions of their emotions or as a means of communicating more or less definite ideas to one another. The development of speech provided a new means for the transmission of experiences from one generation to the next, and as a consequence knowledge began to accumulate in the minds of the human race. When oral tradition

1 Huxley, "Man's Place in Nature," p. 140 (Collected Essays, Vol. VII). 
gave place to the establishment of written records, and methods were invented for the indefinite multiplication of these, the accumulation of knowledge took place at a much more rapid rate and it became possible for every human being, at any rate in civilized communities, to benefit from the experience of all his fellow men. The acceleration of intellectual and moral progress which has been brought about in this way has led to results which may well have deluded man into the belief that he is the centre of the universe and that between himself and the lower animals there is a great gulf fixed.

Man has indeed acquired a degree of control over his environment and over his own destiny which distinguishes him from any of the lower animals, but at the same time the conditions of his life have become far more complex and the young, at any rate in civilized communities, have to go through a long course of education before they are fit to enter upon the struggle for existence on their own account. Amongst the lower animals all, or almost all, the faculties necessary for existence are directly inherited from the parents, incorporated in the organism itself, but man inherits in this way only a relatively small proportion of the powers which he requires to carry on his life. The greater part of human experience is of too recent origin to have become heritable; it has to be acquired afresh by education in every generation, and in this respect is strikingly contrasted with the instincts of the lower animals.

The immense advance which civilized man has made since he parted company with his ape-like progenitors is shown, not only by the fact that he has already to a large extent subjugated the remainder of the organic world and directed the forces of inanimate nature into new channels to serve his own purposes, but also by the intelligent forethought which he exercises for the future welfare of his own race. At the present time this forethought is being exercised in new directions, and a determined effort is being made to apply our knowledge of the principles of organic evolution to the furtherance of human progress. In spite of many differences of opinion, such as that which still prevails with regard to the relative importance of breeding and education, we have undoubtedly arrived already at many results which are of vital significance in this connection, and the intelligent application of scientific principles must here, as always, lead to further progress. We cannot, however, 
control the future of the human race until we have familiarized ourselves with the past, and learned to recognize the part played by the numerous different factors which have been concerned in the evolution, not only of mankind, but of the whole organic world.

It must always be remembered that the problem before us is one of extreme complexity, and that we cannot afford to neglect any of the factors involved. Above all, we must avoid dogmatizing on an insufficient basis of fact. If, for example, the very modern doctrine of the non-inheritance of acquired characters is allowed to influence the actions of men and women, and if, after all, this doctrine should prove to be erroneous, as seems highly probable at the present time, the attempt to apply biological principles to the welfare of humanity may well end in disaster. The penalty which each generation has to pay, in regard to bodily and mental organization, for the mistakes and misfortunes of its ancestors, may, in most cases, be a very small one; but if there is any penalty at all, and if the mistakes are continued from generation to generation, it will surely be a cumulative one.

In dealing with problems of this kind a rational conservatism, with a mind always open to conviction, seems the only safe attitude to adopt. 



\section{N DEX}

A.

AbBreviation of ontogeny, 273.

abiogenesis, $214-216$.

Acacia pycnantha, seedling, Fig. 132.

acacias, life-history, 280.

Acanthodes, 291, Fig. 138.

accessory chromosomes, 135 .

accessory idioplasm, 167.

accretion, 20.

achromatic figure, 74 .

acquired characters and human

progress, 427.

Bordage on, 182.

\begin{tabular}{|c|c|c|}
\hline " & , & Bordage on, 182. \\
\hline " & " & $\begin{array}{l}\text { Brewer on, 178, } \\
180 .\end{array}$ \\
\hline " & ", & $\begin{array}{l}\text { Brown-Séquard } \\
\text { on, } 180 .\end{array}$ \\
\hline " & ", & $\begin{array}{l}\text { Buffon on, } 36 \% \text {, } \\
368 \text {. }\end{array}$ \\
\hline
\end{tabular}

, " Charles Darwin on, $180,393$.

de Vries on, 414.

Eigenmann on, 183.

Erasmus Darwin on, 372 .

Henslow on, 183

HerbertSpencer on, 185.

Hilgard on, 178.

inheritance of, $165,169,176$ $193,404$.

Lamarck on, $379,380,382$.

meaning of term, 156, 176, 177.

Sumner on, 182.

", $\quad$, $\quad$ Weismann on,

actinula larva, 122 . $176,179$.

activity of male, 84,85 .

adaptation, 235,365 .

$$
\begin{gathered}
, \quad \text { and design, } 212 . \\
\text {, fluctuating varia- } \\
\text { tion, } 414,415 .
\end{gathered}
$$

adaptation and mutation, 414, 415 . " natural selection, $396,404,405$.

, average, 184.

, co-operation of factors in bringing about, 405 .

", embryonic, 267
Erasmus Darwin on, $372,373$.

in animals, $334-349$.

individual, 184.

in inorganic world, 395.

in leaves of acacias, 280.

in plants, $350-363$.

mutual, 414, 415 .

origin of, 183.

resulting from survival of fittest, 391.

Robert Chambers on, $383,384$.

adipose tissue, 56, Fig. 19.

resthetic development of man, in-

fluenced by insects, 364 .

resthetic sense, in courtship, 349 .

affinities, natural, 228, 229.

after-effects, 191.

age of habitable earth, $285-287$.

age of occan, 287.

aggregate species, 224 .

aggressive resemblance, 337 .

Agnatha, 290.

Agnostus princeps, Fig. 134.

air-bladders of Fucus, 99.

air-breathing vertebrates, origin, 256.

ala spuria, 305 .

albumen, 23.

alcoholism, 157.

alimentary canal, 124 .

allantois, 270 .

allelomorphs, 200, 206.

aloes, 350 .

Alpine plants, $181,262,350$.

Alps, Arctic vegetation on, 333 .

alternation of generations, 101 .

in aphides, 144 .

in coelenterates, 121.

in ferns, 101-106.

in flowering plants, 106-112. 
Amauris niavius, 346, Fig. 177.

Amblyopsis, 183.

ambulatory appendages of arthropods, $246,247$.

ambulatory legs of vertebrates, 235 . Ameghino, 253.

amitotic nuclear division, 80, Fig. 36. in Copromonas, 83.

amnion, 270.

Amoba, 12-21, Fig. 2.

, mitosis in, 79 .

, sensitive to stimuli, 188.

Amphibia, geological range, 284.

, limbs, 235.

". origin, 292.

amphimixis, 146, 171, Fig. 78.

and variation, 173.

Amphioxus, adult, 266, 267, Fig. 118.

, early development, 45 48, 265, Fig. 13.

, free swimming embryo, 271.

ovum, 140.

Amphisbænidæ, 250.

Amphitherium, 302.

anabolism, 9, Fig. 1.

analogy, $235,2+7$.

anatomy, evidence afforded by, 232 -262 .

ancestral history, 229.

Anchitherium, 310.

Ancon sheep, 154.

Andalusian fowls, blue, 200, 201.

Andrews, C. W., 312.

androecium, 106.

anemophilous flowers, 111, 353 .

Anguis frugilis, 250, Fig. 105.

animals, compared with plants, 34, 35.

dependent on green plants, 35.

anisogamous conjugation, 84 .

anisogamy in Fueus, 101.

Anomodontia, 296.

Antarctic continent, 328 .

anteaters, 333.

Antedon, hybridization in, 174, 175 .

, recapitulation in development, 273, 275, Figs. 123, $12 t$.

antheridia, of Fern, 103, 104, Fig. 51. ". "Fucus, 100, Fig. 47. antheridial cell of pollen grains, 108 ,

Fig. 54.

anthers, 107 .

anticryptic coloration, 337 .

antiquity of man, $422,423$. antlers, 125.

apatetic colours, 336 .

apes, Buffon on, 367, 368 .

, and man, Buffon on, 370 .

, , " Lamarck on, 382.

, ," , relationship of, 422 425.

aphides, parthenogenesis in, 143.

Aphrodite, story of, 214.

apogamy in ferns, $10 j$.

aposenatic coloration, 342 .

Apteryx, 258, Figs. 111, 112.

Apus, dispersal of, 327 .

arborescent colonies, 40.

Archæopteryx, 300, 305-307, Fig. 151.

archegonium, of Fern, 103, 104, Fig. 52.

, , flowering plant, 108.

architype, 232.

Arctic climate, retreat of, 333 .

, vegetation on Alps and Pyrenees, 333.

areas of distribution, $319,320,330$.

Argus pheasant, 349.

Aristotle, 163, 385 .

arm, of man, 23i, 238, Fig. 94.

armadillos, 333.

arrow worm, origin of germ cells, 130.

Artemia, chromosomes, 73.

Arthropoda, limbs, 246, 247.

arthropods and vertebrates, 255.

artificially produced characters, 156, 157.

artificial parthenogenesis, 145 .

, selection, $395,396,410$ 413.

, , $\quad$ and fertility, 41s, 419.

artiodactyl limbs, 241, Fig. 98.

Arum, fertilization, $3 \bar{j} 5$.

Ascaris, chromosomes in, 73.

" distinction between somatic and germ cells, 166 .

fertilization of egg, 131, Fig. 64.

mitosis, 79 .

, origin of germ cells, 129 .

ascidian, adult, 277, Fig. 129.

, degeneration in, 400 .

" larva, 277, Fig. 130.

asexual reproduction, in Eudorina, 91.

, , $\quad$ in Hydra, 116. 
asexual reproduction, in Obelia, 119.

in Pandorina, 89.

Aspidium, Figs. 48, 50.

asters, in mitosis, 71 .

atavism, 261, 262.

attraction of gametes, 143 .

\begin{tabular}{|c|c|c|c|}
\hline & " & " & $\begin{array}{c}\text { in Coccidium, } \\
141,142 .\end{array}$ \\
\hline & " & " & $\begin{array}{l}\text { in ferns and } \\
\text { mosses, 141, } \\
142 .\end{array}$ \\
\hline & " & " & $\begin{array}{c}\text { in Spirogyra, } \\
142 .\end{array}$ \\
\hline
\end{tabular}

" , sexes, $125-127$.

" sphere, 71.

Aucuba, male and female plants, 111.

Aurelia, meristic variation in, 149 .

Australia, fauna and flora of, 250, $328,329,331$.

Australian climate, adaptation to, 280.

automatism, 19 .

Avebury, Lord, 281.

average adaptation, 184 .

axial filament, of spermatozoon, 140 . Axoniderma mirabile, Fig. 168.

B.

Babirusa, tusks, 406, Fig. 185.

baboons, Buffon on, 367, 368 .

Bacillıs saccıbranchi, 66, Fig. 27.

Bacteria, 217, 218, Fig. 83.

" alleged spontaneous generation, 215. origin, 219.

" origin, 219.

Badhamia, Fig. 29.

Bakewell, 387.

Bakena mysticetus, 245, Fig. 101.

Balænidre, teeth, 260.

barnacles, degeneration, 401.

barriers to migration, 320 .

basal cell, 48 .

bast, 65 .

Bates, H. W., 346.

Batesian mimicry, 346.

Bateson, W., 195, 205, 207, 208.

bats, 301 .

" dispersal, 323 .

", wings, 236, 243, Fig. 99.

battle, Charles Darwin on law of, 388 . ," Erasmus Darwin on law of, 373 . beech, evergreen, 329 . beech, seedling, 281, Fig. 133 .

bees, alleged spontaneous generation, $214,215,343$.

,, as pollen carriers, 354,355 , \&c.

", parthenogenesis, 144.

,, proboscis, 354, Fig. 179.

beetles, mutation in, 159 .

Behring Strait, former land connection, 309.

, , former mild climate

Bell, 340. 328.

Biffen, R. H., 205.

binomial nomenclature, 226.

biogenesis, 216.

biogenetic law, 265 .

Biology, origin of name, 373 .

Biophoridæ, 219.

biophors, 22, 66, 168, 219.

birds, compared with reptiles, 305 .

" dispersal, 323, 324.

, egg, 140, Fig. 70.

, evolution, $306,307$.

," geological range, 284.

, limbs, 235 .

" origin, 299,300 .

, wing, 243, 244, Fig. 99.

blackberries, white, 204.

blastocœl, 47.

blastoderm, 270.

blastogenic characters, 176 .

$$
\begin{array}{ccc}
, & \text { inheritance, } \\
& & 169 . \\
\text { origin, } 159, & 160 . \\
& & \text { variations, } 149,157-160 .
\end{array}
$$

blastomeres, 45,79 .

blastopore, 47, 265, 269.

blastosphere, $4 \tilde{5}, 265$.

blastostyles, 120.

blastula, 45, 265 .

", interpretation, 279.

Fig. 13.

of frog, 268, Fig. 119.

, of Hydra, 118, Fig. 59.

blind worm, 250, Fig. 105.

blood, 51, Fig. 15.

blue Andalısian fowls, 200, 201.

boa constrictor, dispersal, 325 .

Bodo, life-history, $85-87$, Fig. 38 .

body cavity, 48, 124.

body wall, 124.

Bombus terrestris, robbing clover, $355,356$.

bone, 57 . 
bony fishes, 292 .

Bordage, E., 182.

Boveri, T., 146, 174.

brachiopods, 289.

brain, in evolution of man, 425 .

," of man and apes, 425 .

branching classification, Charles Darwin on, 387. evolutionary series, La. marck on, 376.

Branchiosaurus, 293, Fig. 139.

Branchipus, dispersal, 327.

breathing, 8 .

breeding experiments with Papilio, 348.

Brewer, 178.

brimstone moth, larva, Fig. 169.

brittle star, 275, Fig. 126.

Brontosaurus, 299, Fig. 144.

Brown-Séquard, 180.

Buceros rhiuoceros, Fig. 407.

budding, 263.

,, in Hydra, 116.

, in Obelia, 119, 120.

Buffon, on sterility of species when crossed, 418.

views of, $365-370$.

Burbank, I., 204.

Butler, Samuel, 3, 186, 191, 369.

Bütschli, O., 19, 21, 22.

butterflies, warning colours, $343-$ 347.

butterfly, leaf, 338, Fig. 171.

,, metamorphosis, 264.

", proboscis, 354 .

\section{C.}

\section{CACTI, 350.}

," thornless, 204.

cæenogenetic characters, $278-281$.

Crenolestes, 301.

Crosar's horse, 262.

Cainozoic Era, 284, 285.

calcareous skeletons, 26 .

Calceolaria, distribution of, 328 .

calcium, influence upon segmentation, 45.

callosities, Buffon on, 367, 368 .

calyx, 106.

cambium, 76.

Cambrian Epoch, 284.

camel, Buffon on, 367.

feet, 241, Fig. 98 .

Camelo-pardalis, Lamarck on, 379.

cancer, nature of, 408 .

Cancer phalangium, 339. candle, analogy of, 2, 5 .

Candollea graminifolia, fertilization, 362.

cane sugar, attracting spermatozoa, 141.

canine teeth, reversion in, 262.

cannon bone, 241.

Capsella bursa-pastoris, development, 48, Fig. 14.

carbohydrates, 27.

carbon, 6.

carbon dioxide, 6,8 .

Carboniferous Epoch, 284.

Carchesium, 40.

carnivores, 301.

carpals, 237.

carpels, 106.

Carpophaga chathamensis, 416.

carrion flies, 355.

nove zealandia, 416.

cartilage, 56, Fig. 20.

casein, 23.

castration, effect, 125 .

Catasetum, fertilization, 362.

caterpillar, larval organs, 275 . stick, 337,338 .

cats, inheritance of mutilation, 178.

,, stump-tailed, 179.

candicle, 361 .

cave-animals, bleaching inherited, 183.

cell, 12.

" body, 13.

, definition of, 38 .

", division, $69-80$.

, , limits of, 81 .

, history of term, 36,37 .

,, membrane, 69.

, plate, 74 .

, sap, 62

, theory, 38 .

, , limitations of, 65 .

,' typical, 69, 70, Fig. 30.

,, wall, 27, 28, 69 .

cellular structure, 36 .

cellulose, $2 \overline{7}, 28$.

centrosome, 71 .

, absence in higher plants, 141.

," , , , ovum, 141.

", as stimulus to development, 146.

, in spermatozoon, 139, Fig. 69.

in unfertilized egg, 146. centrosphere, 71 . 
Cephalaspix, 290, Fig. 135.

Ceratodus (see Neoceratodus).

cercariæ, 144.

cereals, fertilization, 412 .

" improvement, $410-413$.

", Mendelian inheritance in, 205.

cervical vertebræ, of giraffe, 248 , Fig. 104.

", whale, 248, 318, Fig. 103.

Cetacea, evolution of, $314-318$.

$$
\text { , limbs, } 236 .
$$

Chætopterus, artificial parthenogenesis, 145 .

chalk, 26.

chamæleon, colour change in, 341 .

Chambers, Robert, views of, 383 385.

change of function, 255, 256, 262.

Chara, gametes, 141.

characters, compound, 208.

"Chatham," H.M.S., 399.

Chatham Islands, $325,398-400,416$, 417.

chelæe, of sponges, 420, Fig. 187.

Chelonia, 295.

chemical affinity, 6,7 .

, processes, 9 .

" stimulus to development, 146.

chemically modified larva, 157 .

chemotaxis, 141, 143, 189.

chick, embryo, 265, Figs. 117, 122.

chimpanzee, brain, 425 .

$$
\text { , Buffon on, 370. }
$$

Chinese women, stmall feet, 156.

Chironomus, predogenesis, 144.

Chlamydomonas, 40.

chlorophyll, 7, 8, 28 .

$$
\text { , cells, } 64 \text {. }
$$

chloroplastids, 32,64 .

$$
\text { cells, } 64 \text {. }
$$

Chordata, 277.

$$
\text { in Spirogyra, } 96 .
$$

chordate condition, 266.

chromatin, 70,71 .

$$
\text { ", in heredity, 166, 169, 174, }
$$
206.

chromatophores, in Spirogyra, 96.

chromomeres, $73,168,206$.

chromosomes, $71,73,168$.

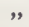

,

B. chromosomes, integral division, 171, Fig. $7 \%$.

maternaland paternal, 137, 207, Fig. 68.

pairing, 133, 137, 206, Fig. 68.

reduction, 132,133 , $137,138,206,207$, Fig. 68 .

chrysalis, 264.

cilia, 39.

Ciona intestinalis, Fig. 129.

circumcision, 179, 180.

Cirolana, 227.

cirripedes, degeneration, 401.

Cladocera, parthenogenesis, 144

Cladorhiza longipinna, Fig. 168.

, (?) trilentata, spicules,

classes, 226. Fig. 187 .

classification, $225-228$.

$$
\begin{aligned}
& \text {, and phylogeny, 230, } \\
& \text { Fig. } 87 . \\
& \text {, artificial, } 228 . \\
& \text {," Buffon on, } 366 \text {. } \\
& \text { " Charles Darwin on, } \\
& 387 . \\
& \text {, Lamarck on, 374, } 375 \text {. } \\
& \text {, natural, 228, Fig. } 86 .
\end{aligned}
$$

clavicle, 234, Figs. $89,90,93$.

clear-winged moths, mimicry, 343 ,

Fig. 175 .

cleavage of ovum, 75 .

climate, adaptation to, 280.

$$
\begin{gathered}
\text { changes in, } 327 . \\
, \quad \text { influence of, Buffon on, } 366 . \\
, \quad \text {, Lamarck on, } \\
376 . \\
\text {, }
\end{gathered}
$$

clover leaf, meristic variation, 149. coal, energy in, 4.

Coccidium, gametes, 87,88 , Fig. 39 . maturation of ovum, 139. coceyx, in man. 261.

cockroaches, dispersal of, 325 .

Codonella, Fig. 9.

Coeciliidæ, 250, Fig. 106.

Coelenterata, 114.

coelenterate and gastrula, 265, 269, $270,27 \%$.

colom, 48, 124, 266.

coelomate and coelenterate types,

124, Fig. 62.

cœlomic epithelium, 124.

, pouches, 266.

cold’-blooded animals, 6 .

colloids, 23.

\section{1, Fig. 77.}

in gametophyte, 138. ,, sporophyte, 139. 
colony formation in Hydra, 116.

" " , Obelia, 119.

" " , Protozoa,40-44. colour changes in animals, 341,342 . colours of animals, 336,337 .

, " , Erasmus Darwin on, 372 .

, ,flowers, $35 \overline{5}$.

column, of flower, 360,363 .

combustion, energy liberated by, 4,6 . nature of, $2,5,6$. communication between cells, 187 . compound characters, 208. conceptacles of Fucus, 100.

Condylarthra, 309, 313.

cone of attraction (or reception) in

Coccidium, 88, 143.

congenital variations(characters), $15 \%$. Conilera, 227.

conjugants, in Paramœeium, 92,

Fig. 41.

conjugation of chromosomes, 137, Fig. 68.

" , gametes, $33,82-85$, 131,141 , 206,207 , Fig. 65.

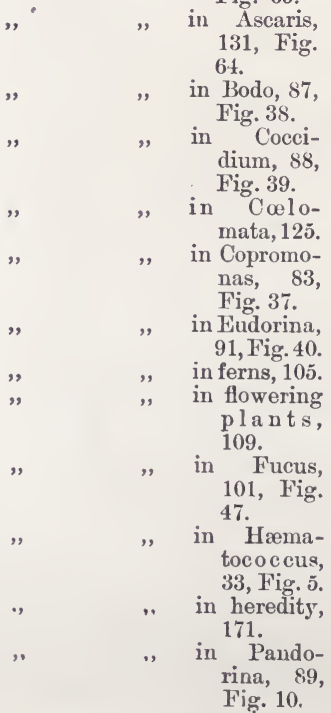

conjugation of gametes in $\mathrm{Para}-$ mocium, 92,93 , Fig. 41.

" $\quad$ in Spirogyra, 96 , 97,142 , Figs. 43, 44.

in Zygogonium, 97 .

of nuclei in zygote, 131, Fig. 64.

, origin of, 127 .

, results of, 84,87 .

connecting links, 232-235, 305318.

destruction of, 222 .

conodonts, 289.

conservatism of germ cells, 184, 190.

continental islands, fauna, 332 .

continents, permanence, 329 .

continuity of germ plasm, 166,168 , Fig. 76 .

, $\quad$, life, 67 . parent and offspring, Erasmus Darwin on, 370 .

, , protoplasin, 66.

eontinuous selection, 411 . variations, 148,150 .

contractile tissue (see muscle fibres). , vacuole, $14,18$.

convergence, $24 \overline{7}-25 \overline{5}$.

" in Acacia, 280.

" , flightless birds, 397 .

", " plants, $2 f 2$.

, , " proboscis of bees and Lepidoptera, 354.

"Convolvulus major," fertilization, $351,352$. co-operation, 40.

" of factors in evolution, 421.

Copromonas, life-history, 83, Fig. 37. coracoid, 234, Figs. 89,93 .

nuclear reduction, 139. corals, $114,26 \tilde{5}$.

cork, cellular structure, 37, Fig. 6. corolla, 106.

corpora lutea, 126.

corpuscles, blood, 51, 52, Fig. 15.

correlation, 409, 415 .

cortical tissues, 50 . 
cosmopolitan distribution, 320 .

Cosmozoa, 216.

cotyledons, caenogenetic characters, 280, 2s1, Figs. 56, 133.

cowslip, fertilization, 356 .

crab, larva, 276, Fig. 128.

"swimming, 247, Fig. 102.

creation, Lamarck on, $375,376$.

" special, 212-214, Fig. 82.

, " Buffon on, 369 .

Creodontia, 3̈8.

Linnæus on, 222.

Crepidula, 323 .

Cretaceous Epoch, 284.

Crinoidea, 274.

Crinorhiza form, 335, 420, Fig. 168 .

Cristatella mucedo, statoblast, Fig. 165.

Crocodilia, 295.

cross-fertilization of flowers, 351363.

crossing (see hybridization).

Crustacea, fresh water, dispersal, 327.

gills, 255 .

cryptic coloration, 337 .

crystalloids, 23.

currents, dispersal by, $321-325$.

curve of frequency of error, 153.

" " variation, 150,153 , Figs. 72 , 73.

Cuscuta europeea, 402, 403, Fig. 184.

eushion plants, $262,350$.

Cyathaspis, 290.

cyclopean larvæ, 157 .

Cyclostomata, 289.

cyst, 20,83 .

cytology, 67 .

cytolysis of ovum, 147.

cytoplasm, 13, 69.

,$\quad$ in heredity, 174, 175.

cytostome, 83.

cytotaxis, 142.

cytotropism, 142.

Cyttaria, 329 .

of gametes, 143.

Cyttarocyclis, Fig. 9.

\section{D.}

Darbishire, A. D., 197.

Darwin, Charles, views of, $164-167$, $176,180,185,195,208,222-$ $224,329,351,-353,356,357$, $359, \quad 385-388, \quad 391-393, \quad 410$, $415,423$.
Darwin, Erasmus, views of, $370-373$. Francis, 192.

death, $21,162,168$.

De Candolle, 386.

decay, nature of, 4.

deep sea animals, adaptation in, 335 , 336.

deer, feet, ?41, Fig. 98.

degeneration, $397-403$.

, in ascidians, 400.

", "flightless birds, 397 .

," ", Morioris, 400. , results of, 398.

Delage, Y., 146.

Delphinus delphis, Figs. 161, 162.

Democritus, 369.

dendrons, 59.

dentition of dog and thylacine, 251253, Figs. 107, 108.

denudation, $285,288$.

dermatogen, 50 .

Descartes, 11, 260.

descent with modification, 221 .

design, doctrine of, 212 .

Desmacidonidæ, spicules, 419, 420, Fig. 187 .

determinants, $16 \overline{7}, 206$.

" $\quad$ effect of stimuli upon, 189.

vibrations in, 189.

deutoplasm, 140, 267.

development, 263-281.

, and unconscious memory, 192.

, Erasmus Darwin on, $371,372$.

, factors in, 192.

,, of birds and reptiles, 270 .

", of flowering plants, 4850, 109, Fig. 14.

of Frog, 268-270, 272,

Figs. 119, 121.

of Hydra, 118, Fig. 29.

Devonian Epoch, 284.

De Vries, Hugo, 154, 204, 224, 410-. 414.

Dianthus, 353.

diatoms, 25 .

dichogamy, 353.

Dictyocysta, Fig. 9.

Didelphyidæe, 301.

differences between plants and animals, 34,35 .

differential division of chromosomes, $17 \%$, Fig. $7 \%$.

differentiation, 39, 43, 44, 60, 119.

sexual, 85 . 
diffusion of gases, 8 .

digestion, in Amøeba, 16.

digestive cavity, of Hydra, 115.

$$
\text { ", ", ", Medusa, } 120 .
$$

digits, reduction, $239-241$.

dihybridism, 201 -203, Figs. 80, 81.

dimorphic flowers, 356.

dimorphism of gametes, 139.

Dinosauria, 297.

Dinotherium, 314, Fig. 160.

diøecious, 99, 105.

Diplodocus, 299.

Diplosoma crystallinum, larva, Fig. 130.

Dipnoi, 255, 291.

Diptera, predogenesis, 199.

direct nuclear division, 80, Fig. 36 .

discontinuity between species, 222, Fig. 85.

in distribution, 319,320 , 333.

in evolutionary series, Lamarck on, 376.

in organic world, 221. discontinuous variation, 149, 153 156.

Dismorphia praxinöe, 345, Fig. 176. dispersal of organisms, $320-327$.

distribution, geographical, $319-333$. disuse (see use and disuse). , of wings, effects of, $397,398$. divergence in evolution, 213.

," A. R. Wallace on, 390.

, Charles Darwin on, 387.

division of labour, $39,43,44,60,85$, 119.

" $\quad$, between male and dodder, 402, 403, Fig. 184. female, 127.

, vestigial leaves, 262, Fig. 184. dodo, 258, 397.

dog, skull and dentition, 251-253,

Figs. 107, 108.

, vestigial teeth, 260.

dogfish, embryo, 270, Fig. 120.

dolphin, Figs. 161, 162.

dolphins, convergence in, 248 .

shark-toothed, 318.

domestication, Charles Darwin on, 410.

Lamarck on, 378.

dominant characters, $197,207$.

Draba verna, elementary species in, $224,412$.

drone flies, mimicking bees, 343 .

, mistaken for bees, 215.
Dubois, E., 423.

Dujardin, 38 .

dynamies of cell-division, 74 .

E.

Ecrimsa, 234, 301, Fig. 92.

echinoids, larval stage, 276.

Echinus, blastomeres, 45.

., hybridization in, 174.

ectoderm, 47.

, of coelomates, 124.

edentates, 301, 333.

, Hydra, 115, 116, 118.

education, 426.

eels, dispersal of, 325,326 .

egg cell (see ovum).

," of Ascaris, mitosis, 79, Fig. 35.

,, ,, bird, 140, Fig. 70 .

,, ,, frog, 268.

eggs, dispersal of fish, 322 .

, similarity in different organisms, 162.

,, size, 267 .

Eigenmaun, 183.

Elasmobranchii, 291.

electrical energy, transmission, 189. electric eel, 256.

," organs, 256.

electro-magnetic theory of mitosis, 74,75 .

elementary species, 224, 412.

elephants, ancestry, 312-314, Fig. 159.

Elephas, 314.

limbs, 239.

élite, 411 .

Elodea, evolution of oxygen, 31 .

embryo, fixation, 126.

, of chick, 265, Figs. 117, 122.

, , Fucus, 101, Fig. 47.

" , mammals, 270 .

," , rabbit, 265, Fig. 117.

embryology, Erasmus Darwin on, 371,372 .

, evidence afforded by, 261-281.

embryonic cell, 48.

embryo-sac, 48, 106, $10 \%, 108$.

embryos of birds and reptiles, 270 .

Emily Henderson, sweet pea, 208.

Empedocles, 369.

Encrinites, 274.

endoderm, 47.

, of cœelomates, 124.

" of Hydra, 115, 116, 118.

endoplasm, 14, 22.

endosare, 14. 
endosperm, 109.

energy, conservation of, 6 .

" manifested in life, 4,5 .

", of chemical affinity, 7 .

" source of, $4,6,7,9$.

, , , in green plants, 30 . engrams, 186.

entelechy, 11 .

enteron, $47,265$.

", in Hydra, 115.

entomophilous flowers, 111,353 .

enucleate eggs, fertilization (see merogony).

environment, control of, 426.

, influence of, $5,6,166$, $182,183$.

A.R.Wallace on, 390 . Buffon on, 366, 367. Charles Darwin on, 387,392 .

Lamarck on, 377-379.

upon development,

193.

", germ plasm,

Eocene Epoch, 284.

159.

Eohippus, 309, 310, Fig. 154.

Ephydatiu fuviatilis, gemmules, Fig. 166.

epiblast, 47, 48, 266.

," of Hydra, 115, 118.

epicoracoid, 234, Figs. 89, 93.

epidermis, 54, Fig. 17.

of plants, 50, 64, Fig. 26.

epigamic ornamentation, 336, 349 .

epigenesis, 163.

Epihippus, 310.

epilepsy, in guinea pigs, 180.

episematic coloration, 342 .

Epistylis, 40.

epithelium, 54, Figs. 16-18, 28.

epochs of earth's history, 284,285 .

equatorial plate, 72 .

Equidæe, pedigree of, $307-312$.

Equus, 309, 311, Fig. 153.

eras of earth's history, 284, 285.

Esperella lapidiformis, spicules, Fig. 187.

Esperiopsis challengeri, 420, 421, Fig. 188. pulchella, spicules, Fig.

eucalypts, 331 . 187.

Eudendrium, migration of germ cells, 123.
Eudorina, 42, 90, Fig. 40.

Eurypteridæ, 291, Fig. 137.

Eutheria, 301. size of, 405.

first appearance of, 302.

evolution, factors of, $365-427$.

" in development, 163.

," individual, 263.

" of sex, 81-147.

" progressive, 192, 334 .

" theory and evidence of, $211-364$.

versus special creation, 212-21t, Fig. 82.

ex-conjugants, in Paramoecium, 93. excretion, 9.

$"$ in Amœba, 17.

experiments in heredity, 174,175 , $194-209$.

explosive character of living mole-

cule, 18.

extinction of groups, 231.

," , species, Buffon on, 366 .

extracted dominants, 198.

, recessives, 198.

eye-colour, Mendelian inheritance of, 205.

eyes, pineal, $258-260$.

F.

FACTORS, co-operation of, 208.

, in development, 192.

" , germ plasm, 206, 207.

" of organic evolution, 365 427.

permutations and combinations of, 206.

freces, 16.

families, in classification, 226.

Farmer, J. B., 206.

fat, $\tilde{6} 6$, Fig. 19.

feather star, hybridization, 174, 175 .

, ", recapitulation in lifehistory, $273-27 \overline{5}$, Figs. 123, 124.

feathers, acquisition of, $17 \tau$.

female animal, 114.

" characters, $84,85,127$.

, dependence on male, 127.

femur, $23 \%$.

fermentation, 218.

fern, life-history, 101-105, Figs. 48 52.

ferns, dispersal, 321 .

fertility and cross-fertilization, 352 , 357.

test of specific identity, 418 
fertilization, adaptation of flowers for, $351-363$. chemical stimulus in, 146.

development without, 145.

in heredity, 171.

membrane, 147 .

of flowers, 110, 351363.

, $\quad, \quad$ ovum, 85, Fig. 65. Fig. 64 .

, , , , , Cœlomata, 125.

, , , , , ferns, 105 .

" " " " "Fucus, 101.

," , , , , Hydra, 118.

fibrillar structure of protoplasm, 22.

fibula, 237.

fig-wort, 353.

filament of stamen, 107.

finger, inheritance of mutilation, 180.

First Cause, Erasmus Darwin on, 372.

fish eggs, dispersal, 322.

fishes, bony, 292.

" deep sea, 336 .

," geological range, 284.

fish-like stage in ontogeny and evolution, $272,273,277,278,279$.

fission, in Amøeba, 21.

, , Bodo, 87.

, , Copromonas, 83.

", "Hromatococcus, 29.

fixation of embryo in uterus, 126.

flagella, in Bodo, 87.

, " Eudorina, 91.

, , Hrmatococcus, 29.

flame, nature of, 2.

Flemming, 69.

flightless birds, 258 .

$$
\begin{array}{ccc}
, \quad & \text { and fluctuating varia- } \\
\text { tion, } 415 .
\end{array}
$$

flint, 24.

floating islands, dispersal by, 324 .

flower, structure of, 106, 10\%, Fig. 53. flowering plants, life-history, $106-$ 112.

flowers, adaptation for fertilization, $351-363$. sexual characters, $110,111$. fluctuating variations, $148,150,155$, Figs. 72,73 . and adaptation, 414, 415.

and natural selection, 414.

De Vries on, $413,414$.

flukes, parthenogenesis in, 144.

foam structure of protoplasm, 21 .

fotal membranes, 270,278 .

foetus, in Mammalia, 270 .

food materials of animals, 16 .

, , , green plants, 3032.

, nature of, 7 .

,, vacuoles, 14.

food-yolk, 267, 278.

influence of, 268,271 .

foot, artiodactyl, 241 .

,, of apes and monkeys, 424 .

, , camel, 241, Fig. 98.

," , deer, 241, Fig. 98.

, ", elephant, 239.

, ", hippopotamus, 241, Fig. 98.

,, ,, horse, 241, Fig. 97.

," ," atavism in, 262.

,, ," , evolution of, $310-312$, Figs. 154-158.

, , Litopterna, 253-255, Fig. 109.

", , llama, 241.

,, ,man, 238, Fig. 94.

, ,, oxen, 241.

," , pig, 241, Fig. 98.

, , rhinoceros, 241 .

,, ,, seal, 244, Fig. 100.

," , sheep, 241.

,, , tapir, 241, Fig. 96.

, , ungulates, 239-241, 253255.

,, pentadactyl, 238.

,, perissodactyl, 241.

Foraminifera, 26, Fig. 4.

fossilization, 287,288 .

fowls, Mendelian inheritance in, 200, 201.

foxgloves, mutation in, 154 .

Francotte, 135.

Freia, Fig. 9.

frequency of error, curve, 153.

fresh water animals, dispersal, 325 , 326.

frog, early development, 268-270, Fig. 119. 
frog, life-history, 272, Fig. 121.

, , interpretation of, 279 , Fig. 131.

, pineal eye in, 260, Figs. 115, 116. frogs and toads, 293.

fruit trees, propagation, 205.

fruits, dispersal, 321.

Fuchsia, distribution, 328 .

Fucus, 99-101, Figs. 45-47.

Functions of organisms, $j$.

Fundulus, cyclopean larvæ, 15\%,

Fig. 75 .

Fungi, 34, 321.

funiculus, of ovule, 108.

furze, recapitulation in seedlings, 280

\section{G.}

Gulaxias nigothoruk, dispersal of, 326.

Gallardo, 75 .

Galton, Francis, 209.

Galton's law of inheritance, 209.

Galton's polygon, 155 .

Galtonia, mitosis in, 76, Figs. 33,34 . gametes, 33, 83 (see also germ cells).

" attraction of, 141, 142, 143.

" conjugation of, 207 .

", evolution of male and female, 84,85 .

of Bodo, 87.

", fern, 104.

"flowering plant, 108, 109, 110.

"Fucus, 100.

", Pandorina, 89.

"Spirogyra, 96, 97, 142, 143.

, Volvox, 91.

purity of, 200, 206.

sexual dimorphism of, 113, 139.

gametic nuclei in Paramœcium, 93. gametogenesis, 132, Fig. 65.

gametophyte, 101.

chromosomes of, 138
of fern, 103, Fig. 50.
" flowering plant, 107,
108.
suppression of,
112.

gamobium, 121.

ganglion, 59.

ganoids, 292.

gastræa, 277.

gastral cavity, 47.

\footnotetext{
", $\quad$ " $\quad$ in Hydra, 115, 118.
}

gastrula, 47, 265, 277.

, interpretation of, $265,279$.

," of Hydra, 118.

, , Sagitta, 130, Fig. 63.

gastrulation, in birds and reptiles, 270.

Geikie, Sir A., 285.

, frog, 269, Fig. 119.

gernmules, dispersal of, 327 .

$$
\begin{gathered}
, \quad \text { in pangenesis, } 164 . \\
\text { of fresh water sponge, } \\
327, \text { Fig. } 166 .
\end{gathered}
$$

genera, 225.

generative cells, 105.

Genesis, Book of, 212.

genital ducts, 125 .

geographical distribution, $319-333$.

", isolation, 416,417 .

geological formations, 284 .

, history of the earth, 284, 285.

," periods, 284.

", range of animal groups, 284.

record, 283, $287-304$.

geometer moths, caterpillars, 337 , 338, Fig. 169.

Geoplana exulans, dispersal, 325.

Geotria, distribution, 329.

germ cells (see also gametes).

, and somatic cells, 97, 98, $99,113,129,166,167$, 168.

conservatism, 184, 190. immortality, $162,168$.

independence, 99.

maturation, 138.

migration in Hydrozoa, 123.

origin in Ascaris, 129.

,

,3

, Coelomates, 124, 130.

Hydrozoa, 123.

,

,

,9

,

,

germinal disk, 140.

potentialities, 163.

" plants, 130.

", Sagitta, 130.

sensitive to stimuli, 188.

," selection, 173.

," variations, $149,157-160$.

germination of fern spore, 103, Fig. 49.

pollen grain, 108, Fig. 54 .

seed, 109, Fig. 56.

germ layer theory, 48. 
germ plasm, complexity, 167 .

composition, 172, Fig. 78.
constitution, 205, 206.
continuity, 166, 168,
Fig. 76.
influenced by environ-
ment, 159.

gestation of nature, $\mathrm{R}$. Chambers on, 384.

gigantic animals, 303, 304, 405-409.

Gila monster, 342.

gill slits, in Amphioxus, 266.

$$
\text { " embryos, 261, 273, Fig. }
$$
122.

gills of crustaceans and fishes, 255 . giraffe, 248, Fig. 104.

,, Lamarck on, 379. glacial periods, $32 \pi, 328$.

glass snakes, 250 .

gliadin, 23.

glucose, $28,31$.

glutinin, 23.

Godlewski, 174.

gonads, 113.

, in Coelomates, 124.

", "Hydrozoa, 124.

," medusæ, 121.

gonoducts, 125.

gonophores, 122 .

gonotheca, 120.

"gorillas," Hanno on, 42:2.

gorse, recapitulation in seedlings, 280 . gradation in nature, Buffon on, 366 .

", , structure, 232.

," of animals, Lamarck on, - 376.

Grantia compressa, larva, 322, Fig. 164.

grape hyacinth, curve of variation, 150 , Fig. 72.

sugar, $28,31$.

grasshopper, spermatogenesis, 134,

Fig. 66.

Gray, Asa, 386.

Gray, J. E., 227.

grazing mechanism, 309.

Great Britain, a continental island, 332.

Greenland, former mild climate, 328. Grew, Nehemiah, 37.

gristle, 56.

growth, 10, 20.

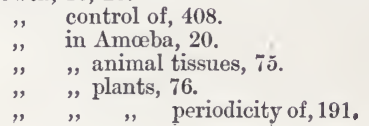

guard cells, 64, Fig. 26.

guinea pigs, Brown-Séquard's experiments, 180.

Gulf Stream, dispersal by, 322, 323 .

Gulick, 417, 418 .

gut wall, 124.

Gymnotus, 256.

gynoecium, 106.

\section{H.}

HABIT, of plants modified by environment, 181.

habitat, 319 ,

habits, adaptation in, 337 .

" influence of, Eamarck on, $377,378,379,380$.

," in plants, 191.

Haeckel, Ernst, 66, 229, 265, 277.

hrematids, 52, Fig. 15.

hæmatochrome, 28.

Hæmatococcus, 27 -34, Fig. 5.

hæmoglobin, 53. conjugation, 89.

hair, vestigial, 261.

hairs of Tradescantia, 61, Fig. 25.

hand of man, 256, 424, 425 .

, ," monkeys and apes, 424 .

Hanno, 422, 424.

harmony, in coloration, 336 .

Harpax tricolor, 339.

Hatteria (see Sphenodon).

Hawaiki, 399.

heat, in living organisms, 6 .

Heidelberg, fossil man, 423.

Heilprin, A., 319.

Heliconinæ, 345.

Heliconius ethilla, 345, Fig. 176.

Heloderma suspectum, 342.

Hemiphaga chathamensis, 416.

Henslow, G., 183.

nove zealandice, 416.

Herbst, 45.

heredity, 161-210 (see also inheritance).

Buffon on, 369

" Charles Darwin'

" Charles Da

fertilization experiments, 174,175 .

Galton on, 209, 210.

in neuter insects, 189, 190. ,, Protista, 161.

Lamarck on, 380, 381.

Mendelian experiments, $194-209$. 
heredity, mnemic theory, 186, 191, 192.

nature of problem, 163.

Pearson on, 210.

Weismann's theory, 166174.

Hering, E., 186.

hermaphrodite, 103, 105, 113, 114.

hermaphroditism, 93, 116, 125, 402.

heterogamy, 144.

Heteromita, life-history, $\quad 85-87$, Fig. 38.

heterosporous ferns, 105 .

heterostyled flowers, 356, Fig. 150 .

heterozygote, $20 \overline{\%}$.

hexadactylism, 154, 413.

Hilgard, E. W., 178.

Hipparion, 311.

Hippocampus antiquorum, 341, Fig. 173.

Hippopotamus, feet, 241, Fig. 98.

histological differentiation, 48 .

histology, 51 .

His, Wilhelm, 178.

hock, of horse, 240, Fig. 95.

holophytic nutrition, 34 .

holozoic nutrition, 34.

Homo heidelhergensis, 423.

Homo, species of, 423.

homologous chromosomes, 137, 206, 207.

homology, 235, 247.

homoplasy, 235, 247.

homosporous ferns, 106.

homozygote, 207.

honey guide, 362 .

honey sucking apparatus, 354 .

Hooke, Robert, 36.

Hooker, J. D., 385.

hormones, 126, 188, 408 .

hornbill, 406, Fig. 186.

hornets, colours of, $342,343$.

horns, excessive development, 406.

horse, evolution of, 307-312, Figs.

$153-158$.

horse's feet (see foot of horse).

, skeleton, Fig. 95.

horses, Mendelian inheritance in, 205.

horse-worm (see Ascaris).

humble bee, robbing clover, 355 , 356.

humerus, 237.

humming birds, as pollen carriers, 353.

distribution, 320 .

" $\quad$ epigamic ornamentation, 349 . hyacinth, mitosis in, 76 , Figs. 33,34 . hybridization, 194-209.

" and evolution, 209.

" and mutation, 413, 414.

in cereals, 413.

Hydra, 114-118, 265, Figs. 5 - -59 .

" dispersal, 327 .

, suppression of medusoid, 123.

hydranth, 119.

hydrocaulus, 119.

hydrotheca, 119.

Iİydrozoa, 123.

hyperphalangy, 244.

hypertonic solution, producing parthenogenesis, 145 .

hypoblast, 47,48 .

" of Amphioxus, 47, 266.

" , Frog, 269.

, , Hydra, 115, 118.

hypostome, 116, 119.

Hypsidx, 345 .

Hyracotherium, 310.

\section{I.}

ICEBERGS, dispersal by, 324 .

Ichthyopterygia (Ichthyosauria), 297 , Fig. 142.

, paddles, 236, 244.

Ichthyosaurus communis, Fig. 142. idants, 168.

identical twins, 173.

idioplasm, 167.

Idolum diabolicum, 339.

ids, 168, 206.

igneous rocks, 282.

Iguanodon, 297, Fig. 143.

illegitimate unions, in Primula, 357. immortality of germ cells, 162, 168 .

." "Protista, 161.

immutability of species,

Buffon on, 369 .

Lamarck on, 374 .

Linnæus on, 222.

individual adaptation, 184 .

" characters, transmission of, 175 .

variations, 150 .

individuality of cells, 68 .

inertia of germ cells, 190.

inflorescences, variation in, 150 .

Infusoria, 40, Fig. 41.

inheritance (see heredity).

of aequired characters,

$165,176-193$.

mutilations, $178,179$. 
insect communities, as individuals, 190.

insectivores, 301 .

insects, as pollen carriers, $353-355$.

, dispersal of, 323 .

,, primordial germ cells, 130.

" selection by, 363 .

", sex determination, 135, 136, Fig. 67.

, spermatogenesis, 135 .

,, wings, 247.

instincts, adaptation in, 337 .

\section{, origin of, 184.}

integral division of chromosomes, 171, Fig. 77.

integration, 44.

integuments of ovule, 108.

intercellular substance, $57,123$.

interclavicle, 234, Figs. 89, 93.

intercrossing, swamping effects, 416 .

interstitial cells, 116.

intussusception, 20.

invagination, 47 .

invertebrates, dispersal, 325 .

, geological range, $28 t$.

Ipomoea purpurea, fertilization, 351 , 352.

irritability, 18.

irritable structures in flowers, 362, 363.

Isoëtes, 105 .

isogamy, 84, 89, 97 .

isolation, $331,416-419$.

Ithomiinæ, 345 .

Lamarck on, $380,381$.

J.

JELLY-FISH, 114, 121, 265.

Joly, 286.

Jurassic Epoch, 284.

K.

KaKAPO, 397.

Kallima inachis, 338, Fig. 171.

kangaroos, 301.

Kant, 178.

karyogamy, 128.

karyokinesis, $69-79$, Figs. $31-33$.

karyoplasm, 14, 69.

karyosome, 70,77 .

katabolism, 9, Fig. 1.

kea, 398 .

khaki clothing, 342 .

kidney, effect of removal, 156 .

kinetic energy, 7 .

kingdoms, 226. kiwi, 258, 331, 397, Figs. 111, 112.

knee, of horse, 240 , Fig. 95 .

L.

LABELLUM, 359.

labyrinthodonts, 243, 406.

Lagena, non-adaptive characters, 419.

Lamarck, views of, 176, 373-382, 418.

Lamarck's four laws, 382.

Lamarckian factors, A. R. Wallace on, 393.

\begin{tabular}{|c|c|c|}
\hline & & \\
\hline ", & $"$ & $\begin{array}{l}\text { Charles Darwin } \\
\text { on, } 166,392 \text {, } \\
393 .\end{array}$ \\
\hline & ," & neglect of, 393 . \\
\hline & , & $\begin{array}{l}\text { Robert Cham- } \\
\text { bers on, } 355 \text {. }\end{array}$ \\
\hline
\end{tabular}

lampreys, dispersal, 325, 326.

" distribution, 329 .

lance-woods, 417.

pineal eye, 258, 260.

land connections, former, 328, 329.

,, planarians, dispersal, 325 .

larva of Antedon, 274.

, Ascidian, 277, Fig. 130.

" crabs, 276, Fig. 128.

, echinoids, 276 .

, frog, 272, Fig. 121.

," Grantia, 322, Fig. 164.

" ophiuroids, 275, Fig. 127.

larvae, 272.

larval forms, dispersal, 321,322 .

Leach, 227.

organs, $272,275,278$.

leaf insects, 338, 339, Figs. 170, 171.

, structure, 63-65, Fig. 26.

leg, of man, 237, 238, Fig. 94 .

legitimate unions, in Primula, 357.

legumin, 23.

Leigh, G. F., 348.

Lemuria, 328.

lemurs, distribution, 328.

leopard, distribution, 320 .

Lepidosiren, 255, 291.

Leptinotarsa, mutation in, 159, 160.

leucocytes, 51, Fig. 15.

level, changes of in land, 328 .

lianes, 350 .

life-history, 263 (see also ontogeny).

life, nature of, $2,3,4,11$.

Lilium, germination of pollen grain, Fig. 54 .

limbs, of arthropods, 246,247 .

", vertebrates, $235-246$.

Limnas chrysipyus, 346, Fig. 177.

linin network, 70 . 
Linnæan species, 412.

Linnæous, 222, 226, 228.

Linnean Society, 340, 385.

lithium larvæe, $15 \%$.

Lithobius, host of Coccidium, 87 .

Litopterna, 253, Fig. 109.

lizards, pineal eye, 258. , shoulder girdle, 234, Fig. 89. llama, Buffon on, 367 . , feet, 241.

Lock, R. H., 152.

locomotion in Amœba, 15.

Loeb, J., 145, 146.

Lubbock, Sir John, 281.

Lull, R. S., 307.

luminous organisms, 6 .

lung-fishes (see Dipnoi).

lungs and swim-bladder, 255, 256.

Lyell, Sir Charles, $324,3 \varangle 5$.

Lygosoma dendyi, 417.

$$
\text { " moco, } 417 .
$$

lyre bird, 349, Fig. 178 .

lysin theory of fertilization, 146, 147 .

\section{M.}

Macculloch, 340 .

machine, analogy of, 2, 3, 10.

Macropodia rostrata, 339,340, Fig.172. magnesium larvæ, 157, Fig. 75 . maize root, section, 37, Fig. 7 . male animal, 114.

„, characters, $84,85,127$.

malic acid, attracting spermatozoa, 141.

Malthus, 386.

Mammalia, compared with Reptilia, $232-235$.

" dispersal, 323.

", geological history, 284, $301-304$.

", gigantic, 406 .

", limbs, 235 .

, nutrition of young, 270 .

" origin, 300 .

" ovum, 140, Fig. 71.

mammoth, Buffon on extinction of, 366.

man, xesthetic development influenced by insects, 364 .

, and apes, relations, 42\%, 423, 424,425 .

Buffon on, 370 .

Lamarck on, 382 .

,, antiquity, 284, 303, 422, 423. man, control of environment, 426 .

, evolution, $422-427$.

" , Charles Darwin on, 423.

, influence on other organisms, 396.

, limbs, 237, Fig. 94.

", Mendelian inheritance in, 205.

", progress of, $422-42 \pi$.

, races of, 423 .

", reversion in, 262.

," vestigial hair, 261.

" " tail, 261.

Mantida, 338, 339.

manubrium, 120.

Maoris and Morioris, 398-400.

marginal canal, 120.

marine animals, dispersal, 321.

fauna and flora, 323 .

Marsh, O. C., 262, 307.

marsh tit, distribution, 319, 320 .

Marsilea, 105.

marsupial mole, 253.

wolf, 251.

Marsupialia, 250, 301, 331.

", distribution, 329, 330, 331,332 .

extinct, 302, 332 .

Mastodon, 314.

Mastodonsaurus, 296.

maternal chromosomes, 137, Fig. 68. functions, a handicap, 127. matter, indestructibility of, 6 .

maturation of germ cells, 132,137 , 138, Figs. 65, 68 .

in heredity, 171.

Mauritius, 258.

Mediterranean fauna, 323.

meduste, 120, 121, 123, Fig. 60.

megagametes, 85, 88, 90.

megalecithal, 267.

meganucleus, 39, 92.

megasporangia, 107 .

megaspores, 105, 106, 107, 108.

Megatherium, 303.

meiosis, 132, 206.

memory, 186, 191, 192.

Mendel, G. J., 195.

Mendelian experiments, 194-209.

" inheritance in Leptinotarsa, 160.

in Primula, 356.

principles, application of, $20 \dot{0}$.

Mend̈elism, 195 proportions, 199.

, and mutations, 415. 
Menura superba, 349, Fig. 178. meristem, 76 .

meristic mutations, 154 .

, variations, $148,149$.

merogony, 146,174 .

Merychippus, 310, Fig. 153.

mesentery, $\tilde{5} 4$.

mesoblast, 48, 266.

mesoblastic somites, 266.

mesoderm, 48, 124.

mesogloea, $115,116$.

Mesohippus, 310, Figs. 153, 156.

mesophyll, 50, 64, Fig. 26.

Mesozoic Era, 284, 285.

metabolism, 9, Fig. 1.

metacarpals, 238.

metagenesis, 121.

metameric segmentation, 149, 266, 279.

metamorphic rocks, 283.

metamorphosis, 263.

Metaphyta, 44, 95.

metapodials, $240,241$.

metatarsals, 238.

Metatheria, 301, 302.

Metazoa, 44.

mice, dispersal, 325.

" experiments in heredity, 179 , 182.

microgametes, $85,88,91$.

microlecithal, 267.

Microlestes, 301, 302.

micronucleus, 39, 92.

micropyle, 109.

microsporangia, $10 \%$.

microspores, 105, 106, 107.

microzooids, 33.

migration from north, 329 .

milk, 271.

mimicry, 343-348.

" and fluctuating variation, 415.

,, and natural selection, 396, 405 .

rings, 345 .

mineral salts, 23.

Miocene Epoch, 284.

Miohippus, 310.

mitosis, $69-79$, Figs. $31-35$.

,, in heredity, 169.

mnemic theory of heredity, 186, 191, 192.

moas, $258,397$.

models and mimics, 345,348 .

Mœritherium, 313, Fig. 159.

moles, marsupial, 253.

Molluscoida, 277 . momentum in evolution, 406-409.

monads, 83 .

Monera, 66.

monoecious, 103, 105.

monohybridism, 201, Fig. 79.

monopodial branching of phylogenetic tree, 230, 231, Fig. 87.

monosome, 135 .

Monotremata, 234, 296, 301, 302, 331. monstrosities, $154,157$.

Morioris, extermination, 398-400. mosses, dispersal, 321.

moths, clear-winged, 343, Fig. 175.

,, proboscis, 354 .

," protective resemblance, 338 .

motion, in living organisms, 6 .

mountain hare, distribution, 333.

mud, dispersal in, 327 .

mud-fishes, 255, 291.

Müller, Fritz, 346.

Müllerian mimicry, 346.

multicellular, 38.

$$
\begin{aligned}
& \text { organisms, origin of, } \\
& 44 .
\end{aligned}
$$

Multituberculata, 302.

multiplication of cells, $69-80$.

Mungoswell's wheat, 412.

Muscari, variation in, 150 .

muscle fibres, $57,58,116$, Figs. 21 , 22,58 .

music and memory, 192.

mutation and adaptation, 414, 415 .

$$
\begin{aligned}
& \text { ", } \quad \begin{array}{l}
\text { evolution, 414. } \\
\text { hybridization, } \\
414 .
\end{array} \\
& \quad, \quad \begin{array}{l}
\text { Mendelism, 415. } \\
\text { natural selection, } 414 .
\end{array} \\
& \text { ", theory, 224. }
\end{aligned}
$$

mutations, 149, 153-156, 224.

, meristic, 154 .

," origin, $159,160$.

" " of species from, $411,412,413$.

substantive, 154 .

mutilations, inheritance of, 178,179 . mutual adaptation, 414,415 .

Mycetozoa (Myxomycetes), 67, Fig. 29.

$$
\text { N. }
$$

NÄGELI, 167.

natural affinities, 228, 229.

" selection, 395-409.

$\begin{array}{cc}, & \text { and mutation, } 414 . \\ \text { A. R. Wallace on, } & 389-391 . \\ & \quad, \quad \text { Buffon on, } 368 .\end{array}$


natural selection, Charles Darwin on, $385-388,392$.

, $\quad \begin{aligned} & \text { Erasmus Darwin } \\ & \text { on, } 373 . \\ & \text { insufficiency of } \\ & 404 .\end{aligned}$
, $\quad$ insufficiency of,
Charles Darwin
on, 392.
summary of theory,
391.

," system, 226.

nectaries, 359, 360, 414, 415.

Neoceratodus, 255, 291, Fig. 110.

Neohipparion, 310, Fig. 157.

Nepenthes, 262.

Nerocila, 227.

nerve-cells, 58, Figs. 23, 24.

, fibres, 58 .

nerves, 59 .

nervous system, 18, 121, 266.

neurons, 58,59 .

neuter insects and heredity, 189, 190 .

newts, 294.

New Zealand, fauna and flora, 258, $328,329,331$.

nipples, vestigial, 345 .

nitrifying bacteria, 218 .

Nitrobacter, 218.

Nitrosomonas, 218, Fig. 84.

nomenclature, $226,22 \%$.

non-adaptive characters, 419-422.

normal curve of variation, 153 . variations, 150 .

notochord, 266.

Notornis, 397.

Notoryctes, 253.

nucellus, 108.

nuclear membrane, 69 .

nucleinic acid, 71 .

nucleolus, 70,77 .

nucleoplasm, 14, 22, 69.

nucleus, 13,69 .

" division (see mitosis and amitotic nuclear division). in heredity, 161, 166, 174 .

", $\quad$ in heredity, 83.

numerical variations, 149 .

nutrition, 7 (see also food materials). " of embryo in Colomata, 125.

O.

ObELia, 119, 265, Fig. 60.

ocean, age of, 287 .

oceanic islands, fauna, $330,332,397$.

oceans, permanence, 329 .
Enothera, mutstion in, 155 .

oil-foam, 19, 21.

Olenus cataractes, Fig. 134.

Oligocene Epoch, 284.

ontogenetic record, obscuring of, $26 \overline{7}, 278$.

ontogeny, 263.

" abbreviation of, 273.

", a habit, 192.

" and phylogeny, 265, 27!, Fig. 131.

" Erasmus Darwin on, 371, 372 .

interpretation of, 277 .

Onychophora, distribution, 330 .

oocytes, 133.

oogenesis, 133, Fig. 65.

oogonia, of Fucus, 100.

,$\quad$ in oogenesis, 133.

oospheres, 90, 100, 101, 108.

ooze, $26,283$.

opal, $24,26$.

Ophisaurus, 250.

Ophiura ciliaris, Fig. 126.

ophiuroids, $275,276$.

opossums, 301.

opposable great toe and thumb, 424 . orang, brain, 425 .

“orang utan," Buffon on, 370 .

Orchidace:e, fertilization, $359-362$.

Orchis mascula, fertilization, 359 362, Fig. 182.

orders, 226.

Ordovician Epoch, 284.

organellæ, 39.

organic evolution, doctrine of, 212 (see also evolution).

organism, 3. units, 38, 65 .

organisms, first appearance of, 212.

" nature of first, 217, 219.

" number and variety of, 211.

origin of, 214-216.

organizing spirits, A. R. Wallace on, 394.

organs, 12, 38.

origin of great groups, 303,304 .

" , living things, $214-216$.

", , sex, 84, 85, 89, 126-128.

Ornithorhynchus, 234, 260, 301,

Fig. 91.

Ornithosauria, 299.

Ornithoscelida, 297.

Orohippus, 310, Figs. 153, 155.

Osborn, 369.

osmosis, 8, 17, 23. 
osmotic pressure, effect on egg, 145. Ostracodermi, 289, 290, Figs. 135, 136.

Otaria hookeri, paddles, Fig. 100.

otter sheep, 154.

ovary, of animals, $113,116$.

", flower, 107.

oviduets, 125.

ovotestis, 113.

ovule, 48, 106, 107, Fig. 5็.

ovum, 85.

, interpretation, 279.

, maturation, 133, Figs. 65, 68.

" of Amphioxus, 45, Fig. 13.

, , Ascaris, 131, Fig. 64.

, , bird, 140, Fig. 70.

, , Chara, 141.

", ", Coccidium, 88, Fig. 39

," "Eudorina, 91, Fig. 40.

", ", fern, 104, Fig. 52 .

" ,flowering plant, 108, 109 , Fig. 55.

,, Fucus, 10n, Fig. 47.

,, Hydra, 116, Figs. 57 59.

, mammals, 71, 271, Fig. 140.

, medusæe, 121.

," plants, 141.

," rabbit, Fig. 71.

", sponges, 113.

, Volvox, 91, Fig. 11.

segmentation of, $263,265$.

similarity in different organisus, 162.

size of, 267.

, typical, 140, Fig. 69.

oxen, feet, 241.

oxidation, 5 .

oxlip, fertilization, 356 .

oxygen, $5,6,8,31$.

oyster, American, introduced, 323.

P.

Paddles, 236, 244, 247.

pademelons, coloration, 337.

predogenesis, 144.

pairing of chromosomes, 133, 137, 206, Figs. 65, 66, 68.

Palæohatteria, 295.

Palæomastodon, 313, Fig. 159.

palsoontology, evidence afforded by, 287-304.

Palæospondylus, 289.

Palæoozoic Era, 284, 285.

palingenetic characters, 277.

palms, climbing, 350 .
Pandorina, 42, 89, Fig. 10.

pangenesis, $164-166$, 369, Fig. 76 .

Papilio, mimicry in, $346-348$, Fig. 177.

parallel modification of body and germ cells, 182.

Paramoecium, 39, 98, 139, Fig. 8.

paraphyses, of Fucus, 100.

parasites, peculiarities of, 401-403. parasitism, of gametophyte, 112.

parenchyma, 65.

Pariasaurus, 297, Fig. 140.

parietal eyes, 258-260, Figs. 114116.

, foramen, 260.

artificial, $144-147$.

Parus palustris, distribution, 319,320 .

passivity of female, 84,85 .

Pasteur, 215.

Patagonia, beech forests, 329 .

paternal chromosomes, 137, Fig. 68. peach trees, inheritance of acquired characters, 182.

peacock, epigamic ornamentation, 349 . Pearson, Karl, 210.

pea, Sicilian, 208.

," structure and germination of seed, Fig. 56 .

peas, Mendelian experiments on, $196-199$.

pedigrees, $229,311$.

Peebles, Florence, 28.

pelagic organisms, $322,336$.

pentacrinoid stage of Antedon, 274, 275, Fig. 124.

Pentacrinus, 274, Fig. 125.

pentadactyl limbs, 237, 294 (see also limbs and feet).

periblem, 50 .

Pericopis angulosa, 345, Fig. 176.

Peripatus, Fig. 167.

perisare, 119.

distribution, 330 .

perissodactyl, 241, 253.

peristaltic movement, 57.

peritoneal epithelium, 54, Fig. 16.

Permian Epoch, 284.

Perrhybris malenka, 345, Fig. 176.

persons in colony, 119.

Petalotricha, Fig. 9.

petals, 106.

phagocytes, phagocytosis, 52.

phalangers, 301 .

phalanges, 238.

Phascolotherium, 302, Fig. 149.

Phenacodus, 309, Fig. 152. 
phloem, 65 .

phosphorescent organs, 336.

phosphorus, 22, 71 .

photosynthesis, 30, 31 .

phyla, 226.

phyllodes, 280.

Phyllopteryx eques, 341, Fig. 174. phylogenetic tree, 229.

phylogeny, 229.

and classification, 230, 231, Fig. 87.

ontogeny, 265,279 ,

Fig. 131.

Erasmus Darwin on, 372.

physical basis of life, 7 .

, conditions of earth, changes in, 327 .

physiological selection, 418 .

unit, the cell, 68 :

Phytoflagellata, colony formation, 40, 42.

" evolution of sex, 89

Pierinæ, 345 .

pig, feet, 241, Fig. 98.

pineal eyes, 258-260, Figs. 114-116.

," gland, 11, 260.

pin-eyed flowers, 356 .

pistil, 106.

Pisum sativum, varieties, 196.

pitcher plants, 262.

Pithecanthropus erectus, 423.

pituitary body, 408.

placenta, 270.

placenta1 mammals, appearance of, 302.

Playianthus betulinus, 417.

Plagiaulacidee, 302.

Plagiaulax, 302, Fig. 148.

Planemu poggei, 346 .

plankton, 322 .

plants, compared with animals, 34,35 .

, dispersal of, 321.

plasma, 51 .

plasmodia, 67 .

plasmogamy, 128.

plasticity of organisms, 335 .

plastids, 32 .

plastogamy, 128.

Platypus (see Ornithorhynchus).

Pleistocene Epoch, 284.

plerome, 50 .

Plesiosauria, 236, 244, 297.

Plesiosaurus macrocephalus, Fig. 141. Pliny, 262.

Pliocene Epoch, 284.

Pliohippus, 310, 311, Figs. 153, 158. ploughshare bone, 305 .

plumage of birds, 349 .

plumcots, 204.

pluteus larva, 275, 276, Fig. 127.

polar bodies, 133, 135, 139, 145, Figs. $35,65,68$.

pole-cell, in insect egg, 130 .

pollen, collection of, 355 .

," grains, 106, $10 \%$.

, sacs, 107.

, tube, 108.

pollination, 110, 111 .

pollinia, 361 . adaptations for, $351-363$.

polyanthus, pollination, 356 .

polydactylism, 149.

polymorphic colony, 119.

polymorphism in Papilio, 346, Fig. 177.

polype, fresh water (see Iydra).

Polypodium, archegonium, Fig. 52.

Polyzoa, dispersal, 327 .

population, Buffon on, 368 .

, Charles Darwin on, 386.

", Lamarck on, 381.

, Wallace on, 389.

porpoises, convergence in, 248.

Portunus, 247, Fig. 102.

postaxial, 238.

larva, Fig. 128.

potential energy, 7 .

Poulton, 1E. B., 336, 337, 346.

preaxial, 238.

Pre-Cambrian Epoch, 284, 285.

pre-formation in development, 163.

prehension, organs of, 256.

prepotency, 3533,358 .

presence or absence hypothesis, 207.

Primary Era, 284, 285.

Primates, 301, 303.

primordia, in germ plasm, 206.

primordial germ cells, 113 (see also germ cells, origin).

utricle, 62, 95.

Primula, fertilization, 356-358.

priority in nomenclature, 227.

Proboscidea, evolution, $312-314$,

Fig. 159.

proboscis, of bee, 354, Fig. 179 .

butterflies and moths,
354.
,. $\quad$ insects, Erasmus Darwin
on, 373.
mutual adaptation,
$414,415$.

procryptic coloration, $337-342$. 
progress, human, 426, 427.

progressive development, $R$. Cham-

bers on, 384.

, evolution, 334 .

, $\quad$ cause of, 192.

", Wallace on, 390 .

, series, 232, 234 .

pronuclei, 131, 145, 146, Fig. 64.

protandrous, 353, 363 .

protective resemblance, $337-342$.

, and fluctuating variation, 415 .

", natural selection, $396,405$.

proteids, 7, 22.

Proterotherium, 253, Fig. 109.

Proteus, 183.

prothallus, of fern, 103.

Protista, 38.' , flowering plant, 108.

, dispersal of, 327 .

" immortality of, 161 .

protogynous, 3033 .

Protohippus, 310.

Protophyta, 38, 95.

,, sexual differentiation in, 91.

protoplasm, 2, 38.

" chemical composition, $22,23$.

, $\quad$ controlling power, 23.

, energy in, 7 .

," physical properties, 21.

, selective action, 26.

" sensitive to stimuli, 188.

, streaming, 62.

Protopterus, 255̃, 291.

Protorohippus, 310, Fig. 153.

Prototheria, 301, 302.

Protozoa, 38, \&c., Figs. 3, 4, 9, \&c.

, non-adaptive characters, 419.

,, nuclear reduction, 139.

", powers of multiplication, 81.

, sexual differentiation, 89.

Prozeuglodon, 318, Fig. 163.

prunes, stoneless, 204.

Pseudopanax chathamicum, 417.

pseudopodia, 15. crassifolium, 417.

ferox, 417.

Pteranodon, 299, Fig. 147.

Pteraspis, 290.

Pterichthys, 290, Fig. 136.

Pterosauria (pterodactyls), 236, 242, 299, Figs. 99, 147.
Pterostylis trullifolia, fertilization, 362.

Pterygotus osiliensis, Fig. 137.

Pulchriphyllium crurifolium, 338, Fig. 170.

Punnett, R. C., 208.

purity of gametes, 200, 206.

putrefaction, 218.

Pyrenees, aretic vegetation on, 333. pyrenoids, 32, Figs. 5, 42.

Q.

QUADRUMANA, 424. quartz, 24.

R.

RABBit, embryo, 265, Fig. 117.

shoulder girdle, 234, Fig. 90 . racial characters, transmission, 175. radial canals, 120 .

Radiolaria, 25, Fig. 3.

radius, 237 .

rafts, dispersal by, 324 .

range in time of animal groups, 284.

Ranunculus aquatilis and Ranunculus hederaceus, Lamarck on, 379.

rats, $179,182,325$.

recapitulation hypothesis, $265-281$.

, ,, anticipated

receptor, 18 .

by Erasmus Darwin, 372 .

recessive characters, $197,207$.

recognition marks, 337 .

red blood corpuscles, 52, 53, Fig. 15.

", clover, robbed by humble bee, $355,356$.

Red Sea, fauna, 323.

red snow, 27 .

reduction of chromosomes (reducing division), 132-139, 206, 207, Figs.

$65-68$.

regeneration, 167 .

regression, filial, 210 .

rejuvenescence, 92 .

reproduction, 10 .

Reptilia, compared with Amphibia, 294.

, compared with Mammalia, $232,235$.

fossil, $295-299$.

", geological range, 284.

", giant, 406 .

" limbs, 235 .

, origin, 294,29 ग. 
respiration, 8 .

$$
\begin{aligned}
& \text {, in Amoba, 17. } \\
& \text {, } \quad \text {,, embryos, } 270 . \\
& \text {, plants, 32, } 33 .
\end{aligned}
$$

response to stimuli, 18.

Réunion, peach trees in, 182.

reversion, 261, 262.

$$
\text { , Mendelian explanation, }
$$
208.

rhinoceros, feet, 241.

Rhynchocephalia, 259, 295 (see also Sphenodon).

ribbon-wood, 417 .

ribs, meristic variation, 149 .

right whale, 245, Fig. 101.

Ritzema Bos, 179.

rock-formation, 282.

rodents, 301 .

"Rodney," brig, 399.

Rodriguez, 258.

Romanes, G. J., 333, 417, 418.

Röntgen rays, $18 \bar{\gamma}$.

root-cap, 50.

root, growing point, 76 , Figs. 33,34 .

Rosenthal, 179.

rostellum, 360 .

rotifers, parthenogenesis, 144 .

rudimentary organs (see vestigial organs).

Charles Darwin on, 392.

Rumia crategata, larva, Fig. 169.

rust, in wheat, 205.

rye, fertilization, 412 .

\section{S.}

SAcculind, degeneration, 401, Fig. 183.

sage (see Salvia).

Sagitta, origin of germ cells, 130 , Fig. 63 .

salt, in sea, $28 \%$.

Salvia, structure of flower and fertilization, 358, 359, Fig. 181.

Salvinia, 105.

Sandwich Islands, oceanic, 332.

sap, 65 .

,, nuclear, 70 .

saprophytic nutrition, 34 .

sarcode, 38.

Sarracenia, 262.

Sauropsida, 300.

Sauropterygia, 297, Fig. 141. scapula, 234, Figs. 89, 90, 93. scents, of flowers, $35 \mathrm{~J}$.
Schleicher, 69.

Schleiden, 38.

Schøtensack, 423.

Schwann, 38.

Scrophulariu nodosa, 353.

seurf, 55.

sea anemones, 114.

,, coast, selection in, 395 .

,, firs, 114.

", urchins, artificial partheno-$$
\text { blastomeres, } 45 .
$$

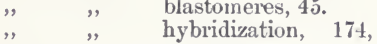

" " 175.

larvie, 276 .

seal, padulles, 244, Fig. 100.

Secondary Era, 284, 285.

$$
\begin{gathered}
\text { sexual characters in } \\
\text { Coclomata, } 125 .
\end{gathered}
$$

secretions, controlling growth, 408 . internal, 126.

sedimentary rocks, $282,286$.

sedimentation, rate of, 285,286 .

seed, 109, Fig. 56.

, coat, 109.

", leaves (ste cotyledons).

seedling of Acacia, 280, Fig. 132.

," , pea, Fig. 56 .

seeds, dispersal, 321 .

Seeley, H. G., 297.

segmentation, metameric, 149, 266.

segmentation of ovum, 4i), 263.

in Amphioxus, 45, Fig. 13.

, birds and reptiles, 270 .

,, frog, 268, Fig. 119.

, Hydra, 118.

," mammais, 271.

interpretation of, 265 .

segregation of germ cells (see grerm cells, origin).

selection, artiticial, 395, 396.

$$
\begin{aligned}
& , \quad, \quad \begin{array}{c}
\text { Charles Darwin } \\
\text { on, } 387 .
\end{array} \\
& , \quad \text { by insects, } 396 . \\
& \text { continuous, } 411 . \\
& , \quad \text { germinal, } 173 . \\
& \text { natural (see natural selec- } \\
& \text { tion). } \\
& \text { not confined to living } \\
& \text { things, } 395 . \\
& \text { sexual (see sexinal selec- } \\
& \text { tion). } \\
& \text { single, } 412 .
\end{aligned}
$$

self-iertilization, $351,352,353$.

sematic colours, 336,342 .

Semon, Richard, 186 .

sonsation, 10. 
sense cells, stimulation of, 188 .

, organs, 18, 121.

sepals, 106.

series, progressive, 232, 234.

Sesia crabroniformis, Fig. 17 .

sex determination in insects, 13j, Fig. 67.

sexes, fundamental distinction

between, 127.

sexual characters iransferred to asexual generation, 110 , 111.

,, differentiation, origin of, 128. sexual phenomena, 84, 85 .

evolution of, 126, 127.

in Bodo, 87, Fig. 38 .

,, Coccidium, 88, Fig. 39.

,Colomata, 125.

,, Copromonas, 83, Fig. 37.

,, Eudoriua, 91, Fig. 40.

", ferns, 104, 105, Figs. 51, 52

", flowering plants, 108, 109, Figs. 54, 55 .

Fucus, 100, 101, Fig. 47.

Hrematococcus, 33, Fig. 5.

, Hydra, 116.

, Obelia, 121.

", Pandorina, 89, Fig. 10.

", Paramœecium, 93, Fig. 41.

,, Spirogyra, 96, 97, Figs. 43, 44.

, Volvox, 91, Fig. 11.

,Zygogonium, 97.

sexual reproduction, 33 .

," selection, Charles Darwin on, 388.

, " Erasmus Darwin

Shand, Alexander, 399. on, 373 .

sharks, fossil, 291.

sheep, Ancon or otter, 154.

feet, 241.

shepherd's purse, 48, Fig. 14.

Shirreff, Patrick, 412.

shoulder girdle, of mammals, 234, Fig. 90 .

\begin{tabular}{|c|c|c|}
\hline & & Ornith \\
\hline ", & " & 234, Fig. 93 . \\
\hline & , & $\begin{array}{l}\text { " reptiles, 233, 234, } \\
\text { Fig. } 89 .\end{array}$ \\
\hline
\end{tabular}

Sicilian pea, 208.

silica, $23,24$.

siliceous skeleton, 25 .

Silurian Epoch, 284.

single selection, 412 .

Sirenia, 301.

paddles, 236, 244.

size, increase of, 309, 405-409. skeleton (see feet, limbs, paddles and wings).

, of extinct animals, Figs. $139-152$.

, · , giraffe, Fig. 104.

, , horse and man, Fig. $9 j$.

, , kiwi, Fig. 112.

," , Obelia, 119.

," , Protista, 23-26, Figs. $3,4$.

whale, Fig. 101.

skull's of "dog and thylacine, 251,

Figs. 107, 108.

slime fungi (see Mycetozoa).

sloths, 333.

slow worm (see Anguis fragilis).

snails, dispersal, 325 .

snakes, mimicry in, 343 .

social problems, de Vries on, 414.

sodium, in sea water, $28 \%$.

sole of foot, Buffon on, 368 .

solitaire, $258,397$.

Sollas, W. J., 286, 287, 311.

coma and germ cells contrasted (see germ cells and somatic cells).

somatic and germ nuclei in Paramoecium, 98.

somatogenic characters, supposed non-inheritance, 169, 176 (see also acquired characters).

somatogenic variations, 149,136 157.

somatopleure, 124.

somites, 266.

soul, 11, 19.

South America, fauna and flora, 328, $329,333$.

Spalacotherium, 302.

special creation, 212-214, 222, Fig. 82.

Buffon on, 369.

," $\quad$ Buffon on, 369.
Lamarck on, 375, 376.

species, aggregate, 224 .

, Charles Darwin on, 223.

, definition of, 223 .

, elementary, 224.

, Lamarck on, 375.

, modification of, Buffon on, 366,367 .

nature of, $222-224$.

number of living, 211.

origin from mutations, 225.

supposed immutability of (see immutability of species).

transformation of, Lamarek on, 376,377 . 
specific characters, non-adaptive, 419-422.

speech, an acquired character, 156, 184. evolution of, 425.

Spencer, Herbert, 2, $185,391$. spermary, 113.

spermatids, 133, Figs. 65, 66. spermatist (Erasmus Darwin), 371. spermatocytes, 133, Figs. 65, 66. spermatogenesis, $132,133,134,135$, Figs. 65, 66.

spermatogonia, 133, Figs. 65, 66. spermatozoa, 85, 139, Fig. 69.

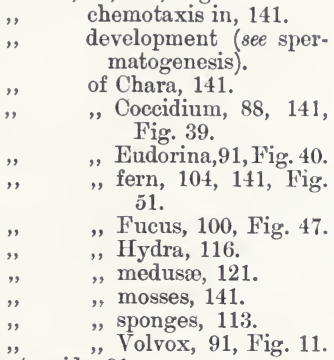
spermatozoids, 91 .

sphærechinus, experiments in hybridization, 174 .

Sphærella (see Hæmatococcus). Sphærozoum, 43, 265, Fig. 12.

Sphenodon punctatus, 259, 260, 295, 320, 331, Figs. 113, 114.

spicules (see sponges, spicules). spider crab, 339, 340, Fig. 172. spindle, nuclear, 71 .

spines, excessive development, 406. spiny anteater (see Echidna).

spireme, 71 .

Spirogyra, 95--97, 142, Figs. 42-44. splanchnopleure, 124.

splint bones, 241,257 .

sponges, deep sea, 335.

" dispersal of fresh water, $32 \%$.

gemmules of fresh water, 327, Fig. 166.

germ cells, 113 .

non - adaptive characters, $419-421$.

spicules, 26, 232, 419, 420, Figs. 88, 187.

Sponyilla fuviatilis, gemmules, Fig. 166. spontaneous generation, 214-216, 219.

Lamarck on, 374.

sporangia of fern, 101, Fig. 48.

spores, 101. , flowering plant, $10 \%$, Fig. 53.

, dispersal, 321.

" . formation, reduction of chromosomes, 138.

germination, in fern, 103.

of Bodo, 8i.

", , Coccidium, 89.

sporophylls, 107 .

sporophyte, 101.

, of fern, 105, Fig. 48.

, , flowering plants, 106.

" number of chromosomes, 139.

sports, 154, 224 (see also mutations).

Squalodontidøe, 318.

Squamata, 295.

stability of organisms, 155 , Fig. 74 .

stag, antlers, 125.

staining reactions, 62,70 .

stamens, 106.

staminodes, 262.

starch, 31.

star-fish, meristic variation, 149 .

statoblasts, 327 , Fig. 165.

stature, variation in human, 152 .

Staurocephalus murchisoni, Fig. 134.

Stebbing, T. R. R., 339.

Stegocephalia, 293.

Stegosaurus, 299, Fig. 145.

Stenobothrus viridulus, spermatogenesis, Fig. 66.

Stentor, Fig. 9.

Stereoguathus, 302.

sterility of species when crossed, 418.

St. Helena, an oceanic island, 332.

stick caterpillars, 337, 338, Fig. 169.

Stieda's organ, 260, Figss. 115, 116.

stigma, of flower, $10 \%$.

stimulation of protoplasm and cells, 188.

stimuli, 18.

to development, $145,146$.

stimulus, transmission between cells. 143.

," , to germ cells, $186-190$.

Stockard, $15 \%$.

stomata, 64, Fig. 26.

storms, dispersal by, 324 . 
stratified epithelium, 56, Fig. 18. rocks, $282-286$.

striped muscle, 57 , Fig. 22.

struggrle for existence,

A. R. Wallace on, 389.

Buffon on, 368.

Charles Darwin on, 386.

importance of, 397, 398, 400 .

Lamarek on, 381 .

stump tails, 179 .

style, of flower, 107 .

Stylidium graminifolia, fertilization, 362 .

subclasses, 226.

subfamilies, 226.

subgenera, 225.

subkingdoms, 226.

suborders, 226.

substantive mutations, 154 .

succulent plants, 350 . variations, 148, 150.

Sumner, F. 13., 182.

sun, dependence of life upon, 5 . suprascapula, 234 .

surface, relation to volume, 20.

, tension, 19.

survival of fittest, 391 .

\begin{tabular}{|c|c|c|}
\hline & & Buffon on $368^{\circ}$ \\
\hline " & & Charles Darwin on, \\
\hline & , & in inorganic world, \\
\hline
\end{tabular}

suspensor, 49 .

Svalöf, 412 .

swcet peas, reversion in, 208.

swim bladder and lungs, 255, 256.

synaposematic groups, $343-346$,

Fig. 176.

synapsis, 133, 137, 206, Figs. 65, 66, 68.

synaptic mates, 135 .

syncytia, 66 .

syneytial epithelium, 67, Fig. 28.

syndesis, 137 (see also synapsis).

syngamy, 83 (see also conjugation). systematist, work of, 226 .

$\mathrm{T}$.

TADPole, ascidian, 277, Fig. 130.

" frog, 272 , Fig. 121.

$\because \quad$ stage, $278,279$.

tail, in man, 261, 262, 424 .

tails, mutilation of, 178,179 .

tapir, feet, 241, Fig. 96.

tarsals, 237.

taxonomic tree, 228, 229, Fig. 86. taxonomy, 226.

aims of, 229.

teeth", of Cetacea, 317,318 , Figs. 162, 163.

" , dog and thylacine, 251253, Figs. 107, 108.

, , elephants, 314.

", , horses, 309.

", vestigial, 260 .

Teleostei, 292.

telescoping of generations, 111, 112, 122.

temperature of body, 6 .

tentacles, 115, 118 .

Tertiary Era, 284, 285.

testis, 113, 116.

Tetrabelodon angustidens, 314, Fig. 159.

, longirostris, 314, Fig. 159.

Tetraxonida, spicules, 232, Figs. 88, 187.

Theosodon, 253, Fig. 109.

Theromorpha, 296.

Thoatherium, 253, Fig. 109.

thrum-eyed flowers, 356.

thylacines, 301.

Thylacinus, 2251, Figs. 107, 108.

tibia, 237 .

time, geological, $285-287$.

,, importance in evolution, Buffon on, 366.

,, in evolution of horse, 311.

," scale, geological, 284.

Tinoceras, 303, 406, Fig. 150.

Tintinnopsis, Fig. 9.

tissue-formation, $45,50,51,75-79$. tissues, $37,51-65$.

Tithorea harmonia, 345, Fig. 176.

tools, use of, $424,425$.

torpedo, 256.

tortoise, 334 .

Tower, W. I., 156, 159, 184.

Tradescantia, histology of, 61-65, Figs. 25, 26.

transformation of species (see species).

transparency, of pelagic animals, $322,336$.

trce-like classification, 228, 229, $\mathrm{Fi}$ 86. evolution, 2:1.

Trematoda, parthenogenesis, 144.

Triassic Epoch, 284.

Triceratops, 299, Fig. 146.

trichocysts, 39 .

Triconodon, 30\%. 
trilobites, 289, Fig. 134.

Trimen, R., 346.

Triticum sativum, Lamarck on, 378 .

Tritylodon, 301, 302.

tuatara (see Splicnodon punctatus).

Tubularia, 121, Fig. 61.

turbellarian, fertilization of polar bods, 135.

turbot, colour changes, 342 .

turtles, dispersal, 323

, limbs, 236, 244.

twins, identical, 173.

types, in wheat, 412.

Tyndall, 215.

Typhlopidæe, 250.

\section{$\mathrm{U}$}

ULNA, $23 \%$.

unconscioús memory, 191, 192.

ungulates, 301.

limbs of, $239-241$.

unguligrade, 240.

unicellular, 38 .

unisexual, 99, 105.

unit characters, 200, 206.

, , $\quad$ origin of, $415,416$. unstriped"muscle, 57, Fig. 21.

Urceotyphlus africanus, Fig. 106.

urea, 9.

Ursus arctos, systematic position, 229 ,

Fig. 86.

use and disuse, effects of, 156,166 .

Buffon on, $36 \bar{\tau}-368$.

Charles Darwin on, 391, 392.

Lamarck on, 377, 378, 379, 380, 382.

R. Chambers on, 385 .

uterus, 125 .

\section{V}

Vacuoles, contractile, 39, 83.

Varanus, shoulder girdle, 234, Fig. 89.

variation, $148-160$.

, cause of, 166.

, curve of, 150, 153, Figs. 72, 73.

in grape hyacinth, 150, Fig. 72.

, human stature, 152, Fig. 73 .

variations, blastogenie, $149,157-$ 160.

congenital, 157.

,

continuous, 148, 150. variations, discontinuous, 149,153 $-156$.

fluctuating, 148, 150, 155, Figs. 72, 73.

germinul, 149, 157-160.

individual, 150 .

inheritance, 403,404 .

meristic, 148, 149.

normal, 150 .

origin, 153, 173, 403, 404.

selective value, 403,404 .

somatugenic, 149,156 , $15 \%$.

substantive, 148, 150 .

vasa deferentia, 125 .

vascular bundles, 65 .

system in plants, 50 .

vegetable marrow, flowers, 111.

vegetative cell in pollen grain, 108.

Vergil, 214.

vertebræ, meristic variation, 149 .

vertebral column, development, 266.

Vertebrata, geological history, 289 -304 .

origin, 291.

Vespa crubro, Fig. 175.

vessels, of plants, 65.

vestigial organs, $257-262,271,345$, 397.

Charles Darwin on, 392.

vibrations, in determinants, 189.

Virchow, 38.

vital force, 11, 143, 216.

vitalism, 19.

vitelline membrane, 140.

volume, increase of, Lamarck on, 382.

relation to surface, 20 .

Volvox, 42, 91, 97, 98, 265, Fig. 11.

Von Baer, 26 .

Ton Mohl, 38.

Vorticella, 40, Fig. 9.

II.

WaIsT's, slender, 156.

Walker, C. E., 175.

Wallace, A. R., views of, 319,329

$359-391,393,394$.

warm-blooded animals, 6 .

warning colours, $342-34 \mathrm{~s}$.

,, ," and natural selec tion, 396 .

wasps, colours, 342 .

waste products, 8,9 . 
wattles, life-history, 280. weapons, use of, 424,425 .

weeds, dispersal, 325 .

Weismann, views of, 146, 166-1it, $176,177,178,179,180,185,189$, $206,219$.

weka, 397,398 .

Wells, W. C., 385.

Western, 387.

whales, ancestry, $314-318$.

" cervical vertebræ, 248, Fig. 103.

,. convergence in, 248 .

,, paddles, 244, Fig. 101.

,, size, 303.

", systematic position, 301 .

", vestigial organs in, 257,260 , Fig. 101 .

wheat, improvement of, 411-413. Lamarck on, 378.

wind, dispersal by, 323.

winged animals, dispersal of, 323 . wings, 2:36.

, convergence in, $248-250$.

," of bat, 243, Fig. 99.

," , bird, 243, 244, Fig. 99.

", " insects, 247.

, , pterodactyls, 242, Fig. 99. wireless telegraphy, analogy of, 187 . Wolff, C. F., 163.

wombats, 301 .

wood, 65 .
Woodward, A. S., 253, 294, 296, 300, $301,302$.

worms, dispersal, 325 .

$x$.

XYLeM, 65.

$$
\text { Y. }
$$

YoLK, 140, 267.

", corpuscles, 117 .

," influence on development, 268 $-2 \pi 0$.

,, sac, 270, 271, Fig. 120.

7.

ZEUGLODONTID E, 318.

zosea larva, 276, Fig. 128.

zona radiata, 140 .

zooids, 43, 119.

zoophytes, 34, 119.

zoospores, 29.

Zoothamnium, 40.

Zosterop ' lateralis, dispersal, 324 .

Zygogonium, conjugation, 97, 143. zygosis, 83,131 .

zygospore, 97.

zygote, 33. 83, 8̄ , 88, 91, 97, 101, $105,109,118$.

conjugation of nuclei in, 131, Fig. 64 . 


\section{FROM CONSTABLE'S LIST}

\section{Life Histories of Northern Animals.}

An Account of the Mammals of Manitoba.

By Ernest Thompson Seton, Naturalist to the Government of Manitoba. In two volumes. Large 8vo. Over 600 pages each. With 7o Maps and 600 Drawings by the Author. Price $73 s$. 6\%. the set. Prospectus on application. basis.

An important book of popular natural history on a strictly scientific

The Origin and Distribution of Life in America.

By Robert Francis Scharff, Ph.D., B.Sc., F.Z.S., M.R.I.A., Keeper of the Natural History Collection at the National Museum of Ireland, Author of "European Animals." Illustrated with Maps. Demy 8vo. Ios. 6d. net.

European Animals : Their Geological History and their Geographical Distribution.

By Robert Francis Scharff, Ph.D., B.Sc., F.Z.S.. M.R.I.A. Numerous Illustrations. Large Crown 8vo. $7 s, 6 d$. net.

\section{Extinct Animals.}

By Sir E. Ray Lankester, F.R.S. With a Portrait of the Author and 21 8 other Illustrations. Demy 8vo. New Popular Edition. 3s. 6 d. net.

NATURE:- "We give the book a hearty welcome, feeling sure that its perusal will draw many young recruits to the army of naturalists, and nany readers to its pages."

\section{A Guide to the Study of Fishes.}

By David Starr Jordan, President of Leland Stanford Junior University. With Coloured Frontispieces and 427 Illustrations. In two volumes. Folio. 5os. net. 


\section{The Birds of the World.}

A popular account by Frank H. Knowlton, M.S., Ph.D., Member American Ornithologists' Union, President Biological Society of Washington, etc., etc. With Chapter on Anatomy of Birds by Frederick A. Lucas, Chief Curator of Brooklyn Museum of Arts and Sciences, and edited by Robert Ridgway, Curator of Birds, U.S. National Museum. 4to. 30 net.

\section{North American Trees:}

Being Descriptions and Illustrations of the Trees growing independently of cultivation in North America, North of Mexico, and the West Indies.

By Nathaniel Lord Britton, Ph.D., Sc.D., Director-in-Chief of the New York Botanical Garden. With the assistance of John Adolph Shafer, Pharm.D., Custodian of the Museums of the New York Botanical Garden. Illustrated. 8vo. 3os. net.

\section{A Manual of the Trees of North America.}

By Charles S. Sargent. With 644 Illustrations by C. E. Faxon. Demy 8vo., 25s. net.

\section{Indian Trees.}

An Account of Trees, Shrubs, Woody Climbers, Bamboos and Palms, Indigenous OR Commonly Cultivated in the British Indian Empire.

By Sir Dietrich Brandis, K.C.I.E., Ph.D. (Bonn), LL.D. (Edin.), F.R.S., F.L.S., F.R.G.S., and Hon. Member of the Royal Scottish Arboricultural Society, of the Society of American Foresters, and of the Pharmaceutical Society of Great Britain. Assisted by Indian Foresters. With many Illustrations. Demy 8vo. 16s. net. Third Impression.

\section{Plant Physiology and Ecology.}

By Frederic Edward Clements, Ph.D., Professor of Botany in the University of Minnesota. IVith 125 Illustrations. Demy 8vo. 8s. 6d. net. 

UNIVERSITY OF CALIFORNIA LIBRARY Los Angeles

This book is DUE on the last date stamped below. 


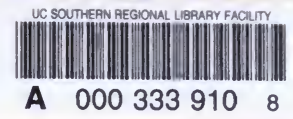


